LA-14405-PR

Progress Report

Approved for public release;

distribution is unlimited.

\title{
Surface Water Data at Los Alamos National Laboratory
}

2008 Water Year 
The three most recent reports in this unclassified series are LA-14376, LA-14328-PR, and LA-14239-PR.

Los Alamos National Laboratory, an affirmative action/ equal opportunity employer, is operated by Los Alamos National Security, LLC, for the National Nuclear Security Administration of the U.S. Department of Energy under contract DE-AC52-06NA25396.

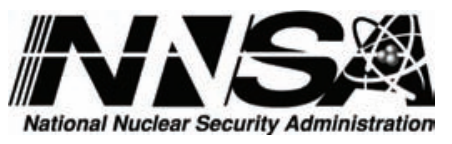

This report was prepared as an account of work sponsored by an agency of the U.S. Government. Neither Los Alamos National Security, LLC, the U.S. Government nor any agency thereof, nor any of their employees make any warranty, express or implied, or assume any legal liability or responsibility for the accuracy, completeness, or usefulness of any information, apparatus, product, or process disclosed, or represent that its use would not infringe privately owned rights. Reference herein to any specific commercial product, process, or service by trade name, trademark, manufacturer, or otherwise does not necessarily constitute or imply its endorsement, recommendation, or favoring by Los Alamos National Security, LLC, the U.S. Government, or any agency thereof. The views and opinions of authors expressed herein do not necessarily state or reflect those of Los Alamos National Security, LLC, the U.S. Government, or any agency thereof. Los Alamos National Laboratory strongly supports academic freedom and a researcher's right to publish; as an institution, however, the Laboratory does not endorse the viewpoint of a publication or guarantee its technical correctness. 
LA-14405-PR

Progress Report

Issued: September 2009

\section{Surface Water Data at Los Alamos National Laboratory \\ 2008 Water Year}

David Ortiz

Betsy Cata

Gregory Kuyumjian 
This page intentionally left blank. 


\section{Contents}

Abbreviations, Acronyms, and Glossary …............................................................. viii

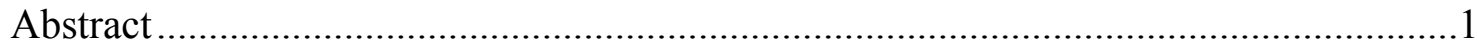

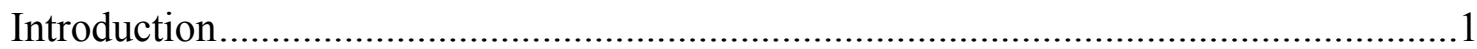

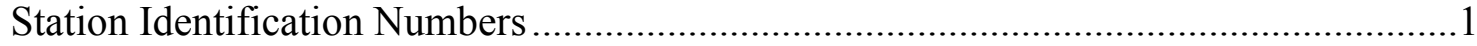

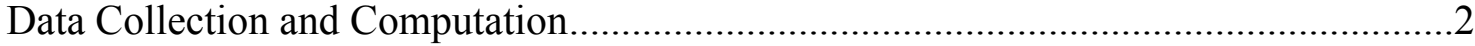

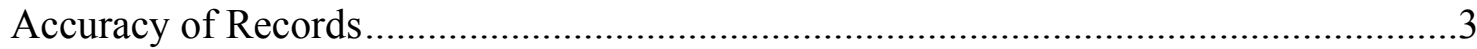

Data Presentation ................................................................................................ 3

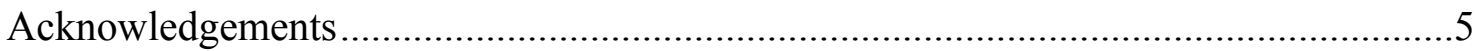

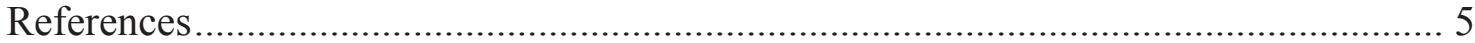

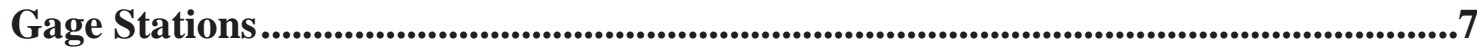

Gage Stations at Los Alamos National Laboratory ................................................. 8

Summary of Discharges from Stream Monitoring

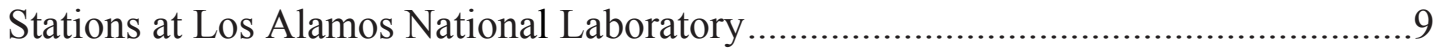

E025 Los Alamos Canyon at Los Alamos ..............................................................11

E026 Los Alamos Canyon below Ice Rink............................................................14

E02685 Los Alamos Canyon at TA-2 ................................................................... 17

E030 Los Alamos Canyon above DP Canyon .........................................................20

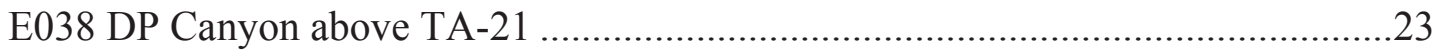

E039 DP Canyon below Meadow at TA-21 ..........................................................27

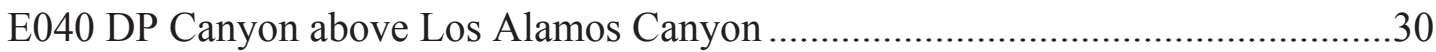

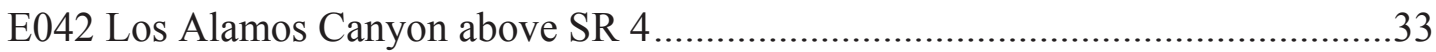

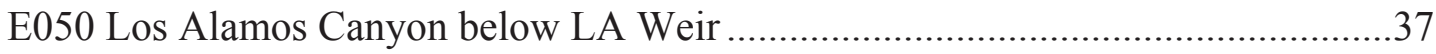

E055 Pueblo Canyon above Acid Canyon ...............................................................40

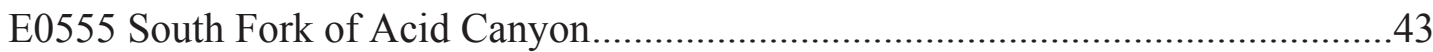

E056 Acid Canyon above Pueblo Canyon ................................................................47

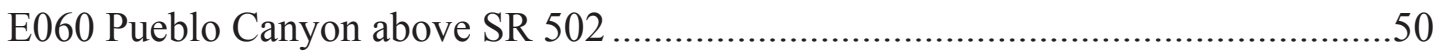

E121 Sandia Canyon Right Fork at Power Plant ..................................................5

E1219 Sandia Canyon East of Power Plant .......................................................56

E122 Sandia Canyon near Roads and Grounds at TA-3 …...................................60

E1222 Sandia Canyon Tributary from Roads and Grounds ...................................63

E1223 Sandia Canyon Tributary from Sigma Building.........................................66

E12235 Sandia Canyon Tributary from MRF ....................................................69

E1225 Sandia Canyon Tributary at Heavy Equipment...........................................72

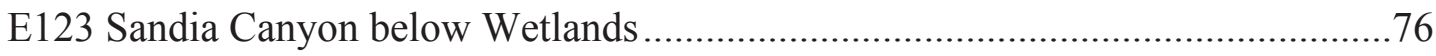


E1234 Sandia Canyon Road and Grounds at Sigma...............................................79

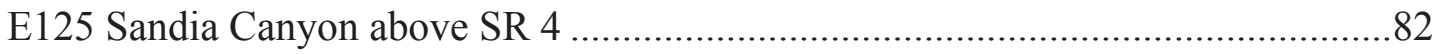

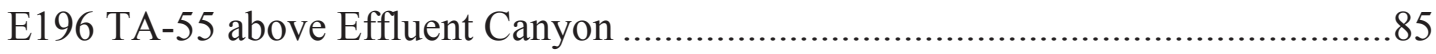

E200 Mortandad Canyon below Effluent Canyon.......................................................8

E2005 Mortandad Canyon Tributary Batch Plant at Sigma …………………….......92

E201 Mortandad Canyon above Ten-Site Canyon...............................96

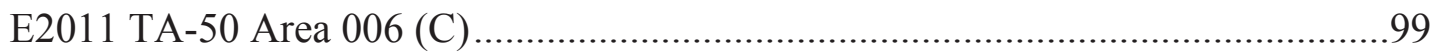

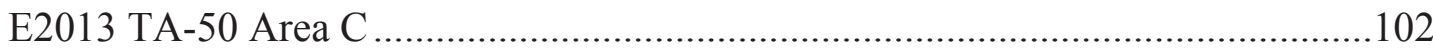

E2015 Ten-Site Canyon above Mortandad Canyon .................................................105

E202 Mortandad Canyon above Sediment Traps ....................................................108

E203 Mortandad Canyon below Sediment Traps ......................................................111

E204 Mortandad Canyon at LANL Boundary .........................................................115

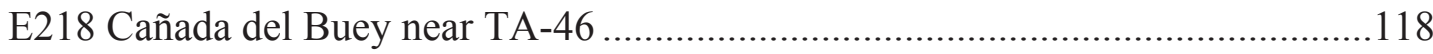

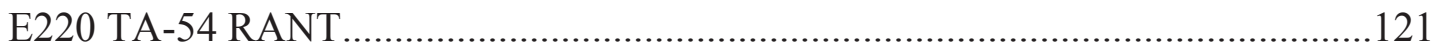

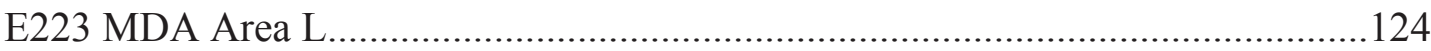

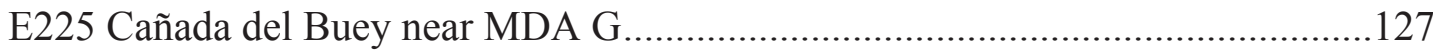

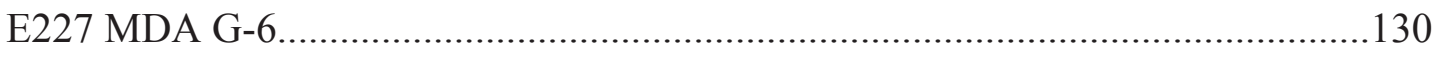

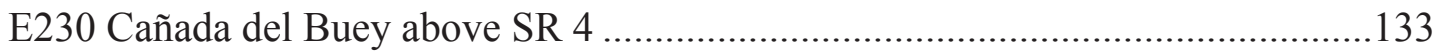

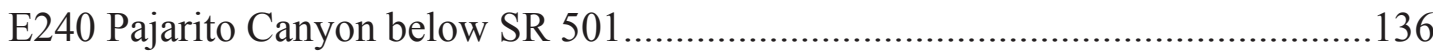

E241 Pajarito Canyon above Starmer's Gulch .......................................................140

E242 Starmer's Gulch above Pajarito Canyon .........................................................143

E2425 Arroyo de la Delfe above Pajarito Canyon....................................................146

E243 Pajarito Canyon above Two-Mile Canyon ......................................................149

E2435 Two-Mile Canyon Tributary at TA-3 .....................................................152

E244 Two-Mile Canyon above Pajarito Canyon ....................................................155

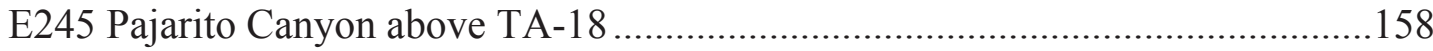

E2455 Pajarito Canyon above Three-Mile Canyon...................................................161

E246 Three-Mile Canyon above Pajarito Canyon .....................................................165

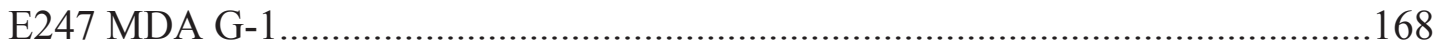

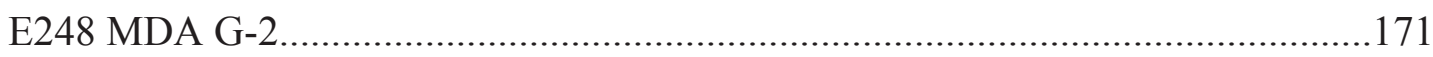

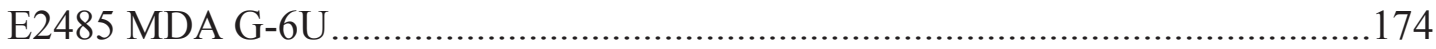

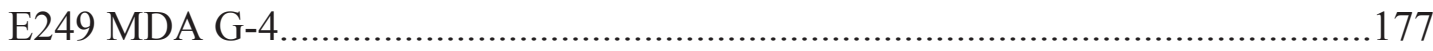

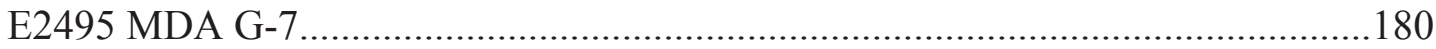

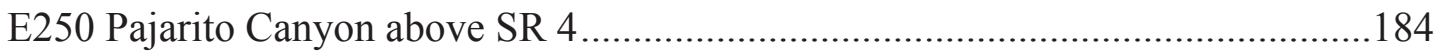




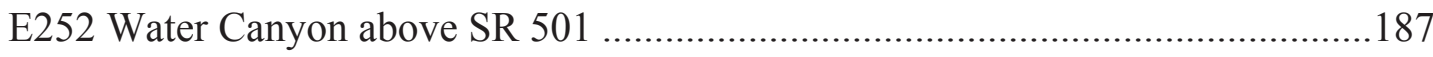

E2528 S Site Canyon above Water Canyon ........................................................190

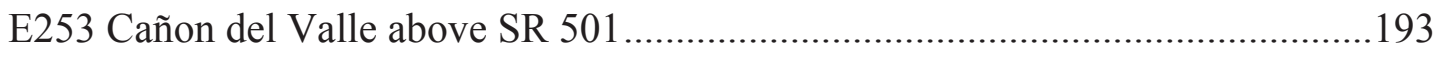

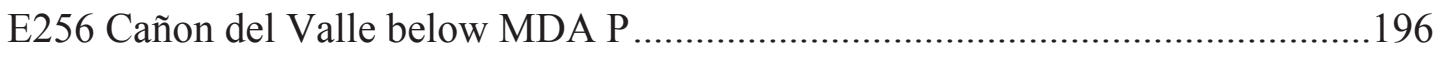

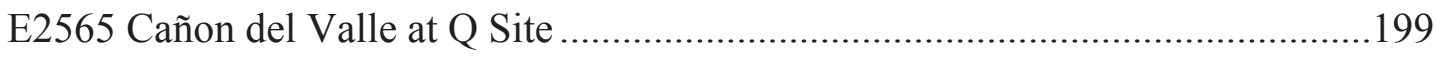

E257 Cañon del Valle Tributary at TA-16 Burn Grounds.........................................202

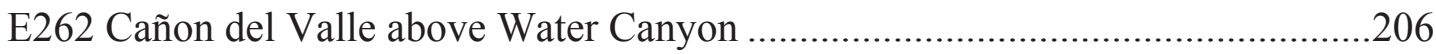

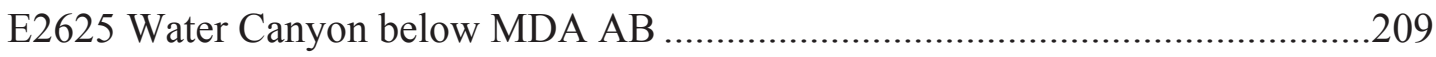

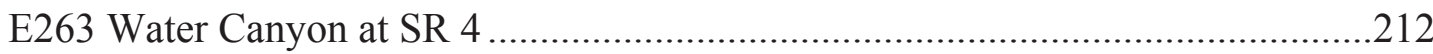

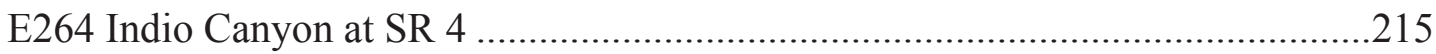

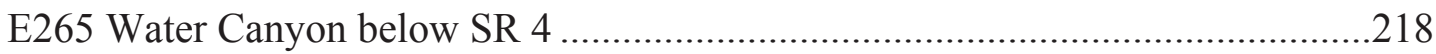

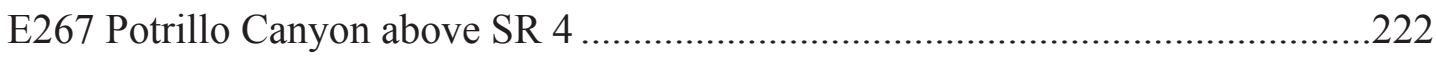

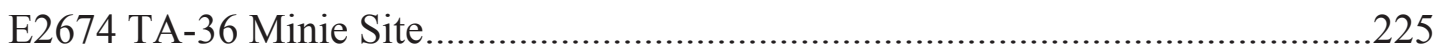

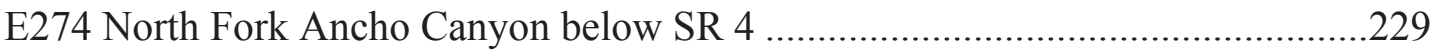

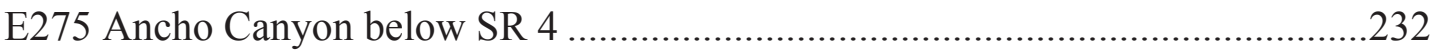

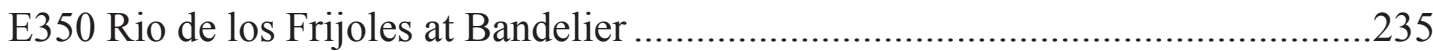

\section{Spring Stations}

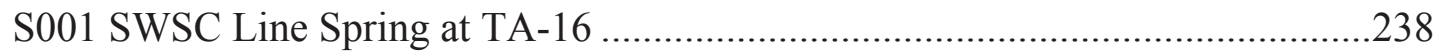

S002 Burn Ground Spring at TA-16 .................................................................240

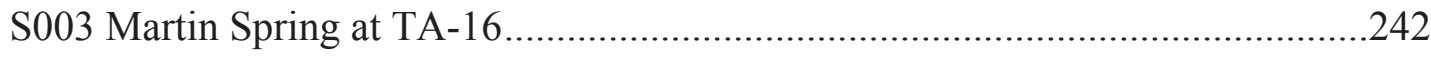

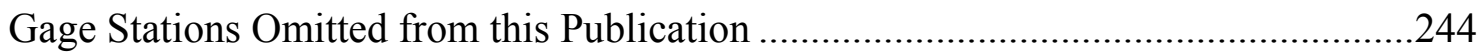

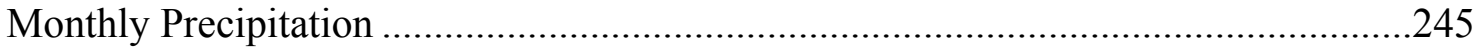




\section{Abbreviations, Acronyms, and Glossary}

Acre-foot (Ac-Ft, acre-ft) is the quantity of water required to cover 1 acre to a depth of 1 foot and is equivalent to 43,560 cubic feet, 325,851 gallons, or 1,233.49 cubic meters.

CFS-day is the volume of water represented by the flow of 1 cubic foot per second for 24 hours. It is equivalent to 86,400 cubic feet, 1.98347 acre-feet, 646,317 gallons, or 2,445 cubic meters.

Control designates a feature downstream from the gage that determines the stagedischarge relation at the gage. This feature may be a natural constriction of the channel, an artificial structure, or a uniform cross section over a long reach of the channel.

Control structure as used in this report, is a structure on a stream or canal used to regulate the flow or stage of the stream or to prevent the intrusion of saltwater.

Cubic feet per second per square mile $\left[\left(\mathrm{ft}^{3} / \mathrm{s}\right) / \mathrm{mi}^{2}\right]$ is the average number of cubic feet of water flowing per second from each square mile of area drained, assuming that the runoff is distributed uniformly in time and area.

Cubic foot per second $\left(\mathrm{ft}^{3} / \mathrm{s}\right)$ is the rate of discharge representing a volume of 1 cubic foot passing a given point during 1 second; it is equivalent to 7.48 gallons per second, 448.8 gallons per minute, or 0.02832 cubic meters per second.

Discharge is the volume of water (or more broadly, the volume of fluid, including suspended sediment) that passes a given point within a given period of time.

Drainage area (DA) of a stream at a specified location is that area measured in a horizontal plane and enclosed by a topographic divide, from which direct surface runoff from precipitation normally drains by gravity into the stream above the specified point. Figures of DA given herein include all closed basins, or noncontributing areas, within the area, unless otherwise noted.

Drainage basin is a part of the surface of the earth that is occupied by a drainage system, which consists of a surface stream or a body of impounded surface water together with all tributary surface streams and bodies of impounded surface water.

Gage height $(\mathrm{GH})$ is the water-surface elevation referred to in some arbitrary gage data. GH is often used interchangeably with the more general term "stage," although GH is more appropriate when used with a reading on a gage.

Gage station is a particular site on a stream, canal, lake, or reservoir in which systematic observations of hydrologic data are obtained. 


\section{Abbreviations, Acronyms, and Glossary (continued)}

GPS is an abbreviation for global positioning system.

HWM is an abbreviation for high-water mark.

Instantaneous discharge is the discharge at a particular instant of time.

LANL is the acronym for Los Alamos National Laboratory.

Mean discharge (MEAN) is the arithmetic mean of individual daily mean discharges during a specific period.

National Geodetic Vertical Datum of 1929 (NGVD) is a geodetic datum derived from a general adjustment of the first-order level nets of both the United States and Canada. It was formerly called Sea Level Datum of 1929, or "mean sea level," in this series of reports. Although the datum was derived from the average sea level over a period of many years at 26 tide stations along the Atlantic, Gulf of Mexico, and Pacific coasts, it does not necessarily represent the local mean sea level at any particular place.

NPDES is the abbreviation for National Pollution Discharge Elimination System.

Point of Zero Flow (PZF) is the gage height at which no flow occurs.

SR means “State Road.”

Stage see Gage Height.

Stage-discharge relation is the relation between the water-surface elevation, termed "gage height," and the volume of water flowing in a channel per unit of time.

Stream flow is the discharge that occurs in a natural channel.

SWSC is an abbreviation for sanitary wastewater systems consolidation.

USGS is the abbreviation for U.S. Geological Survey.

Water year in reports dealing with surface water supply is the 12-month period, October 1 through September 30. The water year is designated by the calendar year in which it ends and which includes 9 of the 12 months. Thus, the year ending September 30, 1980, is called the "1980 water year."

WDR is an abbreviation for "Water-Data Report" in the "Revised Records" paragraph to refer to annual hydrologic-data reports.

WSP is an abbreviation for "Water-Supply Paper" in references to previously published reports. 
This page left blank intentionally. 


\author{
Surface Water Data at Los Alamos National Laboratory: 2008 Water Year \\ by \\ D. Ortiz, B. Cata, and G. Kuyumjian
}

\begin{abstract}
The principal investigators collected and computed surface water discharge data from 69 stream-gage stations that cover most of Los Alamos National Laboratory and one at Bandelier National Monument. Also included are discharge data from three springstwo that flow into Cañon de Valle and one that flows into Water Canyon.
\end{abstract}

\title{
Introduction
}

This annual water data report from Los Alamos National Laboratory (LANL) contains flow data from 69 stream-gage stations that cover most of the Laboratory's property. Data collected on the Laboratory's downstream boundary approximates New Mexico State Road (SR) 4; the upstream boundary is approximated by New Mexico SR 501. Some gage stations are within Laboratory boundaries and were originally installed to assist groups other than the Water Stewardship Programs (EP-WSP), which also conducts site-specific earth science research.

Precipitation records were added to publication. Rainfall/precipitation data collection began at selected stations starting March 2007-July 2007 and continued collection of data to the end of the water year. Tipping-bucket rain gages measured precipitation, with data recorded every 5 minutes. Calendar totals (midnight to midnight) are tabulated and presented with the associated station record. Data gaps were estimated using surrounding stations.

Water chemistry data from selected storm events occurring at some stations will be published in the 2007 “Los Alamos National Laboratory Environmental Surveillance Report.” Such data are also available on the Internet.

\section{Station Identification Numbers}

The U.S. Geological Survey (USGS), Water Resources Division, assigns a unique identification number to each stream-gage station it establishes. All sites numbered since 1950 are part of the downstream-order system. The downstream-order system increases station numbers in the downstream direction along main streams, and in the case of this report, their respective mouths to the Rio Grande.

This report adheres to the USGS convention of downstream order. Because of the proximity of stations in this network, the first 5 digits of all station numbers are 08313. We have replaced this number string with the letter $\mathrm{E}$ in the station number partly to abbreviate and also to accommodate instrumentation. 


\section{Data Collection and Computation}

A complete record at a gage station gathers records of stage and discharge measurements from streams or canals. In addition to gathering these stage and discharge measurements, we directly observe factors affecting the stage/discharge relation, consult weather records, and use other information that supplements base data in determining daily flow. Direct readings on a nonrecording gage or from the data logger provide integrated (5-minute) records of stage. We measure discharge with current meters, using methods the USGS adapted as a result of experience accumulated since 1880. Standard textbooks describe these methods, as do WaterSupply Paper 2175 and the U.S. Geological Survey Technique of Water Resources Investigations, Book 3, Chapter A6.

We use stage/discharge relation curves to prepare rating tables that give the discharge for any stage measured at a stream-gage station. When it is necessary to define discharge extremes outside the range of current meter measurements, we extend the curves using logarithmic plotting; velocity area studies; results of indirect measurements of peak discharge, such as slope area or contracted opening measurements and computations of flow over dams or weirs; or step backwater techniques.

Daily mean discharges are computed by applying daily mean gage height (stage) to the stage discharge curves or tables. If the stage/discharge relation is subject to change because of frequent or continual change in the physical features that form the control, the daily mean discharge is computed by the shifting-control method. In the shifting-control method, correction factors based on individual discharge measurements and notes by personnel taking the measurements are applied to the gage heights before discharges are determined from the curves or tables.

The shifting-control method is also used if the stage/discharge relation for a station is temporarily changed by the presence of aquatic growth or debris on the control. At some northern stream-gage stations, the stage/discharge relation is affected by ice in the winter, and it becomes impossible to compute discharge in the usual manner. Discharge for the period of ice effect is computed on the basis of gage height record and occasional winter discharge measurements. Consideration is given to the available information about temperature and precipitation, notes of observations, and comparable discharge records for other stations in the same or nearby basins for comparable periods of time.

For some gage stations, periods occur when no gage height record is obtained, or the recorded gage height is so faulty that it cannot be used to compute daily discharge or contents. This happens when the recorder stops or otherwise fails to operate properly, intakes are plugged, the float is frozen in the well, etc. For such periods, the daily discharges are estimated on the basis of recorded range-in-stage, prior and subsequent records, discharge measurements, weather records, and record comparisons made against other stations in the same or nearby basins. Likewise, daily contents may be estimated from operator logs, prior and subsequent records, inflow-outflow studies, and other information. 


\section{Accuracy of Records}

The following two factors determine the accuracy of stream flow records:

- stability of the stage-discharge relation or, if the control is unstable, the frequency of discharge measurements and

- accuracy of measurements or stage, accuracy of discharge measurements, and interpretations of records.

Accuracy attributed to records is noted under "Remarks."

- Excellent-95\% of daily discharges are within 5\% of the true value.

- Good-95\% of daily discharges are within $10 \%$ of the true value.

- Fair-95\% of daily discharges are within $15 \%$ of the true value.

- Poor-records do not meet the criteria mentioned.

Accuracy determination is only based on days with flow.

The number of significant figures used to report daily mean discharges is based solely on the magnitude of the discharge value:

\begin{tabular}{|l|l|}
\hline If - the value $\left(\mathrm{ft}^{3} / \mathrm{s}\right)$ is & Then-it is reported as \\
\hline less than $1 \mathrm{ft}^{3} / \mathrm{s}$ & nearest hundredth \\
\hline $1-10 \mathrm{ft}^{3} / \mathrm{s}$ & nearest tenth \\
\hline $10-1,000 \mathrm{ft}^{3} / \mathrm{s}$ & whole number \\
\hline above $1,000 \mathrm{ft}^{3} / \mathrm{s}$ & three significant figures \\
\hline
\end{tabular}

\section{Data Presentation}

The records published in this report are for each gage station and consist of three parts:

- $\quad$ station analysis summary,

- station manuscript description with photo, and

- data table for the water year (October 1, 2006, to September 30, 2007).

The station analysis supplements each daily values table and includes a description of monitoring equipment, problems associated with data collection during the water year, and other information used to compute stream flow discharge.

The station manuscript provides data under various headings: station location, period of record, average discharge, historical extremes, record accuracy, and other points pertinent to station operation and regulation. Each continuous record of discharge includes the following categories of descriptions:

Location. The most accurate and available maps, coupled with global positioning system (GPS) technology, provide location information. The location of the gage with respect to the vicinity's 
cultural and physical features is given, as well as a name that refers to place. For a few stations, the U.S. Army Corps of Engineers or the Water Resources Council (River Mileage Measurement, Bulletin 14, rev. October 1968) provided river mileage. We define left and right banks from the perspective of facing downstream.

Drainage Area. The most accurate and available maps provide drainage area measurements. The accuracy of drainage area measurements varies, depending on the type of map available for this purpose.

Revised Records. Because of new information, published records occasionally are found to be incorrect and revisions are printed in later reports. If a revision did not include daily, monthly, or annual figures of discharge, that fact is noted after the year as follows: (M) means that only instantaneous maximum discharge was revised; (m) means that only the instantaneous minimum was revised; and (P) means that only the peak discharge was revised. If the drainage area has been revised, the report in which the most recently revised figure was first published is given.

Period of Record. The period of record is the time during which published records exist for a station or its equivalent station. An equivalent station is one that was in operation when the present station was not in operation and was located so that records from it can be reasonably considered equivalent to records from the present station.

Gage. This section describes the type of gage in current use. The datum of the current gage referred to in the National Geodetic Vertical Datum (NGVD) of 1929 (see Abbreviations, Acronyms, and Glossary); given under this heading are a condensed history of the types, locations, and data of previous gages.

Remarks. The text presents information relative to the accuracy of the records, special methods of computation, conditions that affect natural flow at the station, and other pertinent information.

Average Discharge. The average discharge is the average of the annual mean discharge published after 5 years of record. Once published, it continues as a moving average.

Extremes for Period of Record. Extremes may include maximum and minimum stages and maximum and minimum discharges or content. Unless otherwise qualified, the maximum discharge or content is the instantaneous maximum corresponding to the highest stage that occurred. The highest stage may have been obtained from a graphic or digital recorder, a crest stage gage, or by direct observation of a nonrecording gage.

If the maximum stage did not occur on the same day as the maximum discharge or content, it is given separately. Similarly, the minimum is the instantaneous minimum discharge, unless otherwise qualified, and was determined and is reported in the same manner as the maximum.

Extremes outside Period of Record. This section contains information concerning major floods or unusually low flows that occurred outside the stated period of record. The information may have been obtained from other agencies, old data files, newspapers, or local citizens. 
Extremes for Current Year. Extremes given here are similar to those for the period of record. The time for occurrence of peaks is expressed in 24-h local standard time. For example, 12:30 A.M. is 0030 and 1:30 P.M. is 1330. The minimum for the current water year appears in this section.

Data Table of Daily Mean Values. The daily table of discharge records for stream-gage stations gives the mean discharge for each day of the water year. In the monthly summary for the table, the line headed "Total" gives the sum of the daily figures for each month; the line headed "Mean" gives the average flow in cubic feet per second for the month; and the lines headed "Max" and "Min" give the maximum and minimum daily mean discharges for each month and in acre feet, respectively, in the line headed "Acre-Ft."

\section{Acknowledgments}

The authors thank the following individuals for their contribution to this report: R. Duran, G. Helland, J. Leo Martinez, B. McKown, W. Porch, R. Romero, and E. Pulliam (PMC Inc.).

\section{References}

Water-Supply Paper 2175 and the U.S. Geological Survey Technique of Water Resources Investigations, Book 3, Chapter A6.

U.S. Army Corps of Engineers, River Mileage Measurement, Bulletin 14, rev. October 1968.

National Geodetic Vertical Datum of 1929.

http://www.esg.montana.edu/gl/glcus.html Used to obtain legal locations.

Previous Los Alamos National Laboratory reports in this series—“'Surface Water Data at Los

Alamos National Laboratory” for Water Years 1995-2004

1995: LA-13177-PR (August 1996)

1996: LA-13234-PR (November 1996)

1997: LA-13403-PR (January 1996)

1998: LA-13551-PR (February 1999)

1999: LA-13706-PR (April 2000)

2000: LA-13814-PR (July 2001)

2001: LA-13905-PR (April 2002) 
2002: LA-14019-PR (March 2003)

2003: LA-14131-PR (March 2004)

2004: LA-14211-PR (April 2005)

2005: LA-14239-PR (May 2006)

2006: LA-14328-PR (August 2007)

2007: LA-14376 (October 2008) 
Gage Stations 


\section{Gage Stations at Los Alamos National Laboratory}

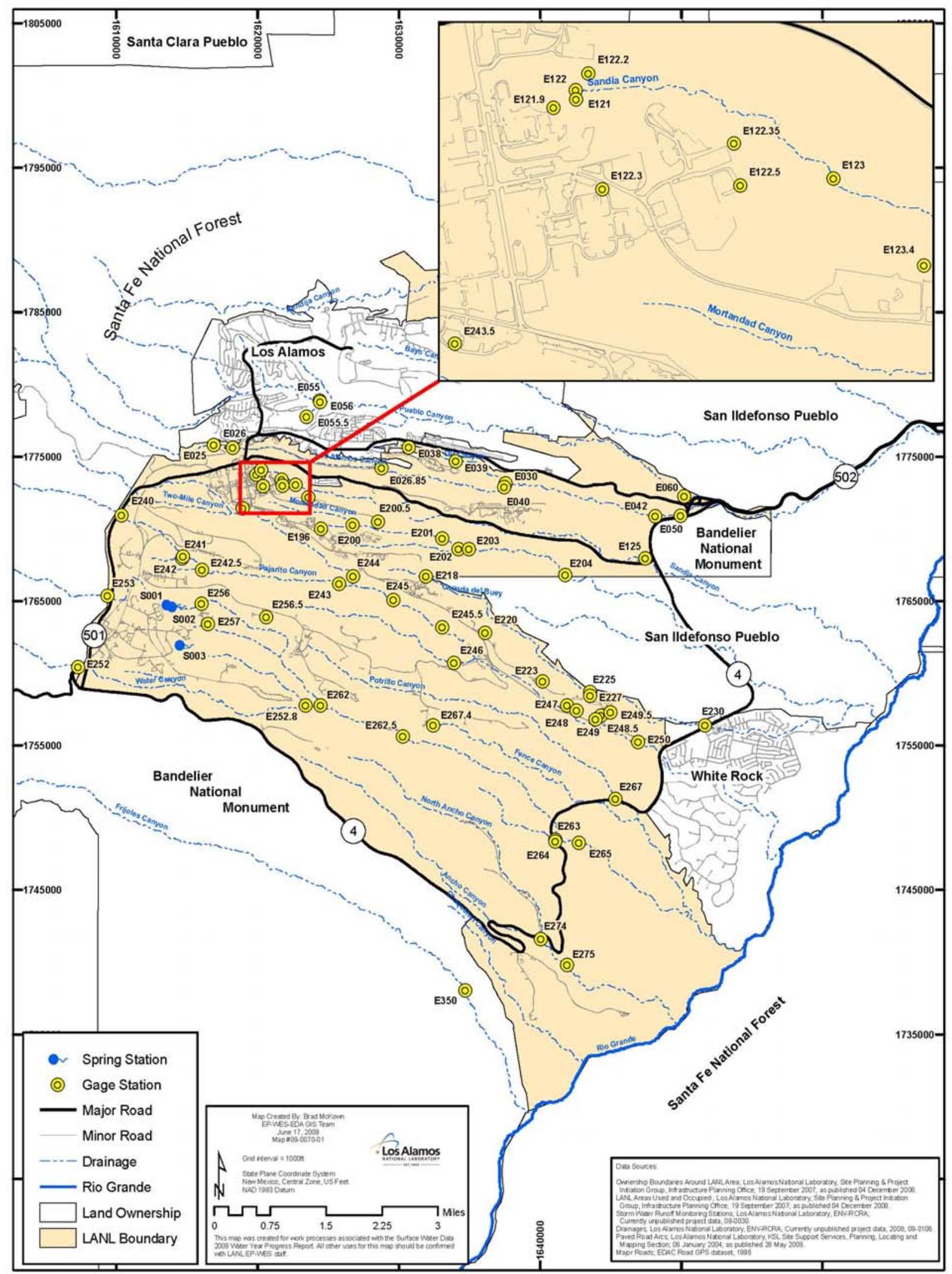




\section{Summary of Discharges from Stream Monitoring Stations at Los Alamos National Laboratory}

Water Year 2008

October 1, 2007 to September 30, 2008

\begin{tabular}{|c|c|c|c|}
\hline Canyon Sites & Days with Flow & Volume in Ac-Ft & Instantaneous Max in $\mathrm{ft}^{3} / \mathrm{s}$ \\
\hline E025 Los Alamos Canyon above Ice Rink & 169 & 301 & 1.9 \\
\hline E026 Los Alamos Canyon below Ice Rink & 150 & 303 & 4.1 \\
\hline E02685 Los Alamos Canyon at TA-2 & 149 & 361 & 28 \\
\hline E030 Los Alamos Canyon above DP Canyon & 140 & 198 & 13 \\
\hline E038 DP Canyon above TA-21 & 35 & 69 & 186 \\
\hline E039 DP Canyon below Meadow at TA-21 & 232 & 185 & 118 \\
\hline E040 DP Canyon above Los Alamos Canyon & 12 & 9.5 & 44 \\
\hline E042 Los Alamos Canyon above SR 4 & 73 & 126 & 61 \\
\hline E050 Los Alamos Canyon below LA Weir & 31 & 35 & 36 \\
\hline E055 Pueblo Canyon above Acid Canyon & 143 & 19 & 68 \\
\hline E0555 South Fork Acid Canyon & 19 & 5.7 & 37 \\
\hline E056 Acid Canyon at Mouth & 32 & 29 & 81 \\
\hline E060 Pueblo Canyon above SR 502 & 167 & 191 & 37 \\
\hline E121 Sandia Canyon Right Fork at Power Plant & 366 & 63 & 16 \\
\hline E1219 Sandia Canyon East of Power Plant & 28 & 2.6 & 5.2 \\
\hline E122 Sandia Canyon near Roads and Grounds at TA-3 & 366 & 63 & 16 \\
\hline E1222 Sandia Canyon Tributary from Roads and Grounds & 13 & 1.4 & 2.2 \\
\hline E1223 Sandia Canyon Tributary from Sigma Building & 3 & 0.14 & 1.1 \\
\hline E12235 Sandia Canyon Tributary from MRF & 8 & 0.20 & 1.8 \\
\hline E1225 Sandia Canyon Tributary at Heavy Equipment & 26 & 2.9 & 8.9 \\
\hline E123 Sandia Canyon below Wetlands & 366 & 439 & 65 \\
\hline E1234 Sandia Canyon Roads and Grounds at Sigma & 25 & 3.0 & 5.7 \\
\hline E125 Sandia Canyon above SR 4 & 1 & 15 & 28 \\
\hline E196 TA-55 above Effluent Canyon & 28 & 2.9 & 0.81 \\
\hline E200 Mortandad Canyon below Effluent Canyon & 107 & 28 & 66 \\
\hline E2005 Mortandad Canyon Tributary Batch Plant at Sigma & 19 & 3.3 & 3.2 \\
\hline E201 Mortandad Canyon above Ten Site Canyon & 4 & 7.4 & 78 \\
\hline E2011 TA-50 Area 006 & 23 & 1.7 & 6.0 \\
\hline E2013 TA-50 Area C & 20 & 2.1 & 7.4 \\
\hline E2015 Ten Site Canyon above Mortandad Canyon & 6 & 17 & 299 \\
\hline E202 Mortandad Canyon above Sediment Traps & 3 & 3.3 & 15 \\
\hline E203 Mortandad Canyon below Sediment Traps & 0 & 0 & 0 \\
\hline E204 Mortandad Canyon at LANL Boundary & 0 & 0 & 0 \\
\hline E218 Cañada del Buey near TA-46 & 13 & 1.9 & 7.4 \\
\hline E220 TA-54 RANT & 6 & 0.16 & 1.0 \\
\hline E223 MDA Area L & 2 & 0.10 & 0.67 \\
\hline E225 Cañada del Buey near MDA G & 1 & 0.04 & 5.0 \\
\hline E227 MDA G-6 & 2 & 0.06 & 0.26 \\
\hline
\end{tabular}




\section{Summary of Discharges from Stream Monitoring Stations at Los Alamos National Laboratory (continued)}

Water Year 2008

October 1, 2007, to September 30, 2008

\begin{tabular}{|c|c|c|c|}
\hline Canyon Sites & Days with Flow & Volume in Ac-Ft & Instantaneous Max in $\mathrm{ft}^{3} / \mathrm{s}$ \\
\hline E230 Cañada del Buey above SR 4 & 5 & 7.9 & 28 \\
\hline E240 Pajarito Canyon below SR 501 & 18 & 1.4 & 1.4 \\
\hline E241 Pajarito Canyon above Starmer’s Gulch & 96 & 14 & 21 \\
\hline E242 Starmer’s Gulch above Pajarito Canyon & 331 & 386 & 100 \\
\hline E2425 Arroyo de la Delfe above Pajarito & 26 & 3.5 & 6.0 \\
\hline E243 Pajarito Canyon above Two Mile Canyon & 289 & 272 & 103 \\
\hline E2435 Two Mile Canyon Tributary at TA-3 & 25 & 3.5 & 9.7 \\
\hline E244 Two Mile Canyon above Pajarito Canyon & 133 & 90 & 223 \\
\hline E245 Pajarito Canyon above TA-18 & 160 & 275 & 119 \\
\hline E2455 Pajarito Canyon above Three Mile Canyon & 165 & 217 & 252 \\
\hline E246 Three Mile Canyon above Pajarito Canyon & 148 & 1.8 & 5.0 \\
\hline E247 MDA G-1 & 2 & 0.12 & 0.93 \\
\hline E248 MDA G-2 & 2 & 0.18 & 4.9 \\
\hline E2485 MDA G-6 & 5 & 0.81 & 3.3 \\
\hline E249 MDA G-4 & 3 & 0.14 & 1.2 \\
\hline E2495 MDA G-7 & 27 & 2.1 & 3.8 \\
\hline E250 Pajarito Canyon above SR 4 & 113 & 67 & 25 \\
\hline E252 Water Canyon above SR 501 & 366 & 167 & 1.8 \\
\hline E2528 S-Site Canyon above Water Canyon & 13 & 19 & 31 \\
\hline E253 Cañon del Valle above SR 501 & 0 & 0 & 0 \\
\hline E256 Cañon del Valle below MDA P & 316 & 35 & 13 \\
\hline E2565 Cañon del Valle at Q Site & 42 & 2.0 & 1.0 \\
\hline E257 Cañon del Valle at Burn Grounds & 15 & 2.5 & 8.5 \\
\hline E262 Cañon del Valle above Water Canyon & 7 & 5.7 & 11 \\
\hline E2625 Water Canyon below MDA AB & 211 & 199 & 75 \\
\hline E263 Water Canyon at SR 4 & 1 & 26 & 75 \\
\hline E264 Indio Canyon at SR 4 & 0 & 0 & 0 \\
\hline E265 Water Canyon below SR 4 & 2 & 17 & 69 \\
\hline E267 Portillo Canyon above SR 4 & 2 & 0.14 & 2.0 \\
\hline E2674 TA-36 Minie Site & 3 & 0.14 & 0.99 \\
\hline E274 North Fork Ancho Canyon below SR 4 & 2 & 2.7 & 89 \\
\hline E275 Ancho Canyon below SR 4 & 6 & 33 & 536 \\
\hline E350 Rito de Los Frijoles at Bandelier & 366 & 1980 & 513 \\
\hline
\end{tabular}




\section{E025 Los Alamos Canyon at Los Alamos}

Location. Lat $35^{\circ}$ 52' 50", long $106^{\circ} 19^{\prime}$ 45", NW 1/4, Sec. 17, T. 19 N., R. 6 E., Los Alamos County.

Drainage Area. $6.92 \mathrm{mi}^{2}$.

Period of Record. October 1, 1993, to September 30, 2001; October 2001 to September 2006 (fragmentary). October 1, 2006, to September 30, 2008.

Revised Record. Drainage area (2007).

Gage. Data logger and 24” Parshall flume. Elevation of gage is 7,237 ft above NGVD.

Remarks. Records are good except estimated daily discharges, which are fair. Flow is partially regulated by Los Alamos Reservoir, which is about 1.52 mi upstream.

Extremes for Period of Record. Maximum discharge, $185 \mathrm{ft}^{3} / \mathrm{s}$, August 9, 2001, gage height not determined. No flow at times.

Extremes for Current Water Year. Maximum discharge $2.90 \mathrm{ft}^{3} / \mathrm{s}$ at $2245 \mathrm{~h}$, March 28, gage height $0.52 \mathrm{ft}$. No peak above base of $5.0 \mathrm{ft}^{3} / \mathrm{s}$. No flow at times.

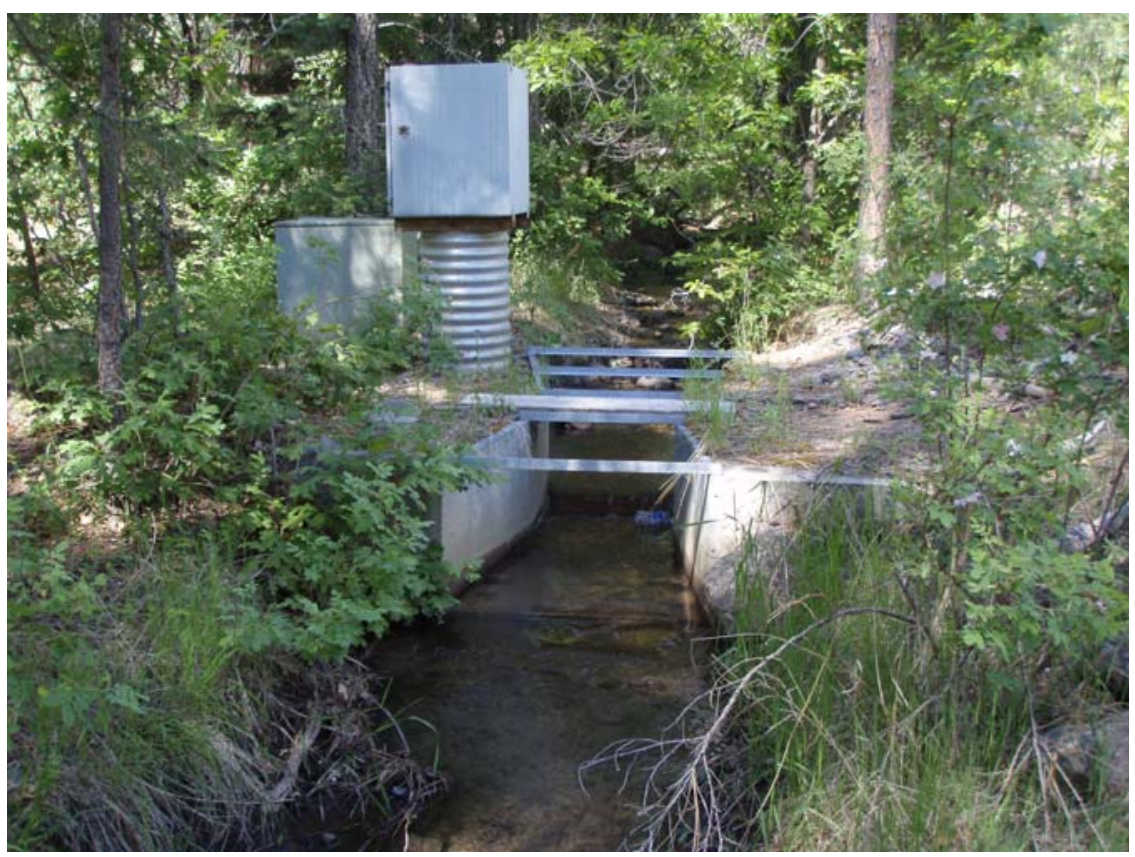




\section{E025 Los Alamos Canyon at Los Alamos}

\section{Station Analysis}

\section{Water Year}

Equipment. Station is equipped with Sutron 8200 (5-min. interval) with a shaft encoder float system. The system is powered by a solar-panel battery system housed in a National Electric Manufacturing Association (NEMA) shelter on top of a 18" Corrugated Metal Pipe (CMP) well attached to a 24" Parshall flume on right bank. No provision for measurements above wading stage.

Field Work. The station was visited 17 times to conduct discharge measurements and service the instrumentation. Field inspections for the gage are listed under site history files on the Hydstra database. Discharge measurements for the gage are listed under site gauging files on the Hydstra database.

Datum Correction. None from levels. Gage height corrections were applied for minor base gage data logger differences.

Gage-Height Record. The data logger referenced to the outside staff gave a complete and satisfactory record, except during the period of December 16 to February 21 when ice affected gage height.

Rating. The low flow control is a 24" Parshall flume. Low flow is subject to minor shifting caused by an accumulation of leaves. The medium to high flow control becomes the channel. Channel is straight for $100^{\prime}$ above and below gage and is subject to overflow at very high stages. The streambed consists of gravel and cobbles, with grass covering over banks.

Rating No. 1 was used for the entire water year.

Discharge. Discharge was computed using rating No. 1. Discharges if frozen were estimated with reference to E026.

Flow is somewhat controlled by Los Alamos Reservoir about 1 River mile upstream of gage.

Remarks. Records are good, except for estimated daily discharges and high flow during the year, which are fair.

Peak flow since the Cerro Grande fire has been too high to record at gage. Station E026 has been established 0.1 mile downstream for high flow. E025 will be functionally operated as low to medium flow partial record station. 
E025 Los Alamos Canyon at Los Alamos

Daily Mean Discharge in Cubic Feet per Second

Water Year October 2007 to September 2008

\begin{tabular}{|c|c|c|c|c|c|c|c|c|c|c|c|c|}
\hline DAY & OCT & NOV & DEC & JAN & FEB & MAR & APR & MAY & JUN & JUL & AUG & SEP \\
\hline 1 & .14 & 0 & 0 & $0^{*}$ & $0^{*}$ & 1.8 & 2.7 & 1.1 & .28 & 0 & 0 & .06 \\
\hline 2 & .15 & 0 & 1.3 & $0^{*}$ & $0^{*}$ & 2.1 & 2.6 & 1.0 & .27 & 0 & 0 & .04 \\
\hline 3 & .15 & 0 & 1.6 & $0^{*}$ & $0^{*}$ & 2.1 & 2.6 & 1.0 & .25 & 0 & 0 & $0^{*}$ \\
\hline 4 & .15 & 0 & 1.6 & $0^{*}$ & $0^{*}$ & 1.9 & 2.5 & .96 & .24 & 0 & .02 & 0 \\
\hline 5 & .15 & 0 & 1.4 & $0^{*}$ & $0^{*}$ & 1.7 & 2.3 & .89 & .25 & .05 & 0 & 0 \\
\hline 6 & .13 & 0 & 1.2 & $0^{*}$ & $0^{*}$ & 1.5 & 2.1 & .80 & .21 & .03 & 0 & 0 \\
\hline 7 & .13 & 0 & .93 & $0^{*}$ & $0^{*}$ & 1.3 & 1.9 & .71 & .21 & 0 & 0 & 0 \\
\hline 8 & .13 & 0 & .95 & $0^{*}$ & $0^{*}$ & 1.1 & 1.9 & .70 & .18 & 0 & .07 & 0 \\
\hline 9 & .12 & 0 & .98 & $0^{*}$ & $0^{*}$ & 1.0 & 1.8 & .71 & .16 & 0 & .08 & 0 \\
\hline 10 & .11 & 0 & 1.1 & $0^{*}$ & $0^{*}$ & .93 & 1.8 & .66 & .13 & 0 & .05 & 0 \\
\hline 11 & .10 & 0 & 1.1 & $0^{*}$ & $0^{*}$ & .87 & 1.7 & .62 & .10 & .03 & 0 & 0 \\
\hline 12 & .10 & 0 & 1.0 & $0^{*}$ & $0^{*}$ & .86 & 1.6 & .55 & .06 & .01 & 0 & 0 \\
\hline 13 & .09 & 0 & .93 & $0^{*}$ & $0^{*}$ & .97 & 1.4 & .54 & .04 & 0 & 0 & 0 \\
\hline 14 & .06 & 0 & .88 & $0^{*}$ & $0^{*}$ & 1.2 & 1.2 & .55 & .02 & 0 & 0 & 0 \\
\hline 15 & .04 & 0 & .80 & $0^{*}$ & $0^{*}$ & 1.4 & 1.0 & .60 & 0 & 0 & 0 & 0 \\
\hline 16 & .02 & 0 & $.66^{*}$ & $0^{*}$ & $0^{*}$ & 1.4 & 1.0 & .54 & 0 & 0 & .04 & 0 \\
\hline 17 & 0 & 0 & $.58^{\star}$ & $0^{*}$ & $0^{*}$ & 1.4 & 1.3 & .51 & 0 & 0 & 0 & 0 \\
\hline 18 & 0 & 0 & $.48^{\star}$ & $0^{*}$ & $0^{*}$ & 1.4 & 1.5 & .49 & 0 & 0 & 0 & 0 \\
\hline 19 & 0 & 0 & $.42^{\star}$ & $0^{*}$ & $0^{*}$ & 1.3 & 1.4 & .47 & 0 & 0 & 0 & 0 \\
\hline 20 & 0 & 0 & $.38^{\star}$ & $0^{*}$ & $0^{*}$ & 1.3 & 1.3 & .45 & 0 & 0 & 0 & 0 \\
\hline 21 & 0 & 0 & $.34^{*}$ & $0^{*}$ & $0^{*}$ & 1.3 & 1.2 & .41 & 0 & 0 & 0 & 0 \\
\hline 22 & 0 & 0 & $.28^{\star}$ & $0^{*}$ & .88 & 1.6 & 1.2 & .42 & 0 & 0 & 0 & 0 \\
\hline 23 & 0 & 0 & $.26^{\star}$ & $0^{*}$ & .79 & 1.9 & 1.3 & .43 & 0 & 0 & .04 & 0 \\
\hline 24 & 0 & 0 & $.10^{\star}$ & $0^{*}$ & .79 & 2.0 & 1.3 & .41 & 0 & 0 & .02 & 0 \\
\hline 25 & 0 & 0 & $.05^{\star}$ & $0^{*}$ & 1.0 & 2.0 & 1.5 & .40 & 0 & 0 & .08 & 0 \\
\hline 26 & 0 & 0 & $0^{*}$ & $0^{*}$ & 1.1 & 2.2 & 1.5 & .38 & 0 & 0 & .04 & 0 \\
\hline 27 & 0 & 0 & $0^{*}$ & $0^{*}$ & 1.2 & 2.5 & 1.5 & .36 & 0 & 0 & 0 & 0 \\
\hline 28 & 0 & 0 & $0^{*}$ & $0^{*}$ & 1.3 & 2.8 & 1.3 & .39 & 0 & 0 & 0 & 0 \\
\hline 29 & 0 & 0 & $0^{*}$ & $0^{*}$ & 1.5 & 2.8 & 1.2 & .36 & 0 & 0 & 0 & 0 \\
\hline 30 & 0 & 0 & $0^{*}$ & $0^{*}$ & ----- & 2.8 & 1.1 & .33 & 0 & 0 & 0 & 0 \\
\hline 31 & 0 & ------ & $0^{*}$ & $0^{*}$ & ------ & 2.8 & ------ & .32 & ------ & 0 & .06 & ----- \\
\hline Total & 1.77 & 0 & 19.32 & 0 & 8.56 & 52.23 & 48.7 & 18.06 & 2.40 & 0.12 & 0.50 & 0.10 \\
\hline Mean & .057 & 0 & .62 & 0 & .30 & 1.68 & 1.62 & .58 & .080 & .004 & .016 & .003 \\
\hline $\operatorname{Max}$ & .15 & 0 & 1.6 & 0 & 1.5 & 2.8 & 2.7 & 1.1 & .28 & .05 & .08 & .06 \\
\hline Min & 0 & 0 & 0 & 0 & 0 & .86 & 1.0 & .32 & 0 & 0 & 0 & 0 \\
\hline Acre-Ft & 3.5 & 0 & 38 & 0 & 17 & 104 & 97 & 36 & 4.8 & .24 & .99 & .20 \\
\hline Wtr Year & 2008 & Total & 151.76 & Mean & & .41 & Max & 2.8 & Min & 0 & Acre-Ft & 301 \\
\hline Cal Year & 2007 & Total & 228.73 & Mean & & .63 & $\operatorname{Max}$ & 4.7 & Min & 0 & Acre-Ft & 454 \\
\hline
\end{tabular}

*Estimated 


\section{E026 Los Alamos Canyon below Ice Rink}

Location. Lat $35^{\circ}$ 52' 49" long $106^{\circ} 19^{\prime} 30^{\prime}$, NE 1/4, Sec. 17, T. 19 N., R. 6 E., Los Alamos County.

Drainage Area. $7.07 \mathrm{mi}^{2}$.

Period of Record. February 26, 2001, to September 30, 2007.

Revised Record. Drainage area (2006); section (2007).

Average Discharge. 7 years, $0.38 \mathrm{ft}^{3} / \mathrm{s}, 276$ acre- $\mathrm{ft} / \mathrm{yr}$.

Gage. Data logger with cellular telemetry. Elevation of gage is 7,183 $\mathrm{ft}$ above NGVD.

Remarks. Records are good except estimated daily discharges, which are fair. Flow partially regulated by Los Alamos Reservoir, which is about 1.55 mi upstream.

Extremes for Period of Record. Maximum discharge, $185 \mathrm{ft}^{3} / \mathrm{s}$, August 9, 2001, gage height $1.52 \mathrm{ft}$. No flow at times.

Extremes for Current Water Year. Maximum discharge, $4.1 \mathrm{ft}^{3} / \mathrm{s}$ at $0520 \mathrm{~h}$, January 2, gage height $0.55 \mathrm{ft}$. No peak above base of $15 \mathrm{ft}^{3} / \mathrm{s}$. No flow at times.

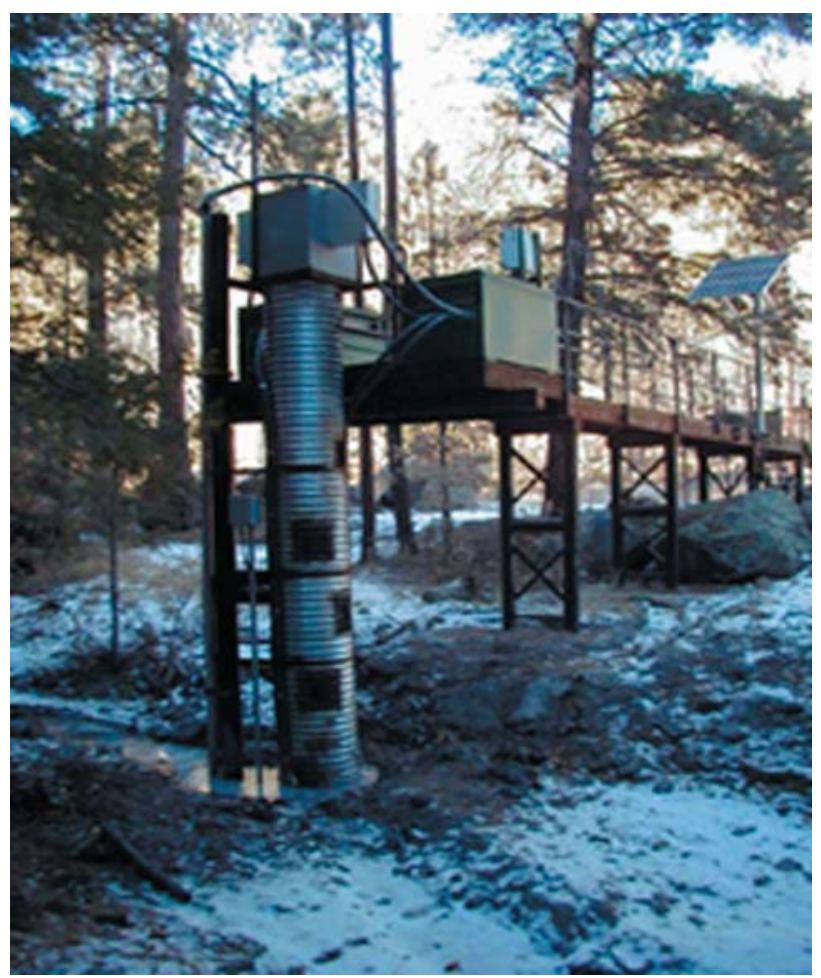




\section{E026 Los Alamos Canyon below Ice Rink}

\section{Station Analysis}

\section{Water Year}

Equipment. Station is equipped with Sutron 8210 (5-min. interval) and shaft encoder float system with cellular telemetry and speech modem. Phone upgraded June 4 from analog to digital service. The system is powered by a solar-panel battery system housed in a NEMA shelter on top of a 24" CMP well, 16' long attached to a 40' steel walkway on left bank. Station is equipped with ISCO samplers for water-quality sample collection. ISCO samplers are housed in a separate $3^{\prime} \times 4^{\prime}$ metal box. Samplers are triggered by stage through the data logger. An outside staff is available for reference. No provision for measurements above wading stage. All high flow measurement will be by slope-area or critical depth computation methods.

Field Work. The station was visited 28 times to conduct discharge measurements and service the instrumentation. Field inspections for the gage are listed under site history files on the Hydstra database. Discharge measurements for the gage are listed under site gauging files on the Hydstra database.

Datum Correction. Levels run Nov. 21, 2001, and datum correction applied as detailed on PCS.

Gage-Height Record. The data logger referenced to the inside staff gave a complete and satisfactory record except for the period of December 22 to January 3 when ice affected gage height.

Rating. The channel at the gage is about $20^{\prime}$ wide and straight for $20^{\prime}$ upstream, where it bends to the left and then straight for about 150' downstream. The streambed through this reach is primary gravel with cobbles. Low flow control is rock and grave riffle 15' downstream from gage. Channel is control for medium and high stages. The buildup and scour of this control leads to changes of shifts during the water year.

Six discharge measurements (Nos. 63-68) and 28 inspections were made during the period.

Rating No. 2 was developed based on measurements made during the period of record.

Flow is partially regulated by Los Alamos Reservoir about 1.5 miles upstream of gage and draining of this reservoir. Gage of reference at this station is the inside reference point (RP measure). Fall exists at all low to medium flow regimes between staff and well.

Discharge. Discharges were computed from Rating No. 2 using variable shift curves that were defined by the measurements and listed on the worksheet.

Remarks. Records are good except for estimated daily discharges, which are fair. 
E026 Los Alamos below Ice Rink

Daily Mean Discharge in Cubic Feet per Second

Water Year October 2007 to September 2008

\begin{tabular}{|c|c|c|c|c|c|c|c|c|c|c|c|c|}
\hline DAY & ОСТ & NOV & DEC & JAN & FEB & MAR & APR & MAY & JUN & JUL & AUG & SEP \\
\hline 1 & .10 & 0 & 1.9 & $0^{*}$ & 0 & 2.1 & 2.4 & .99 & .17 & 0 & 0 & 0 \\
\hline 2 & .10 & 0 & 1.7 & $0^{*}$ & 0 & 2.5 & 2.3 & .98 & .15 & 0 & 0 & 0 \\
\hline 3 & .06 & 0 & 1.9 & $0^{*}$ & 0 & 2.4 & 2.2 & .96 & .14 & 0 & 0 & 0 \\
\hline 4 & .04 & 0 & 1.9 & 0 & 0 & 2.3 & 2.1 & .95 & .13 & 0 & .01 & 0 \\
\hline 5 & .03 & 0 & 1.8 & 0 & 0 & 2.0 & 2.0 & .88 & .10 & .01 & 0 & 0 \\
\hline 6 & .02 & 0 & 1.5 & 0 & 0 & 1.8 & 1.9 & .78 & .07 & 0 & 0 & 0 \\
\hline 7 & .03 & 0 & 1.3 & 0 & 0 & 1.6 & 1.7 & .70 & .06 & 0 & 0 & 0 \\
\hline 8 & .04 & 0 & 1.3 & 0 & 0 & 1.4 & 1.7 & .66 & .04 & 0 & .01 & 0 \\
\hline 9 & .02 & 0 & 1.4 & 0 & 0 & 1.2 & 1.7 & .67 & .03 & 0 & .05 & 0 \\
\hline 10 & 0 & 0 & 1.5 & 0 & 0 & 1.1 & 1.6 & .64 & .02 & 0 & 0 & 0 \\
\hline 11 & 0 & 0 & 1.5 & 0 & 0 & .94 & 1.5 & .61 & .01 & 0 & 0 & 0 \\
\hline 12 & 0 & 0 & 1.3 & 0 & 0 & .96 & 1.4 & .52 & .01 & 0 & 0 & 0 \\
\hline 13 & 0 & 0 & 1.2 & 0 & 0 & 1.1 & 1.1 & .50 & .01 & 0 & 0 & 0 \\
\hline 14 & 0 & 0 & 1.1 & 0 & 0 & 1.5 & .96 & .52 & 0 & 0 & 0 & 0 \\
\hline 15 & 0 & 0 & .92 & 0 & 0 & 1.6 & .81 & .60 & 0 & 0 & 0 & 0 \\
\hline 16 & 0 & 0 & .70 & 0 & 0 & 1.7 & .76 & .50 & 0 & 0 & 0 & 0 \\
\hline 17 & 0 & 0 & .72 & 0 & 0 & 1.6 & 1.1 & .50 & 0 & 0 & 0 & 0 \\
\hline 18 & 0 & 0 & .66 & 0 & 0 & 1.6 & 1.3 & .51 & 0 & 0 & 0 & 0 \\
\hline 19 & 0 & 0 & .61 & 0 & 0 & 1.4 & 1.3 & .43 & $0^{*}$ & 0 & 0 & 0 \\
\hline 20 & 0 & 0 & .56 & 0 & 0 & 1.2 & 1.1 & .43 & $0^{*}$ & 0 & 0 & 0 \\
\hline 21 & 0 & 0 & .56 & 0 & .47 & 1.3 & .98 & .41 & 0 & 0 & 0 & 0 \\
\hline 22 & 0 & 0 & $0^{*}$ & 0 & .92 & 1.6 & 1.0 & .42 & 0 & 0 & 0 & 0 \\
\hline 23 & 0 & 0 & $0^{*}$ & 0 & .84 & 2.0 & 1.1 & .43 & 0 & 0 & 0 & 0 \\
\hline 24 & 0 & 0 & $0^{*}$ & 0 & .93 & 2.0 & 1.1 & .41 & 0 & 0 & 0 & 0 \\
\hline 25 & 0 & 0 & $0^{*}$ & 0 & 1.1 & 2.0 & 1.3 & .37 & 0 & 0 & 0 & 0 \\
\hline 26 & 0 & 0 & $0^{*}$ & 0 & 1.2 & 2.1 & 1.3 & .35 & 0 & 0 & 0 & 0 \\
\hline 27 & 0 & 0 & $0^{*}$ & 0 & 1.3 & 2.3 & 1.3 & .30 & 0 & 0 & 0 & 0 \\
\hline 28 & 0 & .02 & $0^{*}$ & 0 & 1.5 & 2.6 & 1.3 & .36 & 0 & 0 & 0 & 0 \\
\hline 29 & 0 & 0 & $0^{*}$ & 0 & 1.7 & 2.5 & 1.2 & .28 & 0 & 0 & 0 & 0 \\
\hline 30 & 0 & .04 & $0^{*}$ & 0 & ----- & 2.5 & 1.1 & .25 & 0 & 0 & 0 & $0^{*}$ \\
\hline 31 & 0 & ----- & $0^{*}$ & 0 & ------ & 2.5 & ----- & .22 & ----- & 0 & .05 & ----- \\
\hline Total & 0.44 & 0.06 & 26.03 & 0 & 9.96 & 55.40 & 42.61 & 17.13 & 0.94 & 0.01 & 0.12 & 0 \\
\hline Mean & .014 & .002 & .84 & 0 & .34 & 1.79 & 1.42 & .55 & .031 & 0 & .004 & 0 \\
\hline $\operatorname{Max}$ & .10 & .04 & 1.9 & 0 & 1.7 & 2.6 & 2.4 & .99 & .17 & .01 & .05 & 0 \\
\hline Min & 0 & 0 & 0 & 0 & 0 & .94 & .76 & .22 & 0 & 0 & 0 & 0 \\
\hline Acre-Ft & .87 & .12 & 52 & 0 & 20 & 110 & 85 & 34 & 1.9 & .02 & .24 & 0 \\
\hline Wtr Year & 2008 & Total & 152.70 & Mean & & .42 & Max & 2.6 & Min & 0 & Acre-Ft & 303 \\
\hline Cal Year & 2007 & Total & 211.09 & Mean & & .58 & Max & 4.6 & Min & 0 & Acre-Ft & 419 \\
\hline
\end{tabular}

*Estimate 


\section{E02685 Los Alamos Canyon at TA-2}

Location. Lat $35^{\circ}$ 52' 34", long $106^{\circ}$ 17' 21", SE 1/4, Sec. 15, T. 19 N., R. 6 E., Los Alamos County.

Drainage Area. $7.97 \mathrm{mi}^{2}$.

Period of Record. March 8, 2006, to September 30, 2008.

Gage. Data logger. Elevation of gage is 6,853 ft above NGVD.

Remarks. Records are good except for estimated daily discharges, which are fair. Flow partially regulated by Los Alamos Reservoir, which is about 3.0 miles upstream.

Extremes for Period of Record. Maximum discharge, $52 \mathrm{ft}^{3} / \mathrm{s}$, September 6, 2007, gage height $1.94 \mathrm{ft}$. No flow most of the time.

Extremes for Current Water Year. Peak discharges above base of $15 \mathrm{ft}^{3} / \mathrm{s}$ and $\operatorname{maximum}(*)$ :

\begin{tabular}{|c|c|c|c|}
\hline Date & Time & Discharge (ft $\left.\mathbf{3}^{\mathbf{3}} \mathbf{s}\right)$ & Gage Height (ft) \\
\hline December 1 & 0110 & 27 & 1.34 \\
\hline August 9 & 0030 & 20 & 1.17 \\
\hline August 9 & 1305 & 24 & 1.27 \\
\hline August 10 & 1155 & 31 & 1.43 \\
\hline August 31 & 1605 & $28^{*}$ & $1.35^{*}$ \\
\hline
\end{tabular}

No flow at times.

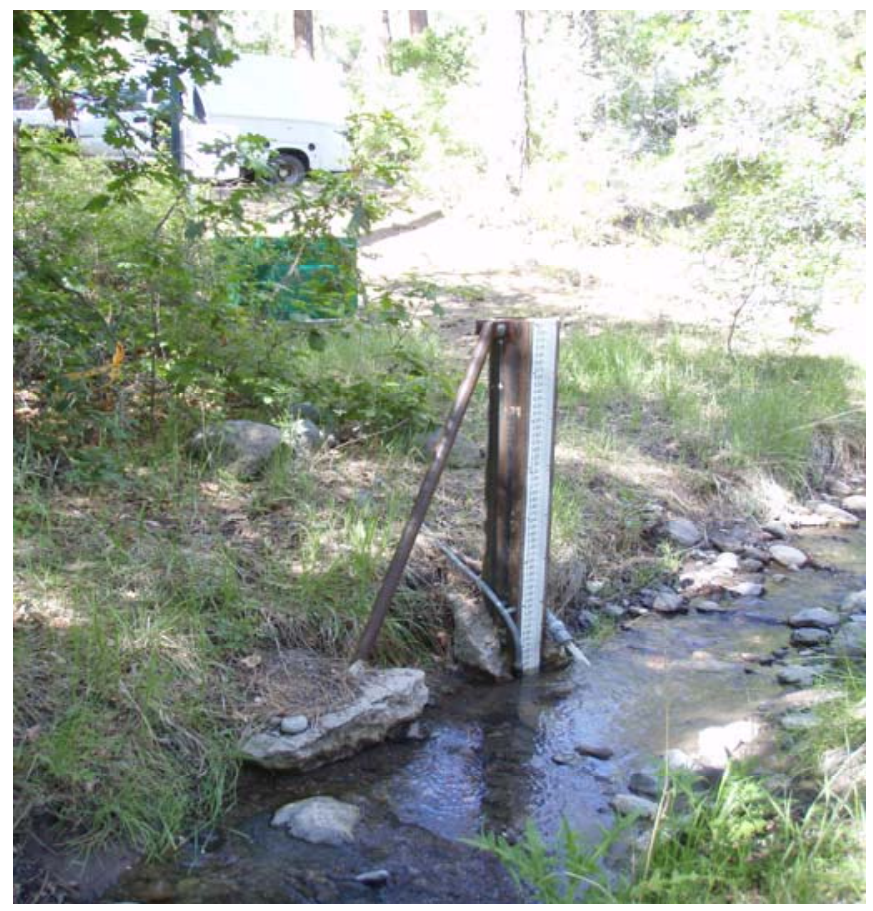




\section{E02685 Los Alamos Canyon at TA-2}

\section{Station Analysis}

\section{Water Year}

Equipment. Station is equipped with Sutron 8210 data logger (5-min. interval) with a Sutron Accubar bubble sensor. System is powered by a solar-panel battery system housed in a NEMA shelter. Station is equipped with an ISCO pump sampler for water-quality sample collection. ISCO is housed in a separate shelter, a $3^{\prime} \times 4^{\prime}$ metal box. Sampler is triggered by stage through the data logger. An outside staff is available for reference. No provision for direct measurements above wading stage.

Field Work. The station was visited 24 times to conduct discharge measurements and service the instrumentation. Field inspections for the gage are listed under site history files on the Hydstra database. Discharge measurements for the gage are listed under site gauging files on the Hydstra database.

Datum Correction. None. Levels of March 30, 2006, found gage within limits, no correction needed.

Gage-Height Record. The data logger referenced to the outside staff gave a complete and satisfactory record except during the period of December 22 to February 20 when ice affected gage height.

Rating. The channel is straight for 100' upstream and downstream. Bed is large gravel and well armored and should not be subject to much movement. Channel is trapezoidal with some vegetation. Flow is regulated somewhat by Los Alamos Reservoir, which is about 3.0 miles upstream.

Eight discharge measurements (Nos. 26-33) and 11 inspections of no flow were made during the year.

Rating No. 2 was used for part of the year. Rating No. 3 was developed based on measurements made during the year. Shifts are small and variable. Flows are very flashy, less than an hour, and as a result mean daily discharges are very small in relation to the instantaneous peak.

Discharge. Discharge was computed from Ratings No. 2 and 3, with shifts applied by stage V diagram.

Remarks. Records are good except for estimated daily discharges, which are fair. 
E02685 Los Alamos Canyon at TA-2

Daily Mean Discharge in Cubic Feet per Second

Water Year October 2007 to September 2008

\begin{tabular}{|c|c|c|c|c|c|c|c|c|c|c|c|c|}
\hline DAY & OCT & NOV & DEC & JAN & FEB & MAR & APR & MAY & JUN & JUL & AUG & SEP \\
\hline 1 & .15 & $0^{*}$ & $6.5^{\star}$ & $0^{*}$ & $0^{*}$ & 2.4 & 2.5 & 1.0 & $0^{*}$ & 0 & 0 & .22 \\
\hline 2 & $.02^{\star}$ & $0^{*}$ & 1.3 & $0^{*}$ & $0^{*}$ & 2.7 & 2.4 & .93 & $0^{*}$ & 0 & 0 & 0 \\
\hline 3 & $0^{*}$ & $0^{*}$ & 1.5 & $0^{*}$ & $0^{*}$ & 2.6 & 2.5 & .87 & 0 & 0 & 0 & 0 \\
\hline 4 & $0^{*}$ & $0^{*}$ & 1.6 & $0^{*}$ & $0^{*}$ & 2.5 & 2.5 & .80 & 0 & 0 & .34 & 0 \\
\hline 5 & $0^{*}$ & $0^{*}$ & 1.5 & $0^{*}$ & $0^{*}$ & 2.3 & 2.4 & .71 & 0 & .11 & 0 & 0 \\
\hline 6 & $0^{*}$ & $0^{*}$ & 1.3 & $0^{*}$ & $0^{*}$ & 2.1 & 2.4 & .60 & 0 & 0 & 0 & 0 \\
\hline 7 & $0^{*}$ & $0^{*}$ & 1.2 & $0^{*}$ & $0^{*}$ & $2.2^{*}$ & 2.2 & .57 & 0 & 0 & .06 & 0 \\
\hline 8 & $0^{*}$ & $0^{*}$ & 1.8 & $0^{*}$ & $0^{*}$ & 1.9 & 2.1 & .50 & 0 & 0 & .04 & 0 \\
\hline 9 & $0^{*}$ & $0^{*}$ & 1.6 & $0^{*}$ & $0^{*}$ & 1.7 & 2.0 & .49 & 0 & 0 & 1.7 & .03 \\
\hline 10 & $0^{*}$ & $0^{*}$ & 1.6 & $0^{*}$ & $0^{*}$ & 1.6 & 1.9 & .41 & 0 & 0 & 1.4 & 0 \\
\hline 11 & $0^{*}$ & $0^{*}$ & 1.7 & $0^{*}$ & $0^{*}$ & 1.5 & 1.7 & .31 & 0 & 0 & 0 & 0 \\
\hline 12 & $0^{*}$ & $0^{*}$ & 1.5 & $0^{*}$ & $0^{*}$ & 1.6 & 1.7 & .24 & 0 & 0 & 0 & 0 \\
\hline 13 & $0^{*}$ & $0^{*}$ & 1.3 & $0^{*}$ & $0^{*}$ & 1.7 & 1.4 & .19 & 0 & 0 & 0 & 0 \\
\hline 14 & $0^{*}$ & $0^{*}$ & 1.3 & $0^{*}$ & $0^{*}$ & 2.1 & 1.2 & .22 & 0 & 0 & 0 & 0 \\
\hline 15 & $0^{*}$ & $0^{*}$ & $1.1^{*}$ & $0^{*}$ & $0^{*}$ & 2.2 & 1.0 & .51 & 0 & 0 & 0 & 0 \\
\hline 16 & $0^{*}$ & $0^{*}$ & $1.2^{*}$ & $0^{*}$ & $0^{*}$ & 2.3 & .86 & .31 & 0 & 0 & .20 & 0 \\
\hline 17 & $0^{*}$ & $0^{*}$ & $.99^{*}$ & $0^{*}$ & $0^{*}$ & 2.0 & 1.1 & .21 & 0 & 0 & 0 & 0 \\
\hline 18 & $0^{*}$ & $0^{*}$ & .87 & $0^{*}$ & $0^{*}$ & 1.7 & 1.2 & .17 & 0 & 0 & 0 & 0 \\
\hline 19 & $0^{*}$ & $0^{*}$ & .81 & $0^{*}$ & $0^{*}$ & 1.6 & 1.3 & .15 & 0 & 0 & 0 & 0 \\
\hline 20 & $0^{*}$ & $0^{*}$ & .75 & $0^{*}$ & $0^{*}$ & 1.6 & 1.2 & .37 & 0 & 0 & 0 & 0 \\
\hline 21 & $0^{*}$ & $0^{*}$ & .73 & $0^{*}$ & $.05^{*}$ & 1.6 & 1.2 & .54 & 0 & 0 & 0 & 0 \\
\hline 22 & $0^{*}$ & $0^{*}$ & $.66^{*}$ & $0^{*}$ & $.10^{*}$ & 1.7 & 1.2 & .50 & 0 & 0 & 0 & 0 \\
\hline 23 & $0^{*}$ & $0^{*}$ & $.46^{*}$ & $0^{*}$ & $.20^{*}$ & 1.8 & 1.3 & .80 & 0 & 0 & .25 & 0 \\
\hline 24 & $0^{*}$ & $0^{*}$ & $.31^{*}$ & $0^{*}$ & $.37^{*}$ & 2.1 & 1.3 & .70 & 0 & 0 & .35 & 0 \\
\hline 25 & $0^{*}$ & $0^{*}$ & $.19^{*}$ & $0^{*}$ & 1.8 & 2.1 & 1.4 & .51 & 0 & 0 & .23 & 0 \\
\hline 26 & $0^{*}$ & $0^{*}$ & $.09^{*}$ & $0^{*}$ & 1.8 & 2.2 & 1.4 & .41 & 0 & 0 & .02 & 0 \\
\hline 27 & $0^{*}$ & $0^{*}$ & $.06^{*}$ & $0^{*}$ & 1.8 & 2.4 & 1.4 & .35 & 0 & 0 & 0 & 0 \\
\hline 28 & $0^{*}$ & $0^{*}$ & $.05^{*}$ & $3.1^{*}$ & 2.0 & 2.6 & 1.3 & $1.1^{*}$ & 0 & 0 & 0 & 0 \\
\hline 29 & $0^{*}$ & $.03^{*}$ & $.03^{*}$ & $.11^{*}$ & 2.1 & 2.7 & 1.2 & $.06^{\star}$ & 0 & 0 & 0 & 0 \\
\hline 30 & $0^{*}$ & $.34^{\star}$ & $.01^{*}$ & $0^{*}$ & ------ & 2.6 & 1.1 & $0^{\star}$ & 0 & 0 & 0 & 0 \\
\hline 31 & $0^{*}$ & ----- & $0^{*}$ & $0^{*}$ & ------ & 2.6 & ------ & $0^{*}$ & ----- & 0 & 1.7 & ------ \\
\hline Total & 0.17 & 0.37 & 34.01 & 3.21 & 10.22 & 64.7 & 48.36 & 14.53 & 0 & 0.11 & 6.29 & 0.25 \\
\hline Mean & .006 & .012 & 1.10 & .10 & .35 & 2.09 & 1.61 & .47 & 0 & .004 & .20 & .008 \\
\hline $\operatorname{Max}$ & .15 & .34 & 6.5 & 3.1 & 2.1 & 2.7 & 2.5 & 1.1 & 0 & .11 & 1.7 & .22 \\
\hline Min & 0 & 0 & 0 & 0 & 0 & 1.5 & .86 & 0 & 0 & 0 & 0 & 0 \\
\hline Acre-Ft & .34 & .73 & 67 & 6.4 & 20 & 128 & 96 & 29 & 0 & .22 & 12 & .50 \\
\hline Wtr Year & 2008 & Total & 182.22 & & & 50 & Max & 6.5 & Min & 0 & Acre-Ft & 361 \\
\hline Cal Year & 2007 & Total & 259.49 & & & 71 & Max & 6.5 & Min & 0 & Acre-Ft & 515 \\
\hline
\end{tabular}

*Estimate 


\section{E030 Los Alamos Canyon above DP Canyon}

Location. Lat $35^{\circ}$ 52' 21", long $106^{\circ} 15^{\prime}$ 36", SW 1/4, sec. 13, T. 19 N., R. 6 E., Los Alamos County.

Drainage Area. $8.57 \mathrm{mi}^{2}$.

Period of Record. July 1994 to September 30, 2008.

Revised Record. Drainage area (2006); township (2007).

Gage. Data logger with concrete control. Elevation of gage is 6,621 ft above NGVD from GPS survey.

Remarks. Records are good. Flow partially regulated by Los Alamos Reservoir, which is about $2.5 \mathrm{mi}$ upstream.

Average Discharge. $14 \mathrm{yr}, 0.30 \mathrm{ft}^{3} / \mathrm{s}, 215$ acre- $\mathrm{ft} / \mathrm{yr}$.

Extremes Outside Period of Record. Flood of July 31, 1968, was $329 \mathrm{ft}^{3} / \mathrm{s}$ from slopearea determination. Gage height was established later at $3.71 \mathrm{ft}$ present datum.

Extremes for Period of Record. Maximum discharge, $125 \mathrm{ft}^{3} / \mathrm{s}$, June 22, 2002, gage height $2.88 \mathrm{ft}$ from peak flow computation. No flow most of the time.

Extremes for Current Year. Peak discharges above base of $10 \mathrm{ft}^{3} / \mathrm{s}$ and maximum (*):

\begin{tabular}{|c|c|c|c|}
\hline Date & Time & Discharge $\left.\mathbf{( f t}^{\mathbf{3}} \mathbf{s}\right)$ & Gage Height (ft.) \\
\hline December 1 & 0245 & 12 & 1.63 \\
\hline September 4 & 1105 & $13^{*}$ & $1.74^{*}$ \\
\hline
\end{tabular}

No flow at times.

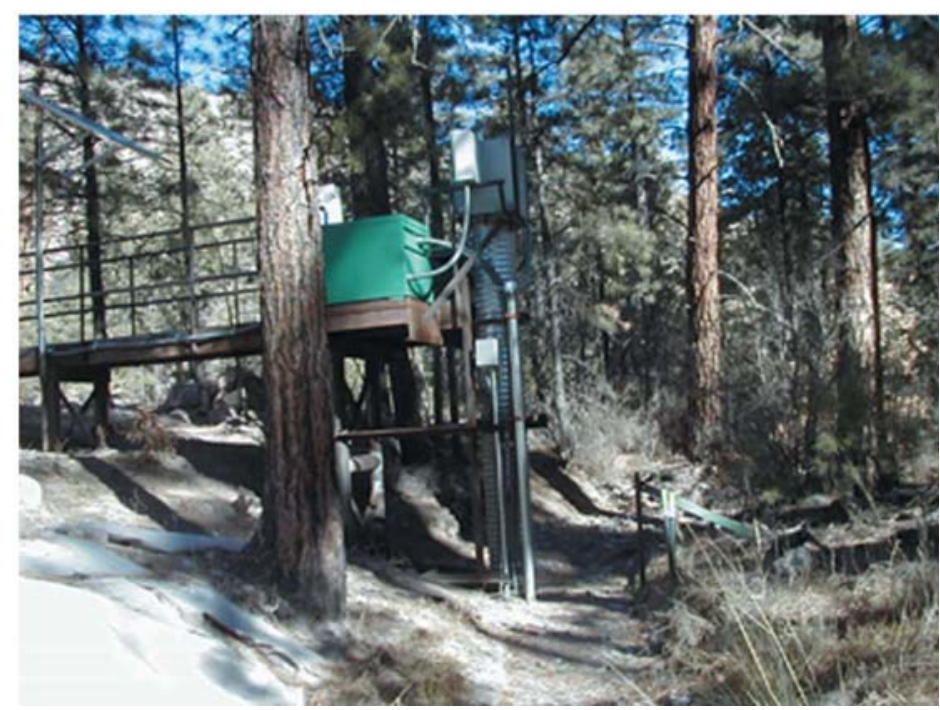




\section{E030 Los Alamos Canyon above DP Canyon}

\section{Station Analysis}

\section{Water Year}

Equipment. Station is equipped with Sutron 8210 (5-min. interval) with shaft encoder float system (5-min. interval). The system is powered by a solar-panel battery system housed in NEMA shelter on 18" CMP well on left bank. Station is equipped with an ISCO pump sampler for water-quality sample collection. ISCO is housed in a separate shelter, a 3' $\times 4^{\prime}$ metal box. Sampler is triggered by stage through the data logger. An outside staff is available for reference. No provision for direct discharge measurements above wading stages.

Cellular telemetry removed March 17, service discontinued.

Field Work. The station was visited 21 times to conduct discharge measurements and service the instrumentation. Field inspections for the gage are listed under site history files on the Hydstra database. Discharge measurements for the gage are listed under site gauging files on the Hydstra database.

Datum Correction. None

Gage-Height Record. The data logger referenced to the outside staff gave a complete and satisfactory record for the year, except during the period of November 31 to February 18 when ice affected gage height.

Rating. Streambed consists of sand and gravel and is subject to slight movement during flow events. The channel is straight for $300^{\prime}$ above gage and $50^{\prime}$ below. Vegetation on bank consists of sparse grass.

Five discharge measurement (Nos. 50-54) and 12 inspections of no flow were made during the year. Shifts were used on the low point of the "V" diagram. Plus zero shifts were defined by measurements 51 and 54 . No. 50 had a shift of -0.24 caused by ice effect on broad-crested weir. Positive shifting at low end is from fill in gage pool, resulting in approach to notch.

Rating No. 2 was used for the entire water year.

Discharge. Discharge was computed from Rating No. 2 using variable shift diagrams.

Remarks. Records are good except for estimated daily discharges, which are fair. 
E030 Los Alamos Canyon above DP Canyon

Daily Mean Discharge in Cubic Feet per Second

Water Year October 2007 to September 2008

\begin{tabular}{|c|c|c|c|c|c|c|c|c|c|c|c|c|}
\hline DAY & OCT & NOV & DEC & JAN & FEB & MAR & APR & MAY & JUN & JUL & AUG & SEP \\
\hline 1 & 0 & 0 & $3.5^{*}$ & $0^{*}$ & $0^{*}$ & 1.3 & 2.0 & .49 & .01 & $0^{*}$ & $0^{*}$ & .10 \\
\hline 2 & .01 & 0 & $0^{*}$ & $0^{*}$ & $0^{*}$ & 1.7 & 1.9 & .48 & .01 & $0^{*}$ & $0^{*}$ & 0 \\
\hline 3 & 0 & 0 & $0^{*}$ & $0^{*}$ & $0^{*}$ & 1.6 & 1.8 & .46 & .01 & $0^{*}$ & $0^{*}$ & 0 \\
\hline 4 & 0 & 0 & $0^{*}$ & $0^{*}$ & $0^{*}$ & 1.4 & 1.8 & .46 & .01 & 2.6 & 0 & 0 * \\
\hline 5 & 0 & 0 & $0^{*}$ & $0^{*}$ & $0^{*}$ & 1.3 & 1.6 & .43 & .01 & $1.0^{*}$ & 0 & 0 \\
\hline 6 & 0 & 0 & $0^{*}$ & $0^{*}$ & $0^{*}$ & 1.1 & 1.4 & .40 & .01 & $0^{*}$ & 0 & 0 \\
\hline 7 & 0 & 0 & $0^{*}$ & $0^{*}$ & $0^{*}$ & 1.8 & 1.2 & .36 & .01 & $0^{*}$ & 0 & 0 \\
\hline 8 & 0 & 0 & $.76^{\star}$ & $0^{*}$ & $0^{*}$ & .93 & 1.1 & .34 & .01 & $0^{*}$ & 0 & 0 \\
\hline 9 & 0 & 0 & .86 & $0^{*}$ & $0^{*}$ & .75 & 1.2 & .35 & .02 & $0^{*}$ & .62 & 0 \\
\hline 10 & 0 & 0 & .91 & $0^{*}$ & $0^{*}$ & .70 & 1.1 & .32 & $0^{*}$ & $0^{*}$ & .59 & 0 \\
\hline 11 & 0 & 0 & 1.1 & $0^{*}$ & $0^{*}$ & .64 & 1.0 & .30 & $0^{*}$ & $0^{*}$ & $0^{*}$ & 0 \\
\hline 12 & 0 & 0 & $.70^{\star}$ & $0^{*}$ & $0^{*}$ & .57 & .97 & .26 & $0^{*}$ & $0^{*}$ & $0^{\star}$ & 0 \\
\hline 13 & 0 & 0 & $.80^{\star}$ & $0^{*}$ & $0^{*}$ & .61 & .91 & .23 & $0^{*}$ & $0^{*}$ & $0^{*}$ & 0 \\
\hline 14 & 0 & 0 & .70 & $0^{*}$ & $0^{*}$ & .80 & .81 & .28 & $0^{*}$ & $0^{*}$ & $0^{*}$ & 0 \\
\hline 15 & 0 & 0 & .44 & $0^{*}$ & $0^{*}$ & .93 & .69 & .48 & $0^{*}$ & $0^{*}$ & $0^{*}$ & 0 \\
\hline 16 & 0 & 0 & .41 & $0^{*}$ & $0^{*}$ & .97 & .58 & .34 & $0^{*}$ & $0^{*}$ & $0^{*}$ & 0 \\
\hline 17 & 0 & 0 & .42 & $0^{*}$ & $0^{*}$ & .96 & .53 & .28 & $0^{*}$ & $0^{*}$ & 0 & 0 \\
\hline 18 & 0 & 0 & .42 & $0^{*}$ & $0^{*}$ & .95 & .76 & .23 & $0^{*}$ & $0^{*}$ & 0 & 0 \\
\hline 19 & 0 & 0 & .40 & $0^{*}$ & .29 & .85 & .74 & .17 & $0^{*}$ & $0^{*}$ & .01 & 0 \\
\hline 20 & 0 & 0 & $.32^{\star}$ & $0^{*}$ & .31 & .80 & .59 & .10 & $0^{*}$ & $0^{*}$ & 0 & 0 \\
\hline 21 & 0 & 0 & $0^{*}$ & $0^{*}$ & .35 & .82 & .54 & .01 & $0^{*}$ & $0^{*}$ & 0 & 0 \\
\hline 22 & 0 & 0 & $0^{*}$ & $0^{*}$ & .33 & 1.0 & .53 & 0 & $0^{\star}$ & $0^{*}$ & 0 & 0 \\
\hline 23 & 0 & 0 & $0^{*}$ & $0^{\star}$ & .35 & 1.3 & .56 & 0 & $0^{*}$ & $0^{*}$ & .07 & 0 \\
\hline 24 & 0 & 0 & $0^{*}$ & $0^{\star}$ & .71 & 1.4 & .57 & .03 & $0^{*}$ & $0^{*}$ & .04 & 0 \\
\hline 25 & 0 & 0 & $0^{*}$ & $0^{*}$ & .80 & 1.4 & .67 & 0 & $0^{*}$ & $0^{*}$ & .06 & 0 \\
\hline 26 & 0 & 0 & $0^{*}$ & $0^{*}$ & .64 & 1.6 & .76 & 0 & $0^{*}$ & $0^{*}$ & .01 & 0 \\
\hline 27 & 0 & 0 & $0^{*}$ & $0^{*}$ & .70 & 1.9 & .72 & 0 & $0^{*}$ & $0^{*}$ & .01 & 0 \\
\hline 28 & 0 & 0 & $0^{*}$ & $1.5^{*}$ & .82 & 2.1 & .65 & .19 & $0^{\star}$ & $0^{*}$ & .01 & 0 \\
\hline 29 & 0 & 0 & $0^{*}$ & $.05^{*}$ & .98 & 2.2 & .58 & .12 & $0^{\star}$ & $0^{*}$ & .01 & 0 \\
\hline 30 & 0 & $.01^{*}$ & $0^{*}$ & $0^{*}$ & ----- & 2.1 & .53 & .01 & $0^{*}$ & $0^{*}$ & .01 & 0 \\
\hline 31 & 0 & ----- & $0^{*}$ & $0^{*}$ & ----- & 2.1 & ----- & .01 & ------ & $0^{*}$ & .50 & ----- \\
\hline Total & 0.01 & 0.01 & 11.74 & 1.55 & 6.28 & 38.58 & 28.79 & 7.13 & 0.10 & 3.6 & 1.94 & 0.10 \\
\hline Mean & 0 & 0 & .38 & .050 & .22 & 1.24 & .96 & .23 & .003 & .12 & .063 & .003 \\
\hline $\operatorname{Max}$ & .01 & .01 & 3.5 & 1.5 & .98 & 2.2 & 2.0 & .49 & .02 & 2.6 & .62 & .10 \\
\hline Min & 0 & 0 & 0 & 0 & 0 & .57 & .53 & 0 & 0 & 0 & 0 & 0 \\
\hline Acre-Ft & .02 & .02 & 23 & 3.1 & 12 & 77 & 57 & 14 & .20 & 7.1 & 3.8 & .20 \\
\hline Wtr Year & 2008 & Total & 99.83 & Mean & & .27 & $\operatorname{Max}$ & 3.5 & Min & 0 & Acre-Ft & 198 \\
\hline Cal Year & 2007 & Total & 162.19 & Mean & & .45 & $\operatorname{Max}$ & 4.3 & Min & 0 & Acre-Ft & 322 \\
\hline
\end{tabular}

*Estimate 


\section{E038 DP Canyon above TA-21}

Location. Lat $35^{\circ}$ 52' 49", long $106^{\circ} 16^{\prime}$ 58", SW 1/4, sec. 14, T. 19 N., R. 6 E., Los Alamos County.

Drainage Area. $0.22 \mathrm{mi}^{2}$.

Period of Record. April 26, 2000, to September 30, 2008.

Revised Record. Drainage area (2006); section (2007).

Average Discharge. 8 years, $0.13 \mathrm{ft}^{3} / \mathrm{s}, 97$ acre- $\mathrm{ft} / \mathrm{yr}$.

Gage. Data logger and rain gage with cellular telemetry. Elevation of gage is 7,087 ft above NGVD.

Remarks. Records are good except estimated daily discharges, which are fair.

Extremes for Period of Record. Maximum discharge, $295 \mathrm{ft}^{3}$ s, July 24, 2004, gage height $4.36 \mathrm{ft}$ from rating curve extended above $10 \mathrm{ft}^{3} / \mathrm{s}$ on basis on peak flow computations. No flow most of the time.

Extremes for Current Water Year. Peak discharges above base of $100 \mathrm{ft}^{3} / \mathrm{s}$ and maximum $(*)$ :

\begin{tabular}{|c|c|c|c|}
\hline Date & Time & Discharge $\mathbf{( f t}^{\mathbf{3}}$ /s) & Gage Height (ft) \\
\hline May 28 & 1555 & 125 & 3.06 \\
\hline August 9 & 1230 & 104 & 2.88 \\
\hline August 10 & 1125 & $186^{*}$ & $3.71^{*}$ \\
\hline
\end{tabular}

No flow at times.

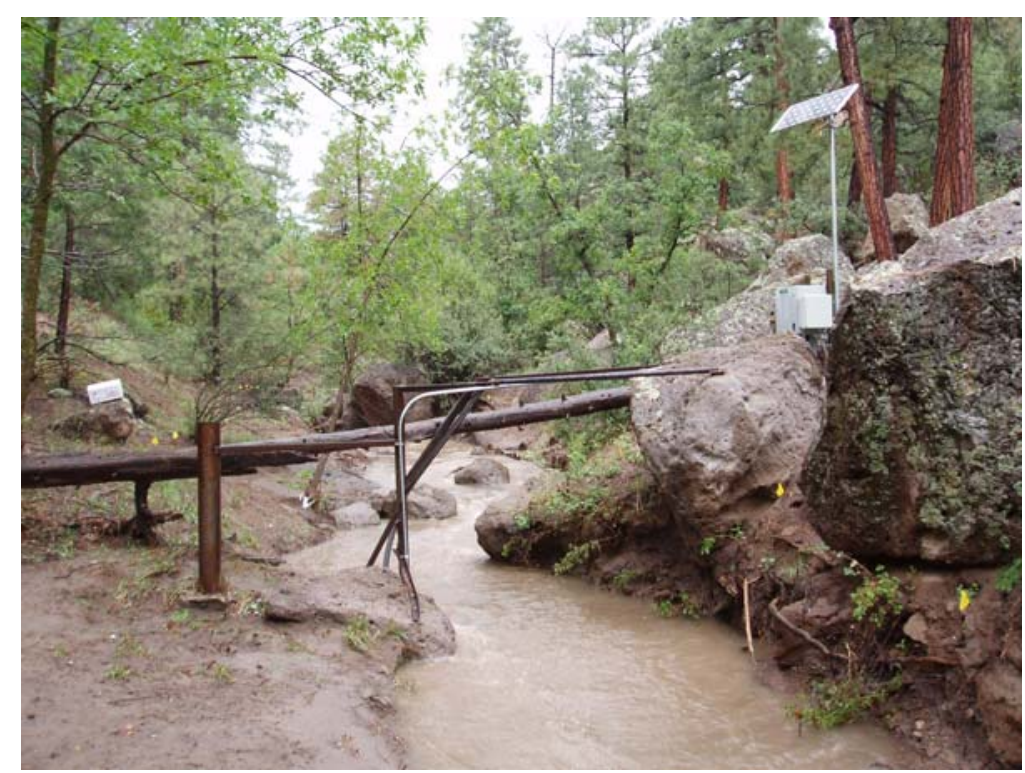




\section{E038 DP Canyon above TA-21}

\section{Station Analysis}

\section{Water Year}

Equipment. Station is equipped with Sutron 8210 data logger (5-min. interval) with a Sutron accububble self-contained bubbler system and cellular telemetry with speech modem. Phone upgraded May 27 from analog to digital service. The system is powered by a solar-panel battery system housed in a NEMA shelter. Station is equipped with an ISCO pump sampler for water-quality sample collection. ISCO is housed in a separate shelter, a $3^{\prime} \times 4^{\prime}$ metal box. Sampler is triggered by stage through the data logger. An outside staff gage is available for reference. No provision for discharge measurements above wading stage. All high flow measurement will be by slope-area or peak flow computation methods.

Station is also equipped with a tipping bucket rain gage, Rain Collection II, which was installed on March 26. All equipment is powered with a solar-panel battery-charging system. Rain data are seasonal.

Field Work. The station was visited 20 times to conduct discharge measurements and service the instrumentation. Field inspections for the gage are listed under site history files on the Hydstra database. Discharge measurements for the gage are listed under site gauging files on the Hydstra database.

Datum Correction. None from levels run July 2005.

Gage-Height Record. The data logger referenced to the outside gage gave a complete and satisfactory record for the year, except for the period of December 5 to March 5 when ice affected gage height.

Rating. The channel is about $10^{\prime}$ wide and straight for about $30^{\prime}$ upstream and downstream. The streambed through this reach consists of primary sand, gravel, and larger boulders. Low flow control is a rock outcrop downstream from gage about 5'. Channel is control for medium and high stages.

One discharge measurement (No. 9) and 16 inspections of no flow were made this year. All inspections of no flow used to develop a "V" diagram shift needed to adjust point of zero flow (PZF).

Rating No. 2 was developed using past discharge measurements and verified with current measurements.

Discharge. Rating No. 2 was used with "V" diagrams to compute this record.

Remarks. Records are good. 
E038 DP Canyon above TA-21

Daily Mean Discharge in Cubic Feet per Second

Water Year October 2007 to September 2008

\begin{tabular}{|c|c|c|c|c|c|c|c|c|c|c|c|c|}
\hline DAY & OCT & NOV & DEC & JAN & FEB & MAR & APR & MAY & JUN & JUL & AUG & SEP \\
\hline 1 & $.65^{\star}$ & $0^{*}$ & $6.6^{*}$ & $0^{*}$ & $0^{*}$ & $0^{*}$ & $0^{*}$ & $0^{*}$ & 0 & .30 & 0 & .64 \\
\hline 2 & $.01^{*}$ & $0^{*}$ & $0^{*}$ & $0^{*}$ & $0^{*}$ & $0^{*}$ & $0^{*}$ & $0^{*}$ & 0 & 0 & 0 & 0 \\
\hline 3 & $0^{*}$ & $0^{*}$ & $0^{*}$ & $0^{*}$ & $0^{*}$ & $0^{*}$ & $0^{*}$ & $0^{*}$ & 0 & 0 & 0 & 0 \\
\hline 4 & $0^{*}$ & $0^{*}$ & $0^{*}$ & $0^{*}$ & $0^{*}$ & $0^{*}$ & $0^{*}$ & $0^{*}$ & 0 & 0 & .61 & 0 \\
\hline 5 & $0^{*}$ & $0^{*}$ & $0^{*}$ & $0^{*}$ & $0^{*}$ & $0^{*}$ & $0^{\star}$ & $0^{*}$ & 0 & 0 & $0^{*}$ & 0 \\
\hline 6 & $0^{*}$ & $0^{*}$ & $0^{*}$ & $0^{*}$ & $0^{*}$ & $0^{*}$ & $0^{*}$ & $0^{*}$ & 0 & 0 & 0 & 0 \\
\hline 7 & $0^{*}$ & $0^{*}$ & $0^{*}$ & $0^{*}$ & $0^{*}$ & $0^{*}$ & $0^{*}$ & $0^{*}$ & 0 & .35 & 0 & 0 \\
\hline 8 & $0^{*}$ & $0^{*}$ & $0^{*}$ & $0^{*}$ & $0^{*}$ & $0^{*}$ & $0^{*}$ & $0^{*}$ & 0 & .25 & .30 & 0 \\
\hline 9 & $0^{*}$ & $0^{*}$ & $0^{*}$ & $0^{*}$ & $0^{*}$ & $0^{*}$ & $0^{*}$ & $0^{*}$ & 0 & $0^{*}$ & 2.6 & 0 \\
\hline 10 & $0^{*}$ & $0^{*}$ & $0^{*}$ & $0^{*}$ & $0^{*}$ & $0^{*}$ & $0^{*}$ & $0^{*}$ & 0 & $0^{*}$ & 2.3 & 0 \\
\hline 11 & $0^{*}$ & $0^{*}$ & $0^{*}$ & $0^{*}$ & $0^{*}$ & $0^{*}$ & $0^{*}$ & $0^{*}$ & 0 & 0 & 0 & 0 \\
\hline 12 & $0^{*}$ & $0^{*}$ & $0^{*}$ & $0^{*}$ & $0^{*}$ & $0^{*}$ & $0^{\star}$ & $0^{*}$ & 0 & 0 & .32 & 0 \\
\hline 13 & $0^{*}$ & $0^{*}$ & $0^{*}$ & $0^{*}$ & $0^{*}$ & $0^{*}$ & $0^{*}$ & $.04^{*}$ & 0 & 0 & 0 & 0 \\
\hline 14 & $0^{*}$ & $0^{*}$ & $0^{*}$ & $0^{*}$ & $0^{*}$ & $0^{*}$ & $0^{*}$ & $.17^{*}$ & 0 & 0 & $.21^{*}$ & 0 \\
\hline 15 & $0^{*}$ & $0^{*}$ & $0^{*}$ & $0^{*}$ & $0^{*}$ & $0^{*}$ & $0^{*}$ & $2.0^{*}$ & 0 & 0 & $0^{*}$ & 0 \\
\hline 16 & $0^{*}$ & $0^{*}$ & $0^{*}$ & $0^{*}$ & $0^{*}$ & $0^{*}$ & $0^{*}$ & $0^{*}$ & 0 & .43 & $1.8^{*}$ & 0 \\
\hline 17 & $0^{*}$ & $0^{*}$ & $0^{*}$ & $0^{*}$ & $0^{*}$ & $0^{*}$ & $0^{*}$ & $0^{*}$ & 0 & $0^{*}$ & $.14^{\star}$ & 0 \\
\hline 18 & $0^{*}$ & $0^{*}$ & $0^{*}$ & $0^{*}$ & $0^{*}$ & $0^{\star}$ & $0^{\star}$ & $0^{\star}$ & 0 & $0^{*}$ & $0^{*}$ & 0 \\
\hline 19 & $0^{*}$ & $0^{*}$ & $0^{*}$ & $0^{*}$ & $0^{*}$ & $0^{*}$ & $0^{*}$ & $0^{*}$ & 0 & $0 *$ & 0 & 0 \\
\hline 20 & $0^{*}$ & $0^{*}$ & $0^{*}$ & $0^{*}$ & $0^{*}$ & $0^{*}$ & $0^{*}$ & $0^{*}$ & 0 & $0^{*}$ & 0 & 0 \\
\hline 21 & $0^{*}$ & $0^{*}$ & $0^{*}$ & $0^{*}$ & $0^{*}$ & $0^{*}$ & $0^{*}$ & $0^{*}$ & 0 & $.52^{*}$ & 0 & 0 \\
\hline 22 & $0^{*}$ & $0^{*}$ & $0^{*}$ & $0^{*}$ & $0^{*}$ & $0^{*}$ & $0^{*}$ & $.01^{*}$ & 0 & $0^{*}$ & 0 & .51 \\
\hline 23 & $0^{*}$ & $.43^{\star}$ & $0^{*}$ & $0^{\star}$ & $0^{*}$ & $0^{*}$ & $0^{*}$ & $.25^{\star}$ & 0 & $0^{*}$ & 1.5 & 0 \\
\hline 24 & $0^{*}$ & $0^{*}$ & $0^{*}$ & $0^{*}$ & $0^{*}$ & $0^{*}$ & $0^{*}$ & $0^{*}$ & 0 & 0 & 1.5 & 0 \\
\hline 25 & $0^{*}$ & $0^{*}$ & $0^{*}$ & $0^{*}$ & $0^{*}$ & $0^{\star}$ & $0^{*}$ & $0^{\star}$ & 0 & 0 & .46 & 0 \\
\hline 26 & $0^{*}$ & $0^{*}$ & $0^{*}$ & $0^{*}$ & $0^{*}$ & $0^{*}$ & $.15^{*}$ & $0^{*}$ & 0 & .03 & $.29 *$ & 0 \\
\hline 27 & $0^{*}$ & $0^{*}$ & $0^{*}$ & $0^{*}$ & $0^{*}$ & $0^{*}$ & $0^{*}$ & $0^{*}$ & 0 & 0 & $0^{*}$ & 0 \\
\hline 28 & $0^{*}$ & $0^{*}$ & $0^{*}$ & $0^{*}$ & $0^{*}$ & $0^{*}$ & $0^{*}$ & $1.2^{*}$ & 0 & 0 & $0^{*}$ & 0 \\
\hline 29 & $0^{*}$ & $0^{*}$ & $0^{*}$ & $0^{*}$ & $0^{*}$ & $0^{*}$ & $0^{*}$ & $0^{\star}$ & 0 & 0 & .17 & 0 \\
\hline 30 & $0^{*}$ & $4.2^{*}$ & $0^{*}$ & $0^{*}$ & ----- & $0^{*}$ & $0^{*}$ & 0 & 0 & 0 & .01 & 0 \\
\hline 31 & $0^{*}$ & ----- & $0^{*}$ & $0^{*}$ & ----- & $0^{*}$ & ----- & 0 & ------ & 0 & 3.6 & ----- \\
\hline Total & 0.66 & 4.63 & 6.6 & 0 & 0 & 0 & 0.15 & 3.67 & 0 & 1.88 & 15.81 & 1.15 \\
\hline Mean & .021 & .15 & .21 & 0 & 0 & 0 & .005 & .12 & 0 & .061 & .51 & .038 \\
\hline Max & .65 & 4.2 & 6.6 & 0 & 0 & 0 & .15 & 2.0 & 0 & .52 & 3.6 & .64 \\
\hline Min & 0 & 0 & 0 & 0 & 0 & 0 & 0 & 0 & 0 & 0 & 0 & 0 \\
\hline Acre-Ft & 1.3 & 9.2 & 13 & 0 & 0 & 0 & .30 & 7.3 & 0 & 3.7 & 31 & 2.3 \\
\hline Wtr Year & 2008 & Total & 34.55 & Mean & & 94 & hax & 6.6 & Min & 0 & Acre-Ft & 69 \\
\hline Cal Year & 2007 & Total & 58.03 & Mean & & 16 & hax & 6.6 & Min & 0 & Acre-Ft & 115 \\
\hline
\end{tabular}

*Estimate 


\section{E038 DP Canyon above TA-21}

Daily Mean Total Rainfall in Inches

Water Year October 2007 to September 2008

\begin{tabular}{|c|c|c|c|c|c|c|c|c|c|c|c|c|}
\hline DAY & OCT & NOV & DEC & JAN & FEB & MAR & APR & MAY & JUN & JUL & AUG & SEP \\
\hline 1 & & & & & & & & 0 & 0 & .29 & 0 & .05 \\
\hline 2 & & & & & & & & 0 & 0 & .01 & 0 & 0 \\
\hline 3 & & & & & & & & 0 & 0 & 0 & 0 & .01 \\
\hline 4 & & & & & & & & 0 & 0 & 0 & .28 & 0 \\
\hline 5 & & & & & & & & 0 & .02 & .02 & 0 & 0 \\
\hline 6 & & & & & & & & 0 & 0 & 0 & 0 & 0 \\
\hline 7 & & & & & & & & 0 & 0 & .35 & .03 & 0 \\
\hline 8 & & & & & & & & 0 & 0 & .20 & .56 & 0 \\
\hline 9 & & & & & & & & 0 & 0 & 0 & .94 & .02 \\
\hline 10 & & & & & & & & 0 & 0 & 0 & .54 & 0 \\
\hline 11 & & & & & & & & 0 & 0 & .04 & 0 & 0 \\
\hline 12 & & & & & & & & 0 & 0 & 0 & .32 & 0 \\
\hline 13 & & & & & & & & .11 & 0 & 0 & .01 & 0 \\
\hline 14 & & & & & & & & .14 & 0 & 0 & .25 & 0 \\
\hline 15 & & & & & & & & .59 & 0 & .03 & 0 & 0 \\
\hline 16 & & & & & & & & 0 & 0 & .17 & .53 & 0 \\
\hline 17 & & & & & & & & 0 & 0 & .01 & .04 & 0 \\
\hline 18 & & & & & & & & 0 & 0 & .03 & 0 & 0 \\
\hline 19 & & & & & & & & 0 & 0 & 0 & 0 & 0 \\
\hline 20 & & & & & & & & 0 & 0 & 0 & .01 & .02 \\
\hline 21 & & & & & & & & 0 & .02 & .13 & 0 & 0 \\
\hline 22 & & & & & & & & .12 & 0 & .02 & 0 & .20 \\
\hline 23 & & & & & & & 0 & .17 & .02 & 0 & .74 & .01 \\
\hline 24 & & & & & & & 0 & .01 & 0 & 0 & .47 & .06 \\
\hline 25 & & & & & & & 0 & 0 & 0 & 0 & .15 & 0 \\
\hline 26 & & & & & & & 0 & 0 & 0 & .10 & .03 & 0 \\
\hline 27 & & & & & & & 0 & 0 & 0 & .02 & 0 & 0 \\
\hline 28 & & & & & & & 0 & .40 & 0 & .03 & 0 & 0 \\
\hline 29 & & & & & & & 0 & 0 & 0 & 0 & .06 & 0 \\
\hline 30 & & & & & ----- & & 0 & 0 & 0 & 0 & 0 & 0 \\
\hline 31 & & ----- & & & ------ & & ----- & 0 & ----- & 0 & 1.03 & ------ \\
\hline Total & & & & & & & 0 & 1.54 & 0.06 & 1.45 & 5.96 & 0.37 \\
\hline Max & & & & & & & 0 & .59 & .02 & .35 & 1.0 & .20 \\
\hline Wtr Year & 2008 & Total & 9.38 & & & .058 & Max & 1.0 & Min & 0 & InstMax & .28 \\
\hline Cal Year & 2007 & Total & & & & & $\operatorname{Max}$ & & Min & & InstMax & \\
\hline
\end{tabular}




\section{E039 DP Canyon below Meadow near TA-21}

Location. Lat $35^{\circ}$ 52' 41", long $106^{\circ}$ 15' 28", SE 1/4, sec. 14, T. 19 N., R. 6 E., Los Alamos County.

Drainage Area. $0.315 \mathrm{mi}^{2}$.

Period of Record. October 1, 1999, to September 30, 2007.

Revised Record. Section, township, and range (2007).

Average Discharge. 8 years, $0.12 \mathrm{ft}^{3} / \mathrm{s}, 85$ acre- $\mathrm{ft} / \mathrm{yr}$.

Gage. Data logger with cellular telemetry. Elevation of gage is 7,010 ft above NGVD from topographic map.

Remarks. Records are good except estimated daily discharges, which are fair.

Extremes for Period of Record. Maximum discharge, $200 \mathrm{ft}^{3}$ s, July 24, 2004, gage height 2.58ft. No flow most of the time.

Extremes for Current Water Year. Peak discharges above base of $50 \mathrm{ft}^{3} / \mathrm{s}$ and maximum (*):

\begin{tabular}{|c|c|c|c|}
\hline Date & Time & Discharge $\left(\mathbf{f t}^{\mathbf{3}} / \mathbf{s}\right)$ & Gage Height (ft) \\
\hline May 28 & 1615 & 62 & 1.39 \\
\hline August 9 & 1250 & 76 & 1.55 \\
\hline August 10 & 1145 & $118^{*}$ & $1.98^{*}$ \\
\hline August 23 & 1650 & 57 & 1.33 \\
\hline August 31 & 1440 & 56 & 1.32 \\
\hline
\end{tabular}

No flow at times.

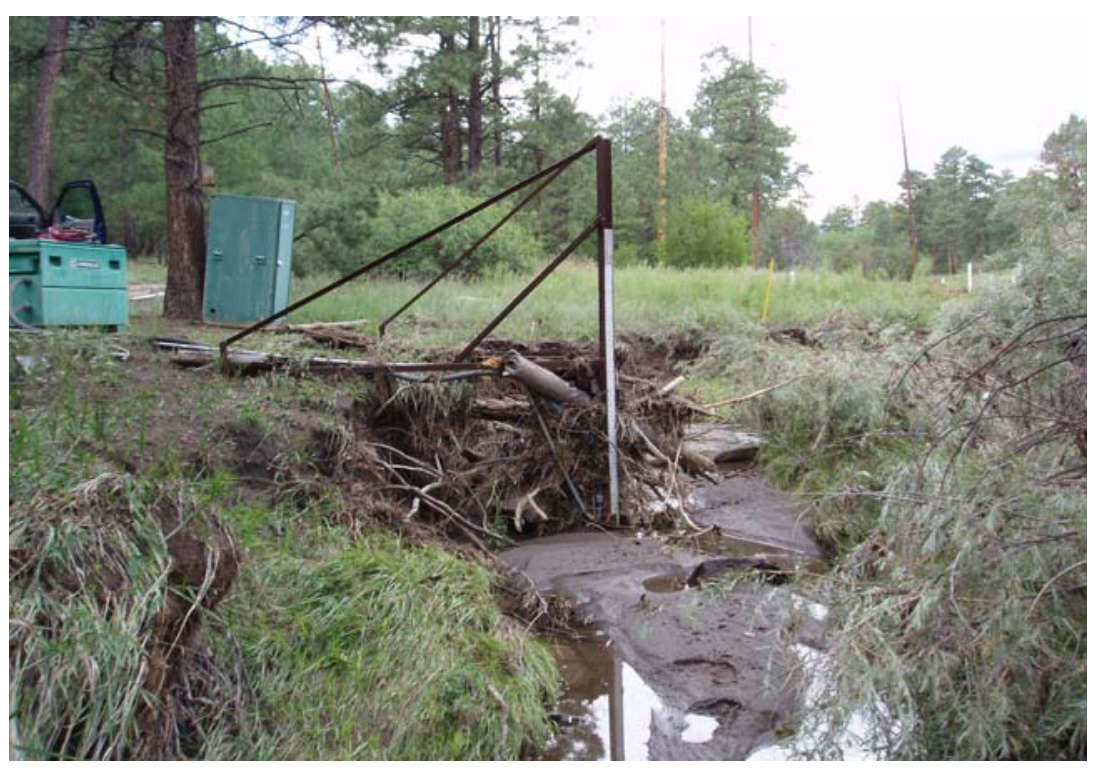




\section{Station Analysis}

\section{Water Year}

Equipment. Station is equipped with Sutron 8210 data logger (5-min. interval) and Sutron Accubar bubble sensor with cellular telemetry and speech modem. Phone upgraded June 5 from analog to digital service. The system is powered by a solar-panel battery system housed in a NEMA shelter. Station is equipped with an ISCO pump sampler for water-quality sample collection. ISCO is housed in a separate shelter, a $3^{\prime} \times$ 4 ' metal box. Sampler is triggered by stage through the data logger. An outside staff is available for reference. Low to medium flow can be waded. High flow will be measured by indirect methods.

Field Work. The station was visited 16 times to conduct discharge measurements and service the instrumentation. Field inspections for the gage are listed under site history files on the Hydstra database. Discharge measurements for the gage are listed under site gauging files on the Hydstra database.

Datum Correction. Levels run July 14, 2005, found gage within acceptable limits. Gage height corrections were applied for minor base gage and recorder difference.

Gage-Height Record. The data logger referenced to the outside staff gave a complete and satisfactory report for the water year except during the periods of December 2 to March 26 when ice affected gage height and July 6-10 and August 25 to September 30, when the data recorder malfunctioned as a result of battery failure.

Rating. The channel has rock outcrop in bed with pockets of sand mostly from deposition below riffles. Channel is straight for 50' above and below gage. A slight left bend $50^{\prime}$ below gage also has a $2^{\prime}$ drop and could act as a broad-crested weir at high flow. Banks and canyon bottom are thickly vegetated with grass.

Three discharge measurements (No. 14-16) and 16 inspections were made during the year.

Rating No. 2 was developed from 3 critical depth computations and a PZF.

Discharge. Discharge was computed by applying gage height to Rating No. 2. The channel in winter is heavily glaciated and choked with ice and snow with flow frozen dry. The winter record is published as zero based on comparison with E038 and E040. Variable shift diagrams were used at lower flows defined by discharge measurements.

Remarks. Records are good except for estimated daily discharges, which are fair 


\section{E039 DP Canyon below Meadow at TA-21}

Daily Mean Discharge in Cubic Feet per Second

Water Year October 2007 to September 2008

\begin{tabular}{|c|c|c|c|c|c|c|c|c|c|c|c|c|}
\hline DAY & OCT & NOV & DEC & JAN & FEB & MAR & APR & MAY & JUN & JUL & AUG & SEP \\
\hline 1 & .62 & .02 & 6.3 & $0^{*}$ & $0^{*}$ & 0 & .05 & .15 & .02 & .01 & .05 & $.10^{*}$ \\
\hline 2 & .04 & .02 & .01 & $0^{*}$ & $0^{*}$ & 0 & .06 & .15 & .04 & .01 & .04 & $.10^{*}$ \\
\hline 3 & .01 & .02 & $.01^{*}$ & $0^{*}$ & $0^{*}$ & .03 & .07 & .14 & .05 & .01 & .05 & $.15^{\star}$ \\
\hline 4 & 0 & .02 & $.01^{*}$ & $0^{*}$ & $0^{*}$ & 0 & .07 & .15 & .03 & .01 & .51 & $.10^{*}$ \\
\hline 5 & 0 & .02 & $.01^{*}$ & $0^{*}$ & $0^{*}$ & 0 & .08 & .18 & .02 & .01 & .03 & $.51^{*}$ \\
\hline 6 & 0 & .02 & $.01^{*}$ & $0^{*}$ & $0^{*}$ & .19 & .09 & .18 & .02 & $.01^{\star}$ & .03 & $.20^{*}$ \\
\hline 7 & 0 & .02 & $.01^{*}$ & $0^{*}$ & $0^{*}$ & $.07^{\star}$ & .10 & .20 & .02 & $.01^{*}$ & .03 & $.10^{*}$ \\
\hline 8 & 0 & .02 & $.01^{*}$ & $0^{*}$ & $0^{*}$ & $.01^{*}$ & .10 & .20 & .03 & $.01^{\star}$ & .12 & $.05^{\star}$ \\
\hline 9 & 0 & .02 & $.01^{*}$ & $0^{*}$ & $0^{*}$ & $.01^{*}$ & .10 & .20 & .04 & $.01^{*}$ & 3.2 & $2.5^{\star}$ \\
\hline 10 & 0 & .02 & $.01^{*}$ & $0^{*}$ & $0^{*}$ & $.01^{*}$ & .11 & .20 & .03 & $.01^{*}$ & 2.4 & $2.2^{*}$ \\
\hline 11 & 0 & .02 & $.01^{*}$ & $0^{*}$ & $0^{*}$ & $.03^{*}$ & .11 & .17 & .03 & .01 & .06 & $.30^{*}$ \\
\hline 12 & 0 & .02 & $.01^{*}$ & $0^{*}$ & $0^{*}$ & $.03^{\star}$ & .12 & .20 & .03 & .01 & .40 & $.10^{*}$ \\
\hline 13 & 0 & .02 & $.01^{*}$ & $0^{*}$ & $0^{*}$ & $.02 *$ & .13 & .20 & .03 & .01 & .04 & $.05^{*}$ \\
\hline 14 & 0 & .02 & $.01^{\star}$ & $0^{*}$ & $0^{*}$ & $0^{*}$ & .14 & .22 & .03 & .01 & .24 & $.01^{*}$ \\
\hline 15 & 0 & .02 & $.01^{*}$ & $0^{*}$ & $0^{*}$ & $0^{*}$ & .15 & 2.9 & .03 & .01 & .04 & $.01^{*}$ \\
\hline 16 & 0 & .02 & $.01^{*}$ & $0^{*}$ & $0^{*}$ & $.02^{*}$ & .16 & .30 & .03 & .26 & 1.7 & $1.3^{*}$ \\
\hline 17 & 0 & .02 & $.01^{*}$ & $0^{*}$ & $0^{*}$ & $.02^{\star}$ & .14 & .28 & .03 & .11 & .11 & $.20^{*}$ \\
\hline 18 & 0 & .02 & $.01^{*}$ & $0^{*}$ & $0^{*}$ & $.02^{*}$ & .13 & .26 & .03 & .03 & .04 & $.10^{*}$ \\
\hline 19 & 0 & .02 & $.01^{*}$ & $0^{*}$ & $0^{*}$ & $.02^{\star}$ & .14 & .26 & .02 & .03 & .04 & $.05^{\star}$ \\
\hline 20 & 0 & .02 & $0^{*}$ & $0^{*}$ & $0^{*}$ & $.02^{\star}$ & .14 & .27 & .02 & .04 & .04 & $.01^{*}$ \\
\hline 21 & 0 & .02 & $0^{*}$ & $0^{*}$ & $0^{*}$ & $.02^{\star}$ & .14 & .27 & .02 & .45 & .04 & $.01^{*}$ \\
\hline 22 & 0 & .02 & $0^{*}$ & $0^{*}$ & $0^{*}$ & $.02^{*}$ & .13 & .27 & .02 & .05 & .04 & $.01^{*}$ \\
\hline 23 & 0 & 0 & $0^{*}$ & $0^{*}$ & $0^{*}$ & $.02^{*}$ & .15 & .44 & .02 & .04 & 1.6 & $1.4^{*}$ \\
\hline 24 & .01 & 0 & $0^{*}$ & $0^{*}$ & $0^{*}$ & $.02^{\star}$ & .17 & .41 & .02 & .04 & 1.4 & $1.5^{\star}$ \\
\hline 25 & .01 & .01 & $0^{*}$ & $0^{*}$ & $.02^{\star}$ & $.02^{*}$ & .17 & .39 & .02 & .04 & $.36^{\star}$ & $.36^{*}$ \\
\hline 26 & .01 & 0 & $0^{*}$ & $0^{*}$ & 0 & $.03^{*}$ & .16 & .39 & .02 & .04 & $.19 *$ & $.19^{*}$ \\
\hline 27 & .01 & 0 & $0^{*}$ & $0^{*}$ & 0 & .04 & .14 & .40 & .02 & .04 & $.15^{\star}$ & $.01^{*}$ \\
\hline 28 & .01 & 0 & $0^{*}$ & $0^{*}$ & 0 & .04 & .14 & 1.5 & .01 & .04 & $.10^{\star}$ & $.01^{*}$ \\
\hline 29 & .01 & 0 & $0^{*}$ & $0^{*}$ & 0 & .05 & .15 & .04 & .01 & .04 & $.17^{*}$ & $.01^{*}$ \\
\hline 30 & .01 & 2.3 & $0^{*}$ & $0^{*}$ & ----- & .05 & .16 & .02 & .01 & .04 & $.10^{*}$ & $.01^{*}$ \\
\hline 31 & .01 & ----- & $0^{*}$ & $0^{*}$ & ----- & .05 & ------ & .02 & ------ & .05 & $.16^{\star}$ & ------ \\
\hline Total & 0.75 & 2.75 & 6.48 & 0 & 0.02 & 0.86 & 3.70 & 10.96 & 0.75 & 1.49 & 13.48 & 11.65 \\
\hline Mean & .024 & .092 & .21 & 0 & .001 & .028 & .12 & .35 & .025 & .048 & .43 & .39 \\
\hline Max & .62 & 2.3 & 6.3 & 0 & .02 & .19 & .17 & 2.9 & .05 & .45 & 3.2 & 2.5 \\
\hline Min & 0 & 0 & 0 & 0 & 0 & 0 & .05 & .02 & .01 & .01 & .03 & .01 \\
\hline Acre-Ft & 1.5 & 5.5 & 13 & 0 & .04 & 1.7 & 7.3 & 22 & 1.5 & 3.0 & 27 & 23 \\
\hline Wtr Year & 2008 & Total & 52.89 & Mean & & 14 & Max & 6.3 & Min & 0 & Acre-Ft & 105 \\
\hline Cal Year & 2007 & Total & 55.80 & Mean & & 15 & Max & 6.3 & Min & 0 & Acre-Ft & 111 \\
\hline
\end{tabular}

*Estimate 


\section{E040 DP Canyon above Los Alamos Canyon}

Location. Lat $35^{\circ}$ 52' 24", long $106^{\circ}$ 15' 34", SW 1/4, sec. 13, T. 19 N., R. 6 E., Los Alamos County.

Drainage Area. $0.60 \mathrm{mi}^{2}$.

Period of Record. May 1999 to September 30, 2008.

Revised Record. Drainage area (2006); section (2007).

Gage. Data logger and concrete control. Elevation of gage is 6,620 ft above NGVD from GPS survey.

Remarks. Records are good except estimated daily discharges, which are fair.

Average Discharge. $9 \mathrm{yr}, 0.031 \mathrm{ft}^{3} / \mathrm{s}, 22$ acre-ft/yr.

Extremes for Period of Record. Maximum discharge, $452 \mathrm{ft}^{3} / \mathrm{s}$, August 8, 2006, gage height $5.65 \mathrm{ft}$. (from slope area measurement). No flow most of the time.

Extremes for Current Water Year. Peak discharge above base of $30 \mathrm{ft}^{3} / \mathrm{s}$ and maximum $(*)$.

\begin{tabular}{|c|c|c|c|}
\hline Date & Time & Discharge (ft $\left.\mathbf{3}^{\mathbf{s}} \mathbf{s}\right)$ & Gage Height (ft) \\
\hline August 31 & 1620 & $44^{*}$ & 3.30 \\
\hline
\end{tabular}

No flow most of the time.

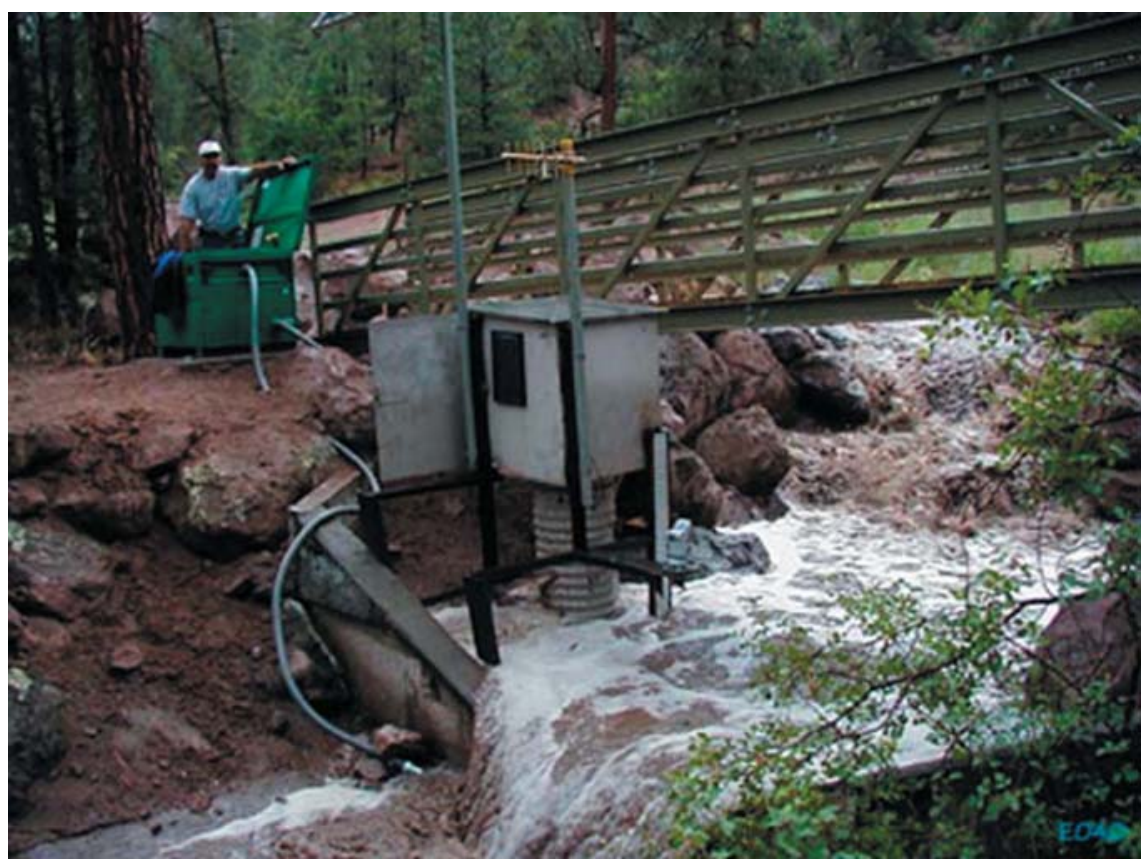




\section{E040 DP Canyon above Los Alamos Canyon}

\section{Station Analysis}

\section{Water Year}

Equipment. Station is equipped with Sutron 8210 data logger (5-min. interval) with milltronics sonic probe. The system is powered by a solar-panel battery system. All equipment is housed in a NEMA shelter. Station is equipped with an ISCO pump sampler for water-quality collection in a $3^{\prime} \times 4^{\prime}$ metal box. Sampler is triggered by stage through the data logger. An outside staff is available for reference. High flow measurements can be made from bridge upstream of gage.

Cellular telemetry removed August 28, discontinued service.

Field Work. The station was visited 29 times to conduct discharge measurements and service the instrumentation. Field inspections for the gage are listed under site history files on the Hydstra database. Discharge measurements for the gage are listed under site gauging files on the Hydstra database.

Datum Corrections. None from levels.

Gage-Height Record. The data logger referenced to the outside staff gave a complete and satisfactory record, except for the period of November 7 to March 17 when ice affected gage height. During the periods of May 15 to June 11 and August 9 to September 31, gage height was estimated because a probe malfunctioned.

Rating. The channel is about $15^{\prime}$ wide and bends to the right above gage and straight for about 100' downstream. The streambed through this reach consists of primarily sand with large boulders. The control at this site is a concrete control with a V notch in the middle for low flow. Channel becomes the control for medium to high flows.

Seven discharge measurements (Nos. 15-21, con-current dye study at site) were made this year. These measurements are based on high flows throughout year. Twenty seven inspections of no flow were made during the year.

Rating No. 3 is good up to $30 \mathrm{ft}^{3} / \mathrm{s}$ and fair above that.

Discharge. Discharge was computed using Rating No. 3. Those days estimated at zero flow were based on comparison with nearby gage stations and precipitation records. No flow most of the time.

Remarks. Records are good except for estimated daily discharges, which are fair. 


\section{E040 DP Canyon above Los Alamos Canyon}

Daily Mean Discharge in Cubic Feet per Second

Water Year October 2007 to September 2008

\begin{tabular}{|c|c|c|c|c|c|c|c|c|c|c|c|c|}
\hline DAY & ОСТ & NOV & DEC & JAN & FEB & MAR & APR & MAY & JUN & JUL & AUG & SEP \\
\hline 1 & $0^{*}$ & 0 & $.29^{*}$ & 0 & $0^{*}$ & 0 & 0 & 0 & $0^{*}$ & 0 & $0^{*}$ & $.05^{*}$ \\
\hline 2 & $0^{*}$ & 0 & $0^{*}$ & 0 & $0^{*}$ & 0 & 0 & 0 & $0^{*}$ & 0 & 0 & $0^{*}$ \\
\hline 3 & 0 & 0 & $0^{*}$ & 0 & $0^{*}$ & 0 & 0 & 0 & $0^{*}$ & 0 & 0 & $0^{*}$ \\
\hline 4 & 0 & 0 & $0^{*}$ & 0 & $0^{*}$ & 0 & 0 & 0 & $0^{*}$ & 0 & 0 & $0^{*}$ \\
\hline 5 & 0 & 0 & $0^{*}$ & 0 & $0^{*}$ & $0^{*}$ & 0 & 0 & $0^{*}$ & 0 & 0 & $0^{*}$ \\
\hline 6 & 0 & 0 & $0^{*}$ & $0^{*}$ & $0^{*}$ & $0^{*}$ & 0 & 0 & $0^{*}$ & 0 & 0 & $0^{*}$ \\
\hline 7 & 0 & $0^{*}$ & $0^{*}$ & $0^{*}$ & $0^{*}$ & 0 & 0 & 0 & $0^{*}$ & 0 & 0 & 0 * \\
\hline 8 & 0 & $0^{*}$ & $0^{*}$ & $0^{*}$ & $0^{*}$ & 0 & 0 & 0 & $0^{*}$ & 0 & .01 & $0^{*}$ \\
\hline 9 & 0 & $0^{*}$ & $0^{*}$ & $0^{*}$ & $0^{*}$ & 0 & 0 & 0 & $0^{*}$ & 0 & $.17^{*}$ & $0^{*}$ \\
\hline 10 & 0 & $0^{*}$ & $0^{*}$ & $0^{*}$ & $0^{*}$ & 0 & 0 & 0 & $0^{*}$ & $0^{*}$ & $.40^{\star}$ & $0^{*}$ \\
\hline 11 & 0 & $0^{*}$ & $0^{*}$ & 0 & $0^{*}$ & 0 & 0 & 0 & $0^{*}$ & $0^{*}$ & $0^{*}$ & $0^{*}$ \\
\hline 12 & 0 & $0^{*}$ & $0^{*}$ & 0 & $0^{*}$ & 0 & 0 & 0 & 0 & $0^{*}$ & $.29 *$ & 0 * \\
\hline 13 & 0 & $0^{*}$ & $0^{*}$ & 0 & $0^{*}$ & 0 & 0 & 0 & 0 & 0 & $.02^{\star}$ & 0 * \\
\hline 14 & 0 & $0^{*}$ & 0 & 0 & $0^{*}$ & $0^{*}$ & 0 & 0 & 0 & 0 & $0^{*}$ & $0^{*}$ \\
\hline 15 & 0 & $0^{*}$ & 0 & 0 & $0^{*}$ & $0^{*}$ & 0 & $.05^{\star}$ & 0 & 0 & $0^{*}$ & $0^{*}$ \\
\hline 16 & 0 & $0^{*}$ & 0 & 0 & $0^{*}$ & $0^{*}$ & 0 & $0^{*}$ & 0 & 0 & $.10^{*}$ & $0^{*}$ \\
\hline 17 & 0 & $0^{*}$ & 0 & 0 & $0^{*}$ & $0^{*}$ & 0 & $0^{*}$ & $0^{*}$ & 0 & $0^{*}$ & $0^{*}$ \\
\hline 18 & 0 & $0^{*}$ & 0 & 0 & $0^{*}$ & 0 & 0 & $0^{*}$ & $0^{*}$ & 0 & $0^{*}$ & $0^{*}$ \\
\hline 19 & 0 & $0^{*}$ & 0 & 0 & $0^{*}$ & 0 & 0 & $0^{*}$ & 0 & 0 & $0^{*}$ & $0^{*}$ \\
\hline 20 & 0 & $0^{*}$ & 0 & 0 & $0^{*}$ & 0 & 0 & $0^{*}$ & 0 & 0 & $0^{*}$ & $0^{*}$ \\
\hline 21 & 0 & $0^{*}$ & 0 & 0 & $0^{*}$ & 0 & 0 & $0^{*}$ & 0 & 0 & $0^{*}$ & $0^{*}$ \\
\hline 22 & 0 & $0^{*}$ & 0 & 0 & $0^{*}$ & 0 & 0 & $0^{*}$ & 0 & 0 & $0^{*}$ & $0^{*}$ \\
\hline 23 & 0 & $0^{*}$ & 0 & 0 & $0^{*}$ & 0 & 0 & $0^{*}$ & 0 & 0 & $.96^{\star}$ & $0^{*}$ \\
\hline 24 & 0 & $0^{*}$ & 0 & 0 & 0 & 0 & 0 & $0^{*}$ & 0 & $0^{*}$ & $0^{*}$ & $0^{*}$ \\
\hline 25 & 0 & 0 & 0 & 0 & 0 & 0 & 0 & $0^{*}$ & 0 & $0^{*}$ & $0^{*}$ & 0 * \\
\hline 26 & 0 & 0 & 0 & 0 & 0 & 0 & 0 & $0^{*}$ & 0 & $0^{*}$ & $0^{*}$ & $0^{*}$ \\
\hline 27 & 0 & 0 & 0 & 0 & 0 & 0 & 0 & $0^{*}$ & 0 & $0^{*}$ & $0^{*}$ & $0^{*}$ \\
\hline 28 & 0 & 0 & 0 & $.34^{\star}$ & 0 & 0 & 0 & $.03^{*}$ & 0 & $0^{*}$ & $0^{*}$ & $0^{*}$ \\
\hline 29 & 0 & 0 & 0 & $0^{*}$ & 0 & 0 & 0 & $0^{*}$ & 0 & $0^{*}$ & $0^{*}$ & $0^{*}$ \\
\hline 30 & 0 & .09 & 0 & $0^{*}$ & ---- & 0 & 0 & $0^{*}$ & 0 & $0^{*}$ & 0 & $0^{*}$ \\
\hline 31 & 0 & ------ & 0 & $0^{*}$ & ----- & 0 & ------ & $0^{*}$ & ------ & $0^{*}$ & $2.0^{\star}$ & ----- \\
\hline Total & 0 & 0.09 & 0.29 & 0.34 & 0 & 0 & 0 & 0.08 & 0 & 0 & 3.95 & 0.05 \\
\hline Mean & 0 & .003 & .009 & .011 & 0 & 0 & 0 & .003 & 0 & 0 & .13 & .002 \\
\hline Max & 0 & .09 & .29 & .34 & 0 & 0 & 0 & .05 & 0 & 0 & 2.0 & .05 \\
\hline Min & 0 & 0 & 0 & 0 & 0 & 0 & 0 & 0 & 0 & 0 & 0 & 0 \\
\hline Acre-Ft & 0 & .18 & .58 & .67 & 0 & 0 & 0 & .16 & 0 & 0 & 7.8 & .10 \\
\hline Wtr Year & 2008 & Total & 4.80 & Mean & & & hax & 2.0 & Min & 0 & Acre-Ft & 9.5 \\
\hline Cal Year & 2007 & Total & 17.83 & Mean & & & hax & .78 & Min & 0 & Acre-Ft & 35 \\
\hline
\end{tabular}

*Estimate 


\section{E042 Los Alamos Canyon above SR 4}

Location. Lat $35^{\circ} 52^{\prime}$ 01", long $106^{\circ} 13^{\prime}$ 25", NW 1/4, sec. 20, T. 19 N., R. 7 E., Santa Fe County.

Drainage Area. $10.11 \mathrm{mi}^{2}$.

Period of Record. November 1970 to June 1971 and October 1991 to September 30, 2008.

Revised Record. Drainage area (2006); quarter (2007); peak discharges for 2006 (2007).

Gage. Data logger and rain gage with cellular telemetry and concrete control. Elevation of gage is $6,300 \mathrm{ft}$ above NGVD from GPS survey.

Remarks. Records are good. Flow partially regulated by Los Alamos Reservoir, which is about $7.8 \mathrm{mi}$ upstream.

Average Discharge. $14 \mathrm{yr}, 0.27 \mathrm{ft}$ /s, 193 acre- $\mathrm{ft} / \mathrm{yr}$.

Extremes for Period of Record. Maximum discharge, $240 \mathrm{ft}^{3} / \mathrm{s}$, August 8, 2006, gage height $3.76 \mathrm{ft}$ (from flood marks). No flow at times.

Extremes for Current Water Year. Peak discharges above base of $40 \mathrm{ft}^{3} / \mathrm{s}$ and maximum $(*)$ :

\begin{tabular}{|c|c|c|c|}
\hline Date & Time & Discharge $\left.\mathbf{( f t}^{\mathbf{3}} \mathbf{s}\right)$ & Gage Height (ft) \\
\hline December 1 & 0310 & $61^{*}$ & $2.41^{*}$ \\
\hline August 10 & 1250 & 58 & 2.39 \\
\hline
\end{tabular}

No flow at times.

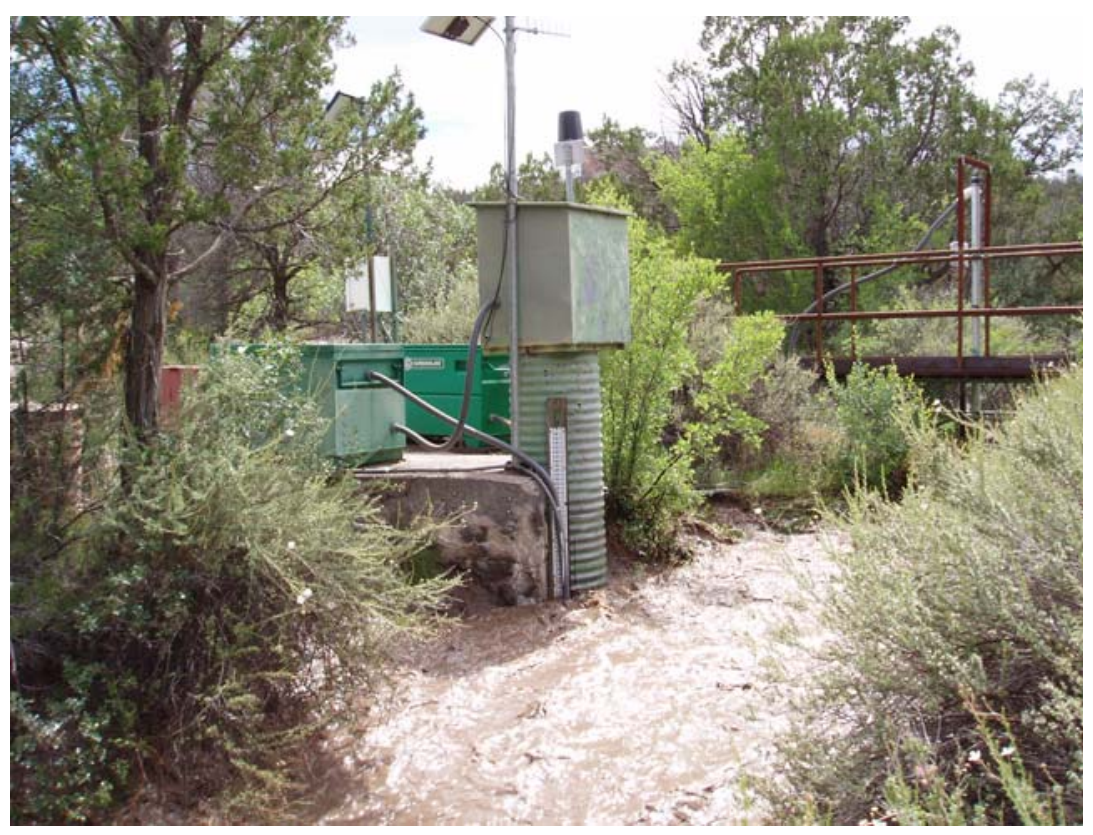




\section{E042 Los Alamos Canyon above SR 4}

\section{Station Analysis}

\section{Water Year}

Equipment. Station is equipped with Sutron 8210 data logger (5-min. interval) with quadrature encoder driven by float tape in stilling well and cellular telemetry with speech modem. Phone upgraded May 9 from analog to digital service. System is powered by a solar-panel battery system housed in a NEMA shelter. Station is equipped with an ISCO pump sampler for water-quality sample collection. ISCO is housed in a separate shelter, a $3^{\prime} \times 4^{\prime}$ metal box. Sampler is triggered by stage through the data logger. Outside staff available for reference. Control is broad-crested weir that has deteriorated somewhat over the years but is still fairly stable. Footbridge is available for high flow discharge measurements.

Station is also equipped with a tipping bucket rain gage, Rain Collection II. All equipment is powered with a solar-panel battery-charging system. Rain data are seasonal.

Field Work. This station was visited 28 times to conduct discharge measurements and service the instrumentation. Field inspections for the gage are listed under site history files on the Hydstra database. Discharge measurements for the gage are listed under site gauging files on the Hydstra database.

Datum Correction. None.

Gage-Height Record. The data logger referenced to the outside staff gave a complete and satisfactory record for the year, except for the period of December to March when ice affected gage and the periods of June 11-30, July 7-16, and August 31 to September 8, when the data logger malfunctioned as a result of battery failure.

Rating. Streambed consists of sand and gravel and channel is straight for over 150' above and below broad-crested weir. Fill and scour, mostly fill, of the gage pool occurs during most flow events.

Nine discharge measurements (Nos. 57-65) were made and 20 inspections of no flow were made. Measurements Nos. 57, 58 were critical depths. Shifts were small and variable and applied only to low flows.

Rating No. 4 was developed from current measurements and some measurements from previous years. No shifts applied above $1.0 \mathrm{ft}^{3} / \mathrm{s}$.

Discharge. Discharge computed from Rating No. 4 using V diagrams, with no shifts on high flows. Those days estimated were based on precipitation and nearby gage stations for verification.

Remarks. Records are good except for estimated daily discharges, which are poor. 
E042 Los Alamos Canyon above SR 4

Daily Mean Discharge in Cubic Feet per Second

Water Year October 2007 to September 2008

\begin{tabular}{|c|c|c|c|c|c|c|c|c|c|c|c|c|}
\hline DAY & OCT & NOV & DEC & JAN & FEB & MAR & APR & MAY & JUN & JUL & AUG & SEP \\
\hline 1 & $0^{*}$ & $0^{*}$ & $10^{*}$ & $0^{*}$ & $0^{*}$ & $0^{*}$ & $1.8^{*}$ & $.20^{*}$ & $0^{*}$ & 0 & 0 & $.20^{*}$ \\
\hline 2 & $0^{*}$ & $0^{*}$ & $0^{*}$ & $0^{*}$ & $0^{*}$ & $0^{*}$ & $1.7^{\star}$ & $.20^{*}$ & $0^{*}$ & 0 & 0 & 0 * \\
\hline 3 & $0^{*}$ & $0^{*}$ & $0^{*}$ & $0^{*}$ & $0^{*}$ & $0^{*}$ & $1.6^{*}$ & $.14^{*}$ & $0^{*}$ & 0 & 0 & 0 * \\
\hline 4 & $0^{*}$ & $0^{*}$ & $0^{*}$ & $0^{*}$ & $0^{*}$ & $0^{*}$ & $1.5^{*}$ & $.06^{*}$ & $0^{*}$ & 1.2 & 0 & $0^{*}$ \\
\hline 5 & $0^{*}$ & $0^{*}$ & $0^{*}$ & $0^{*}$ & $0^{*}$ & $0^{*}$ & $1.4^{*}$ & $.01^{*}$ & $0^{*}$ & .88 & 0 & $0^{*}$ \\
\hline 6 & $0^{*}$ & $0^{*}$ & $0^{*}$ & $0^{*}$ & $0^{*}$ & $0^{*}$ & $1.1^{*}$ & 0 * & $0^{*}$ & 0 & 0 & $0^{*}$ \\
\hline 7 & $0^{*}$ & $0^{*}$ & $0^{*}$ & $0^{*}$ & $0^{*}$ & $0^{*}$ & $.98^{\star}$ & 0 * & $0^{*}$ & $0^{*}$ & 0 & 0 * \\
\hline 8 & $0^{*}$ & $0^{*}$ & $.30^{\star}$ & $0^{*}$ & $0^{*}$ & $0^{*}$ & $.92^{\star}$ & $0^{*}$ & 0 * & $0^{*}$ & 0 & 0 * \\
\hline 9 & $0^{*}$ & $0^{*}$ & $.27^{\star}$ & $0^{*}$ & $0^{*}$ & $0^{*}$ & $1.0^{*}$ & $0^{*}$ & $0^{*}$ & $0^{*}$ & 1.5 & 0 \\
\hline 10 & $0^{*}$ & $0^{*}$ & $3.9^{*}$ & $0^{*}$ & $0^{*}$ & $0^{*}$ & $.99^{*}$ & $0^{*}$ & $0^{*}$ & $0^{*}$ & 1.8 & 0 \\
\hline 11 & $0^{*}$ & $0^{*}$ & $2.7^{*}$ & $0^{*}$ & $0^{*}$ & $0^{*}$ & $.82^{\star}$ & $0^{*}$ & $0^{*}$ & $0^{*}$ & 0 & 0 \\
\hline 12 & $0^{*}$ & $0^{*}$ & $0^{*}$ & $0^{*}$ & $0^{*}$ & $0^{*}$ & $.67^{\star}$ & $0^{*}$ & $0^{*}$ & $0^{*}$ & 0 & 0 \\
\hline 13 & $0^{*}$ & $0^{*}$ & $0^{*}$ & $0^{*}$ & $0^{*}$ & $0^{*}$ & $.49^{\star}$ & 0 * & $0^{*}$ & $0^{*}$ & 0 & 0 \\
\hline 14 & $0^{*}$ & $0^{*}$ & $0^{*}$ & $0^{*}$ & $0^{*}$ & $0^{*}$ & $.34^{*}$ & $0^{*}$ & $0^{*}$ & $0^{*}$ & 0 & 0 \\
\hline 15 & $0^{*}$ & $0^{*}$ & $0^{*}$ & $0^{*}$ & $0^{*}$ & $.19 *$ & $.17^{*}$ & $0^{*}$ & $0^{*}$ & $0^{*}$ & 0 & 0 \\
\hline 16 & $0^{*}$ & $0^{*}$ & $0^{*}$ & $0^{*}$ & $0^{*}$ & $.35^{*}$ & $.07^{*}$ & $.06^{*}$ & $0^{*}$ & $0^{*}$ & .41 & 0 \\
\hline 17 & $0^{*}$ & $0^{*}$ & $0^{*}$ & $0^{*}$ & $0^{*}$ & $.48^{\star}$ & $.22^{*}$ & $0^{*}$ & $0^{*}$ & 0 & 0 & 0 \\
\hline 18 & $0^{*}$ & $0^{*}$ & $0^{*}$ & $0^{*}$ & $0^{*}$ & $.42^{\star}$ & $.47^{*}$ & $0^{*}$ & $0^{*}$ & 0 & 0 & 0 \\
\hline 19 & $0^{*}$ & $0^{*}$ & $0^{*}$ & $0^{*}$ & $0^{*}$ & $.22^{\star}$ & $.49^{*}$ & 0 * & 0 * & 0 & 0 & 0 \\
\hline 20 & $0^{*}$ & $0^{*}$ & $0^{*}$ & $0^{*}$ & $0^{*}$ & $.18^{*}$ & $.34^{*}$ & 0 * & $0^{*}$ & 0 & 0 & 0 \\
\hline 21 & $0^{*}$ & $0^{*}$ & $0^{*}$ & $0^{*}$ & $0^{*}$ & $.04^{*}$ & $.23^{*}$ & $0^{*}$ & $0^{*}$ & 0 & 0 & 0 \\
\hline 22 & $0^{*}$ & $0^{*}$ & $0^{*}$ & $0^{*}$ & $0^{*}$ & $.37^{\star}$ & $.20^{*}$ & $0^{*}$ & $0^{*}$ & 0 & 0 & 0 \\
\hline 23 & $0^{*}$ & $0^{*}$ & $0^{*}$ & $0^{*}$ & $0^{*}$ & $.59^{*}$ & $.24^{*}$ & $0^{*}$ & $0^{*}$ & 0 & .58 & 0 \\
\hline 24 & $0^{*}$ & $0^{*}$ & $0^{*}$ & $0^{*}$ & $0^{*}$ & $.77^{\star}$ & $.24^{*}$ & $0^{*}$ & $0^{*}$ & 0 & .30 & 0 \\
\hline 25 & $0^{*}$ & $0^{*}$ & $0^{*}$ & $0^{*}$ & $0^{*}$ & $.82^{\star}$ & $.37^{\star}$ & $0^{*}$ & $0^{*}$ & 0 & .30 & 0 \\
\hline 26 & $0^{*}$ & $0^{*}$ & $0^{*}$ & $0^{*}$ & $0^{*}$ & $.95^{*}$ & $.50^{*}$ & $0^{*}$ & $0^{*}$ & 0 & 0 & 0 \\
\hline 27 & $0^{*}$ & $0^{*}$ & $0^{*}$ & $0^{*}$ & $0^{*}$ & $1.3^{*}$ & $.49^{*}$ & $0^{*}$ & $0^{*}$ & 0 & 0 & 0 \\
\hline 28 & $0^{*}$ & $0^{*}$ & $0^{*}$ & $2.4^{*}$ & $0^{*}$ & $1.7^{*}$ & $.47^{*}$ & $.48^{\star}$ & $0^{*}$ & 0 & 0 & 0 \\
\hline 29 & $0^{*}$ & $0^{*}$ & $0^{*}$ & $.10^{*}$ & $0^{*}$ & $1.8^{*}$ & $.39 *$ & .01 & $0^{*}$ & 0 & 0 & 0 \\
\hline 30 & $0^{*}$ & $.10^{*}$ & $0^{*}$ & $0^{*}$ & ----- & $1.8^{*}$ & $.27^{*}$ & 0 & $0^{\star}$ & 0 & 0 & 0 \\
\hline 31 & $0^{*}$ & ----- & $0^{*}$ & $0^{*}$ & ------ & $1.7^{*}$ & ----- & $0^{*}$ & ----- & 0 & $1.4^{*}$ & ----- \\
\hline Total & 0 & 0.10 & 17.17 & 2.50 & 0 & 13.68 & 20.47 & 1.16 & 0 & 2.08 & 6.29 & 0.20 \\
\hline Mean & 0 & .003 & .55 & .081 & 0 & .44 & .68 & .037 & 0 & .067 & .20 & .007 \\
\hline Max & 0 & .10 & 10 & 2.4 & 0 & 1.8 & 1.8 & .48 & 0 & 1.2 & 1.8 & .20 \\
\hline Min & 0 & 0 & 0 & 0 & 0 & 0 & .07 & 0 & 0 & 0 & 0 & 0 \\
\hline Acre-Ft & 0 & .20 & 34 & 5.0 & 0 & 27 & 41 & 2.3 & 0 & 4.1 & 12 & .40 \\
\hline Wtr Year & 2008 & Total & 63.65 & Mean & & 17 & $\operatorname{Max}$ & 10 & Min & 0 & Acre-Ft & 126 \\
\hline Cal Year & 2007 & Total & 128.66 & Mean & & 35 & $\operatorname{Max}$ & 10 & Min & 0 & Acre-Ft & 255 \\
\hline
\end{tabular}

*Estimate 
E042 Los Alamos Canyon above SR 4

Daily Total Rainfall in Inches

Water Year October 2007 to September 2008

\begin{tabular}{|c|c|c|c|c|c|c|c|c|c|c|c|c|}
\hline DAY & ОСТ & NOV & DEC & JAN & FEB & MAR & APR & MAY & JUN & JUL & AUG & SEP \\
\hline 1 & & & & & & & & & 0 & 0 & 0 & .04 \\
\hline 2 & & & & & & & & & 0 & .06 & 0 & $0^{*}$ \\
\hline 3 & & & & & & & & & 0 & 0 & 0 & $0^{*}$ \\
\hline 4 & & & & & & & & & 0 & .01 & .16 & $0^{*}$ \\
\hline 5 & & & & & & & & & .03 & .02 & 0 & $0^{*}$ \\
\hline 6 & & & & & & & & & 0 & 0 & .01 & $0^{*}$ \\
\hline 7 & & & & & & & & & 0 & .28 & 0 & $0^{*}$ \\
\hline 8 & & & & & & & & & 0 & .18 & .27 & 0 \\
\hline 9 & & & & & & & & 0 & 0 & .01 & .31 & 0 \\
\hline 10 & & & & & & & & 0 & $0^{*}$ & 0 & .15 & 0 \\
\hline 11 & & & & & & & & 0 & $0^{*}$ & .04 & .01 & 0 \\
\hline 12 & & & & & & & & 0 & $0^{*}$ & 0 & .11 & .05 \\
\hline 13 & & & & & & & & 0 & $0^{*}$ & 0 & 0 & 0 \\
\hline 14 & & & & & & & & .11 & $0^{*}$ & 0 & .14 & 0 \\
\hline 15 & & & & & & & & .49 & $0^{*}$ & .28 & 0 & 0 \\
\hline 16 & & & & & & & & .01 & $0^{*}$ & .07 & .34 & 0 \\
\hline 17 & & & & & & & & 0 & $0^{*}$ & .03 & .07 & 0 \\
\hline 18 & & & & & & & & 0 & $0^{*}$ & .01 & 0 & 0 \\
\hline 19 & & & & & & & & 0 & $0^{*}$ & 0 & 0 & 0 \\
\hline 20 & & & & & & & & 0 & $0^{*}$ & 0 & .02 & 0 \\
\hline 21 & & & & & & & & 0 & $0^{*}$ & .05 & 0 & 0 \\
\hline 22 & & & & & & & & .09 & $0^{*}$ & .58 & 0 & .03 \\
\hline 23 & & & & & & & & .11 & $.01^{*}$ & 0 & .09 & 0 \\
\hline 24 & & & & & & & & 0 & $0^{*}$ & .01 & .27 & 0 \\
\hline 25 & & & & & & & & 0 & $0^{*}$ & 0 & 0 & 0 \\
\hline 26 & & & & & & & & 0 & $0^{*}$ & .25 & 0 & 0 \\
\hline 27 & & & & & & & & 0 & $0^{*}$ & .14 & 0 & 0 \\
\hline 28 & & & & & & & & .07 & $0^{*}$ & .01 & 0 & 0 \\
\hline 29 & & & & & & & & 0 & $0^{*}$ & 0 & .16 & 0 \\
\hline 30 & & & & & ----- & & & 0 & $0^{*}$ & 0 & 0 & 0 \\
\hline 31 & & ------ & & & ----- & & ----- & 0 & ------ & 0 & .42 & ----- \\
\hline Total & 0.22 & 0 & & & & & & 0.39 & 0.03 & 2.02 & 2.53 & 0.12 \\
\hline $\operatorname{Max}$ & .19 & 0 & & & & & & .11 & .03 & .58 & .42 & .05 \\
\hline Wtr Year & 2008 & Total & 5.31 & Mean & & .035 & Max & .58 & Min & 0 & InstMax & .19 \\
\hline Cal Year & 2007 & Total & 11.20 & Mean & & .044 & Max & .87 & Min & 0 & InstMax & .35 \\
\hline
\end{tabular}

*Estimate 


\section{E050 Los Alamos Canyon below Los Alamos Weir}

Location. Lat $35^{\circ}$ 52' 71", long $106^{\circ} 13^{\prime}$ 0.03", NE 1/4, sec. 20, T. 19N., R. 7E., Los Alamos County.

Drainage Area. $10.42 \mathrm{mi}^{2}$.

Period of Record. May 2001 to September 30, 2008.

Revised Record. Drainage area (2006).

Gage. Data logger. Elevation of gage is 6,345 ft above NGVD from GPS survey.

Remarks. Records are good except for estimated daily discharges, which are fair. Flows partially regulated by broad-crested weir $200 \mathrm{ft}$ upstream.

Average Discharge. $7 \mathrm{yr}, 0.22 \mathrm{ft}^{3} / \mathrm{s}, 157$ acre-ft/yr.

Extremes for Period of Record. Maximum discharge, $252 \mathrm{ft}^{3} / \mathrm{s}$ August 08, 2006, gage height $3.20 \mathrm{ft}$ (from slope area measurement). No flow most of the time.

Extremes for Current Year. Peak discharge above base of $30 \mathrm{ft}^{3} / \mathrm{s}$ and maximum (*):

\begin{tabular}{|c|c|c|c|}
\hline Date & Time & Discharge $\left(\mathbf{f t}^{\mathbf{3}} / \mathbf{s}\right)$ & Gage Height (ft) \\
\hline December 1 & 0325 & $36^{*}$ & $1.52^{*}$ \\
\hline
\end{tabular}

No flow most of the time.

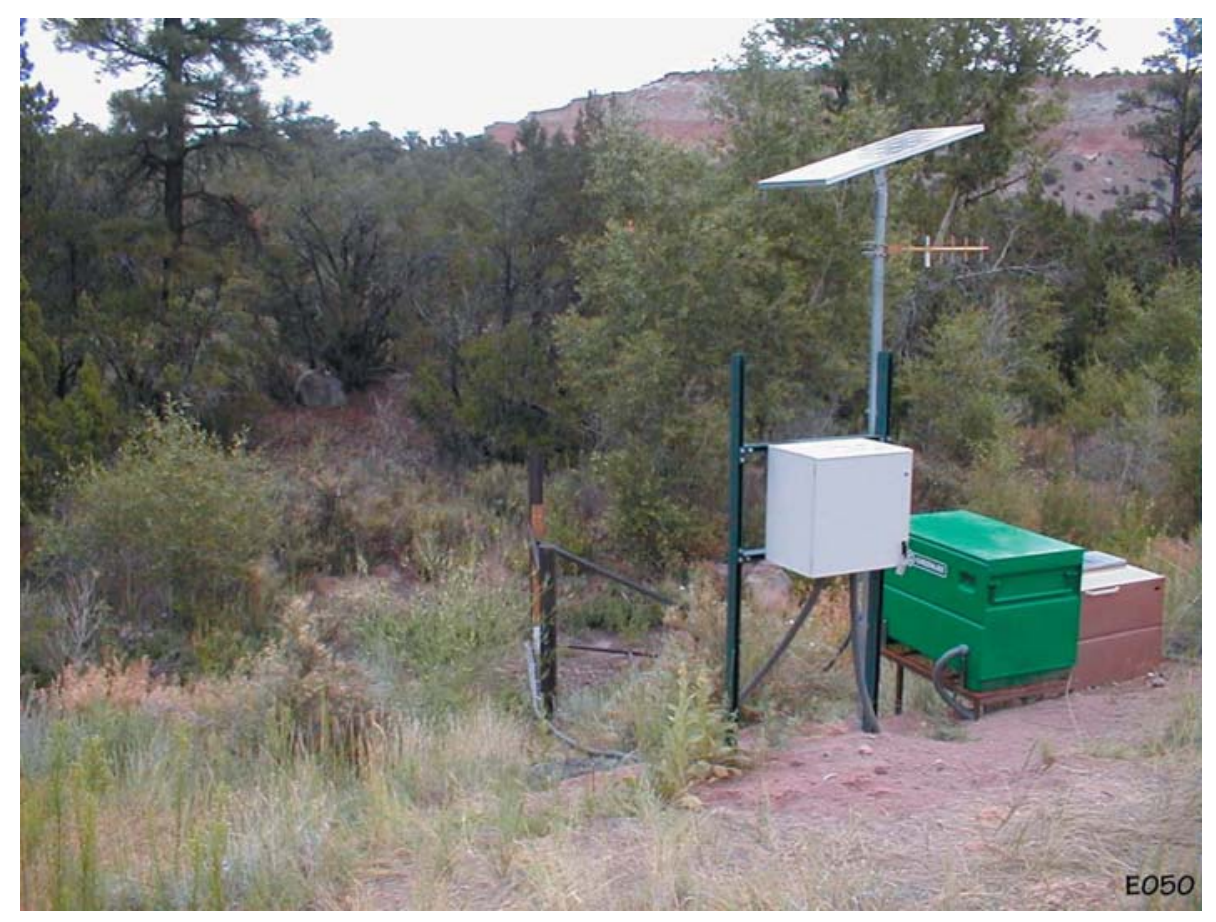




\section{E050 Los Alamos Canyon below Los Alamos Weir}

\section{Station Analysis}

\section{Water Year}

Equipment. Station is equipped with Sutron 8210 data logger (5-min. interval) and Sutron Accubar bubble sensor. System is powered by a solar-panel battery system housed in a NEMA shelter. Station is equipped with an ISCO pump sampler for waterquality sample collection. ISCO is housed in a separate shelter, a $3^{\prime} \times 4^{\prime}$ metal box. Sampler is triggered by stage through the data logger. No provision for discharge measurements above wading stage.

Cellular telemetry removed March 17, service discontinued.

Field Work. The station was visited 24 times to conduct discharge measurements and service the instrumentation. Field inspections for the gage are listed under site history files on the Hydstra database. Discharge measurements for the gage are listed under site gauging files on the Hydstra database.

Datum Correction. None. Levels of May 31, 2001, found gage within limits; no correction needed.

Gage-Height Record. The data logger referenced to the outside staff gave a complete and satisfactory record except during the periods of December 1-19, January 10-29, and February 2-11, when ice affected gage height.

Rating. The channel is straight for 100' upstream and downstream. Bed consists of large gravel and is well armored and should not be subject to much movement. Channel is trapezoidal with little or no vegetation. Flow is regulated somewhat by detention weir just upstream.

Two discharge measurements (Nos. 36-37) and 21 inspections of no flow were made during the year.

Rating No. 2 was developed based on all measurements made during the period of record. Shifts are small and variable. Flows are very flashy, less than an hour, and daily mean discharges are very small in relation to the instantaneous peak.

Discharge. Discharge was computed from Rating No. 2, with shifts applied by stage diagram.

Remarks. Records are good except for estimated daily discharges, which are fair. 
E050 Los Alamos Canyon below Los Alamos Weir

Daily Mean Discharge in Cubic Feet per Second

Water Year October 2007 to September 2008

\begin{tabular}{|c|c|c|c|c|c|c|c|c|c|c|c|c|}
\hline DAY & OCT & NOV & DEC & JAN & FEB & MAR & APR & MAY & JUN & JUL & AUG & SEP \\
\hline 1 & 0 & 0 & $7.5^{*}$ & 0 & 0 & 0 & .65 & 0 & 0 & 0 & 0 & .05 \\
\hline 2 & 0 & 0 & $0^{*}$ & 0 & 0 & 0 & .60 & 0 & 0 & 0 & 0 & 0 \\
\hline 3 & 0 & 0 & $0^{*}$ & 0 & 0 & 0 & .52 & 0 & 0 & 0 & 0 & 0 \\
\hline 4 & 0 & 0 & $0^{*}$ & 0 & $0^{*}$ & 0 & .43 & 0 & 0 & .21 & 0 & 0 \\
\hline 5 & 0 & 0 & $0^{*}$ & 0 & $0^{*}$ & 0 & .36 & 0 & 0 & .44 & 0 & 0 \\
\hline 6 & 0 & 0 & $0^{*}$ & 0 & $0^{*}$ & 0 & .10 & 0 & 0 & 0 & 0 & 0 \\
\hline 7 & 0 & 0 & $0^{*}$ & 0 & $0^{*}$ & 0 & $.08^{\star}$ & 0 & 0 & 0 & 0 & 0 \\
\hline 8 & 0 & 0 & $0^{*}$ & 0 & $0^{*}$ & 0 & 0 & 0 & 0 & 0 & 0 & 0 \\
\hline 9 & 0 & 0 & $0^{*}$ & 0 & $0^{*}$ & 0 & $0^{*}$ & 0 & 0 & 0 & .30 & 0 \\
\hline 10 & 0 & 0 & $0^{*}$ & $0^{*}$ & $0^{*}$ & 0 & $0^{*}$ & 0 & 0 & 0 & .73 & 0 \\
\hline 11 & 0 & 0 & $0^{*}$ & $0^{*}$ & $0^{*}$ & 0 & 0 & 0 & 0 & 0 & 0 & 0 \\
\hline 12 & 0 & 0 & $0^{*}$ & $0^{*}$ & 0 & 0 & 0 & 0 & 0 & 0 & 0 & 0 \\
\hline 13 & 0 & 0 & $0^{*}$ & $0^{*}$ & 0 & 0 & 0 & 0 & 0 & 0 & 0 & 0 \\
\hline 14 & 0 & 0 & $0^{*}$ & $0^{*}$ & 0 & 0 & 0 & 0 & 0 & 0 & 0 & 0 \\
\hline 15 & 0 & 0 & $0^{*}$ & $0^{*}$ & 0 & 0 & 0 & 0 & 0 & 0 & 0 & 0 \\
\hline 16 & 0 & 0 & $0^{*}$ & $0^{*}$ & 0 & 0 & 0 & 0 & 0 & 0 & .06 & 0 \\
\hline 17 & 0 & 0 & $0^{*}$ & $0^{*}$ & 0 & 0 & 0 & 0 & 0 & 0 & 0 & 0 \\
\hline 18 & 0 & 0 & $0^{*}$ & $0^{\star}$ & 0 & 0 & 0 & 0 & 0 & 0 & 0 & 0 \\
\hline 19 & 0 & 0 & $0^{*}$ & $0^{*}$ & 0 & 0 & .04 & 0 & 0 & 0 & 0 & 0 \\
\hline 20 & 0 & 0 & 0 & $0^{*}$ & 0 & 0 & .02 & 0 & 0 & 0 & 0 & 0 \\
\hline 21 & 0 & 0 & 0 & $0^{*}$ & 0 & 0 & 0 & 0 & 0 & 0 & 0 & 0 \\
\hline 22 & 0 & 0 & 0 & $0^{*}$ & 0 & 0 & 0 & 0 & 0 & 0 & 0 & 0 \\
\hline 23 & 0 & 0 & 0 & $0^{*}$ & 0 & 0 & 0 & 0 & 0 & 0 & .14 & 0 \\
\hline 24 & 0 & 0 & 0 & $0^{*}$ & 0 & 0 & 0 & 0 & 0 & 0 & .07 & 0 \\
\hline 25 & 0 & 0 & 0 & $0^{*}$ & 0 & 0 & 0 & 0 & 0 & 0 & .07 & 0 \\
\hline 26 & 0 & 0 & 0 & $0^{*}$ & 0 & .05 & .10 & 0 & 0 & 0 & 0 & 0 \\
\hline 27 & 0 & 0 & 0 & $0^{*}$ & 0 & .21 & .08 & 0 & 0 & 0 & 0 & 0 \\
\hline 28 & 0 & 0 & 0 & $1.8^{*}$ & 0 & .55 & .05 & 0 & 0 & 0 & 0 & 0 \\
\hline 29 & 0 & 0 & 0 & $0^{*}$ & 0 & .72 & 0 & 0 & 0 & 0 & 0 & 0 \\
\hline 30 & 0 & .03 & 0 & $0^{*}$ & ---- & .67 & 0 & 0 & 0 & 0 & 0 & 0 \\
\hline 31 & 0 & ----- & 0 & 0 & ---- & .62 & ------ & 0 & ------ & 0 & .42 & ----- \\
\hline Total & 0 & 0.03 & 7.5 & 1.8 & 0 & 2.82 & 3.03 & 0 & 0 & 0.65 & 1.79 & 0.05 \\
\hline Mean & 0 & .001 & .24 & .058 & 0 & .091 & .10 & 0 & 0 & .021 & .058 & .002 \\
\hline Max & 0 & .03 & 7.5 & 1.8 & 0 & .72 & .65 & 0 & 0 & .44 & .73 & .05 \\
\hline Min & 0 & 0 & 0 & 0 & 0 & 0 & 0 & 0 & 0 & 0 & 0 & 0 \\
\hline Acre-Ft & 0 & .06 & 15 & 3.6 & 0 & 5.6 & 6.0 & 0 & 0 & 1.3 & 3.6 & .10 \\
\hline Wtr Year & 2008 & Total & 17.67 & Mean & & & hax & 7.5 & Min & 0 & Acre-Ft & 35 \\
\hline Cal Year & 2007 & Total & 59.49 & Mean & & 16 & hax & 7.5 & Min & 0 & Acre-Ft & 118 \\
\hline
\end{tabular}

*Estimate 


\section{E055 Pueblo Canyon above Acid Canyon}

Location. Lat. $35^{\circ}$ 53' 20", long $106^{\circ}$ 18' 14", SE 1/4, Sec. 9, T 19 N., R 6 E., Los Alamos County.

Drainage Area. $3.42 \mathrm{mi}^{2}$.

Period of Record. October 1, 2002, to September 30, 2008.

Revised Record. Average discharge (this report). Average discharge for 2007, 5 yr, $0.30 \mathrm{ft}^{3} / \mathrm{s}, 219$ acre-ft/yr.

Gage. Data logger. Elevation of gage is 6,945 ft above NGVD from topographic map.

Remarks. Records are fair to poor.

Average Discharge. $6 \mathrm{yr}, 0.26 \mathrm{ft}^{3} / \mathrm{s}, 186$ acre-ft/yr.

Extremes for Period Outside of Record. Maximum discharge, 1,600 $\mathrm{ft}^{3} / \mathrm{s}$ (from slopearea computation), July 2, 2001, gage height $7.3 \mathrm{ft}$ (from flood mark). No flow most of the time.

Extremes for Period of Record. Maximum discharge, 1,780 ft ${ }^{3} / \mathrm{s}$, August 8, 2006, gage height $7.50 \mathrm{ft}$ (from critical depth computation). No flow most of time.

Extremes for Current Year. Peak discharge above base of $20 \mathrm{ft}^{3} / \mathrm{s}$ and maximum (*):

\begin{tabular}{|c|c|c|c|}
\hline Date & Time & Discharge $\left.\mathbf{( f t}^{\mathbf{3}} / \mathbf{s}\right)$ & Gage Height (ft) \\
\hline January 28 & 1445 & $68^{*}$ & $3.21^{*}$ \\
\hline
\end{tabular}

No flow most of the time.

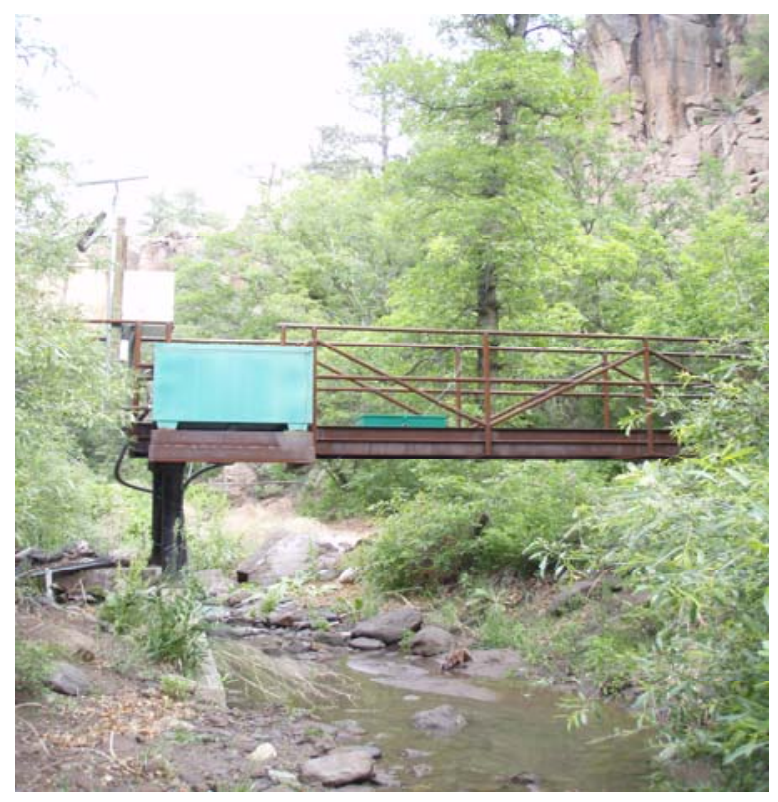




\section{E055 Pueblo Canyon above Acid Canyon}

\section{Station Analysis}

\section{Water Year}

Equipment. Station is equipped with Sutron 8210 data logger (5-min. interval) and Sutron Accubar bubble sensor. System is powered by a solar-panel battery system housed in a NEMA shelter. Station is equipped with ISCO pump sampler for waterquality sample collection. ISCO is housed in separate shelter, a $3^{\prime} \times 4^{\prime}$ metal box. Sampler is triggered by stage through the data logger. An outside staff is available for reference. No provision for direct discharge measurements above wading stage.

Cellular telemetry removed March 19, service discontinued.

Field Work. The station was visited 18 times conduct discharge measurements and service the instrumentation. Field inspections for the gage are listed under site history files on the Hydstra database. Discharge measurements for the gage are listed under site gauging files on the Hydstra database.

Datum Correction. None.

Gage-Height Record. The data logger referenced to the outside staff gave a complete and satisfactory record, except during the period of November 30 to March 19 when ice affected gage height.

Rating. Channel comes into gage from a left to right bend and bends hard left about 100' below gage. Bed consists of unstable sand and gravel with some boulders. The left bank downstream from gage is heavily wooded and that tends to hold the flow to the right, away from the reach of the gage. Lower end of any stage-discharge relation here will be unstable. The upper end could be stable, but floods and construction have enabled the flow to jump channel at the bend above the gage and allow significant flow to go down the road not in accordance with recorded gage heights. This jump-out occurs at approximately $500 \mathrm{ft}^{3} / \mathrm{s}$. This channel problem was corrected in March 2007.

Twelve discharge measurements (Nos. 34-45) were made during the year, measurements (Nos. 36-38) being dye study and only used to verify rating at upper end. Five inspections of no flow were observed

Rating No. 1 was developed using a slope- area computation of about $1,400 \mathrm{ft}^{3} / \mathrm{s}$, one critical depth measurement of $850 \mathrm{ft}^{3} / \mathrm{s}$, and various low flow measurements. Low water definition is poor and high end needs to be confirmed. Low end of rating was verified by dye study and used to enhance rating. At the time of this analysis, Rating No. 1 is fair.

Discharge. Discharge was computed using Rating No. 1 and a series of " $V$ " diagrams. High flows were computed directly.

Remarks. Records are fair to poor. 


\section{E055 Pueblo Canyon above Acid Canyon}

Daily Mean Discharge in Cubic Feet per Second

Water Year October 2007 to September 2008

\begin{tabular}{|c|c|c|c|c|c|c|c|c|c|c|c|c|}
\hline DAY & ОСТ & NOV & DEC & JAN & FEB & MAR & APR & MAY & JUN & JUL & AUG & SEP \\
\hline 1 & .41 & 0 & $.02^{\star}$ & $0^{*}$ & 0 & $.01^{*}$ & .04 & 0 & $0^{*}$ & 0 & 0 & .18 \\
\hline 2 & $.38^{\star}$ & 0 & $.01^{*}$ & $0^{*}$ & 0 & $.02^{\star}$ & .05 & 0 & 0 & 0 & 0 & .14 \\
\hline 3 & $.31^{*}$ & 0 & $.02^{\star}$ & $0^{*}$ & 0 & $.02^{*}$ & .06 & 0 & 0 & 0 & 0 & .11 \\
\hline 4 & .28 & 0 & $.01^{*}$ & $0^{*}$ & 0 & .02 & .06 & 0 & 0 & 0 & .03 & .09 \\
\hline 5 & .24 & 0 & $.01^{*}$ & $0^{*}$ & 0 & .01 & .07 & 0 & 0 & 0 & .05 & .07 \\
\hline 6 & .20 & 0 & $0^{*}$ & $0^{*}$ & 0 & .01 & .07 & 0 & 0 & 0 & .05 & .06 \\
\hline 7 & .14 & 0 & $0^{*}$ & $0^{*}$ & 0 & 0 & .07 & $.07^{\star}$ & 0 & 0 & .05 & .05 \\
\hline 8 & .09 & 0 & $.01^{*}$ & $0^{*}$ & 0 & 0 & .08 & $0^{*}$ & 0 & 0 & .11 & .04 \\
\hline 9 & .03 & 0 & $.02^{\star}$ & $0^{*}$ & 0 & 0 & .08 & $0^{*}$ & 0 & 0 & .20 & .02 \\
\hline 10 & .02 & 0 & $.01^{*}$ & $0^{*}$ & .02 & 0 & .08 & $0^{*}$ & 0 & 0 & .12 & .01 \\
\hline 11 & .02 & 0 & $.02^{\star}$ & $.01^{*}$ & .06 & 0 & .09 & 0 & 0 & 0 & .06 & 0 \\
\hline 12 & .01 & 0 & $.02^{\star}$ & $.01^{*}$ & .02 & 0 & .09 & 0 & 0 & 0 & .07 & 0 \\
\hline 13 & 0 & 0 & $.01^{*}$ & $.01^{*}$ & .03 & 0 & .09 & 0 & 0 & 0 & .05 & 0 \\
\hline 14 & 0 & 0 & $.01^{*}$ & $.01^{*}$ & .09 & .01 & .09 & 0 & 0 & 0 & .06 & 0 \\
\hline 15 & 0 & 0 & $.01^{*}$ & $.01^{*}$ & .08 & 0 & .08 & .08 & 0 & 0 & .08 & 0 \\
\hline 16 & 0 & 0 & $.02^{*}$ & $0^{*}$ & $.02^{\star}$ & $.02 *$ & .06 & $.04^{\star}$ & 0 & 0 & .16 & 0 \\
\hline 17 & 0 & 0 & $.02^{*}$ & 0 & $.02^{\star}$ & $.01^{*}$ & .06 & $0^{*}$ & 0 & 0 & .15 & 0 \\
\hline 18 & 0 & 0 & $.02^{\star}$ & 0 & $.02^{\star}$ & $.10^{\star}$ & .05 & $0^{*}$ & 0 & 0 & .13 & 0 \\
\hline 19 & 0 & 0 & $.02^{\star}$ & 0 & .03 & $.15^{\star}$ & .04 & $0^{*}$ & 0 & 0 & .09 & 0 \\
\hline 20 & 0 & 0 & $.01^{*}$ & 0 & .02 & 0 & .03 & $0^{*}$ & 0 & 0 & .04 & 0 \\
\hline 21 & 0 & 0 & $.01^{*}$ & 0 & .02 & 0 & .01 & 0 & 0 & 0 & .02 & 0 \\
\hline 22 & 0 & 0 & $.01^{*}$ & 0 & .01 & 0 & 0 & 0 & 0 & 0 & .01 & 0 \\
\hline 23 & 0 & 0 & $.01^{*}$ & 0 & .03 & 0 & 0 & 0 & 0 & 0 & .05 & 0 \\
\hline 24 & 0 & 0 & $.01^{*}$ & 0 & $.01^{*}$ & 0 & 0 & 0 & 0 & 0 & .11 & 0 \\
\hline 25 & 0 & 0 & $.02^{\star}$ & 0 & $.02^{\star}$ & 0 & 0 & 0 & 0 & 0 & .22 & 0 \\
\hline 26 & 0 & 0 & $.01^{*}$ & 0 & $.03^{*}$ & .01 & 0 & 0 & 0 & 0 & .14 & 0 \\
\hline 27 & 0 & 0 & $0^{*}$ & 0 & $.02^{\star}$ & .02 & 0 & 0 & 0 & 0 & .09 & 0 \\
\hline 28 & 0 & 0 & $0^{*}$ & .99 & $.01^{*}$ & .02 & 0 & .02 & 0 & 0 & .06 & 0 \\
\hline 29 & 0 & 0 & $0^{*}$ & 0 & $.01^{*}$ & .03 & 0 & $.01^{\star}$ & 0 & 0 & .06 & 0 \\
\hline 30 & 0 & $0^{*}$ & $0^{*}$ & 0 & ----- & .03 & 0 & $0^{*}$ & 0 & 0 & .06 & 0 \\
\hline 31 & 0 & ----- & $0^{*}$ & 0 & ----- & .04 & ----- & $0^{*}$ & ----- & 0 & .27 & ----- \\
\hline Total & 2.13 & 0 & 0.34 & 1.04 & 0.57 & 0.53 & 1.35 & 0.22 & 0 & 0 & 2.59 & 0.77 \\
\hline Mean & .069 & 0 & .011 & .034 & .020 & .017 & .045 & .007 & 0 & 0 & .084 & .026 \\
\hline Max & .41 & 0 & .02 & .99 & .09 & .15 & .09 & .08 & 0 & 0 & .27 & .18 \\
\hline Min & 0 & 0 & 0 & 0 & 0 & 0 & 0 & 0 & 0 & 0 & 0 & 0 \\
\hline Acre-Ft & 4.2 & 0 & .67 & 2.1 & 1.1 & 1.1 & 2.7 & .44 & 0 & 0 & 5.1 & 1.5 \\
\hline Wtr Year & 2008 & Total & 9.54 & Mean & & 26 & hax & .99 & Min & 0 & Acre-Ft & 19 \\
\hline Cal Year & 2007 & Total & 31.43 & Mean & & & Иax & .93 & Min & 0 & Acre-Ft & 62 \\
\hline
\end{tabular}

*Estimate 


\section{E0555 South Fork of Acid Canyon}

Location. Lat $35^{\circ}$ 53' 10", long $106^{\circ} 18^{\prime}$ 26", SE 1/4, Sec. 9, T 19 N., R 6 E., Los Alamos County.

Drainage Area. $0.08 \mathrm{mi}^{2}$.

Period of Record. August 18, 2004, to September 30, 2008.

Revised Record. Period of record (this report).

Gage. Data logger and rain gage with cellular telemetry. Elevation of gage is $7,100 \mathrm{ft}$ above NGVD from GPS survey.

Remarks. Records are good.

Extremes for Period of Record. Maximum discharge, $108 \mathrm{ft}^{3} / \mathrm{s}$, August 08, 2006, gage height $6.22 \mathrm{ft}$. (from critical depth computation). No flow most of the time.

Extremes for Current Year. Peak discharges above base of $20 \mathrm{ft}^{3} / \mathrm{s}$ and maximum (*):

\begin{tabular}{|c|c|c|c|}
\hline Date & Time & Discharge $\left(\mathbf{f t}^{\mathbf{3}} / \mathbf{s}\right)$ & Gage Height (ft) \\
\hline December 26 & 1700 & 35 & 5.64 \\
\hline February 24 & 1905 & $37^{*}$ & $5.67^{*}$ \\
\hline May 28 & 1545 & 23 & 5.45 \\
\hline
\end{tabular}

No flow most of the time.

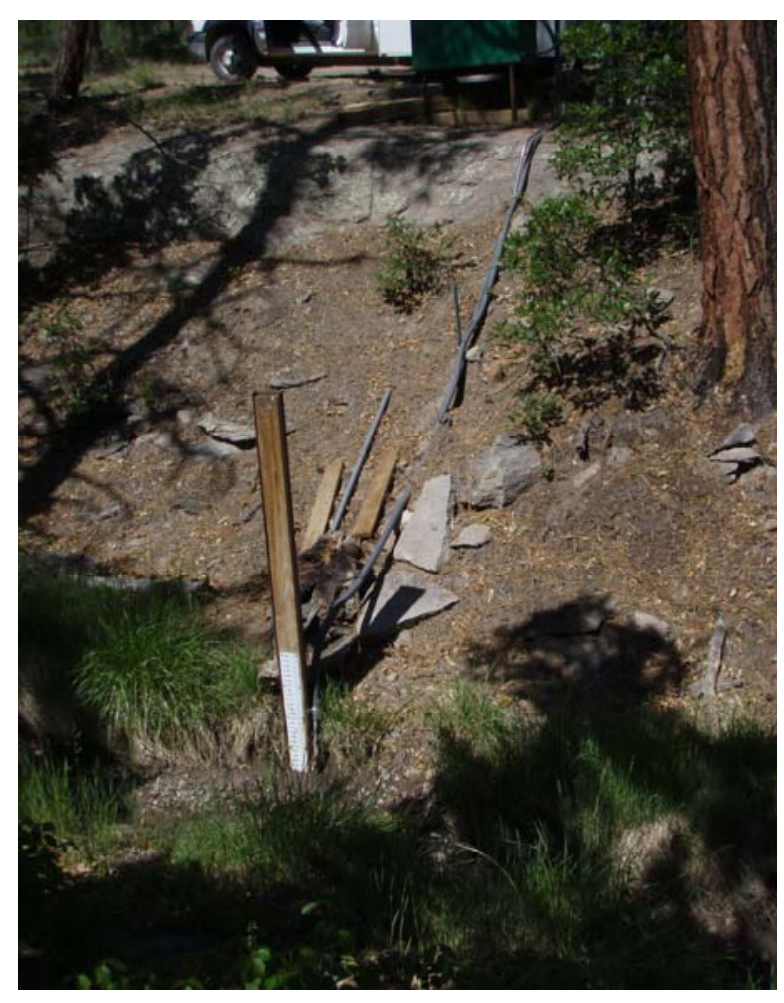




\section{E0555 South Fork of Acid Canyon}

\section{Station Analysis}

\section{Water Year}

Equipment. Station is equipped with Sutron 8210 data logger (5-min. interval) with a Sutron Accubar bubble sensor and cellular telemetry with speech modem housed in a NEMA shelter on left bank. Phone upgraded May 9 from analog to digital service. The system is powered by a solar-panel battery system. The station is equipped with ISCO pump samplers for water-quality sample collection. Samples are trigged by stage through the data logger. Samplers are housed in a separate shelter, a $3^{\prime} \times 4^{\prime}$ metal box. An outside staff gage is available for reference. No provision for discharge measurements above wading stage.

Station is also equipped with a tipping bucket rain gage, Rain Collection II. All equipment is powered with a solar-panel battery-charging system.

Field Work. The station was visited 40 times to conduct discharge measurements and service the instrumentation. Field inspections for the gage are listed under site history files on the Hydstra database. Discharge measurements for the gage are listed under site gauging files on the Hydstra database.

Datum Correction. None. Levels from November 8, 2005, found gage within limits. No corrections needed.

Gage-Height Record. The data logger referenced to the outside staff gave a complete and satisfactory record, except for the periods of December 23-29, January 6-10, and January 27 to March 1 when ice affected gage height and the periods of July 7-20 and July 28 to August 18 when the data logger malfunctioned as a result of battery failure.

Rating. The channel is straight for about $75^{\prime}$ upstream and $100^{\prime}$ downstream. The channel is trapezoidal with little vegetation. Bed consists of rock with gravel and should not be subject to very much movement.

Three discharge measurements (Nos. 5-7) were made and 23 inspections of no flow were made.

Rating No. 1 was developed by one discharge measurement of low flow and one slope area measurement of peak flow. Rating curve was extended to 6.22, based on a critical depth computation.

Discharge. Discharge was computed by applying Rating No. 1. Shift was applied with a "V" diagram for the entire year. Days estimated were based on field notes.

Remarks. Records are good except for estimated daily discharges, which are fair. 
E0555 South Fork of Acid Canyon

Daily Mean Discharge in Cubic Feet per Second

Water Year October 2007 to September 2008

\begin{tabular}{|c|c|c|c|c|c|c|c|c|c|c|c|c|}
\hline DAY & OCT & NOV & DEC & JAN & FEB & MAR & APR & MAY & JUN & JUL & AUG & SEP \\
\hline 1 & 0 & 0 & .62 & 0 & $0^{*}$ & $0^{*}$ & 0 & 0 & 0 & 0 & $0^{*}$ & 0 \\
\hline 2 & 0 & 0 & 0 & 0 & $0^{*}$ & 0 & 0 & 0 & 0 & 0 & $0^{*}$ & 0 \\
\hline 3 & 0 & 0 & 0 & 0 & $0^{*}$ & 0 & 0 & 0 & 0 & 0 & $0^{*}$ & 0 \\
\hline 4 & 0 & 0 & 0 & 0 & $0^{*}$ & 0 & 0 & 0 & 0 & 0 & $0^{*}$ & 0 \\
\hline 5 & 0 & 0 & 0 & 0 & $0^{*}$ & 0 & 0 & 0 & 0 & 0 & $0^{*}$ & 0 \\
\hline 6 & 0 & 0 & 0 & $0^{*}$ & $0^{*}$ & 0 & 0 & 0 & 0 & 0 & $0^{*}$ & 0 \\
\hline 7 & 0 & 0 & 0 & $0^{*}$ & $0^{*}$ & 0 & 0 & 0 & 0 & $0^{*}$ & $0^{*}$ & 0 \\
\hline 8 & 0 & 0 & 0 & $0^{*}$ & $0^{*}$ & 0 & 0 & 0 & 0 & $0^{*}$ & $0^{*}$ & 0 \\
\hline 9 & 0 & 0 & 0 & $0^{*}$ & $0^{*}$ & 0 & 0 & 0 & 0 & $0^{*}$ & $.50^{\star}$ & 0 \\
\hline 10 & 0 & 0 & 0 & $0^{*}$ & $0^{*}$ & .08 & 0 & 0 & 0 & $0^{*}$ & $.30^{\star}$ & 0 \\
\hline 11 & 0 & 0 & 0 & 0 & $0^{*}$ & .03 & 0 & 0 & 0 & $0^{*}$ & $0^{*}$ & 0 \\
\hline 12 & 0 & 0 & 0 & 0 & $0^{*}$ & 0 & 0 & 0 & 0 & $0^{*}$ & $0^{*}$ & 0 \\
\hline 13 & 0 & 0 & 0 & 0 & $0^{*}$ & 0 & 0 & 0 & 0 & $0^{*}$ & $0^{*}$ & 0 \\
\hline 14 & 0 & 0 & 0 & 0 & $0^{*}$ & 0 & 0 & 0 & 0 & $0^{*}$ & $0^{*}$ & 0 \\
\hline 15 & 0 & 0 & 0 & 0 & $0^{*}$ & 0 & 0 & $.49^{\star}$ & 0 & $0^{*}$ & $0^{*}$ & 0 \\
\hline 16 & 0 & 0 & 0 & 0 & $0^{*}$ & 0 & 0 & 0 & 0 & $0^{*}$ & $0^{*}$ & 0 \\
\hline 17 & 0 & 0 & .20 & 0 & $0^{*}$ & 0 & 0 & 0 & 0 & $0^{*}$ & $.10^{*}$ & 0 \\
\hline 18 & 0 & 0 & 0 & 0 & $0^{*}$ & 0 & 0 & 0 & 0 & $0^{*}$ & $.05^{\star}$ & 0 \\
\hline 19 & 0 & 0 & 0 & 0 & $0^{*}$ & 0 & 0 & 0 & 0 & $0^{*}$ & 0 & 0 \\
\hline 20 & 0 & 0 & 0 & 0 & $0^{*}$ & 0 & 0 & 0 & 0 & $0^{*}$ & 0 & 0 \\
\hline 21 & 0 & 0 & 0 & 0 & $0^{*}$ & 0 & 0 & 0 & 0 & 0 & 0 & 0 \\
\hline 22 & 0 & 0 & 0 & 0 & $.01^{*}$ & 0 & 0 & 0 & 0 & 0 & 0 & 0 \\
\hline 23 & 0 & 0 & $0^{*}$ & 0 & $.02^{\star}$ & 0 & 0 & 0 & 0 & 0 & 0 & 0 \\
\hline 24 & 0 & 0 & $0^{*}$ & 0 & $.05^{\star}$ & 0 & 0 & 0 & 0 & 0 & 0 & 0 \\
\hline 25 & 0 & 0 & $0^{*}$ & 0 & $.03^{\star}$ & 0 & 0 & 0 & 0 & 0 & $0^{*}$ & 0 \\
\hline 26 & 0 & 0 & $0^{*}$ & 0 & $.02^{\star}$ & 0 & 0 & 0 & 0 & 0 & 0 & 0 \\
\hline 27 & 0 & 0 & $0^{*}$ & $0^{*}$ & $0^{*}$ & 0 & 0 & 0 & 0 & 0 & 0 & 0 \\
\hline 28 & 0 & 0 & $0^{*}$ & $0^{*}$ & $0^{*}$ & 0 & 0 & .18 & 0 & $0^{*}$ & 0 & 0 \\
\hline 29 & 0 & 0 & $0^{*}$ & $0^{*}$ & $0^{*}$ & 0 & 0 & 0 & 0 & $0^{*}$ & 0 & 0 \\
\hline 30 & 0 & .18 & 0 & $0^{*}$ & ------ & 0 & 0 & 0 & 0 & $0^{*}$ & 0 & 0 \\
\hline 31 & 0 & ------ & 0 & $0^{*}$ & ------ & 0 & ------ & 0 & ----- & $0^{*}$ & .16 & ------ \\
\hline Total & 0 & 0.18 & 0.82 & 0 & 0.13 & 0.11 & 0 & 0.67 & 0 & 0 & 1.11 & 0 \\
\hline Mean & 0 & .006 & .026 & 0 & .005 & .004 & 0 & .022 & 0 & 0 & .036 & 0 \\
\hline Max & 0 & .18 & .62 & 0 & .05 & .08 & 0 & .49 & 0 & 0 & .50 & 0 \\
\hline Min & 0 & 0 & 0 & 0 & 0 & 0 & 0 & 0 & 0 & 0 & 0 & 0 \\
\hline Acre-Ft & 0 & .36 & 1.6 & 0 & .26 & .22 & 0 & 1.3 & 0 & 0 & 2.2 & 0 \\
\hline Wtr Year & 2008 & Total & 3.02 & Mean & & 08 & hax & .62 & Min & 0 & Acre-Ft & 6.0 \\
\hline Cal Year & 2007 & Total & 15.23 & Mean & & 42 & hax & 1.7 & Min & 0 & Acre-Ft & 30 \\
\hline
\end{tabular}

*Estimate 
E0555 South Fork Acid Canyon

Daily Mean Discharge in Cubic Feet per Second

Water Year October 2007 to September 2008

\begin{tabular}{|c|c|c|c|c|c|c|c|c|c|c|c|c|}
\hline DAY & OCT & NOV & DEC & JAN & FEB & MAR & APR & MAY & JUN & JUL & AUG & SEP \\
\hline 1 & & & & & & & 0 & 0 & 0 & $.23^{*}$ & 0 & .06 \\
\hline 2 & & & & & & & 0 & 0 & 0 & $0^{*}$ & 0 & 0 \\
\hline 3 & & & & & & & 0 & 0 & 0 & $0^{*}$ & 0 & .01 \\
\hline 4 & & & & & & & 0 & 0 & 0 & $0^{*}$ & .32 & 0 \\
\hline 5 & & & & & & & 0 & 0 & .04 & $.02^{*}$ & $0^{*}$ & 0 \\
\hline 6 & & & & & & & 0 & 0 & 0 & $0^{*}$ & $0^{*}$ & 0 \\
\hline 7 & & & & & & & 0 & 0 & 0 & $.33^{*}$ & $.27^{\star}$ & 0 \\
\hline 8 & & & & & & & 0 & 0 & 0 & $.32^{*}$ & .43 & 0 \\
\hline 9 & & & & & & & 0 & 0 & 0 & $0^{*}$ & .43 & 0 \\
\hline 10 & & & & & & & .01 & 0 & 0 & $0^{*}$ & .44 & 0 \\
\hline 11 & & & & & & & 0 & 0 & 0 & $.04^{*}$ & 0 & 0 \\
\hline 12 & & & & & & & .04 & 0 & 0 & $0^{*}$ & .10 & 0 \\
\hline 13 & & & & & & & 0 & $.13^{*}$ & 0 & $0^{*}$ & .01 & 0 \\
\hline 14 & & & & & & & 0 & $.13^{*}$ & 0 & $0^{*}$ & .04 & 0 \\
\hline 15 & & & & & & 0 & 0 & $.63^{\star}$ & 0 & $.04^{*}$ & 0 & 0 \\
\hline 16 & & & & & & 0 & 0 & 0 & 0 & $.20^{*}$ & .38 & 0 \\
\hline 17 & & & & & & 0 & 0 & 0 & 0 & $.02^{*}$ & .06 & 0 \\
\hline 18 & & & & & & 0 & 0 & 0 & 0 & $.09^{*}$ & 0 & .03 \\
\hline 19 & & & & & & 0 & 0 & $0^{*}$ & 0 & $0^{*}$ & 0 & .03 \\
\hline 20 & & & & & & 0 & 0 & 0 & 0 & $0^{*}$ & .03 & 0 \\
\hline 21 & & & & & & 0 & 0 & 0 & 0 & $.22^{\star}$ & .01 & 0 \\
\hline 22 & & & & & & 0 & 0 & .28 & 0 & 0 & 0 & .13 \\
\hline 23 & & & & & & 0 & 0 & .16 & .02 & 0 & .42 & 0 \\
\hline 24 & & & & & & 0 & 0 & 0 & $0^{*}$ & $.01^{*}$ & .34 & .11 \\
\hline 25 & & & & & & 0 & 0 & 0 & $0^{*}$ & 0 & .19 & 0 \\
\hline 26 & & & & & & 0 & 0 & 0 & $0^{*}$ & $.01^{*}$ & .10 & 0 \\
\hline 27 & & & & & & 0 & 0 & 0 & $0^{*}$ & $.06^{*}$ & 0 & 0 \\
\hline 28 & & & & & & 0 & 0 & .46 & $0^{*}$ & $.05^{\star}$ & 0 & 0 \\
\hline 29 & & & & & & 0 & 0 & 0 & $.09^{*}$ & 0 & .03 & 0 \\
\hline 30 & & & & & ------ & 0 & 0 & 0 & $0^{*}$ & 0 & .08 & 0 \\
\hline 31 & & ----- & & & ----- & 0 & ----- & 0 & ----- & 0 & .91 & ----- \\
\hline Total & & & & & & 0 & 0.17 & 1.79 & 0.15 & 1.64 & 4.32 & 0.37 \\
\hline Mean & & & & & & 0 & .006 & .058 & .005 & 0.053 & .15 & .012 \\
\hline $\operatorname{Max}$ & & & & & & 0 & .12 & .63 & .09 & .33 & .91 & .13 \\
\hline Min & & & & & & 0 & 0 & 0 & 0 & 0 & 0 & 0 \\
\hline Wtr Year & 2008 & Total & 8.44 & & & .023 & Max & .91 & Min & 0 & InstMax & .24 \\
\hline Cal Year & 2007 & Total & & & & & Max & & Min & & InstMax & \\
\hline
\end{tabular}

*Estimate 


\section{E056 Acid Canyon above Pueblo Canyon}

Location. Lat $35^{\circ}$ 53' 19", long $106^{\circ} 18^{\prime}$ 14" SE 1/4, Sec. 9, T 19 N., R 6 E., Los Alamos County.

Drainage Area. $0.452 \mathrm{mi}^{2}$.

Period of Record. October 1, 2006, to September 30, 2008.

Gage. Data logger. Elevation of gage is 6,944 above NGVD.

Remarks. Records are fair to poor. Records for this site existed before period of record but are not reliable.

Average Discharge. $6 \mathrm{yr}, 0.68 \mathrm{ft}^{3} / \mathrm{s}, 491$ acre-ft/yr.

Extremes for Period of Record. Maximum discharge, $216 \mathrm{ft}^{3} / \mathrm{s}$, July 26, 2007, gage height $2.81 \mathrm{ft}$. No flow most of the time.

Extremes for Current Year. Peak discharges above base of $50 \mathrm{ft}^{3} / \mathrm{s}$ and maximum (*):

\begin{tabular}{|c|c|c|c|}
\hline Date & Time & Discharge $\left.\mathbf{( f t}^{\mathbf{3}} / \mathbf{s}\right)$ & Gage Height (ft) \\
\hline December 1 & 0320 & $81^{*}$ & $2.17^{*}$ \\
\hline
\end{tabular}

No flow most of the time.

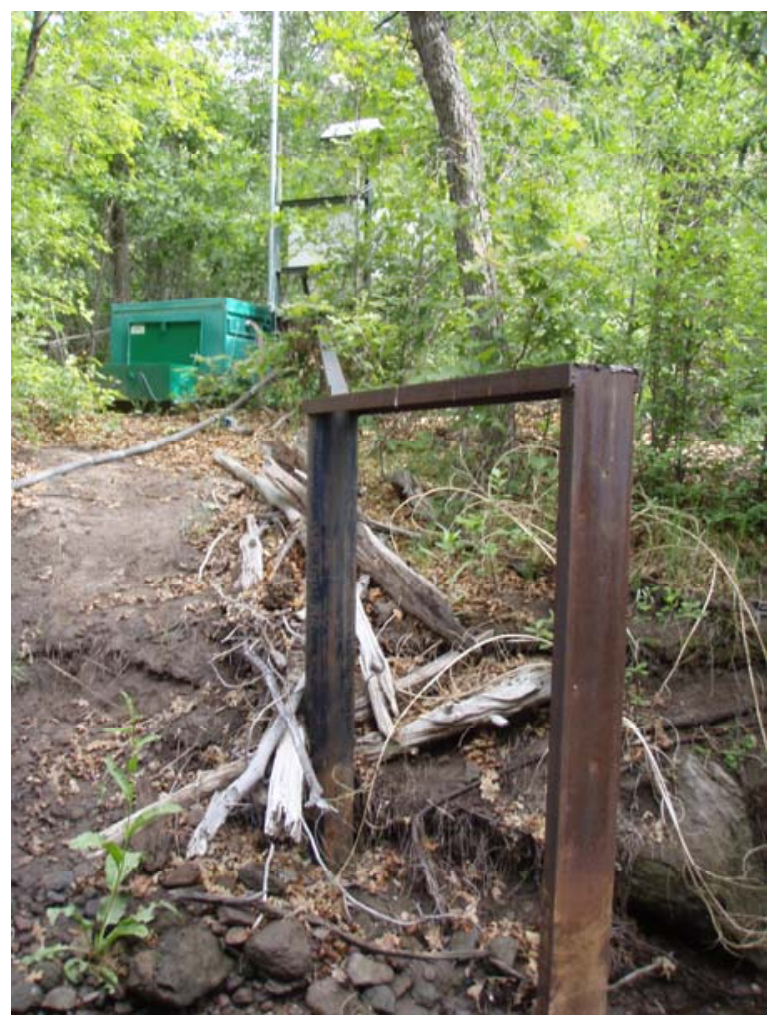




\section{E056 Acid Canyon above Pueblo Canyon}

\section{Station Analysis}

\section{Water Year}

Equipment. Station is equipped with Sutron 8210 data logger (5-min. interval) with a Sutron Accubar bubble sensor mounted on a 6" channel cantilevered over streambed. The system is powered by a solar-panel battery system housed in a NEMA shelter. Station is equipped with an ISCO pump sampler for water-quality sample collection. ISCO is housed in a separate shelter, a $3^{\prime} \times 4^{\prime}$ metal box. Sampler is triggered by stage through the data logger. Outside staff is available for reference. No provision for measurements above wading stage.

Cellular telemetry removed March 19, service discontinued.

Field Work. The station was visited 17 times to conduct discharge measurements and service the instrumentation. Field inspections for the gage are listed under site history files on the Hydstra database. Discharge measurements for the gage are listed under site gauging files on the Hydstra database.

Datum Correction. None. Levels from June 6, 2006. Gage is within acceptable limits.

Gage-Height Record. The data logger referenced to the outside staff gave a complete and satisfactory record for the year except for the periods of December 9-11, February 24-27, and March 16-30 when ice affected gage height and during May 13-29 when the orifice was isolated from flow.

Rating. The channel is about $20^{\prime}$ wide and straight for about $15^{\prime}$ upstream and straight for about 40' downstream and 20' above the confluence of Pueblo Canyon. The streambed through this reach consists of primarily sand and cobbles. The low water control is a 90-degree shaped weir. At high flow, the channel becomes the control.

Ten discharge measurements (Nos. 6-15) and four visits of no flow. All inspections of no flow were used to develop a "V" diagram shift needed to adjust for PZF.

Rating No. 1 is based on all discharge measurements made during the period of record. Shifts were applied to low flow using "V" diagrams.

Discharge. Discharge was computed by applying gage height to Rating No.1 through shift adjustment based on "V" diagrams. Estimated daily discharges were based on precipitation record, field notes, and some comparisons with E0555.

Remarks. Records are good except for estimated daily discharges, which are poor. 


\section{E056 Acid Canyon above Pueblo Canyon}

Daily Mean Discharge in Cubic Feet per Second

Water Year October 2007 to September 2008

\begin{tabular}{|c|c|c|c|c|c|c|c|c|c|c|c|c|}
\hline DAY & OCT & NOV & DEC & JAN & FEB & MAR & APR & MAY & JUN & JUL & AUG & SEP \\
\hline 1 & .01 & 0 & 11 & 0 & 0 & 0 & 0 & 0 & 0 & 0 & 0 & 0 \\
\hline 2 & 0 & 0 & .02 & 0 & 0 & 0 & 0 & 0 & 0 & 0 & 0 & 0 \\
\hline 3 & 0 & 0 & .02 & 0 & 0 & 0 & 0 & 0 & 0 & 0 & 0 & 0 \\
\hline 4 & 0 & 0 & .01 & 0 & 0 & 0 & 0 & 0 & 0 & 0 & .06 & 0 \\
\hline 5 & 0 & 0 & .01 & 0 & 0 & 0 & 0 & 0 & 0 & 0 & 0 & 0 \\
\hline 6 & 0 & 0 & .01 & $0^{*}$ & 0 & 0 & 0 & 0 & 0 & 0 & 0 & 0 \\
\hline 7 & 0 & 0 & .01 & 0 & 0 & 0 & 0 & 0 & 0 & $.01^{*}$ & 0 & 0 \\
\hline 8 & 0 & 0 & .10 & 0 & 0 & 0 & 0 & 0 & 0 & 0 & $.01^{*}$ & 0 \\
\hline 9 & $.01^{*}$ & 0 & $0^{*}$ & 0 & 0 & 0 & 0 & 0 & 0 & 0 & .10 & 0 \\
\hline 10 & 0 & 0 & $0^{*}$ & 0 & 0 & 0 & 0 & 0 & 0 & 0 & .17 & 0 \\
\hline 11 & 0 & 0 & $0^{*}$ & 0 & 0 & 0 & 0 & 0 & 0 & 0 & 0 & 0 \\
\hline 12 & 0 & 0 & 0 & 0 & 0 & 0 & 0 & 0 & 0 & 0 & 0 & 0 \\
\hline 13 & 0 & 0 & 0 & 0 & 0 & 0 & 0 & $.01^{*}$ & 0 & 0 & 0 & 0 \\
\hline 14 & 0 & 0 & 0 & 0 & 0 & 0 & 0 & $.03^{\star}$ & 0 & 0 & 0 & 0 \\
\hline 15 & 0 & 0 & 0 & 0 & 0 & 0 & 0 & $.02^{\star}$ & 0 & 0 & 0 & 0 \\
\hline 16 & 0 & 0 & 0 & 0 & 0 & $.01^{*}$ & 0 & $0^{*}$ & 0 & 0 & 0 & 0 \\
\hline 17 & 0 & 0 & 0 & 0 & 0 & $.01^{*}$ & 0 & $0^{*}$ & 0 & 0 & 0 & 0 \\
\hline 18 & 0 & 0 & 0 & 0 & 0 & $.02^{\star}$ & 0 & $0^{*}$ & 0 & 0 & 0 & 0 \\
\hline 19 & 0 & 0 & 0 & 0 & 0 & $.02^{\star}$ & 0 & $0^{*}$ & 0 & 0 & 0 & 0 \\
\hline 20 & 0 & 0 & 0 & 0 & 0 & $0^{*}$ & 0 & $0^{*}$ & 0 & 0 & 0 & 0 \\
\hline 21 & 0 & 0 & 0 & 0 & 0 & $0^{*}$ & 0 & $0^{*}$ & 0 & 0 & 0 & 0 \\
\hline 22 & 0 & 0 & 0 & 0 & 0 & $0^{*}$ & 0 & $0^{*}$ & 0 & 0 & 0 & 0 \\
\hline 23 & 0 & 0 & 0 & 0 & 0 & $0^{*}$ & 0 & $.03^{\star}$ & 0 & 0 & 0 & 0 \\
\hline 24 & 0 & 0 & 0 & 0 & $.09^{*}$ & $0^{*}$ & 0 & $0^{*}$ & 0 & 0 & .01 & 0 \\
\hline 25 & 0 & 0 & 0 & 0 & $.06^{*}$ & $0^{*}$ & 0 & $0^{*}$ & 0 & 0 & 0 & 0 \\
\hline 26 & 0 & 0 & 0 & 0 & $.05^{\star}$ & $0^{*}$ & 0 & $0^{*}$ & 0 & 0 & 0 & 0 \\
\hline 27 & 0 & 0 & 0 & 0 & $.01^{*}$ & $0^{*}$ & 0 & $0^{*}$ & 0 & 0 & 0 & 0 \\
\hline 28 & 0 & 0 & 0 & 1.9 & 0 & $0^{*}$ & 0 & $.05^{\star}$ & 0 & 0 & 0 & 0 \\
\hline 29 & 0 & 0 & 0 & 0 & 0 & $0^{*}$ & 0 & $0^{*}$ & 0 & 0 & 0 & 0 \\
\hline 30 & 0 & .32 & 0 & 0 & ----- & $0^{*}$ & 0 & 0 & 0 & 0 & 0 & 0 \\
\hline 31 & 0 & ------ & 0 & 0 & ----- & 0 & ----- & 0 & ------ & 0 & .26 & ----- \\
\hline Total & 0.02 & 0.32 & 11.18 & 1.9 & 0.21 & 0.06 & 0 & 0.14 & 0 & 0.01 & 0.61 & 0 \\
\hline Mean & .001 & .011 & .36 & .061 & .007 & .002 & 0 & .005 & 0 & 0 & .020 & 0 \\
\hline $\operatorname{Max}$ & .01 & .32 & 11 & 1.9 & .09 & .02 & 0 & .05 & 0 & .01 & .26 & 0 \\
\hline Min & 0 & 0 & 0 & 0 & 0 & 0 & 0 & 0 & 0 & 0 & 0 & 0 \\
\hline $\begin{array}{r}\text { Acre- } \\
\mathrm{Ft}\end{array}$ & .04 & .63 & 22 & 3.8 & .42 & .12 & 0 & .28 & 0 & .02 & 1.2 & 0 \\
\hline Wtr Year & 2008 & Total & 14.45 & Mean & & 39 & $\operatorname{Max}$ & 11 & Min & 0 & Acre-Ft & 29 \\
\hline Cal Year & 2007 & Total & 50.30 & Mean & & 14 & $\operatorname{Max}$ & 11 & Min & 0 & Acre-Ft & 100 \\
\hline
\end{tabular}




\section{E060 Pueblo Canyon above SR 502}

Location. Lat $35^{\circ}$ 52' 15", long $106^{\circ}$ 13' 1", NE 1/4, Sec. 20, T. 19 N., R. 7 E., Santa Fe County.

Drainage Area. $8.21 \mathrm{mi}^{2}$.

Period of Record. January 1992 to September 30, 2008.

Revised Record. Drainage area (2006).

Gage. Data logger with cellular telemetry. Elevation of gage is 6,341 ft above NGVD from GPS survey.

Remarks. Records are fair. No diversion above station. Perennial flow is primarily from effluent.

Average Discharge. $14 \mathrm{yr}, 0.87 \mathrm{ft}^{3} / \mathrm{s}, 631$ acre- $\mathrm{ft} / \mathrm{yr}$.

Extremes for Period of Record. Maximum discharge, 1,930 ft ${ }^{3} / \mathrm{s}$, August 24, 2006, gage height $11.75 \mathrm{ft}$ (from slope area measurement). Rating curve extended above 130 $\mathrm{ft}^{3} / \mathrm{s}$ on basis of slope area measurement. No flow at times.

Extremes for Current Water Year. Maximum discharge $37 \mathrm{ft}^{3} / \mathrm{s}$ at $0720 \mathrm{~h}$, December 1 , gage height $8.98 \mathrm{ft}$. No peak above base of $75 \mathrm{ft}^{3} / \mathrm{s}$. No flow at times.

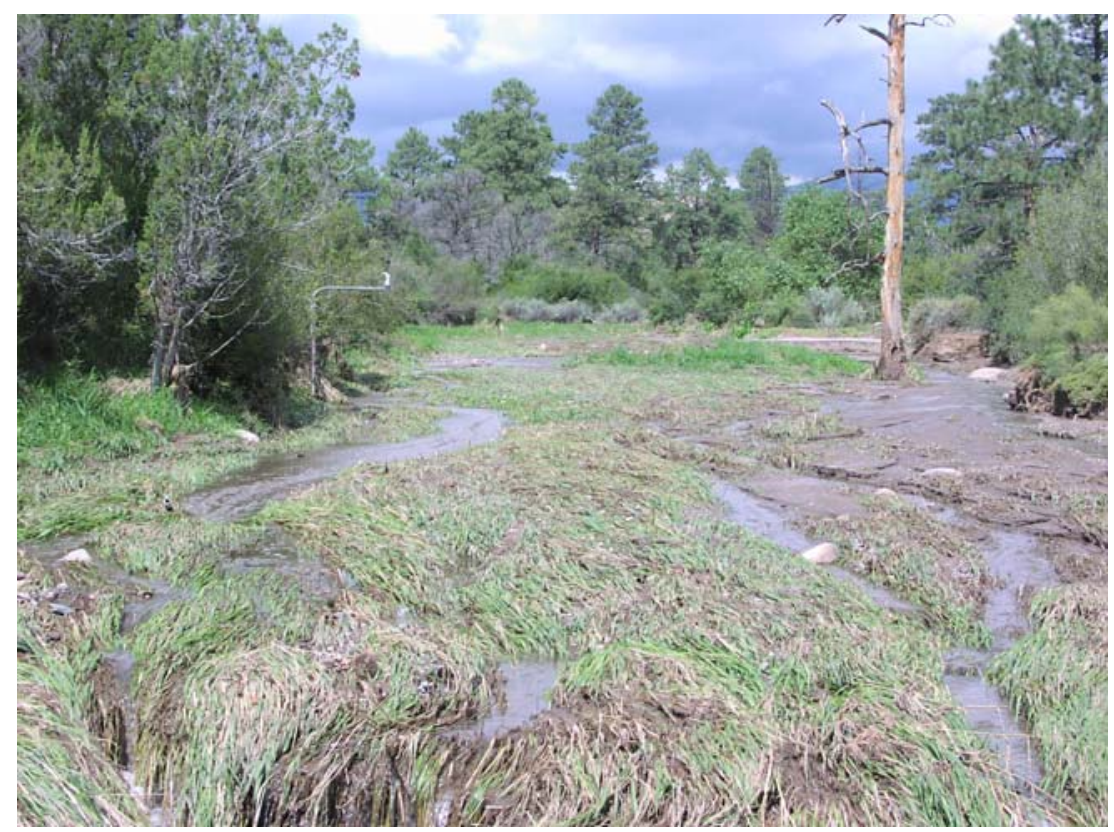




\section{Station Analysis}

\section{Water Year}

Equipment. Station is equipped with Sutron 8210 (5-min. interval) with a Sutron Accubar nonsubmersible transducer housed in a NEMA shelter on right bank. A milltronics sonic probe auxiliary stage sensor is mounted on top of a 4 " $\times 4$ "steel channel that is used to support outside staff and was installed June 1, 2006. The station is also equipped with a pair of ISCO samplers for water-quality sample collection. ISCO is housed in a separate shelter, a $3^{\prime} \times 4^{\prime}$ metal box. Sampler is triggered by stage through the data logger. An outside staff is available for reference gage. No provision for discharge measurements above wading stage.

Field Work. This station was visited 22 times to conduct discharge measurements and service the instrumentation. Field inspections for the gage are listed under site history files on the Hydstra database. Discharge measurements for the gage are listed under site gauging files on the Hydstra database.

Datum Correction. None. Levels of May 7, 2007, found gage to be correct.

Gage-Height Record. The data logger referenced to the outside staff gave a complete and satisfactory record, except for the periods of December 1-5 and December 14 to February 20 when ice affected gage height.

Rating. Channel consists of sand gravel with hard pan bed. Sand degrades easily and will change with flow events. Channel is straight at high flow but incised in sand and meandering at low flow. Channel bottom elevation or PZF may change many times throughout the year and is critical in determining shift values. Grasses become 4-5' tall and cause considerable backwater at most stages. These grasses are usually flattened at high flow.

Five discharge measurements (Nos. 121-125) and 11 inspections of no flow were made this year.

Ratings No. 8 was developed based on measurements made this year. The upper end of the rating is based on slope area measurement from the previous year.

Measuring conditions at this site are poor. Generally, low flows are shallow and characterized by high velocities and uneven measuring sections. Peak flows are flashy and change quickly; mean gage heights for measurements are difficult to determine.

Discharge. Discharge was computed by applying gage height to Rating No. 8 using V diagrams. Various ice days occurred in December, so flow was estimated.

Remarks. Records are fair except for estimated daily discharges, which are poor. 
E060 Pueblo Canyon above SR 502

Daily Mean Discharge in Cubic Feet per Second

Water Year October 2007 to September 2008

\begin{tabular}{|c|c|c|c|c|c|c|c|c|c|c|c|c|}
\hline DAY & OCT & NOV & DEC & JAN & FEB & MAR & APR & MAY & JUN & JUL & AUG & SEP \\
\hline 1 & .01 & 0 & $7.3^{*}$ & $0^{*}$ & $.66^{*}$ & .86 & .58 & .21 & 0 & 0 & 0 & 0 \\
\hline 2 & 0 & 0 & $.58^{\star}$ & $0^{*}$ & $.67^{\star}$ & .87 & .69 & .21 & 0 & 0 & 0 & 0 \\
\hline 3 & 0 & 0 & $.32^{*}$ & $0^{*}$ & $.70^{*}$ & .87 & .76 & .20 & 0 & 0 & 0 & 0 \\
\hline 4 & 0 & 0 & $.09^{*}$ & $0^{*}$ & $.71^{\star}$ & .85 & .59 & .20 & 0 & 0 & 0 & 0 \\
\hline 5 & 0 & 0 & $.13^{\star}$ & $0^{*}$ & $.68^{\star}$ & .86 & .67 & .21 & 0 & 0 & 0 & 0 \\
\hline 6 & 0 & 0 & .08 & $0^{*}$ & $.64^{*}$ & .86 & .63 & .19 & 0 & 0 & 0 & 0 \\
\hline 7 & 0 & 0 & .05 & $0^{*}$ & $.64^{\star}$ & .87 & .77 & .13 & 0 & 0 & 0 & 0 \\
\hline 8 & 0 & 0 & .16 & $0^{*}$ & $.64^{\star}$ & .83 & .58 & .13 & 0 & 0 & .01 & 0 \\
\hline 9 & 0 & 0 & 0 & 0 * & $.67^{*}$ & .84 & .63 & .13 & 0 & 0 & 0 & 0 \\
\hline 10 & 0 & 0 & .01 & $0^{*}$ & $.72^{\star}$ & .83 & .59 & .13 & 0 & 0 & 0 & 0 \\
\hline 11 & 0 & 0 & 0 & $0^{*}$ & $.72^{\star}$ & .80 & .52 & .12 & 0 & 0 & 0 & 0 \\
\hline 12 & 0 & 0 & .03 & $0^{*}$ & $.71^{*}$ & .80 & .48 & .12 & 0 & 0 & 0 & 0 \\
\hline 13 & 0 & 0 & .11 & $0^{*}$ & $.73^{\star}$ & .78 & .56 & .13 & 0 & 0 & 0 & 0 \\
\hline 14 & 0 & 0 & $.73^{*}$ & $0^{*}$ & $.67^{*}$ & .78 & .59 & .15 & 0 & 0 & 0 & 0 \\
\hline 15 & 0 & 0 & $.75^{\star}$ & $0^{*}$ & $.69^{*}$ & .78 & .51 & .10 & 0 & 0 & 0 & 0 \\
\hline 16 & 0 & 0 & $.79^{*}$ & $0^{*}$ & $.70^{*}$ & .79 & .41 & .12 & 0 & 0 & .02 & 0 \\
\hline 17 & 0 & 0 & $.71^{\star}$ & $.76^{\star}$ & $.65^{*}$ & .81 & .39 & .11 & 0 & 0 & .02 & 0 \\
\hline 18 & 0 & 0 & $.68^{\star}$ & $.68^{*}$ & $.71^{*}$ & .81 & .43 & .10 & 0 & 0 & 0 & 0 \\
\hline 19 & 0 & 0 & $.67^{\star}$ & $.67^{*}$ & $.70^{*}$ & .83 & .68 & .08 & 0 & 0 & 0 & 0 \\
\hline 20 & 0 & 0 & $.66^{\star}$ & $.69^{\star}$ & $.70^{*}$ & .83 & .75 & .07 & 0 & 0 & 0 & 0 \\
\hline 21 & 0 & 0 & $.67^{\star}$ & $.75^{*}$ & .92 & .75 & .58 & .06 & 0 & 0 & 0 & 0 \\
\hline 22 & 0 & 0 & $.61^{*}$ & $.71^{*}$ & .92 & .77 & .55 & .03 & 0 & 0 & 0 & 0 \\
\hline 23 & 0 & 0 & $.58^{\star}$ & $.75^{*}$ & .92 & .80 & .33 & .01 & 0 & 0 & 0 & 0 \\
\hline 24 & 0 & 0 & $.64^{\star}$ & $.74^{\star}$ & .87 & .75 & .29 & .03 & 0 & 0 & 0 & 0 \\
\hline 25 & 0 & 0 & $.54^{\star}$ & $.71^{\star}$ & .92 & .63 & .28 & .03 & 0 & 0 & 0 & 0 \\
\hline 26 & 0 & 0 & $.69^{*}$ & $0^{*}$ & .89 & .62 & .29 & .01 & 0 & 0 & 0 & 0 \\
\hline 27 & 0 & 0 & $.62^{\star}$ & $0^{*}$ & .89 & .58 & .29 & 0 & 0 & 0 & 0 & 0 \\
\hline 28 & 0 & .71 & $.54^{\star}$ & $.47^{*}$ & .89 & .71 & .25 & 0 & 0 & 0 & 0 & 0 \\
\hline 29 & 0 & .94 & $.57^{\star}$ & $.66^{*}$ & .88 & .77 & .20 & .01 & 0 & 0 & 0 & 0 \\
\hline 30 & 0 & .93 & $.60^{\star}$ & .65 & ----- & .80 & .20 & 0 & 0 & 0 & 0 & 0 \\
\hline 31 & 0 & ----- & $.66^{\star}$ & .65 & ----- & .59 & ------ & 0 & ----- & 0 & 0 & ----- \\
\hline Total & 0.01 & 2.58 & 20.57 & 8.89 & 21.81 & 24.32 & 15.07 & 3.02 & 0 & 0 & 0.05 & 0 \\
\hline Mean & 0 & .086 & .66 & .29 & .75 & .78 & .50 & .097 & 0 & 0 & .002 & 0 \\
\hline Max & .01 & .94 & 7.3 & .76 & .92 & .87 & .77 & .21 & 0 & 0 & .02 & 0 \\
\hline Min & 0 & 0 & 0 & 0 & .64 & .58 & .20 & 0 & 0 & 0 & 0 & 0 \\
\hline Acre-Ft & .02 & 5.1 & 41 & 18 & 43 & 48 & 30 & 6.0 & 0 & 0 & .10 & 0 \\
\hline Wtr Year & 2008 & Total & 96.32 & & & .26 & $\operatorname{Max}$ & 7.3 & Min & 0 & Acre-Ft & 191 \\
\hline Cal Year & 2007 & Total & 231.44 & & & .63 & $\operatorname{Max}$ & 7.3 & Min & 0 & Acre-Ft & 459 \\
\hline
\end{tabular}

*Estimate 


\section{E121 Sandia Canyon Right Fork at Power Plant}

Location. Lat $35^{\circ}$ 52' 31", long $106^{\circ}$ 19' 7", SW 1/4, Sec. 16, T. 19 N., R. 6 E., Los Alamos County.

Drainage Area. $0.08 \mathrm{mi}^{2}$.

Period of Record. October 1, 2006, to September 30, 2008.

Revised Record. Period of record (this report).

Gage. Data logger. Elevation of gage is 7,283, ft above NGVD from GPS survey.

Remarks. Records are good. Records for this site existed before period of record but are not reliable.

Extremes for Period of Record. Maximum discharge, $191 \mathrm{ft}^{3} / \mathrm{s}$, June 21, 2002, from peak flow computation, gage height $8.13 \mathrm{ft}$. Minimum daily $0.05 \mathrm{ft}^{3} / \mathrm{s}$, August 12, 2008 .

Extremes for Current Year. Peak discharges above $35 \mathrm{ft}^{3} / \mathrm{s}$ and maximum (*):

\begin{tabular}{|c|c|c|c|}
\hline Date & Time & Discharge $\left(\mathbf{f t}^{\mathbf{3}} / \mathbf{s}\right)$ & Gage Height (ft) \\
\hline May 28 & 1545 & 47 & 6.74 \\
\hline August 4 & 1750 & 46 & 6.72 \\
\hline August 8 & 2340 & 57 & 6.98 \\
\hline August 10 & 1115 & $70 *$ & $7.19^{*}$ \\
\hline August 31 & 1525 & 41 & 6.62 \\
\hline
\end{tabular}

Minimum daily discharge, $0.05 \mathrm{ft}^{3} / \mathrm{s}$ August 12 .

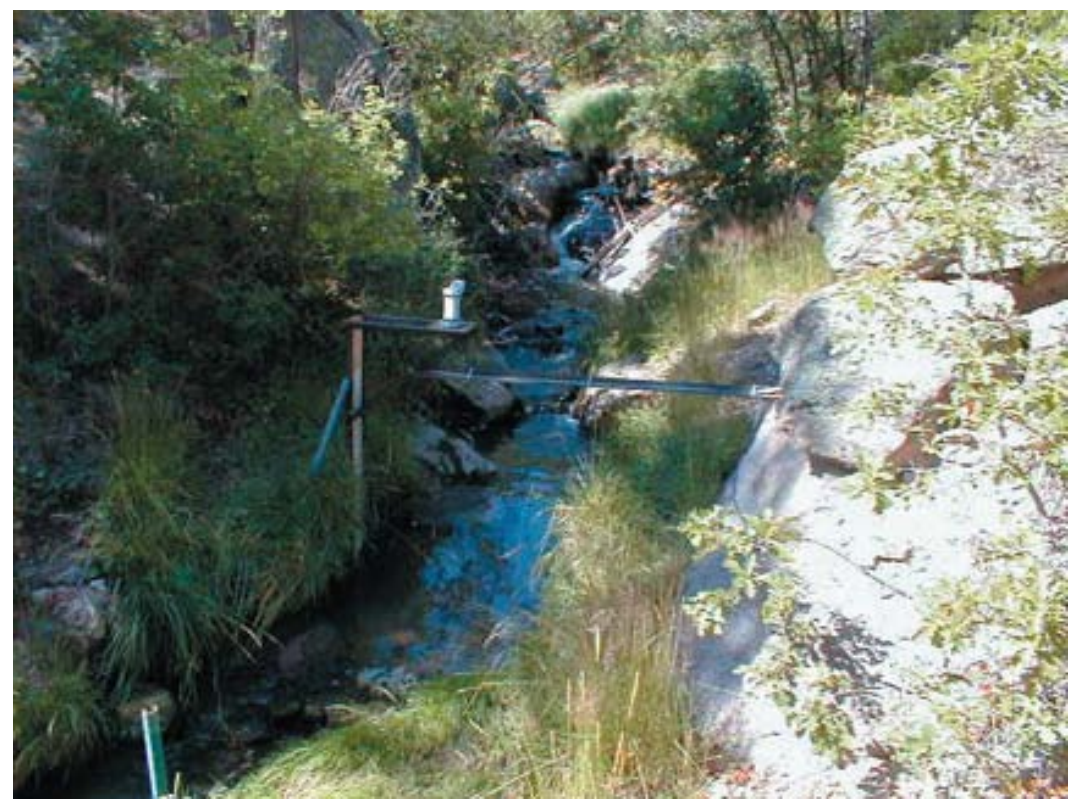




\section{E121 Sandia Canyon Right Fork at Power Plant}

\section{Station Analysis}

\section{Water Year}

Equipment. Station is equipped with Sutron 8210 data logger (5-min. interval) with a Sutron Accubar bubble sensor. System is powered by a solar-panel battery system housed in a NEMA shelter. Station is equipped with an ISCO pump sampler for water-quality sample collection. ISCO is housed in a separate shelter, a $3^{\prime} \times 4^{\prime}$ metal box. Sampler is triggered by stage through the data logger. An outside staff is available for reference. No provision for direct measurements above wading stage.

Field Work. The station was visited 22 times to conduct discharge measurements and service the instrumentation. Field inspections for the gage are listed under site history files on the Hydstra database. Discharge measurements for the gage are listed under site gauging files on the Hydstra database.

Datum Correction. None.

Gage-Height. The data logger reference to the outside staff gave a complete and satisfactory record for the year.

Rating. The channel is straight for about $30^{\prime}$, with a step upstream slope and straight for $50^{\prime}$ downstream with a sharp slope downstream. The streambed through this reach consists of primarily sand, gravel, and cobbles, more so below the gage. The low water control is a bedrock riffle below gage.

Ten discharge measurements (Nos. 72-82) were made during the year. Discharge measurements were used to define a "V" diagram. Range in stage is fairly limited because most flow is effluent.

Rating No. 4 was developed On June 26, 2007, based on the measurements made during the previous water year and used until the end of the current water year.

Discharge. Discharge was computed by applying Rating No. 4 with variable shifts defined by measurements and applied by "V" diagram. No shifts were applied to high flows. There were no ice affected discharges.

Remarks. Records are good. 


\section{E121 Sandia Canyon Right Fork at Power Plant}

Daily Mean Discharge in Cubic Feet per Second

Water Year October 2007 to September 2008

\begin{tabular}{|c|c|c|c|c|c|c|c|c|c|c|c|c|}
\hline DAY & ОСТ & NOV & DEC & JAN & FEB & MAR & APR & MAY & JUN & JUL & AUG & SEP \\
\hline 1 & .58 & .44 & 2.7 & .35 & .58 & .67 & .56 & .64 & .20 & .33 & .67 & .82 \\
\hline 2 & .41 & .61 & .62 & .39 & .50 & .96 & .45 & .75 & .21 & .37 & .69 & .53 \\
\hline 3 & .44 & .55 & .47 & .35 & .58 & .74 & .51 & .82 & .21 & .34 & .62 & .87 \\
\hline 4 & .34 & .52 & .42 & .36 & .67 & .77 & .52 & .62 & .21 & .44 & 1.7 & .61 \\
\hline 5 & .50 & .57 & .54 & .35 & .57 & .56 & .73 & .80 & .16 & .54 & .59 & .65 \\
\hline 6 & .54 & .54 & .46 & .65 & .53 & .69 & .42 & .58 & .17 & .51 & .73 & .55 \\
\hline 7 & .47 & .57 & .49 & .45 & .56 & .85 & .93 & .77 & .30 & .62 & .87 & .74 \\
\hline 8 & .56 & .52 & .80 & .42 & .63 & .81 & .51 & .47 & .27 & .50 & 1.5 & .56 \\
\hline 9 & .56 & .52 & .49 & .27 & .65 & .43 & .47 & .38 & .31 & .55 & 1.2 & .57 \\
\hline 10 & .49 & .47 & .54 & .33 & .73 & .57 & .48 & .30 & .21 & .48 & 1.2 & .53 \\
\hline 11 & .46 & .39 & .62 & .36 & .56 & .46 & .58 & .39 & .26 & .55 & .15 & .60 \\
\hline 12 & .43 & .49 & .47 & .34 & .53 & .81 & .63 & .35 & .36 & .47 & .05 & .61 \\
\hline 13 & .45 & .48 & .45 & .39 & .43 & .63 & .75 & .38 & .26 & .56 & .11 & .56 \\
\hline 14 & .49 & .47 & .54 & .49 & .56 & .42 & .69 & .41 & .35 & .55 & .09 & .50 \\
\hline 15 & .48 & .44 & .43 & .32 & .54 & .57 & .64 & .99 & .37 & .54 & .11 & .65 \\
\hline 16 & .50 & .41 & .56 & .36 & .73 & .64 & .82 & .33 & .37 & .52 & .50 & .50 \\
\hline 17 & .49 & .40 & .53 & .36 & .49 & .48 & .66 & .49 & .35 & .46 & .32 & .56 \\
\hline 18 & .46 & .43 & .39 & .38 & .70 & .48 & .59 & .30 & .31 & .50 & .27 & .50 \\
\hline 19 & .48 & .45 & .47 & .40 & .61 & .48 & .55 & .29 & .28 & .54 & .34 & .45 \\
\hline 20 & .59 & .51 & .40 & .38 & .47 & .61 & .43 & .26 & .26 & .52 & .26 & .46 \\
\hline 21 & .62 & .43 & .47 & .36 & .53 & .78 & .61 & .39 & .34 & .78 & .23 & .56 \\
\hline 22 & .66 & .29 & .36 & .44 & .66 & .38 & .44 & .31 & .33 & .70 & .28 & .65 \\
\hline 23 & .47 & .42 & .47 & .31 & .59 & .42 & .47 & .46 & .32 & .67 & .64 & .54 \\
\hline 24 & .60 & .49 & .36 & .38 & .88 & .76 & .50 & .31 & .31 & .74 & .48 & .63 \\
\hline 25 & .55 & .41 & .41 & .38 & .87 & .63 & .44 & .31 & .33 & .73 & .40 & .57 \\
\hline 26 & .49 & .45 & .38 & .53 & .90 & .48 & .53 & .23 & .31 & .71 & .22 & .52 \\
\hline 27 & .52 & .34 & .36 & .50 & .69 & .37 & .46 & .30 & .34 & .73 & .15 & .50 \\
\hline 28 & .40 & .31 & .37 & .95 & .56 & .53 & .52 & .81 & .37 & .52 & .17 & .49 \\
\hline 29 & .54 & .32 & .33 & .53 & .68 & .47 & .41 & .24 & .39 & .54 & .23 & .49 \\
\hline 30 & .52 & 1.5 & .33 & .79 & ----- & .44 & .37 & .26 & .35 & .61 & .22 & .51 \\
\hline 31 & .40 & ------ & .29 & .49 & ------ & .66 & ------ & .23 & ------ & .59 & 1.2 & ------ \\
\hline Total & 15.49 & 14.74 & 16.52 & 13.36 & 17.98 & 18.55 & 16.67 & 14.17 & 8.81 & 17.21 & 16.19 & 17.28 \\
\hline Mean & .50 & .49 & .53 & .43 & .62 & .60 & .56 & .46 & .29 & .56 & .52 & .58 \\
\hline $\operatorname{Max}$ & .66 & 1.5 & 2.7 & .95 & .90 & .96 & .93 & .99 & .39 & .78 & 1.7 & .87 \\
\hline Min & .34 & .29 & .29 & .27 & .43 & .37 & .37 & .23 & .16 & .33 & .05 & .45 \\
\hline Acre-Ft & 31 & 29 & 33 & 26 & 36 & 37 & 33 & 28 & 17 & 34 & 32 & 34 \\
\hline Wtr Year & 2008 & Total & 186.97 & & & .51 & Max & 2.7 & Min & .05 & Acre-Ft & 371 \\
\hline Cal Year & 2007 & Total & 249.61 & & & .68 & Max & 2.7 & Min & .17 & Acre-Ft & 495 \\
\hline
\end{tabular}




\section{E1219 Sandia Canyon East of Power Plant}

Location. Lat. $35^{\circ}$ 52' 30", long. 106 19' 10", SW 1/4, Sec. 16, T 19 N., R 6 E., Los Alamos County.

Drainage Area. $0.002 \mathrm{mi}^{2}$.

Period of Record. March 3, 2006, to September 30, 2008.

Gage. Data logger, 9” Parshall flume, rain gage with cellular telemetry. Elevation of gage is 7,337 ft above NGVD from land survey.

Remarks. Records are good.

Extremes for Period of Record. Maximum discharge, $5.2 \mathrm{ft}^{3} / \mathrm{s}$, August 10, 2008, gage height $1.41 \mathrm{ft}$. No flow most of the time.

Extremes for Current Year. Peak discharges above base of $2.0 \mathrm{ft}^{3} / \mathrm{s}$ and maximum (*):

\begin{tabular}{|c|c|c|c|}
\hline Date & Time & Discharge (ft $\left.\mathbf{3}^{\mathbf{3}} \mathbf{s}\right)$ & Gage Height (ft) \\
\hline May 28 & 1535 & 2.1 & 0.77 \\
\hline August 4 & 1755 & 2.1 & 0.78 \\
\hline August 8 & 2340 & 3.1 & 1.01 \\
\hline August 10 & 1115 & $5.2^{*}$ & $1.41^{*}$
\end{tabular}

No flow most of the time.

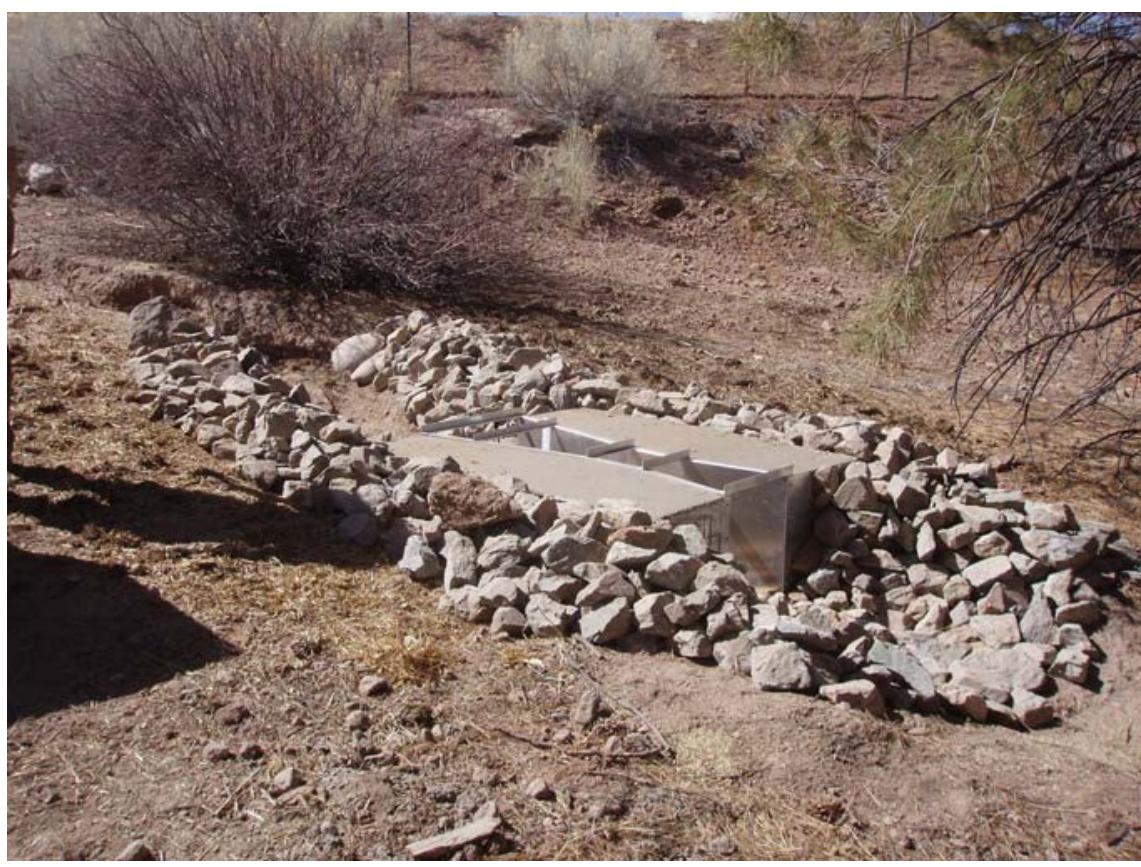




\section{E1219 Sandia Canyon East of Power Plant}

\section{Station Analysis}

\section{Water Year}

Equipment. Station is equipped with Sutron 8210 data logger (5-min. interval) and milltronics sonic probe mounted on a 9" Parshall flume and cellular telemetry with speech modem. Phone upgraded May 8 from analog to digital service. System is powered by a solar-panel battery system housed in a NEMA shelter. Station is equipped with an ISCO pump sampler for water-quality sample collection. ISCO is housed in a separate shelter, a $3^{\prime} \times 4^{\prime}$ metal box. Sampler is triggered by stage through the data logger. The staff in the $9 "$ Parshall flume is the reference gage. No provision for discharge measurements above wading stage.

Station is also equipped with a tipping bucket rain gage, Rain Collection II. All equipment is powered with a solar-panel battery-charging system. Rain data are seasonal.

Field Work. The station was visited 26 times to conduct discharge measurements and service the instrumentation. Field inspections for the gage are listed under site history files on the Hydstra database. Discharge measurements for the gage are listed under site gauging files on the Hydstra database.

Datum Correction. None.

Gage-Height Record. The data logger referenced to the outside staff gave a complete and satisfactory record for the water year, except for the periods of January 6 to February 23, March 2-7, and March 12 to April 8 when ice affected gage height.

Rating. The channel is straight above and below gage and is confined to the main channel by cut banks on both sides. The bottom is a $4^{\prime}$ wide channel prone to some shifting with vegetation on each bank. Low water control is the 9' Parshall flume.

Twenty-five inspections of no flow and one day of snow melt were made during the year.

Rating No. 1 was developed based on the computation of 9' Parshall flume. Point of zero flow is 0.00 gage height.

Discharge. Discharge was computed by applying Rating No. 1 directly.

Remarks. Records are good. 


\section{E1219 Sandia Canyon East of Power Plant}

Daily Mean Discharge in Cubic Feet per Second

Water Year October 2007 to September 2008

\begin{tabular}{|c|c|c|c|c|c|c|c|c|c|c|c|c|}
\hline DAY & OCT & NOV & DEC & JAN & FEB & MAR & APR & MAY & JUN & JUL & AUG & SEP \\
\hline 1 & .01 & 0 & .10 & 0 & $0^{*}$ & 0 & $0^{*}$ & 0 & 0 & 0 & 0 & .01 \\
\hline 2 & 0 & 0 & 0 & 0 & $0^{*}$ & $0^{*}$ & $0^{*}$ & 0 & 0 & 0 & 0 & 0 \\
\hline 3 & 0 & 0 & 0 & 0 & $0^{*}$ & $0^{*}$ & $0^{*}$ & 0 & 0 & 0 & 0 & 0 \\
\hline 4 & 0 & 0 & 0 & 0 & $0^{*}$ & $0^{*}$ & $0^{*}$ & 0 & 0 & 0 & .04 & 0 \\
\hline 5 & 0 & 0 & 0 & 0 & $0^{*}$ & $0^{*}$ & $0^{*}$ & 0 & 0 & .01 & 0 & 0 \\
\hline 6 & 0 & 0 & 0 & $0^{*}$ & $0^{*}$ & $0^{*}$ & $0^{*}$ & 0 & 0 & 0 & 0 & 0 \\
\hline 7 & 0 & 0 & 0 & $0^{*}$ & $0^{*}$ & $0^{*}$ & $0^{*}$ & 0 & 0 & .01 & .02 & 0 \\
\hline 8 & 0 & 0 & .03 & $0^{*}$ & $0^{*}$ & 0 & $0^{*}$ & 0 & 0 & .01 & .03 & 0 \\
\hline 9 & 0 & 0 & 0 & $0^{*}$ & $0^{*}$ & 0 & 0 & 0 & 0 & 0 & .02 & 0 \\
\hline 10 & 0 & 0 & $0^{*}$ & $0^{*}$ & $0^{*}$ & 0 & 0 & 0 & 0 & 0 & .05 & 0 \\
\hline 11 & 0 & 0 & $0^{*}$ & $0^{*}$ & $0^{*}$ & 0 & 0 & 0 & 0 & 0 & .01 & 0 \\
\hline 12 & 0 & 0 & 0 & $0^{*}$ & $0^{*}$ & $0^{*}$ & 0 & 0 & 0 & 0 & .01 & 0 \\
\hline 13 & 0 & 0 & 0 & $0^{*}$ & $0^{*}$ & $0^{*}$ & 0 & 0 & 0 & 0 & .01 & 0 \\
\hline 14 & 0 & 0 & 0 & $0^{*}$ & $0^{*}$ & $0^{*}$ & 0 & 0 & 0 & 0 & .01 & 0 \\
\hline 15 & 0 & 0 & 0 & $0^{*}$ & $0^{*}$ & $0^{*}$ & 0 & .03 & 0 & 0 & 0 & 0 \\
\hline 16 & 0 & 0 & 0 & $0^{*}$ & $0^{*}$ & $0^{*}$ & 0 & 0 & 0 & .01 & .02 & 0 \\
\hline 17 & 0 & 0 & 0 & $0^{*}$ & $0^{*}$ & $0^{*}$ & 0 & 0 & 0 & 0 & 0 & 0 \\
\hline 18 & 0 & 0 & 0 & $0^{*}$ & $0^{*}$ & $0^{*}$ & 0 & 0 & 0 & 0 & 0 & 0 \\
\hline 19 & 0 & 0 & 0 & $0^{*}$ & $0^{*}$ & $0^{*}$ & 0 & 0 & 0 & 0 & 0 & 0 \\
\hline 20 & 0 & 0 & 0 & $0^{*}$ & $0^{*}$ & $0^{*}$ & 0 & 0 & 0 & 0 & 0 & 0 \\
\hline 21 & 0 & 0 & 0 & $0^{*}$ & $0^{*}$ & $0^{*}$ & 0 & 0 & 0 & .01 & 0 & 0 \\
\hline 22 & 0 & 0 & 0 & $0^{*}$ & $0^{*}$ & $0^{*}$ & 0 & 0 & 0 & 0 & 0 & 0 \\
\hline 23 & 0 & $0^{*}$ & 0 & $0^{*}$ & $0^{*}$ & $0^{*}$ & 0 & .01 & 0 & 0 & .02 & 0 \\
\hline 24 & 0 & 0 & 0 & $0^{*}$ & .13 & $0^{*}$ & 0 & 0 & 0 & 0 & .02 & 0 \\
\hline 25 & 0 & 0 & 0 & $0^{*}$ & 0 & $0^{*}$ & 0 & 0 & 0 & 0 & 0 & 0 \\
\hline 26 & 0 & 0 & 0 & $0^{*}$ & 0 & $0^{*}$ & 0 & 0 & 0 & 0 & 0 & 0 \\
\hline 27 & 0 & 0 & 0 & $0^{*}$ & 0 & $0^{*}$ & 0 & 0 & 0 & .01 & 0 & 0 \\
\hline 28 & 0 & 0 & 0 & $0^{*}$ & 0 & $0^{*}$ & 0 & .02 & 0 & 0 & 0 & 0 \\
\hline 29 & 0 & 0 & 0 & $0^{*}$ & 0 & $0^{*}$ & 0 & 0 & 0 & 0 & 0 & 0 \\
\hline 30 & 0 & .06 & 0 & $0^{*}$ & ------ & $0^{*}$ & 0 & 0 & 0 & 0 & 0 & 0 \\
\hline 31 & 0 & ------ & 0 & $0^{*}$ & ------ & $0^{*}$ & ------ & 0 & ----- & 0 & .05 & ------ \\
\hline Total & 0.01 & 0.06 & 0.13 & 0 & 0.13 & 0 & 0 & 0.06 & 0 & 0.06 & 0.31 & 0.01 \\
\hline Mean & 0 & .002 & .004 & 0 & .005 & 0 & 0 & .002 & 0 & .002 & .010 & 0 \\
\hline Max & .01 & .06 & .10 & 0 & .13 & 0 & 0 & .03 & 0 & .01 & .05 & .01 \\
\hline Min & 0 & 0 & 0 & 0 & 0 & 0 & 0 & 0 & 0 & 0 & 0 & 0 \\
\hline Acre-Ft & .02 & .12 & .26 & 0 & .26 & 0 & 0 & .12 & 0 & .12 & .61 & .02 \\
\hline Wtr Year & 2008 & Total & 0.77 & Mean & & 02 & hax & .13 & Min & 0 & Acre-Ft & 1.5 \\
\hline Cal Year & 2007 & Total & 0.84 & Mean & & 02 & hax & .10 & Min & 0 & Acre-Ft & 1.7 \\
\hline
\end{tabular}

*Estimate 
E1219 Sandia Canyon East of Power Plant

Daily Mean Discharge in Cubic Feet per Second

Water Year October 2007 to September 2008

\begin{tabular}{|c|c|c|c|c|c|c|c|c|c|c|c|c|}
\hline DAY & ОСт & NOV & DEC & JAN & FEB & MAR & APR & MAY & JUN & JUL & AUG & SEP \\
\hline 1 & & & & & & & & 0 & 0 & .08 & 0 & .11 \\
\hline 2 & & & & & & & & 0 & 0 & .02 & 0 & $0^{*}$ \\
\hline 3 & & & & & & & & 0 & 0 & .01 & 0 & 0 \\
\hline 4 & & & & & & & & 0 & 0 & 0 & .65 & 0 \\
\hline 5 & & & & & & & & 0 & .07 & .18 & .01 & 0 \\
\hline 6 & & & & & & & & 0 & 0 & .01 & 0 & 0 \\
\hline 7 & & & & & & & & 0 & 0 & .37 & .35 & 0 \\
\hline 8 & & & & & & & & 0 & 0 & .26 & .68 & 0 \\
\hline 9 & & & & & & & 0 & 0 & 0 & 0 & .32 & 0 \\
\hline 10 & & & & & & & .04 & 0 & 0 & 0 & .48 & 0 \\
\hline 11 & & & & & & & 0 & 0 & 0 & .04 & 0 & 0 \\
\hline 12 & & & & & & & .03 & 0 & 0 & .01 & .03 & 0 \\
\hline 13 & & & & & & & 0 & .14 & 0 & 0 & 0 & 0 \\
\hline 14 & & & & & & & 0 & .14 & 0 & 0 & .01 & 0 \\
\hline 15 & & & & & & & 0 & .56 & 0 & .02 & 0 & 0 \\
\hline 16 & & & & & & & 0 & 0 & 0 & .21 & .41 & 0 \\
\hline 17 & & & & & & & .11 & 0 & 0 & .01 & .07 & 0 \\
\hline 18 & & & & & & & 0 & 0 & 0 & .04 & 0 & .06 \\
\hline 19 & & & & & & & 0 & 0 & 0 & 0 & 0 & .03 \\
\hline 20 & & & & & & & 0 & 0 & 0 & 0 & .05 & 0 \\
\hline 21 & & & & & & & 0 & 0 & .01 & .22 & 0 & 0 \\
\hline 22 & & & & & & & 0 & .16 & 0 & 0 & 0 & .09 \\
\hline 23 & & & & & & & 0 & .19 & 0 & 0 & .47 & 0 \\
\hline 24 & & & & & & & 0 & 0 & 0 & 0 & .36 & .06 \\
\hline 25 & & & & & & & 0 & 0 & 0 & .01 & $.17^{*}$ & 0 \\
\hline 26 & & & & & & & 0 & 0 & 0 & .05 & $.04^{*}$ & 0 \\
\hline 27 & & & & & & & 0 & 0 & 0 & .19 & 0 & 0 \\
\hline 28 & & & & & & & 0 & .40 & 0 & .05 & 0 & 0 \\
\hline 29 & & & & & & & 0 & 0 & 0 & 0 & .01 & 0 \\
\hline 30 & & & & & ------ & & 0 & 0 & 0 & 0 & .08 & 0 \\
\hline 31 & & ----- & & & ------ & & ----- & 0 & ----- & 0 & .85 & ----- \\
\hline Total & & & & & & & 0.18 & 1.59 & 0.08 & 1.78 & 5.01 & 0.35 \\
\hline Max & & & & & & & .11 & .56 & .07 & .37 & .85 & .11 \\
\hline Wtr Year & 2008 & Total & 8.99 & Mean & & .050 & Max & .85 & Min & 0 & InstMax & .18 \\
\hline Cal Year & 2007 & Total & 13.09 & Mean & & .070 & $\operatorname{Max}$ & 1.2 & Min & 0 & InstMax & .28 \\
\hline
\end{tabular}

*Estimate 
Location. Lat $35^{\circ}$ 52' 31”, long $106^{\circ}$ 9' 6", SW 1/4, Sec. 16, T. 19 N., R. 6 E., Los Alamos County.

Drainage Area. $0.08 \mathrm{mi}^{2}$.

Period of Record. October 1, 2006, to September 30, 2008.

Gage. Data logger. Elevation of gage is 7,290 ft above NGVD.

Remarks. Records are good. Records for this site existed before period of record but are not reliable.

Extremes for Period of Record. Maximum discharge, $88 \mathrm{ft}^{3} / \mathrm{s}$, August 23, 2003, gage height $4.23 \mathrm{ft}$. Minimum daily discharge $0.00 \mathrm{ft}^{3} / \mathrm{s}$, September 30, 2008.

Extremes for Current Year. Peak discharges above base of $15 \mathrm{ft}^{3} / \mathrm{s}$ and maximum (*):

\begin{tabular}{|c|c|c|c|}
\hline Date & Time & Discharge $\left(\mathbf{f t}^{\mathbf{3}} / \mathbf{s}\right)$ & Gage Height (ft) \\
\hline August 8 & 2340 & $16^{*}$ & $2.95^{*}$ \\
\hline August 10 & 1115 & 16 & 2.91 \\
\hline August 31 & 1520 & 15 & 2.84 \\
\hline
\end{tabular}

Minimum daily discharge, $0.0 \mathrm{ft}^{3} / \mathrm{s}$ September 30 .

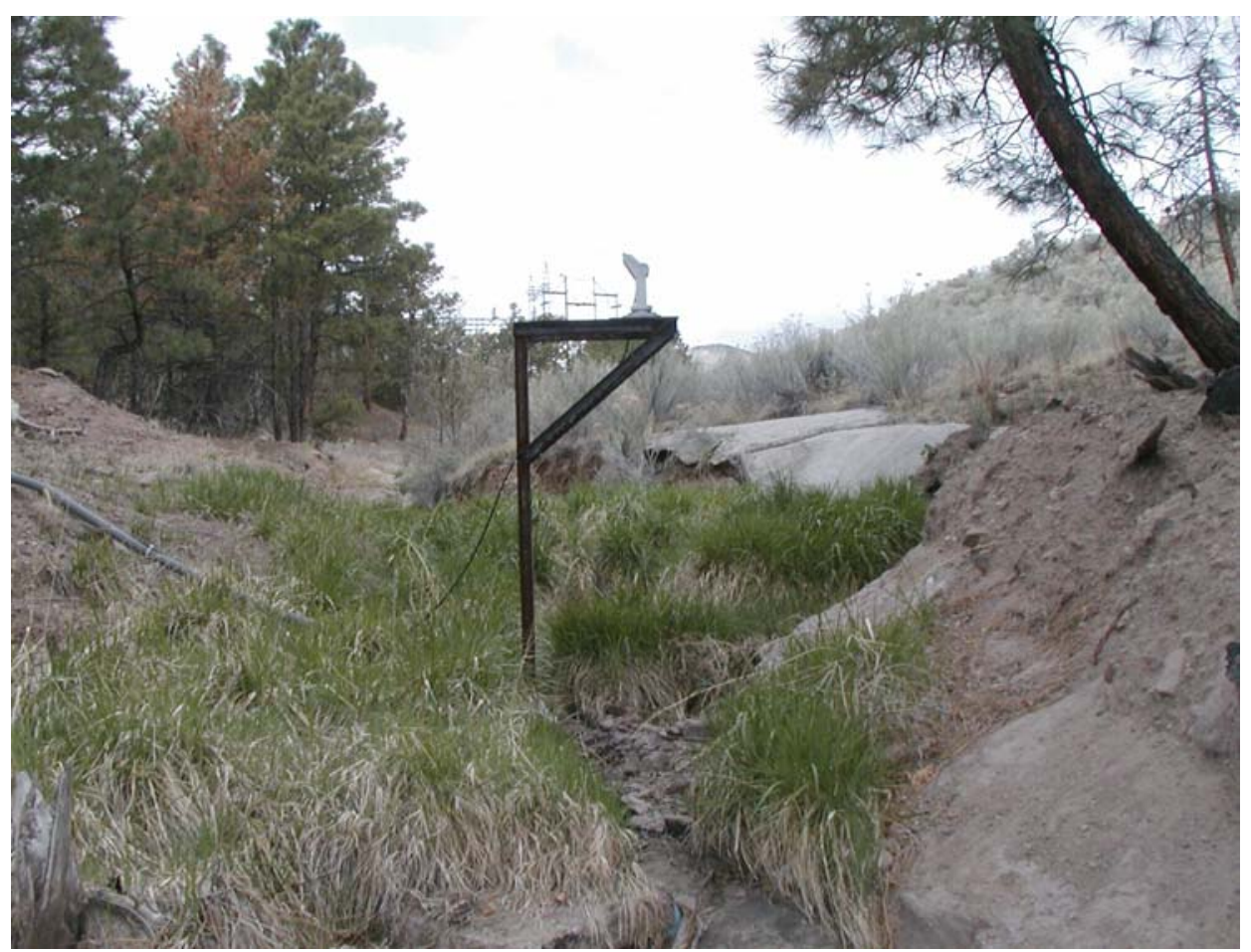




\section{E122 Sandia Canyon near Roads and Grounds at TA-3}

\section{Station Analysis}

\section{Water Year}

Equipment. Station is equipped with Sutron 8210 data logger (5-min. interval) and a milltronics sonic probe. The system is powered by a solar-panel battery system housed in a NEMA shelter. Station is also equipped with ISCO pump sampler for water-quality sample collection. ISCO is housed in a separate shelter, a $3^{\prime} \times 4^{\prime}$ metal box. Sampler is triggered by stage through the data logger. An outside staff is available for reference. No provision for discharge measurements above wading stage.

Field Work. The station was visited 24 times to conduct discharge measurements and service the instrumentation. Field inspections for the gage are listed under site history files on the Hydstra database. Discharge measurements for the gage are listed under site gauging files on the Hydstra database.

Datum Correction. None. Levels of July 25, 2005, found gage to be within limits.

Gage-Height Record. The data logger referenced to the outside staff gave a complete and satisfactory record for the year.

Rating. The channel is straight for about 20' above with a step downstream slope and straight for $15^{\prime}$ downstream, with a sharp slope $5^{\prime}$ downstream. The streambed through this reach consists of primarily bedrock with some cobbles below gage. The low water control is bedrock riffle below gage.

Seven discharge measurements (Nos. 41-47) were made this year.

Rating No. 2 was developed based on the measurements made the previous year and verified with measurements made this year. Shifts are small and mostly negative caused by small amounts of deposition near gage or some bank slough during high flows. They have been distributed using variable diagrams, with no shifts applied on the peak flows.

Discharge. Discharge computed from Rating No. 2, with shifts applied by "V" diagrams.

Remarks. Records are good. 


\section{E122 Sandia Canyon near Roads and Grounds at TA-3}

Daily Mean Discharge in Cubic Feet per Second

Water Year October 2007 to September 2008

\begin{tabular}{|c|c|c|c|c|c|c|c|c|c|c|c|c|}
\hline DAY & OCT & NOV & DEC & JAN & FEB & MAR & APR & MAY & JUN & JUL & AUG & SEP \\
\hline 1 & .14 & .08 & 1.8 & .06 & .05 & .07 & .05 & .09 & .06 & .18 & .02 & .13 \\
\hline 2 & .03 & .08 & .07 & .05 & .05 & .06 & .06 & .09 & .07 & .21 & .02 & .06 \\
\hline 3 & .02 & .08 & .04 & .04 & .05 & .08 & .06 & .09 & .06 & .20 & .01 & .04 \\
\hline 4 & .04 & .08 & .03 & .04 & .05 & .07 & .06 & .10 & .07 & .21 & .48 & .03 \\
\hline 5 & .02 & .08 & .05 & $.03^{*}$ & .04 & .07 & .07 & .11 & .08 & .38 & .17 & .03 \\
\hline 6 & .02 & .08 & .05 & $.03^{*}$ & .04 & .08 & .07 & .11 & .05 & .12 & .07 & .04 \\
\hline 7 & .01 & .07 & .08 & $.04^{*}$ & .06 & .08 & .07 & .09 & .06 & .23 & .24 & .03 \\
\hline 8 & .01 & .04 & .40 & .05 & .06 & .07 & .06 & .14 & .06 & .27 & .48 & .04 \\
\hline 9 & .01 & .04 & .09 & .06 & .06 & .06 & .07 & .12 & .05 & .09 & .54 & .04 \\
\hline 10 & .03 & .04 & .13 & .05 & .06 & .06 & .07 & .12 & .06 & .15 & .44 & $.05^{\star}$ \\
\hline 11 & .05 & .04 & .16 & .04 & .06 & $.06^{*}$ & .06 & .11 & .07 & .12 & .10 & $.04^{*}$ \\
\hline 12 & .03 & .03 & .06 & .03 & .05 & .07 & .06 & .12 & .10 & .23 & .09 & $.04^{*}$ \\
\hline 13 & .02 & .04 & .05 & .04 & .09 & .07 & .06 & .14 & .05 & .06 & .08 & $.04^{*}$ \\
\hline 14 & .02 & .05 & .05 & .04 & .09 & .06 & .06 & .17 & .05 & .07 & .08 & $.03^{*}$ \\
\hline 15 & .02 & .05 & .03 & .04 & .08 & .06 & .07 & .44 & .06 & .07 & .07 & $.03^{*}$ \\
\hline 16 & .02 & .05 & .04 & .04 & .08 & .07 & .07 & .10 & .07 & .14 & $.27^{\star}$ & $.03^{\star}$ \\
\hline 17 & .03 & .04 & .04 & $.04^{*}$ & .07 & .07 & .11 & .09 & .06 & .07 & .08 & $.03^{*}$ \\
\hline 18 & .04 & .04 & .04 & .07 & .06 & .07 & .07 & .08 & .06 & .08 & .05 & .05 \\
\hline 19 & .02 & .03 & .04 & .05 & .06 & .07 & .08 & .09 & .07 & .06 & .04 & $.04^{\star}$ \\
\hline 20 & .03 & .02 & .04 & .05 & .07 & .08 & .07 & .09 & .08 & .07 & .05 & .03 \\
\hline 21 & .02 & .01 & .04 & .05 & .08 & .06 & .07 & .08 & .07 & .17 & .03 & .04 \\
\hline 22 & .03 & .02 & .03 & .05 & .07 & .06 & .07 & .11 & .07 & .05 & .02 & .07 \\
\hline 23 & .03 & .04 & .03 & .05 & .10 & .06 & .08 & .14 & .07 & .05 & .43 & .05 \\
\hline 24 & .05 & .04 & .04 & .04 & .20 & .06 & .08 & .08 & .08 & .04 & .29 & .06 \\
\hline 25 & .07 & .03 & .04 & .05 & .07 & .06 & .07 & .06 & .07 & .05 & .17 & .04 \\
\hline 26 & .07 & .04 & .08 & .04 & .07 & .07 & .07 & .06 & .07 & .08 & .02 & .05 \\
\hline 27 & .07 & .10 & .08 & .15 & .06 & .06 & .07 & .05 & $.07^{\star}$ & .19 & .02 & .04 \\
\hline 28 & .07 & .06 & .04 & .58 & .06 & .07 & .11 & .29 & .07 & .08 & .02 & .05 \\
\hline 29 & .08 & .04 & .03 & .03 & .06 & .07 & .10 & .09 & $.09^{*}$ & .06 & .01 & .06 \\
\hline 30 & .08 & 1.1 & .04 & .05 & ------ & .07 & .10 & .06 & .08 & .05 & .05 & .06 \\
\hline 31 & .08 & ------ & .03 & .05 & ------ & .07 & ------ & .06 & ------ & .04 & .71 & ----- \\
\hline Total & 1.26 & 2.54 & 3.77 & 2.03 & 2.00 & 2.09 & 2.17 & 3.57 & 2.03 & 3.87 & 5.15 & 1.37 \\
\hline Mean & .041 & .085 & .12 & .065 & .069 & .067 & .072 & .12 & .068 & .12 & .17 & .046 \\
\hline Max & .14 & 1.1 & 1.8 & .58 & .20 & .08 & .11 & .44 & .10 & .38 & .71 & .13 \\
\hline Min & .01 & .01 & .03 & .03 & .04 & .06 & .05 & .05 & .05 & .04 & .01 & .03 \\
\hline Acre-Ft & 2.5 & 5.0 & 7.5 & 4.0 & 4.0 & 4.1 & 4.3 & 7.1 & 4.0 & 7.7 & 10 & 2.7 \\
\hline Wtr Year & 2008 & Total & 31.85 & & & & Max & 1.8 & Min & .01 & Acre-Ft & 63 \\
\hline Cal Year & 2007 & Total & 21.18 & & & & Max & 1.8 & Min & 0 & Acre-Ft & 42 \\
\hline
\end{tabular}

*Estimate 


\section{E1222 Sandia Canyon Tributary from Roads and Grounds}

Location. Lat. $35^{\circ}$ 52' 33", long. 106 19' 5", Sec. 16, T. 19 N., R. 6 E., Los Alamos County.

Drainage Area. $0.01 \mathrm{mi}^{2}$.

Period of Record. October 1, 2006, to September 30, 2008.

Gage. Data logger and 9” Parshall flume. Elevation of gage is 7,326 ft above NGVD.

Remarks. Records are good. Records for this site existed before period of record but are not reliable.

Extremes for Period of Record. Maximum discharge, $4.4 \mathrm{ft}^{3} / \mathrm{s}$, September 2, 2007, gage height $1.26 \mathrm{ft}$. No flow most of the time.

Extremes for Current Year. Peak discharges above base of $2.0 \mathrm{ft}^{3} / \mathrm{s}$ and maximum (*):

\begin{tabular}{|c|c|c|c|}
\hline Date & Time & Discharge $\left.\mathbf{( f t}^{3} / \mathbf{s}\right)$ & Gage Height (ft) \\
\hline December 1 & 0135 & $2.2^{*}$ & $0.80^{*}$
\end{tabular}

No flow most of the time.

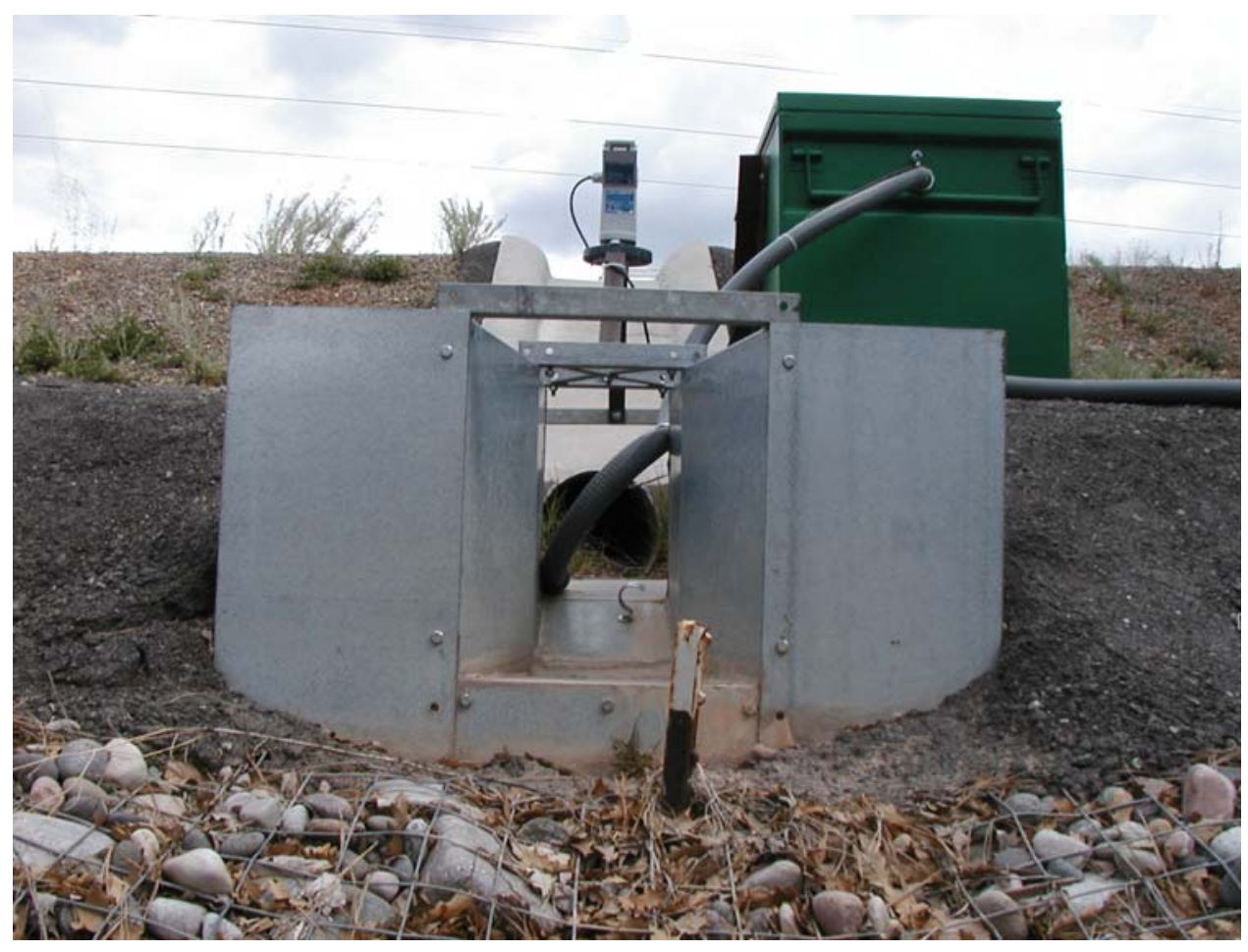




\section{E1222 Sandia Canyon Tributary from Roads and Grounds}

\section{Station Analysis}

\section{Water Year}

Equipment. Station is equipped with Sutron 8210 data logger (5-min. interval) and milltronics sonic probe mounted on a 9" Parshall flume. The system is powered by a solar-panel battery system housed in a NEMA shelter. Station is equipped with an ISCO pump sampler for water-quality sample collection. ISCO is housed in a separate shelter, a 3' $\times 4^{\prime}$ metal box. Sampler is triggered by stage through the data logger. The staff in the 9" Parshall flume is the reference gage. No provision for discharge measurements above wading stage. All high measurements will be by slope area or peak flow computation methods.

Field Work. This station was visited 23 times to conduct discharge measurements and service the instrumentation. Field inspections for the gage are listed under site history files on the Hydstra database. Discharge measurements for the gage are listed under site gauging files on the Hydstra database.

Datum Correction. None.

Gage-Height Record. The data logger referenced to the outside staff gave a complete and satisfactory record, except during the period of January 7 to February 15 when ice in flume affected gage height.

Rating. Channel is straight above and below gage. Channel bottom consists of vegetation, bedrock and gravel.

Twenty-one inspections of no flow and two inspection of snow melt were made this water year.

Rating No. 1 was developed based on the computation of 9" Parshall flume. Point of zero flow is 0.00 gage height.

Discharge. Discharge was computed by applying Rating No. 1 directly. Those days estimated at zero flow were based on precipitation and nearby gage stations.

Remarks. Records are good. 
E1222 Sandia Canyon Tributary from Roads and Grounds

Daily Mean Discharge in Cubic Feet per Second

Water Year October 2007 to September 2008

\begin{tabular}{|c|c|c|c|c|c|c|c|c|c|c|c|c|}
\hline DAY & ОСТ & NOV & DEC & JAN & FEB & MAR & APR & MAY & JUN & JUL & AUG & SEP \\
\hline 1 & 0 & 0 & .32 & 0 & $0^{*}$ & 0 & 0 & 0 & 0 & 0 & 0 & .01 \\
\hline 2 & 0 & 0 & 0 & 0 & $0^{*}$ & 0 & 0 & 0 & 0 & 0 & 0 & 0 \\
\hline 3 & 0 & 0 & 0 & 0 & $0^{*}$ & $0^{*}$ & 0 & 0 & 0 & 0 & 0 & 0 \\
\hline 4 & 0 & 0 & 0 & 0 & $0^{*}$ & 0 & 0 & 0 & 0 & 0 & .05 & 0 \\
\hline 5 & 0 & 0 & 0 & 0 & $0^{*}$ & 0 & 0 & 0 & 0 & 0 & 0 & 0 \\
\hline 6 & 0 & 0 & 0 & 0 & $0^{*}$ & $0^{*}$ & 0 & 0 & 0 & 0 & $0^{*}$ & 0 \\
\hline 7 & 0 & 0 & 0 & $0^{*}$ & $0^{*}$ & $0^{*}$ & 0 & 0 & 0 & 0 & $0^{*}$ & 0 \\
\hline 8 & 0 & 0 & .02 & $0^{*}$ & $0^{*}$ & 0 & 0 & 0 & 0 & 0 & $0^{*}$ & 0 \\
\hline 9 & 0 & 0 & 0 & $0^{*}$ & $0^{*}$ & 0 & 0 & 0 & 0 & 0 & 0 & 0 \\
\hline 10 & 0 & 0 & 0 & $0^{*}$ & $0^{*}$ & 0 & 0 & 0 & 0 & 0 & 0 & 0 \\
\hline 11 & 0 & 0 & 0 & $0^{*}$ & $0^{*}$ & 0 & 0 & 0 & 0 & 0 & 0 & 0 \\
\hline 12 & 0 & 0 & 0 & $0^{*}$ & $0^{*}$ & 0 & 0 & 0 & 0 & 0 & 0 & 0 \\
\hline 13 & 0 & 0 & 0 & $0^{*}$ & $0^{*}$ & 0 & 0 & 0 & 0 & 0 & 0 & 0 \\
\hline 14 & 0 & 0 & 0 & $0^{*}$ & $0^{*}$ & 0 & 0 & 0 & 0 & 0 & 0 & 0 \\
\hline 15 & 0 & 0 & 0 & $0^{*}$ & $0^{*}$ & 0 & 0 & 0 & 0 & 0 & 0 & 0 \\
\hline 16 & 0 & 0 & 0 & $0^{*}$ & 0 & 0 & 0 & 0 & 0 & 0 & .01 & 0 \\
\hline 17 & 0 & 0 & 0 & $0^{*}$ & 0 & 0 & 0 & 0 & 0 & 0 & 0 & 0 \\
\hline 18 & 0 & 0 & 0 & $0^{*}$ & 0 & 0 & 0 & 0 & 0 & 0 & 0 & 0 \\
\hline 19 & 0 & 0 & 0 & $0^{*}$ & 0 & 0 & 0 & 0 & 0 & 0 & 0 & 0 \\
\hline 20 & 0 & 0 & 0 & $0^{*}$ & 0 & 0 & 0 & 0 & 0 & 0 & .01 & 0 \\
\hline 21 & 0 & 0 & 0 & $0^{*}$ & 0 & 0 & 0 & 0 & 0 & 0 & 0 & 0 \\
\hline 22 & 0 & 0 & 0 & $0^{*}$ & 0 & 0 & 0 & 0 & 0 & 0 & 0 & 0 \\
\hline 23 & 0 & 0 & 0 & $0^{*}$ & $0^{*}$ & 0 & 0 & 0 & 0 & 0 & .02 & 0 \\
\hline 24 & 0 & 0 & 0 & $0^{*}$ & 0 & 0 & 0 & 0 & 0 & 0 & .03 & 0 \\
\hline 25 & 0 & 0 & 0 & $0^{*}$ & 0 & 0 & 0 & 0 & 0 & 0 & .01 & 0 \\
\hline 26 & 0 & 0 & 0 & $0^{*}$ & 0 & 0 & 0 & 0 & 0 & 0 & 0 & 0 \\
\hline 27 & 0 & 0 & 0 & $0^{*}$ & 0 & 0 & 0 & 0 & 0 & .01 & 0 & 0 \\
\hline 28 & 0 & 0 & 0 & $0^{*}$ & 0 & 0 & 0 & .02 & 0 & 0 & 0 & 0 \\
\hline 29 & 0 & 0 & 0 & $0^{*}$ & 0 & 0 & 0 & 0 & 0 & 0 & 0 & 0 \\
\hline 30 & 0 & .09 & 0 & $0^{*}$ & ----- & 0 & 0 & 0 & 0 & 0 & 0 & 0 \\
\hline 31 & 0 & ----- & 0 & $0^{*}$ & ------ & 0 & ------ & 0 & ----- & 0 & .08 & ------ \\
\hline Total & 0 & 0.09 & 0.34 & 0 & 0 & 0 & 0 & 0.02 & 0 & 0.01 & 0.21 & 0.01 \\
\hline Mean & 0 & .003 & .011 & 0 & 0 & 0 & 0 & .001 & 0 & 0 & .007 & 0 \\
\hline Max & 0 & .09 & .32 & 0 & 0 & 0 & 0 & .02 & 0 & .01 & .08 & .01 \\
\hline Min & 0 & 0 & 0 & 0 & 0 & 0 & 0 & 0 & 0 & 0 & 0 & 0 \\
\hline Acre-Ft & 0 & .18 & .67 & 0 & 0 & 0 & 0 & .04 & 0 & .02 & .42 & .02 \\
\hline Wtr Year & 2008 & Total & 0.68 & Mean & & 02 & Max & .32 & Min & 0 & Acre-Ft & 1.3 \\
\hline Cal Year & 2007 & Total & 1.55 & Mean & & 04 & Max & .32 & Min & 0 & Acre-Ft & 3.1 \\
\hline
\end{tabular}

*Estimate 


\section{E1223 Sandia Canyon Tributary from Sigma Building}

Location. Lat $35^{\circ}$ 52' 22", long $106^{\circ}$ 19' 3", SW 1/4, Sec. 16, T. 19 N., R. 6 E., Los

Alamos County.

Drainage Area. $0.003 \mathrm{mi}^{2}$.

Period of Record. October 1, 2006, to September 30, 2008.

Gage. Data logger and 9” Parshall flume. Elevation of gage is 7,368 ft above NGVD.

Remarks. Records are good. Records for this site existed before period of record but are not reliable.

Extremes for Period of Record. Maximum discharge, $1.1 \mathrm{ft}^{3} / \mathrm{s}$, August 10, 2008, gage height $0.50 \mathrm{ft}$. No flow most of the time.

Extremes for Current Year. Peak discharges above base of $1.0 \mathrm{ft}^{3} / \mathrm{s}$ and maximum (*):

\begin{tabular}{|c|c|c|c|}
\hline Date & Time & Discharge (ft $\left.{ }^{3} / \mathbf{s}\right)$ & Gage Height (ft) \\
\hline August 10 & 1115 & $1.1^{*}$ & $0.50^{*}$
\end{tabular}

No flow most of the time.

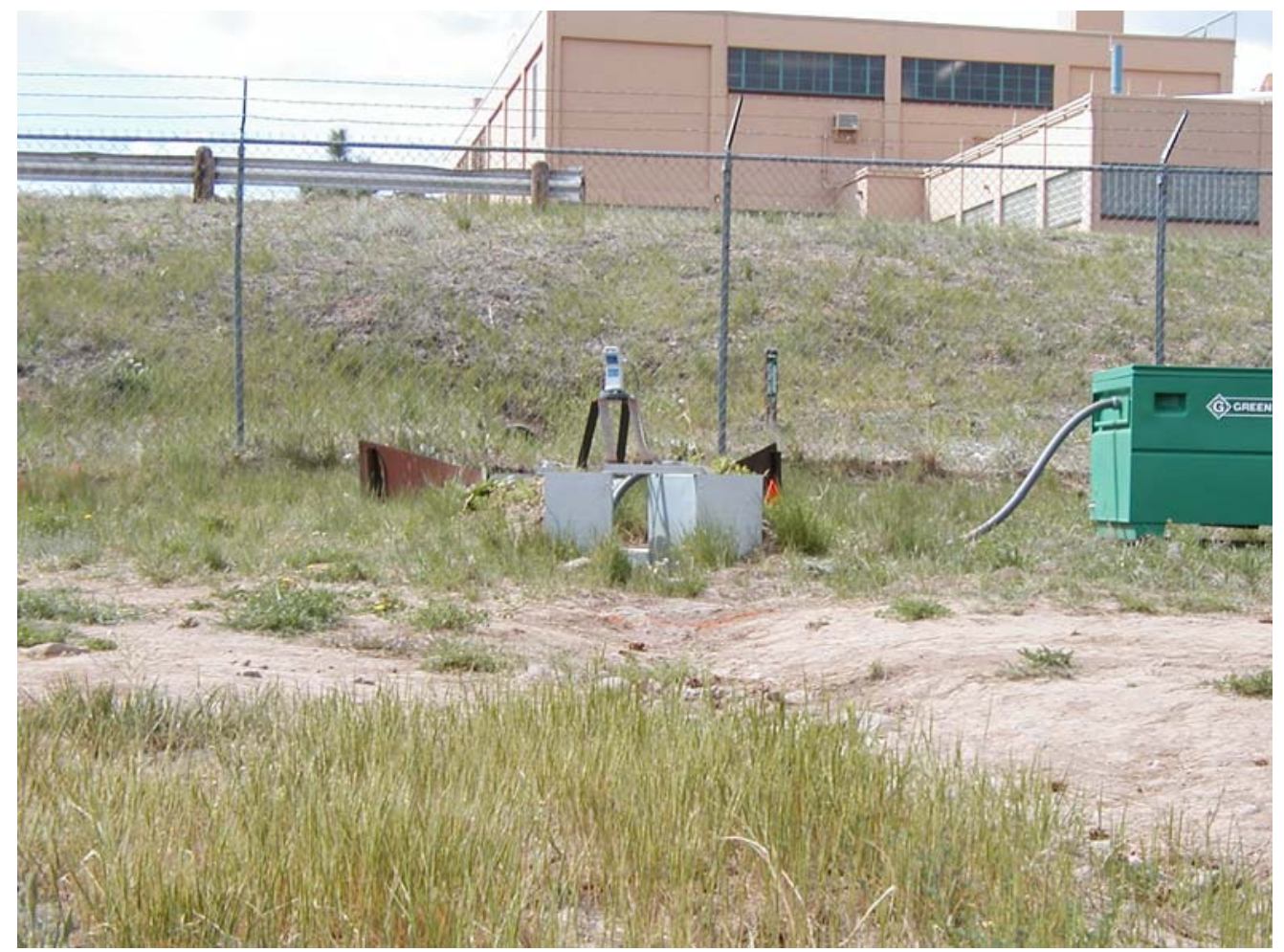




\section{E1223 Sandia Canyon Tributary from Sigma Building \\ Station Analysis}

2008 Water Year

Equipment. Station is equipped with Sutron 8210 data logger (5-min. interval) and milltronics sonic probe mounted on a 9" Parshall flume. The system is powered by a solar-panel battery system housed in a NEMA shelter. Station is equipped with an ISCO pump sampler for water-quality sample collection. ISCO is housed in a separate shelter, a $3^{\prime} \times 4^{\prime}$ metal box. Sampler is triggered by stage through the data logger. The staff in the 9 " Parshall flume is the reference gage. No provision for discharge measurements above wading stage.

Field Work. This station was visited 18 times to conduct discharge measurements and service the instrumentation. Field inspections for the gage are listed under site history files on the Hydstra database. Discharge measurements for the gage are listed under site gauging files on the Hydstra database.

Datum Correction. None.

Gage-Height Record. The data logger referenced to the outside staff gave a complete and satisfactory record, except during the periods of January 6-9 and February 2-16 when snow and ice affected gage height and May 30 to June 30 when data were lost.

Rating. Above 9" Parshall flume is asphalt lot. Flume is located southwest of roadway on the shoulder of the road. Below flume is asphalt roadway.

Eighteen inspections of no flow were made this year.

Rating No. 1 was developed based on the computation of 9" Parshall flume. Point of zero flow is 0.00 gage height.

Discharge. Discharge was computed by applying Rating No. 1 directly. Those days estimated at zero flow were based on precipitation and nearby gage stations.

Remarks. Records are good. 
E1223 Sandia Canyon tributary from Sigma Building

Daily Mean Discharge in Cubic Feet per Second

Water Year October 2007 to September 2008

\begin{tabular}{|c|c|c|c|c|c|c|c|c|c|c|c|c|}
\hline DAY & OCT & NOV & DEC & JAN & FEB & MAR & APR & MAY & JUN & JUL & AUG & SEP \\
\hline 1 & 0 & 0 & .02 & 0 & 0 & 0 & 0 & $0^{*}$ & $0^{*}$ & 0 & 0 & 0 \\
\hline 2 & 0 & 0 & 0 & 0 & $0^{*}$ & $0^{*}$ & 0 & 0 & $0^{*}$ & 0 & 0 & 0 \\
\hline 3 & 0 & 0 & 0 & 0 & $0^{*}$ & $0^{*}$ & 0 & 0 & $0^{*}$ & 0 & 0 & 0 \\
\hline 4 & 0 & 0 & 0 & 0 & $0^{*}$ & 0 & 0 & 0 & $0^{*}$ & 0 & 0 & 0 \\
\hline 5 & 0 & 0 & 0 & 0 & $0^{*}$ & 0 & 0 & 0 & $0^{*}$ & 0 & 0 & 0 \\
\hline 6 & 0 & 0 & 0 & $0^{*}$ & $0^{*}$ & $0^{*}$ & 0 & 0 & $0^{*}$ & 0 & 0 & 0 \\
\hline 7 & 0 & 0 & 0 & $0^{*}$ & $0^{*}$ & 0 & 0 & 0 & $0^{*}$ & 0 & 0 & 0 \\
\hline 8 & 0 & 0 & 0 & $0^{*}$ & $0^{*}$ & 0 & 0 & 0 & $0^{*}$ & 0 & .01 & 0 \\
\hline 9 & 0 & 0 & 0 & $0^{*}$ & $0^{*}$ & 0 & 0 & 0 & $0^{*}$ & 0 & 0 & 0 \\
\hline 10 & 0 & 0 & 0 & 0 & $0^{*}$ & 0 & 0 & 0 & $0^{*}$ & 0 & .01 & 0 \\
\hline 11 & 0 & 0 & 0 & 0 & $0^{*}$ & 0 & 0 & 0 & $0^{*}$ & 0 & 0 & 0 \\
\hline 12 & 0 & 0 & 0 & 0 & $0^{*}$ & 0 & 0 & 0 & $0^{*}$ & 0 & 0 & 0 \\
\hline 13 & 0 & 0 & 0 & 0 & $0^{*}$ & 0 & 0 & 0 & $0^{*}$ & 0 & 0 & 0 \\
\hline 14 & 0 & 0 & 0 & 0 & $0^{*}$ & 0 & 0 & 0 & $0^{*}$ & 0 & 0 & 0 \\
\hline 15 & 0 & 0 & 0 & 0 & $0^{*}$ & 0 & 0 & 0 & $0^{*}$ & 0 & 0 & 0 \\
\hline 16 & 0 & 0 & 0 & 0 & $0^{*}$ & 0 & 0 & 0 & $0^{*}$ & 0 & 0 & 0 \\
\hline 17 & 0 & 0 & 0 & 0 & 0 & 0 & 0 & 0 & $0^{*}$ & 0 & 0 & 0 \\
\hline 18 & 0 & 0 & 0 & 0 & 0 & 0 & 0 & 0 & $0^{*}$ & 0 & 0 & 0 \\
\hline 19 & 0 & 0 & 0 & 0 & 0 & 0 & 0 & 0 & $0^{\star}$ & 0 & 0 & 0 \\
\hline 20 & 0 & 0 & 0 & 0 & 0 & 0 & 0 & 0 & $0^{*}$ & 0 & 0 & 0 \\
\hline 21 & 0 & 0 & 0 & 0 & 0 & 0 & 0 & 0 & $0^{*}$ & 0 & 0 & 0 \\
\hline 22 & 0 & 0 & 0 & 0 & 0 & 0 & 0 & 0 & $0^{*}$ & 0 & 0 & 0 \\
\hline 23 & 0 & $0^{*}$ & 0 & 0 & 0 & 0 & 0 & 0 & $0^{*}$ & 0 & 0 & 0 \\
\hline 24 & 0 & $0^{*}$ & 0 & 0 & 0 & 0 & 0 & 0 & $0^{*}$ & 0 & 0 & 0 \\
\hline 25 & 0 & 0 & 0 & 0 & 0 & 0 & 0 & 0 & $0^{*}$ & 0 & 0 & 0 \\
\hline 26 & 0 & 0 & 0 & 0 & 0 & 0 & 0 & 0 & $0^{*}$ & 0 & 0 & 0 \\
\hline 27 & 0 & 0 & 0 & 0 & 0 & 0 & 0 & 0 & $0^{*}$ & 0 & 0 & 0 \\
\hline 28 & 0 & 0 & 0 & .01 & 0 & 0 & 0 & 0 & $0^{*}$ & 0 & 0 & 0 \\
\hline 29 & 0 & 0 & 0 & 0 & 0 & 0 & 0 & 0 & $0^{*}$ & 0 & 0 & 0 \\
\hline 30 & 0 & .01 & 0 & 0 & ------ & 0 & 0 & $0^{*}$ & $0^{*}$ & 0 & 0 & 0 \\
\hline 31 & 0 & ----- & 0 & 0 & ------ & 0 & ------ & $0^{*}$ & ------ & 0 & .01 & ------ \\
\hline Total & 0 & 0.01 & 0.02 & 0.01 & 0 & 0 & 0 & 0 & 0 & 0 & 0.03 & 0 \\
\hline Mean & 0 & 0 & .001 & 0 & 0 & 0 & 0 & 0 & 0 & 0 & .001 & 0 \\
\hline Max & 0 & .01 & .02 & .01 & 0 & 0 & 0 & 0 & 0 & 0 & .01 & 0 \\
\hline Min & 0 & 0 & 0 & 0 & 0 & 0 & 0 & 0 & 0 & 0 & 0 & 0 \\
\hline Acre-Ft & 0 & .02 & .04 & .02 & 0 & 0 & 0 & 0 & 0 & 0 & .06 & 0 \\
\hline Wtr Year & 2008 & Total & 0.07 & Mean & & 0 & lax & .02 & Min & 0 & Acre-Ft & 0.14 \\
\hline Cal Year & 2007 & Total & 0.24 & Mean & & 01 & hax & .10 & Min & 0 & Acre-Ft & 0.48 \\
\hline
\end{tabular}

*Estimate 


\section{E12235 Sandia Canyon Tributary from MRF}

Location. Lat. $35^{\circ}$ 52' 26", long $106^{\circ} 18^{\prime}$ 47", SW 1/4, Sec. 16, T. 19 N., R. 6 E., Los Alamos County.

Drainage Area. $0.001 \mathrm{mi}^{2}$.

Period of Record. October 1, 2006, to September 30, 2008.

Gage. Data logger and 9” Parshall flume. Elevation of gage is 7,285 ft above NGVD.

Remarks. Records are good. Records for this site existed before period of record but are not reliable.

Extremes for Period of Record. Maximum discharge, $2.50 \mathrm{ft}^{3} / \mathrm{s}$, August 26, 2007, gage height $1.13 \mathrm{ft}$. No flow most of the time.

Extremes for Current Year. Maximum discharge is $1.81 \mathrm{ft}^{3} / \mathrm{s}$ at $1110 \mathrm{~h}$, August 10, gage height $0.92 \mathrm{ft}$. No peak above base of $2.0 \mathrm{ft}^{3} / \mathrm{s}$. No flow most of the time.

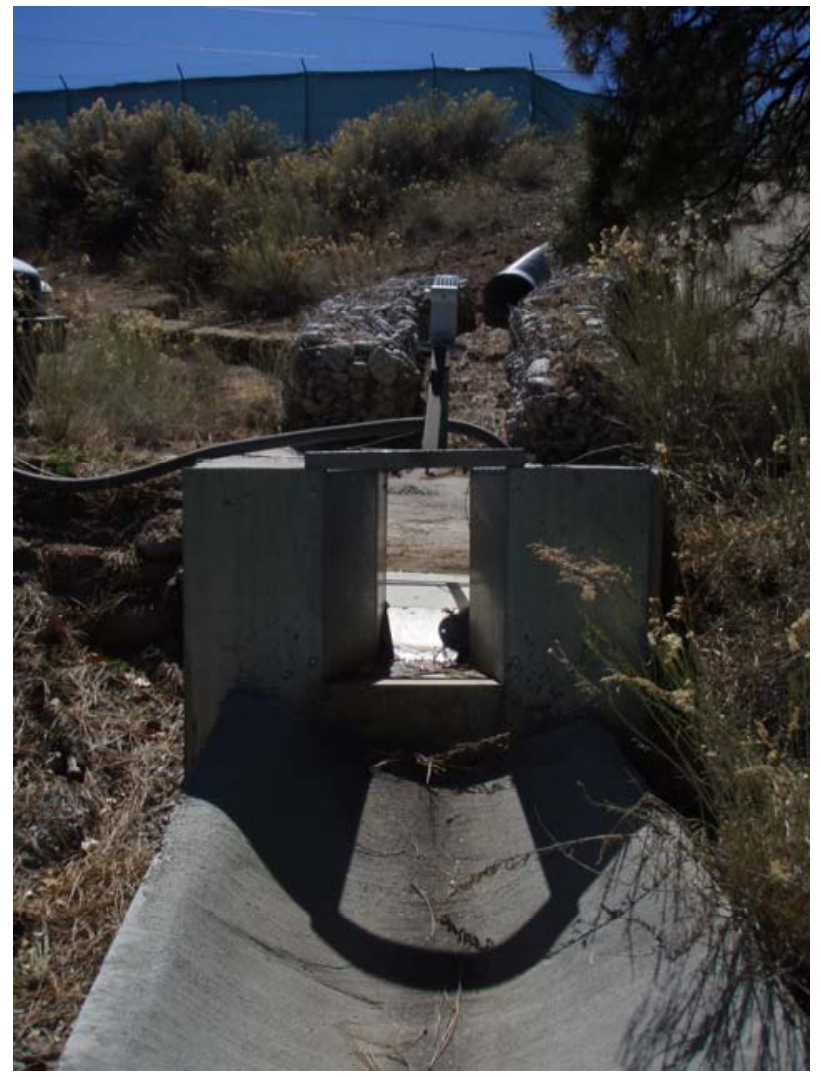




\section{E12235 Sandia Canyon Tributary from MRF \\ Station Analysis}

\section{Water Year}

Equipment. Station is equipped with Sutron 8210 data logger (5-min. interval) and milltronics sonic probe mounted on a 9" Parshall flume. The system is powered by a solar-panel battery system housed in a NEMA shelter. Station is equipped with an ISCO pump sampler for water-quality sample collection. ISCO is housed in a separate shelter, a $3^{\prime} \times 4^{\prime}$ metal box. Sampler is triggered by stage through the data logger. The staff in the 9" Parshall flume is the reference gage. No provision for discharge measurements above wading stage.

Field Work. This station was visited 20 times to conduct discharge measurements and service the instrumentation. Field inspections for the gage are listed under site history files on the Hydstra database. Discharge measurements for the gage are listed under site gauging files on the Hydstra database.

Datum Correction. None.

Gage-Height Record. The data logger referenced to the outside staff gave a complete and satisfactory record, except for January 7-9 and February 3-12 when snow and ice affected gage height.

Rating. Channel is straight above and below 9" Parshall flume with large coble.

Eighteen inspections of no flow and two days of snow melt were made this year.

Rating No. 1 was developed based on the computation of 9" Parshall flume. Point of zero flow is 0.00 gage height.

Discharge. Discharge was computed by applying Rating No. 1 directly. Those days estimated at zero flow were based on precipitation and nearby gage stations.

Remarks. Records are good. 
E12235 Sandia Canyon Tributary from MRF

Daily Mean Discharge in Cubic Feet per Second

Water Year October 2007 to September 2008

\begin{tabular}{|c|c|c|c|c|c|c|c|c|c|c|c|c|}
\hline DAY & OCT & NOV & DEC & JAN & FEB & MAR & APR & MAY & JUN & JUL & AUG & SEP \\
\hline 1 & 0 & 0 & .02 & 0 & 0 & 0 & 0 & 0 & 0 & 0 & 0 & 0 \\
\hline 2 & 0 & 0 & 0 & 0 & 0 & 0 & 0 & 0 & 0 & 0 & 0 & 0 \\
\hline 3 & 0 & 0 & 0 & 0 & $0^{*}$ & 0 & 0 & 0 & 0 & 0 & 0 & 0 \\
\hline 4 & 0 & 0 & 0 & 0 & $0^{*}$ & 0 & 0 & 0 & 0 & 0 & .01 & 0 \\
\hline 5 & 0 & 0 & 0 & 0 & $0^{*}$ & 0 & 0 & 0 & 0 & 0 & 0 & 0 \\
\hline 6 & 0 & 0 & 0 & 0 & $0^{*}$ & 0 & 0 & 0 & 0 & 0 & 0 & 0 \\
\hline 7 & 0 & 0 & 0 & $0^{*}$ & $0^{*}$ & 0 & 0 & 0 & 0 & 0 & 0 & 0 \\
\hline 8 & 0 & 0 & 0 & $0^{*}$ & $0^{*}$ & 0 & 0 & 0 & 0 & 0 & .01 & 0 \\
\hline 9 & 0 & 0 & 0 & $0^{*}$ & $0^{*}$ & 0 & 0 & 0 & 0 & 0 & .01 & 0 \\
\hline 10 & 0 & 0 & 0 & 0 & $0^{*}$ & 0 & 0 & 0 & 0 & 0 & .02 & 0 \\
\hline 11 & 0 & 0 & 0 & 0 & $0^{*}$ & 0 & 0 & 0 & 0 & 0 & 0 & 0 \\
\hline 12 & 0 & 0 & 0 & 0 & $0^{*}$ & 0 & 0 & 0 & 0 & 0 & 0 & 0 \\
\hline 13 & 0 & 0 & 0 & 0 & 0 & 0 & 0 & 0 & 0 & 0 & 0 & 0 \\
\hline 14 & 0 & 0 & 0 & 0 & 0 & 0 & 0 & 0 & 0 & 0 & 0 & 0 \\
\hline 15 & 0 & 0 & 0 & 0 & 0 & 0 & 0 & 0 & 0 & 0 & 0 & 0 \\
\hline 16 & 0 & 0 & 0 & 0 & 0 & 0 & 0 & 0 & 0 & 0 & 0 & 0 \\
\hline 17 & 0 & 0 & 0 & 0 & 0 & 0 & 0 & 0 & 0 & 0 & 0 & 0 \\
\hline 18 & 0 & 0 & 0 & 0 & 0 & 0 & 0 & 0 & 0 & 0 & 0 & 0 \\
\hline 19 & 0 & 0 & 0 & 0 & 0 & 0 & 0 & 0 & 0 & 0 & 0 & 0 \\
\hline 20 & 0 & 0 & 0 & 0 & 0 & 0 & 0 & 0 & 0 & 0 & 0 & 0 \\
\hline 21 & 0 & 0 & 0 & 0 & 0 & 0 & 0 & 0 & 0 & 0 & 0 & 0 \\
\hline 22 & 0 & 0 & 0 & 0 & 0 & 0 & 0 & 0 & 0 & 0 & 0 & 0 \\
\hline 23 & 0 & 0 & 0 & 0 & 0 & 0 & 0 & 0 & 0 & 0 & 0 & 0 \\
\hline 24 & 0 & 0 & 0 & 0 & 0 & 0 & 0 & 0 & 0 & 0 & 0 & 0 \\
\hline 25 & 0 & 0 & 0 & 0 & 0 & 0 & 0 & 0 & 0 & 0 & 0 & 0 \\
\hline 26 & 0 & 0 & 0 & 0 & 0 & 0 & 0 & 0 & 0 & 0 & 0 & 0 \\
\hline 27 & 0 & 0 & 0 & 0 & 0 & 0 & 0 & 0 & 0 & 0 & 0 & 0 \\
\hline 28 & 0 & 0 & 0 & 0 & 0 & 0 & 0 & .01 & 0 & 0 & 0 & 0 \\
\hline 29 & 0 & 0 & 0 & 0 & 0 & 0 & 0 & 0 & 0 & 0 & 0 & 0 \\
\hline 30 & 0 & .01 & 0 & 0 & ------ & 0 & 0 & 0 & 0 & 0 & 0 & 0 \\
\hline 31 & 0 & ----- & 0 & 0 & ------ & 0 & ------ & 0 & ----- & 0 & .01 & ------ \\
\hline Total & 0 & 0.01 & 0.02 & 0 & 0 & 0 & 0 & 0.01 & 0 & 0 & 0.06 & 0 \\
\hline Mean & 0 & 0 & .001 & 0 & 0 & 0 & 0 & 0 & 0 & 0 & .002 & 0 \\
\hline Max & 0 & .01 & .02 & 0 & 0 & 0 & 0 & .01 & 0 & 0 & .02 & 0 \\
\hline Min & 0 & 0 & 0 & 0 & 0 & 0 & 0 & 0 & 0 & 0 & 0 & 0 \\
\hline Acre-Ft & 0 & .02 & .04 & 0 & 0 & 0 & 0 & .02 & 0 & 0 & .12 & 0 \\
\hline Wtr Year & 2008 & Total & 0.10 & Mean & & 0 & Max & .02 & Min & 0 & Acre-Ft & 0.20 \\
\hline Cal Year & 2007 & Total & 0.15 & Mean & & 0 & Max & .02 & Min & 0 & Acre-Ft & 0.30 \\
\hline
\end{tabular}

*Estimate 


\section{E1225 Sandia Canyon Tributary at Heavy Equipment}

Location. Lat $35^{\circ}$ 52' 22", long $106^{\circ} 18^{\prime} 46^{\prime \prime}$, SW 1/4, Sec. 16, T.19 N., R 6 E., Los Alamos County.

Drainage Area. $0.008 \mathrm{mi}^{2}$.

Period of Record. October 1, 2002, to September 30, 2008.

Gage. Data logger, 12" Parshall flume, rain gage with cellular telemetry. Elevation of gage is 7,322 ft above NGVD from land survey.

Remarks. Records are good.

Average Discharge. $6 \mathrm{yr}, 0.003 \mathrm{ft}^{3} / \mathrm{s}, 2.30$ acre-ft/yr.

Extremes for Period of Record. Maximum discharge, $8.90 \mathrm{ft}^{3} / \mathrm{s}$, August 10, 2008, gage height $1.69 \mathrm{ft}$. No Flow most of the time.

Extremes for Current Year. Peak discharges above base of $5.0 \mathrm{ft}^{3} / \mathrm{s}$ and maximum (*):

\begin{tabular}{|c|c|c|c|}
\hline Date & Time & Discharge $\left(\mathbf{f t}^{\mathbf{3}} / \mathbf{s}\right)$ & Gage Height (ft) \\
\hline May 28 & 1545 & 7.3 & 1.49 \\
\hline August 8 & 2345 & 6.0 & 1.30 \\
\hline August 9 & 1215 & 7.4 & 1.50 \\
\hline August 10 & 1120 & $8.9^{*}$ & $1.69^{*}$ \\
\hline
\end{tabular}

No flow most of the time.

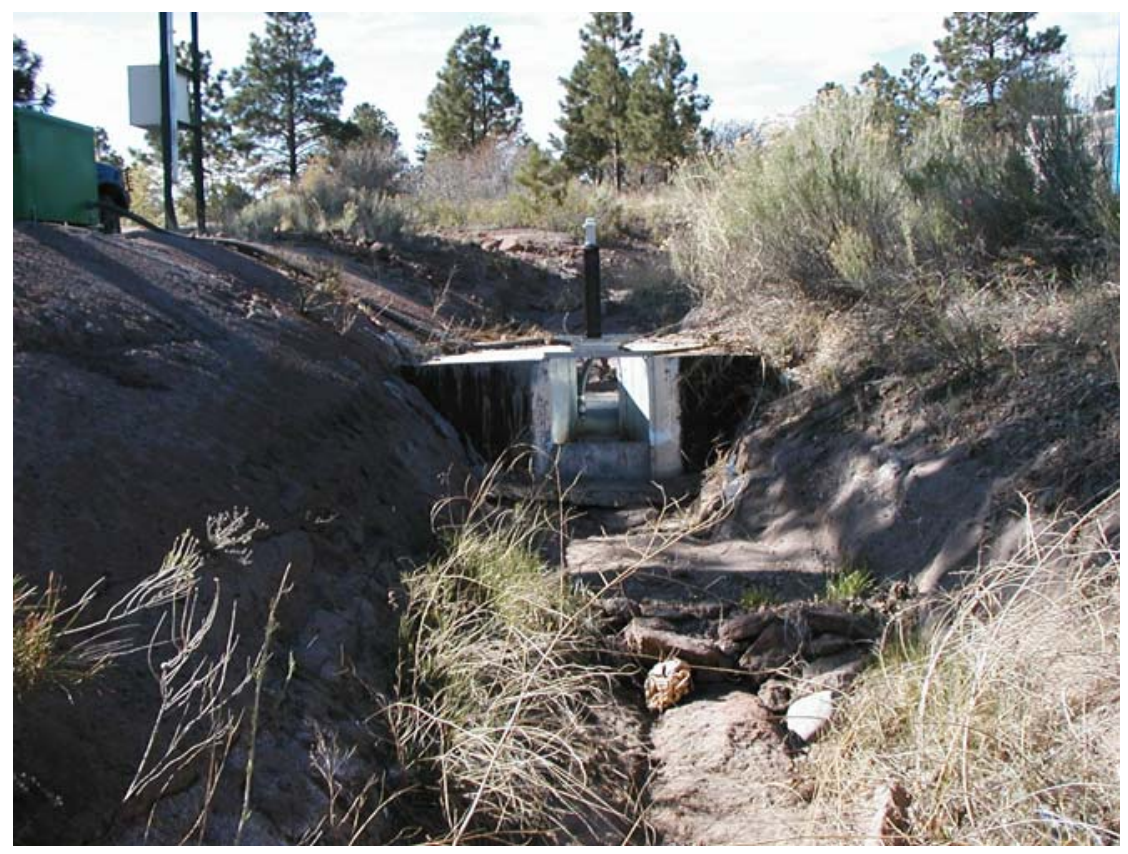




\section{E1225 Sandia Canyon Tributary at Heavy Equipment}

\section{Station Analysis}

\section{Water Year}

Equipment. Station is equipped with Sutron 8210 data logger (5-min. interval) with milltronics sonic probe mounted on a 12" Parshall flume and cellular telemetry with speech modem. Phone upgraded May 29 from analog to digital service. System is powered by a solar-panel battery system housed in a NEMA shelter. The gage station is also equipped with an ISCO pump sampler for water-quality data collection. ISCO is housed in a separate shelter, a $3^{\prime} \times 4^{\prime}$ metal box. Sampler is triggered by stage through the data logger. The staff in the $12^{\prime \prime}$ Parshall flume is the reference gage. There is no provision for direct discharge measurements above wading stage.

Station is also equipped with a tipping bucket rain gage, Rain collection II, which was installed on March 27, 2008. All equipment is powered with a solar-panel batterycharging system. Rain data are seasonal.

Field Work. The station was visited 22 times to conduct discharge measurements and service the instrumentation. Field inspections for the gage are listed under site history files on the Hydstra database. Discharge measurements for the gage are listed under site gauging files on the Hydstra database.

Datum Correction. None.

Gage-Height Record. The data logger referenced to the outside staff gave a complete and satisfactory record for the year, except for the periods of December 10-18, January 5-12, and January 29 to March 10 when ice in the flume affected gage height.

Rating. The channel is straight above and below gage. It is confined to the main channel by cut banks on both sides. The bottom is a 10 -ft-wide channel prone to no shifting. Low water control is the 12" Parshall flume.

Nineteen inspections of no flow and three inspections of snow melt were made this year.

Rating No. 1 was developed based on the computation of 12" Parshall flume. Point of zero flow is 0.00 gage height.

Discharge. Discharge was computed by applying Rating No. 1 directly. Those days estimated at zero flow were based on precipitation and nearby gage stations.

Remarks. Records are good. 
E1225 Sandia Canyon Tributary at Heavy Equipment

Daily Mean Discharge in Cubic Feet per Second

Water Year October 2007 to September 2008

\begin{tabular}{|c|c|c|c|c|c|c|c|c|c|c|c|c|}
\hline DAY & ОСТ & NOV & DEC & JAN & FEB & MAR & APR & MAY & JUN & JUL & AUG & SEP \\
\hline 1 & .02 & 0 & .28 & 0 & 0 & $0^{*}$ & 0 & 0 & 0 & 0 & 0 & .01 \\
\hline 2 & 0 & 0 & 0 & 0 & 0 & $0^{*}$ & 0 & 0 & 0 & 0 & 0 & 0 \\
\hline 3 & 0 & 0 & 0 & 0 & $0^{*}$ & $0^{*}$ & 0 & 0 & 0 & 0 & 0 & 0 \\
\hline 4 & 0 & 0 & 0 & 0 & $0^{*}$ & $0^{*}$ & 0 & 0 & 0 & 0 & .07 & 0 \\
\hline 5 & 0 & 0 & 0 & $0^{*}$ & $0^{*}$ & $0^{*}$ & 0 & 0 & 0 & 0 & 0 & 0 \\
\hline 6 & 0 & 0 & 0 & $0^{*}$ & $0^{*}$ & $0^{*}$ & 0 & 0 & 0 & 0 & 0 & 0 \\
\hline 7 & 0 & 0 & 0 & $0^{*}$ & $0^{*}$ & $0^{*}$ & 0 & 0 & 0 & .01 & .01 & 0 \\
\hline 8 & 0 & 0 & .07 & $0^{*}$ & $0^{*}$ & $0^{*}$ & 0 & 0 & 0 & .02 & .08 & 0 \\
\hline 9 & 0 & 0 & 0 & $0^{*}$ & $0^{*}$ & $0^{*}$ & 0 & 0 & 0 & 0 & .10 & 0 \\
\hline 10 & 0 & 0 & $0^{*}$ & $0^{*}$ & $0^{*}$ & $0^{*}$ & 0 & 0 & 0 & 0 & .13 & 0 \\
\hline 11 & 0 & 0 & $0^{*}$ & $0^{*}$ & $0^{*}$ & 0 & 0 & 0 & 0 & 0 & 0 & 0 \\
\hline 12 & 0 & 0 & 0 & $0^{*}$ & $0^{*}$ & 0 & 0 & 0 & 0 & 0 & 0 & 0 \\
\hline 13 & 0 & 0 & $0^{*}$ & 0 & $0^{*}$ & 0 & 0 & 0 & 0 & 0 & 0 & 0 \\
\hline 14 & 0 & 0 & $0^{*}$ & 0 & $0^{*}$ & 0 & 0 & 0 & 0 & 0 & 0 & 0 \\
\hline 15 & 0 & 0 & $0^{*}$ & 0 & $0^{*}$ & 0 & 0 & .05 & 0 & 0 & 0 & 0 \\
\hline 16 & 0 & 0 & $0^{*}$ & 0 & $0^{*}$ & 0 & 0 & 0 & 0 & 0 & .04 & 0 \\
\hline 17 & 0 & 0 & $0^{*}$ & 0 & $0^{*}$ & 0 & 0 & 0 & 0 & 0 & 0 & 0 \\
\hline 18 & 0 & 0 & $0^{*}$ & 0 & $0^{*}$ & 0 & $0^{*}$ & 0 & 0 & 0 & 0 & 0 \\
\hline 19 & 0 & 0 & 0 & 0 & $0^{*}$ & 0 & 0 & 0 & 0 & 0 & 0 & 0 \\
\hline 20 & 0 & 0 & 0 & 0 & $0^{*}$ & 0 & 0 & 0 & 0 & 0 & .01 & 0 \\
\hline 21 & 0 & 0 & 0 & 0 & $0^{*}$ & 0 & 0 & 0 & 0 & .02 & 0 & 0 \\
\hline 22 & 0 & 0 & 0 & 0 & $0^{*}$ & 0 & 0 & 0 & 0 & 0 & 0 & .01 \\
\hline 23 & 0 & $0^{*}$ & 0 & 0 & $0^{*}$ & 0 & 0 & 0 & 0 & 0 & .03 & 0 \\
\hline 24 & 0 & 0 & 0 & 0 & $0^{*}$ & 0 & 0 & 0 & 0 & 0 & .02 & 0 \\
\hline 25 & 0 & 0 & 0 & 0 & $0^{*}$ & 0 & 0 & 0 & 0 & 0 & .01 & 0 \\
\hline 26 & 0 & 0 & 0 & 0 & $0^{*}$ & 0 & 0 & 0 & 0 & 0 & 0 & 0 \\
\hline 27 & 0 & 0 & 0 & .02 & $0^{*}$ & 0 & 0 & 0 & 0 & .01 & 0 & 0 \\
\hline 28 & 0 & 0 & 0 & .10 & $0^{*}$ & 0 & 0 & .07 & 0 & 0 & 0 & 0 \\
\hline 29 & 0 & 0 & 0 & $0^{*}$ & $0^{*}$ & 0 & 0 & 0 & 0 & 0 & 0 & 0 \\
\hline 30 & 0 & .16 & 0 & $0^{*}$ & ------ & 0 & 0 & 0 & 0 & 0 & .01 & 0 \\
\hline 31 & 0 & ----- & 0 & 0 & ----- & 0 & ------ & 0 & ------ & 0 & .12 & ----- \\
\hline Total & 0.02 & 0.16 & 0.35 & 0.12 & 0 & 0 & 0 & 0.12 & 0 & 0.06 & 0.63 & 0.02 \\
\hline Mean & .001 & .005 & .011 & .004 & 0 & 0 & 0 & .004 & 0 & .002 & .020 & .001 \\
\hline Max & .02 & .16 & .28 & .10 & 0 & 0 & 0 & .07 & 0 & .02 & .13 & .01 \\
\hline Min & 0 & 0 & 0 & 0 & 0 & 0 & 0 & 0 & 0 & 0 & 0 & 0 \\
\hline Acre-Ft & .04 & .32 & .69 & .24 & 0 & 0 & 0 & .24 & 0 & .12 & 1.2 & .04 \\
\hline Wtr Year & 2008 & Total & 1.48 & Mean & & 04 & Max & .28 & Min & 0 & Acre-Ft & 2.9 \\
\hline Cal Year & 2007 & Total & 2.50 & Mean & & 07 & hax & .28 & Min & 0 & Acre-Ft & 5.0 \\
\hline
\end{tabular}

*Estimate 
E1225 Sandia Canyon Tributary at Heavy Equipment

Daily Total Rainfall in Inches

Water Year October 2007 to September 2008

\begin{tabular}{|c|c|c|c|c|c|c|c|c|c|c|c|c|}
\hline DAY & ОСТ & NOV & DEC & JAN & FEB & MAR & APR & MAY & JUN & JUL & AUG & SEP \\
\hline 1 & & & & & & & & 0 & 0 & .07 & 0 & .10 \\
\hline 2 & & & & & & & & 0 & 0 & .05 & 0 & 0 \\
\hline 3 & & & & & & & & 0 & 0 & .03 & 0 & 0 \\
\hline 4 & & & & & & & & 0 & 0 & 0 & .78 & 0 \\
\hline 5 & & & & & & & & 0 & .04 & $.13^{*}$ & .01 & 0 \\
\hline 6 & & & & & & & & 0 & 0 & $.01^{*}$ & .01 & 0 \\
\hline 7 & & & & & & & & 0 & 0 & $.35^{*}$ & .28 & 0 \\
\hline 8 & & & & & & & & 0 & 0 & .24 & .61 & 0 \\
\hline 9 & & & & & & & & 0 & 0 & .06 & .44 & .03 \\
\hline 10 & & & & & & & & 0 & 0 & 0 & .95 & 0 \\
\hline 11 & & & & & & & & 0 & 0 & .03 & .01 & 0 \\
\hline 12 & & & & & & & & 0 & 0 & .01 & .02 & 0 \\
\hline 13 & & & & & & & & .12 & 0 & 0 & 0 & 0 \\
\hline 14 & & & & & & & 0 & .13 & 0 & 0 & 0 & 0 \\
\hline 15 & & & & & & & 0 & .59 & 0 & .01 & 0 & 0 \\
\hline 16 & & & & & & & 0 & .01 & 0 & .15 & .29 & 0 \\
\hline 17 & & & & & & & 0 & 0 & 0 & .01 & .16 & 0 \\
\hline 18 & & & & & & & 0 & 0 & 0 & .03 & .05 & .02 \\
\hline 19 & & & & & & & 0 & 0 & 0 & 0 & 0 & .05 \\
\hline 20 & & & & & & & 0 & 0 & 0 & 0 & .04 & 0 \\
\hline 21 & & & & & & & 0 & 0 & 0 & .34 & .01 & 0 \\
\hline 22 & & & & & & & 0 & .20 & 0 & 0 & 0 & .17 \\
\hline 23 & & & & & & & 0 & .21 & .02 & 0 & $.48^{\star}$ & 0 \\
\hline 24 & & & & & & & 0 & .01 & 0 & 0 & $.38^{\star}$ & .05 \\
\hline 25 & & & & & & & 0 & 0 & 0 & 0 & .11 & 0 \\
\hline 26 & & & & & & & 0 & 0 & 0 & .05 & .03 & 0 \\
\hline 27 & & & & & & & 0 & 0 & 0 & .17 & 0 & 0 \\
\hline 28 & & & & & & & 0 & .49 & 0 & .05 & 0 & 0 \\
\hline 29 & & & & & & & 0 & 0 & 0 & 0 & 0 & 0 \\
\hline 30 & & & & & ------ & & 0 & 0 & 0 & 0 & .18 & 0 \\
\hline 31 & & ----- & & & ------ & & ----- & 0 & ------ & 0 & .95 & ----- \\
\hline Total & & & & & & & 0 & 1.76 & 0.06 & 1.45 & 4.99 & 0.42 \\
\hline Max & & & & & & & 0 & .59 & .04 & .34 & .95 & .17 \\
\hline Wtr Year & 2008 & Total & 8.68 & Mean & & .051 & Max & .95 & Min & 0 & InstMax & .13 \\
\hline Cal Year & 2007 & Total & & Mean & & & $\operatorname{Max}$ & & Min & & InstMax & \\
\hline
\end{tabular}

*Estimate 


\section{E123 Sandia Canyon below Wetlands}

Location. Lat $35^{\circ}$ 52' 23", long $106^{\circ} 18^{\prime}$ 35", SE 1/4, Sec. 16, T. 19 N., R. 6 E., Los Alamos County.

Drainage Area. $0.29 \mathrm{mi}^{2}$.

Period of Record. August 1, 1999, to September 30, 2008.

Revised Record. Drainage area (2006); section (2007).

Gage. Data logger with cellular telemetry. Elevation of gage is 7,204 ft above NGVD from GPS survey.

Remarks. Records are fair to good.

Average Discharge. $8 \mathrm{yr}, 0.62 \mathrm{ft}^{3} / \mathrm{s}, 447$ acre-ft/year.

Extremes for Period of Record. Maximum discharge, $88 \mathrm{ft}^{3} / \mathrm{s}$, August 23, 2003, gage height $4.23 \mathrm{ft}$. Minimum daily discharge, $0.08 \mathrm{ft}^{3} / \mathrm{s}$, June 22, 2003.

Extremes for Current Year. Peak discharges above base of $30 \mathrm{ft}^{3} / \mathrm{s}$ and maximum (*):

\begin{tabular}{|c|c|c|c|}
\hline Date & Time & Discharge $\left(\mathbf{f t}^{\mathbf{3}} \mathbf{s}\right)$ & Gage Height (ft) \\
\hline May 28 & 1625 & 37 & 3.37 \\
\hline July 5 & 1550 & 32 & 3.20 \\
\hline August 4 & 1830 & 60 & 3.76 \\
\hline August 9 & 0020 & 50 & 3.59 \\
\hline August 9 & 1300 & 47 & 3.53 \\
\hline August 10 & 1150 & 65 & 3.85 \\
\hline August 31 & 1600 & 41 & 3.40 \\
\hline
\end{tabular}

Minimum daily discharge, $0.15 \mathrm{ft}^{3} / \mathrm{s}$, August 14 .

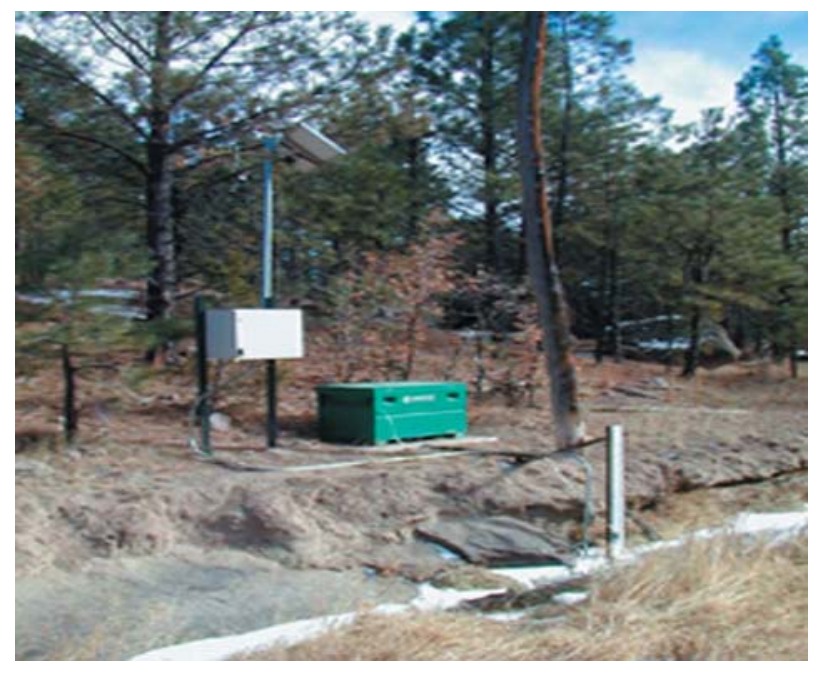




\section{E123 Sandia Canyon below Wetlands}

\section{Station Analysis}

\section{Water Year}

Equipment. Station is equipped with Sutron 8210 data logger (5-min. interval) with a Sutron Accubar bubble sensor. Data logger is equipped with cellular speech modem telemetry. Phone upgraded May 13 from analog to digital service. The system is powered by a solar-panel battery system housed in a NEMA shelter. Station is also equipped with ISCO pump sampler for water-quality sample collection. ISCO is housed in a separate shelter, a $3^{\prime} \times 4^{\prime}$ metal box. Sampler is triggered by stage through the data logger. An outside staff is available for reference. No provision for discharge measurements above wading stage.

An auxiliary 6" Parshall flume is located downstream from E123 and is used to verify the low flow record.

Field Work. The station was visited 46 times to conduct discharge measurements and service the instrumentation. Field inspections for the gage are listed under site history files on the Hydstra database. Discharge measurements for the gage are listed under site gauging files on the Hydstra database.

Datum Correction. None. Levels run June 27 found within limits.

Gage-Height Record. The data logger referenced to the inside gage height gave a complete and satisfactory record for the year, except for several days in January when ice affected gage height.

Rating. Channel is trapezoidal with rock outcrop and small depositional bars within pools. Banks have some grass, which is not very tall or thick. Channel is straight for about $100^{\prime}$ above and below gage.

Fourteen discharge measurements (Nos. 96-109) and 46 inspections of flow were made this year.

Rating Nos. 2, 3, and 4 were developed based on measurements made this year. Shifts are small and mostly negative caused by small amounts of deposition near gage or some bank slough during high flows. They have been distributed using variable diagrams with no shifts applied on the peak flows.

Discharge. Discharge computed from Rating Nos. 2, 3, and 4, with shifts applied by "V" diagrams.

Remarks. Records are good except for estimated days, which are fair. 
E123 Sandia Canyon below Wetlands

Daily Mean Discharge in Cubic Feet per Second

Water Year October 2007 to September 2008

\begin{tabular}{|c|c|c|c|c|c|c|c|c|c|c|c|c|}
\hline DAY & OCT & NOV & DEC & JAN & FEB & MAR & APR & MAY & JUN & JUL & AUG & SEP \\
\hline 1 & .89 & .39 & 2.2 & 1.3 & .45 & .48 & .45 & .40 & .51 & .59 & .72 & .69 \\
\hline 2 & .29 & .62 & .94 & 2.4 & .36 & .63 & .35 & .42 & .50 & .54 & .71 & .21 \\
\hline 3 & .29 & .55 & .62 & 2.0 & .43 & .56 & .40 & .47 & $.43^{\star}$ & .48 & .69 & .56 \\
\hline 4 & .28 & .47 & .50 & 1.4 & .51 & .61 & .39 & .38 & .40 & .66 & 1.5 & .39 \\
\hline 5 & .45 & .53 & .68 & .66 & .43 & .42 & .50 & .47 & .32 & .59 & .93 & .50 \\
\hline 6 & .38 & .48 & .63 & 1.5 & .49 & .57 & .35 & .35 & .31 & .65 & $.59^{\star}$ & .35 \\
\hline 7 & .26 & .49 & .73 & .73 & .56 & .67 & .57 & .45 & .59 & .94 & $.93^{\star}$ & .63 \\
\hline 8 & .42 & .41 & 2.0 & .67 & .40 & .64 & .43 & .31 & .53 & $1.9^{*}$ & $1.6^{\star}$ & .40 \\
\hline 9 & .48 & .40 & .88 & .45 & .41 & .48 & .41 & .30 & .58 & .68 & $3.6^{*}$ & .45 \\
\hline 10 & .33 & .35 & .95 & .54 & .52 & .47 & .40 & .24 & .41 & .57 & $6.7^{*}$ & .40 \\
\hline 11 & .36 & .24 & 1.3 & .69 & .40 & .43 & .38 & .35 & .42 & .67 & $3.7^{*}$ & .48 \\
\hline 12 & .26 & .38 & .68 & .72 & .34 & .59 & .41 & .34 & .55 & .48 & $1.3^{*}$ & .52 \\
\hline 13 & .29 & .37 & .65 & .83 & .34 & .51 & .45 & .34 & .38 & .64 & $1.8^{*}$ & .45 \\
\hline 14 & .30 & .37 & .79 & $.70^{\star}$ & .40 & .45 & .39 & .50 & .51 & .65 & $.15^{\star}$ & .36 \\
\hline 15 & .29 & .37 & .62 & $.80^{\star}$ & .32 & .49 & .30 & $1.7^{\star}$ & .56 & .62 & .40 & .55 \\
\hline 16 & .28 & .35 & .85 & .97 & .44 & .50 & .42 & .40 & .55 & .72 & $1.6^{*}$ & .35 \\
\hline 17 & .29 & .35 & .80 & $.73^{*}$ & .25 & .38 & .45 & .53 & .55 & $.67^{*}$ & .46 & .45 \\
\hline 18 & .25 & .37 & .56 & $.52^{*}$ & .41 & .40 & .43 & .39 & .47 & .60 & .31 & .42 \\
\hline 19 & .29 & .43 & .71 & $.71^{\star}$ & .32 & .40 & .42 & .35 & .42 & .61 & .49 & .32 \\
\hline 20 & .43 & .52 & .59 & $.49^{*}$ & .27 & .48 & .34 & .33 & .40 & .58 & .29 & .31 \\
\hline 21 & .48 & .44 & .70 & $.60^{\star}$ & .32 & .57 & .44 & .44 & .57 & $1.4^{*}$ & .25 & .44 \\
\hline 22 & .59 & .20 & .49 & $.48^{\star}$ & .40 & .32 & .35 & $.80^{\star}$ & .52 & .63 & .34 & .62 \\
\hline 23 & .45 & .40 & .72 & .24 & .39 & .36 & .36 & $.78^{\star}$ & .54 & .58 & $1.4^{*}$ & .43 \\
\hline 24 & .61 & .53 & .56 & $.26^{\star}$ & .69 & .58 & .37 & .40 & .53 & .66 & $1.6^{*}$ & .64 \\
\hline 25 & .51 & .47 & .63 & $.40^{\star}$ & .49 & .53 & .35 & .40 & .56 & .66 & .91 & .54 \\
\hline 26 & .45 & .51 & .63 & .47 & .49 & .41 & .44 & .30 & .53 & .71 & .49 & .47 \\
\hline 27 & .59 & .41 & .61 & .55 & .35 & .37 & .39 & .35 & .59 & .83 & .33 & .39 \\
\hline 28 & .35 & .36 & .70 & $2.2^{\star}$ & .34 & .44 & .46 & .79 & .60 & .60 & .36 & .40 \\
\hline 29 & .50 & .32 & .66 & .41 & .51 & .43 & .39 & $.72^{*}$ & .64 & .61 & .47 & .45 \\
\hline 30 & .66 & 1.8 & .78 & .56 & ------ & .38 & .36 & .60 & .54 & .60 & .55 & .48 \\
\hline 31 & .35 & ------ & .53 & .31 & ------ & .51 & ------ & .54 & ------ & .59 & $4.8^{\star}$ & ------ \\
\hline Total & 12.65 & 13.88 & 24.69 & 25.29 & 12.03 & 15.06 & 12.15 & 15.14 & 15.01 & 21.71 & 39.97 & 13.65 \\
\hline Mean & .41 & .46 & .80 & .82 & .41 & .49 & .41 & .49 & .50 & .70 & 1.29 & .46 \\
\hline Max & .89 & 1.8 & 2.2 & 2.4 & .69 & .67 & .57 & 1.7 & .64 & 1.9 & 6.7 & .69 \\
\hline Min & .25 & .20 & .49 & .24 & .25 & .32 & .30 & .24 & .31 & .48 & .15 & .21 \\
\hline Acre-Ft & 25 & 28 & 49 & 50 & 24 & 30 & 24 & 30 & 30 & 43 & 79 & 27 \\
\hline Wtr Year & 2008 & Total & 221.23 & & & .60 & Max & 6.7 & Min & .15 & Acre-Ft & 439 \\
\hline Cal Year & 2007 & Total & 74.52 & & & .56 & Max & 2.2 & Min & .20 & Acre-Ft & 148 \\
\hline
\end{tabular}

*Estimate 


\section{E1234 Sandia Canyon Roads and Grounds at Sigma}

Location. Lat $35^{\circ}$ 52' 14", long $106^{\circ}$ 18' 24", Sec. 21, T. 19 N., R. 6 E., Los Alamos Country.

Drainage Area. $0.001 \mathrm{mi}^{2}$.

Period of Record. August 2, 2007, to September 30, 2008.

Gage. Data logger and 24” Parshall flume. Elevation 7,311 ft above NGVD.

Remarks. Records are good.

Extremes for Period of Record. Maximum discharge is $5.77 \mathrm{ft}^{3} / \mathrm{s}$, August 10, 2008, gage height $0.81 \mathrm{ft}$. No flow most of the time.

Extremes for Current Year. Peak discharges above base of $2.0 \mathrm{ft}^{3} / \mathrm{s}$ and maximum (*):

\begin{tabular}{|c|c|c|c|}
\hline Date & Time & Discharge $\left(\mathbf{f t}^{\mathbf{3}} / \mathbf{s}\right)$ & Gage Height (ft) \\
\hline May 28 & 1540 & 2.6 & 0.49 \\
\hline August 4 & 1735 & 2.2 & 0.44 \\
\hline August 10 & 1115 & $5.7^{*}$ & $0.81^{*}$
\end{tabular}

No flow most of the time.

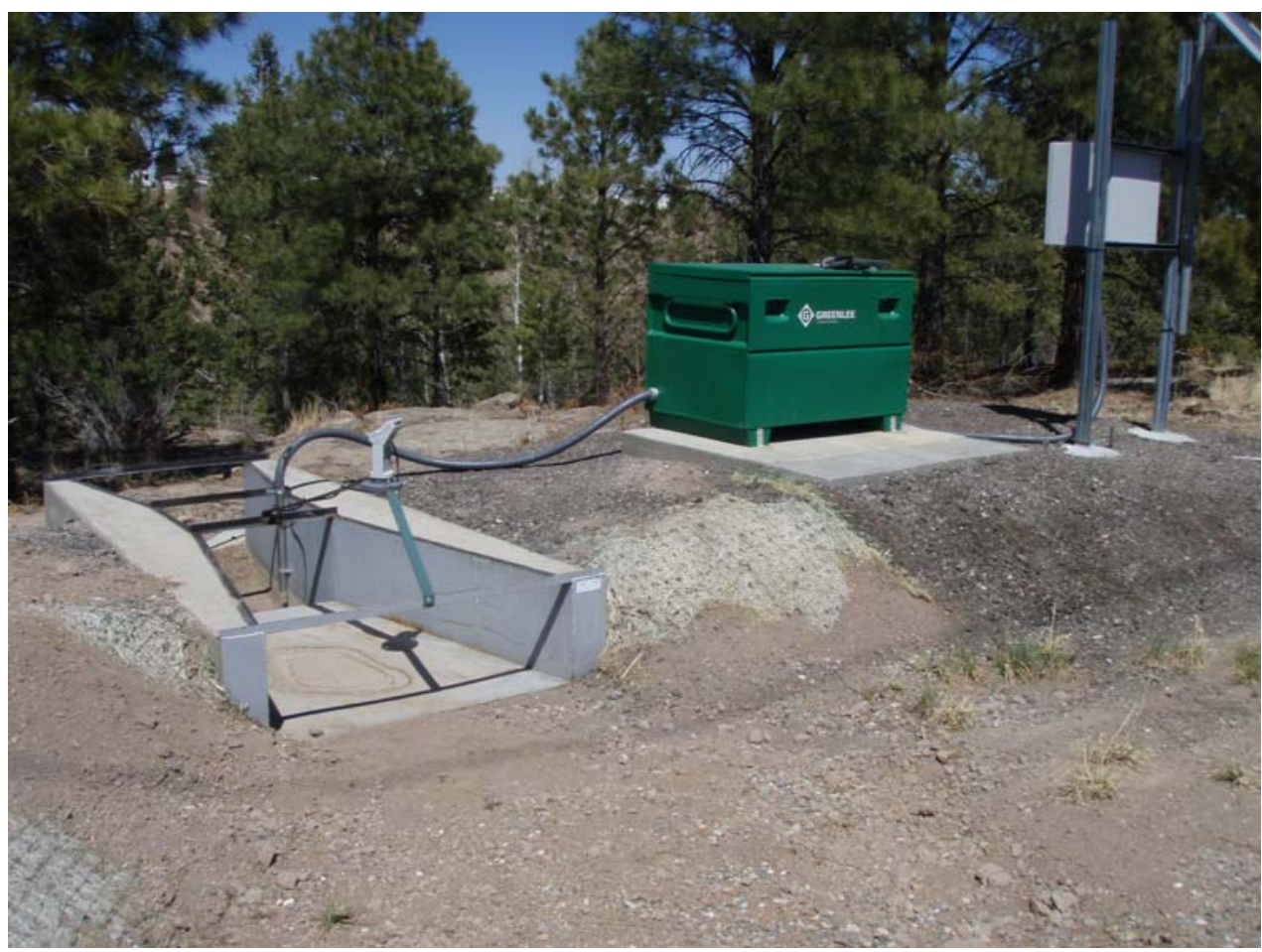




\section{E1234 Sandia Canyon Roads and Grounds at Sigma}

\section{Station Analysis}

\section{Water Year}

Equipment. Station is equipped with Sutron 8210 data logger (5-min. interval) and milltronics sonic probe mounted on a 24" Parshall flume. The system is powered by a solar-panel battery system housed in a NEMA shelter. Station is equipped with an ISCO pump sampler for water-quality sample collection. ISCO is housed in a separate shelter, a $3^{\prime} \times 4^{\prime}$ metal box. Sampler is triggered by stage through the data logger. The staff in the 24" Parshall flume is the reference gage. No provision for discharge measurements above wading stage.

Field Work. This station was visited 20 times to conduct discharge measurements and service the instrumentation. Field inspections for the gage are listed under site history files on the Hydstra database. Discharge measurements for the gage are listed under site gauging files on the Hydstra database.

Datum Correction. None.

Gage-Height Record. The data logger referenced to the outside staff gave a complete and satisfactory record for the year, except for the period of November 6 to March 19 when ice affected gage height and May 15 to May 27 and a few days in June when flume was silted.

Rating. The control for this station is the 24" Parshall flume.

Nineteen inspections of no flow and one day of snow melt were made this year.

Rating No. 1 was developed based on the computation of 24" Parshall flume. Point of zero flow is 0.00 gage height.

Discharge. Discharge was computed by applying Rating No. 1 directly. Those days estimated at zero flow were based on precipitation and nearby gage stations.

Remarks. Records are good. 


\section{E1234 Sandia Canyon Roads and Grounds at Sigma}

Daily Mean Discharge in Cubic Feet per Second

Water Year October 2007 to September 2008

\begin{tabular}{|c|c|c|c|c|c|c|c|c|c|c|c|c|}
\hline DAY & OCT & NOV & DEC & JAN & FEB & MAR & APR & MAY & JUN & JUL & AUG & SEP \\
\hline 1 & .03 & 0 & .11 & $0^{*}$ & $0^{*}$ & $0^{*}$ & 0 & 0 & $0^{*}$ & .03 & 0 & 0 \\
\hline 2 & 0 & 0 & 0 & $0^{*}$ & $0^{*}$ & $0^{*}$ & 0 & 0 & 0 & 0 & 0 & 0 \\
\hline 3 & 0 & 0 & $0^{*}$ & $0^{*}$ & $0^{*}$ & $0^{*}$ & 0 & 0 & 0 & 0 & 0 & 0 \\
\hline 4 & 0 & 0 & $0^{*}$ & $0^{*}$ & $0^{*}$ & $0^{*}$ & 0 & 0 & 0 & 0 & .06 & 0 \\
\hline 5 & 0 & 0 & $0^{*}$ & $0^{*}$ & $0^{*}$ & $0^{*}$ & 0 & $0^{*}$ & 0 & 0 & 0 & 0 \\
\hline 6 & 0 & $0^{*}$ & $0^{*}$ & $0^{*}$ & $0^{*}$ & $0^{*}$ & 0 & 0 & 0 & 0 & 0 & 0 \\
\hline 7 & 0 & $0^{*}$ & $0^{*}$ & $0^{*}$ & $0^{*}$ & $0^{*}$ & 0 & 0 & 0 & .03 & .03 & 0 \\
\hline 8 & 0 & $0^{*}$ & $0^{*}$ & $0^{*}$ & $0^{*}$ & $0^{*}$ & 0 & 0 & 0 & .03 & .04 & 0 \\
\hline 9 & 0 & $0^{*}$ & $0^{*}$ & $0^{*}$ & $0^{*}$ & $0^{*}$ & 0 & 0 & $0^{*}$ & 0 & .06 & 0 \\
\hline 10 & 0 & $0^{*}$ & $0^{*}$ & $0^{*}$ & $0^{*}$ & $0^{*}$ & $0^{*}$ & 0 & 0 & 0 & .07 & 0 \\
\hline 11 & 0 & $0^{*}$ & $0^{*}$ & $0^{*}$ & $0^{*}$ & $0^{*}$ & 0 & 0 & 0 & 0 & 0 & 0 \\
\hline 12 & 0 & $0^{*}$ & $0^{*}$ & $0^{*}$ & $0^{*}$ & 0 & $0^{*}$ & 0 & $0^{*}$ & 0 & 0 & 0 \\
\hline 13 & 0 & $0^{*}$ & $0^{*}$ & $0^{*}$ & $0^{*}$ & 0 & $0^{*}$ & .01 & 0 & 0 & 0 & 0 \\
\hline 14 & 0 & $0^{*}$ & $0^{*}$ & $0^{*}$ & $0^{*}$ & 0 & 0 & .04 & 0 & 0 & 0 & 0 \\
\hline 15 & 0 & $0^{*}$ & $0^{*}$ & $0^{*}$ & $0^{*}$ & $0^{*}$ & 0 & $0^{*}$ & 0 & 0 & 0 & 0 \\
\hline 16 & 0 & $0^{*}$ & $0^{*}$ & $0^{*}$ & $0^{*}$ & 0 & 0 & $0^{*}$ & 0 & .01 & .02 & 0 \\
\hline 17 & 0 & $0^{*}$ & $0^{*}$ & $0^{*}$ & $0^{*}$ & 0 & $0^{*}$ & $0^{*}$ & 0 & 0 & 0 & 0 \\
\hline 18 & 0 & $0^{*}$ & $0^{*}$ & $0^{*}$ & $0^{*}$ & 0 & $0^{*}$ & $0^{*}$ & 0 & 0 & 0 & 0 \\
\hline 19 & 0 & $0^{*}$ & $0^{*}$ & $0^{*}$ & $0^{*}$ & $0^{*}$ & 0 & $0^{*}$ & 0 & 0 & 0 & 0 \\
\hline 20 & 0 & $0^{*}$ & $0^{*}$ & $0^{*}$ & $0^{*}$ & 0 & 0 & $0^{*}$ & 0 & 0 & 0 & 0 \\
\hline 21 & 0 & $0^{*}$ & $0^{*}$ & $0^{*}$ & $0^{*}$ & 0 & 0 & $0^{*}$ & $0^{*}$ & .01 & 0 & 0 \\
\hline 22 & 0 & $0^{*}$ & $0^{*}$ & $0^{*}$ & $0^{*}$ & 0 & 0 & $0^{*}$ & 0 & 0 & 0 & 0 \\
\hline 23 & 0 & $0^{*}$ & $0^{*}$ & $0^{*}$ & $0^{*}$ & 0 & $0^{*}$ & $0^{*}$ & 0 & 0 & .03 & 0 \\
\hline 24 & 0 & $0^{*}$ & $0^{*}$ & $0^{*}$ & $0^{*}$ & 0 & 0 & $0^{*}$ & 0 & 0 & .01 & 0 \\
\hline 25 & 0 & $0^{*}$ & $0^{*}$ & $0^{*}$ & $0^{*}$ & 0 & 0 & $0^{*}$ & 0 & 0 & .04 & 0 \\
\hline 26 & 0 & $0^{*}$ & $0^{*}$ & $0^{*}$ & $0^{*}$ & 0 & 0 & $0^{*}$ & 0 & 0 & 0 & 0 \\
\hline 27 & 0 & $0^{*}$ & $0^{*}$ & .13 & $0^{*}$ & 0 & 0 & $0^{*}$ & 0 & .05 & 0 & 0 \\
\hline 28 & 0 & $0^{*}$ & $0^{*}$ & .25 & $0^{*}$ & 0 & $0^{*}$ & .07 & 0 & .08 & 0 & 0 \\
\hline 29 & 0 & $0^{*}$ & $0^{*}$ & $0^{*}$ & $0^{*}$ & 0 & 0 & 0 & 0 & 0 & 0 & 0 \\
\hline 30 & 0 & .16 & $0^{*}$ & $0^{*}$ & ------ & 0 & 0 & 0 & 0 & 0 & 0 & 0 \\
\hline 31 & 0 & ------ & $0^{*}$ & $0^{*}$ & ------ & 0 & ------ & $0^{*}$ & ----- & 0 & .12 & ------ \\
\hline Total & 0.03 & 0.16 & 0.11 & 0.38 & 0 & 0 & 0 & 0.12 & 0 & 0.24 & 0.48 & 0 \\
\hline Mean & .001 & .005 & .004 & .012 & 0 & 0 & 0 & .004 & 0 & .008 & .015 & 0 \\
\hline Max & .03 & .16 & .11 & .25 & 0 & 0 & 0 & .07 & 0 & .08 & .12 & 0 \\
\hline Min & 0 & 0 & 0 & 0 & 0 & 0 & 0 & 0 & 0 & 0 & 0 & 0 \\
\hline Acre-Ft & .06 & .32 & .22 & .75 & 0 & 0 & 0 & .24 & 0 & .48 & .95 & 0 \\
\hline Wtr Year & 2008 & Total & 1.52 & Mean & & 04 & hax & .25 & Min & 0 & Acre-Ft & 3.0 \\
\hline Cal Year & 2007 & Total & 0.93 & Mean & & 06 & hax & .18 & Min & 0 & Acre-Ft & 1.8 \\
\hline
\end{tabular}

*Estimate 


\section{E125 Sandia Canyon above SR 4}

Location. Lat $35^{\circ}$ 51' 32", long $106^{\circ} 13^{\prime}$ 34", SW 1/4, Sec. 20, T. 19 N., R.7 E., Santa Fe County.

Drainage Area. $2.05 \mathrm{mi}^{2}$.

Period of Record. October 1, 1994, to September 30, 2008.

Revised Record. Drainage area (2006).

Gage. Data logger and concrete control. Elevation of gage is 6,495 ft above NGVD from GPS survey.

Remarks. Records are fair.

Average Discharge. $14 \mathrm{yr}, 0.003 \mathrm{ft}^{3} / \mathrm{s}, 1.96$ acre- $\mathrm{ft} / \mathrm{yr}$.

Extremes for Period of Record. Maximum discharge, $59 \mathrm{ft}^{3} / \mathrm{s}$, August 25, 2006, gage height $3.60 \mathrm{ft}$ (from slope-area measurement). No flow most of the time.

Extremes for Current Year. Peak discharge above base of $3.0 \mathrm{ft}^{3} / \mathrm{s}$ and maximum (*):

\begin{tabular}{|c|c|c|c|}
\hline Date & Time & Discharge (ft $\left.{ }^{\mathbf{3}} / \mathbf{s}\right)$ & Gage Height (ft) \\
\hline January 28 & 0835 & $28^{*}$ & $2.36^{*}$
\end{tabular}

No flow most of the time.

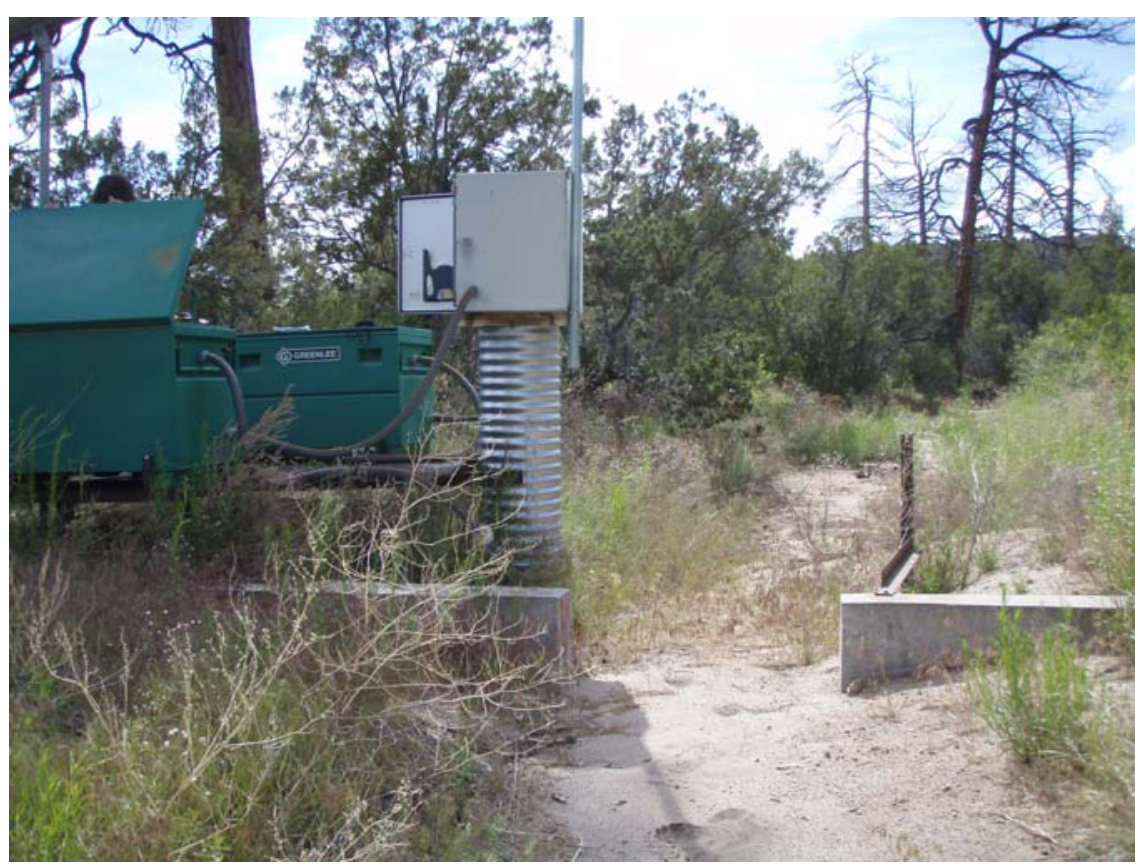




\section{E125 Sandia Canyon above SR 4}

\section{Station Analysis}

\section{Water Year}

Equipment. Station is equipped with Sutron 8210 data logger (5-min. interval) and a shaft encoder float system. The system is powered by a solar-panel battery system. All equipment is housed in NEMA shelter on 18" CMP well. Station is equipped with an ISCO pump sampler for water-quality sample collection. ISCO is housed in a separate shelter, a 3' $\times 4^{\prime}$ metal box. Sampler is triggered by stage through the data logger. Control is a concrete broad-crested weir. No provision for measurements above wading stage.

Cellular telemetry removed March 13, discontinued service.

Field Work. The station was visited 14 times to conduct discharge measurements and service the instrumentation. Field inspections for the gage are listed under site history files on the Hydstra database. Discharge measurements for the gage are listed under site gauging files on the Hydstra database.

Datum Correction. None.

Gage-Height Record. The data logger referenced to the outside staff gave a complete and satisfactory record, except for the period of December 28 to March 14 when ice affected gage height.

Rating. Channel is straight for 150' above and 100' below gage. Bed material consists of sand with vegetation on banks, with the bottom well supported.

Thirteen inspections of no flow and one inspection of flow were made this year.

Rating No. 1 is from theoretical points computed from broad-crested weir formula from USGS-TWRI. Worksheet in record folder shows computations, including estimated velocity head used in computation because " $\mathrm{p}$ " in equation was functionally zero. Rating No. 1 is considered fair.

Discharge. Discharge computed directly from Rating No.1 without shift correction.

Remarks. Records are good. 
E125 Sandia Canyon above SR 4

Daily Mean Discharge in Cubic Feet per Second

Water Year October 2007 to September 2008

\begin{tabular}{|c|c|c|c|c|c|c|c|c|c|c|c|c|}
\hline DAY & OCT & NOV & DEC & JAN & FEB & MAR & APR & MAY & JUN & JUL & AUG & SEP \\
\hline 1 & 0 & 0 & 0 & 0 & $0^{*}$ & $0^{*}$ & 0 & 0 & 0 & 0 & 0 & 0 \\
\hline 2 & 0 & 0 & 0 & 0 & $0^{*}$ & $0^{*}$ & 0 & 0 & 0 & 0 & 0 & 0 \\
\hline 3 & 0 & 0 & 0 & 0 & $0^{*}$ & $0^{*}$ & 0 & 0 & 0 & 0 & 0 & 0 \\
\hline 4 & 0 & 0 & 0 & 0 & $0^{*}$ & $0^{*}$ & 0 & 0 & 0 & 0 & 0 & 0 \\
\hline 5 & 0 & 0 & 0 & 0 & $0^{*}$ & $0^{*}$ & 0 & 0 & 0 & 0 & 0 & 0 \\
\hline 6 & 0 & 0 & 0 & 0 & $0^{*}$ & $0^{*}$ & 0 & 0 & 0 & 0 & 0 & 0 \\
\hline 7 & 0 & 0 & 0 & 0 & $0^{*}$ & $0^{*}$ & 0 & 0 & 0 & 0 & 0 & 0 \\
\hline 8 & 0 & 0 & 0 & 0 & $0^{*}$ & $0^{*}$ & 0 & 0 & 0 & 0 & 0 & 0 \\
\hline 9 & 0 & 0 & 0 & 0 & $0^{*}$ & $0^{*}$ & 0 & 0 & 0 & 0 & 0 & 0 \\
\hline 10 & 0 & 0 & 0 & 0 & $0^{*}$ & $0^{*}$ & 0 & 0 & 0 & 0 & 0 & 0 \\
\hline 11 & 0 & 0 & 0 & 0 & $0^{*}$ & $0^{*}$ & 0 & 0 & 0 & 0 & 0 & 0 \\
\hline 12 & 0 & 0 & 0 & 0 & $0^{*}$ & $0^{*}$ & 0 & 0 & 0 & 0 & 0 & 0 \\
\hline 13 & 0 & 0 & 0 & 0 & $0^{*}$ & $0^{*}$ & 0 & 0 & 0 & 0 & 0 & 0 \\
\hline 14 & 0 & 0 & 0 & 0 & $0^{*}$ & $0^{*}$ & 0 & 0 & 0 & 0 & 0 & 0 \\
\hline 15 & 0 & 0 & 0 & 0 & $0^{*}$ & 0 & 0 & 0 & 0 & 0 & 0 & 0 \\
\hline 16 & 0 & 0 & 0 & 0 & $0^{*}$ & 0 & 0 & 0 & 0 & 0 & 0 & 0 \\
\hline 17 & 0 & 0 & 0 & 0 & $0^{*}$ & 0 & 0 & 0 & 0 & 0 & 0 & 0 \\
\hline 18 & 0 & 0 & 0 & 0 & $0^{*}$ & 0 & 0 & 0 & 0 & 0 & 0 & 0 \\
\hline 19 & 0 & 0 & 0 & 0 & $0^{*}$ & 0 & 0 & 0 & 0 & 0 & 0 & 0 \\
\hline 20 & 0 & 0 & 0 & 0 & $0^{*}$ & 0 & 0 & 0 & 0 & 0 & 0 & 0 \\
\hline 21 & 0 & 0 & 0 & 0 & $0^{*}$ & 0 & 0 & 0 & 0 & 0 & 0 & 0 \\
\hline 22 & 0 & 0 & 0 & 0 & $0^{*}$ & 0 & 0 & 0 & 0 & 0 & 0 & 0 \\
\hline 23 & 0 & 0 & 0 & 0 & $0^{*}$ & 0 & 0 & 0 & 0 & 0 & 0 & 0 \\
\hline 24 & 0 & 0 & 0 & 0 & $0^{*}$ & 0 & 0 & 0 & 0 & 0 & 0 & 0 \\
\hline 25 & 0 & 0 & 0 & 0 & $0^{*}$ & 0 & 0 & 0 & 0 & 0 & 0 & 0 \\
\hline 26 & 0 & 0 & 0 & 0 & $0^{*}$ & 0 & 0 & 0 & 0 & 0 & 0 & 0 \\
\hline 27 & 0 & 0 & 0 & 0 & $0^{*}$ & 0 & 0 & 0 & 0 & 0 & 0 & 0 \\
\hline 28 & 0 & 0 & 0 & 7.6 & $0^{*}$ & 0 & 0 & 0 & 0 & 0 & 0 & 0 \\
\hline 29 & 0 & 0 & 0 & $0^{*}$ & $0^{*}$ & 0 & 0 & 0 & 0 & 0 & 0 & 0 \\
\hline 30 & 0 & 0 & 0 & $0^{*}$ & ------ & 0 & 0 & 0 & 0 & 0 & 0 & 0 \\
\hline 31 & 0 & ----- & 0 & $0^{*}$ & ------ & 0 & ------ & 0 & ------ & 0 & 0 & ------ \\
\hline Total & 0 & 0 & 0 & 7.6 & 0 & 0 & 0 & 0 & 0 & 0 & 0 & 0 \\
\hline Mean & 0 & 0 & 0 & .25 & 0 & 0 & 0 & 0 & 0 & 0 & 0 & 0 \\
\hline Max & 0 & 0 & 0 & 7.6 & 0 & 0 & 0 & 0 & 0 & 0 & 0 & 0 \\
\hline Min & 0 & 0 & 0 & 0 & 0 & 0 & 0 & 0 & 0 & 0 & 0 & 0 \\
\hline Acre-Ft & 0 & 0 & 0 & 15 & 0 & 0 & 0 & 0 & 0 & 0 & 0 & 0 \\
\hline Wtr Year & 2008 & Total & 7.6 & Mean & & 21 & hax & 7.6 & Min & 0 & Acre-Ft & 15 \\
\hline Cal Year & 2007 & Total & 0 & Mean & & 0 & hax & 0 & Min & 0 & Acre-Ft & 0 \\
\hline
\end{tabular}

*Estimate 


\section{E196 TA-55 above Effluent Canyon}

Location. Lat $35^{\circ}$ 51' 52", long $106^{\circ} 18^{\prime}$ 13", NE 1/4, Sec. 21, T. 19 N., R. 6 E., Los Alamos County.

Drainage Area. $0.008 \mathrm{mi}^{2}$.

Period of Record. October 1, 2006, to September 30, 2008.

Gage. Data logger and 12" Parshall flume, as well as rain gage with cellular telemetry. Elevation of gage is 7,270 ft above NGVD.

Remarks. Records are good except for estimated daily discharges, which are fair. Records for this site existed before period of record but are not reliable.

Extremes for Period of Record. Maximum discharge, $0.95 \mathrm{ft}^{3} / \mathrm{s}$, September 2, 2007, gage height $0.42 \mathrm{ft}$. No flow most of the time.

Extremes for Current Year. Maximum discharge, $0.81 \mathrm{ft}^{3} / \mathrm{s}$ at $1115 \mathrm{~h}$, August 10, gage height $0.38 \mathrm{ft}$. No peak above base of $1.0 \mathrm{ft}^{3} / \mathrm{s}$. No flow most of the time.

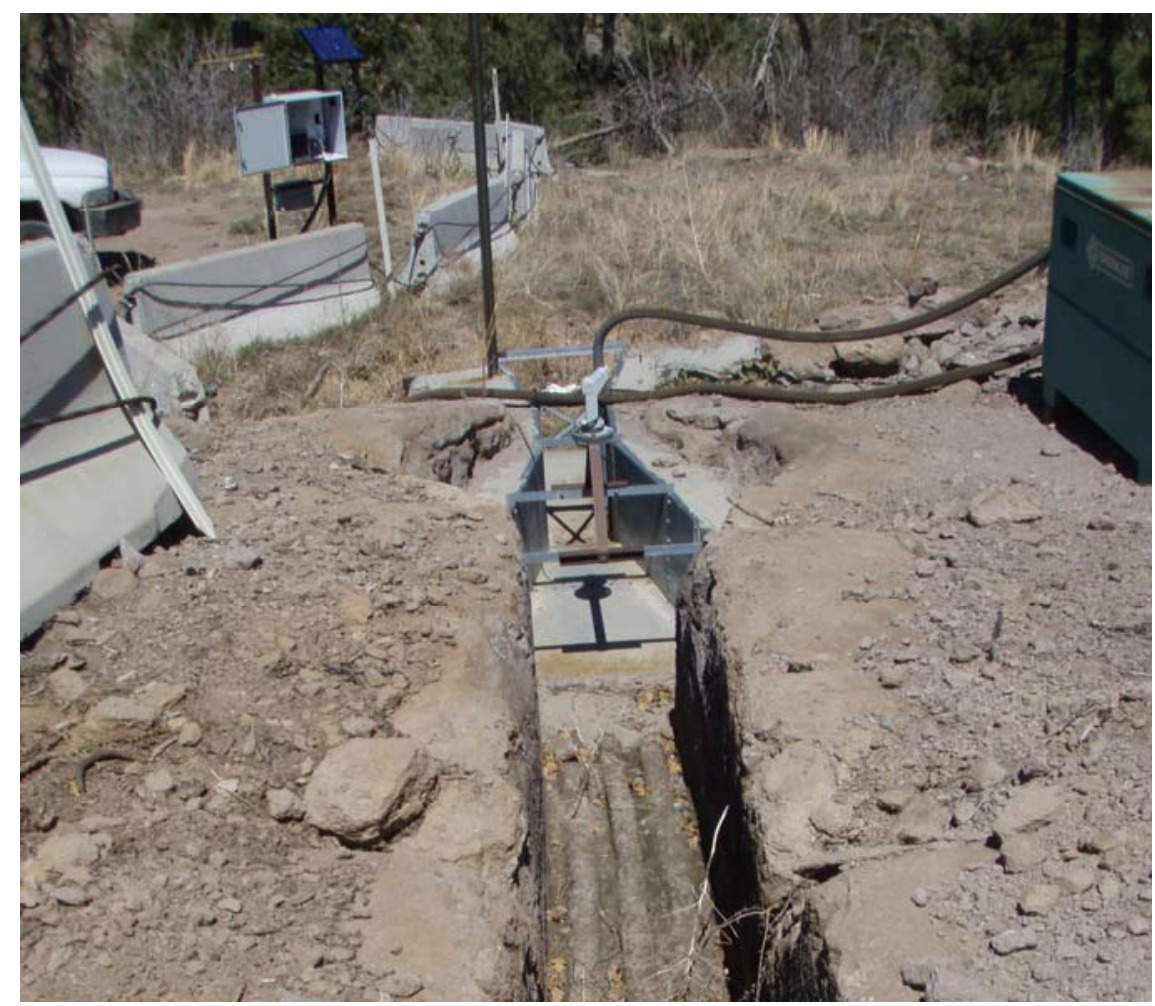




\section{E196 TA-55 above Effluent Canyon}

\section{Station Analysis}

\section{Water Year}

Equipment. Station is equipped with Sutron 8210 data logger (5-min. interval) and milltronics sonic probe mounted on a 12" Parshall flume and cellular telemetry with speech modem. Phone upgraded May 8 from analog to digital service. The system is powered by a solar-panel battery system housed in a NEMA shelter. Station is equipped with an ISCO pump sampler for water-quality sample collection. ISCO is housed in a separate shelter, a $3^{\prime} \times 4^{\prime}$ metal box. Sampler is triggered by stage through the data logger. The staff in the 12" Parshall flume is the reference gage. No provision for discharge measurements above wading stage.

Station is also equipped with a tipping bucket rain gage, Rain Collection II. All equipment is powered with a solar-panel battery-charging system. Rain data are seasonal.

Field Work. This station was visited 22 times to conduct discharge measurements and service the instrumentation. Field inspections for the gage are listed under site history files on the Hydstra database. Discharge measurements for the gage are listed under site gauging files on the Hydstra database.

Datum Correction. None.

Gage-Height Record. The data logger referenced to the outside staff gave a complete and satisfactory record, except during the periods of December 13 to February 27 and March 2-9 when ice affected gage height and May 7-19 and June 8-18 when the data logger malfunctioned as a result of battery failure.

Rating. The control for the station is a 12" Parshall flume.

Twenty inspections of no flow and two inspections of snow melt were made this year.

Rating No. 1 was developed based on the computation of 12" Parshall flume. Point of zero flow is 0.00 gage height.

Discharge. Discharge was computed by applying Rating No. 1 directly. Those days estimated at zero flow were based on precipitation and nearby gage stations.

Remarks. Records are good except for estimated daily discharges, which are fair. 
E196 TA-55 above Effluent Canyon

Daily Mean Discharge in Cubic Feet per Second

Water Year October 2007 to September 2008

\begin{tabular}{|c|c|c|c|c|c|c|c|c|c|c|c|c|}
\hline DAY & OCT & NOV & DEC & JAN & FEB & MAR & APR & MAY & JUN & JUL & AUG & SEP \\
\hline 1 & 0 & 0 & .03 & $0^{*}$ & $0^{*}$ & 0 & 0 & 0 & 0 & 0 & 0 & 0 \\
\hline 2 & 0 & 0 & .01 & $0^{*}$ & $0^{*}$ & $.02^{\star}$ & 0 & 0 & 0 & 0 & 0 & 0 \\
\hline 3 & 0 & 0 & $0^{*}$ & $0^{*}$ & $0^{*}$ & $.13^{*}$ & 0 & 0 & 0 & 0 & 0 & 0 \\
\hline 4 & 0 & 0 & $0^{*}$ & $0^{*}$ & $0^{*}$ & $.02^{\star}$ & 0 & 0 & 0 & 0 & .02 & 0 \\
\hline 5 & 0 & 0 & 0 & $0^{*}$ & $0^{*}$ & $.02^{*}$ & 0 & 0 & 0 & 0 & 0 & 0 \\
\hline 6 & 0 & 0 & 0 & $0^{*}$ & $0^{*}$ & $.06^{*}$ & 0 & 0 & 0 & 0 & 0 & 0 \\
\hline 7 & 0 & 0 & 0 & $0^{*}$ & $0^{*}$ & $.08^{\star}$ & 0 & $0^{*}$ & 0 & 0 & 0 & 0 \\
\hline 8 & 0 & 0 & .01 & $0^{*}$ & $0^{*}$ & $.01^{*}$ & 0 & $0^{*}$ & $0^{*}$ & 0 & 0 & 0 \\
\hline 9 & 0 & 0 & 0 & $0^{*}$ & $0^{*}$ & $0^{*}$ & 0 & $0^{*}$ & $0^{*}$ & 0 & .01 & 0 \\
\hline 10 & 0 & 0 & 0 & $0^{*}$ & $0^{*}$ & 0 & $0^{*}$ & $0^{*}$ & $0^{*}$ & 0 & .02 & 0 \\
\hline 11 & 0 & 0 & 0 & $0^{*}$ & $0^{*}$ & 0 & $0^{*}$ & $0^{*}$ & $0^{*}$ & 0 & 0 & 0 \\
\hline 12 & 0 & 0 & 0 & $0^{*}$ & $.02^{\star}$ & 0 & $0^{*}$ & $0^{*}$ & $0^{*}$ & 0 & 0 & 0 \\
\hline 13 & 0 & 0 & $0^{*}$ & $0^{*}$ & $.02^{\star}$ & 0 & $0^{*}$ & $0^{*}$ & $0^{*}$ & 0 & 0 & 0 \\
\hline 14 & 0 & 0 & $0^{*}$ & $0^{*}$ & $0^{*}$ & 0 & $0^{*}$ & $0^{*}$ & $0^{*}$ & 0 & 0 & 0 \\
\hline 15 & 0 & 0 & $0^{*}$ & $0^{*}$ & $0^{*}$ & 0 & 0 & $0^{*}$ & $0^{*}$ & 0 & 0 & 0 \\
\hline 16 & 0 & 0 & $0^{*}$ & $0^{*}$ & $0^{*}$ & 0 & 0 & $0^{*}$ & $0^{*}$ & 0 & .01 & 0 \\
\hline 17 & 0 & 0 & $0^{*}$ & $0^{*}$ & $0^{*}$ & 0 & 0 & $0^{*}$ & $0^{*}$ & 0 & 0 & 0 \\
\hline 18 & 0 & 0 & $0^{*}$ & $0^{*}$ & $0^{*}$ & 0 & 0 & $0^{*}$ & $0^{*}$ & 0 & 0 & 0 \\
\hline 19 & 0 & 0 & $0^{*}$ & $0^{*}$ & $0^{*}$ & 0 & 0 & $0^{*}$ & 0 & 0 & 0 & 0 \\
\hline 20 & 0 & 0 & $0^{*}$ & $0^{*}$ & $0^{*}$ & 0 & 0 & 0 & 0 & 0 & 0 & 0 \\
\hline 21 & 0 & 0 & $0^{*}$ & $0^{*}$ & $0^{*}$ & 0 & 0 & 0 & 0 & .01 & 0 & 0 \\
\hline 22 & 0 & 0 & $0^{*}$ & $0^{*}$ & $0^{*}$ & 0 & 0 & 0 & 0 & 0 & 0 & 0 \\
\hline 23 & 0 & .39 & $0^{*}$ & $0^{*}$ & $0^{*}$ & 0 & 0 & 0 & 0 & 0 & .01 & 0 \\
\hline 24 & 0 & .14 & $0^{*}$ & $0^{*}$ & $0^{*}$ & 0 & 0 & 0 & 0 & 0 & .01 & 0 \\
\hline 25 & 0 & .09 & $0^{*}$ & $0^{*}$ & $0^{*}$ & 0 & 0 & 0 & 0 & 0 & 0 & 0 \\
\hline 26 & 0 & .09 & $0^{*}$ & $0^{*}$ & $0^{*}$ & 0 & 0 & 0 & 0 & 0 & 0 & 0 \\
\hline 27 & 0 & .08 & $0^{*}$ & $0^{*}$ & $0^{*}$ & 0 & 0 & 0 & 0 & 0 & 0 & 0 \\
\hline 28 & 0 & .08 & $0^{*}$ & $0^{*}$ & 0 & 0 & 0 & .01 & 0 & 0 & 0 & 0 \\
\hline 29 & 0 & 0 & $0^{*}$ & $0^{*}$ & 0 & 0 & 0 & 0 & 0 & 0 & 0 & 0 \\
\hline 30 & 0 & .03 & $0^{*}$ & $0^{*}$ & ----- & 0 & 0 & 0 & 0 & 0 & 0 & 0 \\
\hline 31 & 0 & ------ & $0^{*}$ & $0^{*}$ & ----- & 0 & ----- & 0 & ------ & 0 & .01 & ----- \\
\hline Total & 0 & 0.90 & 0.05 & 0 & 0.04 & 0.34 & 0 & 0.01 & 0 & 0.01 & 0.09 & 0 \\
\hline Mean & 0 & .030 & .002 & 0 & .001 & .011 & 0 & 0 & 0 & 0 & .003 & 0 \\
\hline $\operatorname{Max}$ & 0 & .39 & .03 & 0 & .02 & .13 & 0 & .01 & 0 & .01 & .02 & 0 \\
\hline Min & 0 & 0 & 0 & 0 & 0 & 0 & 0 & 0 & 0 & 0 & 0 & 0 \\
\hline Acre-Ft & 0 & 1.8 & .10 & 0 & .08 & .67 & 0 & .02 & 0 & .02 & .18 & 0 \\
\hline Wtr Year & 2008 & Total & 1.44 & Mean & & 04 & Max & .39 & Min & 0 & Acre-Ft & 2.9 \\
\hline Cal Year & 2007 & Total & 1.06 & Mean & & 03 & $\operatorname{Max}$ & .39 & Min & 0 & Acre-Ft & 2.1 \\
\hline
\end{tabular}

*Estimate 


\section{E196 TA-55 above Effluent Canyon}

Daily Total Rainfall in Inches

Water Year October 2007 to September 2008

\begin{tabular}{|c|c|c|c|c|c|c|c|c|c|c|c|c|}
\hline DAY & ОСт & NOV & DEC & JAN & FEB & MAR & APR & MAY & JUN & JUL & AUG & SEP \\
\hline 1 & & & & & & & & $0^{*}$ & 0 & $.16^{\star}$ & 0 & .02 \\
\hline 2 & & & & & & & & $0^{*}$ & 0 & $.03^{*}$ & 0 & 0 \\
\hline 3 & & & & & & & & $0^{*}$ & 0 & $.03^{*}$ & 0 & 0 \\
\hline 4 & & & & & & & & $0^{*}$ & 0 & $0^{*}$ & .97 & 0 \\
\hline 5 & & & & & & & & $0^{*}$ & .04 & $.09^{*}$ & 0 & 0 \\
\hline 6 & & & & & & & & $0^{*}$ & 0 & $0^{*}$ & 0 & 0 \\
\hline 7 & & & & & & & & $0^{*}$ & 0 & $.37^{*}$ & .09 & 0 \\
\hline 8 & & & & & & & & $0^{*}$ & 0 & .23 & .43 & 0 \\
\hline 9 & & & & & & & & $0^{*}$ & 0 & 0 & .31 & .03 \\
\hline 10 & & & & & & & & $0^{*}$ & 0 & 0 & 1.2 & 0 \\
\hline 11 & & & & & & & & $0^{*}$ & 0 & .05 & 0 & 0 \\
\hline 12 & & & & & & & & $0^{*}$ & 0 & 0 & .02 & 0 \\
\hline 13 & & & & & & & & $.08^{*}$ & 0 & 0 & 0 & 0 \\
\hline 14 & & & & & & & & $.12^{*}$ & 0 & 0 & 0 & 0 \\
\hline 15 & & & & & & & & $.58^{\star}$ & 0 & .04 & 0 & 0 \\
\hline 16 & & & & & & & & 0 & 0 & .14 & .45 & 0 \\
\hline 17 & & & & & & & & 0 & 0 & .04 & .12 & 0 \\
\hline 18 & & & & & & & & 0 & 0 & .02 & 0 & 0 \\
\hline 19 & & & & & & & & 0 & 0 & 0 & 0 & .01 \\
\hline 20 & & & & & & & & 0 & 0 & 0 & .06 & .06 \\
\hline 21 & & & & & & & & 0 & 0 & .41 & 0 & .01 \\
\hline 22 & & & & & & & & .11 & 0 & .01 & 0 & .15 \\
\hline 23 & & & & & & & & .13 & .02 & 0 & .58 & 0 \\
\hline 24 & & & & & & & & 0 & 0 & 0 & .37 & .05 \\
\hline 25 & & & & & & & & 0 & 0 & 0 & .09 & 0 \\
\hline 26 & & & & & & & & 0 & 0 & .05 & 0 & .01 \\
\hline 27 & & & & & & & & 0 & 0 & .14 & 0 & 0 \\
\hline 28 & & & & & & & & .35 & 0 & .07 & 0 & 0 \\
\hline 29 & & & & & & & & 0 & 0 & 0 & .02 & 0 \\
\hline 30 & & & & & ------ & & & 0 & 0 & 0 & 0 & 0 \\
\hline 31 & & ----- & & & ------ & & ----- & 0 & ------ & 0 & .71 & ------ \\
\hline Total & & & & & & & & 0.59 & 0.06 & 0.97 & 5.42 & 0.34 \\
\hline Max & & & & & & & & .35 & .04 & .41 & 1.2 & .15 \\
\hline Wtr Year & 2008 & Total & 7.73 & Mean & & .047 & Max & 1.2 & Min & 0 & InstMax & .36 \\
\hline Cal Year & 2007 & Total & 12.50 & Mean & & .065 & Max & 1.2 & Min & 0 & InstMax & .67 \\
\hline
\end{tabular}

*Estimate 


\section{E200 Mortandad Canyon below Effluent Canyon}

Location. Lat $35^{\circ}$ 51' 55", long $106^{\circ}$ 17' 46", NW 1/4, Sec. 22, T. 19 N., R. 6 E., Los Alamos County.

Drainage Area. $0.49 \mathrm{mi}^{2}$.

Period of Record. May 10, 1995, to September 30, 2008.

Gage. Data logger with cellular telemetry and steel "fabricated" nonstandard flume as low-water control. Elevation of gage is 7,062.50 ft above NGVD from survey.

Remarks. Records are good except estimated daily discharges, which are fair. Flow is mostly effluent from LANL TA-50, liquid radiological waste plant.

Average Discharge. $14 \mathrm{yr}, 0.053 \mathrm{ft}^{3} / \mathrm{s}, 38$ acre- $\mathrm{ft} / \mathrm{yr}$.

Extremes outside Period of Record. Maximum discharge, $34 \mathrm{ft}^{3} / \mathrm{s}$, August 19, 1970, gage height $3.07 \mathrm{ft}$, from old USGS data files.

Extremes for Period of Record. Maximum discharge, $448 \mathrm{ft}^{3} / \mathrm{s}$, August 24, 2006, gage height $5.38 \mathrm{ft}$ (from critical depth computation of flood marks). No flow at times.

Extremes for Current Year. Peak discharges above base of $15 \mathrm{ft}^{3} / \mathrm{s}$ and maximum (*):

\begin{tabular}{|c|c|c|c|}
\hline Date & Time & Discharge $\left.\mathbf{( f t}^{\mathbf{3}} \mathbf{s}\right)$ & Gage Height (ft) \\
\hline November 29 & 1040 & 33 & 3.05 \\
\hline December 1 & 0215 & 26 & 2.81 \\
\hline December 19 & 0945 & 22 & 2.63 \\
\hline August 10 & 1135 & $66^{*}$ & $3.86^{*}$
\end{tabular}

No flow at times.

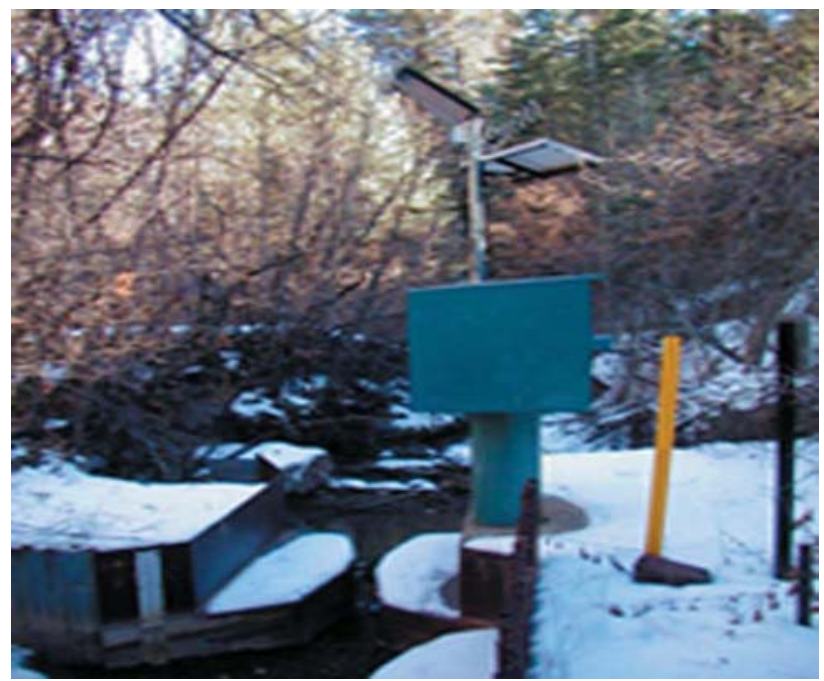




\section{E200 Mortandad Canyon below Effluent Canyon}

\section{Station Analysis}

\section{Water Year}

Equipment. Station is equipped with Sutron 8210 data logger (5-min. interval) and Sutron Accubar bubble sensor with cellular telemetry and speech modem. The system is powered by a solar-panel battery system. All equipment is housed in a NEMA shelter mounted on a 2' CMP well. Station is equipped with an ISCO pump sampler for waterquality sample collection. ISCO is housed in a separate shelter, a $3^{\prime} \times 4^{\prime}$ metal box. Sampler is trigger by stage through the data logger. The staff in the 9 " flume is the reference gage; a larger staff is also available for high flow. No provision for measurements above wading stage.

Field Work. The station was visited 18 times to conduct discharge measurements and service the instrumentation. Field inspections for the gage are listed under site history files on the Hydstra database. Discharge measurements for the gage are listed under site gauging files on the Hydstra database.

Datum Correction. None.

Gage-Height Record. The data logger referenced to the outside staff gave a complete and satisfactory record for the water year except during the periods of December 4-7 and 12-20 when ice affected gage height.

Rating. The channel at the gage is about $33^{\prime}$ wide and straight for about 50' upstream and straight for about 75' below gage. The streambed consists of sand and gravel and is subject to fill behind flume from flow events and gage silting problem from effluent release. Flow is mostly effluent from TA-50 water treatment plant. The control is a fabricated steel flume about $5 "$ at the throat.

Eighteen inspections were made; nine of these inspections observed no flow during the year.

Developed using measurements made during previous years, Rating No. 1 is effective and was used with a variable shift diagram from the last measurement made at the gage station.

Discharge. Discharge was computed by applying gage height to Rating No. 1 through shift adjustment based on a variable shift diagram. Partial days were computed based on TA-50 release and analysis of precipitation records.

Remarks. Records are good except for estimated daily discharges, which are fair. 


\section{E200 Mortandad Canyon below Effluent Canyon}

Daily Mean Discharge in Cubic Feet per Second

Water Year October 2007 to September 2008

\begin{tabular}{|c|c|c|c|c|c|c|c|c|c|c|c|c|}
\hline DAY & OCT & NOV & DEC & JAN & FEB & MAR & APR & MAY & JUN & JUL & AUG & SEP \\
\hline 1 & .08 & 0 & 5.1 & 0 & 0 & 0 & .02 & $.02^{\star}$ & 0 & .01 & 0 & .27 \\
\hline 2 & .02 & 0 & .08 & 0 & 0 & 0 & 0 & 0 & 0 & 0 & 0 & .17 \\
\hline 3 & .04 & 0 & .04 & 0 & 0 & .04 & 0 & 0 & 0 & 0 & 0 & .01 \\
\hline 4 & .01 & 0 & $0^{*}$ & 0 & 0 & 0 & 0 & 0 & .01 & 0 & .28 & .03 \\
\hline 5 & .01 & 0 & $0^{*}$ & 0 & 0 & 0 & 0 & 0 & 0 & 0 & .01 & 0 \\
\hline 6 & 0 & 0 & $0^{*}$ & 0 & $.03^{*}$ & 0 & 0 & 0 & 0 & 0 & .04 & 0 \\
\hline 7 & 0 & 0 & $0^{*}$ & .03 & 0 & 0 & 0 & 0 & 0 & .03 & 0 & 0 \\
\hline 8 & 0 & 0 & .50 & 0 & 0 & 0 & 0 & $.03^{*}$ & 0 & .08 & .07 & 0 \\
\hline 9 & .03 & 0 & .13 & 0 & 0 & 0 & 0 & 0 & .01 & .01 & .12 & .06 \\
\hline 10 & .01 & 0 & 0 & $.03^{\star}$ & 0 & .03 & 0 & 0 & 0 & .02 & 2.4 & .01 \\
\hline 11 & .02 & 0 & 0 & $.03^{\star}$ & 0 & 0 & 0 & 0 & 0 & 0 & .07 & .01 \\
\hline 12 & .01 & 0 & $.03^{\star}$ & 0 & 0 & 0 & 0 & 0 & .01 & 0 & .01 & 0 \\
\hline 13 & 0 & .02 & $.03^{*}$ & 0 & $.03^{\star}$ & 0 & 0 & .03 & 0 & 0 & .05 & 0 \\
\hline 14 & 0 & 0 & $0^{*}$ & 0 & 0 & 0 & $.03^{\star}$ & .01 & 0 & 0 & .01 & 0 \\
\hline 15 & 0 & 0 & $0^{*}$ & 0 & 0 & 0 & $.01^{\star}$ & .17 & 0 & .01 & 0 & .02 \\
\hline 16 & 0 & 0 & $0^{*}$ & 0 & 0 & 0 & 0 & .04 & 0 & 0 & .10 & .03 \\
\hline 17 & 0 & 0 & $0^{*}$ & 0 & 0 & 0 & 0 & 0 & 0 & 0 & .05 & .08 \\
\hline 18 & 0 & 0 & $0^{*}$ & 0 & 0 & .03 & 0 & 0 & 0 & 0 & .03 & .11 \\
\hline 19 & 0 & .02 & $.03^{*}$ & 0 & 0 & .01 & 0 & 0 & 0 & 0 & .01 & .01 \\
\hline 20 & 0 & .03 & $.03^{*}$ & 0 & 0 & 0 & 0 & .01 & 0 & 0 & 0 & 0 \\
\hline 21 & 0 & 0 & 0 & 0 & 0 & 0 & 0 & .01 & 0 & .09 & 0 & 0 \\
\hline 22 & 0 & 0 & 0 & 0 & 0 & 0 & .01 & .01 & 0 & 0 & 0 & 0 \\
\hline 23 & .01 & 0 & 0 & 0 & 0 & 0 & 0 & .01 & 0 & 0 & .29 & 0 \\
\hline 24 & .03 & 0 & 0 & 0 & $0^{*}$ & .02 & 0 & 0 & 0 & .01 & .24 & 0 \\
\hline 25 & 0 & 0 & 0 & 0 & $.03^{\star}$ & 0 & 0 & 0 & .01 & .01 & .09 & 0 \\
\hline 26 & 0 & 0 & 0 & 0 & 0 & 0 & 0 & 0 & 0 & 0 & .05 & 0 \\
\hline 27 & 0 & 0 & 0 & 0 & 0 & 0 & 0 & .01 & 0 & .01 & 0 & 0 \\
\hline 28 & 0 & 0 & 0 & 0 & 0 & 0 & 0 & .13 & 0 & .02 & .02 & 0 \\
\hline 29 & 0 & .50 & 0 & $.03^{\star}$ & 0 & 0 & 0 & .03 & 0 & .02 & 0 & .01 \\
\hline 30 & .02 & .84 & 0 & 0 & ----- & 0 & 0 & 0 & 0 & 0 & 0 & .06 \\
\hline 31 & 0 & ------ & 0 & $.02^{*}$ & ----- & .02 & ----- & 0 & ----- & 0 & .41 & ----- \\
\hline Total & 0.29 & 1.41 & 5.97 & 0.14 & 0.09 & 0.15 & 0.07 & 0.51 & 0.04 & 0.32 & 4.35 & 0.88 \\
\hline Mean & .009 & .047 & .19 & .005 & .003 & .005 & .002 & .016 & .001 & .010 & .14 & .029 \\
\hline Max & .08 & .84 & 5.1 & .03 & .03 & .04 & .03 & .17 & .01 & .09 & 2.4 & .27 \\
\hline Min & 0 & 0 & 0 & 0 & 0 & 0 & 0 & 0 & 0 & 0 & 0 & 0 \\
\hline Acre-Ft & .58 & 2.8 & 12 & .28 & .18 & .30 & .14 & 1.0 & .08 & .63 & 8.6 & 1.7 \\
\hline Wtr Year & 2008 & Total & 14.22 & Mean & & & Max & 5.1 & Min & 0 & Acre-Ft & 28 \\
\hline Cal Year & 2007 & Total & 21.64 & Mean & & & Max & 5.1 & Min & 0 & Acre-Ft & 43 \\
\hline
\end{tabular}

*Estimate 


\section{E2005 Mortandad Canyon Tributary Batch Plant at Sigma}

Location. Lat $35^{\circ}$ 51' 57", long $106^{\circ}$ 17' 24", NE 1/4, Sec. 22, T.19 N., R. 6 E., Los Alamos County.

Drainage Area. $7.69 \mathrm{mi}^{2}$.

Period of Record. July 24, 2007, to September 30, 2008.

Gage. Data logger and 24” Parshall flume, as well as rain gage with cellular telemetry. Elevation of gage is 7,215 ft above NGVD.

Remarks. Records are good.

Extremes for Period of Record. Maximum discharge, $3.17 \mathrm{ft}^{3} / \mathrm{s}$, July 26, 2008, gage height $0.55 \mathrm{ft}$. No flow most of the time.

Extremes for Current Year. Peak discharge above base of $1.0 \mathrm{ft}^{3} / \mathrm{s}$ and maximum (*):

\begin{tabular}{|c|c|c|c|}
\hline Date & Time & Discharge $\left(\mathbf{f t}^{\mathbf{3}} / \mathbf{s}\right)$ & Gage Height (ft) \\
\hline July 9 & 0100 & 2.08 & 0.42 \\
\hline July 26 & 0155 & $3.17^{*}$ & $0.55^{*}$ \\
\hline July 27 & 1650 & 1.64 & 0.36 \\
\hline August 9 & 2315 & 1.37 & 0.32 \\
\hline August 10 & 1135 & 1.71 & 0.37
\end{tabular}

No flow most of the time.

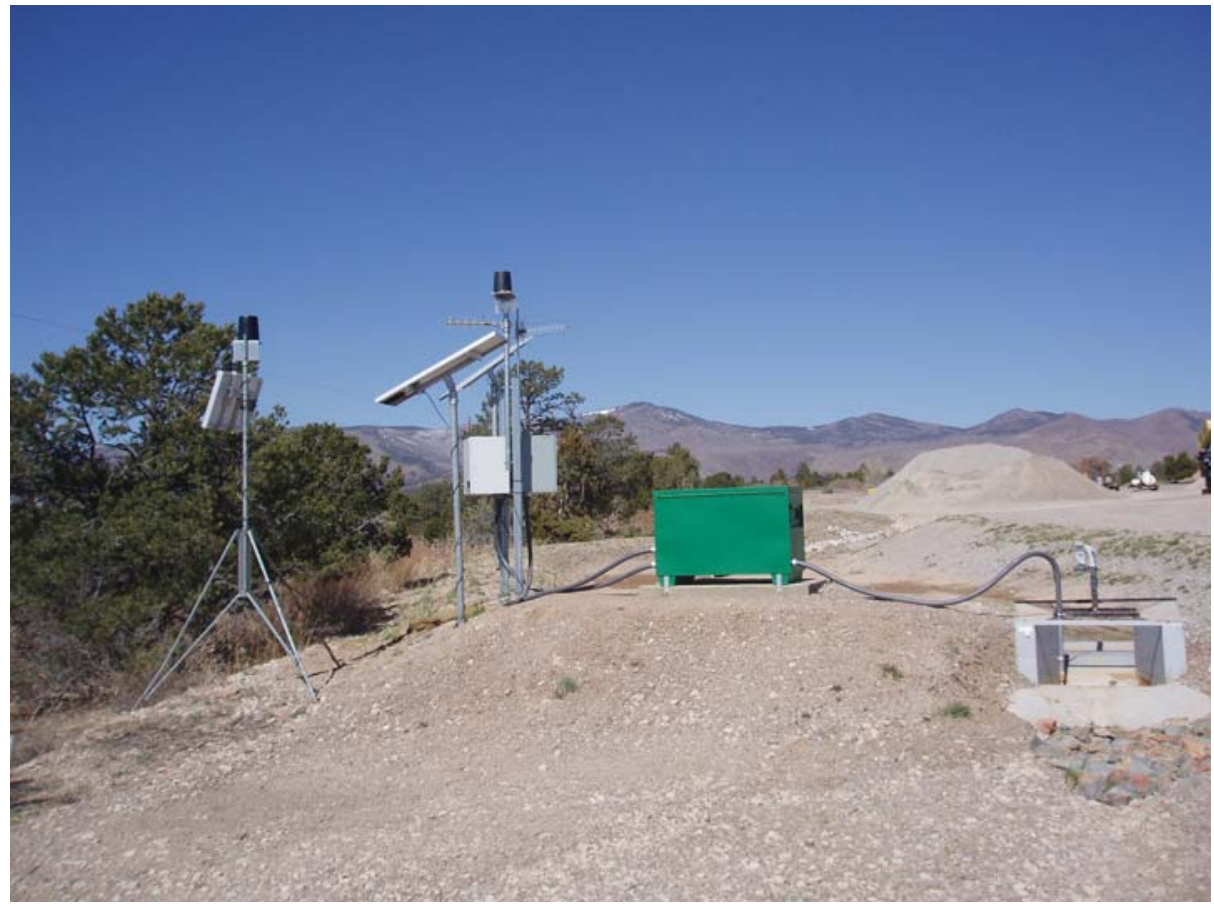




\section{E2005 Mortandad Canyon Tributary Batch Plant at Sigma}

\section{Station Analysis}

\section{Water Year}

Equipment. Station is equipped with Sutron 8210 data logger (5-min. interval) and milltronics sonic probe mounted on a 24" Parshall flume and cellular telemetry with speech modem. Phone upgraded May 29 from analog to digital service. The system is powered by a solar-panel battery system housed in a NEMA shelter. Station is equipped with an ISCO pump sampler for water-quality sample collection. ISCO is housed in a separate shelter, a $3^{\prime} \times 4^{\prime}$ metal box. Sampler is triggered by stage through the data logger. The staff in the 24" Parshall flume is the reference gage. No provision for discharge measurements above wading stage.

Station is also equipped with a tipping bucket rain gage, Rain Collection II. All equipment is powered with a solar-panel battery-charging system. Rain data are seasonal.

Field Work. This station was visited 35 times to conduct discharge measurements and service the instrumentation. Field inspections for the gage are listed under site history files on the Hydstra database. Discharge measurements for the gage are listed under site gauging files on the Hydstra database.

Datum Correction. None.

Gage-Height Record. The data logger referenced to the outside staff gave a complete and satisfactory record except for the period of December 9-12 when ice affected gage height.

Rating. The site has an upstream catchment pond. The channel is straight for $25^{\prime}$ below gage.

Thirty-four inspections of no flow and one inspection of snow melt were made this year.

Rating No. 1 was developed based on the computation of 24" Parshall flume. Point of zero flow is 0.00 gage height.

Discharge. Discharge was computed by applying Rating No 1 directly. Those days estimated at zero flow were based on precipitation and nearby gage stations.

Remarks. Records are good. 
E2005 Mortandad Canyon Tributary Batch Plant at Sigma

Daily Mean Discharge in Cubic Feet per Second

Water Year October 2007 to September 2008

\begin{tabular}{|c|c|c|c|c|c|c|c|c|c|c|c|c|}
\hline DAY & ОСТ & NOV & DEC & JAN & FEB & MAR & APR & MAY & JUN & JUL & AUG & SEP \\
\hline 1 & .01 & 0 & .18 & 0 & 0 & 0 & 0 & 0 & 0 & 0 & 0 & .08 \\
\hline 2 & 0 & 0 & 0 & 0 & 0 & 0 & 0 & 0 & 0 & 0 & 0 & 0 \\
\hline 3 & 0 & 0 & 0 & 0 & 0 & 0 & 0 & 0 & 0 & 0 & 0 & 0 \\
\hline 4 & 0 & 0 & 0 & 0 & 0 & 0 & 0 & 0 & 0 & 0 & 0 & 0 \\
\hline 5 & 0 & 0 & 0 & 0 & 0 & 0 & 0 & 0 & 0 & 0 & 0 & 0 \\
\hline 6 & 0 & 0 & 0 & 0 & 0 & 0 & 0 & 0 & 0 & 0 & 0 & 0 \\
\hline 7 & 0 & 0 & 0 & 0 & 0 & 0 & 0 & 0 & 0 & 0 & 0 & 0 \\
\hline 8 & 0 & 0 & .09 & 0 & 0 & 0 & 0 & 0 & 0 & 0 & 0 & 0 \\
\hline 9 & 0 & 0 & $.05^{\star}$ & 0 & 0 & 0 & 0 & 0 & 0 & .02 & .01 & 0 \\
\hline 10 & 0 & 0 & $0^{*}$ & 0 & 0 & 0 & 0 & 0 & 0 & 0 & .12 & 0 \\
\hline 11 & 0 & 0 & $0^{*}$ & 0 & 0 & 0 & 0 & 0 & 0 & 0 & .02 & 0 \\
\hline 12 & 0 & 0 & $0^{*}$ & 0 & 0 & 0 & 0 & 0 & 0 & 0 & 0 & 0 \\
\hline 13 & 0 & 0 & 0 & 0 & 0 & 0 & 0 & 0 & 0 & 0 & 0 & 0 \\
\hline 14 & 0 & 0 & 0 & 0 & 0 & 0 & 0 & 0 & 0 & 0 & 0 & 0 \\
\hline 15 & 0 & 0 & 0 & 0 & 0 & 0 & 0 & 0 & 0 & 0 & 0 & 0 \\
\hline 16 & 0 & 0 & 0 & 0 & 0 & 0 & 0 & 0 & 0 & 0 & .02 & 0 \\
\hline 17 & 0 & 0 & 0 & 0 & 0 & 0 & 0 & 0 & 0 & 0 & 0 & 0 \\
\hline 18 & 0 & 0 & 0 & 0 & 0 & 0 & 0 & 0 & 0 & 0 & 0 & 0 \\
\hline 19 & 0 & 0 & 0 & 0 & 0 & 0 & 0 & 0 & 0 & 0 & 0 & 0 \\
\hline 20 & 0 & 0 & 0 & 0 & 0 & 0 & 0 & 0 & 0 & 0 & 0 & 0 \\
\hline 21 & 0 & 0 & 0 & 0 & 0 & 0 & 0 & 0 & 0 & 0 & 0 & 0 \\
\hline 22 & 0 & 0 & 0 & 0 & 0 & 0 & 0 & 0 & 0 & 0 & 0 & 0 \\
\hline 23 & 0 & 0 & 0 & 0 & 0 & 0 & 0 & 0 & 0 & 0 & .09 & 0 \\
\hline 24 & 0 & 0 & 0 & 0 & 0 & 0 & 0 & 0 & 0 & 0 & .10 & 0 \\
\hline 25 & 0 & 0 & 0 & 0 & 0 & 0 & 0 & 0 & 0 & 0 & .11 & 0 \\
\hline 26 & 0 & 0 & 0 & 0 & 0 & 0 & 0 & 0 & 0 & .01 & .02 & 0 \\
\hline 27 & 0 & 0 & 0 & 0 & 0 & 0 & 0 & 0 & 0 & .01 & 0 & 0 \\
\hline 28 & 0 & 0 & 0 & .08 & 0 & 0 & 0 & 0 & 0 & 0 & 0 & 0 \\
\hline 29 & 0 & 0 & 0 & 0 & 0 & 0 & 0 & 0 & 0 & 0 & 0 & 0 \\
\hline 30 & 0 & .01 & 0 & 0 & ---- & 0 & 0 & 0 & 0 & 0 & 0 & 0 \\
\hline 31 & 0 & ----- & 0 & 0 & ----- & 0 & ------ & 0 & ------ & 0 & .11 & ----- \\
\hline Total & 0.01 & 0.01 & 0.32 & 0.08 & 0 & 0 & 0 & 0 & 0 & 0.04 & 0.60 & 0.08 \\
\hline Mean & 0 & 0 & .010 & .003 & 0 & 0 & 0 & 0 & 0 & .001 & .019 & .003 \\
\hline Max & .01 & .01 & .18 & .08 & 0 & 0 & 0 & 0 & 0 & .02 & .12 & .08 \\
\hline Min & 0 & 0 & 0 & 0 & 0 & 0 & 0 & 0 & 0 & 0 & 0 & 0 \\
\hline Acre-Ft & .02 & .02 & .63 & .16 & 0 & 0 & 0 & 0 & 0 & .08 & 1.2 & .16 \\
\hline Wtr Year & 2008 & Total & 1.14 & Mean & & 03 & Max & .18 & Min & 0 & Acre-Ft & 2.3 \\
\hline Cal Year & 2007 & Total & 1.07 & Mean & & 07 & hax & .18 & Min & 0 & Acre-Ft & 2.1 \\
\hline
\end{tabular}

${ }^{\star}$ Estimate 


\section{E2005 Mortandad Canyon Tributary Batch Plant at Sigma}

Daily Total Rainfall in Inches

Water Year October 2007 to September 2008

\begin{tabular}{|c|c|c|c|c|c|c|c|c|c|c|c|c|}
\hline DAY & ОСт & NOV & DEC & JAN & FEB & MAR & APR & MAY & JUN & JUL & AUG & SEP \\
\hline 1 & & & & & & & 0 & 0 & 0 & .32 & 0 & .02 \\
\hline 2 & & & & & & & 0 & 0 & 0 & 0 & 0 & 0 \\
\hline 3 & & & & & & & 0 & 0 & 0 & 0 & 0 & 0 \\
\hline 4 & & & & & & & 0 & 0 & 0 & 0 & $.70^{*}$ & 0 \\
\hline 5 & & & & & & & 0 & 0 & .03 & 0 & 0 & 0 \\
\hline 6 & & & & & & & 0 & 0 & 0 & 0 & 0 & 0 \\
\hline 7 & & & & & & & 0 & 0 & 0 & .37 & .03 & 0 \\
\hline 8 & & & & & & & 0 & 0 & 0 & .17 & .33 & 0 \\
\hline 9 & & & & & & & 0 & 0 & 0 & 0 & .36 & .01 \\
\hline 10 & & & & & & & .01 & 0 & 0 & 0 & .84 & 0 \\
\hline 11 & & & & & & & 0 & 0 & .02 & .06 & 0 & 0 \\
\hline 12 & & & & & & & .01 & 0 & 0 & 0 & .13 & 0 \\
\hline 13 & & & & & & & 0 & .04 & 0 & 0 & 0 & 0 \\
\hline 14 & & & & & & & 0 & .12 & 0 & 0 & .06 & 0 \\
\hline 15 & & & & & & & 0 & .53 & 0 & .10 & 0 & 0 \\
\hline 16 & & & & & & & 0 & 0 & 0 & .17 & .43 & 0 \\
\hline 17 & & & & & & & .15 & 0 & 0 & .03 & .10 & 0 \\
\hline 18 & & & & & & & 0 & 0 & 0 & .02 & 0 & .03 \\
\hline 19 & & & & & & & 0 & 0 & 0 & 0 & 0 & .02 \\
\hline 20 & & & & & & & 0 & 0 & .01 & 0 & .06 & .07 \\
\hline 21 & & & & & & & 0 & 0 & 0 & .16 & 0 & 0 \\
\hline 22 & & & & & & & 0 & .07 & 0 & .03 & 0 & .02 \\
\hline 23 & & & & & & & 0 & .13 & .06 & 0 & .85 & 0 \\
\hline 24 & & & & & & & 0 & 0 & 0 & 0 & .34 & .01 \\
\hline 25 & & & & & & & 0 & 0 & $0^{*}$ & 0 & .03 & 0 \\
\hline 26 & & & & & & & 0 & 0 & $0^{*}$ & 0 & .03 & .05 \\
\hline 27 & & & & & & & 0 & 0 & 0 & 0 & 0 & 0 \\
\hline 28 & & & & & & & 0 & .40 & 0 & .01 & 0 & 0 \\
\hline 29 & & & & & & & 0 & 0 & 0 & 0 & .03 & 0 \\
\hline 30 & & & & & ------ & & 0 & 0 & 0 & 0 & 0 & 0 \\
\hline 31 & & ----- & & & ------ & & ------ & 0 & ----- & 0 & .91 & ------ \\
\hline Total & & & & & & & 0.17 & 1.29 & 0.12 & 1.44 & 4.53 & 0.23 \\
\hline Max & & & & & & & .15 & .53 & .06 & .37 & .91 & .07 \\
\hline Wtr Year & 2008 & Total & 8.09 & Mean & & .034 & Max & .91 & Min & 0 & InstMax & .22 \\
\hline Cal Year & 2007 & Total & 6.80 & Mean & & .065 & Max & 1.4 & Min & 0 & InstMax & .31 \\
\hline
\end{tabular}

*Estimate 


\section{E201 Mortandad Canyon above Ten Site Canyon}

Location. Lat $35^{\circ}$ 51' 46", long $106^{\circ}$ 16' 29", SW 1/4, Sec. 22, T. 19 N., R. 6 E., Los Alamos County.

Drainage Area. $0.25 \mathrm{mi}^{2}$.

Period of Record. October 1, 2006, to September 30, 2008.

Revised Record. Period of record (this report).

Gage. Data logger with steel "fabricated" nonstandard flume. Elevation of gage is 6,864 $\mathrm{ft}$ above NGVD.

Remarks. Records are good. Records for this site existed before period of record but are not reliable.

Extremes for Period of Record. Maximum discharge, $78 \mathrm{ft}^{3} / \mathrm{s}$, August 10, 2008, gage height $2.43 \mathrm{ft}$. No flow most of the time.

Extremes for Current Year. Peak discharge above base of $5.0 \mathrm{ft}^{3} / \mathrm{s}$ and maximum (*):

\begin{tabular}{|c|c|c|c|}
\hline Date & Time & Discharge $\left(\mathbf{f t}^{\mathbf{3}} / \mathbf{s}\right)$ & Gage Height (ft) \\
\hline December 1 & 0225 & 16 & 1.19 \\
\hline August 10 & 1205 & $78^{*}$ & $2.43^{*}$
\end{tabular}

No flow most of the time.

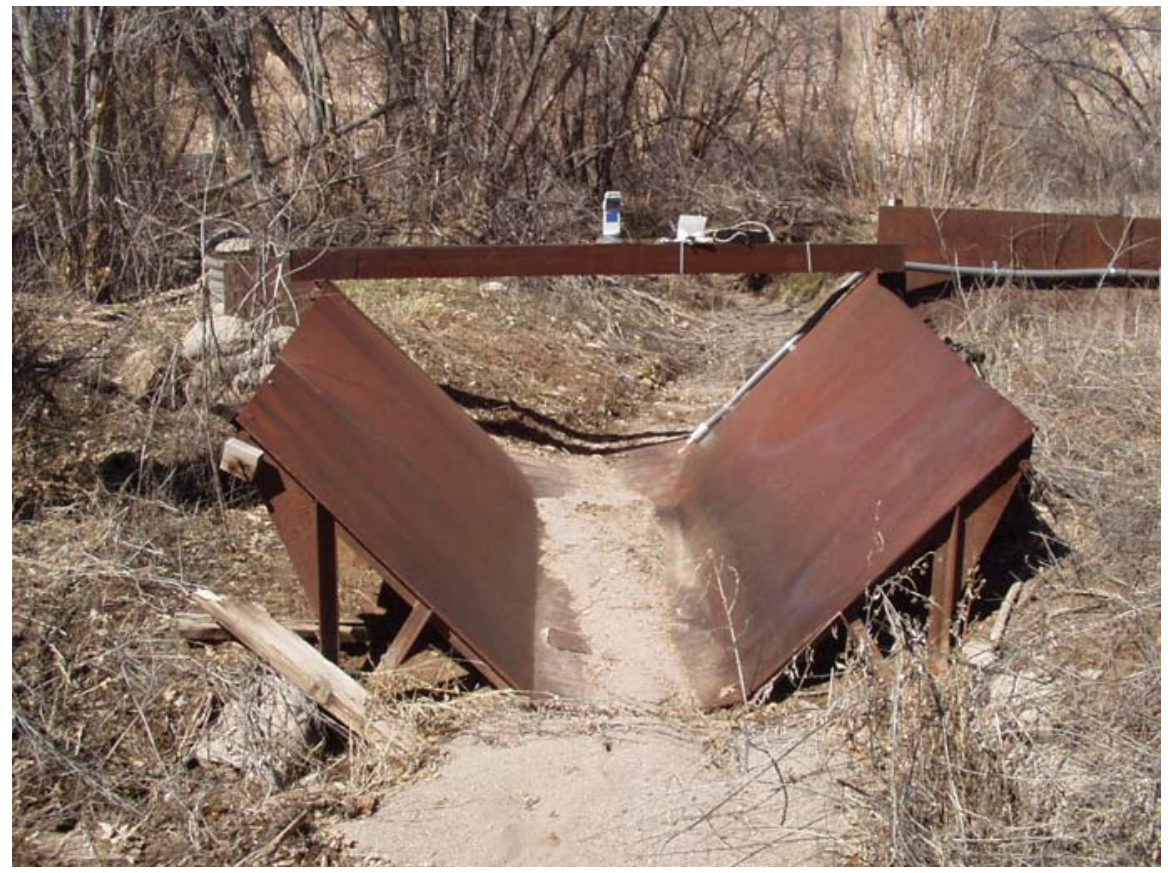




\section{E201 Mortandad Canyon above Ten Site Canyon}

\section{Station Analysis}

\section{Water Year}

Equipment. Station is equipped with Sutron 8210 data logger (5-min. interval) and milltronics sonic probe mounted on a $10^{\prime}$ flume. The system is powered by a solar-panel battery system housed in a NEMA shelter. Station is equipped with an ISCO pump sampler for water-quality sample collection. ISCO is housed in a separate shelter, a $3^{\prime} \times$ $4^{\prime}$ metal box. Sampler is triggered by stage through the data logger. An outside staff is available for reference. No provision for measurements above wading stage.

Cellular telemetry removed March 12, discontinued service.

Field Work. The station was visited 16 times to conduct discharge measurements and service the instrumentation. Field inspections for the gage are listed under site history files on the Hydstra database. Discharge measurements for the gage are listed under site gauging files on the Hydstra database.

Datum Correction. None.

Gage-Height Record. The data logger referenced to the outside staff gave a complete and satisfactory report for the water year.

Rating. Channel is straight above and below modified flume. Flow is confined to cut banks. Channel bottom is $3^{\prime} \mathrm{ft}$ wide with some vegetation above and below flume.

The streambed consists of sand and gravel and is subject to fill flume from low flow events. The control is a fabricated steel flume $10^{\prime}$ at the throat.

Fifteen inspections of no flow and one inspection of flow were made this year.

Rating No. 1 was developed based on slope area computations and discharge measurements

Discharge. Discharge was computed by applying Rating No. 1 directly.

Remarks. Records are good. 


\section{E201 Mortandad Canyon above Ten Site Canyon}

Daily Mean Discharge in Cubic Feet per Second

Water Year October 2007 to September 2008

\begin{tabular}{|c|c|c|c|c|c|c|c|c|c|c|c|c|}
\hline DAY & OCT & NOV & DEC & JAN & FEB & MAR & APR & MAY & JUN & JUL & AUG & SEP \\
\hline 1 & 0 & 0 & .96 & 0 & 0 & 0 & 0 & 0 & 0 & 0 & 0 & 0 \\
\hline 2 & 0 & 0 & 0 & 0 & 0 & 0 & 0 & 0 & 0 & 0 & 0 & 0 \\
\hline 3 & 0 & 0 & 0 & 0 & 0 & 0 & 0 & 0 & 0 & 0 & 0 & 0 \\
\hline 4 & 0 & 0 & 0 & 0 & 0 & 0 & 0 & 0 & 0 & 0 & 0 & 0 \\
\hline 5 & 0 & 0 & 0 & 0 & 0 & 0 & 0 & 0 & 0 & 0 & 0 & 0 \\
\hline 6 & 0 & 0 & 0 & 0 & 0 & 0 & 0 & 0 & 0 & 0 & 0 & 0 \\
\hline 7 & 0 & 0 & 0 & 0 & 0 & 0 & 0 & 0 & 0 & 0 & 0 & 0 \\
\hline 8 & 0 & 0 & 0 & 0 & 0 & 0 & 0 & 0 & 0 & 0 & 0 & 0 \\
\hline 9 & 0 & 0 & 0 & 0 & 0 & 0 & 0 & 0 & 0 & 0 & 0 & 0 \\
\hline 10 & 0 & 0 & 0 & 0 & 0 & 0 & 0 & 0 & 0 & 0 & 2.4 & 0 \\
\hline 11 & 0 & 0 & 0 & 0 & 0 & 0 & 0 & 0 & 0 & 0 & 0 & 0 \\
\hline 12 & 0 & 0 & 0 & 0 & 0 & 0 & 0 & 0 & 0 & 0 & 0 & 0 \\
\hline 13 & 0 & 0 & 0 & 0 & 0 & 0 & 0 & 0 & 0 & 0 & 0 & 0 \\
\hline 14 & 0 & 0 & 0 & 0 & 0 & 0 & 0 & 0 & 0 & 0 & 0 & 0 \\
\hline 15 & 0 & 0 & 0 & 0 & 0 & 0 & 0 & 0 & 0 & 0 & 0 & 0 \\
\hline 16 & 0 & 0 & 0 & 0 & 0 & 0 & 0 & 0 & 0 & 0 & 0 & 0 \\
\hline 17 & 0 & 0 & 0 & 0 & 0 & 0 & 0 & 0 & 0 & 0 & 0 & 0 \\
\hline 18 & 0 & 0 & 0 & 0 & 0 & 0 & 0 & 0 & 0 & 0 & 0 & 0 \\
\hline 19 & 0 & 0 & 0 & 0 & 0 & 0 & 0 & 0 & 0 & 0 & 0 & 0 \\
\hline 20 & 0 & 0 & 0 & 0 & 0 & 0 & 0 & 0 & 0 & 0 & 0 & 0 \\
\hline 21 & 0 & 0 & 0 & 0 & 0 & 0 & 0 & 0 & 0 & 0 & 0 & 0 \\
\hline 22 & 0 & 0 & 0 & 0 & 0 & 0 & 0 & 0 & 0 & 0 & 0 & 0 \\
\hline 23 & 0 & 0 & 0 & 0 & 0 & 0 & 0 & 0 & 0 & 0 & 0 & 0 \\
\hline 24 & 0 & 0 & 0 & 0 & .03 & 0 & 0 & 0 & 0 & 0 & 0 & 0 \\
\hline 25 & 0 & 0 & 0 & 0 & 0 & 0 & 0 & 0 & 0 & 0 & 0 & 0 \\
\hline 26 & 0 & 0 & 0 & 0 & 0 & 0 & 0 & 0 & 0 & 0 & 0 & 0 \\
\hline 27 & 0 & 0 & 0 & 0 & 0 & 0 & 0 & 0 & 0 & 0 & 0 & 0 \\
\hline 28 & 0 & 0 & 0 & .32 & 0 & 0 & 0 & 0 & 0 & 0 & 0 & 0 \\
\hline 29 & 0 & 0 & 0 & 0 & 0 & 0 & 0 & 0 & 0 & 0 & 0 & 0 \\
\hline 30 & 0 & 0 & 0 & 0 & ----- & 0 & 0 & 0 & 0 & 0 & 0 & 0 \\
\hline 31 & 0 & ------ & 0 & 0 & ----- & 0 & ------ & 0 & ----- & 0 & 0 & ------ \\
\hline Total & 0 & 0 & 0.96 & 0.32 & 0.03 & 0 & 0 & 0 & 0 & 0 & 2.4 & 0 \\
\hline Mean & 0 & 0 & .031 & .010 & .001 & 0 & 0 & 0 & 0 & 0 & .077 & 0 \\
\hline Max & 0 & 0 & .96 & .32 & .03 & 0 & 0 & 0 & 0 & 0 & 2.4 & 0 \\
\hline Min & 0 & 0 & 0 & 0 & 0 & 0 & 0 & 0 & 0 & 0 & 0 & 0 \\
\hline Acre-Ft & 0 & 0 & 1.9 & .63 & .06 & 0 & 0 & 0 & 0 & 0 & 4.8 & 0 \\
\hline Wtr Year & 2008 & Total & 3.71 & Mean & & & Max & 2.4 & Min & 0 & Acre-Ft & 7.4 \\
\hline Cal Year & 2007 & Total & 1.99 & Mean & & & Max & 1.0 & Min & 0 & Acre-Ft & 3.9 \\
\hline
\end{tabular}




\section{E2011 TA-50 Area 006 (C)}

Location. Lat $35^{\circ}$ 51' 41", long $106^{\circ}$ 17' 50", SW 1/4, Sec. 22, T. 19 N., R. 6 E., Los Alamos County.

Drainage Area. $0.019 \mathrm{mi}^{2}$.

Period of Record. October 1, 2006, to September 30, 2008.

Gage. Data logger and 24” Parshall flume. Elevation of gage is 7,220 ft above NGVD.

Remarks. Records are good. Records for this site existed before period of record but are not reliable.

Extremes for Period of Record. Maximum discharge, $6.0 \mathrm{ft}^{3} / \mathrm{s}$, August 10, 2008, gage height $0.83 \mathrm{ft}$. No flow most of the time.

Extremes for Current Year. Peak discharges above base of $1.0 \mathrm{ft}^{3} / \mathrm{s}$ and maximum (*):

\begin{tabular}{|c|c|c|c|}
\hline Date & Time & Discharge $\left(\mathrm{ft}^{3} / \mathrm{s}\right)$ & Gage Height (ft) \\
\hline May 25 & 0530 & 2.0 & 0.41 \\
\hline May 28 & 1545 & 4.3 & 0.67 \\
\hline July 21 & 1110 & 1.6 & 0.35 \\
\hline August 4 & 1735 & 3.8 & 0.62 \\
\hline August 9 & 1225 & 1.0 & 0.27 \\
\hline August 10 & 1115 & $6.0 *$ & $0.83 *$ \\
\hline August 23 & 1615 & 3.0 & 0.53 \\
\hline August 31 & 1515 & 2.3 & 0.45 \\
\hline
\end{tabular}

No flow most of the time.

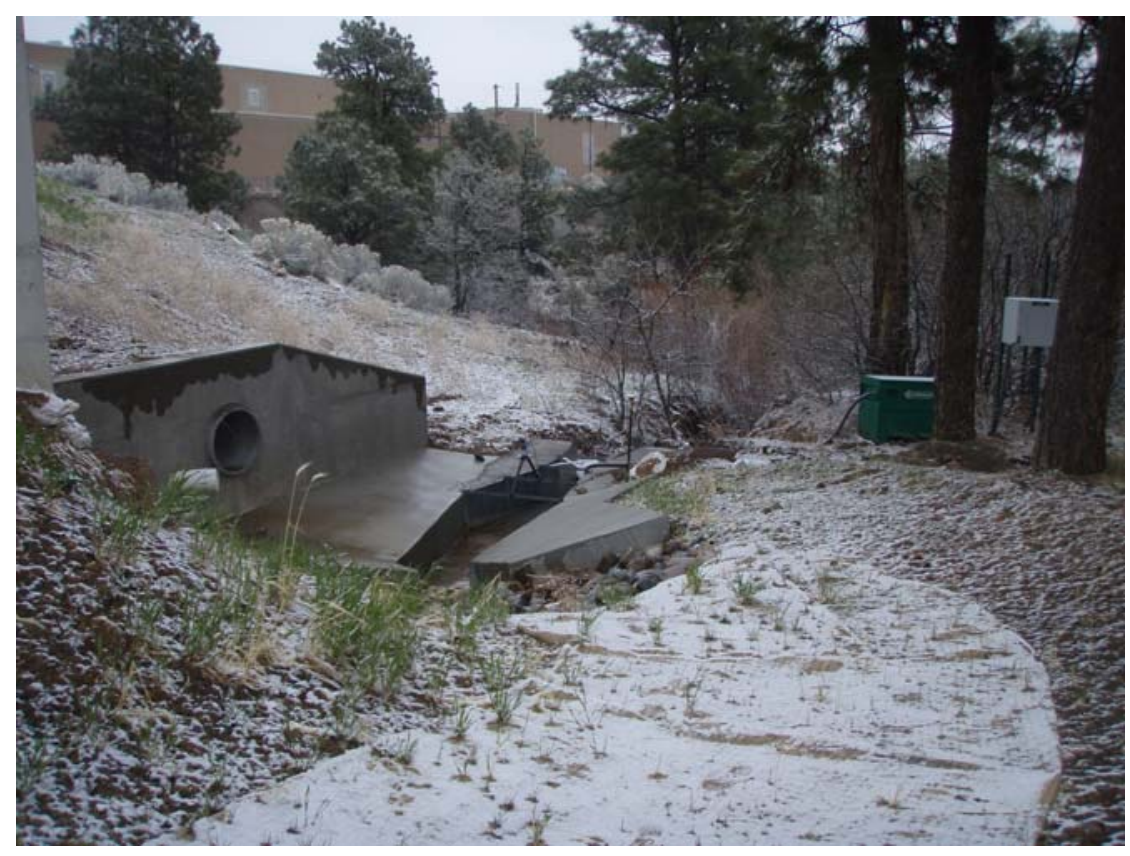




\section{E2011 TA-50 Area 006 (C)}

\section{Station Analysis}

\section{Water Year}

Equipment. Station is equipped with Sutron 8210 data logger (5-min. interval) and milltronics sonic probe mounted on a 24" Parshall flume. The system is powered by a solar-panel battery system housed in a NEMA shelter. Station is equipped with an ISCO pump sampler for water-quality sample collection. ISCO is housed in a separate shelter, a $3^{\prime} \times 4^{\prime}$ metal box. Sampler is triggered by stage through the data logger. The staff in the 24 " Parshall flume is the reference gage. No provision for discharge measurements above wading stage.

Field Work. This station was visited 21 times to conduct discharge measurements and service the instrumentation. Field inspections for the gage are listed under site history files on the Hydstra database. Discharge measurements for the gage are listed under site gauging files on the Hydstra database.

Datum Correction. None

Gage-Height Record. The data logger referenced to the outside staff gave a complete and satisfactory record.

Rating. Upstream and downstream sections consist of formed cement, a result of recent construction to the building adjacent to the site.

Twenty-one inspections of no flow were made this year

Rating No. 1 was developed based on the computation of 24" Parshall flume. Point of zero flow is 0.00 gage height.

Discharge. Discharge was computed by applying Rating No. 1 directly.

Remarks. Records are good. 
E2011 TA-50 Area 006(C)

Daily Mean Discharge in Cubic Feet per Second

Water Year October 2007 to September 2008

\begin{tabular}{|c|c|c|c|c|c|c|c|c|c|c|c|c|}
\hline DAY & OCT & NOV & DEC & JAN & FEB & MAR & APR & MAY & JUN & JUL & AUG & SEP \\
\hline 1 & .01 & 0 & .10 & 0 & 0 & 0 & 0 & 0 & 0 & .01 & 0 & 0 \\
\hline 2 & 0 & 0 & 0 & 0 & 0 & 0 & 0 & 0 & 0 & 0 & 0 & 0 \\
\hline 3 & 0 & 0 & 0 & 0 & 0 & 0 & 0 & 0 & 0 & 0 & 0 & 0 \\
\hline 4 & 0 & 0 & 0 & 0 & 0 & 0 & 0 & 0 & 0 & 0 & .07 & 0 \\
\hline 5 & 0 & 0 & 0 & 0 & 0 & 0 & 0 & 0 & 0 & 0 & 0 & 0 \\
\hline 6 & 0 & 0 & 0 & 0 & 0 & 0 & 0 & 0 & 0 & 0 & 0 & 0 \\
\hline 7 & 0 & 0 & .01 & 0 & 0 & 0 & 0 & 0 & 0 & .01 & 0 & 0 \\
\hline 8 & 0 & 0 & .04 & 0 & 0 & 0 & 0 & 0 & 0 & .01 & .02 & 0 \\
\hline 9 & 0 & 0 & 0 & 0 & 0 & 0 & 0 & 0 & 0 & 0 & .02 & 0 \\
\hline 10 & 0 & 0 & 0 & 0 & 0 & 0 & 0 & 0 & 0 & 0 & .14 & 0 \\
\hline 11 & 0 & 0 & 0 & 0 & 0 & 0 & 0 & 0 & 0 & 0 & 0 & 0 \\
\hline 12 & 0 & 0 & 0 & 0 & 0 & 0 & 0 & 0 & 0 & 0 & 0 & 0 \\
\hline 13 & 0 & 0 & 0 & 0 & 0 & 0 & 0 & 0 & 0 & 0 & 0 & 0 \\
\hline 14 & 0 & 0 & 0 & 0 & 0 & 0 & 0 & 0 & 0 & 0 & 0 & 0 \\
\hline 15 & 0 & 0 & 0 & 0 & 0 & 0 & 0 & .03 & 0 & 0 & 0 & 0 \\
\hline 16 & 0 & 0 & 0 & 0 & 0 & 0 & 0 & 0 & 0 & 0 & .02 & 0 \\
\hline 17 & 0 & 0 & 0 & 0 & 0 & 0 & 0 & 0 & 0 & 0 & 0 & 0 \\
\hline 18 & 0 & 0 & 0 & 0 & 0 & 0 & 0 & 0 & 0 & 0 & 0 & 0 \\
\hline 19 & 0 & 0 & 0 & 0 & 0 & 0 & 0 & 0 & 0 & 0 & 0 & 0 \\
\hline 20 & 0 & 0 & 0 & 0 & 0 & 0 & 0 & 0 & 0 & 0 & 0 & 0 \\
\hline 21 & 0 & 0 & 0 & 0 & 0 & 0 & 0 & 0 & 0 & .02 & 0 & 0 \\
\hline 22 & 0 & 0 & 0 & 0 & 0 & 0 & 0 & 0 & 0 & 0 & 0 & 0 \\
\hline 23 & 0 & 0 & 0 & 0 & 0 & 0 & 0 & 0 & 0 & 0 & .06 & 0 \\
\hline 24 & 0 & 0 & 0 & 0 & .02 & 0 & 0 & 0 & 0 & 0 & .03 & 0 \\
\hline 25 & 0 & 0 & 0 & 0 & 0 & 0 & 0 & .04 & 0 & 0 & 0 & 0 \\
\hline 26 & 0 & 0 & 0 & 0 & 0 & 0 & 0 & 0 & 0 & 0 & 0 & 0 \\
\hline 27 & 0 & 0 & 0 & 0 & 0 & 0 & 0 & 0 & 0 & .01 & 0 & 0 \\
\hline 28 & 0 & 0 & 0 & .06 & 0 & 0 & 0 & .03 & 0 & 0 & 0 & 0 \\
\hline 29 & 0 & 0 & 0 & 0 & 0 & 0 & 0 & 0 & 0 & 0 & 0 & 0 \\
\hline 30 & 0 & .08 & 0 & 0 & ----- & 0 & 0 & 0 & 0 & 0 & 0 & 0 \\
\hline 31 & 0 & ------ & 0 & 0 & ----- & 0 & ----- & 0 & ----- & 0 & .03 & ------ \\
\hline Total & 0.01 & 0.08 & 0.15 & 0.06 & 0.02 & 0 & 0 & 0.10 & 0 & 0.06 & 0.39 & 0 \\
\hline Mean & 0 & .003 & .005 & .002 & .001 & 0 & 0 & .003 & 0 & .002 & .013 & 0 \\
\hline Max & .01 & .08 & .10 & .06 & .02 & 0 & 0 & .04 & 0 & .02 & .14 & 0 \\
\hline Min & 0 & 0 & 0 & 0 & 0 & 0 & 0 & 0 & 0 & 0 & 0 & 0 \\
\hline Acre-Ft & .02 & .16 & .30 & .12 & .04 & 0 & 0 & .20 & 0 & .12 & .77 & 0 \\
\hline Wtr Year & 2008 & Total & 0.87 & Mean & & & Max & .14 & Min & 0 & Acre-Ft & 1.7 \\
\hline Cal Year & 2007 & Total & 0.98 & Mean & & & Max & .34 & Min & 0 & Acre-Ft & 1.9 \\
\hline
\end{tabular}




\section{E2013 TA-50 Area C}

Location. Lat $35^{\circ}$ 51' 41", long $106^{\circ}$ 17' 49", SW 1/4, Sec. 22, T. 19 N., R. 6 E., Los Alamos Country.

Drainage Area. $0.002 \mathrm{mi}^{2}$.

Period of Record. October 1, 2006, to September 30, 2008.

Gage. Data logger and 12” Parshall flume. Elevation of gage is 7,213 ft above NGVD.

Remarks. Records are good. Records for this site existed before period of record but are not reliable.

Extremes for Period of Record. Maximum discharge, $7.50 \mathrm{ft}^{3} / \mathrm{s}$, September 20, 2007, gage height $1.51 \mathrm{ft}$. No flow most of the time.

Extremes for Current Year. Peak discharges above base of $1.0 \mathrm{ft}^{3} / \mathrm{s}$ and maximum (*):

\begin{tabular}{|c|c|c|c|}
\hline Date & Time & Discharge $\left.\mathbf{( f t}^{\mathbf{3}} \mathbf{s}\right)$ & Gage Height (ft) \\
\hline December 1 & 0130 & 1.2 & 0.46 \\
\hline January 28 & 0350 & 1.7 & 0.57 \\
\hline May 15 & 1010 & 5.2 & 1.19 \\
\hline May 28 & 1545 & 3.5 & 0.92 \\
\hline August 4 & 1735 & 2.3 & 0.69 \\
\hline August 7 & 0930 & $7.4^{*}$ & $1.50^{*}$ \\
\hline August 16 & 0343 & 1.1 & 0.42 \\
\hline
\end{tabular}

No flow most of the time.

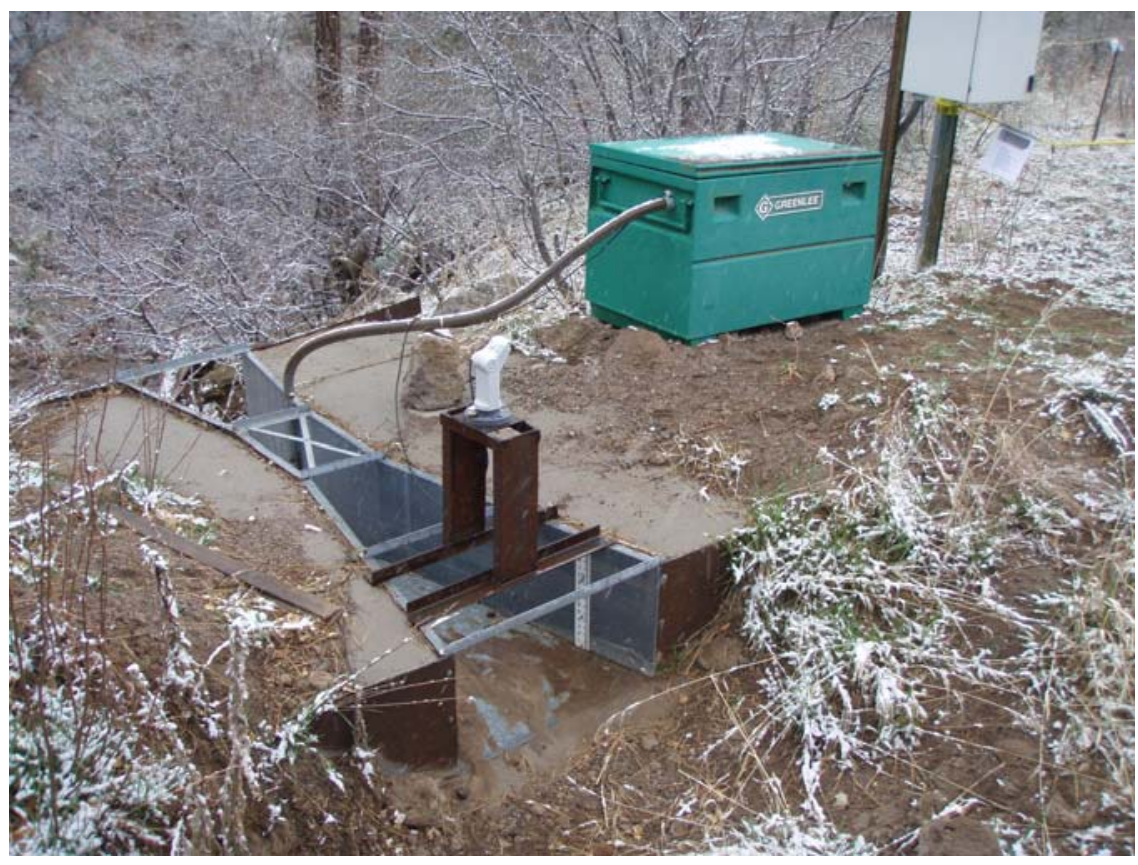




\section{E2013 TA-50 Area C}

\section{Station Analysis}

\section{Water Year}

Equipment. Station is equipped with Sutron 8210 data logger (5-min. interval) and milltronics sonic probe mounted on a 12" Parshall flume. The system is powered by a solar-panel battery system housed in a NEMA shelter. Station is equipped with an ISCO pump sampler for water-quality sample collection. ISCO is housed in a separate shelter, a $3^{\prime} \times 4^{\prime}$ metal box. Sampler is triggered by stage through the data logger. The staff in the 12 " Parshall flume is the reference gage. No provision for discharge measurements above wading stage.

Field Work. This station was visited 23 times to conduct discharge measurements and service the instrumentation. Field inspections for the gage are listed under site history files on the Hydstra database. Discharge measurements for the gage are listed under site gauging files on the Hydstra database.

Datum Correction. None

Gage-Height Record. The data logger referenced to the outside staff gave a complete and satisfactory record except during the period of June 2 to July 3 when the data logger malfunctioned as a result of battery failure and several days in August when flume became silted.

Rating. Rating No. 1 was developed based on the computation of 12 " Parshall flume. Point of zero flow is 0.00 gage height.

Twenty-three inspections of no flow were made.

Discharge. Discharge was computed by applying Rating No. 1 directly. Those days estimated at zero flow were based on precipitation and nearby gage stations.

Remarks. Records are good. 


\section{E2013 TA-50 Area C}

Daily Mean Discharge in Cubic Feet per Second

Water Year October 2007 to September 2008

\begin{tabular}{|c|c|c|c|c|c|c|c|c|c|c|c|c|}
\hline DAY & OCT & NOV & DEC & JAN & FEB & MAR & APR & MAY & JUN & JUL & AUG & SEP \\
\hline 1 & .01 & 0 & .11 & 0 & 0 & 0 & 0 & 0 & 0 & $0^{*}$ & 0 & 0 \\
\hline 2 & 0 & 0 & 0 & 0 & 0 & 0 & 0 & 0 & $0^{*}$ & $0^{*}$ & 0 & 0 \\
\hline 3 & 0 & 0 & 0 & 0 & 0 & 0 & 0 & 0 & $0^{*}$ & $0^{*}$ & 0 & 0 \\
\hline 4 & 0 & 0 & 0 & 0 & 0 & 0 & 0 & 0 & $0^{*}$ & 0 & .05 & 0 \\
\hline 5 & 0 & 0 & 0 & 0 & 0 & 0 & 0 & 0 & $0^{*}$ & 0 & 0 & 0 \\
\hline 6 & 0 & 0 & 0 & 0 & 0 & 0 & 0 & 0 & $0^{*}$ & 0 & $.02^{*}$ & 0 \\
\hline 7 & 0 & 0 & 0 & 0 & 0 & 0 & 0 & 0 & $0^{*}$ & 0 & $.06^{*}$ & 0 \\
\hline 8 & 0 & 0 & .02 & 0 & 0 & 0 & 0 & 0 & $0^{*}$ & 0 & $0^{*}$ & 0 \\
\hline 9 & 0 & 0 & 0 & 0 & 0 & 0 & 0 & 0 & $0^{*}$ & 0 & 0 & 0 \\
\hline 10 & 0 & 0 & 0 & 0 & 0 & 0 & 0 & 0 & $0^{*}$ & 0 & 0 & 0 \\
\hline 11 & 0 & 0 & 0 & 0 & 0 & 0 & 0 & 0 & $0^{*}$ & 0 & 0 & 0 \\
\hline 12 & 0 & 0 & 0 & 0 & 0 & 0 & 0 & 0 & $0^{*}$ & 0 & 0 & 0 \\
\hline 13 & 0 & 0 & 0 & 0 & 0 & 0 & 0 & 0 & $0^{*}$ & 0 & 0 & 0 \\
\hline 14 & 0 & 0 & 0 & 0 & 0 & 0 & 0 & 0 & $0^{*}$ & 0 & 0 & 0 \\
\hline 15 & 0 & 0 & 0 & 0 & 0 & 0 & 0 & .11 & $0^{*}$ & 0 & 0 & 0 \\
\hline 16 & 0 & 0 & 0 & 0 & 0 & 0 & 0 & .01 & $0^{*}$ & 0 & .01 & 0 \\
\hline 17 & 0 & 0 & 0 & 0 & 0 & 0 & 0 & 0 & $0^{*}$ & 0 & 0 & 0 \\
\hline 18 & 0 & 0 & 0 & 0 & 0 & 0 & 0 & 0 & $0^{*}$ & 0 & $0^{*}$ & 0 \\
\hline 19 & 0 & 0 & 0 & 0 & 0 & 0 & 0 & 0 & $0^{*}$ & 0 & $.03^{\star}$ & 0 \\
\hline 20 & 0 & 0 & 0 & 0 & 0 & 0 & 0 & 0 & $0^{*}$ & 0 & $.01^{*}$ & 0 \\
\hline 21 & 0 & 0 & 0 & 0 & 0 & 0 & 0 & 0 & $0^{*}$ & .01 & $.02^{*}$ & 0 \\
\hline 22 & 0 & 0 & 0 & 0 & 0 & 0 & 0 & 0 & $0^{*}$ & 0 & 0 & 0 \\
\hline 23 & 0 & 0 & 0 & 0 & 0 & 0 & 0 & 0 & $0^{*}$ & 0 & 0 & 0 \\
\hline 24 & 0 & 0 & 0 & 0 & .01 & 0 & 0 & 0 & $0^{*}$ & 0 & 0 & 0 \\
\hline 25 & 0 & 0 & 0 & 0 & 0 & 0 & 0 & 0 & $0^{*}$ & 0 & 0 & 0 \\
\hline 26 & 0 & 0 & 0 & 0 & 0 & 0 & 0 & 0 & $0^{*}$ & 0 & $0^{*}$ & 0 \\
\hline 27 & 0 & 0 & 0 & .18 & 0 & 0 & 0 & 0 & $0^{*}$ & 0 & $.03^{*}$ & 0 \\
\hline 28 & 0 & 0 & 0 & .25 & 0 & 0 & 0 & .02 & $0^{*}$ & 0 & $.01^{*}$ & 0 \\
\hline 29 & 0 & 0 & 0 & 0 & 0 & 0 & 0 & 0 & $0^{*}$ & 0 & 0 & 0 \\
\hline 30 & 0 & .09 & 0 & 0 & ----- & 0 & 0 & 0 & $0^{*}$ & 0 & 0 & 0 \\
\hline 31 & 0 & ----- & 0 & 0 & ----- & 0 & ----- & 0 & ------ & 0 & $.01^{\star}$ & ------ \\
\hline Total & 0.01 & 0.09 & 0.13 & 0.43 & 0.01 & 0 & 0 & 0.14 & 0 & 0.01 & 0.25 & 0 \\
\hline Mean & 0 & .003 & .004 & .014 & 0 & 0 & 0 & .005 & 0 & 0 & .008 & 0 \\
\hline Max & .01 & .09 & .11 & .25 & .01 & 0 & 0 & .11 & 0 & .01 & .06 & 0 \\
\hline Min & 0 & 0 & 0 & 0 & 0 & 0 & 0 & 0 & 0 & 0 & 0 & 0 \\
\hline Acre-Ft & .02 & .18 & .26 & .85 & .02 & 0 & 0 & .28 & 0 & .02 & .50 & 0 \\
\hline Wtr Year & 2008 & Total & 1.07 & Mean & & 03 & Max & .25 & Min & 0 & Acre-Ft & 2.1 \\
\hline Cal Year & 2007 & Total & 0.66 & Mean & & 02 & Max & .11 & Min & 0 & Acre-Ft & 1.3 \\
\hline
\end{tabular}

*Estimate 


\section{E2015 Ten Site Canyon above Mortandad Canyon}

Location. Lat. $35^{\circ}$ 51' 38", long. $106^{\circ}$ 16' 30", SE 1/4, Sec. 23, T. 19 N., R. 6 E., Los Alamos County.

Drainage Area. $0.32 \mathrm{mi}$.

Period of Record. October 2000 to September 30, 2008.

Revised Record. Drainage area (2006).

Gage. Data logger with $90^{\circ}$ sharp-crested weir. Elevation of gage is $6,858 \mathrm{ft}$ above NGVD from GPS survey.

Remarks. Records are good.

Average Discharge. $8 \mathrm{yr}, 0.006 \mathrm{ft}^{3} / \mathrm{s}, 4.07$ acre- $\mathrm{ft} / \mathrm{yr}$.

Extremes for Period of Record. Maximum discharge, $303 \mathrm{ft}^{3} / \mathrm{s}$, August 25, 2006, gage height $4.60 \mathrm{ft}$ (from slope-area measurement of peak flow). No flow most of the time.

Extremes for Current Year. Peak discharge above base of $10.0 \mathrm{ft}^{3} / \mathrm{s}$ and maximum (*):

\begin{tabular}{|c|c|c|c|}
\hline Date & Time & Discharge $\left.\mathbf{( f t}^{\mathbf{3}} / \mathbf{s}\right)$ & Gage Height (ft) \\
\hline January 28 & 0410 & $299 *$ & $4.59 *$ \\
\hline August 10 & 1210 & 23 & 3.36 \\
\hline
\end{tabular}

No flow most of the time.

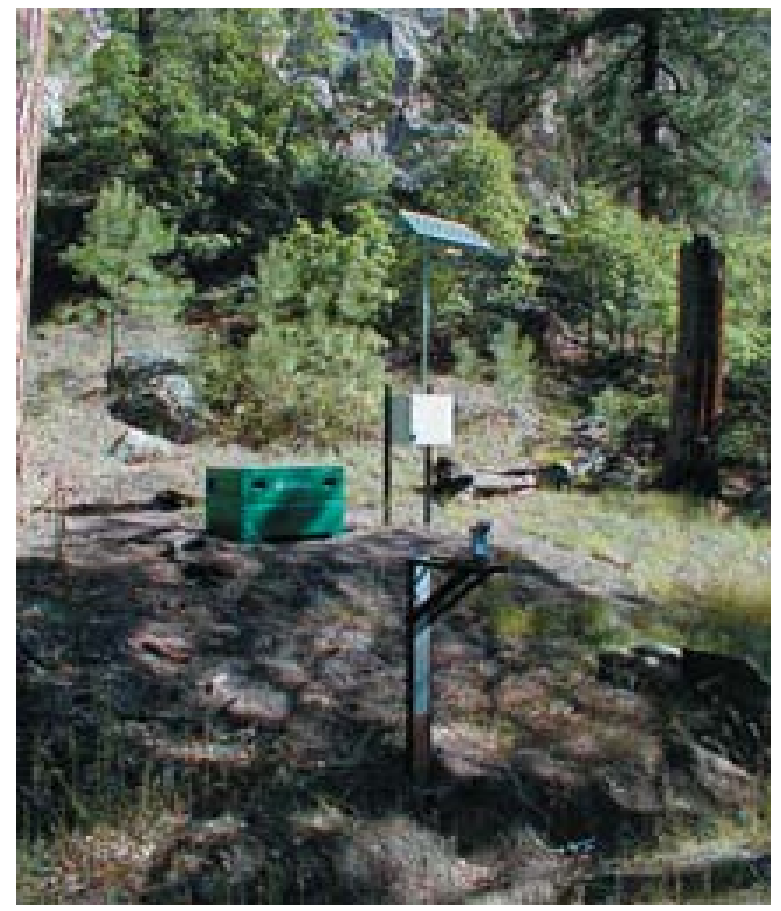




\section{E2015 Ten Site Canyon above Mortandad Canyon}

\section{Station Analysis}

\section{Water Year}

Equipment. Station is equipped with Sutron 8210 data logger (5-min. interval) and Sutron Accubar bubble sensor. The system is powered by a solar-panel battery system housed in a NEMA shelter. Station is also equipped with an ISCO pump sampler for water-quality collection. ISCO is housed in a separate shelter, a $3^{\prime} \times 4^{\prime}$ metal box. Sampler is triggered by stage through the data logger. An outside staff is available for reference. No provisions are made for measurements above wading stage.

Cellular telemetry removed March 14, discontinued service.

Field Work. This station was visited 16 times to conduct discharge measurements and service the instrumentation. Field inspections for the gage are listed under site history files on the Hydstra database. Discharge measurements for the gage are listed under site gauging files on the Hydstra database.

Datum Correction. Levels run on May 12, 2005 and November 17, 2005, found the gage to be $0.05 \mathrm{ft}$ higher. A datum correction of 0.05 was added from May 12 until outside staff is reset to correct datum. On May 24, 2007, all gages set to correct datum. Gage destroyed as a result of a flood on August 25, 2006. Bubbler outlet reset to gage datum of $1.33 \mathrm{ft}$.

Gage-Height Record. The data logger reference to the outside staff gave a complete and satisfactory record.

Rating. The channel is about $8^{\prime}$ wide and straight for about $60^{\prime}$ upstream and straight for about 30' downstream. The streambed through this reach consists of primarily sand with gravel.

Sixteen inspections of no flow were made this year.

Rating No. 2 is based on a theoretical computation for $90^{\circ}$ sharp-crested weir, as well as one critical depth computation.

Discharge. Discharge was computed by applying Rating No. 2 directly.

Remarks. Records are good. 


\section{E2015 Ten Site Canyon above Mortandad Canyon}

Daily Mean Discharge in Cubic Feet per Second

Water Year October 2007 to September 2008

\begin{tabular}{|c|c|c|c|c|c|c|c|c|c|c|c|c|}
\hline DAY & OCT & NOV & DEC & JAN & FEB & MAR & APR & MAY & JUN & JUL & AUG & SEP \\
\hline 1 & 0 & 0 & .58 & 0 & 0 & 0 & 0 & 0 & 0 & 0 & 0 & 0 \\
\hline 2 & 0 & 0 & 0 & 0 & 0 & 0 & 0 & 0 & 0 & 0 & 0 & 0 \\
\hline 3 & 0 & 0 & 0 & 0 & 0 & 0 & 0 & 0 & 0 & 0 & 0 & 0 \\
\hline 4 & 0 & 0 & 0 & 0 & 0 & 0 & 0 & 0 & 0 & 0 & 0 & 0 \\
\hline 5 & 0 & 0 & 0 & 0 & 0 & 0 & 0 & 0 & 0 & 0 & 0 & 0 \\
\hline 6 & 0 & 0 & 0 & 0 & 0 & 0 & 0 & 0 & 0 & 0 & 0 & 0 \\
\hline 7 & 0 & 0 & 0 & 0 & 0 & 0 & 0 & 0 & 0 & 0 & 0 & 0 \\
\hline 8 & 0 & 0 & 0 & 0 & 0 & 0 & 0 & 0 & 0 & 0 & 0 & 0 \\
\hline 9 & 0 & 0 & 0 & 0 & 0 & 0 & 0 & 0 & 0 & 0 & .02 & 0 \\
\hline 10 & 0 & 0 & 0 & 0 & 0 & 0 & 0 & 0 & 0 & 0 & .39 & 0 \\
\hline 11 & 0 & 0 & 0 & 0 & 0 & 0 & 0 & 0 & 0 & 0 & 0 & 0 \\
\hline 12 & 0 & 0 & 0 & 0 & 0 & 0 & 0 & 0 & 0 & 0 & 0 & 0 \\
\hline 13 & 0 & 0 & 0 & 0 & 0 & 0 & 0 & 0 & 0 & 0 & 0 & 0 \\
\hline 14 & 0 & 0 & 0 & 0 & 0 & 0 & 0 & 0 & 0 & 0 & 0 & 0 \\
\hline 15 & 0 & 0 & 0 & 0 & 0 & 0 & 0 & 0 & 0 & 0 & 0 & 0 \\
\hline 16 & 0 & 0 & 0 & 0 & 0 & 0 & 0 & 0 & 0 & 0 & 0 & 0 \\
\hline 17 & 0 & 0 & 0 & 0 & 0 & 0 & 0 & 0 & 0 & 0 & 0 & 0 \\
\hline 18 & 0 & 0 & 0 & 0 & 0 & 0 & 0 & 0 & 0 & 0 & 0 & 0 \\
\hline 19 & 0 & 0 & 0 & 0 & 0 & 0 & 0 & 0 & 0 & 0 & 0 & 0 \\
\hline 20 & 0 & 0 & 0 & 0 & 0 & 0 & 0 & 0 & 0 & 0 & 0 & 0 \\
\hline 21 & 0 & 0 & 0 & 0 & 0 & 0 & 0 & 0 & 0 & 0 & 0 & 0 \\
\hline 22 & 0 & 0 & 0 & 0 & 0 & 0 & 0 & 0 & 0 & 0 & 0 & 0 \\
\hline 23 & 0 & 0 & 0 & 0 & 0 & 0 & 0 & 0 & 0 & 0 & .02 & 0 \\
\hline 24 & 0 & 0 & 0 & 0 & .36 & 0 & 0 & 0 & 0 & 0 & 0 & 0 \\
\hline 25 & 0 & 0 & 0 & 0 & 0 & 0 & 0 & 0 & 0 & 0 & 0 & 0 \\
\hline 26 & 0 & 0 & 0 & 0 & 0 & 0 & 0 & 0 & 0 & 0 & 0 & 0 \\
\hline 27 & 0 & 0 & 0 & 0 & 0 & 0 & 0 & 0 & 0 & 0 & 0 & 0 \\
\hline 28 & 0 & 0 & 0 & 7.2 & 0 & 0 & 0 & 0 & 0 & 0 & 0 & 0 \\
\hline 29 & 0 & 0 & 0 & 0 & 0 & 0 & 0 & 0 & 0 & 0 & 0 & 0 \\
\hline 30 & 0 & 0 & 0 & 0 & ---- & 0 & 0 & 0 & 0 & 0 & 0 & 0 \\
\hline 31 & 0 & ----- & 0 & 0 & ---- & 0 & ------ & 0 & ----- & 0 & .02 & ----- \\
\hline Total & 0 & 0 & 0.58 & 7.2 & 0.36 & 0 & 0 & 0 & 0 & 0 & 0.45 & 0 \\
\hline Mean & 0 & 0 & .019 & .23 & .012 & 0 & 0 & 0 & 0 & 0 & .015 & 0 \\
\hline Max & 0 & 0 & .58 & 7.2 & .36 & 0 & 0 & 0 & 0 & 0 & .39 & 0 \\
\hline Min & 0 & 0 & 0 & 0 & 0 & 0 & 0 & 0 & 0 & 0 & 0 & 0 \\
\hline Acre-Ft & 0 & 0 & 1.2 & 14 & .71 & 0 & 0 & 0 & 0 & 0 & .89 & 0 \\
\hline Wtr Year & 2008 & Total & 8.59 & Mean & & 23 & hax & 7.2 & Min & 0 & Acre-Ft & 17 \\
\hline Cal Year & 2007 & Total & 0.66 & Mean & & 02 & hax & .58 & Min & 0 & Acre-Ft & 1.3 \\
\hline
\end{tabular}




\section{E202 Mortandad Canyon above Sediment Traps}

Location. Lat 35 51' 39", long $106^{\circ}$ 16' 15", SE 1/4, Sec. 23, T. 19 N., R. 6 E., Los Alamos County.

Drainage Area. $1.14 \mathrm{mi}^{2}$.

Period of Record. October 1, 1997, to September 30, 2008.

Revised Record. Average discharge 2007 (this report), 10 yr, 0.002ft 3 s, 1.45 acre-ft/yr.

Gage. Data logger with 30" Parshall flume. Elevation of gage is 6,833 ft above NGVD from land survey.

Remarks. Records are good.

Average Discharge. $11 \mathrm{yr}, 0.003 \mathrm{ft}^{3} / \mathrm{s}, 2.17$ acre- $\mathrm{ft} / \mathrm{yr}$.

Extremes for Period of Record. Maximum discharge, $292 \mathrm{ft}^{3} / \mathrm{s}$, gage height unknown, August 25, 2006 (from critical depth computation of peak flow). No flow most of the time.

Extremes for Current Year. Peak discharge above base of $10.0 \mathrm{ft}^{3} / \mathrm{s}$ and maximum (*):

\begin{tabular}{|c|c|c|c|}
\hline Date & Time & Discharge $\left(\mathbf{f t}^{\mathbf{3}} / \mathbf{s}\right)$ & Gage Height (ft) \\
\hline August 10 & 1240 & $15^{*}$ & $1.35^{*}$ \\
\hline
\end{tabular}

No flow most of the time.

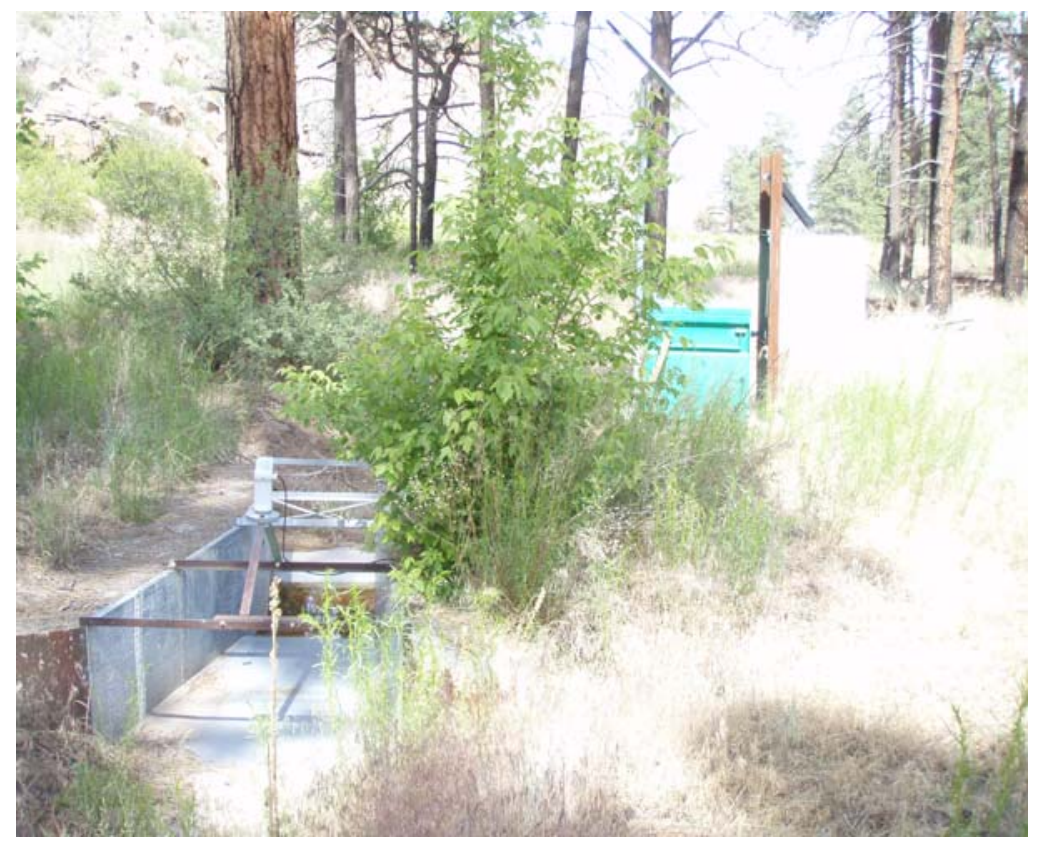




\section{E202 Mortandad Canyon above Sediment Traps \\ Station Analysis}

\section{Water Year}

Equipment. Station is equipped with Sutron 8210 data logger (5-min. interval) and milltronics sonic probe mounted on a 30" Parshall flume. The system is powered by a solar-panel battery system housed in a NEMA shelter. Station is equipped with an ISCO pump sampler for water-quality sample collection. ISCO is housed in a separate shelter, a $3^{\prime} \times 4^{\prime}$ metal box. Sampler is triggered by stage through the data logger. The staff in the 30" Parshall Flume is the reference gage. No provision for discharge measurements above wading stage.

Cellular telemetry removed March 14, discontinued service.

Field Work. This station was visited 16 times to conduct discharge measurements and service the instrumentation. Field inspections for the gage are listed under site history files on the Hydstra database. Discharge measurements for the gage are listed under site gauging files on the Hydstra database.

Datum Correction. None

Gage-Height Record. The data logger referenced to the outside staff gave a complete and satisfactory record.

Rating. Approach and escape sections are spread out because of overgrowth and debris in channel.

Fifteen inspections of no flow and one inspection with observed flow were made this year.

Rating No.1 was developed based on the computation of 30" Parshall flume. Point of zero flow is 0.00 gage height.

Discharge. Discharge was computed by applying Rating No. 1 directly.

Remarks. Records are good. 
E202 Mortandad Canyon above Sediment Traps

Daily Mean Discharge in Cubic Feet per Second

Water Year October 2007 to September 2008

\begin{tabular}{|c|c|c|c|c|c|c|c|c|c|c|c|c|}
\hline DAY & OCT & NOV & DEC & JAN & FEB & MAR & APR & MAY & JUN & JUL & AUG & SEP \\
\hline 1 & 0 & 0 & .61 & 0 & 0 & 0 & 0 & 0 & 0 & 0 & 0 & 0 \\
\hline 2 & 0 & 0 & 0 & 0 & 0 & 0 & 0 & 0 & 0 & 0 & 0 & 0 \\
\hline 3 & 0 & 0 & 0 & 0 & 0 & 0 & 0 & 0 & 0 & 0 & 0 & 0 \\
\hline 4 & 0 & 0 & 0 & 0 & 0 & 0 & 0 & 0 & 0 & 0 & 0 & 0 \\
\hline 5 & 0 & 0 & 0 & 0 & 0 & 0 & 0 & 0 & 0 & 0 & 0 & 0 \\
\hline 6 & 0 & 0 & 0 & 0 & 0 & 0 & 0 & 0 & 0 & 0 & 0 & 0 \\
\hline 7 & 0 & 0 & 0 & 0 & 0 & 0 & 0 & 0 & 0 & 0 & 0 & 0 \\
\hline 8 & 0 & 0 & 0 & 0 & 0 & 0 & 0 & 0 & 0 & 0 & 0 & 0 \\
\hline 9 & 0 & 0 & 0 & 0 & 0 & 0 & 0 & 0 & 0 & 0 & 0 & 0 \\
\hline 10 & 0 & 0 & 0 & 0 & 0 & 0 & 0 & 0 & 0 & 0 & .73 & 0 \\
\hline 11 & 0 & 0 & 0 & 0 & 0 & 0 & 0 & 0 & 0 & 0 & 0 & 0 \\
\hline 12 & 0 & 0 & 0 & 0 & 0 & 0 & 0 & 0 & 0 & 0 & 0 & 0 \\
\hline 13 & 0 & 0 & 0 & 0 & 0 & 0 & 0 & 0 & 0 & 0 & 0 & 0 \\
\hline 14 & 0 & 0 & 0 & 0 & 0 & 0 & 0 & 0 & 0 & 0 & 0 & 0 \\
\hline 15 & 0 & 0 & 0 & 0 & 0 & 0 & 0 & 0 & 0 & 0 & 0 & 0 \\
\hline 16 & 0 & 0 & 0 & 0 & 0 & 0 & 0 & 0 & 0 & 0 & 0 & 0 \\
\hline 17 & 0 & 0 & 0 & 0 & 0 & 0 & 0 & 0 & 0 & 0 & 0 & 0 \\
\hline 18 & 0 & 0 & 0 & 0 & 0 & 0 & 0 & 0 & 0 & 0 & 0 & 0 \\
\hline 19 & 0 & 0 & 0 & 0 & 0 & 0 & 0 & 0 & 0 & 0 & 0 & 0 \\
\hline 20 & 0 & 0 & 0 & 0 & 0 & 0 & 0 & 0 & 0 & 0 & 0 & 0 \\
\hline 21 & 0 & 0 & 0 & 0 & 0 & 0 & 0 & 0 & 0 & 0 & 0 & 0 \\
\hline 22 & 0 & 0 & 0 & 0 & 0 & 0 & 0 & 0 & 0 & 0 & 0 & 0 \\
\hline 23 & 0 & 0 & 0 & 0 & 0 & 0 & 0 & 0 & 0 & 0 & 0 & 0 \\
\hline 24 & 0 & 0 & 0 & 0 & 0 & 0 & 0 & 0 & 0 & 0 & 0 & 0 \\
\hline 25 & 0 & 0 & 0 & 0 & 0 & 0 & 0 & 0 & 0 & 0 & 0 & 0 \\
\hline 26 & 0 & 0 & 0 & 0 & 0 & 0 & 0 & 0 & 0 & 0 & 0 & 0 \\
\hline 27 & 0 & 0 & 0 & 0 & 0 & 0 & 0 & 0 & 0 & 0 & 0 & 0 \\
\hline 28 & 0 & 0 & 0 & .30 & 0 & 0 & 0 & 0 & 0 & 0 & 0 & 0 \\
\hline 29 & 0 & 0 & 0 & 0 & 0 & 0 & 0 & 0 & 0 & 0 & 0 & 0 \\
\hline 30 & 0 & 0 & 0 & 0 & ----- & 0 & 0 & 0 & 0 & 0 & 0 & 0 \\
\hline 31 & 0 & ------ & 0 & 0 & ------ & 0 & ----- & 0 & ----- & 0 & 0 & ------ \\
\hline Total & 0 & 0 & 0.61 & 0.30 & 0 & 0 & 0 & 0 & 0 & 0 & 0.73 & 0 \\
\hline Mean & 0 & 0 & .020 & .010 & 0 & 0 & 0 & 0 & 0 & 0 & .024 & 0 \\
\hline Max & 0 & 0 & .61 & .30 & 0 & 0 & 0 & 0 & 0 & 0 & .73 & 0 \\
\hline Min & 0 & 0 & 0 & 0 & 0 & 0 & 0 & 0 & 0 & 0 & 0 & 0 \\
\hline Acre-Ft & 0 & 0 & 1.2 & .60 & 0 & 0 & 0 & 0 & 0 & 0 & 1.4 & 0 \\
\hline Wtr Year & 2008 & Total & 1.64 & Mean & & & Max & .73 & Min & 0 & Acre-Ft & 3.3 \\
\hline Cal Year & 2007 & Total & 0.92 & Mean & & & Max & .61 & Min & 0 & Acre-Ft & 1.8 \\
\hline
\end{tabular}




\section{E203 Mortandad Canyon below Sediment Traps}

Location. Lat $35^{\circ}$ 51' 39", long. 106 16' 6", SE 1/4, Sec. 23, T. 19 N., R. 6 E., Los Alamos County.

Drainage Area. $1.17 \mathrm{mi}^{2}$.

Period of Record. October 1, 1996, to August 25, 2006 (destroyed by flood); September 2006 to September 30, 2008.

Revised Record. Drainage area (2006).

Gage. Data logger and 6" Parshall flume, as well as rain gage with cellular telemetry. Elevation of gage is $6,817 \mathrm{ft}$ above NGVD from land survey.

Remarks. Records are good.

Average Discharge. $12 \mathrm{yr}, 0.002 \mathrm{ft}^{3} / \mathrm{s}, 1.45$ acre $\mathrm{ft} / \mathrm{yr}$.

Extremes for Period of Record. Maximum discharge, $220 \mathrm{ft}^{3} / \mathrm{s}$, August 25, 2006 (from critical depth computation), gage height unknown. No flow most of the time.

Extremes for Current Year. No peak discharge above base of $1.0 \mathrm{ft}^{3} / \mathrm{s}$. No flow for current year.

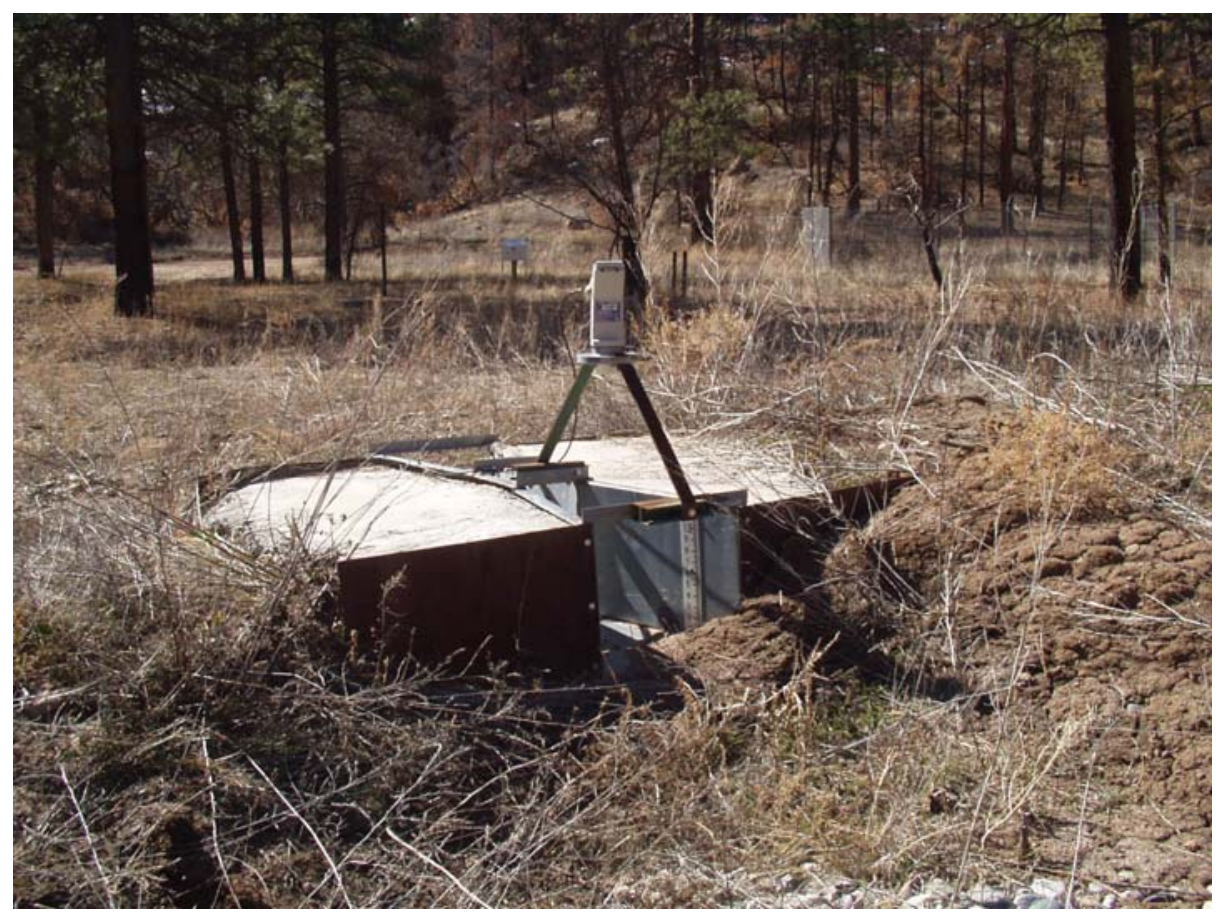




\section{E203 Mortandad Canyon below Sediment Traps \\ Station Analysis}

\section{Water Year}

Equipment. Station is equipped with Sutron 8210 data logger (5-min. interval) and milltronics sonic probe mounted on a 6" Parshall flume and cellular telemetry with speech modem. Phone upgraded May 14 from analog to digital service. The system is powered by a solar-panel battery system housed in a NEMA shelter. Station is equipped with an ISCO pump sampler for water-quality sample collection. ISCO is housed in a separate shelter, a $3^{\prime} \times 4^{\prime}$ metal box. Sampler is triggered by stage through the data logger. The staff in the $6 "$ Parshall flume is the reference gage. No provision for discharge measurements above wading stage.

Station is also equipped with a rain gage, Rain Collection II. All equipment is powered with a solar-panel battery-charging system. Rain data are seasonal.

Field Work. This station was visited 16 times to conduct discharge measurements and service the instrumentation. Field inspections for the gage are listed under site history files on the Hydstra database. Discharge measurements for the gage are listed under site gauging files on the Hydstra database.

Datum Correction. None.

Gage-Height Record. The data logger referenced to the outside staff gave a complete and satisfactory record.

Rating. Approach and escape sections spread out because channel is somewhat undefined.

Sixteen inspections of no flow were made this year.

Rating No. 1 was developed based on the computation of 6" Parshall flume. Point of zero flow is 0.00 gage height.

Discharge. Discharge was computed by applying Rating No. 1 directly.

Remarks. Records are good. No discharge for the year. 


\section{E203 Mortandad Canyon below Sediment Traps}

Daily Mean Discharge in Cubic Feet per Second

Water Year October 2007 to September 2008

\begin{tabular}{|c|c|c|c|c|c|c|c|c|c|c|c|c|}
\hline DAY & OCT & NOV & DEC & JAN & FEB & MAR & APR & MAY & JUN & JUL & AUG & SEP \\
\hline 1 & 0 & 0 & 0 & 0 & 0 & 0 & 0 & 0 & 0 & 0 & 0 & 0 \\
\hline 2 & 0 & 0 & 0 & 0 & 0 & 0 & 0 & 0 & 0 & 0 & 0 & 0 \\
\hline 3 & 0 & 0 & 0 & 0 & 0 & 0 & 0 & 0 & 0 & 0 & 0 & 0 \\
\hline 4 & 0 & 0 & 0 & 0 & 0 & 0 & 0 & 0 & 0 & 0 & 0 & 0 \\
\hline 5 & 0 & 0 & 0 & 0 & 0 & 0 & 0 & 0 & 0 & 0 & 0 & 0 \\
\hline 6 & 0 & 0 & 0 & 0 & 0 & 0 & 0 & 0 & 0 & 0 & 0 & 0 \\
\hline 7 & 0 & 0 & 0 & 0 & 0 & 0 & 0 & 0 & 0 & 0 & 0 & 0 \\
\hline 8 & 0 & 0 & 0 & 0 & 0 & 0 & 0 & 0 & 0 & 0 & 0 & 0 \\
\hline 9 & 0 & 0 & 0 & 0 & 0 & 0 & 0 & 0 & 0 & 0 & 0 & 0 \\
\hline 10 & 0 & 0 & 0 & 0 & 0 & 0 & 0 & 0 & 0 & 0 & 0 & 0 \\
\hline 11 & 0 & 0 & 0 & 0 & 0 & 0 & 0 & 0 & 0 & 0 & 0 & 0 \\
\hline 12 & 0 & 0 & 0 & 0 & 0 & 0 & 0 & 0 & 0 & 0 & 0 & 0 \\
\hline 13 & 0 & 0 & 0 & 0 & 0 & 0 & 0 & 0 & 0 & 0 & 0 & 0 \\
\hline 14 & 0 & 0 & 0 & 0 & 0 & 0 & 0 & 0 & 0 & 0 & 0 & 0 \\
\hline 15 & 0 & 0 & 0 & 0 & 0 & 0 & 0 & 0 & 0 & 0 & 0 & 0 \\
\hline 16 & 0 & 0 & 0 & 0 & 0 & 0 & 0 & 0 & 0 & 0 & 0 & 0 \\
\hline 17 & 0 & 0 & 0 & 0 & 0 & 0 & 0 & 0 & 0 & 0 & 0 & 0 \\
\hline 18 & 0 & 0 & 0 & 0 & 0 & 0 & 0 & 0 & 0 & 0 & 0 & 0 \\
\hline 19 & 0 & 0 & 0 & 0 & 0 & 0 & 0 & 0 & 0 & 0 & 0 & 0 \\
\hline 20 & 0 & 0 & 0 & 0 & 0 & 0 & 0 & 0 & 0 & 0 & 0 & 0 \\
\hline 21 & 0 & 0 & 0 & 0 & 0 & 0 & 0 & 0 & 0 & 0 & 0 & 0 \\
\hline 22 & 0 & 0 & 0 & 0 & 0 & 0 & 0 & 0 & 0 & 0 & 0 & 0 \\
\hline 23 & 0 & 0 & 0 & 0 & 0 & 0 & 0 & 0 & 0 & 0 & 0 & 0 \\
\hline 24 & 0 & 0 & 0 & 0 & 0 & 0 & 0 & 0 & 0 & 0 & 0 & 0 \\
\hline 25 & 0 & 0 & 0 & 0 & 0 & 0 & 0 & 0 & 0 & 0 & 0 & 0 \\
\hline 26 & 0 & 0 & 0 & 0 & 0 & 0 & 0 & 0 & 0 & 0 & 0 & 0 \\
\hline 27 & 0 & 0 & 0 & 0 & 0 & 0 & 0 & 0 & 0 & 0 & 0 & 0 \\
\hline 28 & 0 & 0 & 0 & 0 & 0 & 0 & 0 & 0 & 0 & 0 & 0 & 0 \\
\hline 29 & 0 & 0 & 0 & 0 & 0 & 0 & 0 & 0 & 0 & 0 & 0 & 0 \\
\hline 30 & 0 & 0 & 0 & 0 & ----- & 0 & 0 & 0 & 0 & 0 & 0 & 0 \\
\hline 31 & 0 & ----- & 0 & 0 & ----- & 0 & ----- & 0 & ----- & 0 & 0 & ----- \\
\hline Total & 0 & 0 & 0 & 0 & 0 & 0 & 0 & 0 & 0 & 0 & 0 & 0 \\
\hline Mean & 0 & 0 & 0 & 0 & 0 & 0 & 0 & 0 & 0 & 0 & 0 & 0 \\
\hline Max & 0 & 0 & 0 & 0 & 0 & 0 & 0 & 0 & 0 & 0 & 0 & 0 \\
\hline Min & 0 & 0 & 0 & 0 & 0 & 0 & 0 & 0 & 0 & 0 & 0 & 0 \\
\hline Acre-Ft & 0 & 0 & 0 & 0 & 0 & 0 & 0 & 0 & 0 & 0 & 0 & 0 \\
\hline Wtr Year & 2008 & Total & & & & 0 & hax & 0 & Min & 0 & Acre-Ft & 0 \\
\hline Cal Year & 2007 & Total & & & & 0 & 1ax & 0 & Min & 0 & Acre-Ft & 0 \\
\hline
\end{tabular}




\section{E203 Mortandad Canyon below Sediment Traps}

Daily Total Rainfall in Inches

Water Year October 2007 to September 2008

\begin{tabular}{|c|c|c|c|c|c|c|c|c|c|c|c|c|}
\hline DAY & ОСТ & NOV & DEC & JAN & FEB & MAR & APR & MAY & JUN & JUL & AUG & SEP \\
\hline 1 & & & & & & & & 0 & 0 & 0 & 0 & 0 \\
\hline 2 & & & & & & & & 0 & 0 & 0 & 0 & 0 \\
\hline 3 & & & & & & & & 0 & 0 & 0 & 0 & 0 \\
\hline 4 & & & & & & & & 0 & 0 & 0 & $.40^{*}$ & 0 \\
\hline 5 & & & & & & & & 0 & 0 & .01 & .04 & 0 \\
\hline 6 & & & & & & & & 0 & 0 & 0 & .01 & 0 \\
\hline 7 & & & & & & & & 0 & 0 & 0 & .07 & 0 \\
\hline 8 & & & & & & & & 0 & 0 & 0 & .03 & 0 \\
\hline 9 & & & & & & & & 0 & 0 & 0 & .52 & 0 \\
\hline 10 & & & & & & & & 0 & 0 & 0 & .38 & 0 \\
\hline 11 & & & & & & & & 0 & 0 & .01 & 0 & 0 \\
\hline 12 & & & & & & & & 0 & 0 & .02 & .04 & 0 \\
\hline 13 & & & & & & & & 0 & 0 & 0 & 0 & 0 \\
\hline 14 & & & & & & & & $.10^{*}$ & 0 & 0 & .12 & 0 \\
\hline 15 & & & & & & & & $.56^{\star}$ & 0 & $.12^{*}$ & 0 & 0 \\
\hline 16 & & & & & & & & 0 & 0 & .01 & .24 & 0 \\
\hline 17 & & & & & & & & 0 & 0 & 0 & .04 & 0 \\
\hline 18 & & & & & & & & 0 & 0 & .13 & 0 & 0 \\
\hline 19 & & & & & & & & 0 & 0 & 0 & 0 & .01 \\
\hline 20 & & & & & & & & 0 & 0 & 0 & .02 & .01 \\
\hline 21 & & & & & & & & 0 & 0 & .04 & 0 & 0 \\
\hline 22 & & & & & & & & .03 & 0 & .04 & 0 & .04 \\
\hline 23 & & & & & & & & .07 & 0 & 0 & .07 & 0 \\
\hline 24 & & & & & & & & 0 & 0 & 0 & .35 & 0 \\
\hline 25 & & & & & & & & 0 & 0 & 0 & .04 & 0 \\
\hline 26 & & & & & & & & 0 & 0 & .06 & .02 & .07 \\
\hline 27 & & & & & & & & 0 & 0 & .02 & 0 & .01 \\
\hline 28 & & & & & & & & 0 & 0 & .02 & .01 & 0 \\
\hline 29 & & & & & & & & 0 & 0 & 0 & 0 & 0 \\
\hline 30 & & & & & ----- & & & 0 & 0 & 0 & 0 & 0 \\
\hline 31 & & ------ & & & ----- & & ------ & 0 & ------ & 0 & $.54^{*}$ & ----- \\
\hline Total & & & & & & & & 0.10 & 0 & 0.36 & 2.06 & 0.14 \\
\hline $\operatorname{Max}$ & & & & & & & & .07 & 0 & .13 & .52 & .07 \\
\hline Wtr Year & 2008 & Total & 2.77 & Mean & & .016 & Max & .52 & Min & 0 & InstMax & .17 \\
\hline Cal Year & 2007 & Total & 6.19 & Mean & & .034 & $\operatorname{Max}$ & .64 & Min & 0 & InstMax & .09 \\
\hline
\end{tabular}

*Estimate 


\section{E204 Mortandad Canyon at LANL Boundary}

Location. Lat $35^{\circ}$ 51' 21", long $106^{\circ}$ 14' 43", NW 1/4, Sec. 30, T. 19 N., R. 7 E., Santa Fe County.

Drainage Area. $1.61 \mathrm{mi}^{2}$.

Period of Record. October 1, 1993, to September 30, 2008.

Revised Record. Drainage area (2006).

Gage. Data logger with cellular telemetry and concrete control. Elevation of gage is 6,651 ft above NGVD from survey.

Remarks. Records are good.

Average Discharge. 14 yrs, zero.

Extremes for Period of Record. No flow for period.

Extremes for Current Year. No flow for the year.

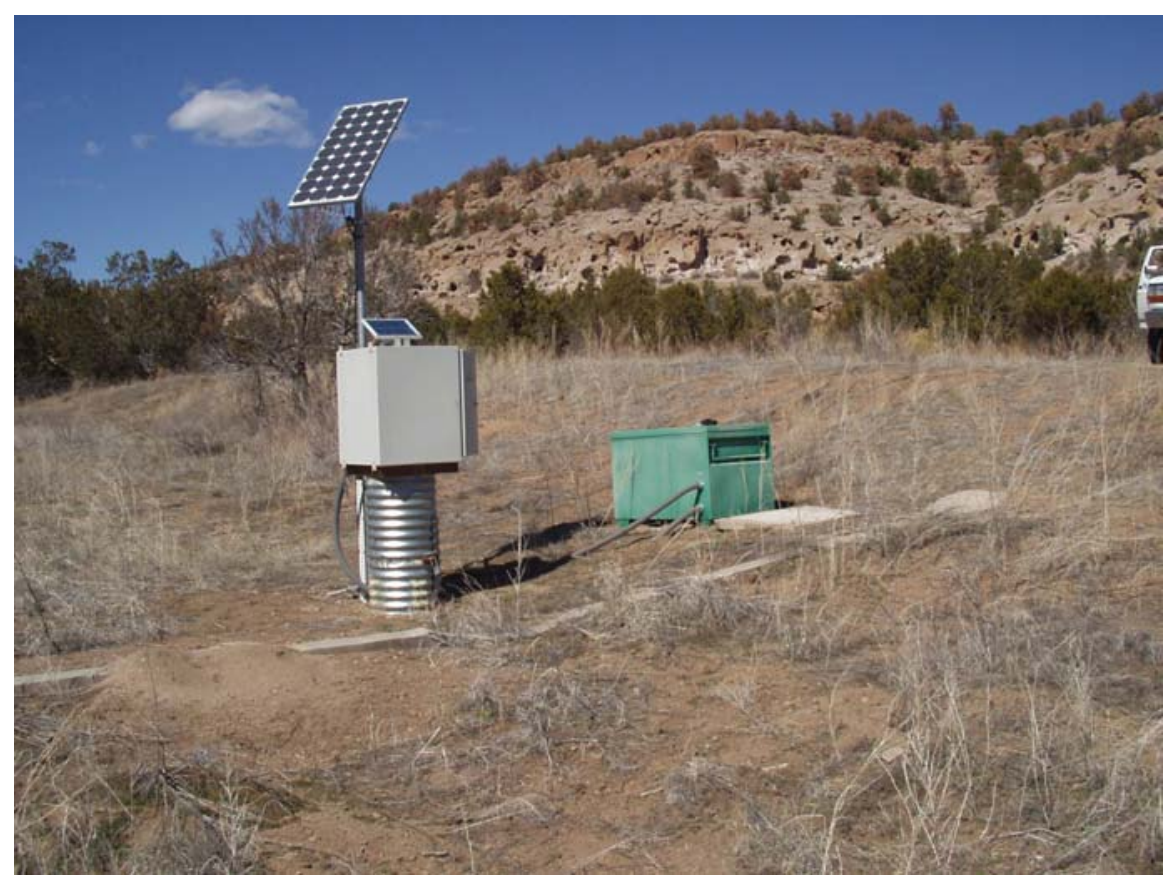




\section{E204 Mortandad Canyon at LANL Boundary}

\section{Station Analysis}

\section{Water Year}

Equipment. Station is equipped with Sutron 8210 (5-min. interval) and shaft encoder float system with cellular phone and speech modem. Phone upgraded June 4 from analog to digital service. The system is powered by a solar-panel battery system housed in a NEMA shelter on top of a 24" CMP well. Station is equipped with an ISCO pump sampler for water-quality sample collection. ISCO is housed in a separate shelter, a $3^{\prime} \times$ $4^{\prime}$ metal box. Samplers are triggered by stage through the data logger. An outside staff is available for reference. No provision for measurements above wading stage. All highflow measurement will be by slope-area or critical depth computation methods.

Field Work. This station was visited 12 times to conduct discharge measurements and service the instrumentation. Field inspections for the gage are listed under site history files on the Hydstra database. Discharge measurements for the gage are listed under site gauging files on the Hydstra database.

Datum Correction. None. Levels run May 24, 2007, showed the gage to read within allowable limits.

Gage-Height Record. The data logger referenced to the outside staff gage gave a complete and satisfactory record.

Rating. Channel is straight above and below gage for $100 \mathrm{ft}$. Channel is not well defined and resembles a low grass covered swale. Flow is infrequent. The control is broad-crested weir with V notch 5' downstream from gage.

Twelve inspections of no flow were made this year.

No rating has been developed; PZF is well defined for concrete broad-crested weir.

Discharge. All recorded values were below PZF. No flow most of the time. Days with rain did not produce enough flow to pass over control.

Remarks. Records are good. 
E204 Mortandad Canyon at LANL Boundary

Daily Mean Discharge in Cubic Feet per Second

Water Year October 2007 to September 2008

\begin{tabular}{|c|c|c|c|c|c|c|c|c|c|c|c|c|}
\hline DAY & OCT & NOV & DEC & JAN & FEB & MAR & APR & MAY & JUN & JUL & AUG & SEP \\
\hline 1 & 0 & 0 & 0 & 0 & 0 & 0 & 0 & 0 & 0 & 0 & 0 & 0 \\
\hline 2 & 0 & 0 & 0 & 0 & 0 & 0 & 0 & 0 & 0 & 0 & 0 & 0 \\
\hline 3 & 0 & 0 & 0 & 0 & 0 & 0 & 0 & 0 & 0 & 0 & 0 & 0 \\
\hline 4 & 0 & 0 & 0 & 0 & 0 & 0 & 0 & 0 & 0 & 0 & 0 & 0 \\
\hline 5 & 0 & 0 & 0 & 0 & 0 & 0 & 0 & 0 & 0 & 0 & 0 & 0 \\
\hline 6 & 0 & 0 & 0 & 0 & 0 & 0 & 0 & 0 & 0 & 0 & 0 & 0 \\
\hline 7 & 0 & 0 & 0 & 0 & 0 & 0 & 0 & 0 & 0 & 0 & 0 & 0 \\
\hline 8 & 0 & 0 & 0 & 0 & 0 & 0 & 0 & 0 & 0 & 0 & 0 & 0 \\
\hline 9 & 0 & 0 & 0 & 0 & 0 & 0 & 0 & 0 & 0 & 0 & 0 & 0 \\
\hline 10 & 0 & 0 & 0 & 0 & 0 & 0 & 0 & 0 & 0 & 0 & 0 & 0 \\
\hline 11 & 0 & 0 & 0 & 0 & 0 & 0 & 0 & 0 & 0 & 0 & 0 & 0 \\
\hline 12 & 0 & 0 & 0 & 0 & 0 & 0 & 0 & 0 & 0 & 0 & 0 & 0 \\
\hline 13 & 0 & 0 & 0 & 0 & 0 & 0 & 0 & 0 & 0 & 0 & 0 & 0 \\
\hline 14 & 0 & 0 & 0 & 0 & 0 & 0 & 0 & 0 & 0 & 0 & 0 & 0 \\
\hline 15 & 0 & 0 & 0 & 0 & 0 & 0 & 0 & 0 & 0 & 0 & 0 & 0 \\
\hline 16 & 0 & 0 & 0 & 0 & 0 & 0 & 0 & 0 & 0 & 0 & 0 & 0 \\
\hline 17 & 0 & 0 & 0 & 0 & 0 & 0 & 0 & 0 & 0 & 0 & 0 & 0 \\
\hline 18 & 0 & 0 & 0 & 0 & 0 & 0 & 0 & 0 & 0 & 0 & 0 & 0 \\
\hline 19 & 0 & 0 & 0 & 0 & 0 & 0 & 0 & 0 & 0 & 0 & 0 & 0 \\
\hline 20 & 0 & 0 & 0 & 0 & 0 & 0 & 0 & 0 & 0 & 0 & 0 & 0 \\
\hline 21 & 0 & 0 & 0 & 0 & 0 & 0 & 0 & 0 & 0 & 0 & 0 & 0 \\
\hline 22 & 0 & 0 & 0 & 0 & 0 & 0 & 0 & 0 & 0 & 0 & 0 & 0 \\
\hline 23 & 0 & 0 & 0 & 0 & 0 & 0 & 0 & 0 & 0 & 0 & 0 & 0 \\
\hline 24 & 0 & 0 & 0 & 0 & 0 & 0 & 0 & 0 & 0 & 0 & 0 & 0 \\
\hline 25 & 0 & 0 & 0 & 0 & 0 & 0 & 0 & 0 & 0 & 0 & 0 & 0 \\
\hline 26 & 0 & 0 & 0 & 0 & 0 & 0 & 0 & 0 & 0 & 0 & 0 & 0 \\
\hline 27 & 0 & 0 & 0 & 0 & 0 & 0 & 0 & 0 & 0 & 0 & 0 & 0 \\
\hline 28 & 0 & 0 & 0 & 0 & 0 & 0 & 0 & 0 & 0 & 0 & 0 & 0 \\
\hline 29 & 0 & 0 & 0 & 0 & 0 & 0 & 0 & 0 & 0 & 0 & 0 & 0 \\
\hline 30 & 0 & 0 & 0 & 0 & ------ & 0 & 0 & 0 & 0 & 0 & 0 & \\
\hline 31 & 0 & ----- & 0 & 0 & ------ & 0 & ------ & 0 & ----- & 0 & 0 & ------ \\
\hline Total & 0 & 0 & 0 & 0 & 0 & 0 & 0 & 0 & 0 & 0 & 0 & 0 \\
\hline Mean & 0 & 0 & 0 & 0 & 0 & 0 & 0 & 0 & 0 & 0 & 0 & 0 \\
\hline $\operatorname{Max}$ & 0 & 0 & 0 & 0 & 0 & 0 & 0 & 0 & 0 & 0 & 0 & 0 \\
\hline Min & 0 & 0 & 0 & 0 & 0 & 0 & 0 & 0 & 0 & 0 & 0 & 0 \\
\hline Acre-Ft & 0 & 0 & 0 & 0 & 0 & 0 & 0 & 0 & 0 & 0 & 0 & 0 \\
\hline Wtr Year & 2008 & Total & & Mean & & 0 & Max & 0 & Min & 0 & Acre-Ft & 0 \\
\hline Cal Year & 2007 & Total & & Mean & & 0 & Max & 0 & Min & 0 & Acre-Ft & 0 \\
\hline
\end{tabular}




\section{E218 Cañada del Buey near TA-46}

Location. Lat $35^{\circ}$ 51' 31", long $106^{\circ} 17^{\prime}$ 17" SW 1/4, Sec. 26, T. 19 N., R. 6 E., Los Alamos County.

Drainage Area. $0.30 \mathrm{mi}^{2}$.

Period of Record. June 1, 2000, to September 30, 2008.

Revised Record. Drainage area (2006).

Gage. Data logger and 24” Parshall flume. Elevation of gage is 6,937 ft above NGVD from GPS survey.

Remarks. Records are good.

Average Discharge. $8 \mathrm{yr}, 0.02 \mathrm{ft}^{3} / \mathrm{s}, 12$ acre-ft/year.

Extremes for Period of Record. Maximum discharge, $228 \mathrm{ft}^{3} / \mathrm{s}$, August 25, 2005, gage height $3.40 \mathrm{ft}$, from critical depth computation of peak flow. No flow most of the time.

Extremes for Current Year. Peak discharge above base of $1.0 \mathrm{ft}^{3} / \mathrm{s}$ and maximum (*):

\begin{tabular}{|c|c|c|c|}
\hline Date & Time & Discharge $\left(\mathbf{f t}^{\mathbf{3}} / \mathbf{s}\right)$ & Gage Height (ft) \\
\hline December 1 & 0140 & 4.3 & 0.71 \\
\hline January 28 & 0435 & 4.4 & 0.72 \\
\hline August 10 & 1145 & $7.4^{*}$ & $0.99^{*}$ \\
\hline August 23 & 1625 & 5.4 & 0.82 \\
\hline August 31 & 1535 & 1.9 & 0.44 \\
\hline
\end{tabular}

No flow most of the time.

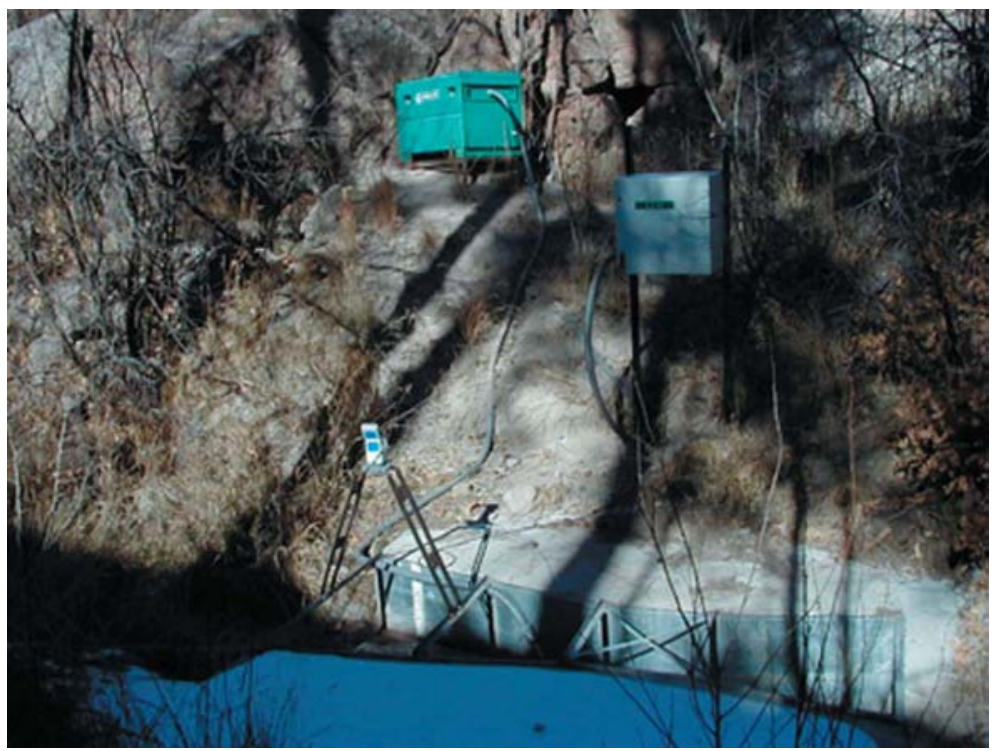




\section{E218 Cañada del Buey near TA-46}

\section{Station Analysis}

\section{Water Year}

Equipment. Station is equipped with Sutron 8210 data logger (5-min. interval) with milltronics sonic probe mounted on a 24" Parshall flume. The system is powered by a solar-panel battery system housed in a NEMA shelter. Station is equipped with ISCO pump sampler for water-quality sample collection. ISCO is housed in a separate shelter, a $3^{\prime} \times 4^{\prime}$ metal box. Samplers are triggered by stage through the data logger. An outside staff is available for reference. No provision for discharge measurements above wading stage. All high-flow measurement will be by slope-area or peak-flow computation methods.

Cellular telemetry removed March 11, discontinued service.

Field Work. This station was visited 11 times to conduct discharge measurements and service the instrumentation. Field inspections for the gage are listed under site history files on the Hydstra database. Discharge measurements for the gage are listed under site gauging files on the Hydstra database.

Datum Correction. None.

Gage-Height Record. The data logger referenced to the outside staff gave a complete and satisfactory record.

Rating. The channel is $15^{\prime}$ wide and straight upstream and downstream for about $100^{\prime}$. The streambed through this reach consists of boulders with sand and gravel. The lowflow control is the 24" Parshall flume; at very high flow the channel becomes control.

Eleven inspections of no flow were made this year.

Rating No. 1 was developed based on the computation of 24" Parshall flume and two peak-flow computations.

Discharge. Discharge was computed by applying gage height to Rating No. 1 directly, with shift adjustment based on PZF.

Remarks. Records are good. 
E218 Cañada del Buey near TA-46

Daily Mean Discharge in Cubic Feet per Second

Water Year October 2007 to September 2008

\begin{tabular}{|c|c|c|c|c|c|c|c|c|c|c|c|c|}
\hline DAY & ОСт & NOV & DEC & JAN & FEB & MAR & APR & MAY & JUN & JUL & AUG & SEP \\
\hline 1 & 0 & 0 & .27 & 0 & 0 & 0 & 0 & 0 & .01 & 0 & 0 & 0 \\
\hline 2 & 0 & 0 & 0 & 0 & 0 & 0 & 0 & 0 & 0 & 0 & 0 & 0 \\
\hline 3 & 0 & 0 & 0 & 0 & 0 & 0 & 0 & 0 & .01 & 0 & 0 & 0 \\
\hline 4 & 0 & 0 & 0 & 0 & 0 & 0 & 0 & 0 & 0 & 0 & 0 & 0 \\
\hline 5 & 0 & 0 & 0 & 0 & 0 & 0 & 0 & 0 & 0 & 0 & 0 & 0 \\
\hline 6 & 0 & 0 & 0 & 0 & 0 & 0 & 0 & 0 & 0 & .01 & 0 & 0 \\
\hline 7 & 0 & 0 & 0 & 0 & 0 & 0 & 0 & 0 & 0 & 0 & 0 & 0 \\
\hline 8 & 0 & 0 & 0 & 0 & 0 & 0 & 0 & 0 & 0 & 0 & 0 & 0 \\
\hline 9 & 0 & 0 & 0 & 0 & 0 & 0 & 0 & 0 & 0 & 0 & 0 & 0 \\
\hline 10 & 0 & 0 & 0 & 0 & 0 & 0 & 0 & 0 & 0 & 0 & .09 & 0 \\
\hline 11 & 0 & 0 & 0 & 0 & 0 & 0 & 0 & 0 & 0 & 0 & 0 & .01 \\
\hline 12 & 0 & 0 & 0 & 0 & 0 & 0 & 0 & 0 & 0 & 0 & 0 & 0 \\
\hline 13 & 0 & 0 & 0 & 0 & 0 & 0 & 0 & 0 & 0 & 0 & 0 & 0 \\
\hline 14 & 0 & 0 & 0 & 0 & 0 & 0 & 0 & 0 & 0 & 0 & .01 & 0 \\
\hline 15 & 0 & 0 & 0 & 0 & 0 & 0 & 0 & 0 & 0 & 0 & 0 & 0 \\
\hline 16 & 0 & 0 & 0 & 0 & 0 & 0 & 0 & 0 & 0 & 0 & 0 & 0 \\
\hline 17 & 0 & 0 & 0 & 0 & 0 & 0 & 0 & 0 & 0 & 0 & 0 & 0 \\
\hline 18 & 0 & 0 & 0 & 0 & 0 & 0 & 0 & 0 & .01 & 0 & 0 & 0 \\
\hline 19 & 0 & 0 & 0 & 0 & 0 & 0 & 0 & 0 & 0 & 0 & 0 & 0 \\
\hline 20 & 0 & 0 & 0 & 0 & 0 & 0 & 0 & 0 & 0 & 0 & 0 & 0 \\
\hline 21 & 0 & 0 & 0 & 0 & 0 & 0 & 0 & 0 & 0 & 0 & 0 & .01 \\
\hline 22 & 0 & 0 & 0 & 0 & 0 & 0 & 0 & 0 & 0 & 0 & 0 & 0 \\
\hline 23 & 0 & 0 & 0 & 0 & 0 & 0 & 0 & 0 & 0 & 0 & .10 & 0 \\
\hline 24 & 0 & 0 & 0 & 0 & 0 & 0 & 0 & 0 & 0 & 0 & 0 & 0 \\
\hline 25 & 0 & 0 & 0 & 0 & 0 & 0 & 0 & 0 & 0 & 0 & .01 & 0 \\
\hline 26 & 0 & 0 & 0 & 0 & 0 & 0 & 0 & 0 & 0 & 0 & 0 & 0 \\
\hline 27 & 0 & 0 & 0 & 0 & 0 & 0 & 0 & 0 & 0 & 0 & 0 & 0 \\
\hline 28 & 0 & 0 & 0 & .42 & 0 & 0 & 0 & 0 & 0 & 0 & 0 & 0 \\
\hline 29 & 0 & 0 & 0 & 0 & 0 & 0 & 0 & 0 & 0 & 0 & 0 & 0 \\
\hline 30 & 0 & 0 & 0 & 0 & ------ & 0 & 0 & 0 & 0 & 0 & 0 & 0 \\
\hline 31 & 0 & ------ & 0 & 0 & ------ & 0 & ------ & 0 & ------ & 0 & .02 & ------ \\
\hline Total & 0 & 0 & 0.27 & 0.42 & 0 & 0 & 0 & 0 & 0.03 & 0.01 & 0.23 & 0.02 \\
\hline Mean & 0 & 0 & .009 & .014 & 0 & 0 & 0 & 0 & .001 & 0 & .007 & .001 \\
\hline Max & 0 & 0 & .27 & .42 & 0 & 0 & 0 & 0 & .01 & .01 & .10 & .01 \\
\hline Min & 0 & 0 & 0 & 0 & 0 & 0 & 0 & 0 & 0 & 0 & 0 & 0 \\
\hline Acre-Ft & 0 & 0 & .54 & .83 & 0 & 0 & 0 & 0 & .06 & .02 & .46 & .04 \\
\hline Wtr Year & 2008 & Total & 0.98 & Mean & & 3 & hax & .42 & Min & 0 & Acre-Ft & 1.9 \\
\hline Cal Year & 2007 & Total & 7.39 & Mean & & 20 & hax & 1.5 & Min & 0 & Acre-Ft & 15 \\
\hline
\end{tabular}


Location. Lat $35^{\circ}$ 50' 41", long $106^{\circ}$ 15' 52", Sec. 25, T. 19 N., R. 6 E., Ramon Vigil Grant, Los Alamos County.

Drainage Area. $0.0004 \mathrm{mi}^{2}$.

Period of Record. October 1, 2006, to September 30, 2008.

Gage. Data logger and 9" Parshall flume. Elevation of gage is 6,877 ft above NGVD.

Remarks. Records are good. Records for this site existed before period of record but are not reliable. Legal location based on projected values.

Extremes for Period of Record. Maximum discharge, $2.09 \mathrm{ft}^{3} / \mathrm{s}$, July 30, 2007, gage height $0.78 \mathrm{ft}$. No flow most of the time.

Extremes for Current Year. Peak discharge above base of $1.0 \mathrm{ft}^{3} / \mathrm{s}$ and maximum (*):

\begin{tabular}{|c|c|c|c|}
\hline Date & Time & Discharge (ft $\left.\mathbf{3}^{\mathbf{s}} \mathbf{s}\right)$ & Gage Height (ft) \\
\hline August 23 & 1605 & $1.0^{*}$ & $0.48^{*}$ \\
\hline
\end{tabular}

No flow most of the time.

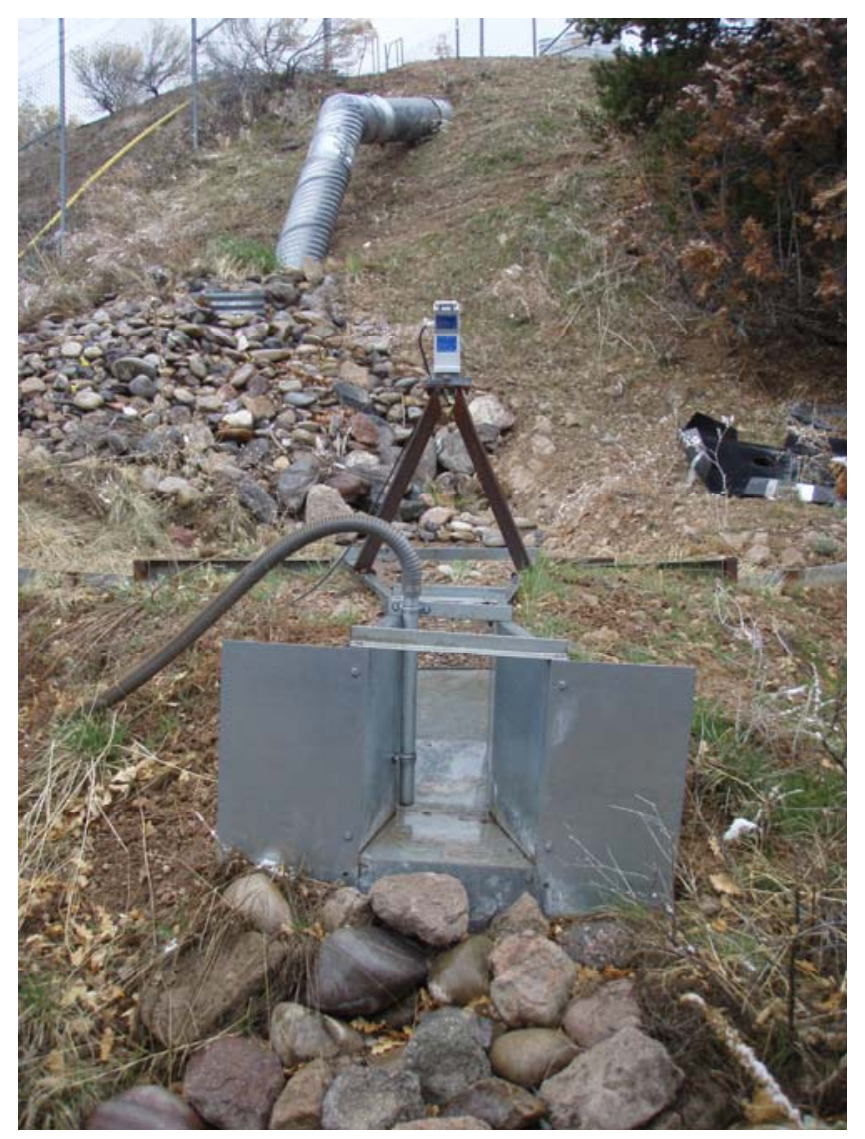




\section{E220 TA-54 RANT}

\section{Station Analysis}

\section{Water Year}

Equipment. Station is equipped with Sutron 8210 data logger (5-min. interval) and milltronics sonic probe mounted on a 9" Parshall flume. The system is powered by a solar-panel battery system housed in a NEMA shelter. Station is equipped with an ISCO pump sampler for water-quality sample collection. ISCO is housed in a separate shelter, a $3^{\prime} \times 4^{\prime}$ metal box. Sampler is triggered by stage through the data logger. The staff in the 9" Parshall flume is the reference gage. No provision for discharge measurements above wading stage.

Field Work. This station was visited 20 times to conduct discharge measurements and service the instrumentation. Field inspections for the gage are listed under site history files on the Hydstra database. Discharge measurements for the gage are listed under site gauging files on the Hydstra database.

Datum Correction. None.

Gage-Height Record. The data logger referenced to the outside staff gave a complete and satisfactory record.

Rating. Channel is straight above and below gage for $10^{\prime}$. The streambed consists of gravel and large coble.

Twenty inspections of no flow were made this year.

Rating No. 1 was developed based on the computation of 9" Parshall flume. Point of zero flow is 0.00 gage height.

Discharge. Discharge was computed by applying gage height to Rating No. 1 directly.

Remarks. Records are good. 
E220 TA-54 RANT

Daily Mean Discharge in Cubic Feet per Second

Water Year October 2007 to September 2008

\begin{tabular}{|c|c|c|c|c|c|c|c|c|c|c|c|c|}
\hline DAY & OCT & NOV & DEC & JAN & FEB & MAR & APR & MAY & JUN & JUL & AUG & SEP \\
\hline 1 & 0 & 0 & .02 & 0 & 0 & 0 & 0 & 0 & 0 & 0 & 0 & 0 \\
\hline 2 & 0 & 0 & 0 & 0 & 0 & 0 & 0 & 0 & 0 & 0 & 0 & 0 \\
\hline 3 & 0 & 0 & 0 & 0 & 0 & 0 & 0 & 0 & 0 & 0 & 0 & 0 \\
\hline 4 & 0 & 0 & 0 & 0 & 0 & 0 & 0 & 0 & 0 & 0 & 0 & 0 \\
\hline 5 & 0 & 0 & 0 & 0 & 0 & 0 & 0 & 0 & 0 & 0 & 0 & 0 \\
\hline 6 & 0 & 0 & 0 & 0 & 0 & 0 & 0 & 0 & 0 & 0 & 0 & 0 \\
\hline 7 & 0 & 0 & 0 & 0 & 0 & 0 & 0 & 0 & 0 & 0 & 0 & 0 \\
\hline 8 & 0 & 0 & 0 & 0 & 0 & 0 & 0 & 0 & 0 & 0 & 0 & 0 \\
\hline 9 & 0 & 0 & 0 & 0 & 0 & 0 & 0 & 0 & 0 & 0 & 0 & 0 \\
\hline 10 & 0 & 0 & 0 & 0 & 0 & 0 & 0 & 0 & 0 & 0 & .01 & 0 \\
\hline 11 & 0 & 0 & 0 & 0 & 0 & 0 & 0 & 0 & 0 & 0 & 0 & 0 \\
\hline 12 & 0 & 0 & 0 & 0 & 0 & 0 & 0 & 0 & 0 & 0 & 0 & 0 \\
\hline 13 & 0 & 0 & 0 & 0 & 0 & 0 & 0 & 0 & 0 & 0 & 0 & 0 \\
\hline 14 & 0 & 0 & 0 & 0 & 0 & 0 & 0 & 0 & 0 & 0 & 0 & 0 \\
\hline 15 & 0 & 0 & 0 & 0 & 0 & 0 & 0 & 0 & 0 & 0 & 0 & 0 \\
\hline 16 & 0 & 0 & 0 & 0 & 0 & 0 & 0 & 0 & 0 & 0 & 0 & 0 \\
\hline 17 & 0 & 0 & 0 & 0 & 0 & 0 & 0 & 0 & 0 & 0 & 0 & 0 \\
\hline 18 & 0 & 0 & 0 & 0 & 0 & 0 & 0 & 0 & 0 & 0 & 0 & 0 \\
\hline 19 & 0 & 0 & 0 & 0 & 0 & 0 & 0 & 0 & 0 & 0 & 0 & 0 \\
\hline 20 & 0 & 0 & 0 & 0 & 0 & 0 & 0 & 0 & 0 & 0 & 0 & 0 \\
\hline 21 & 0 & 0 & 0 & 0 & 0 & 0 & 0 & 0 & 0 & 0 & 0 & 0 \\
\hline 22 & 0 & 0 & 0 & 0 & 0 & 0 & 0 & 0 & 0 & 0 & 0 & 0 \\
\hline 23 & 0 & 0 & 0 & 0 & 0 & 0 & 0 & 0 & 0 & 0 & .01 & 0 \\
\hline 24 & 0 & 0 & 0 & 0 & 0 & 0 & 0 & 0 & 0 & 0 & 0 & 0 \\
\hline 25 & 0 & 0 & 0 & 0 & 0 & 0 & 0 & 0 & 0 & 0 & 0 & 0 \\
\hline 26 & 0 & 0 & 0 & 0 & 0 & 0 & 0 & 0 & 0 & 0 & 0 & 0 \\
\hline 27 & 0 & 0 & 0 & 0 & 0 & 0 & 0 & 0 & 0 & 0 & 0 & 0 \\
\hline 28 & 0 & 0 & 0 & .01 & 0 & 0 & 0 & 0 & 0 & 0 & 0 & 0 \\
\hline 29 & 0 & 0 & 0 & 0 & 0 & 0 & 0 & 0 & 0 & 0 & 0 & 0 \\
\hline 30 & 0 & .02 & 0 & 0 & ------ & 0 & 0 & 0 & 0 & 0 & 0 & 0 \\
\hline 31 & 0 & ------ & 0 & 0 & ------ & 0 & ------ & 0 & ----- & 0 & .01 & ------ \\
\hline Total & 0 & 0.02 & 0.02 & 0.01 & 0 & 0 & 0 & 0 & 0 & 0 & 0.03 & 0 \\
\hline Mean & 0 & .001 & .001 & 0 & 0 & 0 & 0 & 0 & 0 & 0 & .001 & 0 \\
\hline Max & 0 & .02 & .02 & .01 & 0 & 0 & 0 & 0 & 0 & 0 & .01 & 0 \\
\hline Min & 0 & 0 & 0 & 0 & 0 & 0 & 0 & 0 & 0 & 0 & 0 & 0 \\
\hline Acre-Ft & 0 & .04 & .04 & .02 & 0 & 0 & 0 & 0 & 0 & 0 & .06 & 0 \\
\hline Wtr Year & 2008 & Total & 0.08 & Mean & & 0 & Max & .02 & Min & 0 & Acre-Ft & 0.16 \\
\hline Cal Year & 2007 & Total & 0.23 & Mean & & 01 & Max & .04 & Min & 0 & Acre-Ft & 0.46 \\
\hline
\end{tabular}




\section{E223 MDA Area L}

Location. Lat 35 50' 8", long $106^{\circ}$ 15' 2", Sec. 36, T. 19 N., R. 6 E., Ramon Vigil Grant, Los Alamos Country.

Drainage Area. $0.003 \mathrm{mi}^{2}$.

Period of Record. October 1, 2006, to September 30, 2008.

Gage. Data logger and 9" Parshall flume. Elevation of gage is 6,776 ft above NGVD.

Remarks. Records are good. Records for this site existed before period of record but are not reliable. Legal location based on projected values.

Extremes for Period of Record. Maximum discharge, $1.80 \mathrm{ft}^{3} / \mathrm{s}$, September 23, 2007, gage height $0.92 \mathrm{ft}$. No flow most of the time.

Extremes for Current Year. Maximum discharge $0.67 \mathrm{ft}^{3} / \mathrm{s}$ at $0445 \mathrm{~h}$, December 1, gage height $0.49 \mathrm{ft}$. No peak above base of $1.0 \mathrm{ft}^{3} / \mathrm{s}$. No flow most of the time.

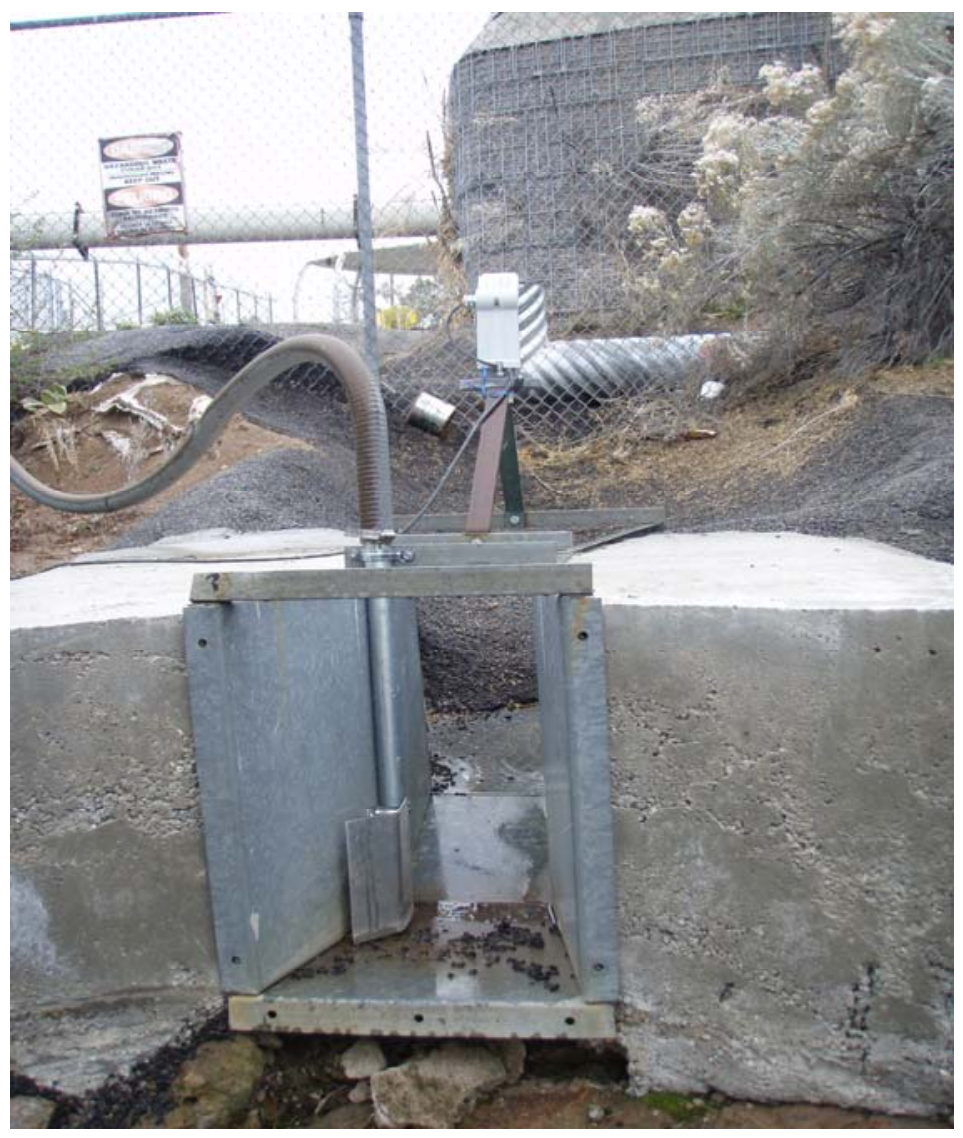




\section{E223 MDA Area L}

\section{Station Analysis}

\section{Water Year}

Equipment. Station is equipped with Sutron 8210 data logger (5-min. interval) and milltronics sonic probe mounted on a 9" Parshall flume. The system is powered by a solar-panel battery system housed in a NEMA shelter. Station is equipped with an ISCO pump sampler for water-quality sample collection. ISCO is housed in a separate shelter, a $3^{\prime} \times 4^{\prime}$ metal box. Sampler is triggered by stage through the data logger. The staff in the 9" Parshall flume is the reference gage. No provision for discharge measurements above wading stage.

Field Work. This station was visited 19 times to conduct discharge measurements and service the instrumentation. Field inspections for the gage are listed under site history files on the Hydstra database. Discharge measurements for the gage are listed under site gauging files on the Hydstra database.

Datum Correction. None.

Gage-Height Record. The data logger referenced to the outside staff gave a complete and satisfactory record.

Rating. Above 9" Parshall flume consists of asphalt paved lot; below gage the channel drops steeply.

Nineteen inspections of no flow were made.

Rating No. 1 was developed based on the computation of 9" Parshall flume. Point of zero flow is 0.00 gage height.

Discharge. Discharge was computed by applying gage height to Rating No. 1 directly.

Remarks. Records are good. 
E223 MDA Area L

Daily Mean Discharge in Cubic Feet per Second

Water Year October 2007 to September 2008

\begin{tabular}{|c|c|c|c|c|c|c|c|c|c|c|c|c|}
\hline DAY & ОСТ & NOV & DEC & JAN & FEB & MAR & APR & MAY & JUN & JUL & AUG & SEP \\
\hline 1 & 0 & 0 & .01 & 0 & 0 & 0 & 0 & 0 & 0 & 0 & 0 & 0 \\
\hline 2 & 0 & 0 & 0 & 0 & 0 & 0 & 0 & 0 & 0 & 0 & 0 & 0 \\
\hline 3 & 0 & 0 & 0 & 0 & 0 & 0 & 0 & 0 & 0 & 0 & 0 & 0 \\
\hline 4 & 0 & 0 & 0 & 0 & 0 & 0 & 0 & 0 & 0 & 0 & 0 & 0 \\
\hline 5 & 0 & 0 & 0 & 0 & 0 & 0 & 0 & 0 & 0 & 0 & 0 & 0 \\
\hline 6 & 0 & 0 & 0 & 0 & 0 & 0 & 0 & 0 & 0 & 0 & 0 & 0 \\
\hline 7 & 0 & 0 & 0 & 0 & 0 & 0 & 0 & 0 & 0 & 0 & 0 & 0 \\
\hline 8 & 0 & 0 & 0 & 0 & 0 & 0 & 0 & 0 & 0 & 0 & 0 & 0 \\
\hline 9 & 0 & 0 & 0 & 0 & 0 & 0 & 0 & 0 & 0 & 0 & 0 & 0 \\
\hline 10 & 0 & 0 & 0 & 0 & 0 & 0 & 0 & 0 & 0 & 0 & 0 & 0 \\
\hline 11 & 0 & 0 & 0 & 0 & 0 & 0 & 0 & 0 & 0 & 0 & 0 & 0 \\
\hline 12 & 0 & 0 & 0 & 0 & 0 & 0 & 0 & 0 & 0 & 0 & 0 & 0 \\
\hline 13 & 0 & 0 & 0 & 0 & 0 & 0 & 0 & 0 & 0 & 0 & 0 & 0 \\
\hline 14 & 0 & 0 & 0 & 0 & 0 & 0 & 0 & 0 & 0 & 0 & 0 & 0 \\
\hline 15 & 0 & 0 & 0 & 0 & 0 & 0 & 0 & 0 & 0 & 0 & 0 & 0 \\
\hline 16 & 0 & 0 & 0 & 0 & 0 & 0 & 0 & 0 & 0 & 0 & 0 & 0 \\
\hline 17 & 0 & 0 & 0 & 0 & 0 & 0 & 0 & 0 & 0 & 0 & 0 & 0 \\
\hline 18 & 0 & 0 & 0 & 0 & 0 & 0 & 0 & 0 & 0 & 0 & 0 & 0 \\
\hline 19 & 0 & 0 & 0 & 0 & 0 & 0 & 0 & 0 & 0 & 0 & 0 & 0 \\
\hline 20 & 0 & 0 & 0 & 0 & 0 & 0 & 0 & 0 & 0 & 0 & 0 & 0 \\
\hline 21 & 0 & 0 & 0 & 0 & 0 & 0 & 0 & 0 & 0 & 0 & 0 & 0 \\
\hline 22 & 0 & 0 & 0 & 0 & 0 & 0 & 0 & 0 & 0 & 0 & 0 & 0 \\
\hline 23 & 0 & 0 & 0 & 0 & 0 & 0 & 0 & 0 & 0 & 0 & 0 & 0 \\
\hline 24 & 0 & 0 & 0 & 0 & .02 & 0 & 0 & 0 & 0 & 0 & 0 & 0 \\
\hline 25 & 0 & 0 & 0 & 0 & 0 & 0 & 0 & 0 & 0 & 0 & 0 & 0 \\
\hline 26 & 0 & 0 & 0 & 0 & 0 & 0 & 0 & 0 & 0 & 0 & 0 & 0 \\
\hline 27 & 0 & 0 & 0 & .01 & 0 & 0 & 0 & 0 & 0 & 0 & 0 & 0 \\
\hline 28 & 0 & 0 & 0 & .01 & 0 & 0 & 0 & 0 & 0 & 0 & 0 & 0 \\
\hline 29 & 0 & 0 & 0 & 0 & 0 & 0 & 0 & 0 & 0 & 0 & 0 & 0 \\
\hline 30 & 0 & 0 & 0 & 0 & ----- & 0 & 0 & 0 & 0 & 0 & 0 & 0 \\
\hline 31 & 0 & ----- & 0 & 0 & ----- & 0 & ------ & 0 & ------ & 0 & 0 & ------ \\
\hline Total & 0 & 0 & 0.01 & 0.02 & 0.02 & 0 & 0 & 0 & 0 & 0 & 0 & 0 \\
\hline Mean & 0 & 0 & 0 & .001 & .001 & 0 & 0 & 0 & 0 & 0 & 0 & 0 \\
\hline Max & 0 & 0 & .01 & .01 & .02 & 0 & 0 & 0 & 0 & 0 & 0 & 0 \\
\hline Min & 0 & 0 & 0 & 0 & 0 & 0 & 0 & 0 & 0 & 0 & 0 & 0 \\
\hline Acre-Ft & 0 & 0 & .02 & .04 & .04 & 0 & 0 & 0 & 0 & 0 & 0 & 0 \\
\hline Wtr Year & 2008 & Total & 0.05 & Mean & & 0 & Max & .02 & Min & 0 & Acre-Ft & 0.10 \\
\hline Cal Year & 2007 & Total & 0.04 & Mean & & 0 & Max & .01 & Min & 0 & Acre-Ft & 0.08 \\
\hline
\end{tabular}




\section{E225 Cañada del Buey near MDA G}

Location. Lat $35^{\circ}$ 50' 1", long $106^{\circ}$ 14' 22", Sec. 31, T. 19 N., R. 7 E., Ramon Vigil Grant, Los Alamos County.

Drainage Area. $1.48 \mathrm{mi}^{2}$.

Period of Record. October 1, 1993, to September 30, 2008.

Revised Record. Drainage area (2006).

Gage. Data logger and concrete control. Elevation of gage is 6,599 ft above NGVD from GPS survey.

Remarks. Records are good. Legal location based on projected values.

Average Discharge. 12 yr, zero.

Extremes for Period of Record. Maximum discharge, $17 \mathrm{ft}^{3} / \mathrm{s}$, September 8, 1995, gage height $2.71 \mathrm{ft}$. No flow most of the time.

Extremes for Current Year. Maximum discharge $0.01 \mathrm{ft}^{3} / \mathrm{s}$ at $0410 \mathrm{~h}$, January 28, gage height $0.94 \mathrm{ft}$. No peak discharge above base of $5.0 \mathrm{ft}^{3} / \mathrm{s}$. No flow most of the time.

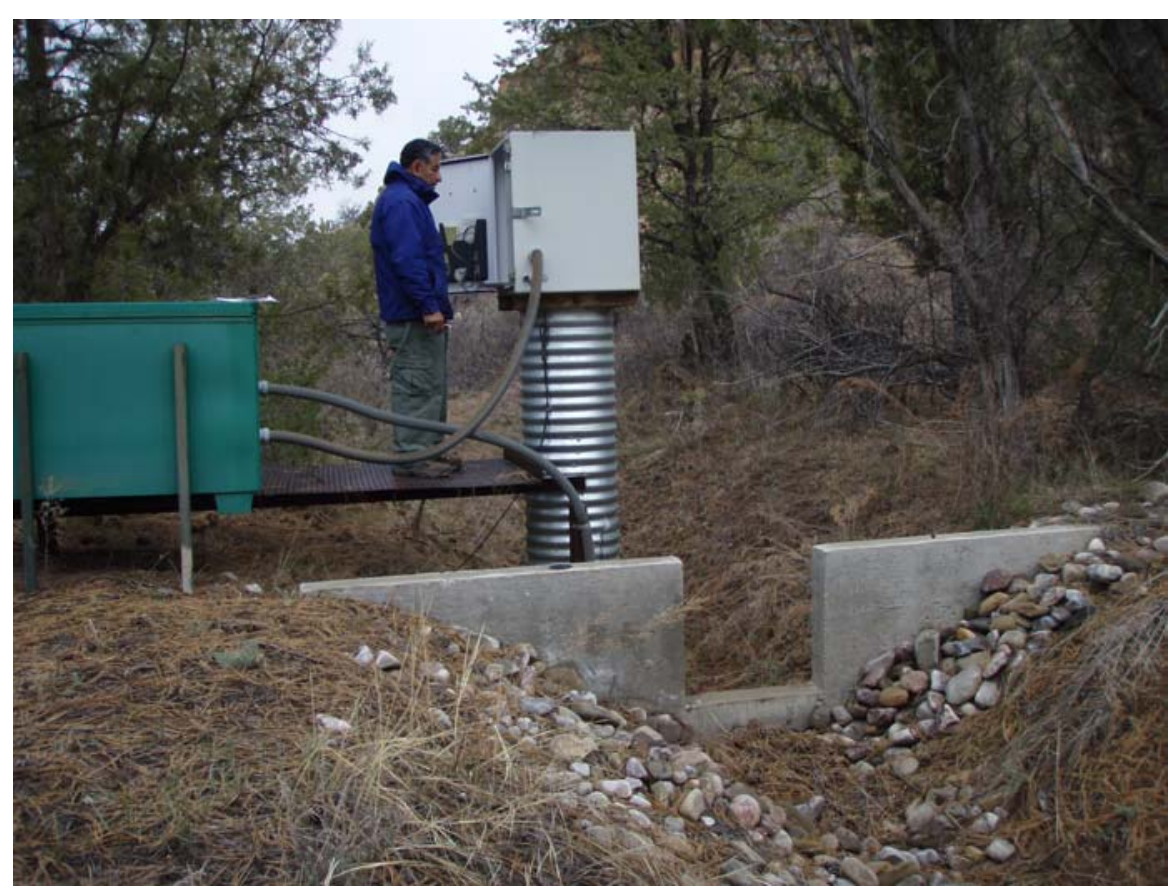




\section{E225 Cañada del Buey near MDA G}

\section{Station Analysis}

\section{Water Year}

Equipment. Station is equipped with Sutron 8210 data logger (5-min. interval) and a shaft encoder float system. The system is powered by a solar-panel battery system. All equipment is housed in a NEMA shelter on 18" CMP well. Station is equipped with an ISCO pump sampler for water-quality sample collection. ISCO is housed in a separate shelter, a $3^{\prime} \times 4^{\prime}$ metal box. Sampler is triggered by stage through the data logger. An outside staff is available for reference. No provision for discharge measurements above wading stage.

Cellular telemetry removed March 11, discontinued service.

Field Work. The station was visited 16 times to conduct discharge measurements and service the instrumentation. Field inspections for the gage are listed under site history files on the Hydstra database. Discharge measurements for the gage are listed under site gauging files on the Hydstra database.

Datum Correction. None.

Gage-Height Record. The data logger referenced to the outside staff gave a complete and satisfactory record.

Rating. Channel is straight for at least $100^{\prime}$ above and below gage. Bed material consists of sand with a thin covering of grass. The control consists of a concrete broad-crested weir 10' downstream of gage.

Sixteen inspections of no flow were made during this period.

Rating No. 1 was developed by broad-crested weir computation.

Discharge. Discharge was computed by applying gage height to Rating No. 1 directly. No flow most of the time.

Remarks. Records are good. 
E225 Cañada del Buey near MDA G

Daily Mean Discharge in Cubic Feet per Second

Water Year October 2007 to September 2008

\begin{tabular}{|c|c|c|c|c|c|c|c|c|c|c|c|c|}
\hline DAY & OCT & NOV & DEC & JAN & FEB & MAR & APR & MAY & JUN & JUL & AUG & SEP \\
\hline 1 & 0 & 0 & 0 & 0 & 0 & 0 & 0 & 0 & 0 & 0 & 0 & 0 \\
\hline 2 & 0 & 0 & 0 & 0 & 0 & 0 & 0 & 0 & 0 & 0 & 0 & 0 \\
\hline 3 & 0 & 0 & 0 & 0 & 0 & 0 & 0 & 0 & 0 & 0 & 0 & 0 \\
\hline 4 & 0 & 0 & 0 & 0 & 0 & 0 & 0 & 0 & 0 & 0 & 0 & 0 \\
\hline 5 & 0 & 0 & 0 & 0 & 0 & 0 & 0 & 0 & 0 & 0 & 0 & 0 \\
\hline 6 & 0 & 0 & 0 & 0 & 0 & 0 & 0 & 0 & 0 & 0 & 0 & 0 \\
\hline 7 & 0 & 0 & 0 & 0 & 0 & 0 & 0 & 0 & 0 & 0 & 0 & 0 \\
\hline 8 & 0 & 0 & 0 & 0 & 0 & 0 & 0 & 0 & 0 & 0 & 0 & 0 \\
\hline 9 & 0 & 0 & 0 & 0 & 0 & 0 & 0 & 0 & 0 & 0 & 0 & 0 \\
\hline 10 & 0 & 0 & 0 & 0 & 0 & 0 & 0 & 0 & 0 & 0 & 0 & 0 \\
\hline 11 & 0 & 0 & 0 & 0 & 0 & 0 & 0 & 0 & 0 & 0 & 0 & 0 \\
\hline 12 & 0 & 0 & 0 & 0 & 0 & 0 & 0 & 0 & 0 & 0 & 0 & 0 \\
\hline 13 & 0 & 0 & 0 & 0 & 0 & 0 & 0 & 0 & 0 & 0 & 0 & 0 \\
\hline 14 & 0 & 0 & 0 & 0 & 0 & 0 & 0 & 0 & 0 & 0 & 0 & 0 \\
\hline 15 & 0 & 0 & 0 & 0 & 0 & 0 & 0 & 0 & 0 & 0 & 0 & 0 \\
\hline 16 & 0 & 0 & 0 & 0 & 0 & 0 & 0 & 0 & 0 & 0 & 0 & 0 \\
\hline 17 & 0 & 0 & 0 & 0 & 0 & 0 & 0 & 0 & 0 & 0 & 0 & 0 \\
\hline 18 & 0 & 0 & 0 & 0 & 0 & 0 & 0 & 0 & 0 & 0 & 0 & 0 \\
\hline 19 & 0 & 0 & 0 & 0 & 0 & 0 & 0 & 0 & 0 & 0 & 0 & 0 \\
\hline 20 & 0 & 0 & 0 & 0 & 0 & 0 & 0 & 0 & 0 & 0 & 0 & 0 \\
\hline 21 & 0 & 0 & 0 & 0 & 0 & 0 & 0 & 0 & 0 & 0 & 0 & 0 \\
\hline 22 & 0 & 0 & 0 & 0 & 0 & 0 & 0 & 0 & 0 & 0 & 0 & 0 \\
\hline 23 & 0 & 0 & 0 & 0 & 0 & 0 & 0 & 0 & 0 & 0 & 0 & 0 \\
\hline 24 & 0 & 0 & 0 & 0 & 0 & 0 & 0 & 0 & 0 & 0 & 0 & 0 \\
\hline 25 & 0 & 0 & 0 & 0 & 0 & 0 & 0 & 0 & 0 & 0 & 0 & 0 \\
\hline 26 & 0 & 0 & 0 & 0 & 0 & 0 & 0 & 0 & 0 & 0 & 0 & 0 \\
\hline 27 & 0 & 0 & 0 & 0 & 0 & 0 & 0 & 0 & 0 & 0 & 0 & 0 \\
\hline 28 & 0 & 0 & 0 & .02 & 0 & 0 & 0 & 0 & 0 & 0 & 0 & 0 \\
\hline 29 & 0 & 0 & 0 & 0 & 0 & 0 & 0 & 0 & 0 & 0 & 0 & 0 \\
\hline 30 & 0 & 0 & 0 & 0 & ------ & 0 & 0 & 0 & 0 & 0 & 0 & 0 \\
\hline 31 & 0 & ----- & 0 & 0 & ----- & 0 & ------ & 0 & ----- & 0 & 0 & ------ \\
\hline Total & 0 & 0 & 0 & 0.02 & 0 & 0 & 0 & 0 & 0 & 0 & 0 & 0 \\
\hline Mean & 0 & 0 & 0 & .001 & 0 & 0 & 0 & 0 & 0 & 0 & 0 & 0 \\
\hline Max & 0 & 0 & 0 & .02 & 0 & 0 & 0 & 0 & 0 & 0 & 0 & 0 \\
\hline Min & 0 & 0 & 0 & 0 & 0 & 0 & 0 & 0 & 0 & 0 & 0 & 0 \\
\hline Acre-Ft & 0 & 0 & 0 & .04 & 0 & 0 & 0 & 0 & 0 & 0 & 0 & 0 \\
\hline Wtr Year & 2008 & Total & 0.02 & Mean & & 0 & Max & .02 & Min & 0 & Acre-Ft & 0.04 \\
\hline Cal Year & 2007 & Total & 0 & Mean & & 0 & Max & 0 & Min & 0 & Acre-Ft & 0 \\
\hline
\end{tabular}


Location. Lat $35^{\circ}$ 49' 58", long $106^{\circ}$ 14' 21", Sec. 31, T. 19 N., R. 7 E., Ramon Vigil Grant, Los Alamos County.

Drainage Area. $0.012 \mathrm{mi}^{2}$.

Period of Record. October 1, 2006, to September 30, 2008.

Gage. Data logger and 9” Parshall flume. Elevation of gage is 6,614 ft above NGVD.

Remarks. Records are good. Records for this site existed before period of record but are not reliable. Legal location based on projected values.

Extremes for Period of Record. Maximum discharge, $1.7 \mathrm{ft}^{3} / \mathrm{s}$, July 26, 2007, gage height $0.67 \mathrm{ft}$. No flow most of the time.

Extremes for Current Year. Maximum discharge, $0.26 \mathrm{ft}^{3} / \mathrm{s}$ at $0415 \mathrm{~h}$, January 28, gage height $0.0 .20 \mathrm{ft}$. No peak discharge above base of $1.0 \mathrm{ft}^{3} / \mathrm{s}$. No flow most of the year.

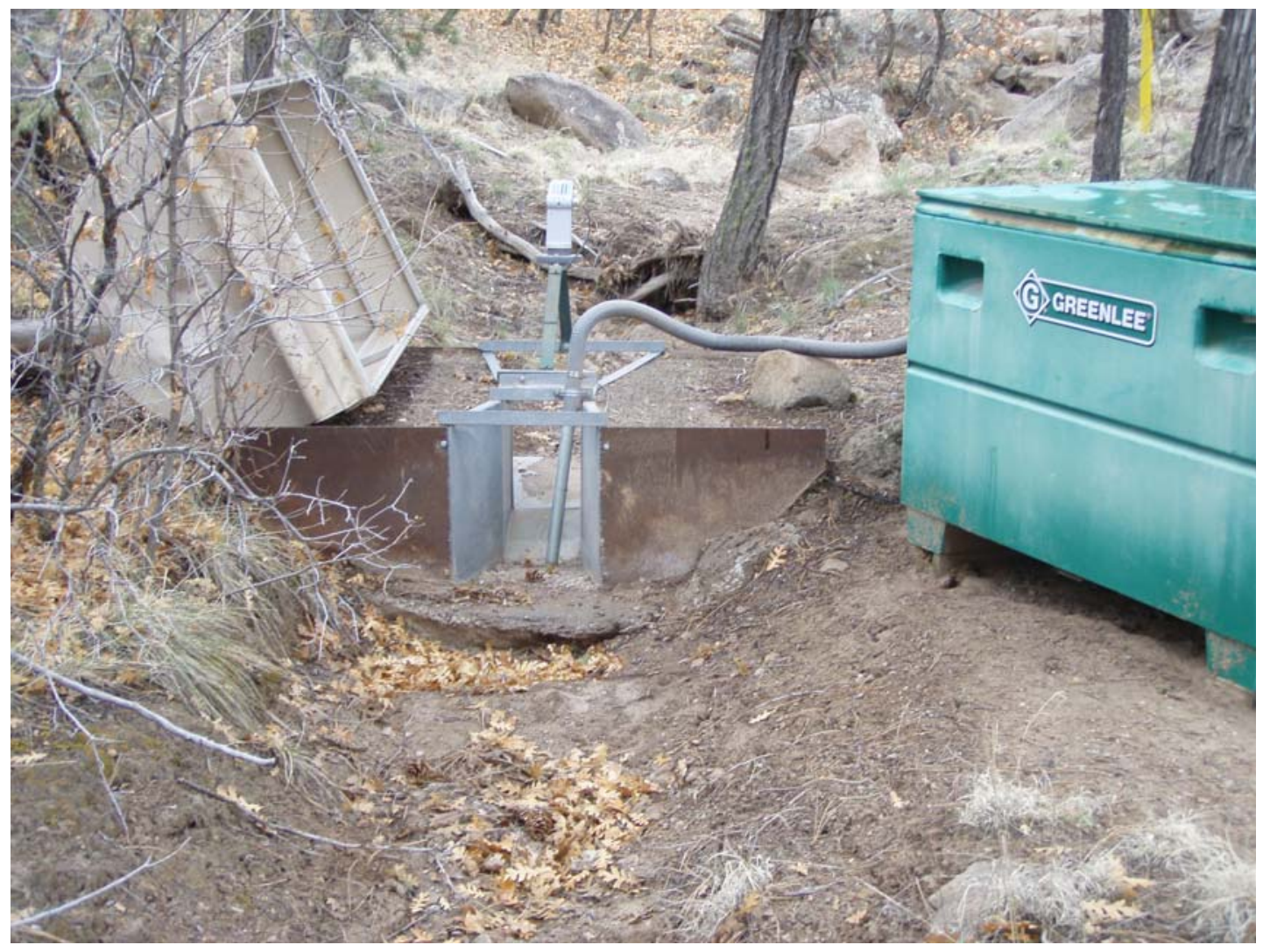




\section{E227 MDA G-6}

\section{Station Analysis}

\section{Water Year}

Equipment. Station is equipped with Sutron 8210 data logger (5-min. interval) and milltronics sonic probe mounted on a 9" Parshall flume. The system is powered by a solar-panel battery system housed in a NEMA shelter. Station is equipped with an ISCO pump sampler for water-quality sample collection. ISCO is housed in a separate shelter, a $3^{\prime} \times 4^{\prime}$ metal box. Sampler is triggered by stage through the data logger. The staff in the 9" Parshall flume is the reference gage. No provision for discharge measurements above wading stage.

Field Work. This station was visited 17 times to conduct discharge measurements and service the instrumentation. Field inspections for the gage are listed under site history files on the Hydstra database. Discharge measurements for the gage are listed under site gauging files on the Hydstra database.

Datum Correction. None.

Gage-Height Record. The data logger referenced to the outside staff gave a complete and satisfactory record.

Rating. Channel is straight above and below flume. Channel is shallow and streambed consists of mostly sand and gravel.

Seventeen inspections of no flow were made this year.

Rating No. 1 was developed based on the computation of 9" Parshall flume. Point of zero flow is 0.00 gage height.

Discharge. Discharge was computed by applying gage height to Rating No 1 directly. No flow most of the time.

Remarks. Records are good. 


\section{E227 MDA G-6}

Daily Mean Discharge in Cubic Feet per Second

Water Year October 2007 to September 2008

\begin{tabular}{|c|c|c|c|c|c|c|c|c|c|c|c|c|}
\hline DAY & OCT & NOV & DEC & JAN & FEB & MAR & APR & MAY & JUN & JUL & AUG & SEP \\
\hline 1 & 0 & 0 & .02 & 0 & 0 & 0 & 0 & 0 & 0 & 0 & 0 & 0 \\
\hline 2 & 0 & 0 & 0 & 0 & 0 & 0 & 0 & 0 & 0 & 0 & 0 & 0 \\
\hline 3 & 0 & 0 & 0 & 0 & 0 & 0 & 0 & 0 & 0 & 0 & 0 & 0 \\
\hline 4 & 0 & 0 & 0 & 0 & 0 & 0 & 0 & 0 & 0 & 0 & 0 & 0 \\
\hline 5 & 0 & 0 & 0 & 0 & 0 & 0 & 0 & 0 & 0 & 0 & 0 & 0 \\
\hline 6 & 0 & 0 & 0 & 0 & 0 & 0 & 0 & 0 & 0 & 0 & 0 & 0 \\
\hline 7 & 0 & 0 & 0 & 0 & 0 & 0 & 0 & 0 & 0 & 0 & 0 & 0 \\
\hline 8 & 0 & 0 & 0 & 0 & 0 & 0 & 0 & 0 & 0 & 0 & 0 & 0 \\
\hline 9 & 0 & 0 & 0 & 0 & 0 & 0 & 0 & 0 & 0 & 0 & 0 & 0 \\
\hline 10 & 0 & 0 & 0 & 0 & 0 & 0 & 0 & 0 & 0 & 0 & 0 & 0 \\
\hline 11 & 0 & 0 & 0 & 0 & 0 & 0 & 0 & 0 & 0 & 0 & 0 & 0 \\
\hline 12 & 0 & 0 & 0 & 0 & 0 & 0 & 0 & 0 & 0 & 0 & 0 & 0 \\
\hline 13 & 0 & 0 & 0 & 0 & 0 & 0 & 0 & 0 & 0 & 0 & 0 & 0 \\
\hline 14 & 0 & 0 & 0 & 0 & 0 & 0 & 0 & 0 & 0 & 0 & 0 & 0 \\
\hline 15 & 0 & 0 & 0 & 0 & 0 & 0 & 0 & 0 & 0 & 0 & 0 & 0 \\
\hline 16 & 0 & 0 & 0 & 0 & 0 & 0 & 0 & 0 & 0 & 0 & 0 & 0 \\
\hline 17 & 0 & 0 & 0 & 0 & 0 & 0 & 0 & 0 & 0 & 0 & 0 & 0 \\
\hline 18 & 0 & 0 & 0 & 0 & 0 & 0 & 0 & 0 & 0 & 0 & 0 & 0 \\
\hline 19 & 0 & 0 & 0 & 0 & 0 & 0 & 0 & 0 & 0 & 0 & 0 & 0 \\
\hline 20 & 0 & 0 & 0 & 0 & 0 & 0 & 0 & 0 & 0 & 0 & 0 & 0 \\
\hline 21 & 0 & 0 & 0 & 0 & 0 & 0 & 0 & 0 & 0 & 0 & 0 & 0 \\
\hline 22 & 0 & 0 & 0 & 0 & 0 & 0 & 0 & 0 & 0 & 0 & 0 & 0 \\
\hline 23 & 0 & 0 & 0 & 0 & 0 & 0 & 0 & 0 & 0 & 0 & 0 & 0 \\
\hline 24 & 0 & 0 & 0 & 0 & 0 & 0 & 0 & 0 & 0 & 0 & 0 & 0 \\
\hline 25 & 0 & 0 & 0 & 0 & 0 & 0 & 0 & 0 & 0 & 0 & 0 & 0 \\
\hline 26 & 0 & 0 & 0 & 0 & 0 & 0 & 0 & 0 & 0 & 0 & 0 & 0 \\
\hline 27 & 0 & 0 & 0 & 0 & 0 & 0 & 0 & 0 & 0 & 0 & 0 & 0 \\
\hline 28 & 0 & 0 & 0 & .01 & 0 & 0 & 0 & 0 & 0 & 0 & 0 & 0 \\
\hline 29 & 0 & 0 & 0 & 0 & 0 & 0 & 0 & 0 & 0 & 0 & 0 & 0 \\
\hline 30 & 0 & 0 & 0 & 0 & ----- & 0 & 0 & 0 & 0 & 0 & 0 & 0 \\
\hline 31 & 0 & ----- & 0 & 0 & ----- & 0 & ------ & 0 & ----- & 0 & 0 & ------ \\
\hline Total & 0 & 0 & 0.02 & 0.01 & 0 & 0 & 0 & 0 & 0 & 0 & 0 & 0 \\
\hline Mean & 0 & 0 & .001 & 0 & 0 & 0 & 0 & 0 & 0 & 0 & 0 & 0 \\
\hline Max & 0 & 0 & .02 & .01 & 0 & 0 & 0 & 0 & 0 & 0 & 0 & 0 \\
\hline Min & 0 & 0 & 0 & 0 & 0 & 0 & 0 & 0 & 0 & 0 & 0 & 0 \\
\hline Acre-Ft & 0 & 0 & .04 & .02 & 0 & 0 & 0 & 0 & 0 & 0 & 0 & 0 \\
\hline Wtr Year & 2008 & Total & 0.03 & Mean & & 0 & Max & .02 & Min & 0 & Acre-Ft & .06 \\
\hline Cal Year & 2007 & Total & 0.04 & Mean & & 0 & Max & .02 & Min & 0 & Acre-Ft & .08 \\
\hline
\end{tabular}




\section{E230 Cañada del Buey above SR 4}

Location. Lat $35^{\circ}$ 49' 38", long $106^{\circ} 12^{\prime}$ 43", Sec. 33, T. 19 N., R. 7 E., Ramon Vigil Grant, Los Alamos County.

Drainage Area. $2.15 \mathrm{mi}^{2}$.

Period of Record. October 1991 to September 30, 2008.

Revised Record. Drainage area (2006).

Gage. Data logger with cellular telemetry and concrete control. Elevation of gage is 6,395 ft above NGVD from GPS survey.

Remarks. Records are good. Legal location based on projected values.

Average Discharge. $14 \mathrm{yr}, 0.009 \mathrm{ft}^{3} / \mathrm{s}, 6.52$ acre- $\mathrm{ft} / \mathrm{yr}$.

Extremes for Period of Record. Maximum discharge $210 \mathrm{ft}^{3} / \mathrm{s}$, June 17, 1999, gage height $3.30 \mathrm{ft}$. No flow most of the time.

Extremes for Current Year. Peak discharges above base of $20 \mathrm{ft}^{3} / \mathrm{s}$ and maximum (*):

\begin{tabular}{|c|c|c|c|}
\hline Date & Time & Discharge (ft $\left.\mathbf{~}^{\mathbf{3}} / \mathbf{s}\right)$ & Gage Height (ft) \\
\hline August 9 & 1300 & $28^{*}$ & $1.22^{*}$ \\
\hline
\end{tabular}

No flow most of the time.

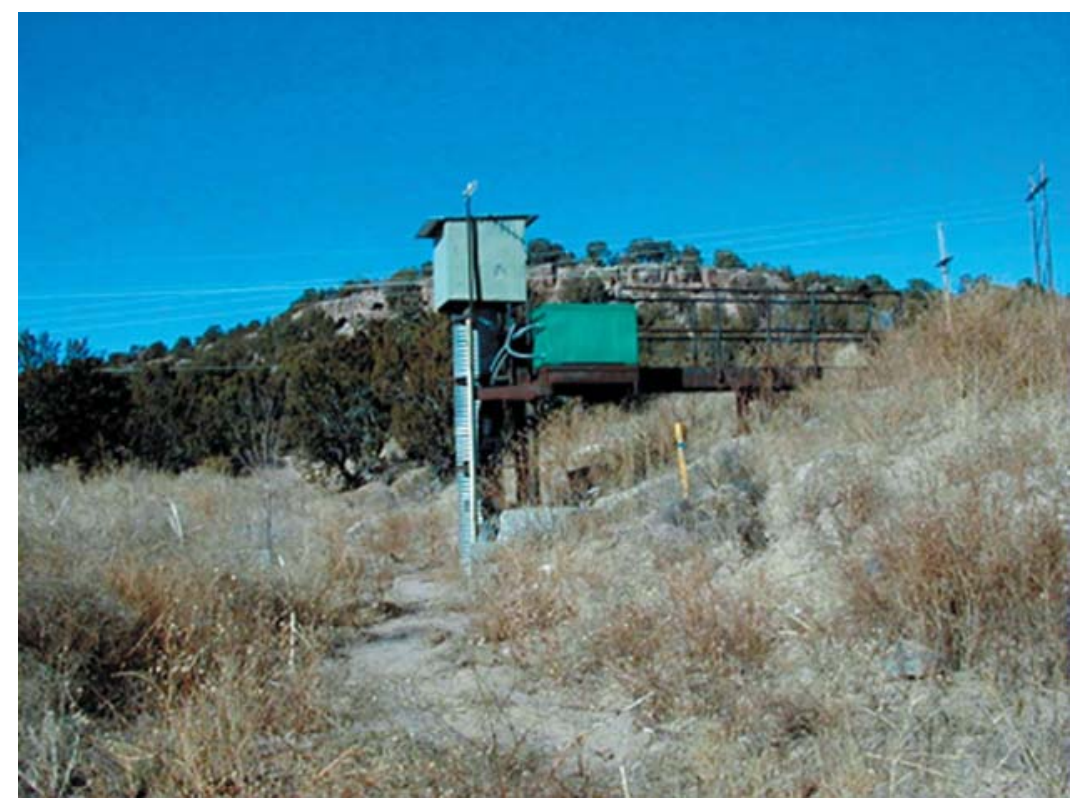




\section{E230 Cañada del Buey above SR 4}

\section{Station Analysis}

\section{Water Year}

Equipment. Station is equipped with Sutron 8210 data logger (5-min. interval) and shaft encoder float system with cellular phone and speech modem. Phone was upgraded May 14 from analog to digital service. The system is powered by a solar-panel battery system housed in a NEMA shelter on top of a 24" CMP well. Station is equipped with an ISCO pump sampler for water-quality sample collection. ISCO is housed in a separate shelter, a $3^{\prime} \times 4^{\prime}$ metal box. Sampler is triggered by stage through the data logger. A second auxiliary shelter can accommodate two additional ISCO samplers. No provision for discharge measurements above wading stage.

Field Work. This station was visited 16 times to conduct discharge measurements and service the instrumentation. Field inspections for the gage are listed under site history files on the Hydstra database. Discharge measurements for the gage are listed under site gauging files on the Hydstra database.

Datum Correction. None.

Gage-Height Record. The data logger reference to the outside staff gave a complete and satisfactory record.

Rating. Channel has fairly sharp right bend 50' above gage where two channels converge. Channel is straight for $100^{\prime}$ below gage, where it enters a rectangular double box culvert under SR 4. Control is a tapered (low-end on left) broad-crested weir.

Fifteen inspections of no flow and one inspection of flow were made during the year.

Rating No. 1 is based on discharge measurements Nos. $1-8$, which were made during previous years.

Discharge. Discharge was computed by applying gage height to Rating No. 1 directly.

Remarks. Records are good. 


\section{E230 Cañada del Buey above SR 4}

Daily Mean Discharge in Cubic Feet per Second

Water Year October 2007 to September 2008

\begin{tabular}{|c|c|c|c|c|c|c|c|c|c|c|c|c|}
\hline DAY & ОСТ & NOV & DEC & JAN & FEB & MAR & APR & MAY & JUN & JUL & AUG & SEP \\
\hline 1 & 0 & 0 & .50 & 0 & 0 & 0 & 0 & 0 & 0 & 0 & 0 & 0 \\
\hline 2 & 0 & 0 & 0 & 0 & 0 & 0 & 0 & 0 & 0 & 0 & 0 & 0 \\
\hline 3 & 0 & 0 & 0 & 0 & 0 & 0 & 0 & 0 & 0 & 0 & 0 & 0 \\
\hline 4 & 0 & 0 & 0 & 0 & 0 & 0 & 0 & 0 & 0 & 0 & 0 & 0 \\
\hline 5 & 0 & 0 & 0 & 0 & 0 & 0 & 0 & 0 & 0 & 0 & 0 & 0 \\
\hline 6 & 0 & 0 & 0 & 0 & 0 & 0 & 0 & 0 & 0 & 0 & 0 & 0 \\
\hline 7 & 0 & 0 & 0 & 0 & 0 & 0 & 0 & 0 & 0 & 0 & 0 & 0 \\
\hline 8 & 0 & 0 & 0 & 0 & 0 & 0 & 0 & 0 & 0 & 0 & 0 & 0 \\
\hline 9 & 0 & 0 & 0 & 0 & 0 & 0 & 0 & 0 & 0 & 0 & .36 & 0 \\
\hline 10 & 0 & 0 & 0 & 0 & 0 & 0 & 0 & 0 & 0 & 0 & 0 & 0 \\
\hline 11 & 0 & 0 & 0 & 0 & 0 & 0 & 0 & 0 & 0 & 0 & 0 & 0 \\
\hline 12 & 0 & 0 & 0 & 0 & 0 & 0 & 0 & 0 & 0 & 0 & 0 & 0 \\
\hline 13 & 0 & 0 & 0 & 0 & 0 & 0 & 0 & 0 & 0 & 0 & 0 & 0 \\
\hline 14 & 0 & 0 & 0 & 0 & 0 & 0 & 0 & 0 & 0 & 0 & 0 & 0 \\
\hline 15 & 0 & 0 & 0 & 0 & 0 & 0 & 0 & 0 & 0 & 0 & 0 & 0 \\
\hline 16 & 0 & 0 & 0 & 0 & 0 & 0 & 0 & 0 & 0 & 0 & 0 & 0 \\
\hline 17 & 0 & 0 & 0 & 0 & 0 & 0 & 0 & 0 & 0 & .21 & 0 & 0 \\
\hline 18 & 0 & 0 & 0 & 0 & 0 & 0 & 0 & 0 & 0 & 0 & 0 & 0 \\
\hline 19 & 0 & 0 & 0 & 0 & 0 & 0 & 0 & 0 & 0 & 0 & 0 & 0 \\
\hline 20 & 0 & 0 & 0 & 0 & 0 & 0 & 0 & 0 & 0 & 0 & 0 & 0 \\
\hline 21 & 0 & 0 & 0 & 0 & 0 & 0 & 0 & 0 & 0 & 0 & 0 & 0 \\
\hline 22 & 0 & 0 & 0 & 0 & 0 & 0 & 0 & 0 & 0 & 0 & 0 & 0 \\
\hline 23 & 0 & 0 & 0 & 0 & 0 & 0 & 0 & 0 & 0 & 0 & 0 & 0 \\
\hline 24 & 0 & 0 & 0 & 0 & 0 & 0 & 0 & 0 & 0 & 0 & 0 & 0 \\
\hline 25 & 0 & 0 & 0 & 0 & 0 & 0 & 0 & 0 & 0 & 0 & 0 & 0 \\
\hline 26 & 0 & 0 & 0 & 0 & 0 & 0 & 0 & 0 & 0 & 0 & 0 & 0 \\
\hline 27 & 0 & 0 & 0 & 0 & 0 & 0 & 0 & 0 & 0 & 0 & 0 & 0 \\
\hline 28 & 0 & 0 & 0 & 2.9 & 0 & 0 & 0 & 0 & 0 & 0 & 0 & 0 \\
\hline 29 & 0 & 0 & 0 & 0 & 0 & 0 & 0 & 0 & 0 & 0 & 0 & 0 \\
\hline 30 & 0 & .02 & 0 & 0 & ------ & 0 & 0 & 0 & 0 & 0 & 0 & 0 \\
\hline 31 & 0 & ----- & 0 & 0 & ------ & 0 & ----- & 0 & ----- & 0 & 0 & ------ \\
\hline Total & 0 & 0.02 & 0.50 & 2.9 & 0 & 0 & 0 & 0 & 0 & 0.21 & 0.36 & 0 \\
\hline Mean & 0 & .001 & .016 & .094 & 0 & 0 & 0 & 0 & 0 & .007 & .012 & 0 \\
\hline Max & 0 & .02 & .50 & 2.9 & 0 & 0 & 0 & 0 & 0 & .21 & .36 & 0 \\
\hline Min & 0 & 0 & 0 & 0 & 0 & 0 & 0 & 0 & 0 & 0 & 0 & 0 \\
\hline Acre-Ft & 0 & .04 & .99 & 5.8 & 0 & 0 & 0 & 0 & 0 & .42 & .71 & 0 \\
\hline Wtr Year & 2008 & Total & 3.99 & Mean & & 11 & hax & 2.9 & Min & 0 & Acre-Ft & 7.9 \\
\hline Cal Year & 2007 & Total & 2.20 & Mean & & 06 & Иax & .80 & Min & 0 & Acre-Ft & 4.4 \\
\hline
\end{tabular}




\section{E240 Pajarito Canyon below SR 501}

Location. Lat $35^{\circ}$ 52' 02", long $106^{\circ}$ 21' 05", NW 1/4, Sec. 19, T. 19 N., R. 6 E., Los Alamos County.

Drainage Area. $1.90 \mathrm{mi}^{2}$.

Period of Record. October 1993 to June 28, 2000 (destroyed by flood); April 2001 to September 30, 2008.

Revised Records. WDR 1997: Gage height “Extremes for Period of Record.” Drainage area (2006). Levels date published as 2004, corrected on December 2001 (this report).

Gage. Data logger with cellular telemetry. Elevation of gage is 7,719 $\mathrm{ft}$ above NGVD from GPS survey. Formerly published as "Pajarito Canyon above Highway 501 near Los Alamos, NM” at different datum.

Remarks. Records are fair.

Average Discharge. $14 \mathrm{yr}, 0.10 \mathrm{ft}^{3} / \mathrm{s}, 70$ acre-ft/yr.

Extremes for Period of Record. Maximum discharge, 1,020 $\mathrm{ft}^{3} / \mathrm{s}$, June 28, 2000, from peak-flow computation, gage height not determined. No flow at times.

Extremes for Current Year. Maximum discharge, $1.42 \mathrm{ft}^{3} / \mathrm{s}$ at $1135 \mathrm{~h}$, August 6, gage height $1.02 \mathrm{ft}$. No peak discharges above base of $5.0 \mathrm{ft}^{3} / \mathrm{s}$. No flow most of the time.

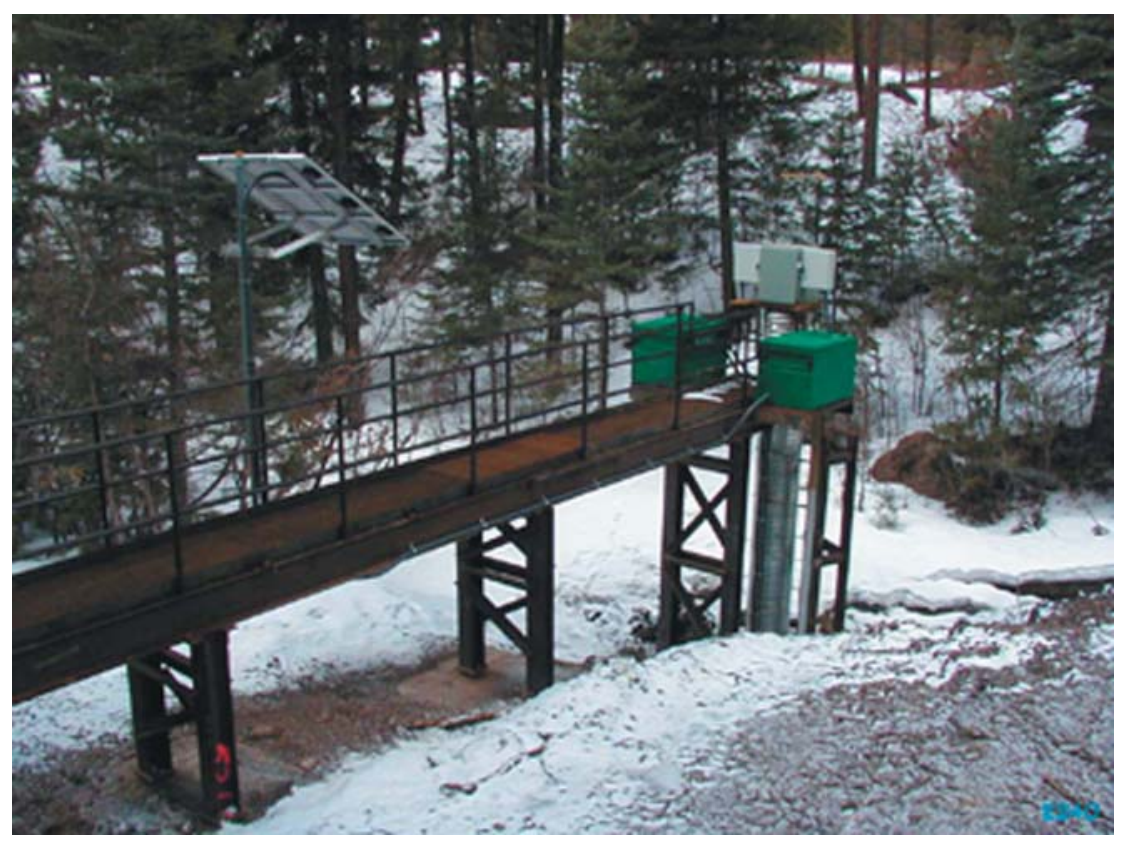




\section{E240 Pajarito Canyon below SR 501}

\section{Station Analysis}

\section{Water Year}

Equipment. Station is equipped with Sutron 8210 data logger (5-min. interval) and shaft encoder float system with cellular phone and speech modem. Phone was upgraded April 8 from analog to digital service. The system is powered by a solar-panel battery system housed in a NEMA shelter on top of a 24" CMP well. Station is equipped with an ISCO pump sampler for water-quality sample collection. ISCO is housed in a separate shelter, a $3^{\prime} \times 4^{\prime}$ metal box. Sampler is triggered by stage through the data logger. An outside staff is available for reference. No provision for discharge measurements above wading stage.

Station is also equipped with a rain gage, Rain Collection II. All equipment is powered with a solar-panel battery-charging system. Rain data are seasonal.

Field Work. This station was visited 23 times to conduct discharge measurements and service the instrumentation. Field inspections for the gage are listed under site history files on the Hydstra database. Discharge measurements for the gage are listed under site gauging files on the Hydstra database.

Datum Correction. None reported from levels run December 11, 2001.

Gage-Height Record. The data logger referenced to the outside staff gave a complete and satisfactory record except for the periods of April 8-29, July 9-17, and July 28 to August 4 when the data logger malfunctioned as a result of battery failure.

Rating. Gage is approximately 300' below outlet of two round culverts through the Highway 501 roadbed. Channel bed consists of sand and gravel and is subject to movement. Grass and brush are fairly thick over banks areas. Banks are not high (approximately 1-2' most places). Two gabions were installed in the fall of 2001; they act as low-water controls. One gabion is $2^{\prime}$ below gage across the entire width of the channel, with a 6" V notch for low water. Another gabion is 50' above gage.

Twenty-two inspections of no flow and one inspection of flow were made this year.

Rating No. 3 was developed based on the six measurements from previous years and slope area made during the year.

Discharge. Discharge was computed by applying gage height to Rating No. 3 using variable shift diagrams. Missing periods were estimated using E241.

Remarks. Records are fair. 


\section{E240 Pajarito Canyon below SR 501}

Daily Mean Discharge in Cubic Feet per Second

Water Year October 2007 to September 2008

\begin{tabular}{|c|c|c|c|c|c|c|c|c|c|c|c|c|}
\hline DAY & OCT & NOV & DEC & JAN & FEB & MAR & APR & MAY & JUN & JUL & AUG & SEP \\
\hline 1 & .02 & 0 & .16 & 0 & 0 & 0 & .03 & 0 & 0 & 0 & $0^{*}$ & 0 \\
\hline 2 & .01 & 0 & 0 & 0 & 0 & 0 & .01 & 0 & 0 & 0 & $0^{*}$ & 0 \\
\hline 3 & 0 & 0 & 0 & 0 & 0 & 0 & .01 & 0 & 0 & 0 & $0^{*}$ & 0 \\
\hline 4 & 0 & 0 & 0 & 0 & 0 & 0 & .04 & 0 & 0 & 0 & $0^{*}$ & 0 \\
\hline 5 & 0 & 0 & 0 & 0 & 0 & 0 & .05 & 0 & 0 & 0 & 0 & 0 \\
\hline 6 & 0 & 0 & 0 & 0 & 0 & 0 & .05 & 0 & 0 & 0 & .01 & 0 \\
\hline 7 & 0 & 0 & 0 & 0 & 0 & 0 & .05 & 0 & 0 & 0 & 0 & 0 \\
\hline 8 & 0 & 0 & 0 & 0 & 0 & 0 & $0^{*}$ & 0 & 0 & 0 & 0 & 0 \\
\hline 9 & 0 & 0 & 0 & 0 & 0 & 0 & $0^{*}$ & 0 & 0 & $0^{*}$ & .02 & 0 \\
\hline 10 & 0 & 0 & 0 & 0 & 0 & 0 & $0^{*}$ & 0 & 0 & $0^{*}$ & 0 & 0 \\
\hline 11 & 0 & 0 & 0 & 0 & 0 & 0 & $0^{*}$ & 0 & 0 & $0^{*}$ & 0 & 0 \\
\hline 12 & 0 & 0 & 0 & 0 & 0 & 0 & $0^{*}$ & 0 & 0 & $0^{*}$ & 0 & 0 \\
\hline 13 & 0 & 0 & 0 & 0 & 0 & 0 & $0^{*}$ & 0 & 0 & $0^{*}$ & 0 & 0 \\
\hline 14 & 0 & 0 & 0 & 0 & 0 & 0 & $0^{*}$ & 0 & 0 & $0^{*}$ & 0 & 0 \\
\hline 15 & 0 & 0 & 0 & 0 & 0 & 0 & $0^{*}$ & 0 & 0 & $0^{*}$ & 0 & 0 \\
\hline 16 & 0 & 0 & 0 & 0 & 0 & 0 & $0^{*}$ & 0 & 0 & $0^{*}$ & 0 & 0 \\
\hline 17 & 0 & 0 & 0 & 0 & 0 & 0 & $0^{*}$ & 0 & 0 & $0^{*}$ & 0 & 0 \\
\hline 18 & 0 & 0 & 0 & 0 & 0 & 0 & $0^{*}$ & 0 & 0 & 0 & 0 & 0 \\
\hline 19 & 0 & 0 & 0 & 0 & 0 & 0 & $0^{*}$ & 0 & 0 & 0 & 0 & 0 \\
\hline 20 & 0 & 0 & 0 & 0 & 0 & 0 & $0^{*}$ & 0 & 0 & 0 & 0 & 0 \\
\hline 21 & 0 & 0 & 0 & 0 & 0 & 0 & $0^{*}$ & 0 & 0 & 0 & 0 & 0 \\
\hline 22 & 0 & 0 & 0 & 0 & 0 & 0 & $0^{*}$ & 0 & 0 & 0 & 0 & 0 \\
\hline 23 & 0 & 0 & 0 & 0 & 0 & 0 & $0^{*}$ & 0 & 0 & 0 & 0 & 0 \\
\hline 24 & 0 & 0 & 0 & 0 & 0 & 0 & $0^{*}$ & 0 & 0 & 0 & 0 & 0 \\
\hline 25 & 0 & 0 & 0 & 0 & 0 & 0 & $0^{*}$ & 0 & 0 & 0 & 0 & 0 \\
\hline 26 & 0 & 0 & 0 & 0 & 0 & .01 & $0^{*}$ & 0 & 0 & 0 & 0 & 0 \\
\hline 27 & 0 & 0 & 0 & 0 & 0 & .04 & $0^{*}$ & 0 & 0 & 0 & 0 & 0 \\
\hline 28 & 0 & 0 & 0 & 0 & 0 & .05 & $0^{*}$ & 0 & 0 & $0^{*}$ & 0 & 0 \\
\hline 29 & 0 & 0 & 0 & 0 & 0 & .05 & $0^{*}$ & 0 & 0 & $0^{*}$ & 0 & 0 \\
\hline 30 & 0 & 0 & 0 & 0 & ------ & .06 & 0 & 0 & 0 & $0^{*}$ & 0 & 0 \\
\hline 31 & 0 & ----- & 0 & 0 & ------ & .06 & ------ & 0 & ------ & $0^{*}$ & 0 & ------ \\
\hline Total & 0.03 & 0 & 0.16 & 0 & 0 & 0.27 & 0.24 & 0 & 0 & 0 & 0.03 & 0 \\
\hline Mean & .001 & 0 & .005 & 0 & 0 & .009 & .008 & 0 & 0 & 0 & .001 & 0 \\
\hline Max & .02 & 0 & .16 & 0 & 0 & .06 & .05 & 0 & 0 & 0 & .02 & 0 \\
\hline Min & 0 & 0 & 0 & 0 & 0 & 0 & 0 & 0 & 0 & 0 & 0 & 0 \\
\hline Acre-Ft & .06 & 0 & .32 & 0 & 0 & .54 & .48 & 0 & 0 & 0 & .06 & 0 \\
\hline Wtr Year & 2008 & Total & 0.73 & Mean & & & $\operatorname{lax}$ & .16 & Min & 0 & Acre-Ft & 1.4 \\
\hline Cal Year & 2007 & Total & 23.91 & Mean & & & $\operatorname{lax}$ & 2.7 & Min & 0 & Acre-Ft & 47 \\
\hline
\end{tabular}

*Estimated 


\section{E240 Pajarito Canyon below SR 501}

Daily Total Rainfall in Inches

Water Year October 2007 to September 2008

\begin{tabular}{|c|c|c|c|c|c|c|c|c|c|c|c|c|}
\hline DAY & OCT & NOV & DEC & JAN & FEB & MAR & APR & MAY & JUN & JUL & AUG & SEP \\
\hline 1 & & & & & & & 0 & 0 & 0 & 0 & $0^{*}$ & .07 \\
\hline 2 & & & & & & & 0 & 0 & 0 & 0 & $0^{*}$ & 0 \\
\hline 3 & & & & & & & 0 & 0 & 0 & .05 & $0^{*}$ & .01 \\
\hline 4 & & & & & & & 0 & 0 & 0 & 0 & $.45^{\star}$ & 0 \\
\hline 5 & & & & & & & 0 & 0 & .06 & .04 & 0 & 0 \\
\hline 6 & & & & & & & 0 & $0^{*}$ & 0 & .04 & .75 & 0 \\
\hline 7 & & & & & & & 0 & 0 & 0 & .47 & .16 & 0 \\
\hline 8 & & & & & & & $0^{*}$ & 0 & 0 & .42 & .55 & 0 \\
\hline 9 & & & & & & & $.03^{*}$ & 0 & 0 & $0^{*}$ & .40 & 0 \\
\hline 10 & & & & & & & $0^{*}$ & 0 & 0 & $0^{*}$ & .07 & 0 \\
\hline 11 & & & & & & & $0^{*}$ & 0 & 0 & $.05^{\star}$ & 0 & 0 \\
\hline 12 & & & & & & & $.01^{*}$ & 0 & 0 & $0^{*}$ & 0 & 0 \\
\hline 13 & & & & & & & $0^{*}$ & .06 & 0 & $0^{*}$ & 0 & 0 \\
\hline 14 & & & & & & & $0^{*}$ & .27 & 0 & $0^{*}$ & 0 & 0 \\
\hline 15 & & & & & & & $0^{*}$ & .64 & 0 & $.04^{\star}$ & .01 & 0 \\
\hline 16 & & & & & & & $0^{*}$ & 0 & 0 & $.29^{\star}$ & .37 & 0 \\
\hline 17 & & & & & & & $.10^{*}$ & 0 & 0 & $.39^{\star}$ & .09 & 0 \\
\hline 18 & & & & & & & $0^{*}$ & 0 & 0 & .11 & 0 & .08 \\
\hline 19 & & & & & & & $0^{*}$ & 0 & 0 & 0 & 0 & .03 \\
\hline 20 & & & & & & & $0^{*}$ & 0 & .01 & 0 & .09 & .02 \\
\hline 21 & & & & & & & $0^{*}$ & 0 & 0 & .67 & 0 & 0 \\
\hline 22 & & & & & & & $0^{*}$ & .27 & 0 & 0 & 0 & .16 \\
\hline 23 & & & & & & & $0^{*}$ & .25 & 0 & 0 & .43 & .01 \\
\hline 24 & & & & & & & $0^{*}$ & .01 & 0 & .09 & .36 & 0 \\
\hline 25 & & & & & & & $0^{*}$ & 0 & 0 & 0 & .44 & 0 \\
\hline 26 & & & & & & & $0^{*}$ & 0 & 0 & .11 & 0 & 0 \\
\hline 27 & & & & & & & $0^{*}$ & 0 & 0 & .01 & 0 & 0 \\
\hline 28 & & & & & & & $0^{*}$ & .35 & 0 & $.02^{\star}$ & 0 & 0 \\
\hline 29 & & & & & & & $0^{*}$ & 0 & .02 & $0^{*}$ & 0 & 0 \\
\hline 30 & & & & & ------ & & 0 & 0 & 0 & $0^{*}$ & .01 & 0 \\
\hline 31 & & ------ & & & ----- & & ------ & 0 & ----- & $0^{*}$ & 1.54 & ------ \\
\hline Total & & & & & & & 0 & 2.44 & 0.09 & 2.05 & 5.23 & 0.38 \\
\hline $\operatorname{Max}$ & & & & & & & 0 & .64 & .06 & .67 & 1.5 & .16 \\
\hline Wtr Year & 2008 & Total & 10.70 & Mean & & .033 & Max & 1.5 & Min & 0 & InstMax & .59 \\
\hline Cal Year & 2007 & Total & 15.92 & Mean & & .045 & Max & 1.3 & Min & 0 & InstMax & .35 \\
\hline
\end{tabular}

*Estimate 


\section{E241 Pajarito Canyon above Starmer's Gulch}

Location. Lat. 3551'33.6", long 106²0'12.6", SW 1/4, Sec. 20, T. 19 N., R. 6 E., Los Alamos County.

Drainage Area. $3.97 \mathrm{mi}^{2}$.

Period of Record. March 1999 to June 28, 2000 (destroyed by flood); July 2001 to September 30, 2008.

Revised Record. Drainage area (2006).

Gage. Data logger and $90^{\circ}$ sharp-crested weir. Elevation of gage is $7,382 \mathrm{ft}$ above NGVD from GPS survey.

Remarks. Records are good.

Average Discharge. $8 \mathrm{yr}, 0.06 \mathrm{ft}^{3} / \mathrm{s}, 43$ acre-ft/yr.

Extremes for Period of Record. Maximum discharge, $300 \mathrm{ft}^{3} / \mathrm{s}$, June 28, 2000, from peak-flow computation, gage height $5.0 \mathrm{ft}$. No flow at times.

Extremes for Current Year. Peak discharges above base of $10 \mathrm{ft}^{3} / \mathrm{s}$ and maximum (*):

\begin{tabular}{|c|c|c|c|}
\hline Date & Time & Discharge $\left.\mathbf{( f t}^{\mathbf{3}} / \mathbf{s}\right)$ & Gage Height (ft) \\
\hline December 1 & 0520 & $21^{*}$ & $2.14^{*}$ \\
\hline August 8 & 1320 & 15 & 1.97 \\
\hline August 31 & 1530 & 14 & 1.92 \\
\hline
\end{tabular}

No flow at times.

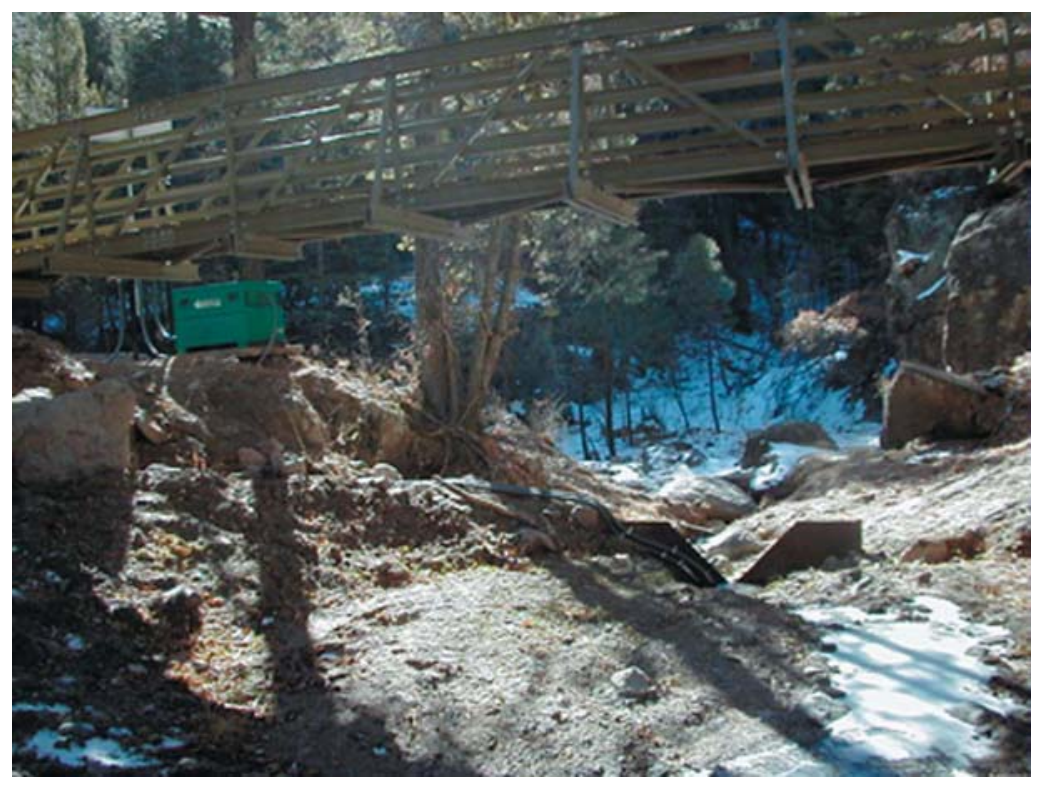




\section{E241 Pajarito Canyon above Starmer's Gulch}

\section{Station Analysis}

\section{Water Year}

Equipment. Station is equipped with Sutron 8210 data logger (5-min. interval) with Sutron Accubar bubble sensor. The system is powered by a solar-panel battery system housed in a NEMA shelter. Station is equipped with an ISCO pump sampler for waterquality sample collection. ISCO is housed in a separate shelter, a $3^{\prime} \times 4^{\prime}$ metal box. An outside staff is available for reference. Gage was rebuilt after destruction by a flood that took place on June 28, 2000. Recording instruments are the same. Footbridge was installed as part of reconstruction and may be used for high-flow measurements and sampling. Bridge also provides access to gage E242 during high-flow periods.

Field Work. The station was visited 15 times to conduct discharge measurements and service the instrumentation. Field inspections for the gage are listed under site history files on the Hydstra database. Discharge measurements for the gage are listed under site gauging files on the Hydstra database.

Datum Correction. Most recent levels run September 22, 2005, found that the wire weight gage and PZF were within limits. No changes necessary.

Gage-Height Record. The data logger referenced to the outside staff gave a complete and satisfactory record.

Rating. The channel is straight for $100^{\prime}$ above and below weir. Banks have significant vegetation and the streambed consists of sand and gravel that is somewhat armored and firm.

Seven discharge measurements (Nos. 48-54) and fifteen inspections of flow were made this year. Observed flow is usually very little flow over the V-notch.

Rating No. 3 is based on standard $90^{\circ}$ sharp-crested weir formula verified with current measurements (No. 49 not used). Point of zero flow is $0.70 \mathrm{ft}$ from levels. Shifts defined by measurements were used for "V" diagrams. Shifts were computed and record worked to inside gage readings. Although wire weight is a reference gage, natural fall occurs between the wire weight and the orifice from filling after flow events. Effectively, the wire weight becomes a general check at this station.

Weir should be very effective, with virtually no approach velocity to interfere.

Discharge. Discharge was computed by applying gage height to Rating No. 3 directly.

Remarks. Records are good. 


\section{E241 Pajarito Canyon above Starmer's Gulch}

Daily Mean Discharge in Cubic Feet per Second

Water Year October 2007 to September 2008

\begin{tabular}{|c|c|c|c|c|c|c|c|c|c|c|c|c|}
\hline DAY & OCT & NOV & DEC & JAN & FEB & MAR & APR & MAY & JUN & JUL & AUG & SEP \\
\hline 1 & 0 & 0 & 3.3 & 0 & 0 & .01 & .01 & 0 & 0 & 0 & 0 & .01 \\
\hline 2 & 0 & 0 & .02 & 0 & 0 & .01 & .01 & 0 & 0 & 0 & 0 & 0 \\
\hline 3 & 0 & 0 & .01 & 0 & 0 & .01 & .01 & 0 & 0 & 0 & 0 & 0 \\
\hline 4 & 0 & 0 & .02 & 0 & 0 & .01 & .01 & 0 & 0 & 0 & 0 & 0 \\
\hline 5 & 0 & 0 & .01 & 0 & 0 & .01 & .01 & 0 & 0 & 0 & 0 & 0 \\
\hline 6 & 0 & 0 & .01 & 0 & 0 & .01 & .01 & 0 & 0 & 0 & 0 & 0 \\
\hline 7 & 0 & 0 & .01 & 0 & 0 & .01 & .01 & 0 & 0 & 0 & 0 & 0 \\
\hline 8 & 0 & 0 & .02 & 0 & 0 & .01 & .01 & 0 & 0 & 0 & .34 & 0 \\
\hline 9 & 0 & 0 & .01 & 0 & 0 & .01 & .01 & 0 & 0 & 0 & .14 & 0 \\
\hline 10 & 0 & 0 & .01 & 0 & 0 & .01 & .01 & 0 & 0 & 0 & .02 & 0 \\
\hline 11 & 0 & 0 & .01 & 0 & 0 & .01 & .01 & 0 & 0 & 0 & 0 & 0 \\
\hline 12 & 0 & 0 & .01 & 0 & 0 & .01 & .01 & 0 & 0 & 0 & 0 & 0 \\
\hline 13 & 0 & 0 & .01 & 0 & 0 & .01 & .01 & 0 & 0 & 0 & 0 & 0 \\
\hline 14 & 0 & 0 & .01 & 0 & .01 & .01 & .01 & 0 & 0 & 0 & 0 & 0 \\
\hline 15 & 0 & 0 & .01 & 0 & .01 & .01 & .01 & 0 & 0 & 0 & 0 & 0 \\
\hline 16 & 0 & 0 & .01 & .01 & .01 & .01 & 0 & 0 & 0 & 0 & 0 & 0 \\
\hline 17 & 0 & 0 & .01 & 0 & .01 & .01 & 0 & 0 & 0 & 0 & 0 & 0 \\
\hline 18 & 0 & 0 & .01 & 0 & .01 & .01 & 0 & 0 & 0 & 0 & 0 & 0 \\
\hline 19 & 0 & 0 & .01 & 0 & .01 & .01 & 0 & 0 & 0 & 0 & 0 & 0 \\
\hline 20 & 0 & 0 & .01 & 0 & .01 & .01 & 0 & 0 & 0 & 0 & 0 & 0 \\
\hline 21 & 0 & 0 & 0 & 0 & .01 & .01 & 0 & 0 & 0 & .02 & 0 & 0 \\
\hline 22 & 0 & 0 & 0 & 0 & .01 & .01 & 0 & 0 & 0 & 0 & 0 & 0 \\
\hline 23 & 0 & 0 & 0 & 0 & .01 & .01 & 0 & 0 & 0 & 0 & .01 & 0 \\
\hline 24 & 0 & 0 & 0 & 0 & .34 & .01 & 0 & 0 & 0 & 0 & .01 & 0 \\
\hline 25 & 0 & 0 & 0 & 0 & .04 & .01 & 0 & 0 & 0 & 0 & .01 & 0 \\
\hline 26 & 0 & 0 & 0 & 0 & .01 & .01 & 0 & 0 & 0 & 0 & 0 & 0 \\
\hline 27 & 0 & 0 & 0 & .43 & .01 & .01 & 0 & 0 & 0 & 0 & 0 & 0 \\
\hline 28 & 0 & 0 & 0 & 1.3 & .01 & .01 & 0 & .03 & 0 & 0 & 0 & 0 \\
\hline 29 & 0 & 0 & 0 & .01 & .01 & .01 & 0 & 0 & 0 & 0 & 0 & 0 \\
\hline 30 & 0 & .15 & 0 & 0 & ------ & .01 & 0 & 0 & 0 & 0 & 0 & 0 \\
\hline 31 & 0 & ----- & 0 & 0 & ----- & .01 & ----- & 0 & ----- & 0 & .29 & ----- \\
\hline Total & 0 & 0.15 & 3.52 & 1.75 & 0.52 & 0.31 & 0.15 & 0.03 & 0 & 0.02 & 0.82 & 0.01 \\
\hline Mean & 0 & .005 & .11 & .056 & .018 & .010 & .005 & .001 & 0 & .001 & .026 & 0 \\
\hline Max & 0 & .15 & 3.3 & 1.3 & .34 & .01 & .01 & .03 & 0 & .02 & .34 & .01 \\
\hline Min & 0 & 0 & 0 & 0 & 0 & .01 & 0 & 0 & 0 & 0 & 0 & 0 \\
\hline Acre-Ft & 0 & .30 & 7.0 & 3.5 & 1.0 & .61 & .30 & .06 & 0 & .04 & 1.6 & .02 \\
\hline Wtr Year & 2008 & Total & 7.28 & Mean & & 20 & Max & 3.3 & Min & 0 & Acre-Ft & 14 \\
\hline Cal Year & 2007 & Total & 12.53 & Mean & & 34 & $\operatorname{Max}$ & 3.3 & Min & 0 & Acre-Ft & 25 \\
\hline
\end{tabular}




\section{E242 Starmer's Gulch above Pajarito Canyon}

Location. Lat $35^{\circ}$ 51' 33", long $106^{\circ} 20^{\prime}$ 13", SW 1/4, Sec. 20, T. 19 N., R. 6 E., Los Alamos County.

Drainage Area. $1.03 \mathrm{mi}^{2}$.

Period of Record. March 1999 to September 30, 2008.

Revised Record. Drainage area (2006).

Gage. Data logger. Elevation of gage is 7,377 ft above NGVD from GPS survey.

Remarks. Records are good except estimated daily discharges, which are poor.

Average Discharge. $8 \mathrm{yr}, 0.24 \mathrm{ft}^{3} / \mathrm{s}, 172$ acre- $\mathrm{ft} / \mathrm{yr}$.

Extremes for Period of Record. Maximum discharge, $628 \mathrm{ft}^{3} / \mathrm{s}$, August 29, 2007, gage height $3.98 \mathrm{ft}$. No flow at times.

Extremes for Current Year. Peak discharges above base of $10 \mathrm{ft}^{3} / \mathrm{s}$ and maximum (*):

\begin{tabular}{|c|c|c|c|}
\hline Date & Time & Discharge $\left(\mathbf{f t}^{\mathbf{3}} / \mathbf{s}\right)$ & Gage Height (ft) \\
\hline December 1 & 0145 & 95 & 2.62 \\
\hline January 28 & 0440 & 69 & 2.48 \\
\hline February 24 & 1530 & 18 & 2.06 \\
\hline August 8 & 1325 & $100^{*}$ & $2.64^{*}$ \\
\hline August 31 & 1530 & 59 & 2.42 \\
\hline
\end{tabular}

Minimum daily discharge, $0.0 \mathrm{ft}^{3} / \mathrm{s}$ February 8.

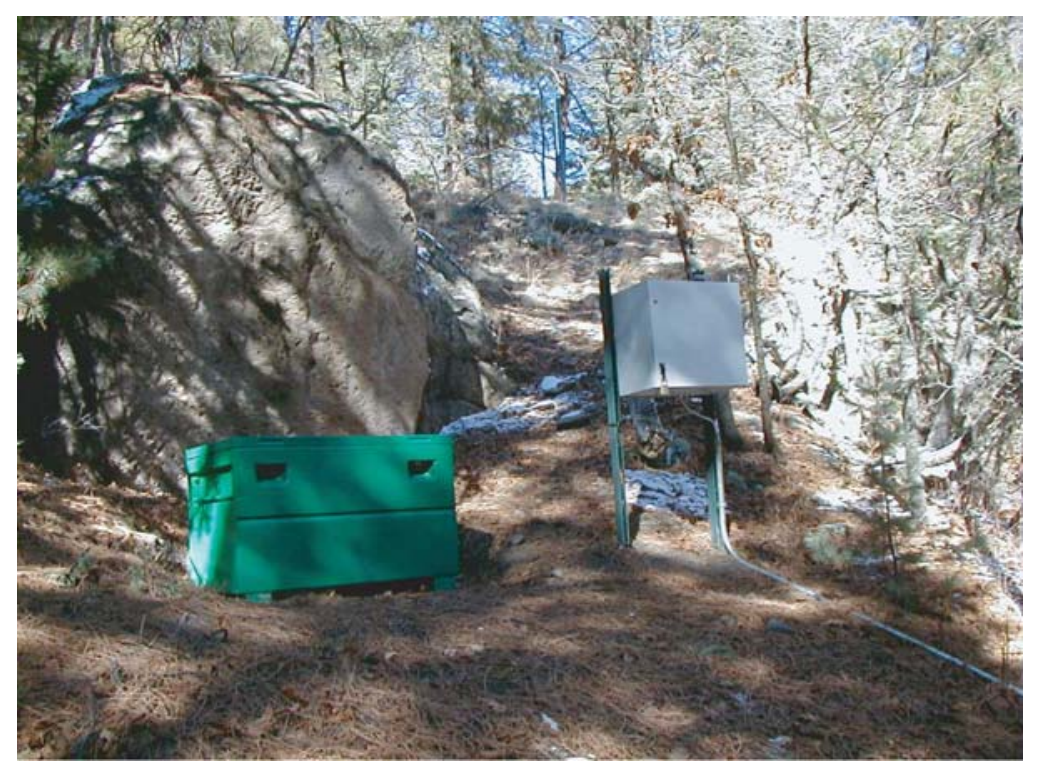




\section{E242 Starmer's Gulch above Pajarito Canyon \\ Station Analysis}

\section{Water Year}

Equipment. Station is equipped with Sutron 8210 data logger (5-min. interval) with Sutron Accubar bubble sensor and milltronics probe sensor. The system is powered by a solar-panel battery system housed in a NEMA shelter. Station is equipped with an ISCO pump sampler for water-quality sample collection. ISCO is housed in a separate shelter, a $3^{\prime} \times 4^{\prime}$ metal box. An outside staff is available for reference. No provision for discharge measurements above wading stage.

Cellular telemetry removed March 19, discontinued service.

Field Work. The station was visited 17 times to conduct discharge measurements and service the instrumentation. Field inspections for the gage are listed under site history files on the Hydstra database. Discharge measurements for the gage are listed under site gauging files on the Hydstra database.

Datum Correction. Last levels run on September 22, 2005, found the gage within limits.

Gage-Height Record. The data logger reference to the outside staff gave a complete and satisfactory record except for the periods of January 2 to February 11, February 17-19, February 22 and 23, and February 27 to March 3 when ice affected gage height and May 30 to June 9 when the gage malfunctioned as a result of probe failure.

Rating. The channel is approximately $13^{\prime}$ wide and straight for about 100 ' upstream and straight for approximately 50' downstream then bends to the left. The streambed through this reach consists of rock with cobbles. The right and left bank are covered with scrub oak and some conifers.

Eight discharge measurements (Nos. 53-60) and 17 inspections were made this water year.

Rating No. 4 was used all year. Stage application of shifts is illustrated by "V" diagrams and time application of shifts. All measurements were low (less than $1.0 \mathrm{ft}^{3} / \mathrm{s}$ ) and checked using Rating 4 within $0.10 \mathrm{ft}$ of shift. Rating 4 is considered good.

Discharge. Discharge was computed by applying gage height to Rating No. 4 directly with "V" diagrams.

Remarks. Records are good except for estimated daily discharges, which are poor. 


\section{E242 Starmer's Gulch above Pajarito Canyon}

Daily Mean Discharge in Cubic Feet per Second

Water Year October 2007 to September 2008

\begin{tabular}{|c|c|c|c|c|c|c|c|c|c|c|c|c|}
\hline DAY & ОСТ & NOV & DEC & JAN & FEB & MAR & APR & MAY & JUN & JUL & AUG & SEP \\
\hline 1 & .04 & .08 & 14 & 0 & $.03^{*}$ & $2.5^{*}$ & 2.4 & .35 & $.18^{*}$ & .26 & .05 & .83 \\
\hline 2 & .04 & .07 & .87 & $0^{*}$ & $0^{*}$ & $2.4^{*}$ & 2.6 & .40 & $.17^{*}$ & .24 & .04 & .39 \\
\hline 3 & .02 & .07 & 1.6 & $0^{*}$ & $0^{*}$ & $2.8^{*}$ & 2.4 & .43 & $.19 *$ & .24 & .04 & .36 \\
\hline 4 & .02 & .08 & 1.4 & $0^{*}$ & $0^{*}$ & 3.2 & 2.4 & .43 & $.20^{\star}$ & .19 & .06 & .19 \\
\hline 5 & .02 & .07 & .81 & $0^{*}$ & $0^{*}$ & 2.7 & 2.5 & .40 & $.17^{*}$ & .20 & .10 & .07 \\
\hline 6 & .01 & .01 & .53 & $0^{*}$ & $0^{*}$ & 2.0 & 2.3 & .37 & $.18^{*}$ & .23 & .10 & .04 \\
\hline 7 & .01 & .01 & .38 & $0^{*}$ & $0^{*}$ & 2.1 & 2.2 & .25 & $.17^{\star}$ & .23 & .10 & .03 \\
\hline 8 & .01 & 0 & .96 & $0^{*}$ & $0^{*}$ & $1.5^{*}$ & 2.0 & .27 & $.19 *$ & .21 & 2.7 & .03 \\
\hline 9 & .01 & 0 & .88 & $0^{*}$ & $.02^{*}$ & $1.3^{*}$ & 2.0 & .33 & $.24^{*}$ & .20 & .56 & .02 \\
\hline 10 & 0 & 0 & .95 & $.01^{*}$ & $.04^{*}$ & $1.2^{*}$ & 2.0 & .20 & .28 & .20 & .26 & .02 \\
\hline 11 & 0 & 0 & .94 & $.02 *$ & $.10^{*}$ & 1.1 & 1.7 & .31 & .52 & .22 & .11 & .02 \\
\hline 12 & 0 & 0 & .96 & $.03^{*}$ & .50 & .99 & 1.7 & .27 & .48 & .18 & .06 & .01 \\
\hline 13 & 0 & 0 & 1.0 & $.04^{*}$ & 1.0 & 1.1 & 1.4 & .26 & .48 & .12 & .05 & .02 \\
\hline 14 & .01 & 0 & .85 & $.02^{*}$ & 1.5 & 1.2 & 1.3 & .36 & .46 & .12 & .04 & .01 \\
\hline 15 & .01 & 0 & .61 & $.03^{*}$ & 1.6 & 1.8 & 1.1 & .45 & .45 & .13 & .04 & .01 \\
\hline 16 & .01 & 0 & .61 & $.04^{*}$ & 1.7 & 2.1 & .76 & .34 & .46 & .16 & .05 & .01 \\
\hline 17 & .01 & .01 & .42 & $.03 *$ & $1.1^{*}$ & 2.0 & 1.0 & .33 & .45 & .21 & .05 & .01 \\
\hline 18 & .02 & 0 & .24 & $.02 *$ & $1.2^{*}$ & 1.9 & .90 & .33 & .41 & .20 & .04 & .01 \\
\hline 19 & .03 & 0 & .18 & $.01^{*}$ & $1.1^{*}$ & 1.7 & 1.0 & .24 & .35 & .13 & .03 & .02 \\
\hline 20 & .03 & .01 & .10 & $.04^{*}$ & 1.2 & 1.6 & .84 & .23 & .42 & .16 & .04 & .02 \\
\hline 21 & .02 & 0 & .06 & $.03^{*}$ & .98 & 1.5 & .78 & .17 & .36 & .25 & .03 & .02 \\
\hline 22 & .04 & 0 & .03 & $.02 *$ & $1.0^{*}$ & 1.5 & .75 & .19 & .36 & .18 & .03 & .01 \\
\hline 23 & .05 & 0 & .03 & $.01^{*}$ & $1.4^{*}$ & 1.6 & .65 & .24 & .34 & .13 & .04 & .01 \\
\hline 24 & .04 & 0 & .03 & $.03^{*}$ & 2.7 & 1.7 & .65 & .25 & .33 & .14 & .05 & .01 \\
\hline 25 & .05 & .01 & .01 & $.02^{*}$ & 1.4 & 1.8 & .51 & .23 & .29 & .10 & .14 & .01 \\
\hline 26 & .04 & 0 & .01 & $.05^{\star}$ & 1.8 & 1.7 & .56 & .23 & .25 & .12 & .09 & .01 \\
\hline 27 & .04 & 0 & 0 & $.02^{*}$ & $2.0^{*}$ & 1.5 & .50 & .14 & .25 & .11 & .05 & .01 \\
\hline 28 & .04 & 0 & 0 & $5.8^{*}$ & $2.2^{*}$ & 1.8 & .55 & .27 & .21 & .08 & .04 & .01 \\
\hline 29 & .05 & 0 & 0 & $1.0^{*}$ & $2.2^{*}$ & 2.1 & .51 & .20 & .25 & .07 & .03 & .01 \\
\hline 30 & .05 & .48 & 0 & $.50^{*}$ & ----- & 2.1 & .39 & $.18^{*}$ & .22 & .06 & .03 & .01 \\
\hline 31 & .05 & ------ & 0 & $.04^{*}$ & ------ & 2.2 & ------ & $.17^{*}$ & ------ & .06 & 2.2 & ------ \\
\hline Total & 0.77 & 0.90 & 28.46 & 7.81 & 26.77 & 56.69 & 40.35 & 8.82 & 9.31 & 5.13 & 7.25 & 2.23 \\
\hline Mean & .025 & .030 & .92 & .25 & .92 & 1.83 & 1.35 & .28 & .31 & .17 & .23 & .074 \\
\hline $\operatorname{Max}$ & .05 & .48 & 14 & 5.8 & 2.7 & 3.2 & 2.6 & .45 & .52 & .26 & 2.7 & .83 \\
\hline Min & 0 & 0 & 0 & 0 & 0 & .99 & .39 & .14 & .17 & .06 & .03 & .01 \\
\hline Acre-Ft & 1.5 & 1.8 & 56 & 15 & 53 & 112 & 80 & 17 & 18 & 10 & 14 & 4.4 \\
\hline Wtr Year & 2008 & Total & 194.49 & & & .53 & Max & 14 & Min & 0 & Acre-Ft & 386 \\
\hline Cal Year & 2007 & Total & 246.10 & & & .67 & $\operatorname{Max}$ & 14 & Min & 0 & Acre-Ft & 488 \\
\hline
\end{tabular}

*Estimate 


\section{E2425 Arroyo de la Delfe above Pajarito Canyon}

Location. Lat 35 51' 25", long $106^{\circ}$ 19' 56", NW 1/4, Sec. 29, T. 19 N., R. 6 E., Ramon Vigil Grant, Los Alamos County.

Drainage Area. $0.15 \mathrm{mi}^{2}$.

Period of Record. June 1, 2000, to September 30, 2008.

Revised Record. Drainage area (2006).

Gage. Data logger and $90^{\circ}$ sharp-crested weir. Elevation of gage is $7,311 \mathrm{ft}$ above NGVD from GPS survey.

Remarks. Records are fair.

Average Discharge. $8 \mathrm{yr}, 0.04 \mathrm{ft}^{3} / \mathrm{s}, 29$ acre-ft/yr.

Extremes for Period of Record. Maximum discharge, $30 \mathrm{ft}^{3} / \mathrm{s}$, August 24, 2005, gage height $2.64 \mathrm{ft}$. No flow at times.

Extremes for Current Year. Peak discharge above base of $5.0 \mathrm{ft}^{3} / \mathrm{s}$ and maximum (*):

\begin{tabular}{|c|c|c|c|}
\hline Date & Time & Discharge (ft $\left.\mathbf{3}^{\mathbf{3}} / \mathbf{s}\right)$ & Gage Height (ft) \\
\hline August 8 & 1335 & $6.0^{*}$ & $1.87^{*}$ \\
\hline
\end{tabular}

No flow most of the time.

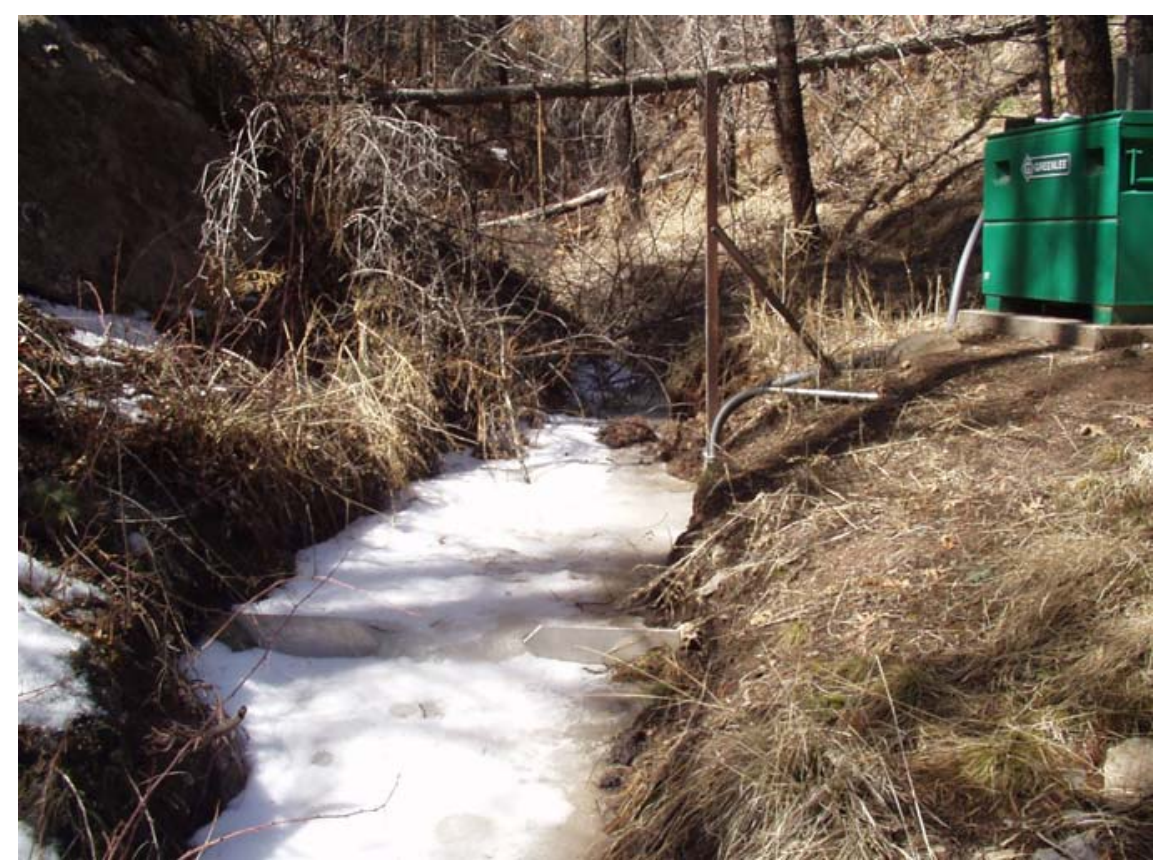




\section{E2425 Arroyo de la Delfe above Pajarito Canyon \\ Station Analysis}

\section{Water Year}

Equipment. Station is equipped with Sutron 8210 data logger (5-min. interval) with Sutron Accubar bubble sensor. The system is powered by a solar-panel battery system housed in a NEMA shelter. Station is equipped with an ISCO pump sampler for waterquality sample collection. ISCO is housed in a separate shelter, a $3^{\prime} \times 4^{\prime}$ metal box. Sampler is triggered by stage through the data logger. An outside staff is available for reference. No provision for measurements above wading stage.

Field Work. The station was visited 15 times to conduct discharge measurements and service the instrumentation. Field inspections for the gage are listed under site history files on the Hydstra database. Discharge measurements for the gage are listed under site gauging files on the Hydstra database.

Datum Correction. None.

Gage-Height Record. The data logger referenced to the outside staff gave a complete and satisfactory record.

Rating. Channel is straight and narrow above and below gage. Bed material consists of sand and coarse gravel. Control is $90^{\circ}$ sharp-crested weir plate.

Seven discharge measurements (No. 24-30) and 15 inspections of flow were made this year.

Rating 1 was continued in use. "V" diagrams were used to apply shift and nothing but low flow occurred during the shifting period.

Discharge. Discharge was computed by applying gage height to Rating No.1 with variable shift diagrams.

Remarks. Records are fair. 


\section{E2425 Arroyo de La Delfe above Pajarito Canyon}

Daily Mean Discharge in Cubic Feet per Second

Water Year October 2007 to September 2008

\begin{tabular}{|c|c|c|c|c|c|c|c|c|c|c|c|c|}
\hline DAY & OCT & NOV & DEC & JAN & FEB & MAR & APR & MAY & JUN & JUL & AUG & SEP \\
\hline 1 & .03 & .01 & 1.0 & .02 & .01 & .11 & .04 & .03 & .02 & .01 & .01 & .07 \\
\hline 2 & .03 & .01 & .15 & .02 & .01 & .11 & .04 & .03 & .02 & .01 & .01 & .03 \\
\hline 3 & .03 & .01 & .08 & .02 & .01 & .09 & .04 & .03 & .02 & .01 & .01 & .03 \\
\hline 4 & .03 & .01 & .07 & .02 & .01 & .08 & .04 & .03 & .02 & .01 & .01 & .02 \\
\hline 5 & .03 & .01 & .06 & .02 & .01 & .08 & .04 & .03 & .02 & .01 & .01 & .02 \\
\hline 6 & .02 & .01 & .05 & .02 & .01 & .07 & .04 & .03 & .02 & .01 & .01 & .02 \\
\hline 7 & .02 & .01 & .05 & .02 & .01 & .06 & .04 & .02 & .02 & .01 & .01 & .01 \\
\hline 8 & .02 & .01 & .08 & .02 & .01 & .06 & .04 & .02 & .02 & .01 & .21 & .01 \\
\hline 9 & .02 & .01 & .13 & .01 & .01 & .06 & .04 & .03 & .02 & .01 & .19 & .01 \\
\hline 10 & .02 & .01 & .10 & .01 & .01 & .06 & .04 & .03 & .02 & .01 & .13 & .01 \\
\hline 11 & .02 & .01 & .09 & .01 & .01 & .06 & .04 & .03 & .02 & .01 & .07 & .01 \\
\hline 12 & .02 & .01 & .09 & .01 & .01 & .07 & .04 & .03 & .02 & .01 & .03 & .01 \\
\hline 13 & .02 & .01 & .07 & .01 & .04 & .07 & .04 & .02 & .02 & .01 & .02 & .01 \\
\hline 14 & .01 & .01 & .07 & .01 & .11 & .08 & .04 & .03 & .01 & .01 & .02 & .01 \\
\hline 15 & .01 & .01 & .06 & .02 & .11 & .07 & .03 & .03 & .01 & .01 & .02 & .01 \\
\hline 16 & .01 & .01 & .05 & .01 & .12 & .06 & .03 & .03 & .01 & .01 & .02 & .01 \\
\hline 17 & .01 & .01 & .04 & .02 & .08 & .06 & .03 & .02 & .01 & .01 & .02 & .01 \\
\hline 18 & .01 & .01 & .04 & .02 & .06 & .06 & .04 & .02 & .01 & .01 & .01 & .01 \\
\hline 19 & .01 & .01 & .03 & .02 & .06 & .05 & .03 & .02 & .01 & .01 & .01 & .01 \\
\hline 20 & .01 & .01 & .03 & .02 & .06 & .05 & .03 & .02 & .01 & .01 & .01 & .01 \\
\hline 21 & .01 & .01 & .03 & .02 & .06 & .05 & .03 & .02 & .01 & .02 & .01 & .01 \\
\hline 22 & .01 & .01 & .03 & .01 & .07 & .05 & .03 & .02 & .01 & .02 & .01 & .01 \\
\hline 23 & .01 & .01 & .03 & 0 & .07 & .05 & .03 & .02 & .01 & .01 & .01 & .01 \\
\hline 24 & .01 & .01 & .03 & 0 & .21 & .05 & .03 & .02 & .01 & .01 & .01 & .01 \\
\hline 25 & .01 & .01 & .03 & 0 & .16 & .04 & .03 & .02 & .01 & .01 & .02 & .01 \\
\hline 26 & .01 & .01 & .03 & 0 & .12 & .04 & .03 & .02 & .01 & .01 & .02 & .01 \\
\hline 27 & .01 & .01 & .02 & .01 & .10 & .04 & .03 & .02 & .01 & .01 & .01 & .01 \\
\hline 28 & .01 & .01 & .02 & .13 & .10 & .04 & .03 & .03 & .01 & .01 & .01 & .01 \\
\hline 29 & .01 & .01 & .02 & .01 & .10 & .04 & .03 & .02 & .01 & .01 & .01 & .01 \\
\hline 30 & .01 & .03 & .02 & .02 & ----- & .04 & .03 & .02 & .01 & .01 & .01 & .01 \\
\hline 31 & .01 & ----- & .02 & .02 & ----- & .04 & ----- & .02 & ----- & .01 & .09 & ----- \\
\hline Total & 0.49 & 0.32 & 2.62 & 0.55 & 1.75 & 1.89 & 1.05 & 0.76 & 0.43 & 0.33 & 1.04 & 0.43 \\
\hline Mean & .016 & .011 & .085 & .018 & .060 & .061 & .035 & .025 & .014 & .011 & .034 & .014 \\
\hline $\operatorname{Max}$ & .03 & .03 & 1.0 & .13 & .21 & .11 & .04 & .03 & .02 & .02 & .21 & .07 \\
\hline Min & .01 & .01 & .02 & 0 & .01 & .04 & .03 & .02 & .01 & .01 & .01 & .01 \\
\hline Acre-Ft & .97 & .63 & 5.2 & 1.1 & 3.5 & 3.7 & 2.1 & 1.5 & .85 & .65 & 2.1 & .85 \\
\hline Wtr Year & 2008 & Total & 11.66 & Mean & & 32 & Max & 1.0 & Min & 0 & Acre-Ft & 23 \\
\hline Cal Year & 2007 & Total & 20.01 & Mean & & 55 & Max & 1.0 & Min & .01 & Acre-Ft & 40 \\
\hline
\end{tabular}




\section{E243 Pajarito Canyon above Two Mile Canyon}

Location. Lat $35^{\circ}$ 51' 14", long $106^{\circ}$ 17' 48", Sec. 27, T. 19N., R. 6 E., Ramon Vigil Grant, Los Alamos County.

Drainage Area. $4.24 \mathrm{mi}^{2}$.

Period of Record. February 2002 to September 30, 2008.

Revisions. Drainage area (2006).

Gage. Data logger with cellular telemetry. Elevation of gage 6,941 ft above NGVD from GPS survey.

Remarks. Records are good except estimated daily discharges, which are fair. Legal location based on projected values.

Average Discharge. $6 y \mathrm{r}, 0.26 \mathrm{ft}^{3} / \mathrm{s}, 187$ acre- $\mathrm{ft} / \mathrm{yr}$.

Extremes for Period of Record. Maximum discharge, $272 \mathrm{ft}^{3} / \mathrm{s}$, August 24, 2005, gage height $4.38 \mathrm{ft}$. No flow most of the time.

Extremes for Current Year. Peak discharges above base of $5.0 \mathrm{ft}^{3} / \mathrm{s}$ and maximum (*):

\begin{tabular}{|c|c|c|c|}
\hline Date & Time & Discharge $\left.\mathbf{( f t}^{\mathbf{3}} / \mathbf{s}\right)$ & Gage Height (ft) \\
\hline December 1 & 0225 & 57 & 2.22 \\
\hline January 28 & 0940 & $102^{*}$ & $2.43^{*}$ \\
\hline February 24 & 1625 & 34 & 2.00 \\
\hline August 8 & 1440 & 19 & 1.86 \\
\hline August 9 & 1400 & 12 & 1.74 \\
\hline August 10 & 1140 & 39 & 2.08 \\
\hline
\end{tabular}

No flow most of the time.

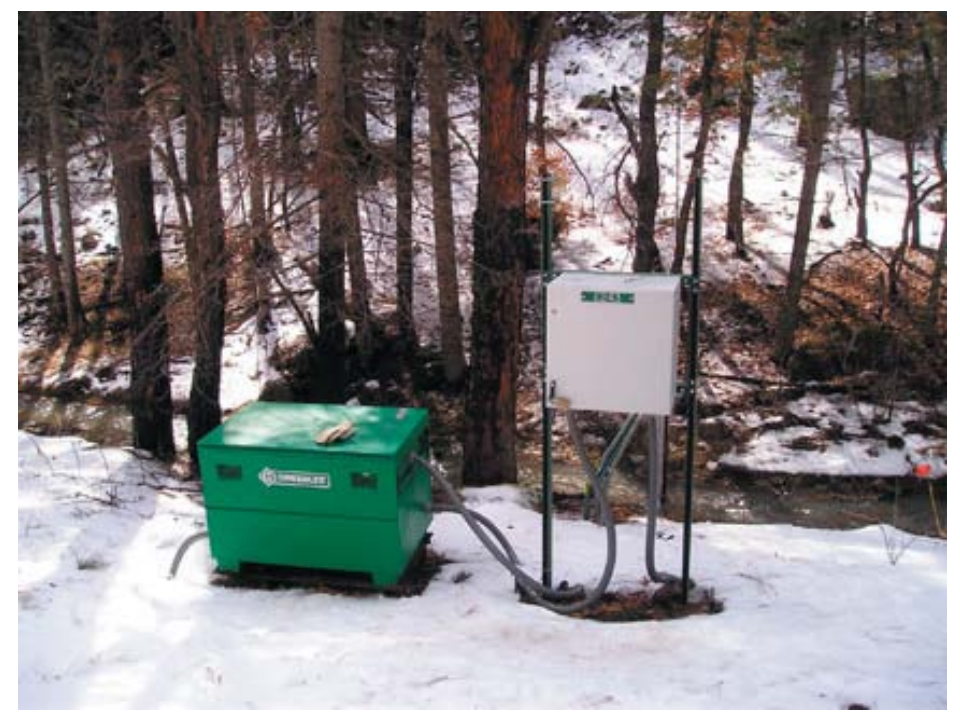




\section{E243 Pajarito Canyon above Two Mile Canyon}

\section{Station Analysis}

\section{Water Year}

Equipment. Station is equipped with Sutron 8210 data logger (5-min. interval) with Sutron Accubar bubble sensor with cellular telemetry and speech modem. Phone upgraded July 24 from analog to digital service. The system is powered by a solar-panel battery system housed in a NEMA shelter. Station is equipped with an ISCO pump sampler for water-quality sample collection. ISCO is housed in a separate shelter, a $3^{\prime} \times$ $4^{\prime}$ metal box. Sampler is triggered by stage through the data logger. Porcelain staff gage was available for outside reference until August 24, 2005. A steel post was installed September 1, 2005, on left bank to datum and was used as a reference point of 2.56 gage height to datum until gage reconstruction was accomplished in April 2006. No provision for direct measurement above wading stage.

Field Work. The station was visited 20 times to conduct discharge measurements and service the instrumentation. Field inspections for the gage are listed under site history files on the Hydstra database. Discharge measurements for the gage are listed under site gauging files on the Hydstra database.

Datum Correction. None. On September 1, 2005, a datum correction of $1.0 \mathrm{ft}$ lower was applied because of the scour in channel after a flood on August 24, 2005. Levels run April 7, 2006, found gage within limits, with no corrections necessary. RM 1 was lost as a result of the flood of August 24, 2005. RM 2 was used to established gage datum.

Gage-Height Record. The data logger referenced to the outside staff gave a complete and satisfactory record except during the periods of December 13 to February 7, February 12-18, and February 25 to March 3 when ice affected gage height.

Rating. The channel is straight for 150 ' above and below gage. It is trapezoidal, with the bed fairly well armored with large gravel and some cobbles. Banks are fairly well vegetated with grasses and should remain stable at all flows.

Four discharge measurements (Nos. 26-29) and five inspections of no flow were made this year.

Rating No. 4 was continued in use and considered fair.

Discharge. Discharge was computed by applying gage height to Rating No. 4, with shifts at low flow applied by "V" diagrams. PZF shows some estimated zero flow in winter from icing over orifice; during this time the stream was frozen dry.

Remarks. This station was originally at a site downstream approximately 0.5 miles. With the building of the Pajarito Flood Control Structure, it had to be moved. That site was never rated, and although gage height record is available in HYDSTA, the record for 1998-2002 will not be published. Records are good except for estimated daily discharges, which are fair. 


\section{E243 Pajarito Canyon above Two Mile Canyon}

Daily Mean Discharge in Cubic Feet per Second

Water Year October 2007 to September 2008

\begin{tabular}{|c|c|c|c|c|c|c|c|c|c|c|c|c|}
\hline DAY & ОСТ & NOV & DEC & JAN & FEB & MAR & APR & MAY & JUN & JUL & AUG & SEP \\
\hline 1 & .10 & 0 & 10 & $.09 *$ & $.10^{*}$ & $1.4^{*}$ & 1.4 & .41 & .25 & .05 & .01 & .19 \\
\hline 2 & .11 & 0 & .80 & $.08^{*}$ & $.10^{*}$ & $1.5^{\star}$ & 1.5 & .41 & .21 & .05 & .01 & .09 \\
\hline 3 & .08 & 0 & .91 & $.07^{*}$ & $.10^{*}$ & $1.5^{\star}$ & 1.5 & .40 & .18 & .05 & .01 & .07 \\
\hline 4 & .06 & 0 & .95 & $.06^{*}$ & $.10^{*}$ & 1.6 & 1.4 & .38 & .18 & .04 & .01 & .05 \\
\hline 5 & .06 & 0 & .65 & $.05^{\star}$ & $.10^{*}$ & 1.6 & 1.4 & .37 & .19 & .04 & .01 & .02 \\
\hline 6 & .05 & 0 & .43 & $.04^{*}$ & $.10^{*}$ & 1.4 & 1.4 & .35 & .18 & .04 & 0 & 0 \\
\hline 7 & .05 & 0 & .31 & $.04^{*}$ & $.10^{*}$ & 1.3 & 1.3 & .33 & .17 & .04 & 0 & 0 \\
\hline 8 & .05 & 0 & .58 & $.09^{*}$ & .08 & 1.1 & 1.3 & .31 & .15 & .03 & .78 & 0 \\
\hline 9 & .04 & 0 & .67 & $.11^{*}$ & .08 & .92 & 1.2 & .31 & .15 & .03 & .92 & 0 \\
\hline 10 & .04 & 0 & .53 & $.10^{*}$ & .08 & .85 & 1.2 & .29 & .14 & .03 & 1.7 & 0 \\
\hline 11 & .03 & 0 & .66 & $.01^{*}$ & .36 & .96 & 1.2 & .33 & .12 & .02 & .16 & 0 \\
\hline 12 & .03 & 0 & .63 & $.01^{*}$ & $.43^{*}$ & 1.1 & 1.1 & .31 & .11 & .02 & .04 & 0 \\
\hline 13 & .02 & 0 & $.60^{*}$ & $.01^{*}$ & $.81^{*}$ & 1.1 & .94 & .31 & .11 & .01 & .02 & 0 \\
\hline 14 & .02 & 0 & $.57^{*}$ & $.01^{*}$ & $.83^{*}$ & 1.3 & .82 & .35 & .10 & .01 & .01 & 0 \\
\hline 15 & .02 & 0 & $.53^{*}$ & $.01^{*}$ & $.83^{*}$ & 1.3 & .70 & .56 & .09 & 0 & .01 & 0 \\
\hline 16 & .02 & 0 & $.48^{*}$ & $.01^{*}$ & $.83^{*}$ & 1.4 & .62 & .46 & .08 & 0 & .01 & 0 \\
\hline 17 & .02 & 0 & $.45^{\star}$ & $.01^{*}$ & $.82^{*}$ & 1.4 & .66 & .42 & .08 & 0 & 0 & 0 \\
\hline 18 & .02 & 0 & $.41^{*}$ & $.01^{*}$ & $.91 *$ & 1.3 & .63 & .42 & .07 & 0 & 0 & 0 \\
\hline 19 & .02 & 0 & $.38^{*}$ & $.01^{*}$ & .96 & 1.3 & .64 & .39 & .08 & 0 & 0 & 0 \\
\hline 20 & .02 & 0 & $.35^{\star}$ & $.01^{*}$ & 1.0 & 1.3 & .63 & .35 & .08 & 0 & 0 & 0 \\
\hline 21 & .02 & 0 & $.32^{\star}$ & $.01^{*}$ & 1.0 & 1.3 & .61 & .33 & .08 & .02 & 0 & 0 \\
\hline 22 & .03 & 0 & $.29^{\star}$ & $.01^{*}$ & .99 & 1.2 & .57 & .37 & .07 & .03 & 0 & 0 \\
\hline 23 & .02 & 0 & $.27^{*}$ & $.01^{*}$ & .97 & 1.3 & .54 & .41 & .07 & .03 & 0 & 0 \\
\hline 24 & .02 & 0 & $.24^{*}$ & $.01^{*}$ & 4.7 & 1.3 & .51 & .40 & .07 & .03 & 0 & 0 \\
\hline 25 & .02 & 0 & $.21^{*}$ & $.01^{*}$ & $2.3^{*}$ & 1.2 & .50 & .38 & .07 & .03 & 0 & 0 \\
\hline 26 & .02 & 0 & $.17^{*}$ & $.01^{*}$ & $1.4^{*}$ & 1.3 & .48 & .36 & .06 & .03 & 0 & 0 \\
\hline 27 & .02 & 0 & $.15^{\star}$ & $.01^{*}$ & $1.4^{*}$ & 1.2 & .47 & .34 & .06 & .04 & 0 & 0 \\
\hline 28 & .02 & 0 & $.14^{*}$ & $1.1^{*}$ & $1.4^{*}$ & 1.2 & .45 & .61 & .06 & .04 & 0 & 0 \\
\hline 29 & .02 & 0 & $.13^{*}$ & $.10^{*}$ & $1.4^{*}$ & 1.3 & .44 & .49 & .06 & .04 & 0 & 0 \\
\hline 30 & .02 & 0 & $.11^{*}$ & $.09 *$ & ------ & 1.3 & .41 & .36 & .05 & .03 & 0 & 0 \\
\hline 31 & .02 & ------ & $.10^{*}$ & $.10^{*}$ & ------ & 1.4 & ------ & .30 & ------ & .02 & .29 & ------ \\
\hline Total & 1.09 & 0 & 23.02 & 2.29 & 24.28 & 39.63 & 26.52 & 11.81 & 3.37 & 0.80 & 3.99 & 0.42 \\
\hline Mean & .035 & 0 & .74 & .074 & .84 & 1.28 & .88 & .38 & .11 & .026 & .13 & .014 \\
\hline Max & .11 & 0 & 10 & 1.1 & 4.7 & 1.6 & 1.5 & .61 & .25 & .05 & 1.7 & .19 \\
\hline Min & .02 & 0 & .10 & .01 & .08 & .85 & .41 & .29 & .05 & 0 & 0 & 0 \\
\hline Acre-Ft & 2.2 & 0 & 46 & 4.5 & 48 & 79 & 53 & 23 & 6.7 & 1.6 & 7.9 & .83 \\
\hline Wtr Year & 2008 & Total & 137.22 & & & .37 & Max & 10 & Min & 0 & Acre-Ft & 272 \\
\hline Cal Year & 2007 & Total & 109.86 & & & .30 & $\operatorname{Max}$ & 10 & Min & 0 & Acre-Ft & 218 \\
\hline
\end{tabular}

*Estimate 
Location. Lat $35^{\circ}$ 52' 08", long $106^{\circ}$ 19' 21", NE 1/4, Sec. 20, T. 19 N., R. 6 E., Ramon Vigil Grant, Los Alamos County.

Drainage Area. $0.02 \mathrm{mi}^{2}$.

Period of Record. October 1, 2005, to September 30, 2008.

Revisions. Drainage area (2006).

Gage. Data logger and 12” Parshall flume. Elevation of gage is 7,402 ft above NGVD from land survey.

Remarks. Records are good. Records for this site existed before period of record but are not reliable.

Extremes for Period of Record. Maximum discharge, $60 \mathrm{ft}^{3} / \mathrm{s}$ July 14, 2007, gage height $2.40 \mathrm{ft}$. No flow most of the time.

Extremes for Current Year. Peak discharges above base of $6.0 \mathrm{ft}^{3} / \mathrm{s}$ and maximum (*):

\begin{tabular}{|c|c|c|c|}
\hline Date & Time & Discharge $\left.\mathbf{( f t}^{\mathbf{3}} / \mathbf{s}\right)$ & Gage Height (ft) \\
\hline May 28 & 1545 & 8.6 & 1.66 \\
\hline August 4 & 1740 & $9.7^{*}$ & $1.79 *$ \\
\hline August 8 & 1345 & 6.2 & 1.34 \\
\hline August 31 & 1520 & 9.6 & 1.78 \\
\hline
\end{tabular}

No flow most of the time.

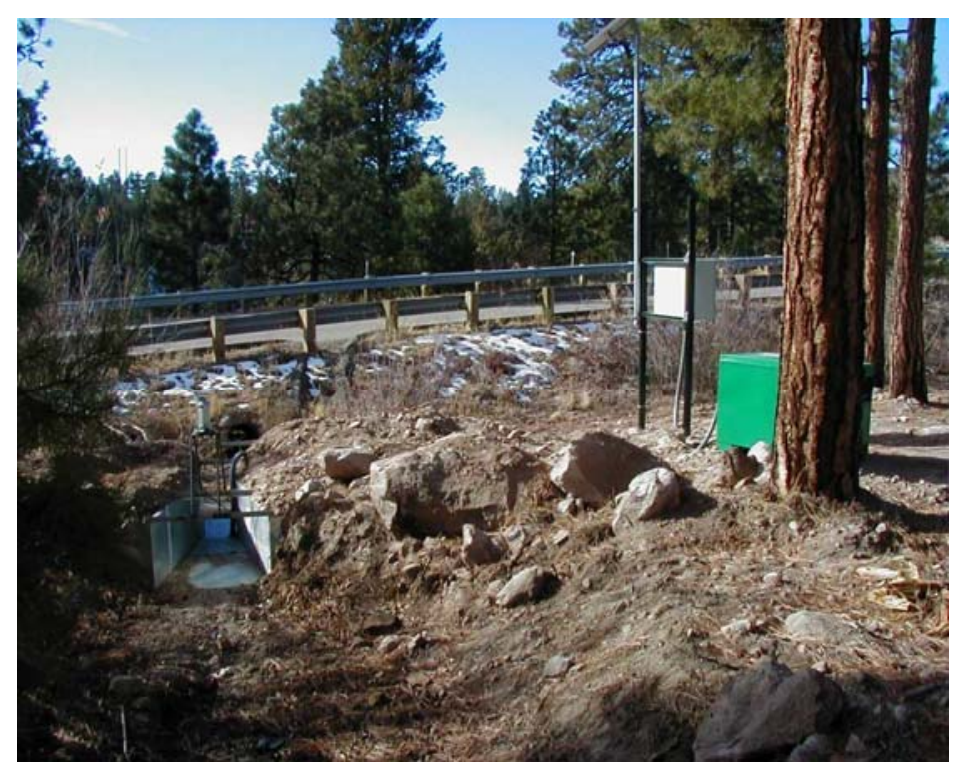




\section{Station Analysis}

\section{Water Year}

Equipment. Station is equipped with Sutron 8210 data logger (5-min. interval) and milltronics sonic probe mounted on a 12" Parshall flume. The system is powered by a solar-panel battery system housed in a NEMA shelter. Station is equipped with an ISCO pump sampler for water-quality sample collection. ISCO is housed in a separate shelter, a $3^{\prime} \times 4^{\prime}$ metal box. Sampler is triggered by stage through the data logger. The staff in the 12 " Parshall flume is the reference gage. No provision for discharge measurements above wading stage.

Field Work. The station was visited 26 times to conduct discharge measurements and service the instrumentation. Field inspections for the gage are listed under site history files on the Hydstra database. Discharge measurements for the gage are listed under site gauging files on the Hydstra database.

Datum Correction. None.

Gage-Height Record. The data logger referenced to the outside staff gave a complete and satisfactory record except for the period of September 4-31 when the data logger malfunctioned as a result of battery failure.

Rating. The channel is straight above and below gage. It is confined to the main channel by cut banks on both sides. The bottom is a $10^{\prime}$ wide channel prone to some shifting with vegetation on each bank. Low-water control is the 12" Parshall flume.

Twenty-two inspections of no flow, two inspections of snow melt, and two inspections of flow were made this year.

Rating No. 1 was developed based on the computation of 12" Parshall flume. Point of zero flow is 0.00 gage height.

Discharge. Discharge was computed by applying gage height to Rating No. 1 directly.

Remarks. Records are good. 
E2435 Two Mile Canyon Tributary at TA-3

Daily Mean Discharge in Cubic Feet per Second

Water Year October 2007 to September 2008

\begin{tabular}{|c|c|c|c|c|c|c|c|c|c|c|c|c|}
\hline DAY & OCT & NOV & DEC & JAN & FEB & MAR & APR & MAY & JUN & JUL & AUG & SEP \\
\hline 1 & .03 & 0 & .29 & 0 & 0 & 0 & 0 & 0 & 0 & 0 & 0 & 0 \\
\hline 2 & 0 & 0 & 0 & 0 & 0 & 0 & 0 & 0 & 0 & 0 & 0 & 0 \\
\hline 3 & 0 & 0 & 0 & 0 & 0 & 0 & 0 & 0 & 0 & 0 & 0 & 0 \\
\hline 4 & 0 & 0 & 0 & 0 & 0 & 0 & 0 & 0 & 0 & 0 & .20 & $0^{*}$ \\
\hline 5 & 0 & 0 & 0 & 0 & 0 & 0 & 0 & 0 & 0 & .02 & 0 & $0^{*}$ \\
\hline 6 & 0 & 0 & 0 & 0 & 0 & 0 & 0 & 0 & 0 & 0 & 0 & $0^{*}$ \\
\hline 7 & 0 & 0 & 0 & 0 & 0 & 0 & 0 & 0 & 0 & .04 & $.04^{*}$ & $0^{\star}$ \\
\hline 8 & 0 & 0 & .06 & 0 & 0 & 0 & 0 & 0 & 0 & .05 & .04 & 0 * \\
\hline 9 & 0 & 0 & 0 & 0 & 0 & 0 & 0 & 0 & 0 & 0 & .01 & $0^{*}$ \\
\hline 10 & 0 & 0 & 0 & 0 & 0 & 0 & 0 & 0 & 0 & 0 & .01 & $0^{*}$ \\
\hline 11 & 0 & 0 & 0 & 0 & 0 & 0 & 0 & 0 & 0 & 0 & 0 & $0^{*}$ \\
\hline 12 & 0 & 0 & 0 & 0 & 0 & 0 & 0 & 0 & 0 & 0 & 0 & $0^{*}$ \\
\hline 13 & 0 & 0 & 0 & 0 & 0 & 0 & 0 & .01 & 0 & 0 & 0 & $0^{*}$ \\
\hline 14 & 0 & 0 & 0 & 0 & 0 & 0 & 0 & 0 & 0 & 0 & 0 & $0^{\star}$ \\
\hline 15 & 0 & 0 & 0 & 0 & 0 & 0 & 0 & .08 & 0 & 0 & 0 & $0^{*}$ \\
\hline 16 & 0 & 0 & 0 & 0 & 0 & 0 & 0 & 0 & 0 & .01 & .07 & $0^{*}$ \\
\hline 17 & 0 & 0 & 0 & 0 & 0 & 0 & 0 & 0 & 0 & 0 & .01 & $0^{*}$ \\
\hline 18 & 0 & 0 & 0 & 0 & 0 & 0 & 0 & 0 & 0 & 0 & 0 & $0^{*}$ \\
\hline 19 & 0 & 0 & 0 & 0 & 0 & 0 & 0 & 0 & 0 & 0 & 0 & $0^{*}$ \\
\hline 20 & 0 & 0 & 0 & 0 & 0 & 0 & 0 & 0 & 0 & 0 & 0 & $0^{*}$ \\
\hline 21 & 0 & 0 & 0 & 0 & 0 & 0 & 0 & 0 & 0 & .07 & 0 & $0^{*}$ \\
\hline 22 & 0 & 0 & 0 & 0 & 0 & 0 & 0 & 0 & 0 & 0 & 0 & $0^{*}$ \\
\hline 23 & 0 & 0 & 0 & 0 & 0 & 0 & 0 & .01 & 0 & 0 & 0 & $0^{*}$ \\
\hline 24 & 0 & 0 & 0 & 0 & .03 & 0 & 0 & 0 & 0 & 0 & 0 & $0^{*}$ \\
\hline 25 & 0 & 0 & 0 & 0 & 0 & 0 & 0 & 0 & 0 & 0 & 0 & $0^{*}$ \\
\hline 26 & 0 & 0 & 0 & 0 & 0 & 0 & 0 & 0 & 0 & 0 & 0 & $0^{*}$ \\
\hline 27 & 0 & 0 & 0 & .02 & 0 & 0 & 0 & 0 & 0 & .03 & 0 & $0^{*}$ \\
\hline 28 & 0 & 0 & 0 & .09 & 0 & 0 & 0 & .09 & 0 & 0 & 0 & $0^{*}$ \\
\hline 29 & 0 & 0 & 0 & 0 & 0 & 0 & 0 & 0 & 0 & 0 & 0 & $0^{*}$ \\
\hline 30 & 0 & .25 & 0 & 0 & ------ & 0 & 0 & 0 & 0 & 0 & .01 & 0 * \\
\hline 31 & 0 & ------ & 0 & 0 & ------ & 0 & ------ & 0 & ----- & 0 & .21 & ------ \\
\hline Total & 0.03 & 0.25 & 0.35 & 0.11 & 0.03 & 0 & 0 & 0.19 & 0 & 0.22 & 0.60 & 0 \\
\hline Mean & .001 & .008 & .011 & .004 & .001 & 0 & 0 & .006 & 0 & .007 & .019 & 0 \\
\hline Max & .03 & .25 & .29 & .09 & .03 & 0 & 0 & .09 & 0 & .07 & .21 & 0 \\
\hline Min & 0 & 0 & 0 & 0 & 0 & 0 & 0 & 0 & 0 & 0 & 0 & 0 \\
\hline Acre-Ft & .06 & .50 & .69 & .22 & .06 & 0 & 0 & .38 & 0 & .44 & 1.2 & 0 \\
\hline Wtr Year & 2008 & Total & 1.78 & Mean & & D5 & hax & .29 & Min & 0 & Acre-Ft & 3.5 \\
\hline Cal Year & 2007 & Total & 3.52 & Mean & & 10 & hax & .36 & Min & 0 & Acre-Ft & 7.0 \\
\hline
\end{tabular}

*Estimate 


\section{E244 Two Mile Canyon above Pajarito Canyon}

Location. Lat $35^{\circ}$ 51’ 15", long $106^{\circ} 17^{\prime}$ 46", Sec. 27, T. 19 N., R. 6 E., Ramon Vigil Grant, Los Alamos County.

Drainage Area. $3.15 \mathrm{mi}^{2}$.

Period of Record. October 1, 2002, to September 30, 2008.

Revised Record. Drainage area (2006). Period of record (this report).

Gage. Data logger with cellular telemetry. Elevation of gage is $6,940 \mathrm{ft}$ above NGVD from GPS survey.

Remarks. Records are good except estimated daily discharges, which are fair. Legal location based on projected values.

Average Discharge. $6 \mathrm{yr}, 0.11 \mathrm{ft}^{2} / \mathrm{s}, 83$ acre-ft/yr.

Extremes for Period of Record. Maximum discharge, $628 \mathrm{ft}^{3} / \mathrm{s}$, August 25, 2006, gage height $6.01 \mathrm{ft}$ (from flood marks). No flow most of the time.

Extremes for Current Year. Peak discharges above base of $30 \mathrm{ft}^{3} / \mathrm{s}$ and maximum (*):

\begin{tabular}{|c|c|c|c|}
\hline Date & Time & Discharge $\left(\mathbf{f t}^{\mathbf{3}} / \mathbf{s}\right)$ & Gage Height (ft) \\
\hline December 1 & 0255 & 63 & 2.62 \\
\hline January 28 & 0500 & 95 & 3.02 \\
\hline August 10 & 1155 & $223^{*}$ & $4.04^{*}$ \\
\hline
\end{tabular}

No flow most of the time.

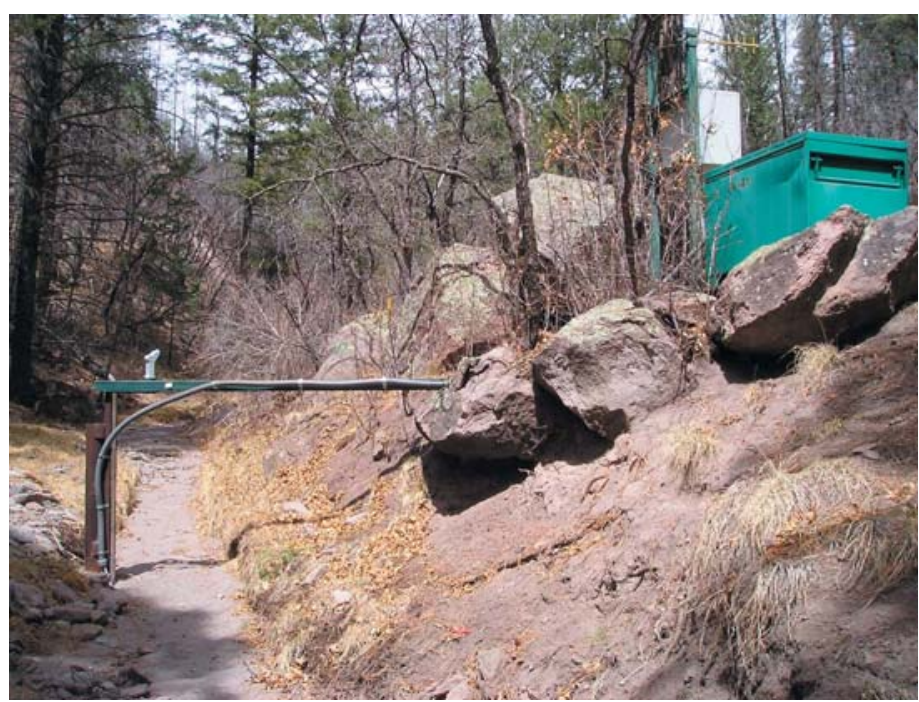




\section{E244 Two Mile Canyon above Pajarito Canyon}

\section{Station Analysis}

\section{Water Year}

Equipment. Station is equipped with Sutron 8210 data logger (5-min. interval) and milltronics sonic probe with cellular phone and speech modem. Phone upgraded July 2 from analog to digital service. The system is powered by a solar-panel battery system housed in a NEMA shelter. Station is equipped with an ISCO pump sampler for waterquality sample collection. ISCO is housed in a separate shelter, a $3^{\prime} \times 4^{\prime}$ metal box. Sampler is triggered by stage through the data logger. An outside staff gage is available for reference. Wading measurements can be in the vicinity of the gage. No provision for measurement above wading stages.

Field Work. The station was visited 21 times to conduct discharge measurements and service the instrumentation. Field inspections for the gage are listed under site history files on the Hydstra database. Discharge measurements for the gage are listed under site gauging files on the Hydstra database.

Datum Correction. None from levels. Gage reset by levels (November 2, 2006) after destruction by flood in August 2006.

Gage-Height Record. The data logger referenced to the outside staff gage gave a complete and satisfactory record except for the period of February 4-23 when ice affected gage height.

Rating. The channel at the gage is straight for about $150^{\prime}$ above gage and $50^{\prime}$ feet below gage. Channel expands quite a bit below gage. Bed material consists of coarse sand and gravel. Banks are grassy, with some small trees and outcrops affecting roughness at higher flows.

Four discharge measurements (Nos. 17-20) and 10 inspections of no flow were made during the year

Rating No. 2 was developed from the current year measurements and one slope-area measurement.

Discharge. Discharge was computed by applying gage height to Rating No. 2 directly. Some periods have large shifts on lower end because dry conditions varied the PZF.

Remarks. Records are good. This station was operated at a site 200' downstream but never rated. It had to be moved because that site is in backwater from Pajarito Flood Control structure, which was built in 2000 just after the Cerro Grande fire. 


\section{E244 Two Mile Canyon above Pajarito Canyon}

Daily Mean Discharge in Cubic Feet per Second

Water Year October 2007 to September 2008

\begin{tabular}{|c|c|c|c|c|c|c|c|c|c|c|c|c|}
\hline DAY & ОСТ & NOV & DEC & JAN & FEB & MAR & APR & MAY & JUN & JUL & AUG & SEP \\
\hline 1 & .15 & 0 & 13 & 0 & .53 & .07 & 0 & 0 & 0 & 0 & 0 & .27 \\
\hline 2 & .13 & 0 & .26 & 0 & .67 & .07 & 0 & 0 & 0 & 0 & 0 & .13 \\
\hline 3 & .11 & 0 & .11 & 0 & .47 & .06 & 0 & 0 & 0 & 0 & 0 & .12 \\
\hline 4 & .10 & 0 & .05 & 0 & $.43^{*}$ & .04 & 0 & 0 & 0 & 0 & .24 & .10 \\
\hline 5 & .10 & 0 & .04 & 0 & $.40^{*}$ & .03 & 0 & 0 & 0 & 0 & 0 & .10 \\
\hline 6 & .09 & 0 & .04 & 0 & $.38^{*}$ & .03 & 0 & 0 & 0 & 0 & 0 & .09 \\
\hline 7 & .08 & 0 & .04 & 0 & $.37^{*}$ & 0 & 0 & 0 & 0 & 0 & 0 & .09 \\
\hline 8 & .07 & 0 & .27 & 0 & $.35^{*}$ & .02 & 0 & 0 & 0 & 0 & .01 & .09 \\
\hline 9 & .06 & 0 & .20 & 0 & $.34^{\star}$ & 0 & 0 & 0 & 0 & 0 & .47 & .08 \\
\hline 10 & .05 & 0 & .08 & 0 & $.32^{*}$ & 0 & 0 & 0 & 0 & 0 & 5.5 & .08 \\
\hline 11 & .04 & 0 & .06 & 0 & $.30^{*}$ & 0 & 0 & 0 & 0 & 0 & .21 & .08 \\
\hline 12 & .04 & 0 & .02 & 0 & $.28^{\star}$ & 0 & 0 & 0 & 0 & 0 & .15 & .07 \\
\hline 13 & .02 & 0 & 0 & 0 & $.27^{\star}$ & 0 & 0 & 0 & 0 & 0 & .13 & .05 \\
\hline 14 & .01 & 0 & 0 & 0 & $.25^{*}$ & .01 & 0 & 0 & 0 & 0 & .12 & .05 \\
\hline 15 & .01 & 0 & 0 & 0 & $.23^{*}$ & 0 & 0 & 0 & 0 & 0 & .11 & .04 \\
\hline 16 & .01 & 0 & 0 & 0 & $.21^{*}$ & .01 & 0 & 0 & 0 & 0 & .18 & .04 \\
\hline 17 & 0 & 0 & 0 & 0 & $.20^{*}$ & .01 & 0 & 0 & 0 & 0 & .15 & .03 \\
\hline 18 & 0 & 0 & 0 & 0 & $.18^{*}$ & .01 & 0 & 0 & 0 & 0 & .14 & .02 \\
\hline 19 & 0 & 0 & 0 & 0 & .12 & .01 & .03 & 0 & 0 & 0 & .12 & .02 \\
\hline 20 & 0 & 0 & 0 & 0 & $.10^{*}$ & .01 & .02 & 0 & 0 & 0 & .09 & .02 \\
\hline 21 & 0 & 0 & 0 & 0 & $.09^{*}$ & 0 & .02 & 0 & 0 & 0 & .05 & .02 \\
\hline 22 & 0 & 0 & 0 & 0 & $.01^{*}$ & 0 & .01 & 0 & 0 & 0 & .04 & .01 \\
\hline 23 & 0 & 0 & 0 & 0 & $.09^{*}$ & 0 & .01 & 0 & 0 & 0 & .17 & .01 \\
\hline 24 & 0 & 0 & 0 & 0 & .84 & 0 & .01 & 0 & 0 & 0 & .19 & .01 \\
\hline 25 & 0 & 0 & 0 & 0 & .48 & 0 & 0 & 0 & 0 & 0 & .23 & .01 \\
\hline 26 & 0 & 0 & 0 & 0 & .15 & 0 & 0 & 0 & 0 & 0 & .08 & 0 \\
\hline 27 & 0 & 0 & 0 & 0 & .09 & 0 & 0 & 0 & 0 & 0 & .05 & 0 \\
\hline 28 & 0 & 0 & 0 & 8.3 & .08 & 0 & 0 & .03 & 0 & 0 & .05 & 0 \\
\hline 29 & 0 & 0 & 0 & 1.1 & .07 & 0 & .01 & 0 & 0 & 0 & .04 & 0 \\
\hline 30 & 0 & .17 & 0 & .75 & ----- & 0 & 0 & 0 & 0 & 0 & .03 & 0 \\
\hline 31 & 0 & ----- & 0 & .39 & ----- & 0 & ----- & 0 & ---- & 0 & .44 & ----- \\
\hline Total & 1.07 & 0.17 & 14.17 & 10.54 & 8.30 & 0.38 & 0.11 & 0.03 & 0 & 0 & 8.99 & 1.63 \\
\hline Mean & .035 & .006 & .46 & .34 & .29 & .012 & .004 & .001 & 0 & 0 & .29 & .054 \\
\hline Max & .15 & .17 & 13 & 8.3 & .84 & .07 & .03 & .03 & 0 & 0 & 5.5 & .27 \\
\hline Min & 0 & 0 & 0 & 0 & .01 & 0 & 0 & 0 & 0 & 0 & 0 & 0 \\
\hline Acre-Ft & 2.1 & .34 & 28 & 21 & 16 & .75 & .22 & .06 & 0 & 0 & 18 & 3.2 \\
\hline Wtr Year & 2008 & Total & 45.39 & Mean & & .12 & Max & 13 & Min & 0 & Acre-Ft & 90 \\
\hline Cal Year & 2007 & Total & 41.87 & Mean & & .11 & $\operatorname{Max}$ & 13 & Min & 0 & Acre-Ft & 83 \\
\hline
\end{tabular}

*Estimate 


\section{E245 Pajarito Canyon above TA-18}

Location. Lat $35^{\circ}$ 51' 4", long $106^{\circ}$ 17' 11", Sec. 27, T. 19 N., R. 6 E., Ramon Vigil Grant, Los Alamos County.

Drainage Area. $7.56 \mathrm{mi}^{2}$.

Period of Record. November 1993 to September 30, 2008.

Revisions. Drainage area (2006).

Gage. Data logger and concrete control. Elevation of gage is 6,880 $\mathrm{ft}$ above NGVD from GPS survey.

Remarks. Records are good except for estimated daily discharges, which are fair. Legal location based on projected values.

Average Discharge. $14 \mathrm{yr}, 0.22 \mathrm{ft}$ /s, 156 acre- $\mathrm{ft} / \mathrm{yr}$.

Extremes for Period of Record. Maximum discharge, $517 \mathrm{ft}^{3} / \mathrm{s}$, June 28, 2000, gage height $5.03 \mathrm{ft}$ (from flood mark). No flow most of the time.

Extremes for Current Year. Peak discharge above base of $25 \mathrm{ft}^{3} / \mathrm{s}$ and maximum (*):

\begin{tabular}{|c|c|c|c|}
\hline Date & Time & Discharge $\left(\mathbf{f t}^{\mathbf{3}} / \mathbf{s}\right)$ & Gage Height (ft) \\
\hline December 1 & 0310 & $119^{*}$ & $3.57^{*}$ \\
\hline January 28 & 1045 & 5 & 1.30 \\
\hline February 24 & 2110 & 13 & 1.65 \\
\hline
\end{tabular}

No flow most of the time.

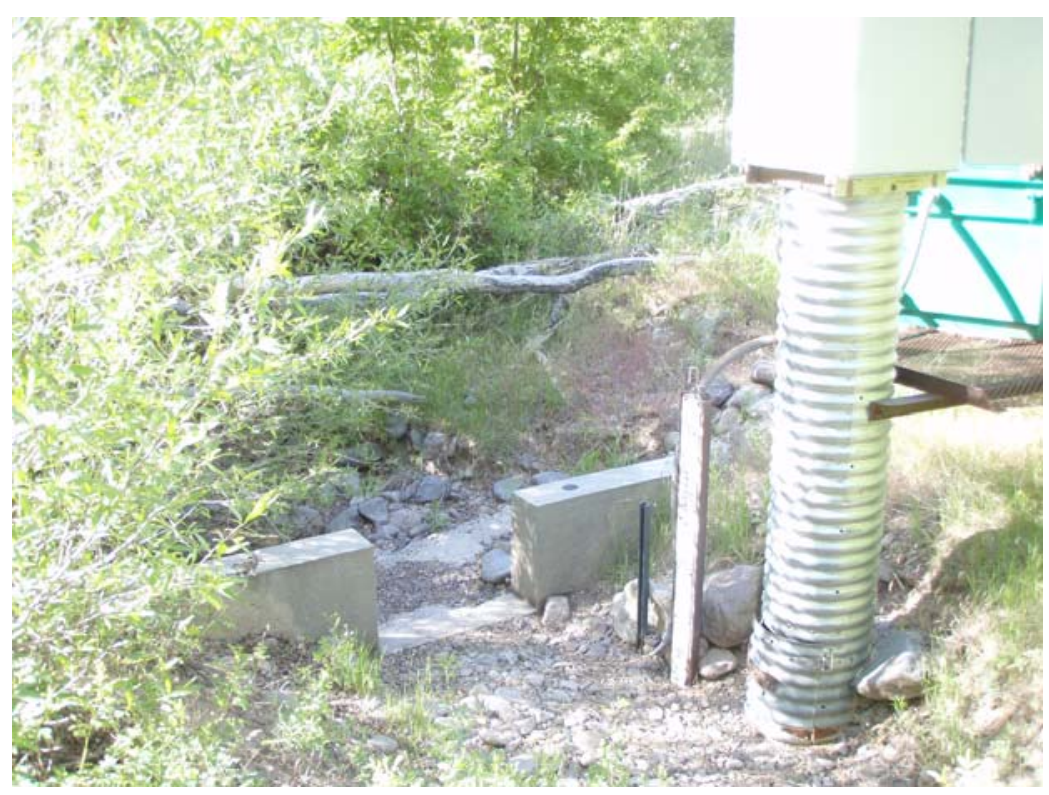




\section{E245 Pajarito Canyon above TA-18}

\section{Station Analysis}

\section{Water Year}

Equipment. Station is equipped with Sutron 8210 (5-min. interval) and shaft encoder float system. The system is powered by a solar-panel battery system housed in NEMA shelter on 18" CMP well on the right bank. Station is equipped with an ISCO pump sampler for water-quality sample collection. ISCO is housed in a separate shelter, a $3^{\prime} \times$ $4^{\prime}$ metal box. Sampler is triggered by stage through the data logger. An outside staff is available for reference. No provision for direct discharge measurements above wading stages.

Cellular telemetry removed March 25, discontinued service.

Field Work. The station was visited 17 times to conduct discharge measurements and service the instrumentation. Field inspections for the gage are listed under site history files on the Hydstra database. Discharge measurements for the gage are listed under site gauging files on the Hydstra database.

Datum Correction. None. Levels run May 12 show gage to read within allowable limits.

Gage-Height Record. The data logger referenced to the outside staff gage gave a complete and satisfactory record except for the period of December 15 to January 27 when gage height was affected by ice and July 14 to September 30 when gage height was incomplete when data logger malfunctioned.

Rating. The channel is straight for $100^{\prime}$ above and below gage. Bed material is gravel with fine sand movement during flow events. Control is concrete broad crested weir.

Seven discharge measurements (Nos. 17-23) and ten visits of no flow were made this year. One critical depth computation was done on peak flow of December 1, 2007.

Rating No. 2 was used all year.

Discharge. Discharge was computed by applying Rating No. 2 using "V" shift diagrams. Long period of lost record was estimated at zero flow based on precipitation and nearby gage stations.

Remarks. Records are good except for estimated daily discharges, which are fair. 


\section{E245 Pajarito Canyon above TA-18}

Daily Mean Discharge in Cubic Feet per Second

Water Year October 2007 to September 2008

\begin{tabular}{|c|c|c|c|c|c|c|c|c|c|c|c|c|}
\hline DAY & OCT & NOV & DEC & JAN & FEB & MAR & APR & MAY & JUN & JUL & AUG & SEP \\
\hline 1 & .47 & 0 & 22 & $.75^{\star}$ & .82 & .86 & .75 & .05 & 0 & 0 & $0^{*}$ & $0^{*}$ \\
\hline 2 & .07 & 0 & .83 & $.75^{\star}$ & .82 & .85 & .75 & .03 & 0 & 0 & $0^{*}$ & $0^{*}$ \\
\hline 3 & 0 & 0 & .83 & $.74^{*}$ & .79 & .83 & .75 & 0 & 0 & 0 & $0^{*}$ & $0^{*}$ \\
\hline 4 & 0 & 0 & .83 & $.75^{*}$ & .80 & .85 & .75 & 0 & 0 & 0 & $0^{*}$ & $0^{*}$ \\
\hline 5 & 0 & 0 & .83 & $.76^{*}$ & .81 & .83 & .75 & 0 & 0 & 0 & $0^{*}$ & $0^{*}$ \\
\hline 6 & 0 & 0 & .81 & $.76^{\star}$ & .78 & .83 & .75 & 0 & 0 & 0 & $0^{*}$ & 0 * \\
\hline 7 & 0 & 0 & .75 & $.77^{*}$ & .81 & .83 & .71 & 0 & 0 & 0 & $0^{*}$ & $0^{*}$ \\
\hline 8 & 0 & 0 & 1.0 & $.78^{*}$ & .80 & .83 & .67 & 0 & 0 & 0 & $0^{*}$ & $0^{*}$ \\
\hline 9 & 0 & 0 & .92 & $.78^{\star}$ & .80 & .83 & .67 & 0 & 0 & 0 & $4.6^{*}$ & $0^{*}$ \\
\hline 10 & 0 & 0 & .83 & $.80^{*}$ & .81 & .83 & .63 & 0 & 0 & 0 & $0^{*}$ & 0 * \\
\hline 11 & 0 & 0 & .83 & $.80^{*}$ & .78 & .82 & .56 & 0 & 0 & 0 & $0^{*}$ & $0^{*}$ \\
\hline 12 & 0 & 0 & .78 & $.81^{*}$ & .79 & .82 & .54 & 0 & 0 & 0 & $0^{*}$ & $0^{*}$ \\
\hline 13 & 0 & 0 & .74 & $.80^{*}$ & .80 & .82 & .50 & 0 & 0 & 0 & $0^{*}$ & $0^{*}$ \\
\hline 14 & 0 & 0 & .63 & $.81^{*}$ & .77 & .81 & .43 & 0 & 0 & $0^{*}$ & $0^{*}$ & $0^{*}$ \\
\hline 15 & 0 & 0 & $.66^{*}$ & $.81^{*}$ & .78 & .81 & .36 & .11 & 0 & $0^{*}$ & $0^{*}$ & $0^{*}$ \\
\hline 16 & 0 & 0 & $.67^{*}$ & $.81^{*}$ & .79 & .80 & .31 & 0 & 0 & $0^{*}$ & $0^{*}$ & $0^{*}$ \\
\hline 17 & 0 & 0 & $.68^{*}$ & $.82^{\star}$ & .75 & .80 & .29 & 0 & 0 & $0^{*}$ & $0^{*}$ & 0 * \\
\hline 18 & 0 & 0 & $.68^{\star}$ & $.82^{*}$ & .76 & .80 & .28 & 0 & 0 & $0^{*}$ & $0^{*}$ & $0^{*}$ \\
\hline 19 & 0 & 0 & $.69^{*}$ & $.82^{*}$ & .77 & .79 & .28 & 0 & 0 & $0^{*}$ & $0^{*}$ & $0^{*}$ \\
\hline 20 & 0 & 0 & $.70^{*}$ & $.83^{*}$ & .76 & .79 & .28 & 0 & 0 & $0^{*}$ & $0^{*}$ & $0^{*}$ \\
\hline 21 & 0 & 0 & $.70^{*}$ & $.83^{*}$ & .75 & .78 & .26 & 0 & 0 & $0^{*}$ & $0^{*}$ & $0^{*}$ \\
\hline 22 & 0 & 0 & $.71^{*}$ & $.84^{*}$ & .75 & .78 & .24 & 0 & 0 & $0^{*}$ & $0^{*}$ & $0^{*}$ \\
\hline 23 & 0 & 0 & $.72^{*}$ & $.84^{*}$ & .75 & .78 & .20 & 0 & 0 & $0^{*}$ & $.70^{*}$ & $0^{*}$ \\
\hline 24 & 0 & 0 & $.73^{*}$ & $.84^{*}$ & 3.2 & .77 & .17 & 0 & 0 & $0^{*}$ & $0^{*}$ & $0^{*}$ \\
\hline 25 & 0 & 0 & $.72^{\star}$ & $.85^{*}$ & 1.4 & .75 & .15 & 0 & 0 & $0^{*}$ & $0^{*}$ & $0^{*}$ \\
\hline 26 & 0 & 0 & $.72^{\star}$ & $.85^{*}$ & .84 & .75 & .13 & 0 & 0 & $0^{*}$ & $0^{*}$ & $0^{*}$ \\
\hline 27 & 0 & 0 & $.74^{\star}$ & $.80^{*}$ & .83 & .75 & .12 & 0 & 0 & $0^{*}$ & $0^{*}$ & $0^{*}$ \\
\hline 28 & 0 & 0 & $.74^{*}$ & .72 & .84 & .75 & .11 & .02 & 0 & $0^{*}$ & $0^{*}$ & $0^{*}$ \\
\hline 29 & 0 & 0 & $.74^{\star}$ & .80 & .84 & .75 & .10 & 0 & 0 & $0^{*}$ & $0^{*}$ & $0^{*}$ \\
\hline 30 & 0 & 0 & $.74^{\star}$ & .84 & ----- & .75 & .08 & 0 & 0 & $0^{*}$ & $0^{*}$ & $0^{*}$ \\
\hline 31 & 0 & ---- & $.75^{\star}$ & .81 & ----- & .75 & ----- & 0 & ---- & $0^{*}$ & $0^{*}$ & ----- \\
\hline Total & 0.54 & 0 & 44.70 & 24.79 & 25.99 & 24.79 & 12.57 & 0.21 & 0 & 0 & 5.30 & 0 \\
\hline Mean & .017 & 0 & 1.44 & .80 & .90 & .80 & .42 & .007 & 0 & 0 & .17 & 0 \\
\hline Max & .47 & 0 & 22 & .85 & 3.2 & .86 & .75 & .11 & 0 & 0 & 4.6 & 0 \\
\hline Min & 0 & 0 & .63 & .72 & .75 & .75 & .08 & 0 & 0 & 0 & 0 & 0 \\
\hline Acre-Ft & 1.1 & 0 & 89 & 49 & 52 & 49 & 25 & .42 & 0 & 0 & 11 & 0 \\
\hline Wtr Year & 2008 & Total & 138.89 & & & .38 & Max & 22 & Min & 0 & Acre-Ft & 275 \\
\hline Cal Year & 2007 & Total & 147.43 & & & .40 & Max & 22 & Min & 0 & Acre-Ft & 292 \\
\hline
\end{tabular}

*Estimate 
Location. Lat $35^{\circ}$ 50' 45.3", long $106^{\circ} 16^{\prime}$ 29", Sec. 16, T. 19N., R. 6 E., Ramon Vigil Grant, Los Alamos County.

Drainage Area. $7.81 \mathrm{mi}^{2}$.

Period of Record. October 1, 2002, to September 30, 2008.

Revisions. Drainage area (this report).

Gage. Data logger and cellular telemetry and rain gage. Elevation of gage is $6796 \mathrm{ft}$ from LIDAR DEM.

Remarks. Records are fair.

Average Discharge. $6 \mathrm{yr}, 0.24 \mathrm{ft}^{3} / \mathrm{s}, 170$ acre- $\mathrm{ft} / \mathrm{yr}$.

Extremes for Period of Record. Maximum discharge, $228 \mathrm{ft}^{3} / \mathrm{s}$, August 24, 2005, gage height $2.86 \mathrm{ft}$.

Extremes for Current Year. Peak discharge above base of $20 \mathrm{ft}^{3} / \mathrm{s}$ and maximum (*):

\begin{tabular}{|c|c|c|c|}
\hline Date & Time & Discharge $\left(\mathbf{f t}^{\mathbf{3}} / \mathbf{s}\right)$ & Gage Height (ft) \\
\hline December 1 & 0355 & 58 & 1.95 \\
\hline January 28 & 0430 & 199 & 2.57 \\
\hline February 24 & 2135 & 22 & 1.62 \\
\hline August 10 & 1225 & $252^{*}$ & $2.78^{*}$ \\
\hline
\end{tabular}

No flow most of the time.

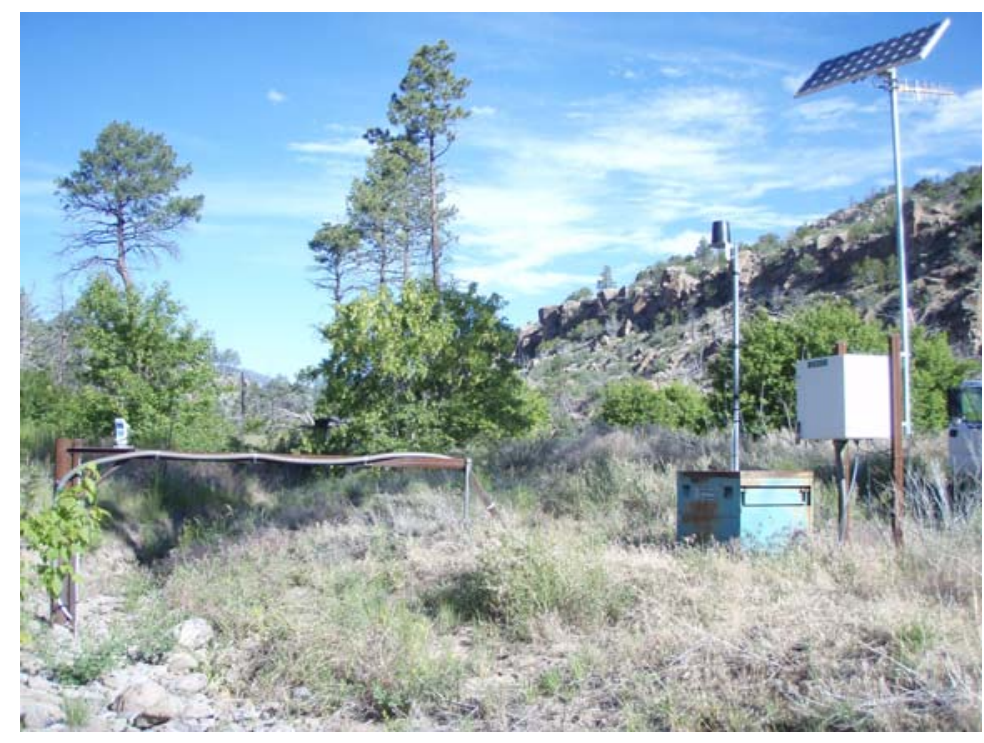




\section{E2455 Pajarito Canyon above Three Mile Canyon}

\section{Station Analysis}

\section{Water Year}

Equipment. Station is equipped with Sutron 8210 (5-min. interval) and milltronics sonic probe and cellular telemetry with speck modem. Phone upgraded May 12 from analog to digital service. The system is powered by a solar-panel battery system housed in NEMA shelter. Station is equipped with an ISCO pump sampler for water-quality sample collection. ISCO is housed in a separate shelter, a $3^{\prime} \times 4^{\prime}$ metal box. Sampler is triggered by stage through the data logger. An outside staff is available for reference. No provision for direct discharge measurements above wading stages.

Station is also equipped with a tipping bucket rain gage, Rain Collection II. All equipment is powered with a solar-panel battery-charging system. Rain data are seasonal.

Field Work. The station was visited 18 times to conduct discharge measurements and service the instrumentation. Field inspections for the gage are listed under site history files on the Hydstra database. Discharge measurements for the gage are listed under site gauging files on the Hydstra database.

Datum Correction. A correction of -0.65 was applied to the current year because of the removal of $90^{\circ}$ crested weir. Levels run May 12, 2008.

Gage-Height Record. The data logger referenced to the outside staff gage gave a complete and satisfactory record for the year.

Rating. The channel is straight for $80^{\prime}$ above and below gage. Banks have some vegetations and streambed consists of sand and gravel. The $90^{\circ}$ sharp-crested weir plate was removed in 2007, requiring new levels and rating.

Four discharge measurements (Nos. 11-14) and 11 inspections of no flow were made this year.

Rating No. 4 was developed using four measurements (Nos. 11-14) and one critical depth computation.

Discharge. Discharge was computed by applying gage height to Rating No. 4 directly.

Remarks. Records are fair. This station was not published last year because of construction near the site that required new rating curve to be developed. The records for 2007 are available but are not reliable. 


\section{E2455 Pajarito Canyon above Three Mile Canyon}

Daily Mean Discharge in Cubic Feet per Second

Water Year October 2007 to September 2008

\begin{tabular}{|c|c|c|c|c|c|c|c|c|c|c|c|c|}
\hline DAY & OCT & NOV & DEC & JAN & FEB & MAR & APR & MAY & JUN & JUL & AUG & SEP \\
\hline 1 & 0 & 0 & 4.0 & .53 & .73 & .47 & .47 & .47 & 0 & 0 & 0 & 0 \\
\hline 2 & 0 & 0 & 0 & .51 & .67 & .47 & .47 & .47 & 0 & 0 & 0 & 0 \\
\hline 3 & 0 & 0 & 0 & .50 & .55 & .47 & .48 & .47 & 0 & 0 & 0 & 0 \\
\hline 4 & 0 & 0 & .24 & .47 & .47 & .47 & .47 & .47 & 0 & 0 & 0 & 0 \\
\hline 5 & 0 & 0 & .47 & .47 & .47 & .47 & .47 & .47 & 0 & 0 & 0 & 0 \\
\hline 6 & 0 & 0 & .47 & .47 & .48 & .47 & .47 & .47 & 0 & 0 & 0 & 0 \\
\hline 7 & 0 & 0 & .47 & .47 & .48 & .49 & .47 & .47 & 0 & 0 & 0 & 0 \\
\hline 8 & 0 & 0 & .78 & .49 & .48 & .48 & .47 & .47 & 0 & 0 & 0 & 0 \\
\hline 9 & 0 & 0 & .64 & .49 & .48 & .47 & .47 & .47 & 0 & 0 & 0 & 0 \\
\hline 10 & 0 & 0 & .47 & .49 & .49 & .48 & .47 & .47 & 0 & 0 & 7.4 & 0 \\
\hline 11 & 0 & 0 & .47 & .49 & .49 & .47 & .47 & .47 & 0 & 0 & 0 & 0 \\
\hline 12 & 0 & 0 & .49 & .50 & .49 & .47 & .47 & .24 & 0 & 0 & 0 & 0 \\
\hline 13 & 0 & 0 & .51 & .50 & .50 & .47 & .47 & 0 & 0 & 0 & 0 & 0 \\
\hline 14 & 0 & 0 & .53 & .50 & .50 & .49 & .47 & 0 & 0 & 0 & 0 & 0 \\
\hline 15 & 0 & 0 & .50 & .50 & .50 & .48 & .47 & 0 & 0 & 0 & 0 & 0 \\
\hline 16 & 0 & 0 & .52 & .51 & .51 & .48 & .47 & 0 & 0 & 0 & 0 & 0 \\
\hline 17 & 0 & 0 & .50 & .51 & .51 & .48 & .47 & 0 & 0 & 0 & 0 & 0 \\
\hline 18 & 0 & 0 & .51 & .51 & .50 & .48 & .47 & 0 & 0 & 0 & 0 & 0 \\
\hline 19 & 0 & 0 & .48 & .51 & .47 & .48 & .47 & 0 & 0 & 0 & 0 & 0 \\
\hline 20 & 0 & 0 & .48 & .50 & .47 & .47 & .47 & 0 & 0 & 0 & 0 & 0 \\
\hline 21 & 0 & 0 & .47 & .50 & .47 & .47 & .47 & 0 & 0 & 0 & 0 & 0 \\
\hline 22 & 0 & 0 & .47 & .49 & .47 & .47 & .47 & 0 & 0 & 0 & 0 & 0 \\
\hline 23 & 0 & 0 & .47 & .49 & .47 & .47 & .47 & 0 & 0 & 0 & 0 & 0 \\
\hline 24 & 0 & 0 & .47 & .47 & 4.4 & .48 & .47 & 0 & 0 & 0 & 0 & 0 \\
\hline 25 & 0 & 0 & .47 & .48 & 2.3 & .47 & .47 & 0 & 0 & 0 & 0 & 0 \\
\hline 26 & 0 & 0 & .48 & .49 & .47 & .47 & .47 & 0 & 0 & 0 & 0 & 0 \\
\hline 27 & 0 & 0 & .48 & .48 & .47 & .47 & .47 & 0 & 0 & 0 & 0 & 0 \\
\hline 28 & 0 & 0 & .47 & 14 & .47 & .47 & .47 & 0 & 0 & 0 & 0 & 0 \\
\hline 29 & 0 & 0 & .48 & 1.2 & .47 & .47 & .47 & 0 & 0 & 0 & 0 & 0 \\
\hline 30 & 0 & 0 & .50 & .73 & ----- & .47 & .47 & 0 & 0 & 0 & 0 & 0 \\
\hline 31 & 0 & ----- & .48 & .75 & ----- & .47 & ------ & 0 & ----- & 0 & .01 & ------ \\
\hline Total & 0 & 0 & 17.77 & 30.00 & 20.23 & 14.69 & 14.11 & 5.41 & 0 & 0 & 7.41 & 0 \\
\hline Mean & 0 & 0 & .57 & .97 & .70 & .47 & .47 & .17 & 0 & 0 & .24 & 0 \\
\hline $\operatorname{Max}$ & 0 & 0 & 4.0 & 14 & 4.4 & .49 & .48 & .47 & 0 & 0 & 7.4 & 0 \\
\hline Min & 0 & 0 & 0 & .47 & .47 & .47 & .47 & 0 & 0 & 0 & 0 & 0 \\
\hline Acre-Ft & 0 & 0 & 35 & 60 & 40 & 29 & 28 & 11 & 0 & 0 & 15 & 0 \\
\hline Wtr Year & 2008 & Total & 109.62 & Mean & & .30 & Max & 14 & Min & 0 & Acre-Ft & 217 \\
\hline Cal Year & 2007 & Total & 21.93 & Mean & & 063 & Max & 4.0 & Min & 0 & Acre-Ft & 43 \\
\hline
\end{tabular}




\section{E2455 Pajarito Canyon above Three Mile Canyon}

Daily Total Rainfall in Inches

Water Year October 2007 to September 2008

\begin{tabular}{|c|c|c|c|c|c|c|c|c|c|c|c|c|}
\hline DAY & ОСт & NOV & DEC & JAN & FEB & MAR & APR & MAY & JUN & JUL & AUG & SEP \\
\hline 1 & & & & & & & & 0 & 0 & .05 & 0 & 0 \\
\hline 2 & & & & & & & & 0 & 0 & .05 & 0 & 0 \\
\hline 3 & & & & & & & & 0 & 0 & .02 & 0 & 0 \\
\hline 4 & & & & & & & & 0 & 0 & .01 & $.55^{\star}$ & 0 \\
\hline 5 & & & & & & & & 0 & .03 & .01 & $0^{\star}$ & 0 \\
\hline 6 & & & & & & & 0 & 0 & 0 & .02 & $0^{*}$ & 0 \\
\hline 7 & & & & & & & 0 & 0 & 0 & .11 & $.04^{\star}$ & 0 \\
\hline 8 & & & & & & & 0 & 0 & 0 & .10 & $.05^{\star}$ & .03 \\
\hline 9 & & & & & & & .04 & 0 & 0 & .01 & $.26^{\star}$ & .01 \\
\hline 10 & & & & & & & 0 & 0 & 0 & 0 & $.44^{\star}$ & 0 \\
\hline 11 & & & & & & & 0 & 0 & 0 & .05 & 0 & 0 \\
\hline 12 & & & & & & & .06 & 0 & 0 & .01 & 0 & .09 \\
\hline 13 & & & & & & & 0 & 0 & 0 & 0 & 0 & 0 \\
\hline 14 & & & & & & & 0 & .16 & 0 & 0 & .23 & 0 \\
\hline 15 & & & & & & & 0 & .59 & 0 & .26 & .04 & 0 \\
\hline 16 & & & & & & & 0 & 0 & 0 & .17 & .03 & 0 \\
\hline 17 & & & & & & & .16 & 0 & 0 & .42 & 0 & 0 \\
\hline 18 & & & & & & & 0 & 0 & 0 & 0 & 0 & 0 \\
\hline 19 & & & & & & & 0 & 0 & 0 & 0 & 0 & 0 \\
\hline 20 & & & & & & & 0 & 0 & 0 & 0 & $.09^{*}$ & 0 \\
\hline 21 & & & & & & & 0 & 0 & 0 & 0 & $0^{*}$ & 0 \\
\hline 22 & & & & & & & 0 & .06 & 0 & 0 & $0^{*}$ & 0 \\
\hline 23 & & & & & & & 0 & .09 & 0 & 0 & .43 & 0 \\
\hline 24 & & & & & & & 0 & 0 & 0 & 0 & .27 & 0 \\
\hline 25 & & & & & & & 0 & 0 & 0 & 0 & .08 & 0 \\
\hline 26 & & & & & & & 0 & 0 & 0 & 0 & 0 & 0 \\
\hline 27 & & & & & & & 0 & 0 & 0 & 0 & 0 & 0 \\
\hline 28 & & & & & & & 0 & .06 & 0 & .19 & 0 & 0 \\
\hline 29 & & & & & & & 0 & 0 & 0 & .02 & .04 & 0 \\
\hline 30 & & & & & ------ & & 0 & 0 & 0 & 0 & .14 & \\
\hline 31 & & ----- & & & ------ & & ------ & 0 & ----- & 0 & $.71^{\star}$ & ------ \\
\hline Total & & & & & & & 0.26 & 0.97 & 0.03 & 1.50 & 1.45 & 0.13 \\
\hline $\operatorname{Max}$ & & & & & & & .16 & .59 & .03 & .42 & .43 & .09 \\
\hline Wtr Year & 2008 & Total & 4.34 & Mean & & .025 & Max & .59 & Min & 0 & InstMax & .35 \\
\hline Cal Year & 2007 & Total & 5.96 & Mean & & .044 & Max & .77 & Min & 0 & InstMax & .11 \\
\hline
\end{tabular}

*Estimate 


\section{E246 Three Mile Canyon above Pajarito Canyon}

Location. Lat $35^{\circ}$ 50' 20", long $106^{\circ}$ 16' 17", Sec. 35, T. 19 N., R. 6 E., Ramon Vigil Grant, Los Alamos County.

Drainage Area. $1.62 \mathrm{mi}^{2}$.

Period of Record. October 1998 to September 30, 2008.

Revised Record. Drainage area (2006).

Gage. Data logger and 9” Parshall flume with cellular telemetry. Elevation of gage is 6,755 ft above NGVD.

Remarks. Records are good except for estimated daily discharges, which are fair.

Average Discharge. $10 \mathrm{yr}, 0.02 \mathrm{ft}^{3} / \mathrm{s}, 14$ acre-ft/yr.

Extremes for Period of Record. Maximum discharge, $536 \mathrm{ft}^{3} / \mathrm{s}$, August 25, 2006, gage height $3.50 \mathrm{ft}$ from critical depth computation of peak flow. No flow most of the time.

Extremes for Current Year. Peak discharge above base of $1.0 \mathrm{ft}^{3} / \mathrm{s}$ and maximum (*):

\begin{tabular}{|c|c|c|c|}
\hline Date & Time & Discharge $\left(\mathbf{f t}^{\mathbf{3}} / \mathbf{s}\right)$ & Gage Height (ft) \\
\hline January 28 & 0635 & $5^{*}$ & $1.45^{*}$ \\
\hline February 24 & 2125 & 4 & 1.14 \\
\hline
\end{tabular}

No flow most of the time.

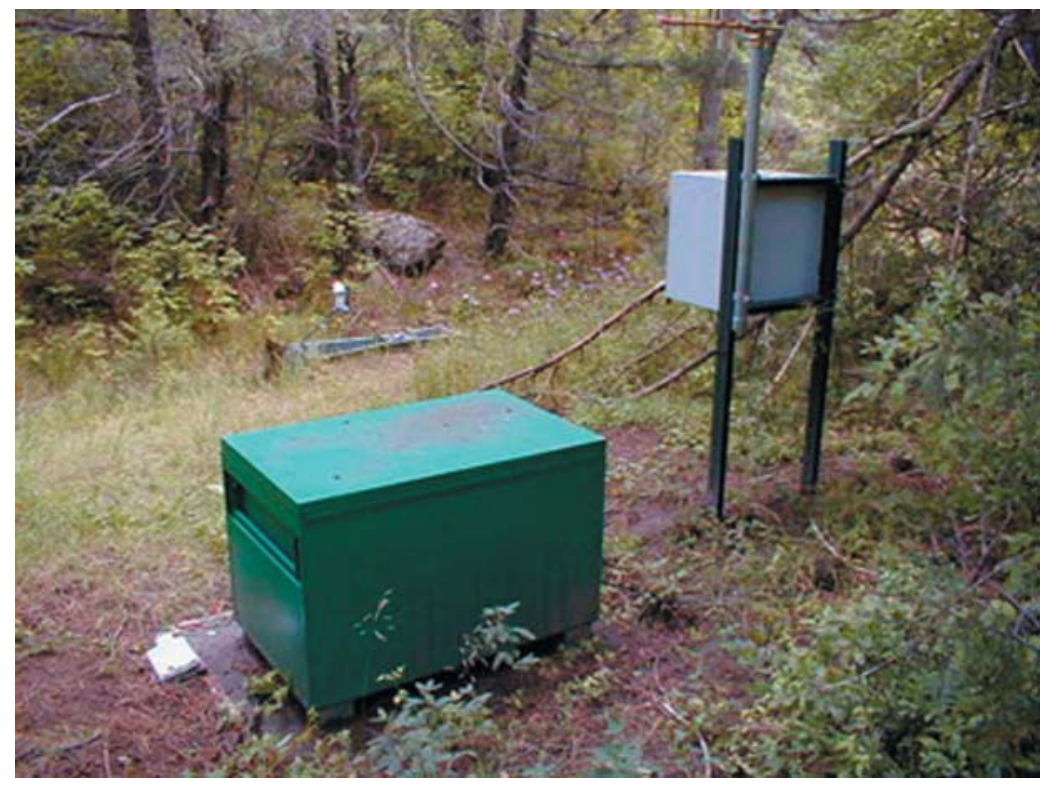




\section{E246 Three Mile Canyon above Pajarito Canyon}

\section{Station Analysis}

\section{Water Year}

Equipment. Station is equipped with Sutron 8210 data logger (5-min. interval) and milltronics sonic probe mounted on 9" Parshall flume and cellular telemetry with speech modem. Phone upgraded June 19 from analog to digital service. The system is powered by a solar-panel battery system housed in a NEMA shelter on right bank. Station is equipped with an ISCO pump sampler for water-quality sample collection. ISCO is housed in a separate shelter, a $3^{\prime} \times 4^{\prime}$ metal box. Sampler is triggered by stage through the data logger. The staff in the 9" Parshall flume is the reference gage. There is no provision for direct discharge measurements above wading stage.

Field Work. The station was visited 14 times to conduct discharge measurements and service the instrumentation. Field inspections for the gage are listed under site history files on the Hydstra database. Discharge measurements for the gage are listed under site gauging files on the Hydstra database.

Datum Correction. None.

Gage-Height Record. The data logger referenced to the outside staff gave a complete and satisfactory record except for the period of January 31 to March 24 when ice affected gage height.

Rating. The channel is straight above and below gage. It is confined to the main channel by cut banks on both sides. The bottom is $10^{\prime}$ wide, with the channel prone to some shifting with vegetation on each bank. Low-water control is the 9" Parshall flume.

Two discharge measurement (Nos. 10-11) and eight inspections of no flow were made this year.

Rating No. 1 was developed based on the computation of 9" Parshall flume and was extended on the basis of two critical depth computations and was used directly. Point of zero flow is 0.00 gage height.

Discharge. Discharge was computed by applying gage height to Rating No. 1 directly.

Remarks. Records are good except for estimated daily discharges, which are fair. 


\section{E246 Three Mile Canyon above Pajarito Canyon}

Daily Mean Discharge in Cubic Feet per Second

Water Year October 2007 to September 2008

\begin{tabular}{|c|c|c|c|c|c|c|c|c|c|c|c|c|}
\hline DAY & ОСт & NOV & DEC & JAN & FEB & MAR & APR & MAY & JUN & JUL & AUG & SEP \\
\hline 1 & 0 & 0 & 0 & 0 & $.08^{*}$ & $.03^{*}$ & .11 & .07 & .02 & 0 & 0 & 0 \\
\hline 2 & 0 & 0 & 0 & 0 & $.08^{\star}$ & $.03^{*}$ & .12 & .07 & .02 & 0 & 0 & 0 \\
\hline 3 & 0 & 0 & 0 & 0 & $.09^{\star}$ & $.03^{*}$ & .11 & .07 & .01 & 0 & 0 & 0 \\
\hline 4 & 0 & 0 & 0 & 0 & $.09^{\star}$ & $.03^{*}$ & .11 & .07 & .01 & 0 & 0 & 0 \\
\hline 5 & 0 & 0 & 0 & 0 & $.08^{\star}$ & $.03^{*}$ & .11 & .07 & .01 & 0 & 0 & 0 \\
\hline 6 & 0 & 0 & 0 & 0 & $.08^{\star}$ & $.03^{*}$ & .10 & .07 & .01 & 0 & 0 & 0 \\
\hline 7 & 0 & 0 & 0 & 0 & $.08^{*}$ & $.03^{*}$ & .10 & .06 & .01 & 0 & 0 & 0 \\
\hline 8 & 0 & 0 & 0 & 0 & $.08^{*}$ & $.03^{*}$ & .10 & .06 & .01 & 0 & 0 & 0 \\
\hline 9 & 0 & 0 & 0 & 0 & $.08^{\star}$ & $.03^{*}$ & .11 & .06 & .01 & 0 & 0 & 0 \\
\hline 10 & 0 & 0 & 0 & 0 & $.08^{\star}$ & $.03^{*}$ & .10 & .05 & .01 & 0 & 0 & 0 \\
\hline 11 & 0 & 0 & 0 & 0 & $.09^{\star}$ & $.03^{*}$ & .09 & .05 & .01 & 0 & 0 & 0 \\
\hline 12 & 0 & 0 & 0 & 0 & $.09^{*}$ & $.03^{*}$ & .09 & .05 & .01 & 0 & 0 & 0 \\
\hline 13 & 0 & 0 & 0 & 0 & $.10^{\star}$ & $.03^{*}$ & .09 & .04 & .01 & 0 & 0 & 0 \\
\hline 14 & 0 & 0 & 0 & 0 & $.11^{*}$ & $.03^{*}$ & .09 & .05 & .01 & 0 & 0 & 0 \\
\hline 15 & 0 & 0 & 0 & 0 & $.11^{*}$ & $.03^{*}$ & .09 & .07 & .01 & 0 & 0 & 0 \\
\hline 16 & 0 & 0 & 0 & 0 & $.11^{*}$ & $.03^{*}$ & .08 & .05 & .01 & 0 & 0 & 0 \\
\hline 17 & 0 & 0 & 0 & 0 & $.11^{*}$ & $.03^{*}$ & .10 & .05 & .01 & 0 & 0 & 0 \\
\hline 18 & 0 & 0 & 0 & 0 & $.13^{*}$ & $.03^{*}$ & .09 & .04 & .01 & 0 & 0 & 0 \\
\hline 19 & 0 & 0 & 0 & 0 & $.17^{*}$ & $.03^{*}$ & .09 & .04 & .01 & 0 & 0 & 0 \\
\hline 20 & 0 & 0 & 0 & 0 & $.19^{*}$ & $.03^{*}$ & .08 & .03 & .01 & 0 & 0 & 0 \\
\hline 21 & 0 & 0 & 0 & 0 & $.22^{\star}$ & $.03^{*}$ & .08 & .03 & .01 & 0 & 0 & 0 \\
\hline 22 & 0 & 0 & 0 & 0 & $.23^{\star}$ & $.03^{*}$ & .08 & .04 & .01 & 0 & 0 & 0 \\
\hline 23 & 0 & 0 & 0 & 0 & $.24^{\star}$ & $.03^{*}$ & .08 & .04 & .01 & 0 & 0 & 0 \\
\hline 24 & 0 & 0 & 0 & 0 & $.97^{\star}$ & $.03^{*}$ & .07 & .03 & 0 & 0 & 0 & 0 \\
\hline 25 & 0 & 0 & 0 & 0 & $.78^{\star}$ & .09 & .07 & .03 & 0 & 0 & 0 & 0 \\
\hline 26 & 0 & 0 & 0 & 0 & $.04^{*}$ & .13 & .08 & .03 & 0 & 0 & 0 & 0 \\
\hline 27 & 0 & 0 & 0 & 0 & $.03^{\star}$ & .13 & .07 & .02 & 0 & 0 & 0 & 0 \\
\hline 28 & 0 & 0 & 0 & 1.8 & $.03^{\star}$ & .13 & .08 & .03 & 0 & 0 & 0 & 0 \\
\hline 29 & 0 & 0 & 0 & .27 & $.03^{\star}$ & .13 & .08 & .02 & 0 & 0 & 0 & 0 \\
\hline 30 & 0 & 0 & 0 & .13 & ------ & .12 & .07 & .02 & 0 & 0 & 0 & 0 \\
\hline 31 & 0 & ------ & 0 & $.08^{\star}$ & ------ & .11 & ------ & .02 & ------ & 0 & 0 & ------ \\
\hline Total & 0 & 0 & 0 & 2.28 & 4.60 & 1.56 & 2.72 & 1.43 & 0.25 & 0 & 0 & 0 \\
\hline Mean & 0 & 0 & 0 & .074 & .16 & .050 & .091 & .046 & .008 & 0 & 0 & 0 \\
\hline Max & 0 & 0 & 0 & 1.8 & .97 & .13 & .12 & .07 & .02 & 0 & 0 & 0 \\
\hline Min & 0 & 0 & 0 & 0 & .03 & .03 & .07 & .02 & 0 & 0 & 0 & 0 \\
\hline Acre-Ft & 0 & 0 & 0 & 4.5 & 9.1 & 3.1 & 5.4 & 2.8 & .50 & 0 & 0 & 0 \\
\hline Wtr Year & 2008 & Total & 12.84 & Mean & & 35 & Max & 1.8 & Min & 0 & Acre-Ft & 25 \\
\hline Cal Year & 2007 & Total & 12.71 & Mean & & 35 & Max & .45 & Min & 0 & Acre-Ft & 25 \\
\hline
\end{tabular}

*Estimate 


\section{E247 MDA G-1}

Location. Lat $35^{\circ}$ 49' 51", long $106^{\circ}$ 14' 41", Sec. 31, T. 19 N., R. 7 E., Ramon Vigil Grant, Los Alamos County.

Drainage Area. $0.002 \mathrm{mi}^{2}$.

Period of Record. October 1, 2004, to September 30, 2008.

Gage. Data logger and 9” Parshall flume. Elevation of gage is 6,626 ft above NGVD.

Remarks. Records are good. Records for this site existed before period of record but are not reliable. Legal location based on projected values.

Extremes for Period of Record. Maximum discharge, $0.94 \mathrm{ft}^{3} / \mathrm{s}$, September 23, 2007, gage height $0.46 \mathrm{ft}$. No flow most of the time.

Extremes for Current Year. Maximum discharge, $0.93 \mathrm{ft}^{3} / \mathrm{s}$ at $0457 \mathrm{~h}$, December 1, gage height $0.46 \mathrm{ft}$. No peak discharge above base of $1.0 \mathrm{ft}^{3} / \mathrm{s}$. No flow most of the time.

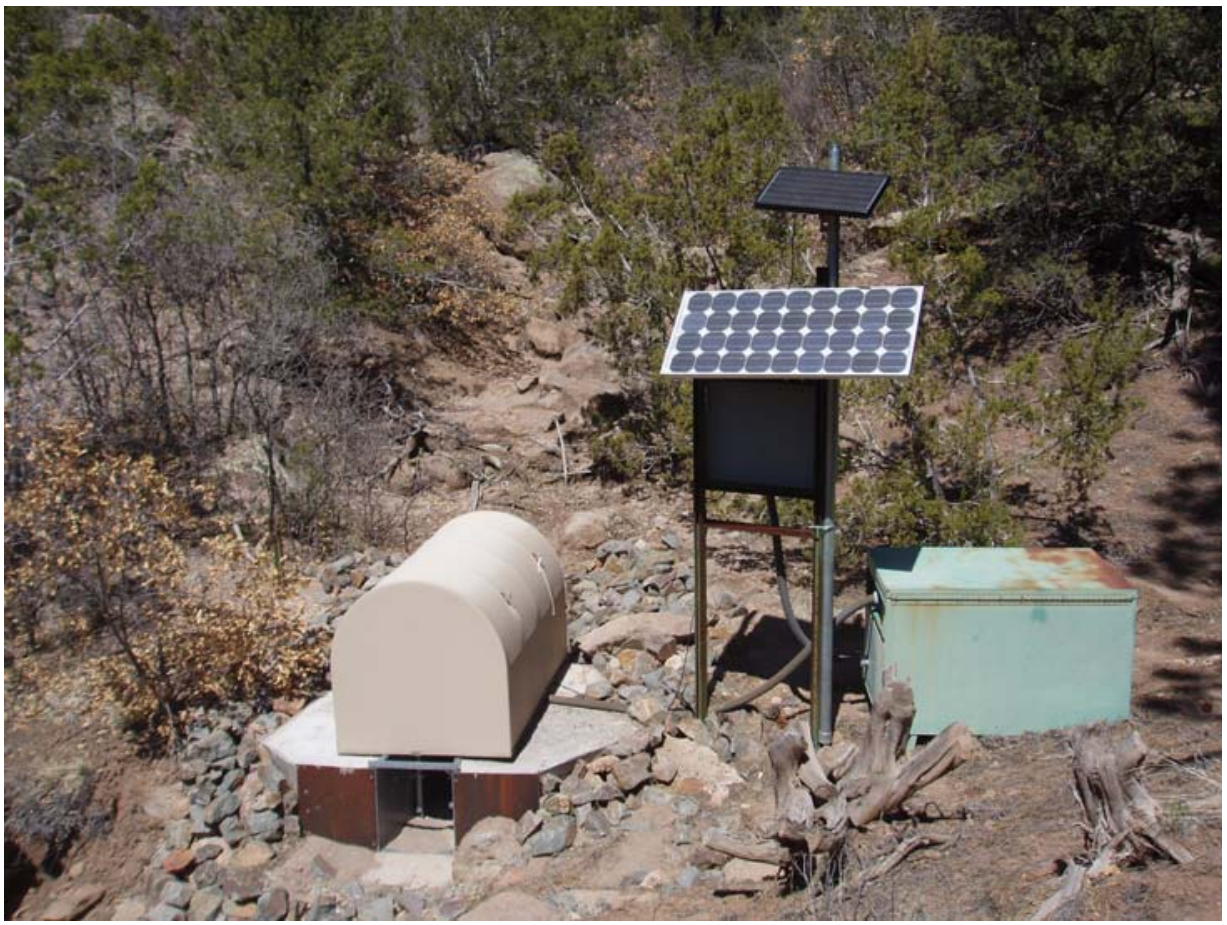




\section{E247 MDA G-1}

\section{Station Analysis}

\section{Water Year}

Equipment. Station is equipped with Sutron 8210 data logger (5-min. interval) and milltronics sonic probe mounted on a 9" Parshall flume. The system is powered by a solar-panel battery system housed in a NEMA shelter. Station is equipped with an ISCO pump sampler for water-quality sample collection. ISCO is housed in a separate shelter, a $3^{\prime} \times 4^{\prime}$ metal box. Sampler is triggered by stage through the data logger. The staff in the 9" Parshall flume is the reference gage. No provision for discharge measurements above wading stage. All high measurements will be by slope-area or peak-flow computation methods.

Field Work. This station was visited 14 times to conduct discharge measurements and service the instrumentation. Field inspections for the gage are listed under site history files on the Hydstra database. Discharge measurements for the gage are listed under site gauging files on the Hydstra database.

Datum Correction. None.

Gage-Height Record. The data logger referenced to the outside staff gave a complete and satisfactory record

Rating. The channel is straight $30^{\prime}$ upstream with sand and gravel. The channel downstream from the flume is $40^{\prime}$ with vegetation on both banks. Streambed consists of sand and gravel. The control for this station is the 9" Parshall flume.

Fourteen inspections of no flow were made this year.

Rating No. 1 was developed based on the computation of 9" Parshall flume. Point of zero flow is 0.00 gage height.

Discharge. Discharge was computed by applying gage height to Rating No. 1 directly.

Remarks. Records are good. 


\section{E247 MDA G-1}

Daily Mean Discharge in Cubic Feet per Second

Water Year October 2007 to September 2008

\begin{tabular}{|c|c|c|c|c|c|c|c|c|c|c|c|c|}
\hline DAY & OCT & NOV & DEC & JAN & FEB & MAR & APR & MAY & JUN & JUL & AUG & SEP \\
\hline 1 & 0 & 0 & .01 & 0 & 0 & 0 & 0 & 0 & 0 & 0 & 0 & 0 \\
\hline 2 & 0 & 0 & 0 & 0 & 0 & 0 & 0 & 0 & 0 & 0 & 0 & 0 \\
\hline 3 & 0 & 0 & 0 & 0 & 0 & 0 & 0 & 0 & 0 & 0 & 0 & 0 \\
\hline 4 & 0 & 0 & 0 & 0 & 0 & 0 & 0 & 0 & 0 & 0 & 0 & 0 \\
\hline 5 & 0 & 0 & 0 & 0 & 0 & 0 & 0 & 0 & 0 & 0 & 0 & 0 \\
\hline 6 & 0 & 0 & 0 & 0 & 0 & 0 & 0 & 0 & 0 & 0 & 0 & 0 \\
\hline 7 & 0 & 0 & 0 & 0 & 0 & 0 & 0 & 0 & 0 & 0 & 0 & 0 \\
\hline 8 & 0 & 0 & 0 & 0 & 0 & 0 & 0 & 0 & 0 & 0 & 0 & 0 \\
\hline 9 & 0 & 0 & 0 & 0 & 0 & 0 & 0 & 0 & 0 & 0 & 0 & 0 \\
\hline 10 & 0 & 0 & 0 & 0 & 0 & 0 & 0 & 0 & 0 & 0 & 0 & 0 \\
\hline 11 & 0 & 0 & 0 & 0 & 0 & 0 & 0 & 0 & 0 & 0 & 0 & 0 \\
\hline 12 & 0 & 0 & 0 & 0 & 0 & 0 & 0 & 0 & 0 & 0 & 0 & 0 \\
\hline 13 & 0 & 0 & 0 & 0 & 0 & 0 & 0 & 0 & 0 & 0 & 0 & 0 \\
\hline 14 & 0 & 0 & 0 & 0 & 0 & 0 & 0 & 0 & 0 & 0 & 0 & 0 \\
\hline 15 & 0 & 0 & 0 & 0 & 0 & 0 & 0 & 0 & 0 & 0 & 0 & 0 \\
\hline 16 & 0 & 0 & 0 & 0 & 0 & 0 & 0 & 0 & 0 & 0 & 0 & 0 \\
\hline 17 & 0 & 0 & 0 & 0 & 0 & 0 & 0 & 0 & 0 & 0 & 0 & 0 \\
\hline 18 & 0 & 0 & 0 & 0 & 0 & 0 & 0 & 0 & 0 & 0 & 0 & 0 \\
\hline 19 & 0 & 0 & 0 & 0 & 0 & 0 & 0 & 0 & 0 & 0 & 0 & 0 \\
\hline 20 & 0 & 0 & 0 & 0 & 0 & 0 & 0 & 0 & 0 & 0 & 0 & 0 \\
\hline 21 & 0 & 0 & 0 & 0 & 0 & 0 & 0 & 0 & 0 & 0 & 0 & 0 \\
\hline 22 & 0 & 0 & 0 & 0 & 0 & 0 & 0 & 0 & 0 & 0 & 0 & 0 \\
\hline 23 & 0 & 0 & 0 & 0 & 0 & 0 & 0 & 0 & 0 & 0 & 0 & 0 \\
\hline 24 & 0 & 0 & 0 & 0 & 0 & 0 & 0 & 0 & 0 & 0 & 0 & 0 \\
\hline 25 & 0 & 0 & 0 & 0 & 0 & 0 & 0 & 0 & 0 & 0 & 0 & 0 \\
\hline 26 & 0 & 0 & 0 & 0 & 0 & 0 & 0 & 0 & 0 & 0 & 0 & 0 \\
\hline 27 & 0 & 0 & 0 & 0 & 0 & 0 & 0 & 0 & 0 & 0 & 0 & 0 \\
\hline 28 & 0 & 0 & 0 & .05 & 0 & 0 & 0 & 0 & 0 & 0 & 0 & 0 \\
\hline 29 & 0 & 0 & 0 & 0 & 0 & 0 & 0 & 0 & 0 & 0 & 0 & 0 \\
\hline 30 & 0 & 0 & 0 & 0 & ----- & 0 & 0 & 0 & 0 & 0 & 0 & 0 \\
\hline 31 & 0 & ------ & 0 & 0 & ----- & 0 & ----- & 0 & ------ & 0 & 0 & ----- \\
\hline Total & 0 & 0 & 0.01 & 0.05 & 0 & 0 & 0 & 0 & 0 & 0 & 0 & 0 \\
\hline Mean & 0 & 0 & 0 & .002 & 0 & 0 & 0 & 0 & 0 & 0 & 0 & 0 \\
\hline Max & 0 & 0 & .01 & .05 & 0 & 0 & 0 & 0 & 0 & 0 & 0 & 0 \\
\hline Min & 0 & 0 & 0 & 0 & 0 & 0 & 0 & 0 & 0 & 0 & 0 & 0 \\
\hline Acre-Ft & 0 & 0 & .02 & .10 & 0 & 0 & 0 & 0 & 0 & 0 & 0 & 0 \\
\hline Wtr Year & 2008 & Total & 0.06 & Mean & & 0 & Max & .05 & Min & 0 & Acre-Ft & 0.12 \\
\hline Cal Year & 2007 & Total & 0.01 & Mean & & 0 & Max & .01 & Min & 0 & Acre-Ft & 0.02 \\
\hline
\end{tabular}




\section{E248 MDA G-2}

Location. Lat $35^{\circ}$ 49' 48", long $106^{\circ}$ 14' 33", Sec. 31. T. 19 N., R. 7 E., Ramon Vigil Grant, Los Alamos County.

Drainage Area. $0.016 \mathrm{mi}^{2}$.

Period of Record. October 1, 2006, to September 30, 2008.

Gage. Data logger and 9” Parshall Flume. Elevation of gage is 6,621 ft above NGVD.

Remarks. Records are good. Records for this site existed before period of record but are not reliable. Legal location based on projected values.

Extremes for Period of Record. Maximum discharge, $4.9 \mathrm{ft}^{3} / \mathrm{s}$, January 28, 2008, gage height $1.72 \mathrm{ft}$. No flow most of the time.

Extremes for Current Year. Peak discharges above base of $1.0 \mathrm{ft}^{3} / \mathrm{s}$ and maximum (*):

\begin{tabular}{|c|c|c|c|}
\hline Date & Time & Discharge $\left(\mathbf{f t}^{\mathbf{3}} \mathbf{s}\right)$ & Gage Height (ft) \\
\hline December 1 & 0505 & 1.1 & 0.67 \\
\hline January 28 & 0315 & $4.9^{*}$ & $1.72^{*}$ \\
\hline
\end{tabular}

No flow most of the time.

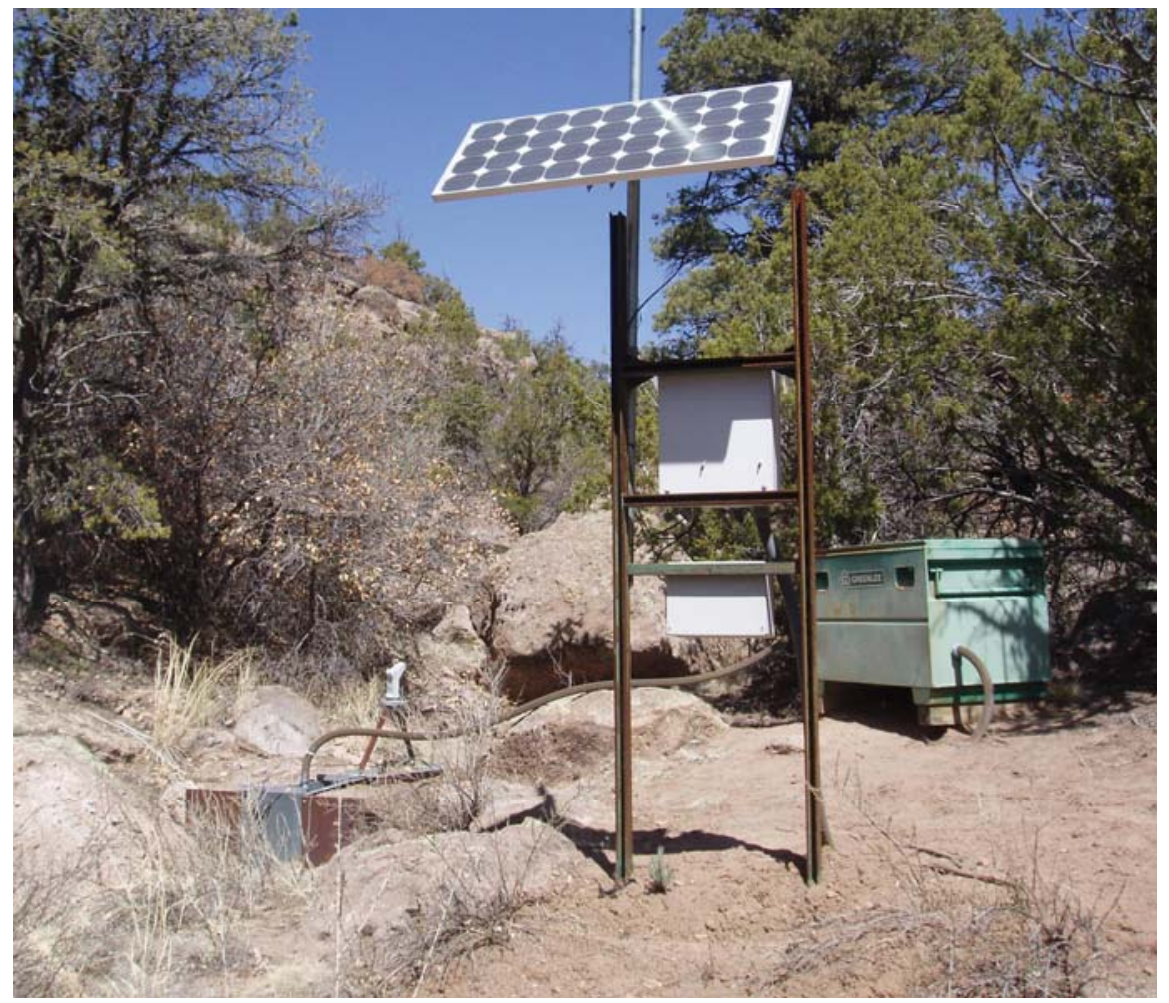




\section{E248 MDA G-2}

\section{Station Analysis}

\section{Water Year}

Equipment. Station is equipped with Sutron 8210 data logger (5-min. interval) and milltronics sonic probe mounted on a 9" Parshall flume. The system is powered by a solar-panel battery system housed in a NEMA shelter. Station is equipped with an ISCO pump sampler for water-quality sample collection. ISCO is housed in a separate shelter, a $3^{\prime} \times 4^{\prime}$ metal box. Sampler is triggered by stage through the data logger. The staff in the 9" Parshall flume is the reference gage. No provision for discharge measurements above wading stage. All high measurements will be by slope-area or peak-flow computation methods.

Field Work. This station was visited 15 times to conduct discharge measurements and service the instrumentation. Field inspections for the gage are listed under site history files on the Hydstra database. Discharge measurements for the gage are listed under site gauging files on the Hydstra database.

Datum Correction. None.

Gage-Height Record. The data logger referenced to the outside staff gave a complete and satisfactory record.

Rating. The control for this station is the 9" Parshall flume. The channel is straight 30' upstream with sand, gravel and vegetation. The channel downstream from the flume is 40 ' long with large boulders on both banks.

Fifteen inspections of no flow were made this water year.

Rating No. 1 was developed based on the computation of 9" Parshall flume. Point of zero flow is 0.00 gage height.

Discharge. Discharge was computed by applying gage height to Rating No. 1 directly.

Remarks. Records are good. 


\section{E248 MDA G-2}

Daily Mean Discharge in Cubic Feet per Second

Water Year October 2007 to September 2008

\begin{tabular}{|c|c|c|c|c|c|c|c|c|c|c|c|c|}
\hline DAY & OCT & NOV & DEC & JAN & FEB & MAR & APR & MAY & JUN & JUL & AUG & SEP \\
\hline 1 & 0 & 0 & .07 & 0 & 0 & 0 & 0 & 0 & 0 & 0 & 0 & 0 \\
\hline 2 & 0 & 0 & 0 & 0 & 0 & 0 & 0 & 0 & 0 & 0 & 0 & 0 \\
\hline 3 & 0 & 0 & 0 & 0 & 0 & 0 & 0 & 0 & 0 & 0 & 0 & 0 \\
\hline 4 & 0 & 0 & 0 & 0 & 0 & 0 & 0 & 0 & 0 & 0 & 0 & 0 \\
\hline 5 & 0 & 0 & 0 & 0 & 0 & 0 & 0 & 0 & 0 & 0 & 0 & 0 \\
\hline 6 & 0 & 0 & 0 & 0 & 0 & 0 & 0 & 0 & 0 & 0 & 0 & 0 \\
\hline 7 & 0 & 0 & 0 & 0 & 0 & 0 & 0 & 0 & 0 & 0 & 0 & 0 \\
\hline 8 & 0 & 0 & 0 & 0 & 0 & 0 & 0 & 0 & 0 & 0 & 0 & 0 \\
\hline 9 & 0 & 0 & 0 & 0 & 0 & 0 & 0 & 0 & 0 & 0 & 0 & 0 \\
\hline 10 & 0 & 0 & 0 & 0 & 0 & 0 & 0 & 0 & 0 & 0 & 0 & 0 \\
\hline 11 & 0 & 0 & 0 & 0 & 0 & 0 & 0 & 0 & 0 & 0 & 0 & 0 \\
\hline 12 & 0 & 0 & 0 & 0 & 0 & 0 & 0 & 0 & 0 & 0 & 0 & 0 \\
\hline 13 & 0 & 0 & 0 & 0 & 0 & 0 & 0 & 0 & 0 & 0 & 0 & 0 \\
\hline 14 & 0 & 0 & 0 & 0 & 0 & 0 & 0 & 0 & 0 & 0 & 0 & 0 \\
\hline 15 & 0 & 0 & 0 & 0 & 0 & 0 & 0 & 0 & 0 & 0 & 0 & 0 \\
\hline 16 & 0 & 0 & 0 & 0 & 0 & 0 & 0 & 0 & 0 & 0 & 0 & 0 \\
\hline 17 & 0 & 0 & 0 & 0 & 0 & 0 & 0 & 0 & 0 & 0 & 0 & 0 \\
\hline 18 & 0 & 0 & 0 & 0 & 0 & 0 & 0 & 0 & 0 & 0 & 0 & 0 \\
\hline 19 & 0 & 0 & 0 & 0 & 0 & 0 & 0 & 0 & 0 & 0 & 0 & 0 \\
\hline 20 & 0 & 0 & 0 & 0 & 0 & 0 & 0 & 0 & 0 & 0 & 0 & 0 \\
\hline 21 & 0 & 0 & 0 & 0 & 0 & 0 & 0 & 0 & 0 & 0 & 0 & 0 \\
\hline 22 & 0 & 0 & 0 & 0 & 0 & 0 & 0 & 0 & 0 & 0 & 0 & 0 \\
\hline 23 & 0 & 0 & 0 & 0 & 0 & 0 & 0 & 0 & 0 & 0 & 0 & 0 \\
\hline 24 & 0 & 0 & 0 & 0 & 0 & 0 & 0 & 0 & 0 & 0 & 0 & 0 \\
\hline 25 & 0 & 0 & 0 & 0 & 0 & 0 & 0 & 0 & 0 & 0 & 0 & 0 \\
\hline 26 & 0 & 0 & 0 & 0 & 0 & 0 & 0 & 0 & 0 & 0 & 0 & 0 \\
\hline 27 & 0 & 0 & 0 & 0 & 0 & 0 & 0 & 0 & 0 & 0 & 0 & 0 \\
\hline 28 & 0 & 0 & 0 & .02 & 0 & 0 & 0 & 0 & 0 & 0 & 0 & 0 \\
\hline 29 & 0 & 0 & 0 & 0 & 0 & 0 & 0 & 0 & 0 & 0 & 0 & 0 \\
\hline 30 & 0 & 0 & 0 & 0 & ----- & 0 & 0 & 0 & 0 & 0 & 0 & 0 \\
\hline 31 & 0 & ----- & 0 & 0 & ----- & 0 & ----- & 0 & ----- & 0 & 0 & ------ \\
\hline Total & 0 & 0 & 0.07 & 0.02 & 0 & 0 & 0 & 0 & 0 & 0 & 0 & 0 \\
\hline Mean & 0 & 0 & .002 & .001 & 0 & 0 & 0 & 0 & 0 & 0 & 0 & 0 \\
\hline Max & 0 & 0 & .07 & .02 & 0 & 0 & 0 & 0 & 0 & 0 & 0 & 0 \\
\hline Min & 0 & 0 & 0 & 0 & 0 & 0 & 0 & 0 & 0 & 0 & 0 & 0 \\
\hline Acre-Ft & 0 & 0 & .14 & .04 & 0 & 0 & 0 & 0 & 0 & 0 & 0 & 0 \\
\hline Wtr Year & 2008 & Total & 0.09 & Mean & & 0 & Max & .07 & Min & 0 & Acre-Ft & 0.18 \\
\hline Cal Year & 2007 & Total & 1.55 & Mean & & 04 & Max & .76 & Min & 0 & Acre-Ft & 3.1 \\
\hline
\end{tabular}




\section{E2485 MDA G-6U}

Location. Lat $35^{\circ}$ 49' 45", long $106^{\circ}$ 14' 12", Sec. 31, T. 19 N., R. 7 E., Ramon Vigil Grant, Los Alamos County.

Drainage Area. $0.03 \mathrm{mi}^{2}$.

Period of Record. October 1, 2006, to September 30, 2008.

Gage. Data logger and 9” Parshall flume. Elevation of gage is 6,602 ft above NGVD.

Remarks. Records are good. Records for this site existed before period of record but are not reliable. Legal location based on projected values.

Extremes for Period of Record. Maximum discharge, $3.8 \mathrm{ft}^{3} / \mathrm{s}$, September 17, 2007, gage height $1.15 \mathrm{ft}$. No flow most of the time.

Extremes for Current Year. Peak discharges above base of $1.0 \mathrm{ft}^{3} / \mathrm{s}$ and maximum (*):

\begin{tabular}{|c|c|c|c|}
\hline Date & Time & Discharge $\left(\mathbf{f t}^{\mathbf{3}} / \mathbf{s}\right)$ & Gage Height (ft) \\
\hline December 1 & 0500 & 2.14 & 0.79 \\
\hline January 28 & 0405 & 2.27 & 0.82 \\
\hline July 17 & 1340 & $3.26^{*}$ & $1.09^{*}$ \\
\hline
\end{tabular}

No flow most of the time.

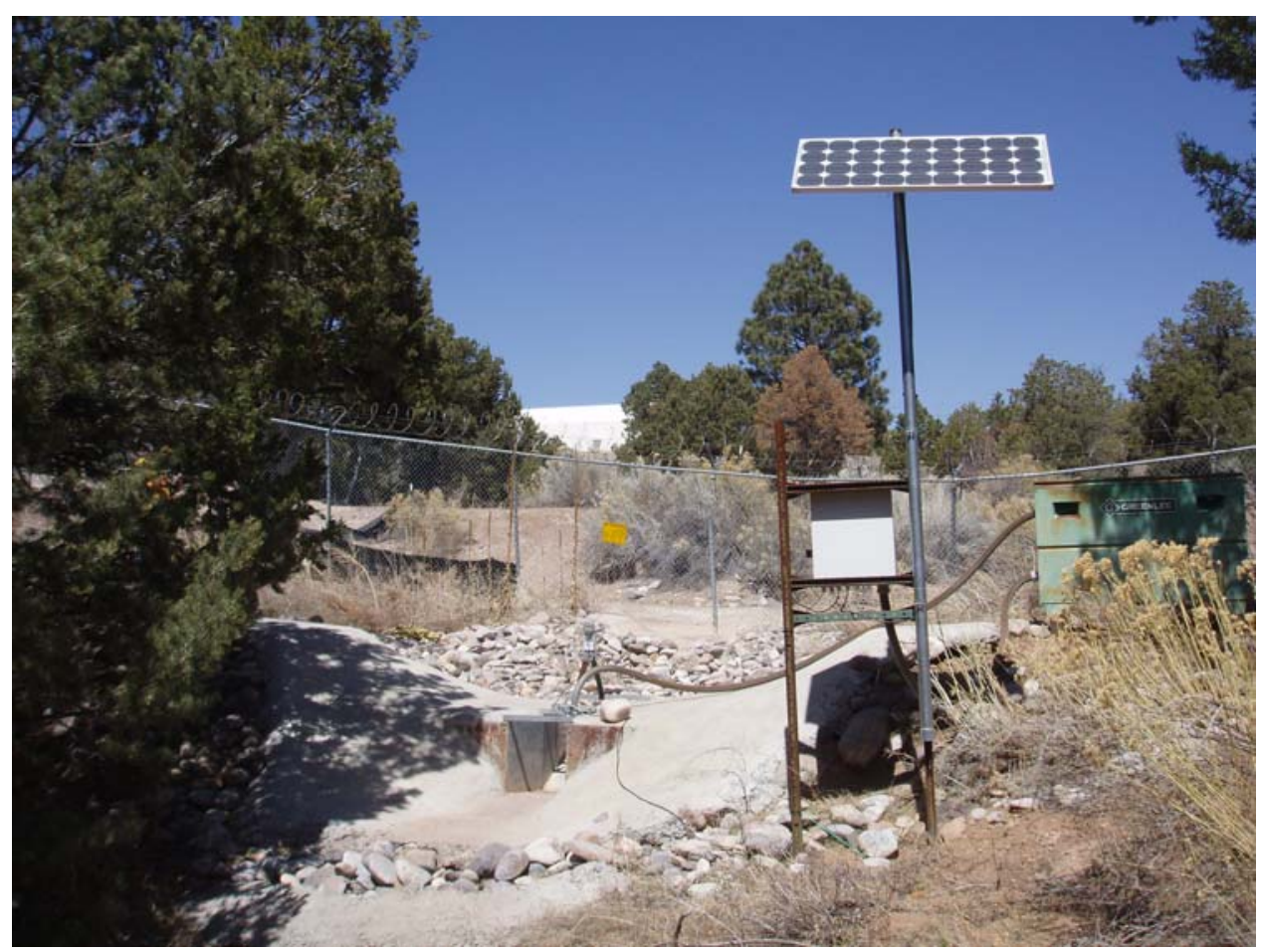




\section{E2485 MDA G-6U}

\section{Station Analysis}

\section{Water Year}

Equipment. Station is equipped with Sutron 8210 data logger (5-min. interval) and milltronics sonic probe mounted on a 9" Parshall flume. The system is powered by a solar-panel battery system housed in a NEMA shelter. Station is equipped with an ISCO pump sampler for water-quality sample collection. ISCO is housed in a separate shelter, a $3^{\prime} \times 4^{\prime}$ metal box. Sampler is triggered by stage through the data logger. The staff in the 9" Parshall flume is the reference gage. No provision for discharge measurements above wading stage. All measurements above wading stage will be by slope-area or peak-flow computation methods.

Field Work. This station was visited 16 times to conduct discharge measurements and service the instrumentation. Field inspections for the gage are listed under site history files on the Hydstra database. Discharge measurements for the gage are listed under site gauging files on the Hydstra database.

Datum Correction. None.

Gage-Height Record. The data logger referenced to the outside staff gave a complete and satisfactory record.

Rating. The control for this station is the 9" Parshall flume. The channel is straight 40' upstream with sand, gravel, and vegetation. The channel downstream from the flume is 70 ' long with vegetation and sand on both banks.

Fifteen inspections of no flow and one inspection of snow melt were made this year.

Rating No. 1 was developed based on the computation of 9" Parshall flume. Point of zero flow is 0.00 gage height.

Discharge. Discharge was computed by applying gage height to Rating No. 1 directly.

Remarks. Records are good. 


\section{E2485 MDA G-6U}

Daily Mean Discharge in Cubic Feet per Second

Water Year October 2007 to September 2008

\begin{tabular}{|c|c|c|c|c|c|c|c|c|c|c|c|c|}
\hline DAY & OCT & NOV & DEC & JAN & FEB & MAR & APR & MAY & JUN & JUL & AUG & SEP \\
\hline 1 & 0 & 0 & .13 & 0 & 0 & 0 & 0 & 0 & 0 & 0 & 0 & 0 \\
\hline 2 & 0 & 0 & 0 & 0 & 0 & 0 & 0 & 0 & 0 & 0 & 0 & 0 \\
\hline 3 & 0 & 0 & 0 & 0 & 0 & 0 & 0 & 0 & 0 & 0 & 0 & 0 \\
\hline 4 & 0 & 0 & 0 & 0 & 0 & 0 & 0 & 0 & 0 & 0 & 0 & 0 \\
\hline 5 & 0 & 0 & 0 & 0 & 0 & 0 & 0 & 0 & 0 & 0 & 0 & 0 \\
\hline 6 & 0 & 0 & 0 & 0 & 0 & 0 & 0 & 0 & 0 & 0 & 0 & 0 \\
\hline 7 & 0 & 0 & 0 & 0 & 0 & 0 & 0 & 0 & 0 & 0 & 0 & 0 \\
\hline 8 & 0 & 0 & 0 & 0 & 0 & 0 & 0 & 0 & 0 & 0 & 0 & 0 \\
\hline 9 & 0 & 0 & 0 & 0 & 0 & 0 & 0 & 0 & 0 & 0 & 0 & 0 \\
\hline 10 & 0 & 0 & 0 & 0 & 0 & 0 & 0 & 0 & 0 & 0 & 0 & 0 \\
\hline 11 & 0 & 0 & 0 & 0 & 0 & 0 & 0 & 0 & 0 & 0 & 0 & 0 \\
\hline 12 & 0 & 0 & 0 & 0 & 0 & 0 & 0 & 0 & 0 & 0 & 0 & 0 \\
\hline 13 & 0 & 0 & 0 & 0 & 0 & 0 & 0 & 0 & 0 & 0 & 0 & 0 \\
\hline 14 & 0 & 0 & 0 & 0 & 0 & 0 & 0 & 0 & 0 & 0 & 0 & 0 \\
\hline 15 & 0 & 0 & 0 & 0 & 0 & 0 & 0 & 0 & 0 & 0 & 0 & 0 \\
\hline 16 & 0 & 0 & 0 & 0 & 0 & 0 & 0 & 0 & 0 & 0 & 0 & 0 \\
\hline 17 & 0 & 0 & 0 & 0 & 0 & 0 & 0 & 0 & 0 & .07 & 0 & 0 \\
\hline 18 & 0 & 0 & 0 & 0 & 0 & 0 & 0 & 0 & 0 & 0 & 0 & 0 \\
\hline 19 & 0 & 0 & 0 & 0 & 0 & 0 & 0 & 0 & 0 & 0 & 0 & 0 \\
\hline 20 & 0 & 0 & 0 & 0 & 0 & 0 & 0 & 0 & 0 & 0 & 0 & 0 \\
\hline 21 & 0 & 0 & 0 & 0 & 0 & 0 & 0 & 0 & 0 & 0 & 0 & 0 \\
\hline 22 & 0 & 0 & 0 & 0 & 0 & 0 & 0 & 0 & 0 & 0 & 0 & 0 \\
\hline 23 & 0 & 0 & 0 & 0 & 0 & 0 & 0 & 0 & 0 & 0 & 0 & 0 \\
\hline 24 & 0 & 0 & 0 & 0 & 0 & 0 & 0 & 0 & 0 & 0 & 0 & 0 \\
\hline 25 & 0 & 0 & 0 & 0 & 0 & 0 & 0 & 0 & 0 & 0 & 0 & 0 \\
\hline 26 & 0 & 0 & 0 & 0 & 0 & 0 & 0 & 0 & 0 & 0 & 0 & 0 \\
\hline 27 & 0 & 0 & 0 & .01 & 0 & 0 & 0 & 0 & 0 & 0 & 0 & 0 \\
\hline 28 & 0 & 0 & 0 & .16 & 0 & 0 & 0 & 0 & 0 & 0 & 0 & 0 \\
\hline 29 & 0 & 0 & 0 & 0 & 0 & 0 & 0 & 0 & 0 & 0 & 0 & 0 \\
\hline 30 & 0 & .04 & 0 & 0 & ------ & 0 & 0 & 0 & 0 & 0 & 0 & 0 \\
\hline 31 & 0 & ------ & 0 & 0 & ------ & 0 & ----- & 0 & ----- & 0 & 0 & ------ \\
\hline Total & 0 & 0.04 & 0.13 & 0.17 & 0 & 0 & 0 & 0 & 0 & 0.07 & 0 & 0 \\
\hline Mean & 0 & .001 & .004 & .006 & 0 & 0 & 0 & 0 & 0 & .002 & 0 & 0 \\
\hline Max & 0 & .04 & .13 & .16 & 0 & 0 & 0 & 0 & 0 & .07 & 0 & 0 \\
\hline Min & 0 & 0 & 0 & 0 & 0 & 0 & 0 & 0 & 0 & 0 & 0 & 0 \\
\hline Acre-Ft & 0 & .08 & .26 & .34 & 0 & 0 & 0 & 0 & 0 & .14 & 0 & 0 \\
\hline Wtr Year & 2008 & Total & 0.41 & Mean & & 01 & hax & .16 & Min & 0 & Acre-Ft & 0.81 \\
\hline Cal Year & 2007 & Total & 0.36 & Mean & & 01 & hax & .13 & Min & 0 & Acre-Ft & 0.71 \\
\hline
\end{tabular}




\section{E249 MDA G-4}

Location. Lat $35^{\circ}$ 49' 42", long $106^{\circ}$ 14' 17", Sec. 31, T. 19 N., R. 7 E., Ramon Vigil Grant, Los Alamos County.

Drainage Area. $0.007 \mathrm{mi}^{2}$.

Period of Record. October 1, 2006, to September 30, 2008.

Gage. Data logger 9” Parshall flume. Elevation of gage is 6,602 ft above NGVD.

Remarks. Records are good. Records for this site existed before period of record but are not reliable. Legal location based on projected values.

Extremes for Period of Record. Maximum discharge, $1.20 \mathrm{ft}^{3} / \mathrm{s}$, July 17, 2008, gage height $0.54 \mathrm{ft}$. No flow most of the time.

Extremes for Current Year. Peak discharges above $1.0 \mathrm{ft}^{3} / \mathrm{s}$ and maximum (*):

\begin{tabular}{|c|c|c|c|}
\hline Date & Time & Discharge $\left(\mathbf{f t}^{\mathbf{3}} / \mathbf{s}\right)$ & Gage Height (ft) \\
\hline January 28 & 0020 & 0.37 & 0.25 \\
\hline July 17 & 1320 & $1.20^{*}$ & $0.54^{*}$ \\
\hline
\end{tabular}

No flow most of the time.

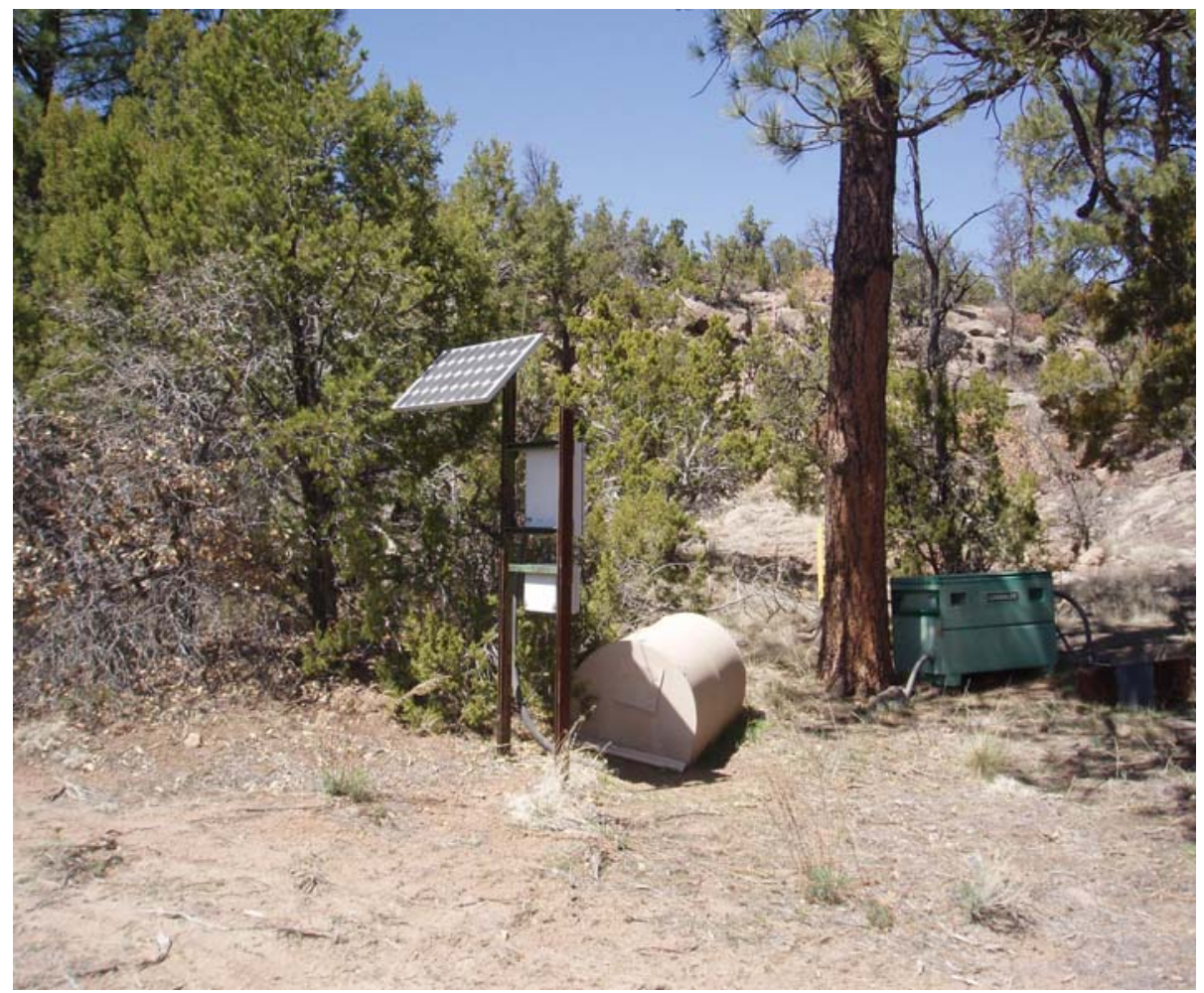




\section{E249 MDA G-4}

\section{Station Analysis}

\section{Water Year}

Equipment. Station is equipped with Sutron 8210 data logger (5-min. interval) and milltronics sonic probe mounted on a 9" Parshall flume. The system is powered by a solar-panel battery system housed in a NEMA shelter. Station is equipped with an ISCO pump sampler for water-quality sample collection. ISCO is housed in a separate shelter, a $3^{\prime} \times 4^{\prime}$ metal box. Sampler is triggered by stage through the data logger. The staff in the 9 " Parshall flume is the reference gage. No provision for discharge measurements above wading stage.

Field Work. The station was visited 16 times to conduct discharge measurements and service the instrumentation. Field inspections for the gage are listed under site history files on the Hydstra database. Discharge measurements for the gage are listed under site gauging files on the Hydstra database.

Datum Correction. None.

Gage-Height Record. The data logger referenced to the outside staff gave a complete and satisfactory record except for the period of November 24 to December 6 when the data logger malfunctioned as a result of a dead battery.

Rating. The control for this station is the 9" Parshall flume. The channel is straight 20' above and below gage. The streambed consists of sand and boulders.

Sixteen inspections of no flow were made this year.

Rating No. 1 was developed based on the computation of a 9" Parshall flume. Point of zero flow is 0.00 gage height.

Discharge. Discharge was computed by applying gage height to Rating No. 1 directly. Those days estimated at zero flow were based on precipitation and nearby gage stations for verification.

Remarks. Records are good. 


\section{E249 MDA G-4}

Daily Mean Discharge in Cubic Feet per Second

Water Year October 2007 to September 2008

\begin{tabular}{|c|c|c|c|c|c|c|c|c|c|c|c|c|}
\hline DAY & OCT & NOV & DEC & JAN & FEB & MAR & APR & MAY & JUN & JUL & AUG & SEP \\
\hline 1 & 0 & 0 & $0^{*}$ & 0 & 0 & 0 & 0 & 0 & 0 & 0 & 0 & 0 \\
\hline 2 & 0 & 0 & $0^{*}$ & 0 & 0 & 0 & 0 & 0 & 0 & 0 & 0 & 0 \\
\hline 3 & 0 & 0 & $0^{*}$ & 0 & 0 & 0 & 0 & 0 & 0 & 0 & 0 & 0 \\
\hline 4 & 0 & 0 & $0^{*}$ & 0 & 0 & 0 & 0 & 0 & 0 & 0 & 0 & 0 \\
\hline 5 & 0 & 0 & $0^{*}$ & 0 & 0 & 0 & 0 & 0 & 0 & 0 & 0 & 0 \\
\hline 6 & 0 & 0 & 0 & 0 & 0 & 0 & 0 & 0 & 0 & 0 & 0 & 0 \\
\hline 7 & 0 & 0 & 0 & 0 & 0 & 0 & 0 & 0 & 0 & 0 & 0 & 0 \\
\hline 8 & 0 & 0 & 0 & 0 & 0 & 0 & 0 & 0 & 0 & 0 & 0 & 0 \\
\hline 9 & 0 & 0 & 0 & 0 & 0 & 0 & 0 & 0 & 0 & 0 & 0 & 0 \\
\hline 10 & 0 & 0 & 0 & 0 & 0 & 0 & 0 & 0 & 0 & 0 & 0 & 0 \\
\hline 11 & 0 & 0 & 0 & 0 & 0 & 0 & 0 & 0 & 0 & 0 & 0 & 0 \\
\hline 12 & 0 & 0 & 0 & 0 & 0 & 0 & 0 & 0 & 0 & 0 & 0 & 0 \\
\hline 13 & 0 & 0 & 0 & 0 & 0 & 0 & 0 & 0 & 0 & 0 & 0 & 0 \\
\hline 14 & 0 & 0 & 0 & 0 & 0 & 0 & 0 & 0 & 0 & 0 & 0 & 0 \\
\hline 15 & 0 & 0 & 0 & 0 & 0 & 0 & 0 & 0 & 0 & 0 & 0 & 0 \\
\hline 16 & 0 & 0 & 0 & 0 & 0 & 0 & 0 & 0 & 0 & 0 & 0 & 0 \\
\hline 17 & 0 & 0 & 0 & 0 & 0 & 0 & 0 & 0 & 0 & .01 & 0 & 0 \\
\hline 18 & 0 & 0 & 0 & 0 & 0 & 0 & 0 & 0 & 0 & 0 & 0 & 0 \\
\hline 19 & 0 & 0 & 0 & 0 & 0 & 0 & 0 & 0 & 0 & 0 & 0 & 0 \\
\hline 20 & 0 & 0 & 0 & 0 & 0 & 0 & 0 & 0 & 0 & 0 & 0 & 0 \\
\hline 21 & 0 & 0 & 0 & 0 & 0 & 0 & 0 & 0 & 0 & 0 & 0 & 0 \\
\hline 22 & 0 & 0 & 0 & 0 & 0 & 0 & 0 & 0 & 0 & 0 & 0 & 0 \\
\hline 23 & 0 & 0 & 0 & 0 & 0 & 0 & 0 & 0 & 0 & 0 & 0 & 0 \\
\hline 24 & 0 & $0^{*}$ & 0 & 0 & 0 & 0 & 0 & 0 & 0 & 0 & 0 & 0 \\
\hline 25 & 0 & $0^{*}$ & 0 & 0 & 0 & 0 & 0 & 0 & 0 & 0 & 0 & 0 \\
\hline 26 & 0 & $0^{*}$ & 0 & 0 & 0 & 0 & 0 & 0 & 0 & 0 & 0 & 0 \\
\hline 27 & 0 & $0^{*}$ & 0 & .02 & 0 & 0 & 0 & 0 & 0 & 0 & 0 & 0 \\
\hline 28 & 0 & $0^{*}$ & 0 & .04 & 0 & 0 & 0 & 0 & 0 & 0 & 0 & 0 \\
\hline 29 & 0 & $0^{*}$ & 0 & 0 & 0 & 0 & 0 & 0 & 0 & 0 & 0 & 0 \\
\hline 30 & 0 & $0^{*}$ & 0 & 0 & ------ & 0 & 0 & 0 & 0 & 0 & 0 & 0 \\
\hline 31 & 0 & ----- & 0 & 0 & ------ & 0 & ----- & 0 & ----- & 0 & 0 & ------ \\
\hline Total & 0 & 0 & 0 & 0.06 & 0 & 0 & 0 & 0 & 0 & 0.01 & 0 & 0 \\
\hline Mean & 0 & 0 & 0 & .002 & 0 & 0 & 0 & 0 & 0 & 0 & 0 & 0 \\
\hline Max & 0 & 0 & 0 & .04 & 0 & 0 & 0 & 0 & 0 & .01 & 0 & 0 \\
\hline Min & 0 & 0 & 0 & 0 & 0 & 0 & 0 & 0 & 0 & 0 & 0 & 0 \\
\hline Acre-Ft & 0 & 0 & 0 & .12 & 0 & 0 & 0 & 0 & 0 & .02 & 0 & 0 \\
\hline Wtr Year & 2008 & Total & 0.07 & Mean & & 0 & Max & .04 & Min & 0 & Acre-Ft & 0.14 \\
\hline Cal Year & 2007 & Total & 0.01 & Mean & & 0 & Max & .01 & Min & 0 & Acre-Ft & 0.02 \\
\hline
\end{tabular}

*Estimate 
Location. Lat $35^{\circ}$ 49' 47", long $106^{\circ}$ 14' 05", Sec. 31, T. 19 N., R. 7 E., Ramon Vigil Grant, Los Alamos County on left bank.

Drainage Area. $0.01 \mathrm{mi}^{2}$.

Period of Record. October 1, 2005, to September 30, 2008.

Gage. Data logger and 9" Parshall flume, as well as rain gage with cellular telemetry. Elevation of gage is 6,633 ft above NGVD from GIS 9.1.

Remarks. Records are good. Records for this site existed before period of record but are not reliable. Legal location based on projected values.

Extremes for Period of Record. Maximum discharge, $5.7 \mathrm{ft}^{3} / \mathrm{s}$, August 7, 2006, gage height $1.50 \mathrm{ft}$. No flow most of the time.

Extremes for Current Year. Peak discharges above $1.0 \mathrm{ft}^{3} / \mathrm{s}$ and maximum (*):

\begin{tabular}{|c|c|c|c|}
\hline Date & Time & Discharge $\left.\mathbf{( f t}^{\mathbf{3}} / \mathbf{s}\right)$ & Gage Height (ft) \\
\hline November 30 & 2305 & 1.90 & 0.73 \\
\hline July 17 & 1320 & $3.80^{*}$ & $1.15^{*}$ \\
\hline July 26 & 1605 & 1.23 & 0.55 \\
\hline August 4 & 1755 & 1.86 & 0.72 \\
\hline August 9 & 1305 & 1.00 & 0.48 \\
\hline August 14 & 1335 & 1.13 & 0.52 \\
\hline September 12 & 1245 & 1.33 & 0.58 \\
\hline
\end{tabular}

No flow most of the time.

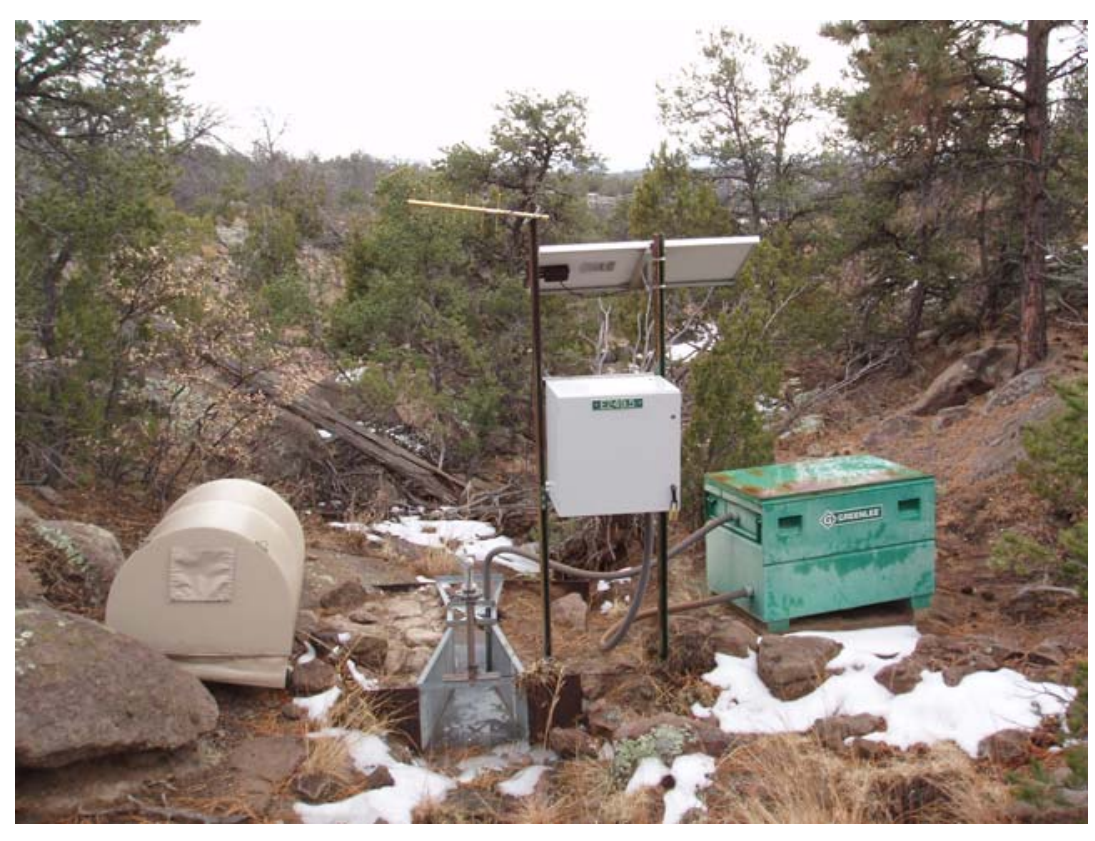




\section{E2495 MDA-G-7}

\section{Station Analysis}

\section{Water Year}

Equipment. Station is equipped with Sutron 8210 data logger (5-min. interval) and milltronics sonic probe mounted on a 9" Parshall flume with cellular phone and speech modem. Phone was upgraded May 8 from analog to digital service. The system is powered by a solar-panel battery system housed in a NEMA shelter. Station is equipped with an ISCO pump sampler for water-quality sample collection. ISCO is housed in a separate shelter, a $3^{\prime} \times 4^{\prime}$ metal box. Sampler is triggered by stage through the data logger. The staff in the 9" Parshall flume is the reference gage. No provision for discharge measurements above wading stage.

Station is also equipped with a rain gage, Rain Collection II, which was installed March 26. All equipment is powered with a solar-panel battery-charging system. Rain data are seasonal.

Field Work. The station was visited 24 times to conduct discharge measurements and service the instrumentation. Field inspections for the gage are listed under site history files on the Hydstra database. Discharge measurements for the gage are listed under site gauging files on the Hydstra database.

Datum Correction. None.

Gage-Height Record. The data logger referenced to the outside staff gave a complete and satisfactory record for the year.

Rating. The channel is straight above and below gage. It is confined to the main channel by cut banks on both sides. The bottom is $4^{\prime}$ wide channel; both banks should be very stable. Low-water control is the 9" Parshall flume.

Twenty-two visits of no flow and two inspections with flow were made this year.

Rating No. 1 was developed based on the computation of 9" Parshall flume. Point of zero flow is 0.00 gage height.

Discharge. Discharge was computed by applying gage height to Rating No. 1 directly.

Remarks. Records are good. 
E2495 MDA G-7

Daily Mean Discharge in Cubic Feet per Second

Water Year October 2007 to September 2008

\begin{tabular}{|c|c|c|c|c|c|c|c|c|c|c|c|c|}
\hline DAY & ОСт & NOV & DEC & JAN & FEB & MAR & APR & MAY & JUN & JUL & AUG & SEP \\
\hline 1 & .01 & 0 & .20 & 0 & 0 & 0 & 0 & 0 & 0 & .01 & 0 & .02 \\
\hline 2 & 0 & 0 & 0 & 0 & 0 & 0 & 0 & 0 & 0 & 0 & 0 & 0 \\
\hline 3 & 0 & 0 & 0 & 0 & 0 & 0 & 0 & 0 & 0 & 0 & 0 & 0 \\
\hline 4 & 0 & 0 & 0 & 0 & 0 & 0 & 0 & 0 & 0 & 0 & .09 & 0 \\
\hline 5 & 0 & 0 & 0 & 0 & 0 & 0 & 0 & 0 & 0 & 0 & 0 & 0 \\
\hline 6 & 0 & 0 & 0 & 0 & 0 & 0 & 0 & 0 & 0 & 0 & 0 & 0 \\
\hline 7 & 0 & 0 & 0 & 0 & 0 & 0 & 0 & 0 & 0 & .02 & 0 & 0 \\
\hline 8 & 0 & 0 & .07 & 0 & 0 & 0 & 0 & 0 & 0 & .01 & .01 & 0 \\
\hline 9 & 0 & 0 & 0 & 0 & 0 & 0 & 0 & 0 & 0 & 0 & .02 & 0 \\
\hline 10 & 0 & 0 & 0 & 0 & 0 & 0 & 0 & 0 & 0 & 0 & .01 & 0 \\
\hline 11 & 0 & 0 & 0 & 0 & 0 & 0 & 0 & 0 & 0 & 0 & 0 & 0 \\
\hline 12 & 0 & 0 & 0 & 0 & 0 & 0 & 0 & 0 & 0 & 0 & 0 & .03 \\
\hline 13 & 0 & 0 & 0 & 0 & 0 & 0 & 0 & 0 & 0 & 0 & 0 & 0 \\
\hline 14 & 0 & 0 & 0 & 0 & 0 & 0 & 0 & .01 & 0 & 0 & .02 & 0 \\
\hline 15 & 0 & 0 & 0 & 0 & 0 & 0 & 0 & .06 & 0 & .01 & 0 & 0 \\
\hline 16 & 0 & 0 & 0 & 0 & 0 & 0 & 0 & 0 & 0 & 0 & .01 & 0 \\
\hline 17 & 0 & 0 & 0 & 0 & 0 & 0 & 0 & 0 & 0 & .07 & 0 & 0 \\
\hline 18 & 0 & 0 & 0 & 0 & 0 & 0 & 0 & 0 & 0 & 0 & 0 & 0 \\
\hline 19 & 0 & 0 & 0 & 0 & 0 & 0 & 0 & 0 & 0 & 0 & 0 & 0 \\
\hline 20 & 0 & 0 & 0 & 0 & 0 & 0 & 0 & 0 & 0 & 0 & 0 & 0 \\
\hline 21 & 0 & 0 & 0 & 0 & 0 & 0 & 0 & 0 & 0 & 0 & 0 & 0 \\
\hline 22 & 0 & 0 & 0 & 0 & 0 & 0 & 0 & 0 & 0 & 0 & 0 & 0 \\
\hline 23 & 0 & 0 & 0 & 0 & 0 & 0 & 0 & .01 & 0 & 0 & .01 & 0 \\
\hline 24 & 0 & 0 & 0 & 0 & .01 & 0 & 0 & 0 & 0 & 0 & .04 & 0 \\
\hline 25 & 0 & 0 & 0 & 0 & 0 & 0 & 0 & 0 & 0 & 0 & 0 & 0 \\
\hline 26 & 0 & 0 & 0 & 0 & 0 & 0 & 0 & 0 & 0 & .03 & 0 & 0 \\
\hline 27 & 0 & 0 & 0 & .01 & 0 & 0 & 0 & 0 & 0 & 0 & 0 & 0 \\
\hline 28 & 0 & 0 & 0 & .08 & 0 & 0 & 0 & 0 & 0 & 0 & 0 & 0 \\
\hline 29 & 0 & 0 & 0 & 0 & 0 & 0 & 0 & 0 & 0 & 0 & 0 & 0 \\
\hline 30 & 0 & .17 & 0 & 0 & ------ & 0 & 0 & 0 & 0 & 0 & 0 & 0 \\
\hline 31 & 0 & ------ & 0 & 0 & ------ & 0 & ------ & 0 & ------ & 0 & .04 & ----- \\
\hline Total & 0.01 & 0.17 & 0.27 & 0.09 & 0.01 & 0 & 0 & 0.08 & 0 & 0.15 & 0.25 & 0.05 \\
\hline Mean & 0 & .006 & .009 & .003 & 0 & 0 & 0 & .003 & 0 & .005 & .008 & .002 \\
\hline Max & .01 & .17 & .20 & .08 & .01 & 0 & 0 & .06 & 0 & .07 & .09 & .03 \\
\hline Min & 0 & 0 & 0 & 0 & 0 & 0 & 0 & 0 & 0 & 0 & 0 & 0 \\
\hline Acre-Ft & .02 & .34 & .54 & .18 & .02 & 0 & 0 & .16 & 0 & .30 & .50 & .10 \\
\hline Wtr Year & 2008 & Total & 1.08 & Mean & & & hax & .20 & Min & 0 & Acre-Ft & 2.1 \\
\hline Cal Year & 2007 & Total & 1.81 & Mean & & 5 & hax & .48 & Min & 0 & Acre-Ft & 3.6 \\
\hline
\end{tabular}


E2495 MDA G-7

Daily Total Rainfall in Inches

Water Year October 2007 to September 2008

\begin{tabular}{|c|c|c|c|c|c|c|c|c|c|c|c|c|}
\hline DAY & ОСТ & NOV & DEC & JAN & FEB & MAR & APR & MAY & JUN & JUL & AUG & SEP \\
\hline 1 & & & & & & & & 0 & 0 & .09 & 0 & .01 \\
\hline 2 & & & & & & & & 0 & 0 & .01 & 0 & 0 \\
\hline 3 & & & & & & & & 0 & 0 & 0 & 0 & 0 \\
\hline 4 & & & & & & & & 0 & 0 & .01 & .52 & 0 \\
\hline 5 & & & & & & & & 0 & .01 & 0 & 0 & 0 \\
\hline 6 & & & & & & & & 0 & 0 & 0 & 0 & 0 \\
\hline 7 & & & & & & & & 0 & 0 & .38 & .02 & 0 \\
\hline 8 & & & & & & & & 0 & 0 & .17 & .10 & 0 \\
\hline 9 & & & & & & & & 0 & 0 & 0 & .13 & 0 \\
\hline 10 & & & & & & & & 0 & 0 & 0 & .10 & 0 \\
\hline 11 & & & & & & & & 0 & 0 & .01 & 0 & 0 \\
\hline 12 & & & & & & & & 0 & 0 & .01 & 0 & $.12^{*}$ \\
\hline 13 & & & & & & & & 0 & 0 & 0 & 0 & 0 \\
\hline 14 & & & & & & & & .09 & 0 & 0 & .07 & 0 \\
\hline 15 & & & & & & & & .51 & 0 & .31 & 0 & 0 \\
\hline 16 & & & & & & & & 0 & 0 & .04 & .09 & 0 \\
\hline 17 & & & & & & & & 0 & 0 & .82 & 0 & 0 \\
\hline 18 & & & & & & & & 0 & 0 & .02 & 0 & 0 \\
\hline 19 & & & & & & & & 0 & 0 & 0 & 0 & 0 \\
\hline 20 & & & & & & & & 0 & .01 & 0 & 0 & 0 \\
\hline 21 & & & & & & & 0 & 0 & 0 & .02 & 0 & 0 \\
\hline 22 & & & & & & & 0 & .06 & 0 & .10 & 0 & 0 \\
\hline 23 & & & & & & & 0 & .11 & .01 & 0 & $.44^{\star}$ & 0 \\
\hline 24 & & & & & & & 0 & 0 & 0 & 0 & $.28^{\star}$ & 0 \\
\hline 25 & & & & & & & 0 & 0 & 0 & 0 & 0 & 0 \\
\hline 26 & & & & & & & 0 & 0 & 0 & .24 & 0 & .11 \\
\hline 27 & & & & & & & 0 & 0 & 0 & .05 & 0 & 0 \\
\hline 28 & & & & & & & 0 & .06 & 0 & .04 & 0 & 0 \\
\hline 29 & & & & & & & 0 & 0 & .01 & 0 & 0 & 0 \\
\hline 30 & & & & & ------ & & 0 & 0 & 0 & 0 & 0 & 0 \\
\hline 31 & & ----- & & & ----- & & ----- & 0 & ----- & 0 & $.41^{*}$ & ----- \\
\hline Total & & & & & & & 0 & 0.83 & 0.04 & 2.32 & 1.05 & 0.15 \\
\hline $\operatorname{Max}$ & & & & & & & 0 & .51 & .01 & .82 & .52 & .11 \\
\hline Wtr Year & 2008 & Total & 4.39 & & & .027 & Max & .82 & Min & 0 & InstMax & .34 \\
\hline Cal Year & 2007 & Total & & & & & $\operatorname{Max}$ & & Min & & InstMax & \\
\hline
\end{tabular}

*Estimate 


\section{E250 Pajarito Canyon above SR 4}

Location. Lat $35^{\circ}$ 49' 26", long $106^{\circ}$ 13' 40", Sec. 5, T. 18 N., R. 7 E., Ramon Vigil Grant, Los Alamos County.

Drainage Area. $10.6 \mathrm{mi}^{2}$.

Period of Record. November 1993 to August 25, 2006 (destroyed by flood); September 2006 to September 30, 2008.

Revised Record. Drainage area (2006).

Gage. Data logger with cellular telemetry and concrete control. Elevation of gage is 6,528 ft. above NGVD from GPS survey.

Remarks. Records are good. Legal location based on projected values.

Average Discharge. $14 \mathrm{yr}, 0.06 \mathrm{ft}^{3} / \mathrm{s}, 40$ acre-ft/yr.

Extremes for Period of Record. Maximum discharge, $206 \mathrm{ft}^{3} / \mathrm{s}$, August 25, 2006, gage height $4.62 \mathrm{ft}$ (from peak-flow computations). No flow most of the time.

Extremes for Current Water Year. Peak discharges above $5.0 \mathrm{ft}^{3} / \mathrm{s}$ and maximum (*):

\begin{tabular}{|c|c|c|c|}
\hline Date & Time & Discharge $\left.\mathbf{( f t}^{\mathbf{3}} / \mathbf{s}\right)$ & Gage Height (ft) \\
\hline December 1 & 0910 & $25^{*}$ & $3.85^{*}$ \\
\hline January 28 & 1415 & 8.59 & 3.08 \\
\hline February 25 & 0205 & 7.02 & 2.96 \\
\hline
\end{tabular}

No flow most of the time.

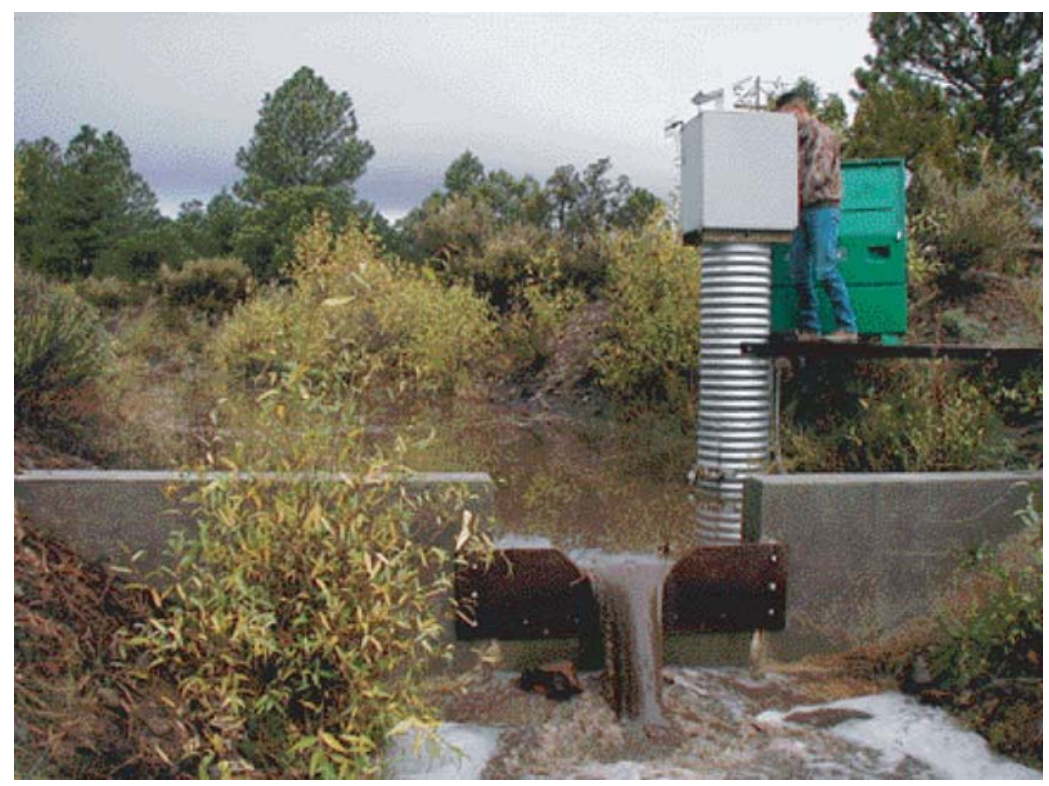




\section{E250 Pajarito Canyon above SR 4}

\section{Station Analysis}

\section{Water Year}

Equipment. Station is equipped with Sutron 8210 (5-min. interval) with shaft encoder float system (5-min. interval) and cellular phone with speech modem. Phone was upgraded July 1 from analog to digital service. The system is powered by a solar-panel battery system housed in NEMA shelter on 18" CMP well. Station is equipped with an ISCO pump sampler for water-quality sample collection. ISCO is housed in a separate shelter, a $3^{\prime} \times 4^{\prime}$ metal box. Sampler is triggered by stage through the data logger. An outside staff is available for reference. No provision for direct discharge measurements above wading stages.

Field Work. The station was visited 18 times to conduct discharge measurements and service the instrumentation. Field inspections for the gage are listed under site history files on the Hydstra database. Discharge measurements for the gage are listed under site gauging files on the Hydstra database.

Datum Correction. None. Most recent levels run November 17, 2004, found gage within acceptable limits.

Gage-Height Record. The data logger referenced to the outside staff gave a complete and satisfactory record except for the period of January 31 to February 24 when ice affected gage height.

Rating. The channel is straight for $50^{\prime}$ above and $100^{\prime}$ below gage. Streambed material consists of gravel. The control consists of concrete with a $90^{\circ}$ weir plate.

One discharge measurement (No. 12) and 18 visits were made this water year.

Rating No. 1 was developed from $90^{\circ}$ weir formula and broad crested weir computation above notch. Rating No. 1 was continued in use and is considered good.

Discharge. Discharge was computed by applying gage height to Rating No. 1 with "V" diagram a shift of +0.01 applied for all low-flow periods. This shift value was defined by previous measurements.

Remarks. Records are good. Access to this station is controlled by Dynamic Experimentation access control personnel because of explosive testing that takes place in parts of the area. 
E250 Pajarito Canyon above SR 4

Daily Mean Discharge in Cubic Feet per Second

Water Year October 2007 to September 2008

\begin{tabular}{|c|c|c|c|c|c|c|c|c|c|c|c|c|}
\hline DAY & OCT & NOV & DEC & JAN & FEB & MAR & APR & MAY & JUN & JUL & AUG & SEP \\
\hline 1 & 0 & 0 & 7.0 & 0 & $.05^{\star}$ & .49 & .15 & .10 & .02 & 0 & 0 & 0 \\
\hline 2 & 0 & 0 & .29 & 0 & $0^{*}$ & 1.0 & .16 & .10 & 0 & 0 & 0 & 0 \\
\hline 3 & 0 & 0 & .06 & 0 & $0^{*}$ & .76 & .20 & .10 & 0 & 0 & 0 & 0 \\
\hline 4 & 0 & 0 & .05 & 0 & $0^{*}$ & .63 & .20 & .10 & 0 & 0 & 0 & 0 \\
\hline 5 & 0 & 0 & .04 & 0 & $0^{*}$ & .60 & .22 & .10 & 0 & 0 & 0 & 0 \\
\hline 6 & 0 & 0 & .04 & 0 & $0^{*}$ & .55 & .21 & .10 & 0 & 0 & 0 & 0 \\
\hline 7 & 0 & 0 & .04 & 0 & $0^{*}$ & .57 & .18 & .10 & 0 & 0 & 0 & 0 \\
\hline 8 & 0 & 0 & .11 & 0 & $0^{*}$ & .49 & .17 & .09 & 0 & 0 & 0 & 0 \\
\hline 9 & 0 & 0 & .05 & 0 & $0^{*}$ & .44 & .17 & .08 & 0 & 0 & 0 & 0 \\
\hline 10 & 0 & 0 & 0 & 0 & $0^{*}$ & .34 & .20 & .08 & 0 & 0 & 0 & 0 \\
\hline 11 & 0 & 0 & 0 & 0 & $0^{*}$ & .30 & .17 & .08 & 0 & 0 & 0 & 0 \\
\hline 12 & 0 & 0 & 0 & 0 & $0^{*}$ & .30 & .17 & .08 & 0 & 0 & 0 & 0 \\
\hline 13 & 0 & 0 & 0 & 0 & $0^{*}$ & .35 & .15 & .07 & 0 & 0 & 0 & 0 \\
\hline 14 & 0 & 0 & 0 & 0 & $0^{*}$ & .47 & .15 & .12 & 0 & 0 & 0 & 0 \\
\hline 15 & 0 & 0 & 0 & 0 & $0^{*}$ & .64 & .14 & .21 & 0 & 0 & 0 & 0 \\
\hline 16 & 0 & 0 & 0 & 0 & $0^{*}$ & .56 & .13 & .12 & 0 & 0 & 0 & 0 \\
\hline 17 & 0 & 0 & 0 & 0 & $0^{*}$ & .54 & .12 & .10 & 0 & 0 & 0 & 0 \\
\hline 18 & 0 & 0 & 0 & 0 & $0^{*}$ & .40 & .15 & .09 & 0 & 0 & 0 & 0 \\
\hline 19 & 0 & 0 & 0 & 0 & $0^{*}$ & .30 & .15 & .07 & 0 & 0 & 0 & 0 \\
\hline 20 & 0 & 0 & 0 & 0 & $0^{*}$ & .28 & .14 & .07 & 0 & 0 & 0 & 0 \\
\hline 21 & 0 & 0 & 0 & 0 & $0^{*}$ & .24 & .13 & .06 & 0 & 0 & 0 & 0 \\
\hline 22 & 0 & 0 & 0 & 0 & $0^{*}$ & .20 & .13 & .08 & 0 & 0 & 0 & 0 \\
\hline 23 & 0 & 0 & 0 & 0 & $.05^{\star}$ & .18 & .13 & .11 & 0 & 0 & 0 & 0 \\
\hline 24 & 0 & 0 & 0 & 0 & $.10^{*}$ & .17 & .12 & .10 & 0 & 0 & 0 & 0 \\
\hline 25 & 0 & 0 & 0 & 0 & 1.0 & .18 & .11 & .08 & 0 & 0 & 0 & 0 \\
\hline 26 & 0 & 0 & 0 & 0 & .92 & .16 & .11 & .06 & 0 & 0 & 0 & 0 \\
\hline 27 & 0 & 0 & 0 & 0 & .35 & .16 & .11 & .05 & 0 & 0 & 0 & 0 \\
\hline 28 & 0 & 0 & 0 & 3.2 & .30 & .16 & .11 & .06 & 0 & 0 & 0 & 0 \\
\hline 29 & 0 & 0 & 0 & .36 & .37 & .16 & .11 & .07 & 0 & 0 & 0 & 0 \\
\hline 30 & 0 & 0 & 0 & .20 & ----- & .15 & .11 & .04 & 0 & 0 & 0 & 0 \\
\hline 31 & 0 & ------ & 0 & $.10^{*}$ & ----- & .15 & ----- & .03 & ----- & 0 & 0 & ------ \\
\hline Total & 0 & 0 & 7.68 & 3.86 & 3.14 & 11.92 & 4.50 & 2.70 & 0.02 & 0 & 0 & 0 \\
\hline Mean & 0 & 0 & .25 & .12 & .11 & .38 & .15 & .087 & .001 & 0 & 0 & 0 \\
\hline Max & 0 & 0 & 7.0 & 3.2 & 1.0 & 1.0 & .22 & .21 & .02 & 0 & 0 & 0 \\
\hline Min & 0 & 0 & 0 & 0 & 0 & .15 & .11 & .03 & 0 & 0 & 0 & 0 \\
\hline Acre-Ft & 0 & 0 & 15 & 7.7 & 6.2 & 24 & 8.9 & 5.4 & .04 & 0 & 0 & 0 \\
\hline Wtr Year & 2008 & Total & 33.82 & Mean & & 092 & Max & 7.0 & Min & 0 & Acre-Ft & 67 \\
\hline Cal Year & 2007 & Total & 7.95 & Mean & & b22 & Max & 7.0 & Min & 0 & Acre-Ft & 16 \\
\hline
\end{tabular}

*Estimate 


\section{E252 Water Canyon above SR 501}

Location. Lat $35^{\circ}$ 50' 18", long $106^{\circ}$ 21' 42", Sec. 36, T. 19 N., R. 5 E., Los Alamos County in Santa Fe National Forest.

Drainage Area. $3.25 \mathrm{mi}^{2}$.

Period of Record. October 1994 to June 2000 (destroyed by flood); April 2001 to September 2008.

Revised Record. Drainage area (2006).

Gage. Data logger with cellular telemetry. Elevation of gage is 7,553 ft above NGVD from GPS survey.

Remarks. Records are good. Legal location based on projected values.

Average Discharge. $14 \mathrm{yr}, 0.12 \mathrm{ft}^{3} / \mathrm{s}, 85$ acre-ft/yr.

Extremes for Period of Record. Maximum discharge, $840 \mathrm{ft}^{3} / \mathrm{s}$ on June 28, 2000, from peak-flow computation, gage height $7.91 \mathrm{ft}$. No flow at times.

Extremes for Current Year. Maximum discharge $1.76 \mathrm{ft}^{3} / \mathrm{s}$ at $1735 \mathrm{~h}$, March 25, gage height $2.70 \mathrm{ft}$. No peak discharges above base of $2.0 \mathrm{ft}^{3} / \mathrm{s}$. No flow most of the time.

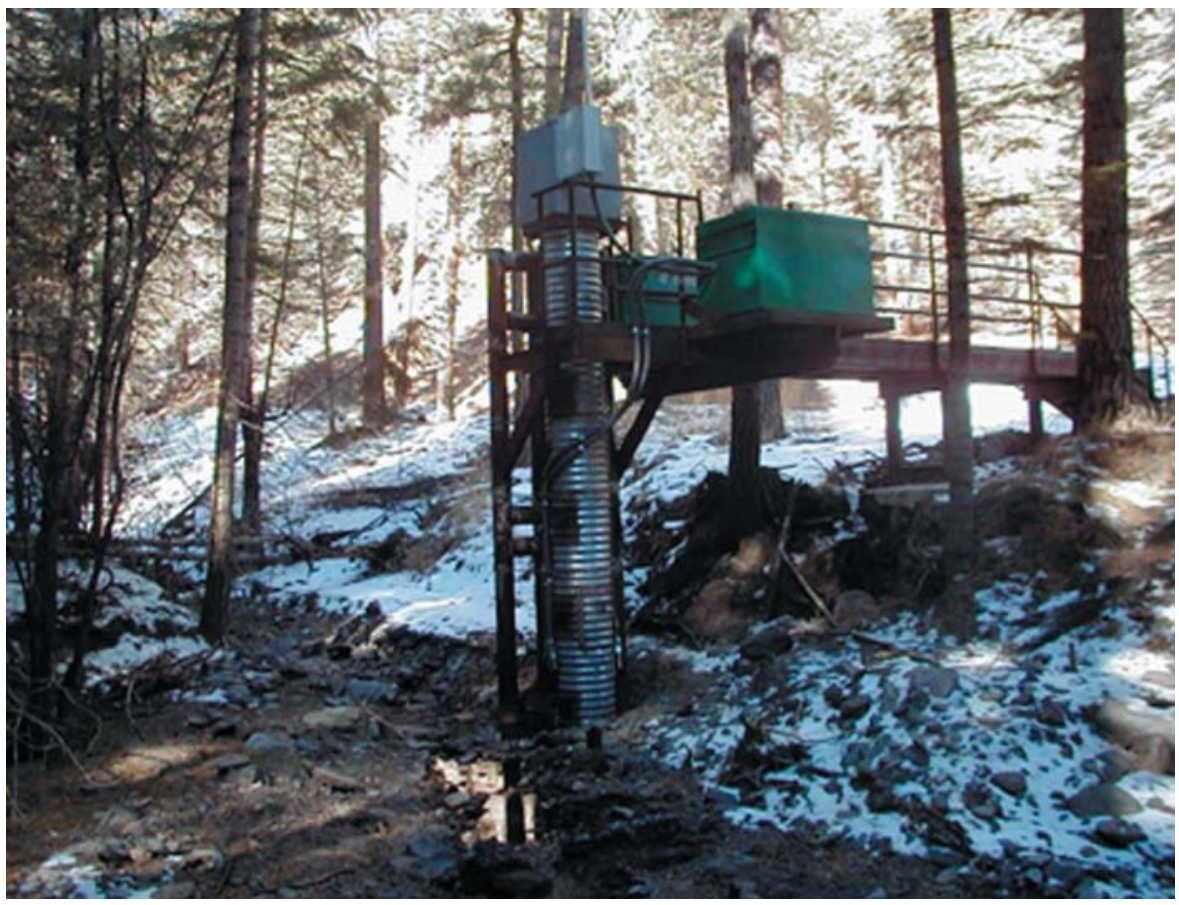




\section{E252 Water Canyon above SR 501}

\section{Station Analysis}

\section{Water Year}

Equipment. Station is equipped with Sutron 8210 (5-min. interval), with shaft encoder float system (5-min. interval) and cellular phone with speech modem. Phone upgraded May 13 from analog to digital service. The system is powered by a solar-panel battery system housed in NEMA shelter on 24" CMP well. Station is equipped with an ISCO pump sampler for water-quality sample collection. ISCO is housed in a separate shelter, a $3^{\prime} \times 4^{\prime}$ metal box. Sampler is triggered by stage through the data logger. An outside staff is available for reference. There is no low-water control or provision for direct discharge measurements above wading stages.

Field Work. The station was visited 16 times to conduct discharge measurement and service the instrumentation. Field inspections for the gage are listed under site history files on the Hydstra database. Discharge measurements for the gage are listed under site gauging files on the Hydstra database.

Datum Correction. None. Levels run when gage was established on April 16, 2001. New gage is at same datum as old and is about $20^{\prime}$ upstream of old well.

Gage-Height Record. The data logger referenced to the inside staff and reference point gave a complete and satisfactory record for the year.

Rating. The channel at the gage is $30^{\prime}$ wide and straight for about $40^{\prime}$ upstream then bends to the left; downstream, the gage is straight for 100'. The streambed through this reach primarily consists of sand, gravel, and cobbles. Low-flow control is rock riffle 5' below gage. The channel has been scoured and filled significantly by high flows that resulted from the Cerro Grande fire.

Five discharge measurements (Nos. 52-56) were made this year. All measurements listed indicated shifts ranging from 0.03 to -0.02 . The shifts were applied using several different variable stage shifts prorated over time.

Rating No. 3 should be considered good except for the extreme lower end (less than 0.5 $\mathrm{ft}^{3} / \mathrm{s}$ ), which will continue to change back and forth in response to high flows. Steep slopes in the gage reach and throughout the region cause considerable movement of material either scours or fills. Low-water records at this site will continue to be a problem until increased runoff from the burned areas returns to something close to prefire conditions.

Discharge. Discharge was computed by applying inside gage height to Rating No. 3 directly.

Remarks. Records are good. 
E252 Water Canyon above SR 501

Daily Mean Discharge in Cubic Feet per Second

Water Year October 2007 to September 2008

\begin{tabular}{|c|c|c|c|c|c|c|c|c|c|c|c|c|}
\hline DAY & OCT & NOV & DEC & JAN & FEB & MAR & APR & MAY & JUN & JUL & AUG & SEP \\
\hline 1 & .03 & .01 & .03 & .17 & .16 & .77 & 1.2 & .29 & .04 & .11 & .07 & .04 \\
\hline 2 & .03 & .01 & .18 & .14 & .23 & .91 & 1.0 & .29 & .12 & .11 & .07 & .04 \\
\hline 3 & .02 & .01 & .31 & .14 & .25 & .82 & .96 & .29 & .21 & .11 & .07 & .04 \\
\hline 4 & .02 & .01 & .26 & .14 & .26 & .81 & .89 & .26 & .21 & .11 & .07 & .05 \\
\hline 5 & .02 & .01 & .25 & .14 & .25 & .82 & .82 & .25 & .21 & .11 & .06 & .06 \\
\hline 6 & .02 & .01 & .23 & .14 & .25 & .76 & .76 & .25 & .21 & .11 & .06 & .06 \\
\hline 7 & .02 & .02 & .21 & .14 & .25 & .65 & .69 & .25 & .21 & .11 & .06 & .07 \\
\hline 8 & .02 & .02 & .23 & .14 & .25 & .65 & .65 & .22 & .21 & .11 & .06 & .07 \\
\hline 9 & .02 & .02 & .33 & .14 & .25 & .60 & .65 & .22 & .20 & .11 & .07 & .07 \\
\hline 10 & .01 & .02 & .33 & .13 & .22 & .52 & .58 & .22 & .17 & .11 & .06 & .07 \\
\hline 11 & .01 & .02 & .33 & .12 & .21 & .53 & .51 & .21 & .17 & .11 & .06 & .07 \\
\hline 12 & .01 & .02 & .33 & .11 & .21 & .60 & .51 & .21 & .17 & .11 & .06 & .07 \\
\hline 13 & .01 & .02 & .32 & .11 & .21 & .68 & .50 & .16 & .17 & .11 & .06 & .07 \\
\hline 14 & .01 & .02 & .29 & .11 & .21 & .84 & .46 & .09 & .15 & .11 & .06 & .07 \\
\hline 15 & .01 & .02 & .29 & .11 & .21 & .97 & .44 & .09 & .14 & .11 & .06 & .06 \\
\hline 16 & .01 & .02 & .28 & .11 & .22 & 1.1 & .44 & .09 & .14 & .11 & .06 & .06 \\
\hline 17 & .01 & .02 & .25 & .11 & .25 & 1.1 & .44 & .08 & .14 & .11 & .06 & .05 \\
\hline 18 & .01 & .02 & .25 & .11 & .37 & 1.0 & .44 & .07 & .14 & .11 & .04 & .03 \\
\hline 19 & .01 & .02 & .25 & .11 & .41 & 1.0 & .41 & .07 & .14 & .11 & .04 & .01 \\
\hline 20 & .01 & .02 & .21 & .11 & .44 & 1.1 & .39 & .07 & .14 & .11 & .04 & .01 \\
\hline 21 & .01 & .02 & .21 & .11 & .44 & 1.2 & .39 & .07 & .14 & .14 & .04 & .01 \\
\hline 22 & .01 & .02 & .21 & .11 & .44 & 1.3 & .37 & .06 & .14 & .17 & .04 & .01 \\
\hline 23 & .02 & .02 & .20 & .11 & .42 & 1.4 & .36 & .06 & .14 & .12 & .04 & .01 \\
\hline 24 & .02 & .02 & .17 & .11 & .42 & 1.5 & .33 & .06 & .13 & .07 & .04 & .01 \\
\hline 25 & .02 & .02 & .19 & .11 & .50 & 1.5 & .29 & .06 & .11 & .07 & .04 & .01 \\
\hline 26 & .02 & .02 & .21 & .09 & .52 & 1.6 & .31 & .06 & .11 & .07 & .03 & .01 \\
\hline 27 & .02 & .02 & .21 & .09 & .52 & 1.5 & .33 & .06 & .11 & .07 & .03 & .01 \\
\hline 28 & .01 & .03 & .17 & .11 & .60 & 1.5 & .33 & .05 & .11 & .07 & .03 & .01 \\
\hline 29 & .01 & .03 & .17 & .11 & .64 & 1.5 & .29 & .05 & .11 & .07 & .03 & .01 \\
\hline 30 & .01 & .03 & .17 & .11 & ------ & 1.4 & .29 & .04 & .11 & .07 & .03 & \\
\hline 31 & .01 & --.-- & .17 & .11 & ---- & 1.3 & ----- & .04 & ----- & .07 & .04 & ----- \\
\hline Total & 0.47 & 0.57 & 7.24 & 3.70 & 9.61 & 31.93 & 16.03 & 4.29 & 4.50 & 3.19 & 1.58 & 1.16 \\
\hline Mean & .015 & .019 & .23 & .12 & .33 & 1.03 & .53 & .14 & .15 & .10 & .051 & .040 \\
\hline $\operatorname{Max}$ & .03 & .03 & .33 & .17 & .64 & 1.6 & 1.2 & .29 & .21 & .17 & .07 & .07 \\
\hline Min & .01 & .01 & .03 & .09 & .16 & .52 & .29 & .04 & .04 & .07 & .03 & .01 \\
\hline Acre-Ft & .93 & 1.1 & 14 & 7.3 & 19 & 63 & 32 & 8.5 & 8.9 & 6.3 & 3.1 & 2.3 \\
\hline Wtr Year & 2008 & Total & 84.27 & Mean & & .23 & Max & 1.6 & Min & .01 & Acre-Ft & 167 \\
\hline Cal Year & 2007 & Total & 103.28 & Mean & & .28 & Max & 5.6 & Min & .01 & Acre-Ft & 205 \\
\hline
\end{tabular}


Location. Lat $35^{\circ}$ 49' 51", long $106^{\circ} 18^{\prime}$ 27", Sec. 33, T. 19 N., R. 6 E., Ramon Vigil Grant, Los Alamos County.

Drainage Area. $0.76 \mathrm{mi}^{2}$.

Period of Record. April 1999 to September 30, 2008.

Revised Record. Drainage area (2006).

Gage. Data logger and $90^{\circ}$ sharp-crested weir. Elevation of gage is $6,840 \mathrm{ft}$ above NGVD from GPS survey.

Remarks. Records are good. Legal location based on projected values.

Average Discharge. $9 \mathrm{yr}, 0.007 \mathrm{ft}^{3} / \mathrm{s}, 5.1$ acre-ft/yr.

Extremes for Period of Record. Maximum discharge, of $162 \mathrm{ft}^{3} / \mathrm{s}$, August 20, 2004, gage height $4.03 \mathrm{ft}$. No flow most of the time.

Extremes for Current Year. Peak discharges above base of $10 \mathrm{ft}^{3} / \mathrm{s}$ and maximum (*):

\begin{tabular}{|c|c|c|c|}
\hline Date & Time & Discharge (ft3/s) & Gage Height (ft) \\
\hline December 1 & 0210 & 18 & 2.99 \\
\hline January 28 & 0500 & $31^{*}$ & $3.30^{*}$ \\
\hline August 10 & 1230 & 25 & 3.17 \\
\hline August 18 & 2235 & 10 & 2.62 \\
\hline August 23 & 1705 & 11 & 2.70 \\
\hline August 24 & 1920 & 10 & 2.60 \\
\hline
\end{tabular}

No flow most of the time.

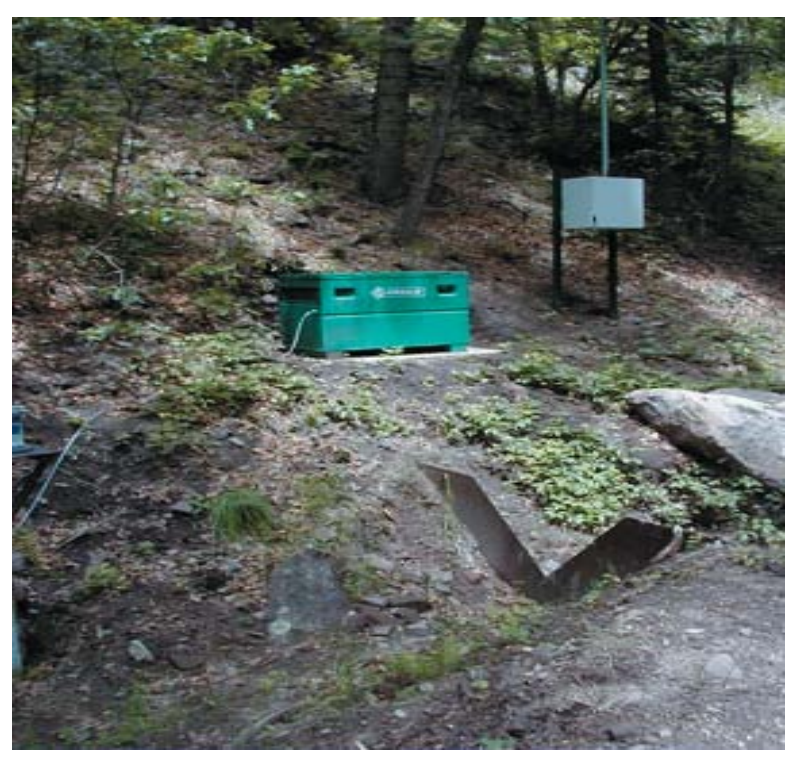




\section{E2528 S Site Canyon above Water Canyon}

\section{Station Analysis}

\section{Water Year}

Equipment. Station is equipped with Sutron 8210 data logger (5-min. interval) with milltronics sonic probe. The system is powered by a solar-panel battery system housed in a NEMA shelter. Station is equipped with an ISCO pump sampler for water-quality sample collection. ISCO is housed in a separate shelter, a $3^{\prime} \times 4^{\prime}$ metal box. Sampler is triggered by stage through the data logger. An outside staff is available for reference. No provision for discharge measurements above wading stage.

Cellular telemetry removed March 28, discontinued service.

Field Work. The station was visited 15 times to conduct discharge measurements and service the instrumentation. Field inspections for the gage are listed under site history files on the Hydstra database. Discharge measurements for the gage are listed under site gauging files on the Hydstra database.

Datum Correction. None.

Gage-Height Record. The data logger referenced to the outside staff gave a complete and satisfactory record except for the periods of January 29 to March 3 when ice affected gage height and March 28 to April 28 when the data logger malfunctioned.

Rating. Control is $90^{\circ}$ weir with $2^{\prime}$ deep notch. Canyon is very steep directly above station, but it does flatten out enough to allow weir to be effective.

Fifteen inspections of no flow were made this year.

Rating No. 1 was developed using weir formula $\mathrm{Q}=2.49$ h 2.48; one critical depth computation was made during the 2004 water year. Rating No. 1 is considered fair. Large shifts were applied to the low end (PZF) because of filling in the pool. These shifts are most likely to change over time when fill conditions occur.

Discharge. Discharge was computed by applying gage height to Rating No. 1 with "V" diagrams adjusting the PZF. Those periods estimated at zero flow were based on precipitation and adjoining stations E2525 and E262.

Remarks. Records are good except for estimated daily discharges, which are fair. 


\section{E2528 S Site Canyon above Water Canyon}

Daily Mean Discharge in Cubic Feet per Second

Water Year October 2007 to September 2008

\begin{tabular}{|c|c|c|c|c|c|c|c|c|c|c|c|c|}
\hline DAY & OCT & NOV & DEC & JAN & FEB & MAR & APR & MAY & JUN & JUL & AUG & SEP \\
\hline 1 & 0 & 0 & 2.6 & 0 & $0^{*}$ & $0^{*}$ & $0^{*}$ & 0 & 0 & 0 & 0 & 0 \\
\hline 2 & 0 & 0 & .02 & 0 & $0^{*}$ & $0^{*}$ & $0^{*}$ & 0 & 0 & 0 & 0 & 0 \\
\hline 3 & 0 & 0 & 0 & 0 & $0^{*}$ & $0^{*}$ & $0^{*}$ & 0 & 0 & 0 & 0 & 0 \\
\hline 4 & 0 & 0 & 0 & 0 & $0^{*}$ & 0 & $0^{*}$ & 0 & 0 & 0 & .28 & 0 \\
\hline 5 & 0 & 0 & 0 & 0 & $0^{*}$ & 0 & $0^{*}$ & 0 & 0 & 0 & 0 & 0 \\
\hline 6 & 0 & 0 & 0 & 0 & $0^{*}$ & 0 & $0^{*}$ & 0 & 0 & 0 & 0 & 0 \\
\hline 7 & 0 & 0 & 0 & 0 & $0^{*}$ & 0 & $0^{*}$ & 0 & 0 & 0 & 0 & 0 \\
\hline 8 & 0 & 0 & 0 & 0 & $0^{*}$ & 0 & $0^{*}$ & 0 & 0 & 0 & .27 & 0 \\
\hline 9 & 0 & 0 & 0 & 0 & $0^{*}$ & 0 & $0^{*}$ & 0 & 0 & 0 & 0 & 0 \\
\hline 10 & 0 & 0 & 0 & 0 & $0^{*}$ & 0 & $0^{*}$ & 0 & 0 & 0 & 1.2 & 0 \\
\hline 11 & 0 & 0 & 0 & 0 & $0^{*}$ & 0 & $0^{*}$ & 0 & 0 & 0 & .02 & 0 \\
\hline 12 & 0 & 0 & 0 & 0 & $0^{*}$ & 0 & $0^{*}$ & 0 & 0 & 0 & 0 & 0 \\
\hline 13 & 0 & 0 & 0 & 0 & $0^{*}$ & 0 & $0^{*}$ & 0 & 0 & 0 & 0 & 0 \\
\hline 14 & 0 & 0 & 0 & 0 & $0^{*}$ & 0 & $0^{*}$ & 0 & 0 & 0 & 0 & 0 \\
\hline 15 & 0 & 0 & 0 & 0 & $0^{*}$ & 0 & $0^{*}$ & 0 & 0 & 0 & 0 & 0 \\
\hline 16 & 0 & 0 & 0 & 0 & $0^{*}$ & 0 & $0^{*}$ & 0 & 0 & 0 & 0 & 0 \\
\hline 17 & 0 & 0 & 0 & 0 & $0^{*}$ & 0 & $0^{*}$ & 0 & 0 & 0 & 0 & 0 \\
\hline 18 & 0 & 0 & 0 & 0 & $0^{*}$ & 0 & $0^{*}$ & 0 & 0 & 0 & .27 & 0 \\
\hline 19 & 0 & 0 & 0 & 0 & $0^{*}$ & 0 & $0^{*}$ & 0 & 0 & 0 & 0 & 0 \\
\hline 20 & 0 & 0 & 0 & 0 & $0^{*}$ & 0 & $0^{*}$ & 0 & 0 & 0 & 0 & 0 \\
\hline 21 & 0 & 0 & 0 & 0 & $0^{*}$ & 0 & $0^{*}$ & 0 & 0 & 0 & 0 & 0 \\
\hline 22 & 0 & 0 & 0 & 0 & $0^{*}$ & 0 & $0^{*}$ & 0 & 0 & 0 & 0 & 0 \\
\hline 23 & 0 & 0 & 0 & 0 & $0^{*}$ & 0 & $0^{*}$ & 0 & 0 & 0 & .63 & 0 \\
\hline 24 & 0 & 0 & 0 & 0 & $0^{*}$ & 0 & $0^{*}$ & 0 & 0 & 0 & .54 & 0 \\
\hline 25 & 0 & 0 & 0 & 0 & $0^{*}$ & 0 & $0^{*}$ & 0 & 0 & 0 & .07 & 0 \\
\hline 26 & 0 & 0 & 0 & 0 & $0^{*}$ & 0 & $0^{*}$ & 0 & 0 & 0 & .01 & 0 \\
\hline 27 & 0 & 0 & 0 & 0 & $0^{*}$ & 0 & $0^{*}$ & 0 & 0 & 0 & .01 & 0 \\
\hline 28 & 0 & 0 & 0 & $3.5^{\star}$ & $0^{*}$ & $0^{*}$ & $0^{*}$ & 0 & 0 & 0 & 0 & 0 \\
\hline 29 & 0 & 0 & 0 & $0^{*}$ & $0^{*}$ & $0^{*}$ & 0 & 0 & 0 & 0 & 0 & 0 \\
\hline 30 & 0 & 0 & 0 & $0^{*}$ & ------ & $0^{*}$ & 0 & 0 & 0 & 0 & 0 & 0 \\
\hline 31 & 0 & ----- & 0 & $0^{*}$ & ------ & $0^{*}$ & ------ & 0 & ------ & 0 & 0 & ------ \\
\hline Total & 0 & 0 & 2.62 & 3.5 & 0 & 0 & 0 & 0 & 0 & 0 & 3.30 & 0 \\
\hline Mean & 0 & 0 & .085 & .11 & 0 & 0 & 0 & 0 & 0 & 0 & .11 & 0 \\
\hline Max & 0 & 0 & 2.6 & 3.5 & 0 & 0 & 0 & 0 & 0 & 0 & 1.2 & 0 \\
\hline Min & 0 & 0 & 0 & 0 & 0 & 0 & 0 & 0 & 0 & 0 & 0 & 0 \\
\hline Acre-Ft & 0 & 0 & 5.2 & 6.9 & 0 & 0 & 0 & 0 & 0 & 0 & 6.5 & 0 \\
\hline Wtr Year & 2008 & Total & 9.42 & Mean & & & $\operatorname{lax}$ & 3.5 & Min & 0 & Acre-Ft & 19 \\
\hline Cal Year & 2007 & Total & 9.34 & Mean & & 26 & $\operatorname{lax}$ & 6.0 & Min & 0 & Acre-Ft & 19 \\
\hline
\end{tabular}

*Estimate 


\section{E253 Cañon del Valle above SR 501}

Location. Lat $35^{\circ}$ 51' 6", long $106^{\circ}$ 21' 17", NE 1/4, Sec. 25, T. 19 N., R. 5 E., Los Alamos County in Santa Fe National Forest.

Drainage Area. $2.27 \mathrm{mi}^{2}$.

Period of Record. October 1994 to June 2000 (gage destroyed by flood); January 31, 2001, to September 30, 2008.

Gage. Data logger and $120^{\circ}$ weir plate, as well as rain gage with cellular telemetry. Elevation of gage is 7,701 ft above NGVD from GPS survey.

Remarks. Records are good.

Average Discharge. $14 \mathrm{yr}, 0.03 \mathrm{ft}^{3} / \mathrm{s}, 18$ acre-ft/yr.

Extremes for Period of Record. Maximum discharge, $740 \mathrm{ft}^{3} / \mathrm{s}$, June 28, 2000, from peak flow computation, gage height $8.42 \mathrm{ft}$. No flow most of the time.

Extremes for Current Year. No peak discharge above base of $5.0 \mathrm{ft}^{3} / \mathrm{s}$. No flow for the year.

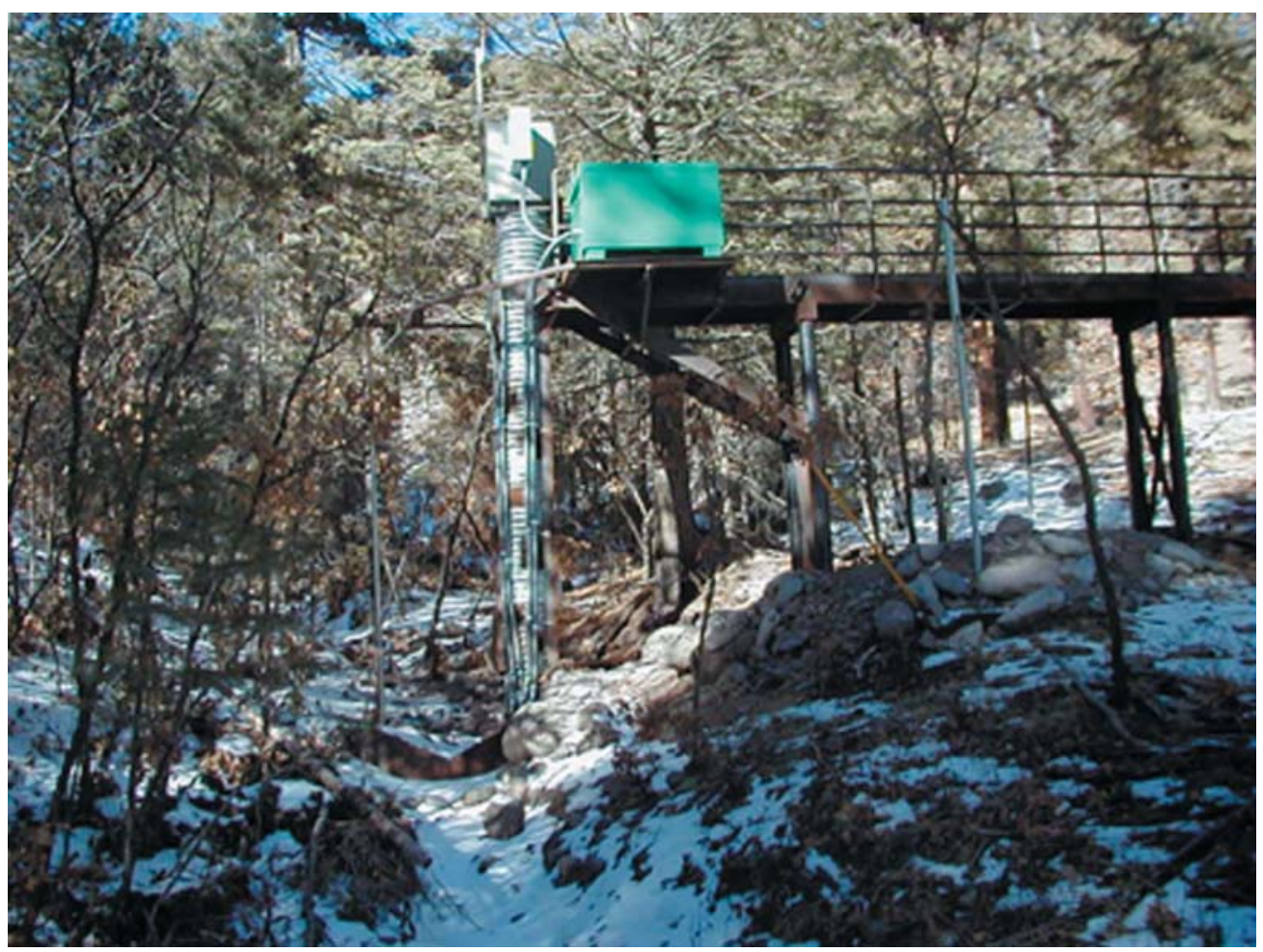




\section{E253 Cañon del Valle above SR 501}

\section{Station Analysis}

\section{Water Year}

Equipment. Station is equipped with Sutron 8210 (5-min. interval) with shaft encoder float system (5-min. interval) and cellular phone with speech modem. Phone upgraded September 25 from analog to digital service. The system is powered by a solar-panel battery system housed in NEMA shelter on 24" CMP well, 16' long attached to a 60' metal walkway. Station is equipped with an ISCO pump sampler for water-quality sample collection. ISCO is housed in a separate shelter, a $3^{\prime} \times 4^{\prime}$ metal box. Sampler is triggered by stage through the data logger. An outside staff is available for reference. No provision for direct discharge measurements above wading stages.

Station is also equipped with a rain gage, Rain Collection II, which was installed on September 25. All equipment is powered with a solar-panel battery-charging system.

Field Work. The station was visited 17 times to conduct discharge measurements and service the instrumentation. Inspections for the gage are listed under site history files on the Hydstra database. Discharge measurements for the gage are listed under site gauging files on the Hydstra database.

Datum Correction. None. Levels were run in April 16, 2001, when gage was reestablished.

Gage-Height Record. The data logger referenced to the outside staff gave a complete and satisfactory record for the year except for the periods of January 18 to February 6 and September 25-28 when the data logger malfunctioned as a result of battery failure.

Rating. The channel at the gage is about $8^{\prime}$ wide and straight for about $50^{\prime}$ upstream then bends to the left and straight for $100^{\prime}$ downstream and bends to the right. The streambed through this reach consists of primarily gravel with cobbles. The low-flow control is a $120^{\circ}$ sharp-crested weir. The channel becomes the control at high flow.

Seventeen inspections of no flow were made this year.

Rating No. 2 is based on four discharge measurements and one critical-depth computation; theoretical computation is based on a $120^{\circ}$ sharp-crested weir to a gage height of 2.30 feet. Broad-crested weir computation is used above that stage.

Discharge. Discharge was computed by applying gage height to Rating No. 2 directly, with one variable shift applied. Those days estimated at zero flow were based on precipitation and station E256, which is downstream at approximately 3 miles.

Remarks. Records are good. 


\section{E253 Cañon del Valle above SR-501}

Daily Mean Discharge in Cubic Feet per Second

Water Year October 2007 to September 2008

\begin{tabular}{|c|c|c|c|c|c|c|c|c|c|c|c|c|}
\hline DAY & OCT & NOV & DEC & JAN & FEB & MAR & APR & MAY & JUN & JUL & AUG & SEP \\
\hline 1 & 0 & 0 & 0 & 0 & $0^{*}$ & 0 & 0 & 0 & 0 & 0 & 0 & 0 \\
\hline 2 & 0 & 0 & 0 & 0 & $0^{*}$ & 0 & 0 & 0 & 0 & 0 & 0 & 0 \\
\hline 3 & 0 & 0 & 0 & 0 & $0^{*}$ & 0 & 0 & 0 & 0 & 0 & 0 & 0 \\
\hline 4 & 0 & 0 & 0 & 0 & $0^{*}$ & 0 & 0 & 0 & 0 & 0 & 0 & 0 \\
\hline 5 & 0 & 0 & 0 & 0 & $0^{*}$ & 0 & 0 & 0 & 0 & 0 & 0 & 0 \\
\hline 6 & 0 & 0 & 0 & 0 & $0^{*}$ & 0 & 0 & 0 & 0 & 0 & 0 & 0 \\
\hline 7 & 0 & 0 & 0 & 0 & 0 & 0 & 0 & 0 & 0 & 0 & 0 & 0 \\
\hline 8 & 0 & 0 & 0 & 0 & 0 & 0 & 0 & 0 & 0 & 0 & 0 & 0 \\
\hline 9 & 0 & 0 & 0 & 0 & 0 & 0 & 0 & 0 & 0 & 0 & 0 & 0 \\
\hline 10 & 0 & 0 & 0 & 0 & 0 & 0 & 0 & 0 & 0 & 0 & 0 & 0 \\
\hline 11 & 0 & 0 & 0 & 0 & 0 & 0 & 0 & 0 & 0 & 0 & 0 & 0 \\
\hline 12 & 0 & 0 & 0 & 0 & 0 & 0 & 0 & 0 & 0 & 0 & 0 & 0 \\
\hline 13 & 0 & 0 & 0 & 0 & 0 & 0 & 0 & 0 & 0 & 0 & 0 & 0 \\
\hline 14 & 0 & 0 & 0 & 0 & 0 & 0 & 0 & 0 & 0 & 0 & 0 & 0 \\
\hline 15 & 0 & 0 & 0 & 0 & 0 & 0 & 0 & 0 & 0 & 0 & 0 & 0 \\
\hline 16 & 0 & 0 & 0 & 0 & 0 & 0 & 0 & 0 & 0 & 0 & 0 & 0 \\
\hline 17 & 0 & 0 & 0 & 0 & 0 & 0 & 0 & 0 & 0 & 0 & 0 & 0 \\
\hline 18 & 0 & 0 & 0 & $0^{*}$ & 0 & 0 & 0 & 0 & 0 & 0 & 0 & 0 \\
\hline 19 & 0 & 0 & 0 & $0^{*}$ & 0 & 0 & 0 & 0 & 0 & 0 & 0 & 0 \\
\hline 20 & 0 & 0 & 0 & $0^{*}$ & 0 & 0 & 0 & 0 & 0 & 0 & 0 & 0 \\
\hline 21 & 0 & 0 & 0 & $0^{*}$ & 0 & 0 & 0 & 0 & 0 & 0 & 0 & 0 \\
\hline 22 & 0 & 0 & 0 & $0^{*}$ & 0 & 0 & 0 & 0 & 0 & 0 & 0 & 0 \\
\hline 23 & 0 & 0 & 0 & $0^{*}$ & 0 & 0 & 0 & 0 & 0 & 0 & 0 & 0 \\
\hline 24 & 0 & 0 & 0 & $0^{*}$ & 0 & 0 & 0 & 0 & 0 & 0 & 0 & 0 \\
\hline 25 & 0 & 0 & 0 & $0^{*}$ & 0 & 0 & 0 & 0 & 0 & 0 & 0 & $0^{*}$ \\
\hline 26 & 0 & 0 & 0 & $0^{*}$ & 0 & 0 & 0 & 0 & 0 & 0 & 0 & $0^{*}$ \\
\hline 27 & 0 & 0 & 0 & $0^{*}$ & 0 & 0 & 0 & 0 & 0 & 0 & 0 & $0^{*}$ \\
\hline 28 & 0 & 0 & 0 & $0^{*}$ & 0 & 0 & 0 & 0 & 0 & 0 & 0 & $0^{*}$ \\
\hline 29 & 0 & 0 & 0 & $0^{*}$ & 0 & 0 & 0 & 0 & 0 & 0 & 0 & 0 \\
\hline 30 & 0 & 0 & 0 & $0^{*}$ & ----- & 0 & 0 & 0 & 0 & 0 & 0 & 0 \\
\hline 31 & 0 & ----- & 0 & $0^{*}$ & ----- & 0 & ------ & 0 & ----- & 0 & 0 & ----- \\
\hline Total & 0 & 0 & 0 & 0 & 0 & 0 & 0 & 0 & 0 & 0 & 0 & 0 \\
\hline Mean & 0 & 0 & 0 & 0 & 0 & 0 & 0 & 0 & 0 & 0 & 0 & 0 \\
\hline $\operatorname{Max}$ & 0 & 0 & 0 & 0 & 0 & 0 & 0 & 0 & 0 & 0 & 0 & 0 \\
\hline Min & 0 & 0 & 0 & 0 & 0 & 0 & 0 & 0 & 0 & 0 & 0 & 0 \\
\hline Acre-Ft & 0 & 0 & 0 & 0 & 0 & 0 & 0 & 0 & 0 & 0 & 0 & 0 \\
\hline Wtr Year & 2008 & Total & 0 & Mean & & 0 & Max & 0 & Min & 0 & Acre-Ft & 0 \\
\hline Cal Year & 2007 & Total & 0.08 & Mean & & 0 & Max & .04 & Min & 0 & Acre-Ft & 0.16 \\
\hline
\end{tabular}

*Estimate 
Location. Lat $35^{\circ}$ 51' 01", long $106^{\circ}$ 19' 57", Sec. 29, T.19 N., R. 6 E., Ramon Vigil Grant, Los Alamos County.

Drainage Area. $3.13 \mathrm{mi}^{2}$.

Period of Record. January 24, 2002, to September 30, 2008.

Revised Record. Drainage area (2006).

Gage. Data logger and 24” Parshall flume. Elevation of gage is 7,329 ft above NGVD from GPS survey.

Remarks. Records are good. Legal location based on projected values.

Average Discharge. $6 \mathrm{yr}, 0.06 \mathrm{ft}^{3} / \mathrm{s}, 44$ acre- $\mathrm{ft} / \mathrm{yr}$.

Extremes for Period of Record. Maximum discharge, $19 \mathrm{ft}^{3} / \mathrm{s}$, August 24, 2005, gage height $1.74 \mathrm{ft}$. No flow most of the time.

Extremes for Current Year. Peak discharge above base of $10 \mathrm{ft}^{3} / \mathrm{s}$ and maximum (*):

\begin{tabular}{|c|c|c|c|}
\hline Date & Time & Discharge $\left(\mathbf{f t}^{\mathbf{3}} / \mathbf{s}\right)$ & Gage Height (ft) \\
\hline January 28 & 0440 & $13^{*}$ & $1.38^{*}$ \\
\hline
\end{tabular}

No flow most of the time.

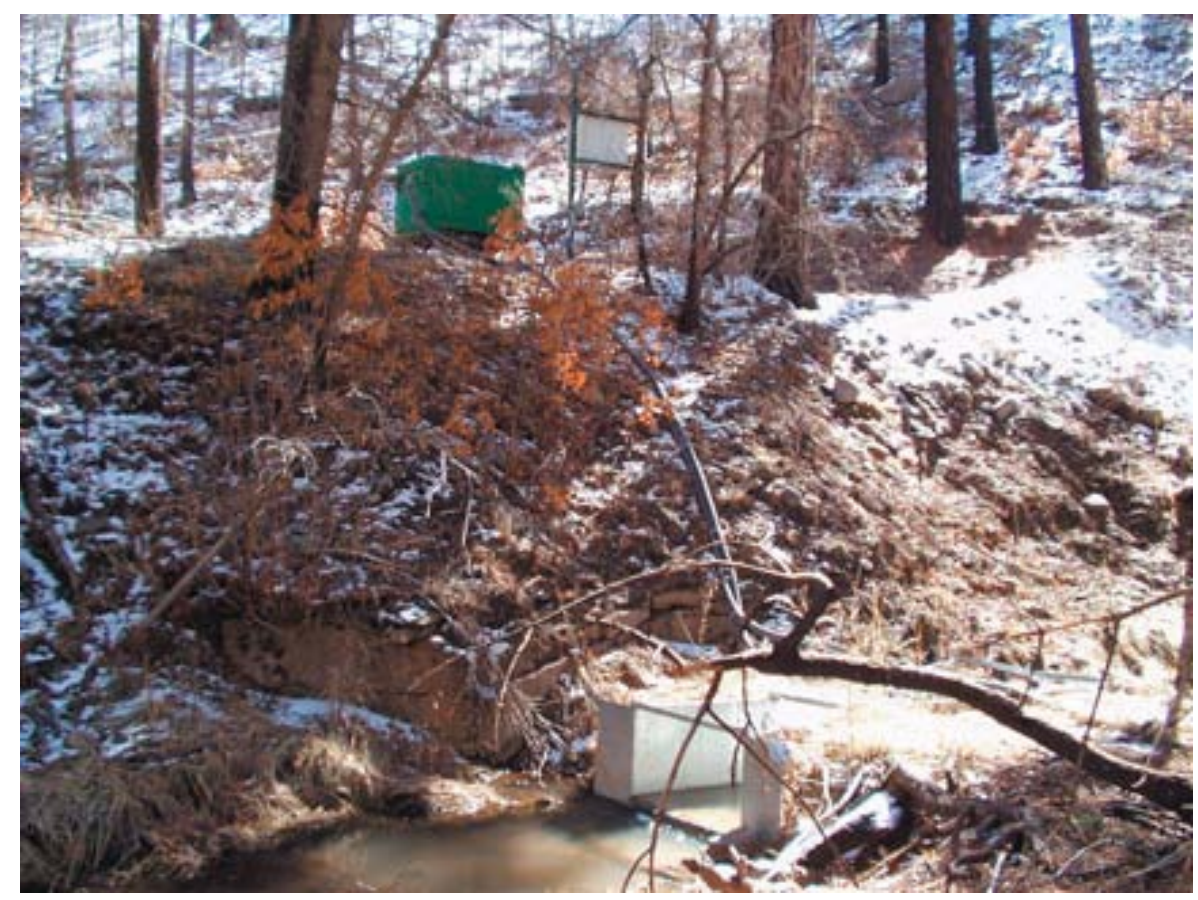




\section{E256 Cañon del Valle below MDA P}

\section{Station Analysis}

\section{Water Year}

Equipment. Station is equipped with Sutron 8210 data logger (5-min. interval) with Sutron Accubar bubble sensor within 24" Parshall flume. The system is powered by a solar-panel battery system housed in a NEMA shelter on left bank. Station is equipped with an ISCO pump sampler for water-quality sample collection. ISCO is housed in a separate shelter, a $3^{\prime} \times 4^{\prime}$ metal box. An outside staff is available for reference. There is no provision for discharge measurement above wading stage.

Field Work. The station was visited 17 times to conduct discharge measurements and service the instrumentation. Field inspections for the gage are listed under site history files on the Hydstra database. Discharge measurements for the gage are listed under site gauging files on the Hydstra database.

Datum Corrections. None.

Gage-Height Record. The data logger referenced to the inside staff gave a complete and satisfactory record.

Rating. The channel is straight for $50^{\prime}$ upstream and $20^{\prime}$ downstream from gage. The streambed consists of sand with gravel subject to fill behind flume from flow events and gage-silting problems. The banks are covered with vegetation.

One discharge measurement (No. 9) and 17 observations of flow were made this year.

Rating No. 1 is based on 24" Parshall flume.

Discharge. Discharge computed by applying gage height to Rating No. 1 using variableshift diagrams.

Remarks. Records are good. 
E256 Cañon del Valle below MDA-P

Daily Mean Discharge in Cubic Feet per Second

Water Year October 2007 to September 2008

\begin{tabular}{|c|c|c|c|c|c|c|c|c|c|c|c|c|}
\hline DAY & OCT & NOV & DEC & JAN & FEB & MAR & APR & MAY & JUN & JUL & AUG & SEP \\
\hline 1 & .03 & 0 & .94 & 0 & 0 & .13 & .07 & .05 & .03 & .01 & .01 & .07 \\
\hline 2 & .02 & 0 & .06 & .02 & 0 & .13 & .06 & .05 & .03 & .01 & .01 & .05 \\
\hline 3 & .02 & 0 & .05 & .03 & 0 & .10 & .06 & .05 & .03 & .01 & 0 & .05 \\
\hline 4 & .02 & 0 & .04 & .03 & .03 & .09 & .06 & .05 & .03 & .01 & .04 & .05 \\
\hline 5 & .02 & 0 & .04 & .02 & .03 & .09 & .06 & .05 & .03 & .01 & .02 & .04 \\
\hline 6 & .02 & 0 & .03 & .02 & .03 & .08 & .07 & .06 & .03 & .01 & .02 & .04 \\
\hline 7 & .02 & 0 & .04 & .02 & .03 & .08 & .06 & .06 & .03 & .02 & .02 & .04 \\
\hline 8 & .02 & 0 & .05 & .03 & .03 & .08 & .06 & .06 & .03 & .02 & .17 & .04 \\
\hline 9 & .02 & 0 & .06 & .02 & .03 & .08 & .06 & .06 & .02 & .02 & .23 & .04 \\
\hline 10 & .02 & 0 & .05 & .02 & .03 & .08 & .06 & .06 & .03 & .01 & .24 & .03 \\
\hline 11 & .02 & 0 & .05 & .02 & .04 & .09 & .07 & .05 & .03 & .01 & .08 & .03 \\
\hline 12 & .02 & 0 & .05 & .02 & .04 & .10 & .07 & .05 & .03 & .01 & .06 & .03 \\
\hline 13 & .02 & 0 & .05 & .02 & .04 & .12 & .08 & .05 & .03 & .01 & .05 & .03 \\
\hline 14 & .02 & 0 & .04 & .02 & .05 & .13 & .08 & .06 & .03 & .01 & .05 & .03 \\
\hline 15 & .02 & 0 & .05 & .02 & .06 & .11 & .07 & .07 & .03 & .01 & .05 & .03 \\
\hline 16 & .02 & 0 & .05 & .02 & .10 & .12 & .07 & .06 & .03 & .01 & .05 & .03 \\
\hline 17 & .02 & 0 & .05 & .02 & .07 & .11 & .07 & .06 & .02 & .02 & .04 & .03 \\
\hline 18 & .02 & 0 & .04 & .02 & .06 & .11 & .06 & .05 & .02 & .02 & .04 & .03 \\
\hline 19 & .02 & 0 & .04 & .02 & .06 & .11 & .06 & .05 & .02 & .01 & .03 & .03 \\
\hline 20 & .02 & 0 & .03 & 0 & .06 & .09 & .06 & .05 & .02 & .01 & .03 & .04 \\
\hline 21 & .03 & 0 & .03 & 0 & .06 & .09 & .06 & .04 & .02 & .06 & .03 & .04 \\
\hline 22 & .03 & 0 & .03 & 0 & .06 & .08 & .06 & .05 & .01 & .03 & .03 & .04 \\
\hline 23 & .03 & 0 & .03 & 0 & .06 & .08 & .06 & .05 & .01 & .02 & .05 & .04 \\
\hline 24 & .03 & 0 & .03 & 0 & .22 & .08 & .05 & .04 & .01 & .02 & .03 & .03 \\
\hline 25 & .03 & 0 & .03 & 0 & .17 & .08 & .05 & .04 & .01 & .02 & .04 & .03 \\
\hline 26 & .03 & 0 & 0 & 0 & .11 & .08 & .05 & .04 & .01 & .02 & .03 & .03 \\
\hline 27 & .03 & 0 & 0 & .14 & .10 & .08 & .05 & .03 & .01 & .02 & .03 & .03 \\
\hline 28 & .03 & 0 & 0 & 2.6 & .10 & .08 & .05 & .05 & .01 & .02 & .03 & .03 \\
\hline 29 & .03 & 0 & 0 & .03 & .11 & .08 & .05 & .05 & .01 & .02 & .03 & .03 \\
\hline 30 & .01 & .03 & 0 & 0 & ------ & .08 & .05 & .04 & .01 & .01 & .03 & .04 \\
\hline 31 & 0 & ------ & 0 & 0 & ------ & .08 & ------ & .03 & ------ & .01 & .09 & ------ \\
\hline Total & 0.69 & 0.03 & 1.96 & 3.16 & 1.78 & 2.92 & 1.84 & 1.56 & 0.66 & 0.50 & 1.66 & 1.10 \\
\hline Mean & .022 & .001 & .063 & .10 & .061 & .094 & .061 & .050 & .022 & .016 & .054 & .037 \\
\hline Max & .03 & .03 & .94 & 2.6 & .22 & .13 & .08 & .07 & .03 & .06 & .24 & .07 \\
\hline Min & 0 & 0 & 0 & 0 & 0 & .08 & .05 & .03 & .01 & .01 & 0 & .03 \\
\hline Acre-Ft & 1.4 & .06 & 3.9 & 6.3 & 3.5 & 5.8 & 3.6 & 3.1 & 1.3 & .99 & 3.3 & 2.2 \\
\hline Wtr Year & 2008 & Total & 17.86 & Mean & & 49 & Max & 2.6 & Min & 0 & Acre-Ft & 35 \\
\hline Cal Year & 2007 & Total & 18.64 & Mean & & 51 & Max & .94 & Min & 0 & Acre-Ft & 37 \\
\hline
\end{tabular}




\section{E2565 Cañon del Valle at Q Site}

Location. Lat $35^{\circ}$ 50' 52", long -106 19' 0.9", Sec. 28, T.19 N., R. 6 E., Ramon Vigil Grant, Los Alamos County.

Drainage Area. $0.00 \mathrm{mi}^{2} ; 0.11$ acre-ft.

Period of Record. October 1, 2007, to September 30, 2008.

Gage. Data logger and 24” Parshall flume. Elevation of gage is 7,346 ft above NGVD from GPS survey.

Remarks. Records are good. Records for this site existed before period of record but are not reliable. Legal location based on projected values.

Extremes for Period of Record. Maximum discharge, $1.0 \mathrm{ft}^{3} / \mathrm{s}$, August 8, 2008, gage height $0.26 \mathrm{ft}$. No flow most of the time.

Extremes for Current Year. Peak discharge above base of $1.0 \mathrm{ft}^{3} / \mathrm{s}$ and maximum (*):

\begin{tabular}{|c|c|c|c|}
\hline Date & Time & Discharge $\left.\mathbf{( f t}^{\mathbf{3}} / \mathbf{s}\right)$ & Gage Height (ft) \\
\hline August 10 & 1140 & $1.0^{*}$ & $0.26^{*}$ \\
\hline
\end{tabular}

No flow most of the time.

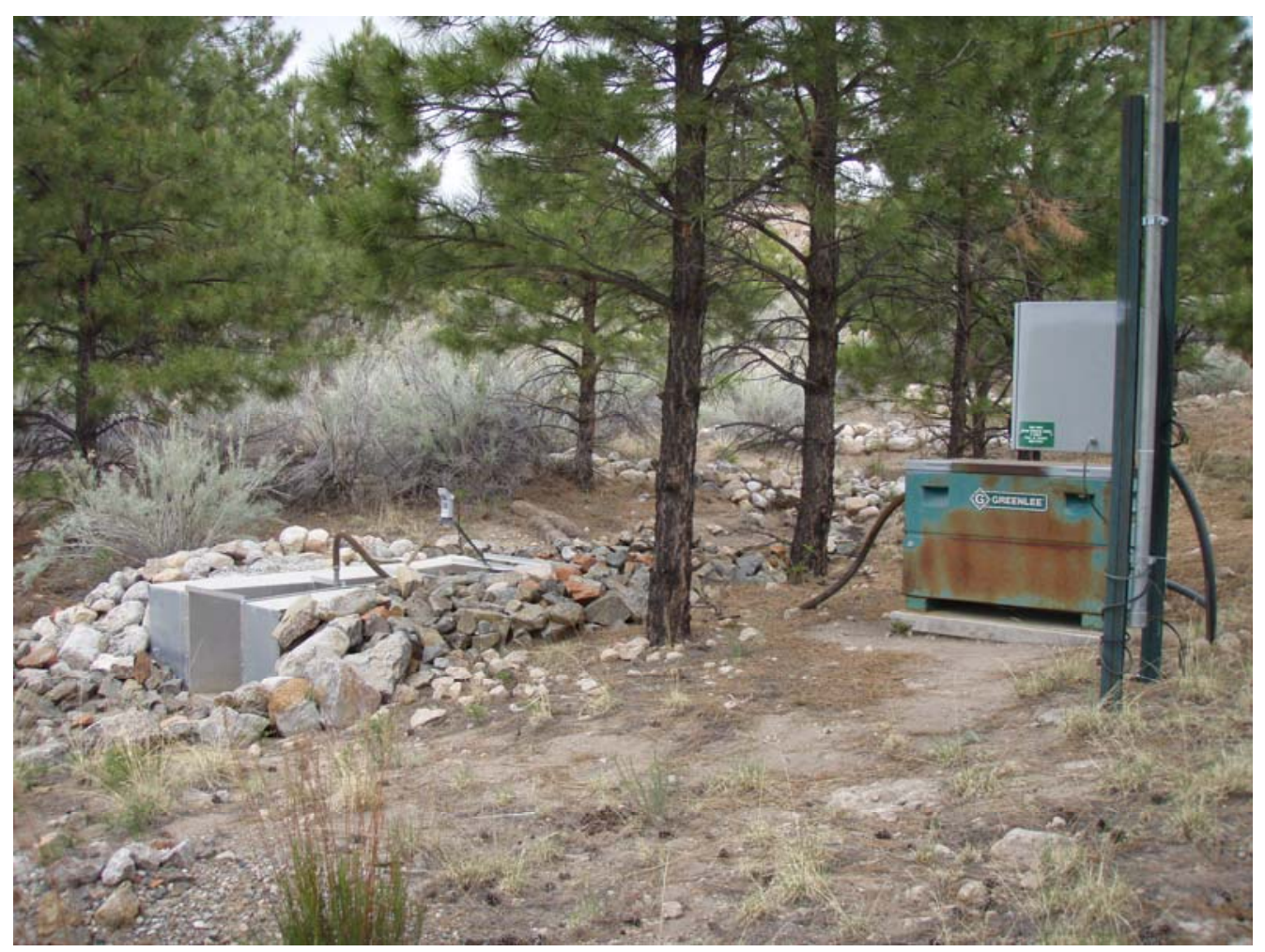




\section{E2565 Cañon del Valle at Q Site}

\section{Station Analysis}

\section{Water Year}

Equipment. Station is equipped with Sutron 8210 data logger (5-min. interval) with milltronics sonic probe mounted on a 12" Parshall flume. The system is powered by a solar-panel battery system housed in a NEMA shelter. Station is equipped with an ISCO pump sampler for water-quality sample collection. ISCO is housed in a separate shelter, a $3^{\prime} \times 4^{\prime}$ metal box. Sampler is triggered by stage through the data logger. An outside staff is available for reference. No provision for discharge measurements above wading stage.

Field Work. The station was visited 17 times to conduct discharge measurements and service the instrumentation. Field inspections for the gage are listed under site history files on the Hydstra database. Discharge measurements for the gage are listed under site gauging files on the Hydstra database.

Datum Corrections. None.

Gage-Height Record. The data logger referenced to the outside staff gave a complete and satisfactory record except for the periods of November 23-27, December 1 to February 14, February 22-24, and February 27 to March 25 when ice affected gage height.

Rating. The channel is lined with angular rock and filter fabric. It is confined to the main channel. The bottom is $4^{\prime}$ wide channel prone to some shifting with vegetation on each bank. Low-water control is the $12^{\prime \prime}$ Parshall flume.

Sixteen inspection of no flow were made this year.

Rating No. 1 was developed based on the computation of 12" Parshall flume. Point of zero flow is 0.00 gage height.

Discharge. Discharge computed by applying gage height to Rating No. 1 directly.

Remarks. Records are good. 


\section{E2565 Canon del Valle at Q Site}

Daily Mean Discharge in Cubic Feet per Second

Water Year October 2007 to September 2008

\begin{tabular}{|c|c|c|c|c|c|c|c|c|c|c|c|c|}
\hline DAY & OCT & NOV & DEC & JAN & FEB & MAR & APR & MAY & JUN & JUL & AUG & SEP \\
\hline 1 & .01 & 0 & $.01^{*}$ & $0^{*}$ & $0^{*}$ & $0^{*}$ & 0 & 0 & 0 & .01 & 0 & 0 \\
\hline 2 & $0^{*}$ & 0 & $0^{*}$ & $0^{*}$ & $0^{*}$ & $0^{*}$ & 0 & 0 & 0 & $0^{*}$ & 0 & 0 \\
\hline 3 & $0^{*}$ & 0 & $0^{*}$ & $0^{*}$ & $0^{*}$ & $0^{*}$ & 0 & 0 & 0 & 0 & 0 & 0 \\
\hline 4 & 0 & 0 & $0^{*}$ & $0^{*}$ & $0^{*}$ & $0^{*}$ & 0 & 0 & 0 & 0 & .02 & $0^{*}$ \\
\hline 5 & $0^{*}$ & 0 & $0^{*}$ & $0^{*}$ & $0^{*}$ & $0^{*}$ & 0 & 0 & .01 & $0^{*}$ & $0^{*}$ & $0^{*}$ \\
\hline 6 & $0^{*}$ & 0 & $0^{*}$ & $0^{*}$ & $0^{*}$ & $0^{*}$ & 0 & 0 & $0^{*}$ & $0^{*}$ & 0 & 0 * \\
\hline 7 & $0^{*}$ & 0 & .01 & $0^{*}$ & $0^{*}$ & $0^{*}$ & 0 & 0 & 0 & .02 & 0 & 0 * \\
\hline 8 & $0^{*}$ & 0 & .02 & $0^{*}$ & $0^{*}$ & $0^{*}$ & 0 & 0 & 0 & .05 & .01 & 0 * \\
\hline 9 & $0^{*}$ & 0 & 0 & $0^{*}$ & $0^{*}$ & $0^{*}$ & 0 & 0 & .01 & .04 & .01 & 0 \\
\hline 10 & $0^{*}$ & 0 & $0^{*}$ & $0^{*}$ & $0^{*}$ & $0^{*}$ & 0 & 0 & 0 & 0 & .03 & $0^{*}$ \\
\hline 11 & .01 & 0 & $0^{*}$ & $0^{*}$ & $0^{*}$ & $0^{*}$ & 0 & 0 & 0 & 0 & .02 & 0 \\
\hline 12 & 0 & 0 & $0^{*}$ & $0^{*}$ & $0^{*}$ & $0^{*}$ & 0 & 0 & $0^{*}$ & .02 & .02 & 0 \\
\hline 13 & 0 & 0 & $0^{*}$ & $0^{*}$ & $0^{*}$ & $0^{*}$ & 0 & 0 & 0 & 0 & .02 & 0 * \\
\hline 14 & 0 & 0 & $0^{*}$ & $0^{*}$ & $0^{*}$ & $0^{*}$ & 0 & .08 & 0 & 0 & .01 & $0^{*}$ \\
\hline 15 & 0 & 0 & $0^{*}$ & $0^{*}$ & 0 & $0^{*}$ & 0 & .08 & 0 & .01 & .02 & $0^{*}$ \\
\hline 16 & 0 & 0 & $0^{*}$ & $0^{*}$ & 0 & $0^{*}$ & 0 & .03 & 0 & .03 & .02 & $0^{*}$ \\
\hline 17 & $0^{*}$ & 0 & $0^{*}$ & $0^{*}$ & 0 & $0^{*}$ & 0 & 0 * & $0^{*}$ & .05 & $0^{*}$ & 0 * \\
\hline 18 & $0^{*}$ & 0 & $0^{*}$ & $0^{*}$ & 0 & $0^{*}$ & 0 & 0 * & 0 & .02 & 0 * & 0 * \\
\hline 19 & 0 & 0 & $0^{*}$ & $0^{*}$ & 0 & $0^{*}$ & 0 & 0 & 0 & 0 & 0 & 0 * \\
\hline 20 & 0 & 0 & $0^{*}$ & $0^{*}$ & 0 & $0^{*}$ & 0 & 0 & $0^{*}$ & 0 & $0^{*}$ & .01 \\
\hline 21 & 0 & 0 & $0^{*}$ & $0^{*}$ & 0 & $0^{*}$ & 0 & 0 & 0 & .01 & 0 & $0^{*}$ \\
\hline 22 & $0^{*}$ & 0 & $0^{*}$ & $0^{*}$ & $0^{*}$ & $0^{*}$ & 0 & .02 & 0 & 0 & 0 & .01 \\
\hline 23 & 0 & $0^{*}$ & $0^{*}$ & $0^{*}$ & $0^{*}$ & $0^{*}$ & 0 & .06 & $0^{*}$ & 0 & .01 & $0^{*}$ \\
\hline 24 & 0 & $0^{*}$ & $0^{*}$ & $0^{*}$ & $0^{*}$ & $0^{*}$ & 0 & .04 & 0 & 0 & .03 & 0 \\
\hline 25 & 0 & $0^{*}$ & $0^{*}$ & $0^{*}$ & 0 & $0^{*}$ & 0 & $0^{*}$ & 0 & 0 & .04 & 0 * \\
\hline 26 & 0 & $0^{*}$ & $0^{*}$ & $0^{*}$ & 0 & 0 & 0 & 0 & 0 & $0^{*}$ & .02 & 0 \\
\hline 27 & $0^{*}$ & $0^{*}$ & $0^{*}$ & $0^{*}$ & $0^{*}$ & 0 & 0 & 0 & 0 & $0^{*}$ & $0^{*}$ & 0 \\
\hline 28 & 0 & 0 & $0^{*}$ & $0^{*}$ & $0^{*}$ & 0 & 0 & $.02^{*}$ & 0 & 0 & .01 & 0 \\
\hline 29 & 0 & 0 & $0^{*}$ & $0^{*}$ & $0^{*}$ & 0 & 0 & $0^{*}$ & 0 & 0 & $0^{*}$ & $0^{*}$ \\
\hline 30 & 0 & 0 & $0^{*}$ & $0^{*}$ & ------ & 0 & 0 & 0 * & $0^{*}$ & 0 & $0^{*}$ & 0 * \\
\hline 31 & 0 & ----- & $0^{*}$ & $0^{*}$ & ----- & 0 & ------ & $0^{*}$ & ----- & 0 & .04 & ----- \\
\hline Total & 0.02 & 0 & 0.04 & 0 & 0 & 0 & 0 & 0.33 & 0.02 & 0.26 & 0.33 & 0.02 \\
\hline Mean & .001 & 0 & .001 & 0 & 0 & 0 & 0 & .011 & .001 & .008 & .011 & .001 \\
\hline Max & .01 & 0 & .02 & 0 & 0 & 0 & 0 & .08 & .01 & .05 & .04 & .01 \\
\hline Min & 0 & 0 & 0 & 0 & 0 & 0 & 0 & 0 & 0 & 0 & 0 & 0 \\
\hline Acre-Ft & .04 & 0 & .08 & 0 & 0 & 0 & 0 & .65 & .04 & .52 & .65 & .04 \\
\hline Wtr Year & 2008 & Total & 1.02 & Mean & & 03 & Max & .08 & Min & 0 & Acre-Ft & 2.0 \\
\hline Cal Year & 2007 & Total & 0.08 & Mean & & 0 & hax & .02 & Min & 0 & Acre-Ft & 0.16 \\
\hline
\end{tabular}

*Estimate 
Location. Lat $35^{\circ}$ 50' 47", long $106^{\circ}$ 19' 50", Sec. 29, T. 19 N., R. 6 E., Ramon Vigil Grant.

Drainage Area. $0.040 \mathrm{mi}^{2}$.

Period of Record. October 1, 2002, to September 30, 2008.

Gage. Data logger and 12" Parshall flume, as well as rain gage with cellular telemetry. Elevation of gage is 7,359 ft above NGVD.

Remarks. Records are good. Records for this site existed before period of record but are not reliable. Legal location based on projected values.

Average Discharge. 6 yr., $0.01 \mathrm{ft}^{3} / \mathrm{s}, 10.6$ acre-ft/yr.

Extremes for Period of Record. Maximum discharge, $9.5 \mathrm{ft}^{3} / \mathrm{s}$, August 29, 2007, gage height $1.77 \mathrm{ft}$. No flow most of the time.

Extremes for Current Year. Peak discharges above base of $3.0 \mathrm{ft}^{3} / \mathrm{s}$ and maximum (*):

\begin{tabular}{|c|c|c|c|}
\hline Date & Time & Discharge $\left.\mathbf{f t}^{\mathbf{3}} / \mathbf{s}\right)$ & Gage Height (ft) \\
\hline December 1 & 0130 & 2.3 & 0.70 \\
\hline January 28 & 0410 & 2.3 & 0.70 \\
\hline July 21 & 1130 & 1.5 & 0.53 \\
\hline August 4 & 1750 & 1.5 & 0.52 \\
\hline August 8 & 1310 & 4.0 & 1.00 \\
\hline August 10 & 1140 & $8.5^{*}$ & $1.64^{*}$ \\
\hline August 23 & 1635 & 1.2 & 0.46 \\
\hline August 31 & 1515 & 1.6 & 0.54 \\
\hline
\end{tabular}

No flow most of the time.

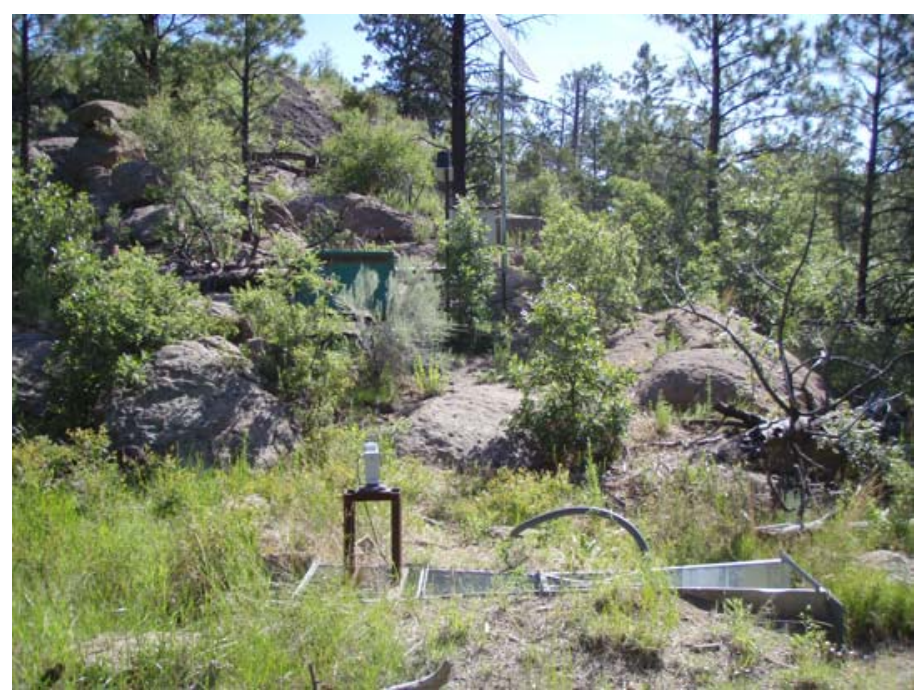




\section{E257 Cañon del Valle Tributary at TA-16 Burn Grounds \\ Station Analysis}

\section{Water Year}

Equipment. Station is equipped with Sutron 8210 data logger (5-min. interval) and milltronics sonic probe mounted on a 12" Parshall flume and cellular phone with speech modem. Phone upgraded June 17 from analog to digital service. The system is powered by a solar-panel battery system housed in a NEMA shelter. Station is equipped with an ISCO pump sampler for water-quality sample collection. ISCO is housed in a separate shelter, a $3^{\prime} \times 4^{\prime}$ metal box. Sampler is triggered by stage through the data logger. The staff in the 12" Parshall flume is the reference gage. No provision for discharge measurements above wading stage.

Station is also equipped with a tipping bucket rain gage, Rain Collection II. All equipment is powered with a solar-panel battery-charging system. Data are seasonal.

Field Work. This station was visited 25 times to conduct discharge measurements and service the instrumentation. Field inspections for the gage are listed under site history files on the Hydstra database. Discharge measurements for the gage are listed under site gauging files on the Hydstra database.

Datum Correction. None.

Gage-Height Record. The data logger referenced to the outside staff gave a complete and satisfactory record for the year.

Rating. The channel is straight above and below gage. It is confined to the main channel by cut banks on both sides. The bottom is $10^{\prime}$ wide; channel is prone to some shifting with vegetation on each bank. Low-water control is the 12" Parshall flume.

Twenty-five inspections of no flow were made this water year.

Rating No. 1 was developed based on the computation of 12" Parshall flume. Point of zero flow is 0.00 gage height.

Discharge. Discharge was computed by applying gage height to Rating No. 1 directly.

Remarks. Records are good. 
E257 Cañon del Valle Tributary at TA-16 Burn Grounds

Daily Mean Discharge in Cubic Feet per Second

Water Year October 2007 to September 2008

\begin{tabular}{|c|c|c|c|c|c|c|c|c|c|c|c|c|}
\hline DAY & OCT & NOV & DEC & JAN & FEB & MAR & APR & MAY & JUN & JUL & AUG & SEP \\
\hline 1 & 0 & 0 & .20 & 0 & 0 & 0 & 0 & 0 & 0 & 0 & 0 & 0 \\
\hline 2 & 0 & 0 & 0 & 0 & 0 & 0 & 0 & 0 & 0 & 0 & 0 & 0 \\
\hline 3 & 0 & 0 & 0 & 0 & 0 & 0 & 0 & 0 & 0 & 0 & 0 & 0 \\
\hline 4 & 0 & 0 & 0 & 0 & 0 & 0 & 0 & 0 & 0 & 0 & .04 & 0 \\
\hline 5 & 0 & 0 & 0 & 0 & 0 & 0 & 0 & 0 & 0 & 0 & 0 & 0 \\
\hline 6 & 0 & 0 & 0 & 0 & 0 & 0 & 0 & 0 & 0 & 0 & 0 & 0 \\
\hline 7 & 0 & 0 & 0 & 0 & 0 & 0 & 0 & 0 & 0 & 0 & 0 & 0 \\
\hline 8 & 0 & 0 & .01 & 0 & 0 & 0 & 0 & 0 & 0 & 0 & .13 & 0 \\
\hline 9 & 0 & 0 & 0 & 0 & 0 & 0 & 0 & 0 & 0 & 0 & .03 & 0 \\
\hline 10 & 0 & 0 & 0 & 0 & 0 & 0 & 0 & 0 & 0 & 0 & .16 & 0 \\
\hline 11 & 0 & 0 & 0 & 0 & 0 & 0 & 0 & 0 & 0 & 0 & 0 & 0 \\
\hline 12 & 0 & 0 & 0 & 0 & 0 & 0 & 0 & 0 & 0 & 0 & 0 & 0 \\
\hline 13 & 0 & 0 & 0 & 0 & 0 & 0 & 0 & 0 & 0 & 0 & 0 & 0 \\
\hline 14 & 0 & 0 & 0 & 0 & 0 & 0 & 0 & 0 & 0 & 0 & 0 & 0 \\
\hline 15 & 0 & 0 & 0 & 0 & 0 & 0 & 0 & 0 & 0 & 0 & 0 & 0 \\
\hline 16 & 0 & 0 & 0 & 0 & 0 & 0 & 0 & 0 & 0 & 0 & 0 & 0 \\
\hline 17 & 0 & 0 & 0 & 0 & 0 & 0 & 0 & 0 & 0 & 0 & 0 & 0 \\
\hline 18 & 0 & 0 & 0 & 0 & 0 & 0 & 0 & 0 & 0 & 0 & 0 & 0 \\
\hline 19 & 0 & 0 & 0 & 0 & 0 & 0 & 0 & 0 & 0 & 0 & 0 & 0 \\
\hline 20 & 0 & 0 & 0 & 0 & 0 & 0 & 0 & 0 & 0 & 0 & 0 & 0 \\
\hline 21 & 0 & 0 & 0 & 0 & 0 & 0 & 0 & 0 & 0 & .02 & 0 & 0 \\
\hline 22 & 0 & 0 & 0 & 0 & 0 & 0 & 0 & 0 & 0 & 0 & 0 & 0 \\
\hline 23 & 0 & 0 & 0 & 0 & 0 & 0 & 0 & 0 & 0 & 0 & .04 & 0 \\
\hline 24 & 0 & 0 & 0 & 0 & .05 & 0 & 0 & 0 & 0 & 0 & .01 & 0 \\
\hline 25 & 0 & 0 & 0 & 0 & .02 & 0 & 0 & 0 & 0 & 0 & 0 & 0 \\
\hline 26 & 0 & 0 & 0 & 0 & 0 & 0 & 0 & 0 & 0 & 0 & 0 & 0 \\
\hline 27 & 0 & 0 & 0 & .06 & 0 & 0 & 0 & 0 & 0 & 0 & 0 & 0 \\
\hline 28 & 0 & 0 & 0 & .40 & 0 & 0 & 0 & 0 & 0 & 0 & 0 & 0 \\
\hline 29 & 0 & 0 & 0 & 0 & 0 & 0 & 0 & 0 & 0 & 0 & 0 & 0 \\
\hline 30 & 0 & .04 & 0 & 0 & ----- & 0 & 0 & 0 & 0 & 0 & 0 & 0 \\
\hline 31 & 0 & ------ & 0 & 0 & ----- & 0 & ----- & 0 & ----- & 0 & .04 & ------ \\
\hline Total & 0 & 0.04 & 0.21 & 0.46 & 0.07 & 0 & 0 & 0 & 0 & 0.02 & 0.45 & 0 \\
\hline Mean & 0 & .001 & .007 & .015 & .002 & 0 & 0 & 0 & 0 & .001 & .015 & 0 \\
\hline Max & 0 & .04 & .20 & .40 & .05 & 0 & 0 & 0 & 0 & .02 & .16 & 0 \\
\hline Min & 0 & 0 & 0 & 0 & 0 & 0 & 0 & 0 & 0 & 0 & 0 & 0 \\
\hline Acre-Ft & 0 & .08 & .42 & .91 & .14 & 0 & 0 & 0 & 0 & .04 & .89 & 0 \\
\hline Wtr Year & 2008 & Total & 1.25 & Mean & & & Max & .40 & Min & 0 & Acre-Ft & 2.5 \\
\hline Cal Year & 2007 & Total & 2.08 & Mean & & & Max & .20 & Min & 0 & Acre-Ft & 4.1 \\
\hline
\end{tabular}


E257 Cañon del Valle Tributary at TA-16 Burn Grounds

Daily Total in Rainfall in Inches

Water Year October 2007 to September 2008

\begin{tabular}{|c|c|c|c|c|c|c|c|c|c|c|c|c|}
\hline DAY & ОСТ & NOV & DEC & JAN & FEB & MAR & APR & MAY & JUN & JUL & AUG & SEP \\
\hline 1 & & & & & & & & 0 & 0 & .09 & 0 & 0 \\
\hline 2 & & & & & & & & 0 & 0 & 0 & 0 & 0 \\
\hline 3 & & & & & & & & 0 & 0 & .02 & 0 & 0 \\
\hline 4 & & & & & & & & 0 & 0 & 0 & 1.35 & 0 \\
\hline 5 & & & & & & & & 0 & .06 & .01 & 0 & 0 \\
\hline 6 & & & & & & & & 0 & 0 & .05 & .01 & 0 \\
\hline 7 & & & & & & & & 0 & 0 & .59 & .35 & 0 \\
\hline 8 & & & & & & & & 0 & 0 & .26 & .96 & 0 \\
\hline 9 & & & & & & & & 0 & 0 & .01 & .19 & 0 \\
\hline 10 & & & & & & & & 0 & 0 & 0 & .85 & 0 \\
\hline 11 & & & & & & & & 0 & 0 & .06 & 0 & 0 \\
\hline 12 & & & & & & & & 0 & 0 & 0 & 0 & .02 \\
\hline 13 & & & & & & & & .15 & 0 & 0 & 0 & 0 \\
\hline 14 & & & & & & & & .14 & 0 & 0 & .01 & 0 \\
\hline 15 & & & & & & & & .60 & 0 & .04 & .05 & 0 \\
\hline 16 & & & & & & & & 0 & 0 & .24 & .35 & 0 \\
\hline 17 & & & & & & & & 0 & 0 & .29 & .09 & 0 \\
\hline 18 & & & & & & & & 0 & 0 & .02 & 0 & .06 \\
\hline 19 & & & & & & & 0 & 0 & 0 & 0 & 0 & .04 \\
\hline 20 & & & & & & & 0 & 0 & .03 & 0 & .05 & .12 \\
\hline 21 & & & & & & & 0 & 0 & 0 & .76 & 0 & 0 \\
\hline 22 & & & & & & & 0 & .18 & 0 & 0 & 0 & .15 \\
\hline 23 & & & & & & & 0 & .13 & 0 & 0 & .86 & 0 \\
\hline 24 & & & & & & & 0 & 0 & 0 & 0 & .46 & 0 \\
\hline 25 & & & & & & & 0 & 0 & 0 & .01 & .15 & 0 \\
\hline 26 & & & & & & & 0 & 0 & 0 & .17 & 0 & 0 \\
\hline 27 & & & & & & & 0 & 0 & 0 & .11 & 0 & 0 \\
\hline 28 & & & & & & & 0 & .45 & 0 & .02 & 0 & 0 \\
\hline 29 & & & & & & & 0 & 0 & 0 & 0 & 0 & 0 \\
\hline 30 & & & & & ------ & & 0 & 0 & 0 & 0 & .03 & 0 \\
\hline 31 & & ----- & & & ------ & & ----- & 0 & ----- & 0 & 1.0 & ----- \\
\hline Total & & & & & & & 0 & 1.65 & 0.09 & 2.70 & 6.71 & 0.23 \\
\hline $\operatorname{Max}$ & & & & & & & 0 & .60 & .06 & .76 & 1.3 & .15 \\
\hline Wtr Year & 2008 & Total & 11.38 & Mean & & .072 & Max & 1.3 & Min & 0 & InstMax & .31 \\
\hline Cal Year & 2007 & Total & 14.78 & Mean & & .094 & Max & 1.6 & Min & 0 & InstMax & .31 \\
\hline
\end{tabular}




\section{E262 Cañon del Valle above Water Canyon}

Location. Lat $35^{\circ}$ 49' 51", long $106^{\circ}$ 18' 14", Sec. 33, T. 19 N., R. 6 E., Ramon Vigil Grant, Los Alamos County.

Drainage Area. $4.32 \mathrm{mi}^{2}$.

Period of Record. October 1, 1998, to September 30, 2008.

Revised Record. Drainage area (2006).

Gage. Data logger and $90^{\circ}$ weir plate. Elevation of gage is 6,840 ft above NGVD.

Remarks. Records are good. Legal location based on projected values.

Average Discharge. $9 \mathrm{yr}, 0.013 \mathrm{ft}^{3} / \mathrm{s}, 9.4 \mathrm{acre}-\mathrm{ft} / \mathrm{yr}$.

Extremes for Period of Record. Maximum discharge, $63 \mathrm{ft}^{3} / \mathrm{s}$, August 20, 2004, gage height $4.10 \mathrm{ft}$. No flow most of the time.

Extremes for Current Year. Peak discharge above base of $5.0 \mathrm{ft}^{3} / \mathrm{s}$ and maximum (*):

\begin{tabular}{|c|c|c|c|}
\hline Date & Time & Discharge $\left(\mathbf{f t}^{\mathbf{3}} / \mathbf{s}\right)$ & Gage Height (ft) \\
\hline December 1 & 0345 & 9.3 & 3.0 \\
\hline January 28 & 0510 & $11^{*}$ & $3.07^{*}$ \\
\hline August 10 & 1300 & 6.1 & 2.81 \\
\hline
\end{tabular}

No flow most of the time

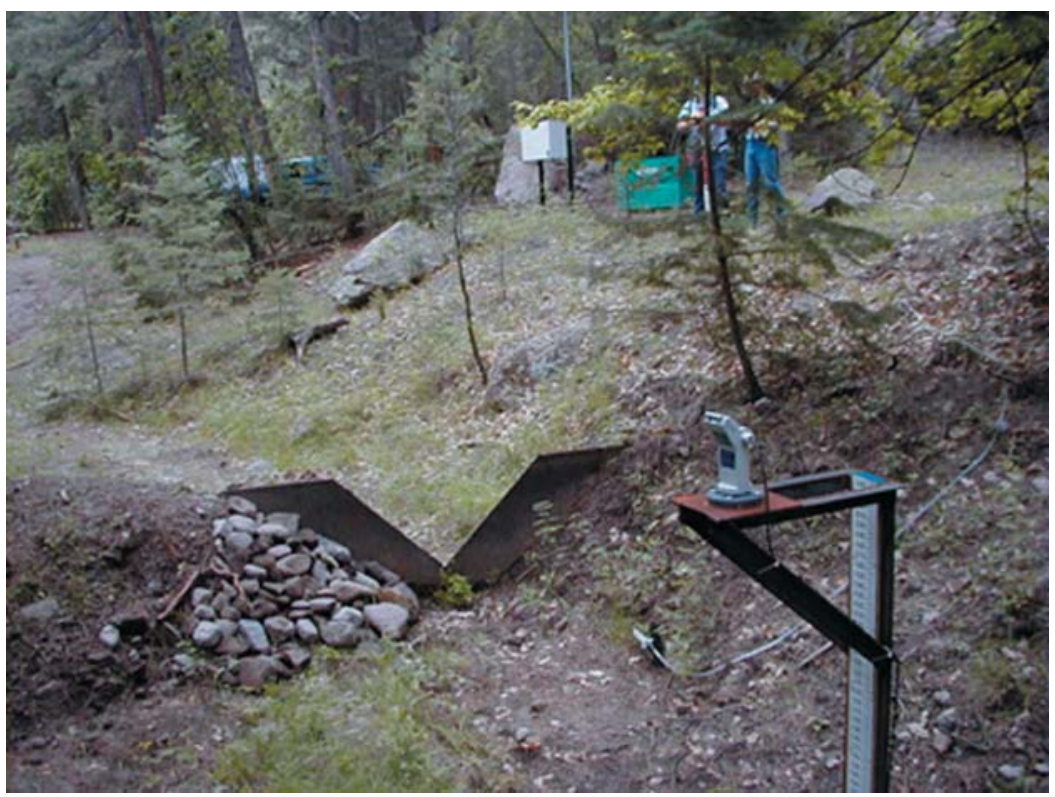




\section{Station Analysis}

\section{Water Year}

Equipment. Station is equipped with Sutron 8210 data logger (5-min. interval) and milltronics sonic probe mounted on a 6" channel cantilevered spanning into stream. The system is powered by a solar-panel battery system housed in a NEMA shelter. Station is equipped with an ISCO pump sampler for water-quality sample collection. ISCO is housed in a $3^{\prime} \times 4^{\prime}$ metal box. Sampler is triggered by stage through the data logger. An outside staff is available for reference. No provision for discharge measurements above wading stage.

Cellular telemetry removed March 28, discontinued service.

Field Work. The station was visited 14 times to conduct discharge measurements and service the instrumentation. Field inspections for the gage are listed under site history files on the Hydstra database. Discharge measurements for the gage are listed under site gauging files on the Hydstra database.

Datum Correction. None.

Gage-Height Record. The data logger referenced to the outside staff gave a complete and satisfactory record except for the periods of January 17-23 and February 24-26 when ice affected gage height.

Rating. The channel is about $10^{\prime}$ wide and straight for about $50^{\prime}$ upstream and straight for about $30^{\prime}$ downstream. The streambed through this reach consists of primarily rock with gravel, sand and cobbles.

One discharge measurement (No. 12) and 12 inspections of no flow were made during this year.

Rating No. 1 is based on a theoretical computation for $90^{\circ}$ sharp-crested weir up to a gage height of $2.95 \mathrm{ft}$. Broad-crested weir computation is used above that stage.

Discharge. Discharge was computed by applying gage height to Rating No. 1 directly.

Remarks. Records are good. 
E262 Cañon del Valle above Water Canyon

Daily Mean Discharge in Cubic Feet per Second

Water Year October 2007 to September 2008

\begin{tabular}{|c|c|c|c|c|c|c|c|c|c|c|c|c|}
\hline DAY & OCT & NOV & DEC & JAN & FEB & MAR & APR & MAY & JUN & JUL & AUG & SEP \\
\hline 1 & 0 & 0 & 1.5 & 0 & 0 & 0 & 0 & 0 & 0 & 0 & 0 & 0 \\
\hline 2 & 0 & 0 & 0 & 0 & 0 & 0 & 0 & 0 & 0 & 0 & 0 & 0 \\
\hline 3 & 0 & 0 & 0 & 0 & 0 & 0 & 0 & 0 & 0 & 0 & 0 & 0 \\
\hline 4 & 0 & 0 & 0 & 0 & 0 & 0 & 0 & 0 & 0 & 0 & 0 & 0 \\
\hline 5 & 0 & 0 & 0 & 0 & 0 & 0 & 0 & 0 & 0 & 0 & 0 & 0 \\
\hline 6 & 0 & 0 & 0 & 0 & 0 & 0 & 0 & 0 & 0 & 0 & 0 & 0 \\
\hline 7 & 0 & 0 & 0 & 0 & 0 & 0 & 0 & 0 & 0 & 0 & 0 & 0 \\
\hline 8 & 0 & 0 & 0 & 0 & 0 & 0 & 0 & 0 & 0 & 0 & 0 & 0 \\
\hline 9 & 0 & 0 & 0 & 0 & 0 & 0 & 0 & 0 & 0 & 0 & 0 & 0 \\
\hline 10 & 0 & 0 & 0 & 0 & 0 & 0 & 0 & 0 & 0 & 0 & .30 & 0 \\
\hline 11 & 0 & 0 & 0 & 0 & 0 & 0 & 0 & 0 & 0 & 0 & 0 & 0 \\
\hline 12 & 0 & 0 & 0 & 0 & 0 & 0 & 0 & 0 & 0 & 0 & 0 & 0 \\
\hline 13 & 0 & 0 & 0 & 0 & 0 & 0 & 0 & 0 & 0 & 0 & 0 & 0 \\
\hline 14 & 0 & 0 & 0 & 0 & 0 & 0 & 0 & 0 & 0 & 0 & 0 & 0 \\
\hline 15 & 0 & 0 & 0 & 0 & 0 & 0 & 0 & 0 & 0 & 0 & 0 & 0 \\
\hline 16 & 0 & 0 & 0 & 0 & 0 & 0 & 0 & 0 & 0 & 0 & 0 & 0 \\
\hline 17 & 0 & 0 & 0 & $0^{*}$ & 0 & 0 & 0 & 0 & 0 & 0 & 0 & 0 \\
\hline 18 & 0 & 0 & 0 & $0^{*}$ & 0 & 0 & 0 & 0 & 0 & 0 & 0 & 0 \\
\hline 19 & 0 & 0 & 0 & $0^{*}$ & 0 & 0 & 0 & 0 & 0 & 0 & 0 & 0 \\
\hline 20 & 0 & 0 & 0 & $0^{*}$ & 0 & 0 & 0 & 0 & 0 & 0 & 0 & 0 \\
\hline 21 & 0 & 0 & 0 & $0^{*}$ & 0 & 0 & 0 & 0 & 0 & 0 & 0 & 0 \\
\hline 22 & 0 & 0 & 0 & $0^{*}$ & 0 & 0 & 0 & 0 & 0 & 0 & 0 & 0 \\
\hline 23 & 0 & 0 & 0 & $0^{*}$ & 0 & 0 & 0 & 0 & 0 & 0 & 0 & 0 \\
\hline 24 & 0 & 0 & 0 & 0 & $0^{*}$ & 0 & 0 & 0 & 0 & 0 & 0 & 0 \\
\hline 25 & 0 & 0 & 0 & 0 & $0^{*}$ & 0 & 0 & 0 & 0 & 0 & 0 & 0 \\
\hline 26 & 0 & 0 & 0 & 0 & $0^{*}$ & 0 & 0 & 0 & 0 & 0 & 0 & 0 \\
\hline 27 & 0 & 0 & 0 & 0 & 0 & 0 & 0 & 0 & 0 & 0 & 0 & 0 \\
\hline 28 & 0 & 0 & 0 & .98 & 0 & .03 & 0 & 0 & 0 & 0 & 0 & 0 \\
\hline 29 & 0 & 0 & 0 & .03 & 0 & 0 & 0 & 0 & 0 & 0 & 0 & 0 \\
\hline 30 & 0 & 0 & 0 & .03 & ------ & 0 & 0 & 0 & 0 & 0 & 0 & 0 \\
\hline 31 & 0 & ------ & 0 & .01 & ------ & 0 & ------ & 0 & ----- & 0 & 0 & ----- \\
\hline Total & 0 & 0 & 1.5 & 1.05 & 0 & 0.03 & 0 & 0 & 0 & 0 & 0.30 & 0 \\
\hline Mean & 0 & 0 & .048 & .034 & 0 & .001 & 0 & 0 & 0 & 0 & .010 & 0 \\
\hline Max & 0 & 0 & 1.5 & .98 & 0 & .03 & 0 & 0 & 0 & 0 & .30 & 0 \\
\hline Min & 0 & 0 & 0 & 0 & 0 & 0 & 0 & 0 & 0 & 0 & 0 & 0 \\
\hline Acre-Ft & 0 & 0 & 3.0 & 2.1 & 0 & .06 & 0 & 0 & 0 & 0 & .60 & 0 \\
\hline Wtr Year & 2008 & Total & 2.88 & Mean & & & Max & 1.5 & Min & 0 & Acre-Ft & 5.7 \\
\hline Cal Year & 2007 & Total & 3.66 & Mean & & 10 & Max & 1.5 & Min & 0 & Acre-Ft & 7.3 \\
\hline
\end{tabular}

*Estimate 


\section{E2625 Water Canyon below MDA AB}

Location. Lat $35^{\circ}$ 49' 31", long $106^{\circ}$ 17' 03", Sec. 3, T. 18 N., R. 6 E., Ramon Vigil Grant, Los Alamos County.

Drainage Area. $11.55 \mathrm{mi}^{2}$.

Period of Record. October 1, 2001, to September 30, 2008.

Revised Record. Drainage area (2006).

Gage. Data logger and $90^{\circ}$ weir plate. Elevation of gage is 6,666 ft above NGVD.

Remarks. Records are fair. Legal location based on projected values. Records existed before period of record but are not reliable.

Average Discharge. $7 \mathrm{yr} ., 0.15 \mathrm{ft}^{3} / \mathrm{s}, 110$ acre-ft/yr.

Extremes for Period of Record. Maximum discharge, $306 \mathrm{ft}^{3} / \mathrm{s}$, August 29, 2007, gage height $4.57 \mathrm{ft}$. No flow most of the time.

Extremes for Current Year. Peak discharges above base of $10 \mathrm{ft}^{3} / \mathrm{s}$ and maximum (*):

\begin{tabular}{|c|c|c|c|}
\hline Date & Time & Discharge $\left(\mathbf{f t}^{\mathbf{3}} \mathbf{s}\right)$ & Gage Height (ft) \\
\hline December 1 & 0305 & 27 & 3.10 \\
\hline January 28 & 0505 & $75^{*}$ & $3.70^{*}$ \\
\hline July 21 & 1255 & 12 & 2.53 \\
\hline August 8 & 1500 & 16 & 2.72 \\
\hline August 10 & 1300 & 19 & 2.84 \\
\hline August 23 & 1740 & 17 & 2.79 \\
\hline August 24 & 1955 & 12 & 2.57 \\
\hline
\end{tabular}

No flow most of the time.

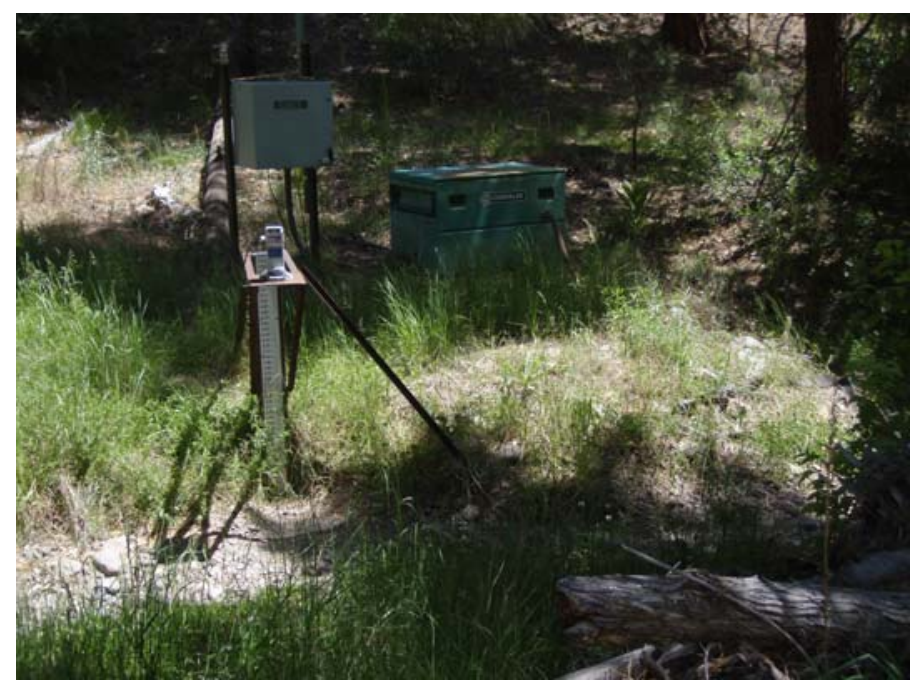




\section{E2625 Water Canyon below MDA AB}

\section{Station Analysis}

\section{Water Year}

Equipment. Station is equipped with Sutron 8210 data logger (5-min. interval) and milltronics sonic probe mounted on a 6" channel cantilevered over the stream. The system is powered by a solar-panel battery system housed in a NEMA shelter. Station is equipped with an ISCO pump sampler for water-quality sample collection. ISCO is housed in a separate shelter, a $3^{\prime} \times 4^{\prime}$ metal box. Sampler is triggered by stage through the data logger. An outside staff is available for reference. No provisions are made for measurement above wading stage.

Cellular telemetry removed March 17, discontinued service.

Field Work. The station was visited 18 times to conduct discharge measurements and service the instrumentation. Field inspections for the gage are listed under site history files on the Hydstra database. Discharge measurements for the gage are listed under site gauging files on the Hydstra database.

Datum Correction. None. Levels on March 4, 2005, found gage within allowable range.

Gage-Height Record. The data logger referenced to the outside staff gave a complete and satisfactory record for the year except for the period of December 18 to January 22 when ice affected gage height.

Rating. The channel is approximately $20^{\prime}$ wide and straight for about $75^{\prime}$ upstream and straight for about $100^{\prime}$ downstream. The streambed through this reach consists of primarily sand and cobbles. The low-water control is a $90^{\circ}$ sharp-crested weir. During high flow, the channel becomes the control.

One discharge measurement (No. 18) and 11 inspections of no flow were made this year. All inspections of no flow were used to develop a "V" diagram shift needed to adjust for PZF.

Rating No. 2 is based on a theoretical computation for $90^{\circ}$ shape-crested weir up to a gage height of $2.75 \mathrm{ft}$, and extended to $4.38 \mathrm{ft}$ gage height based on a slope-area measurement.

Shifts were applied to low flow using "V" diagrams. Large negatives (about $0.76 \mathrm{ft}$ ) were also applied to zero flow observations.

Discharge. Discharge was computed by applying gage height to Rating No. 2 through shift adjustment based on "V" diagrams. Estimated daily discharges were based on precipitation record, field notes, and some comparison with E262 and E2525.

Remarks. Records are fair. 
E2625 Water Canyon below MDA AB

Daily Mean Discharge in Cubic Feet per Second

Water Year October 2007 to September 2008

\begin{tabular}{|c|c|c|c|c|c|c|c|c|c|c|c|c|}
\hline DAY & OCT & NOV & DEC & JAN & FEB & MAR & APR & MAY & JUN & JUL & AUG & SEP \\
\hline 1 & 0 & 0 & 8.8 & $.02^{*}$ & .24 & .71 & 1.2 & .16 & .01 & 0 & 0 & .11 \\
\hline 2 & 0 & 0 & 1.0 & $.02^{*}$ & .19 & .75 & 1.1 & .15 & .01 & 0 & .01 & 0 \\
\hline 3 & 0 & 0 & .68 & $.02^{\star}$ & .16 & .75 & .88 & .15 & .01 & 0 & 0 & 0 \\
\hline 4 & 0 & 0 & .38 & $.02^{\star}$ & .09 & .75 & .80 & .13 & .01 & 0 & .39 & 0 \\
\hline 5 & 0 & 0 & .26 & $.02^{\star}$ & .05 & .77 & .86 & .11 & .01 & 0 & .01 & 0 \\
\hline 6 & 0 & 0 & .18 & $.02^{\star}$ & .02 & .76 & .63 & .11 & .01 & 0 & .01 & 0 \\
\hline 7 & 0 & 0 & .16 & $.02^{*}$ & .02 & .64 & .65 & .09 & .01 & 0 & .01 & 0 \\
\hline 8 & 0 & 0 & .79 & $.02^{*}$ & .01 & .68 & .58 & .07 & .01 & 0 & 1.5 & 0 \\
\hline 9 & 0 & 0 & 1.3 & $.01^{*}$ & .01 & .53 & .67 & .06 & 0 & 0 & .03 & 0 \\
\hline 10 & 0 & 0 & 1.1 & $.01^{*}$ & .02 & .56 & .54 & .04 & 0 & 0 & 1.9 & 0 \\
\hline 11 & 0 & 0 & .92 & $.01^{*}$ & .15 & .55 & .46 & .05 & 0 & 0 & .01 & 0 \\
\hline 12 & 0 & 0 & .67 & $.01^{*}$ & .40 & .75 & .40 & .04 & 0 & 0 & 0 & 0 \\
\hline 13 & 0 & 0 & .57 & $.01^{*}$ & .24 & .75 & .35 & .04 & 0 & 0 & 0 & 0 \\
\hline 14 & 0 & 0 & .48 & $.01^{*}$ & .15 & .75 & .34 & .09 & 0 & 0 & 0 & 0 \\
\hline 15 & 0 & 0 & .29 & $.01^{*}$ & .15 & .79 & .33 & .32 & 0 & .04 & 0 & 0 \\
\hline 16 & 0 & 0 & .21 & $.01^{*}$ & .15 & 1.3 & .26 & .12 & 0 & 0 & 0 & 0 \\
\hline 17 & 0 & 0 & .21 & $.01^{*}$ & .24 & 1.3 & .38 & .06 & 0 & 0 & 0 & 0 \\
\hline 18 & 0 & 0 & $.19^{\star}$ & $.01^{*}$ & .22 & 1.2 & .26 & .04 & 0 & 0 & 0 & 0 \\
\hline 19 & 0 & 0 & $.19^{*}$ & $.01^{*}$ & .22 & 1.1 & .25 & .03 & 0 & 0 & 0 & 0 \\
\hline 20 & 0 & 0 & $.16^{\star}$ & $.01^{*}$ & .26 & 1.2 & .20 & .02 & 0 & 0 & 0 & 0 \\
\hline 21 & 0 & 0 & $.15^{*}$ & $.01^{*}$ & .34 & 1.4 & .20 & .02 & 0 & .56 & 0 & 0 \\
\hline 22 & 0 & 0 & $.07^{\star}$ & $.01^{*}$ & .33 & 1.5 & .17 & .02 & 0 & .01 & 0 & 0 \\
\hline 23 & 0 & 0 & $.02^{*}$ & .01 & .36 & 1.5 & .17 & .05 & 0 & 0 & 1.5 & 0 \\
\hline 24 & 0 & 0 & $.02^{*}$ & .01 & 1.8 & 1.5 & .18 & .03 & 0 & 0 & .89 & 0 \\
\hline 25 & 0 & 0 & $.02^{*}$ & .01 & 2.5 & 1.5 & .17 & .03 & 0 & 0 & .11 & 0 \\
\hline 26 & 0 & 0 & $.02^{\star}$ & .01 & .95 & 1.5 & .18 & .02 & 0 & .01 & 0 & 0 \\
\hline 27 & 0 & 0 & $.02^{\star}$ & .01 & .68 & 1.5 & .18 & .01 & 0 & .01 & 0 & 0 \\
\hline 28 & 0 & 0 & $.02^{*}$ & 11 & .76 & 1.6 & .26 & .03 & 0 & 0 & 0 & 0 \\
\hline 29 & 0 & 0 & $.02^{*}$ & 1.5 & .75 & 1.6 & .25 & .06 & 0 & .01 & 0 & 0 \\
\hline 30 & 0 & 0 & $.02^{\star}$ & .81 & ----- & 1.5 & .21 & .01 & 0 & 0 & 0 & 0 \\
\hline 31 & 0 & ----- & $.02^{*}$ & .31 & ---- & 1.3 & ------ & .01 & ----- & 0 & .27 & ----- \\
\hline Total & 0 & 0 & 18.94 & 13.97 & 11.46 & 32.99 & 13.11 & 2.17 & 0.08 & 0.64 & 6.64 & 0.11 \\
\hline Mean & 0 & 0 & .61 & .45 & .40 & 1.06 & .44 & .070 & .003 & .021 & .21 & .004 \\
\hline Max & 0 & 0 & 8.8 & 11 & 2.5 & 1.6 & 1.2 & .32 & .01 & .56 & 1.9 & .11 \\
\hline Min & 0 & 0 & .02 & .01 & .01 & .53 & .17 & .01 & 0 & 0 & 0 & 0 \\
\hline Acre-Ft & 0 & 0 & 38 & 28 & 23 & 65 & 26 & 4.3 & .16 & 1.3 & 13 & .22 \\
\hline Wtr Year & 2008 & Total & 100.11 & & & .27 & $\operatorname{Max}$ & 11 & Min & 0 & Acre-Ft & 199 \\
\hline Cal Year & 2007 & Total & 54.29 & & & .15 & $\operatorname{Max}$ & 8.8 & Min & 0 & Acre-Ft & 108 \\
\hline
\end{tabular}

*Estimate 


\section{E263 Water Canyon at SR 4}

Location. Lat $35^{\circ} 48^{\prime} 20^{\prime \prime}$, long $106^{\circ} 14^{\prime}$ 52" Sec. 12, T. 18 N., R. 6 E., Ramon Vigil Grant, Los Alamos County.

Drainage Area. $12.43 \mathrm{mi}^{2}$.

Period of Record. April 1999 to September 30, 2008.

Revised Record. Drainage area (2006).

Gage. Data logger and cellular telemetry. Elevation of gage is 6,365 $\mathrm{ft}$ above NGVD from GPS survey.

Remarks. Records are good. Legal location based on projected values.

Average Discharge: $8 \mathrm{yr}, 0.08 \mathrm{ft}^{3} / \mathrm{s}, 57 \mathrm{acre}-\mathrm{ft} / \mathrm{yr}$.

Extremes for Period of Record. Maximum discharge, $306 \mathrm{ft}^{3} / \mathrm{s}$, June 28, 2000, gage height $3.78 \mathrm{ft}$. No flow most of the time.

Extremes for Current Year. Peak discharge above base of $35 \mathrm{ft}^{3} / \mathrm{s}$ and maximum (*):

\begin{tabular}{|c|c|c|c|}
\hline Date & Time & Discharge (ft $\left.{ }^{\mathbf{3}} / \mathbf{s}\right)$ & Gage Height (ft) \\
\hline January 28 & 0650 & $75^{*}$ & $1.94^{*}$ \\
\hline
\end{tabular}

No flow most of the time.

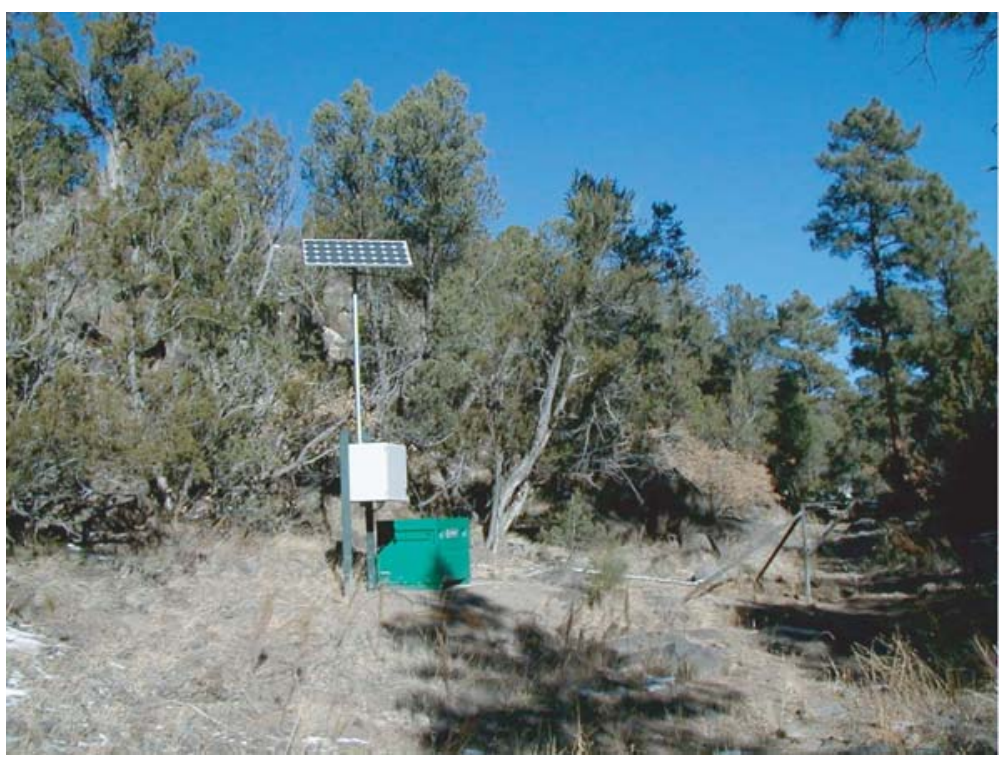




\section{E263 Water Canyon at SR 4}

\section{Station Analysis}

\section{Water Year}

Equipment. Station is equipped with Sutron 8210 data logger (5-min. interval) and milltronics sonic probe and cellular telemetry. Phone upgraded June 5 from analog to digital service. The system is powered by a solar-panel battery system housed in a NEMA shelter. Station is equipped with an ISCO pump sampler for water-quality sample collection. ISCO is housed in a separate shelter, a $3^{\prime} \times 4^{\prime}$ metal box. Sampler is triggered by stage through the data logger. An outside staff is available for reference. No provision for discharge measurements above wading stage.

Field Work. This station was visited 15 times to conduct discharge measurements and service the instrumentation. Field inspections for the gage are listed under site history files on the Hydstra database. Discharge measurements for the gage are listed under site gauging files on the Hydstra database.

Datum Correction. None.

Gage-Height Record. The data logger referenced to the outside staff gave a complete and satisfactory record except for the period of January 28 and 29 when ice affected gage height.

Rating. The channel is straight for 400' upstream and 200' downstream. Streambed is well armored with bedrock and boulders. Some pockets of sand exist from sediment deposition. Vegetation is sparse in the channel bottom and the banks. Scour should be minimal to non-existent.

Rating No. 1 continued in use.

Discharge. Discharge was computed by applying gage height to Rating No. 1 through shift adjustment based on "V" diagrams.

Remarks. Records are good. 


\section{E263 Water Canyon at SR 4}

Daily Mean Discharge in Cubic Feet per Second

Water Year October 2007 to September 2008

\begin{tabular}{|c|c|c|c|c|c|c|c|c|c|c|c|c|}
\hline DAY & OCT & NOV & DEC & JAN & FEB & MAR & APR & MAY & JUN & JUL & AUG & SEP \\
\hline 1 & 0 & 0 & 0 & 0 & 0 & 0 & 0 & 0 & 0 & 0 & 0 & 0 \\
\hline 2 & 0 & 0 & 0 & 0 & 0 & 0 & 0 & 0 & 0 & 0 & 0 & 0 \\
\hline 3 & 0 & 0 & 0 & 0 & 0 & 0 & 0 & 0 & 0 & 0 & 0 & 0 \\
\hline 4 & 0 & 0 & 0 & 0 & 0 & 0 & 0 & 0 & 0 & 0 & 0 & 0 \\
\hline 5 & 0 & 0 & 0 & 0 & 0 & 0 & 0 & 0 & 0 & 0 & 0 & 0 \\
\hline 6 & 0 & 0 & 0 & 0 & 0 & 0 & 0 & 0 & 0 & 0 & 0 & 0 \\
\hline 7 & 0 & 0 & 0 & 0 & 0 & 0 & 0 & 0 & 0 & 0 & 0 & 0 \\
\hline 8 & 0 & 0 & 0 & 0 & 0 & 0 & 0 & 0 & 0 & 0 & 0 & 0 \\
\hline 9 & 0 & 0 & 0 & 0 & 0 & 0 & 0 & 0 & 0 & 0 & 0 & 0 \\
\hline 10 & 0 & 0 & 0 & 0 & 0 & 0 & 0 & 0 & 0 & 0 & 0 & 0 \\
\hline 11 & 0 & 0 & 0 & 0 & 0 & 0 & 0 & 0 & 0 & 0 & 0 & 0 \\
\hline 12 & 0 & 0 & 0 & 0 & 0 & 0 & 0 & 0 & 0 & 0 & 0 & 0 \\
\hline 13 & 0 & 0 & 0 & 0 & 0 & 0 & 0 & 0 & 0 & 0 & 0 & 0 \\
\hline 14 & 0 & 0 & 0 & 0 & 0 & 0 & 0 & 0 & 0 & 0 & 0 & 0 \\
\hline 15 & 0 & 0 & 0 & 0 & 0 & 0 & 0 & 0 & 0 & 0 & 0 & 0 \\
\hline 16 & 0 & 0 & 0 & 0 & 0 & 0 & 0 & 0 & 0 & 0 & 0 & 0 \\
\hline 17 & 0 & 0 & 0 & 0 & 0 & 0 & 0 & 0 & 0 & 0 & 0 & 0 \\
\hline 18 & 0 & 0 & 0 & 0 & 0 & 0 & 0 & 0 & 0 & 0 & 0 & 0 \\
\hline 19 & 0 & 0 & 0 & 0 & 0 & 0 & 0 & 0 & 0 & 0 & 0 & 0 \\
\hline 20 & 0 & 0 & 0 & 0 & 0 & 0 & 0 & 0 & 0 & 0 & 0 & 0 \\
\hline 21 & 0 & 0 & 0 & 0 & 0 & 0 & 0 & 0 & 0 & 0 & 0 & 0 \\
\hline 22 & 0 & 0 & 0 & 0 & 0 & 0 & 0 & 0 & 0 & 0 & 0 & 0 \\
\hline 23 & 0 & 0 & 0 & 0 & 0 & 0 & 0 & 0 & 0 & 0 & 0 & 0 \\
\hline 24 & 0 & 0 & 0 & 0 & 0 & 0 & 0 & 0 & 0 & 0 & 0 & 0 \\
\hline 25 & 0 & 0 & 0 & 0 & 0 & 0 & 0 & 0 & 0 & 0 & 0 & 0 \\
\hline 26 & 0 & 0 & 0 & 0 & 0 & 0 & 0 & 0 & 0 & 0 & 0 & 0 \\
\hline 27 & 0 & 0 & 0 & 0 & 0 & 0 & 0 & 0 & 0 & 0 & 0 & 0 \\
\hline 28 & 0 & 0 & 0 & $13^{*}$ & 0 & 0 & 0 & 0 & 0 & 0 & 0 & 0 \\
\hline 29 & 0 & 0 & 0 & $0^{*}$ & 0 & 0 & 0 & 0 & 0 & 0 & 0 & 0 \\
\hline 30 & 0 & 0 & 0 & 0 & ------ & 0 & 0 & 0 & 0 & 0 & 0 & 0 \\
\hline 31 & 0 & ----- & 0 & 0 & ------ & 0 & ------ & 0 & ------ & 0 & 0 & ------ \\
\hline Total & 0 & 0 & 0 & 13 & 0 & 0 & 0 & 0 & 0 & 0 & 0 & 0 \\
\hline Mean & 0 & 0 & 0 & .42 & 0 & 0 & 0 & 0 & 0 & 0 & 0 & 0 \\
\hline Max & 0 & 0 & 0 & 13 & 0 & 0 & 0 & 0 & 0 & 0 & 0 & 0 \\
\hline Min & 0 & 0 & 0 & 0 & 0 & 0 & 0 & 0 & 0 & 0 & 0 & 0 \\
\hline Acre-Ft & 0 & 0 & 0 & 26 & 0 & 0 & 0 & 0 & 0 & 0 & 0 & 0 \\
\hline Wtr Year & 2008 & Total & 13 & Mean & & & hax & 13 & Min & 0 & Acre-Ft & 26 \\
\hline Cal Year & 2007 & Total & 8.28 & Mean & & & hax & 5.5 & Min & 0 & Acre-Ft & 16 \\
\hline
\end{tabular}

*Estimate 


\section{E264 Indio Canyon at SR 4}

Location. Lat $35^{\circ}$ 48' 18", long $106^{\circ}$ 14' 51", Sec. 12, T. 18 N., R. 6 E., Ramon Vigil Grant, Los Alamos County.

Drainage Area. $0.49 \mathrm{mi}^{2}$.

Period of Record. October 1, 2006, to September 30, 2008.

Gage. Data logger. Elevation of gage is 6,366 ft above NGVD.

Remarks. Records are good. Records for this site existed before the period of record but are not reliable.

Extremes for Period of Record. Maximum discharge, $0.03 \mathrm{ft}^{3} / \mathrm{s}$, March 2, 2007, gage height $0.76 \mathrm{ft}$. No flow most of the time.

Extremes for Current Year. Maximum discharge of $0.01 \mathrm{ft}^{3} / \mathrm{s}$ at $2005 \mathrm{~h}$, August 31, gage height $0.71 \mathrm{ft}$. No peak discharge above $1.0 \mathrm{ft}^{3} / \mathrm{s}$. No flow most of the time.

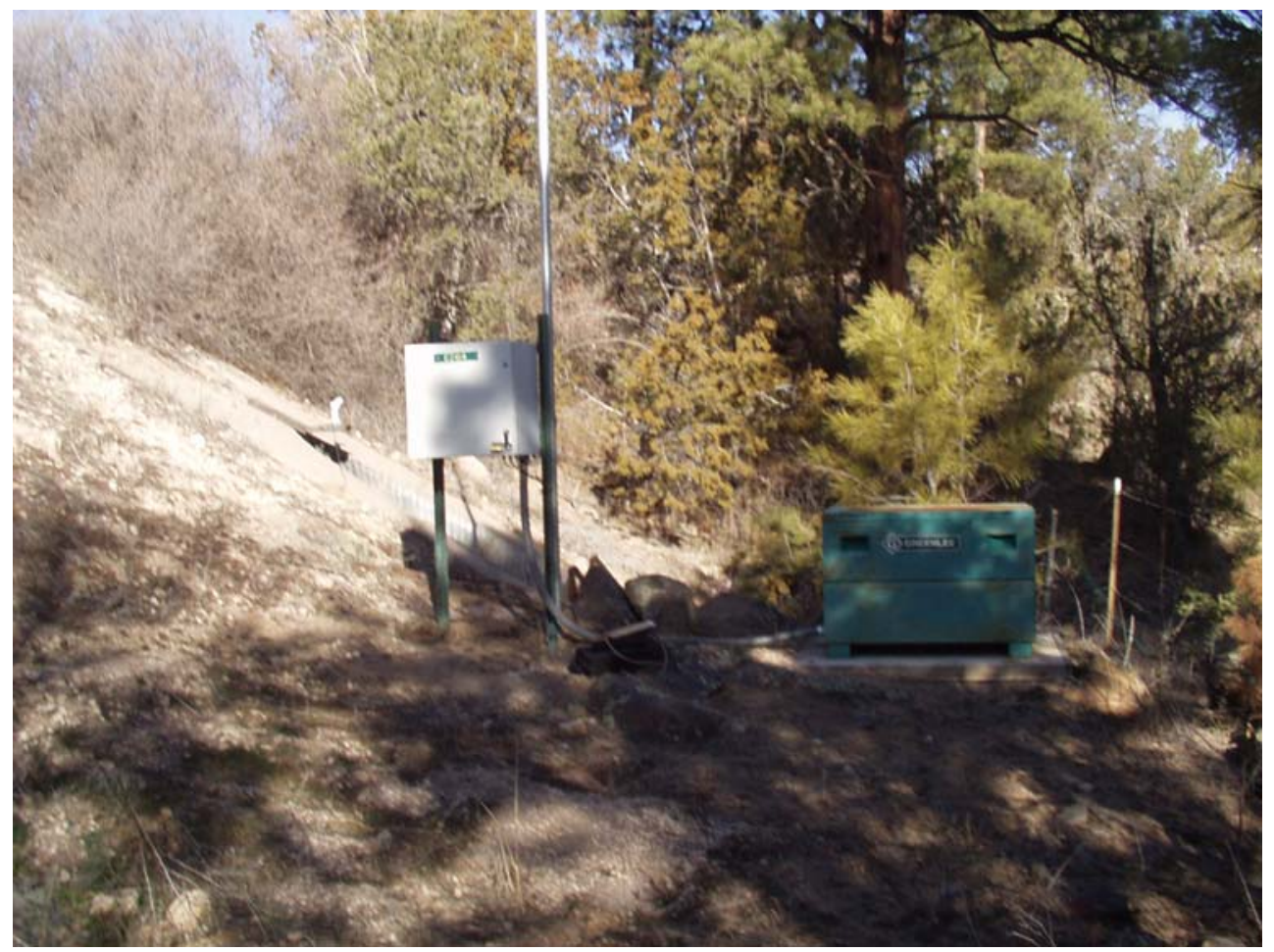




\section{E264 Indio Canyon at SR 4}

\section{Station Analysis}

\section{Water Year}

Equipment. Station is equipped with Sutron 8210 (5-min. interval) with Sutron Accubar bubble sensor. The system is powered by a solar-panel battery system housed in a NEMA shelter. Station is equipped with an ISCO pump sampler for water-quality sample collection. ISCO is housed in a separate shelter, a $3^{\prime} \times 4^{\prime}$ metal box. An outside staff is available for reference. No provision for discharge measurement above wading stage.

Cellular telemetry removed March 17, discontinued service.

Field Work. The station was visited 12 times to conduct discharge measurements and service the instrumentation. Inspections for the gage are listed under site history files on the Hydstra database. Discharge measurements for the gage are listed under site-gauging files on the Hydstra database.

Datum Correction. None. Levels were run May 13, 2005, found gage correct to datum.

Gage-Height Record. The data logger referenced to the outside staff gave a complete and satisfactory record for the year.

Rating. The channel at the gage is approximately $8^{\prime}$ wide and straight for about 50' upstream 25' downstream and bends to the right. The streambed through this reach consists of primarily sand. The low-flow control is a $120^{\circ}$ sharp-crested weir. The channel becomes the control at high flow.

Twelve inspections of no flow were made this year.

Rating No.1 is based on a theoretical computation for $120^{\circ}$ sharp-crested weir to a gage height of $2.60 \mathrm{ft}$. Broad-crested weir computation is used above that stage.

Discharge. Discharge was computed by applying gage height to Rating No. 1 directly.

Remarks. Records are good. 
E264 Indio Canyon at SR 4

Daily Mean Discharge in Cubic Feet per Second

Water Year October 2007 to September 2008

\begin{tabular}{|c|c|c|c|c|c|c|c|c|c|c|c|c|}
\hline DAY & OCT & NOV & DEC & JAN & FEB & MAR & APR & MAY & JUN & JUL & AUG & SEP \\
\hline 1 & 0 & 0 & 0 & 0 & 0 & 0 & 0 & 0 & 0 & 0 & 0 & 0 \\
\hline 2 & 0 & 0 & 0 & 0 & 0 & 0 & 0 & 0 & 0 & 0 & 0 & 0 \\
\hline 3 & 0 & 0 & 0 & 0 & 0 & 0 & 0 & 0 & 0 & 0 & 0 & 0 \\
\hline 4 & 0 & 0 & 0 & 0 & 0 & 0 & 0 & 0 & 0 & 0 & 0 & 0 \\
\hline 5 & 0 & 0 & 0 & 0 & 0 & 0 & 0 & 0 & 0 & 0 & 0 & 0 \\
\hline 6 & 0 & 0 & 0 & 0 & 0 & 0 & 0 & 0 & 0 & 0 & 0 & 0 \\
\hline 7 & 0 & 0 & 0 & 0 & 0 & 0 & 0 & 0 & 0 & 0 & 0 & 0 \\
\hline 8 & 0 & 0 & 0 & 0 & 0 & 0 & 0 & 0 & 0 & 0 & 0 & 0 \\
\hline 9 & 0 & 0 & 0 & 0 & 0 & 0 & 0 & 0 & 0 & 0 & 0 & 0 \\
\hline 10 & 0 & 0 & 0 & 0 & 0 & 0 & 0 & 0 & 0 & 0 & 0 & 0 \\
\hline 11 & 0 & 0 & 0 & 0 & 0 & 0 & 0 & 0 & 0 & 0 & 0 & 0 \\
\hline 12 & 0 & 0 & 0 & 0 & 0 & 0 & 0 & 0 & 0 & 0 & 0 & 0 \\
\hline 13 & 0 & 0 & 0 & 0 & 0 & 0 & 0 & 0 & 0 & 0 & 0 & 0 \\
\hline 14 & 0 & 0 & 0 & 0 & 0 & 0 & 0 & 0 & 0 & 0 & 0 & 0 \\
\hline 15 & 0 & 0 & 0 & 0 & 0 & 0 & 0 & 0 & 0 & 0 & 0 & 0 \\
\hline 16 & 0 & 0 & 0 & 0 & 0 & 0 & 0 & 0 & 0 & 0 & 0 & 0 \\
\hline 17 & 0 & 0 & 0 & 0 & 0 & 0 & 0 & 0 & 0 & 0 & 0 & 0 \\
\hline 18 & 0 & 0 & 0 & 0 & 0 & 0 & 0 & 0 & 0 & 0 & 0 & 0 \\
\hline 19 & 0 & 0 & 0 & 0 & 0 & 0 & 0 & 0 & 0 & 0 & 0 & 0 \\
\hline 20 & 0 & 0 & 0 & 0 & 0 & 0 & 0 & 0 & 0 & 0 & 0 & 0 \\
\hline 21 & 0 & 0 & 0 & 0 & 0 & 0 & 0 & 0 & 0 & 0 & 0 & 0 \\
\hline 22 & 0 & 0 & 0 & 0 & 0 & 0 & 0 & 0 & 0 & 0 & 0 & 0 \\
\hline 23 & 0 & 0 & 0 & 0 & 0 & 0 & 0 & 0 & 0 & 0 & 0 & 0 \\
\hline 24 & 0 & 0 & 0 & 0 & 0 & 0 & 0 & 0 & 0 & 0 & 0 & 0 \\
\hline 25 & 0 & 0 & 0 & 0 & 0 & 0 & 0 & 0 & 0 & 0 & 0 & 0 \\
\hline 26 & 0 & 0 & 0 & 0 & 0 & 0 & 0 & 0 & 0 & 0 & 0 & 0 \\
\hline 27 & 0 & 0 & 0 & 0 & 0 & 0 & 0 & 0 & 0 & 0 & 0 & 0 \\
\hline 28 & 0 & 0 & 0 & 0 & 0 & 0 & 0 & 0 & 0 & 0 & 0 & 0 \\
\hline 29 & 0 & 0 & 0 & 0 & 0 & 0 & 0 & 0 & 0 & 0 & 0 & 0 \\
\hline 30 & 0 & 0 & 0 & 0 & ------ & 0 & 0 & 0 & 0 & 0 & 0 & 0 \\
\hline 31 & 0 & ------ & 0 & 0 & ------ & 0 & ----- & 0 & ----- & 0 & 0 & ------ \\
\hline Total & 0 & 0 & 0 & 0 & 0 & 0 & 0 & 0 & 0 & 0 & 0 & 0 \\
\hline Mean & 0 & 0 & 0 & 0 & 0 & 0 & 0 & 0 & 0 & 0 & 0 & 0 \\
\hline Max & 0 & 0 & 0 & 0 & 0 & 0 & 0 & 0 & 0 & 0 & 0 & 0 \\
\hline Min & 0 & 0 & 0 & 0 & 0 & 0 & 0 & 0 & 0 & 0 & 0 & 0 \\
\hline Acre-Ft & 0 & 0 & 0 & 0 & 0 & 0 & 0 & 0 & 0 & 0 & 0 & 0 \\
\hline Wtr Year & 2008 & Total & 0 & Mean & & 0 & Max & 0 & Min & 0 & Acre-Ft & 0 \\
\hline Cal Year & 2007 & Total & 0.09 & Mean & & 0 & Max & .01 & Min & 0 & Acre-Ft & 0.18 \\
\hline
\end{tabular}




\section{E265 Water Canyon below SR 4}

Location. Lat $35^{\circ} 48^{\prime}$ 18", long $106^{\circ} 14^{\prime}$ 31" Sec. 7, T. 18 N., R. 7 E., Ramon Vigil Grant, Los Alamos County.

Drainage Area. $13.11 \mathrm{mi}^{2}$.

Period of Record. October 1993 to September 30, 2008.

Revised Records. Drainage area (2006).

Gage. Data logger with cellular telemetry, rain gage, and stabilized natural-rock control. Elevation of gage is 6,309 $\mathrm{ft}$ above NGVD from GPS survey.

Remarks. Records good except for estimated daily discharges, which are fair. Legal location based on projected values.

Average Discharge. $14 \mathrm{yr}, 0.04 \mathrm{ft}^{3} / \mathrm{s}, 32$ acre-ft/yr.

Extremes for Period of Record. Maximum discharge, $274 \mathrm{ft}^{3} / \mathrm{s}$, June 28, 2000, gage height $5.13 \mathrm{ft}$ (from flood mark). No flow most of the time.

Extremes for Current Year. Peak discharge above base of $50 \mathrm{ft}^{3} / \mathrm{s}$ and maximum (*):

\begin{tabular}{|c|c|c|c|}
\hline Date & Time & Discharge (ft $\left.{ }^{\mathbf{3}} / \mathbf{s}\right)$ & Gage Height (ft.) \\
\hline January 28 & 0730 & $69^{*}$ & $2.00^{*}$ \\
\hline
\end{tabular}

No flow most of the time.

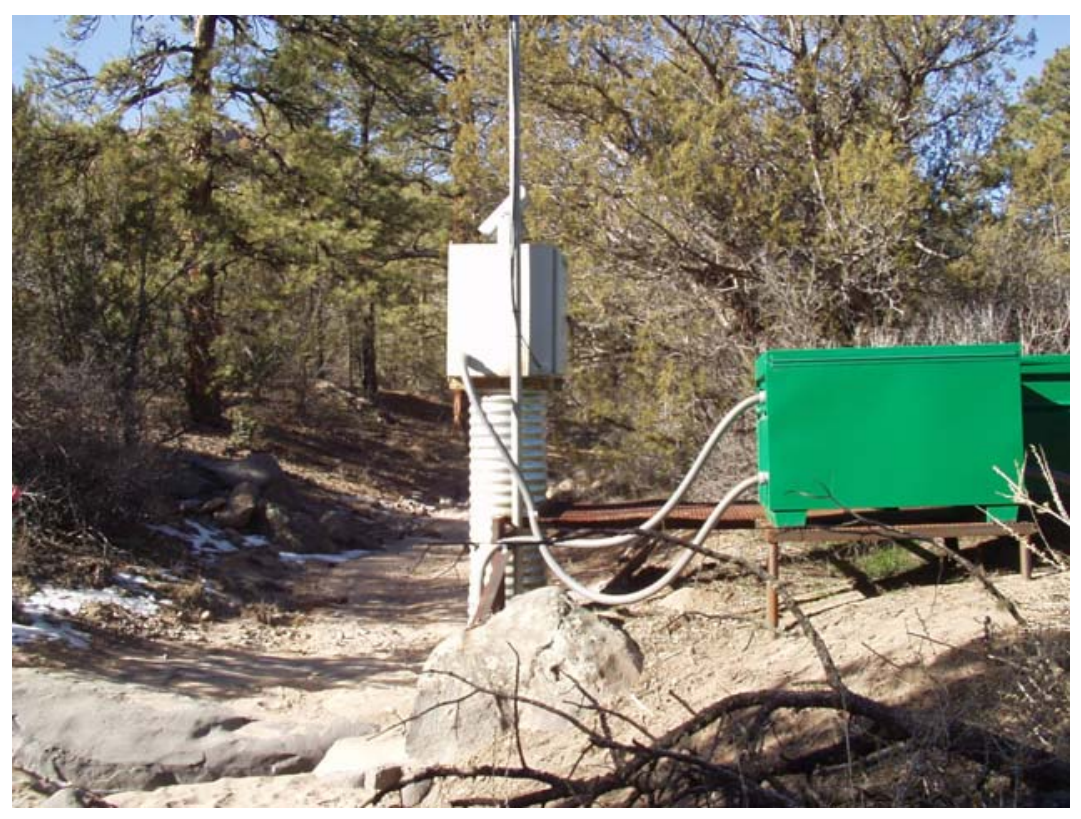




\section{E265 Water Canyon below SR 4}

\section{Station Analysis}

\section{Water Year}

Equipment. Station is equipped with Sutron 8210 (5-min. interval) with shaft encoder float system (5-min. interval) and cellular phone with speech modem. Phone upgraded April 9 from analog to digital service. The system is powered by a solar-panel battery system housed in a NEMA shelter on 24" CMP well. Station is equipped with an ISCO pump sampler for water-quality sample collection. ISCO is housed in a separate shelter, a $3^{\prime} \times 4^{\prime}$ metal box. Sampler is triggered by stage through the data logger. An outside staff is available for reference. No provision for measurements above wading stage.

Station is also equipped with a tipping bucket rain gage, Rain Collection II. All equipment is powered with a solar-panel battery-charging system. Data are seasonal.

Field Work. The station was visited 23 times to conduct discharge measurements and service the instrumentation. Field inspections for the gage are listed under site history files on the Hydstra database. Discharge measurements for the gage are listed under site gauging files on the Hydstra database.

Datum Correction. None.

Gage-Height Record. The data logger referenced to the outside staff gave complete and satisfactory record except for the periods of October 1-29 and August 5-8 when the data logger malfunctioned as a result of battery failure and November 30 to December 4 and January 28 when ice affected gage height.

Rating. The channel is straight for 100' above and below gage. Banks are low and have very little vegetation. Streambed consists of mostly rock with lenses of sand.

Twenty-three inspections of no flow were made this year.

Rating No. 4 was used for the entire water year.

Discharge. Discharge was computed by applying gage height to Rating No. 4 directly.

Remarks. Records are good except for estimated daily discharges, which are fair. 


\section{E265 Water Canyon below SR 4}

Daily Mean Discharge in Cubic Feet per Second

Water Year October 2007 to September 2008

\begin{tabular}{|c|c|c|c|c|c|c|c|c|c|c|c|c|}
\hline DAY & OCT & NOV & DEC & JAN & FEB & MAR & APR & MAY & JUN & JUL & AUG & SEP \\
\hline 1 & $0^{*}$ & 0 & $0^{*}$ & 0 & 0 & 0 & 0 & 0 & 0 & 0 & 0 & 0 \\
\hline 2 & $0^{*}$ & 0 & $0^{*}$ & 0 & 0 & 0 & 0 & 0 & 0 & 0 & 0 & 0 \\
\hline 3 & $0^{*}$ & 0 & $0^{*}$ & 0 & 0 & 0 & 0 & 0 & 0 & 0 & 0 & 0 \\
\hline 4 & $0^{*}$ & 0 & 0 & 0 & 0 & 0 & 0 & 0 & 0 & 0 & 0 & 0 \\
\hline 5 & $0^{*}$ & 0 & 0 & 0 & 0 & 0 & 0 & 0 & 0 & 0 & $0^{*}$ & 0 \\
\hline 6 & $0^{*}$ & 0 & 0 & 0 & 0 & 0 & 0 & 0 & 0 & 0 & $0^{*}$ & 0 \\
\hline 7 & $0^{*}$ & 0 & 0 & 0 & 0 & 0 & 0 & 0 & 0 & 0 & $0^{*}$ & 0 \\
\hline 8 & $0^{*}$ & 0 & 0 & 0 & 0 & 0 & 0 & 0 & 0 & 0 & $0^{*}$ & 0 \\
\hline 9 & $0^{*}$ & 0 & 0 & 0 & 0 & 0 & 0 & 0 & 0 & 0 & 0 & 0 \\
\hline 10 & $0^{*}$ & 0 & 0 & 0 & 0 & 0 & 0 & 0 & 0 & 0 & 0 & 0 \\
\hline 11 & $0^{*}$ & 0 & 0 & 0 & 0 & 0 & 0 & 0 & 0 & 0 & 0 & 0 \\
\hline 12 & $0^{*}$ & 0 & 0 & 0 & 0 & 0 & 0 & 0 & 0 & 0 & 0 & 0 \\
\hline 13 & $0^{*}$ & 0 & 0 & 0 & 0 & 0 & 0 & 0 & 0 & 0 & 0 & 0 \\
\hline 14 & $0^{*}$ & 0 & 0 & 0 & 0 & 0 & 0 & 0 & 0 & 0 & 0 & 0 \\
\hline 15 & $0^{*}$ & 0 & 0 & 0 & 0 & 0 & 0 & .01 & 0 & 0 & 0 & 0 \\
\hline 16 & $0^{*}$ & 0 & 0 & 0 & 0 & 0 & 0 & 0 & 0 & 0 & 0 & 0 \\
\hline 17 & $0^{*}$ & 0 & 0 & 0 & 0 & 0 & 0 & 0 & 0 & 0 & 0 & 0 \\
\hline 18 & $0^{*}$ & 0 & 0 & 0 & 0 & 0 & 0 & 0 & 0 & 0 & 0 & 0 \\
\hline 19 & $0^{*}$ & 0 & 0 & 0 & 0 & 0 & 0 & 0 & 0 & 0 & 0 & 0 \\
\hline 20 & $0^{*}$ & 0 & 0 & 0 & 0 & 0 & 0 & 0 & 0 & 0 & 0 & 0 \\
\hline 21 & $0^{*}$ & 0 & 0 & 0 & 0 & 0 & 0 & 0 & 0 & 0 & 0 & 0 \\
\hline 22 & $0^{*}$ & 0 & 0 & 0 & 0 & 0 & 0 & 0 & 0 & 0 & 0 & 0 \\
\hline 23 & $0^{*}$ & 0 & 0 & 0 & 0 & 0 & 0 & 0 & 0 & 0 & 0 & 0 \\
\hline 24 & $0^{*}$ & 0 & 0 & 0 & 0 & 0 & 0 & 0 & 0 & 0 & 0 & 0 \\
\hline 25 & $0^{*}$ & 0 & 0 & 0 & 0 & 0 & 0 & 0 & 0 & 0 & 0 & 0 \\
\hline 26 & $0^{*}$ & 0 & 0 & 0 & 0 & 0 & 0 & 0 & 0 & 0 & 0 & 0 \\
\hline 27 & $0^{*}$ & 0 & 0 & 0 & 0 & 0 & 0 & 0 & 0 & 0 & 0 & 0 \\
\hline 28 & $0^{*}$ & 0 & 0 & $8.6^{\star}$ & 0 & 0 & 0 & 0 & 0 & 0 & 0 & 0 \\
\hline 29 & $0^{*}$ & 0 & 0 & 0 & 0 & 0 & 0 & 0 & 0 & 0 & 0 & 0 \\
\hline 30 & 0 & $0^{*}$ & 0 & 0 & ------ & 0 & 0 & 0 & 0 & 0 & 0 & 0 \\
\hline 31 & 0 & ----- & 0 & 0 & ------ & 0 & ------ & 0 & ------ & 0 & 0 & ------ \\
\hline Total & 0 & 0 & 0 & 8.6 & 0 & 0 & 0 & 0.01 & 0 & 0 & 0 & 0 \\
\hline Mean & 0 & 0 & 0 & .28 & 0 & 0 & 0 & 0 & 0 & 0 & 0 & 0 \\
\hline Max & 0 & 0 & 0 & 8.6 & 0 & 0 & 0 & .01 & 0 & 0 & 0 & 0 \\
\hline Min & 0 & 0 & 0 & 0 & 0 & 0 & 0 & 0 & 0 & 0 & 0 & 0 \\
\hline Acre-Ft & 0 & 0 & 0 & 17 & 0 & 0 & 0 & .02 & 0 & 0 & 0 & 0 \\
\hline Wtr Year & 2008 & Total & 8.61 & Mean & & 24 & hax & 8.6 & Min & 0 & Acre-Ft & 17 \\
\hline Cal Year & 2007 & Total & 7.04 & Mean & & 19 & hax & 4.2 & Min & 0 & Acre-Ft & 14 \\
\hline
\end{tabular}

*Estimate 


\section{E265 Water Canyon below SR 4}

Daily Total Rainfall in Inches

Water Year October 2007 to September 2008

\begin{tabular}{|c|c|c|c|c|c|c|c|c|c|c|c|c|}
\hline DAY & OCT & NOV & DEC & JAN & FEB & MAR & APR & MAY & JUN & JUL & AUG & SEP \\
\hline 1 & & & & & & & & 0 & 0 & .52 & 0 & .15 \\
\hline 2 & & & & & & & & 0 & 0 & .01 & 0 & 0 \\
\hline 3 & & & & & & & & 0 & 0 & .01 & 0 & 0 \\
\hline 4 & & & & & & & & 0 & 0 & .01 & .77 & 0 \\
\hline 5 & & & & & & & & 0 & .02 & .03 & $0^{*}$ & 0 \\
\hline 6 & & & & & & & & 0 & 0 & .06 & $.07^{\star}$ & 0 \\
\hline 7 & & & & & & & & 0 & 0 & .35 & $.14^{\star}$ & 0 \\
\hline 8 & & & & & & & & 0 & 0 & .15 & $.01^{\star}$ & 0 \\
\hline 9 & & & & & & & & 0 & 0 & .01 & 0 & 0 \\
\hline 10 & & & & & & & .01 & 0 & 0 & 0 & .28 & 0 \\
\hline 11 & & & & & & & 0 & 0 & 0 & .03 & 0 & 0 \\
\hline 12 & & & & & & & .13 & 0 & 0 & 0 & 0 & .05 \\
\hline 13 & & & & & & & 0 & .02 & 0 & 0 & 0 & 0 \\
\hline 14 & & & & & & & 0 & .15 & 0 & 0 & .16 & 0 \\
\hline 15 & & & & & & & 0 & .45 & 0 & .40 & .16 & .01 \\
\hline 16 & & & & & & & 0 & 0 & 0 & .06 & .13 & 0 \\
\hline 17 & & & & & & & .08 & 0 & 0 & .11 & .07 & 0 \\
\hline 18 & & & & & & & 0 & 0 & 0 & .01 & 0 & .02 \\
\hline 19 & & & & & & & 0 & 0 & 0 & 0 & .01 & 0 \\
\hline 20 & & & & & & & 0 & 0 & .01 & .17 & .02 & .10 \\
\hline 21 & & & & & & & 0 & 0 & 0 & .15 & 0 & .01 \\
\hline 22 & & & & & & & 0 & .08 & 0 & .02 & 0 & .04 \\
\hline 23 & & & & & & & 0 & .12 & 0 & 0 & .24 & 0 \\
\hline 24 & & & & & & & 0 & .01 & 0 & 0 & .03 & 0 \\
\hline 25 & & & & & & & 0 & 0 & 0 & 0 & 0 & 0 \\
\hline 26 & & & & & & & 0 & 0 & 0 & .18 & .06 & .37 \\
\hline 27 & & & & & & & 0 & 0 & 0 & .16 & 0 & 0 \\
\hline 28 & & & & & & & 0 & .14 & 0 & 0 & 0 & 0 \\
\hline 29 & & & & & & & 0 & 0 & 0 & 0 & .17 & 0 \\
\hline 30 & & & & & ------ & & 0 & 0 & 0 & 0 & 0 & 0 \\
\hline 31 & & ------ & & & ------ & & ------ & 0 & ----- & 0 & .40 & ------ \\
\hline Total & & & & & & & 0.22 & 0.97 & 0.03 & 2.44 & 2.50 & 0.75 \\
\hline $\operatorname{Max}$ & & & & & & & .13 & .45 & .02 & .52 & .77 & .37 \\
\hline Wtr Year & 2008 & Total & 6.91 & Mean & & .041 & Max & . 77 & Min & 0 & InstMax & .18 \\
\hline Cal Year & 2007 & Total & 6.64 & Mean & & .048 & $\operatorname{Max}$ & .74 & Min & 0 & InstMax & .35 \\
\hline
\end{tabular}

*Estimate 


\section{E267 Potrillo Canyon above SR 4}

Location. Lat $35^{\circ} 48^{\prime}$ 48", long $106^{\circ}$ 14' 00", Sec. 6, T. 18 N., R. 7 E., Ramon Vigil Grant, Los Alamos County.

Drainage Area. $2.26 \mathrm{mi}^{2}$.

Period of Record. October 1, 1995, to September 30, 2008.

Revised Record. LA-13551-PR (1998): station number; drainage area (2006).

Gage. Data logger with cellular telemetry and concrete control. Elevation of gage is 6,454 ft above NGVD from GPS survey.

Remarks. Records are good. Legal location based on projected values.

Average Discharge. $14 \mathrm{yr}, 0.003 \mathrm{ft}^{3} / \mathrm{s}, 2.17$ acre- $\mathrm{ft} / \mathrm{yr}$.

Extremes for Period of Record. Maximum discharge, $63 \mathrm{ft}^{3} / \mathrm{s}$, August 29, 1995, gage height $2.70 \mathrm{ft}$ (from slope-area determination). No flow most of the time.

Extremes for Current Year. Maximum discharge, $2.0 \mathrm{ft}^{3} / \mathrm{s} 1410 \mathrm{~h}$, July 17, gage height $0.98 \mathrm{ft}$. No peak discharge above base of $5.0 \mathrm{ft}^{3} / \mathrm{s}$. No flow most of the time.

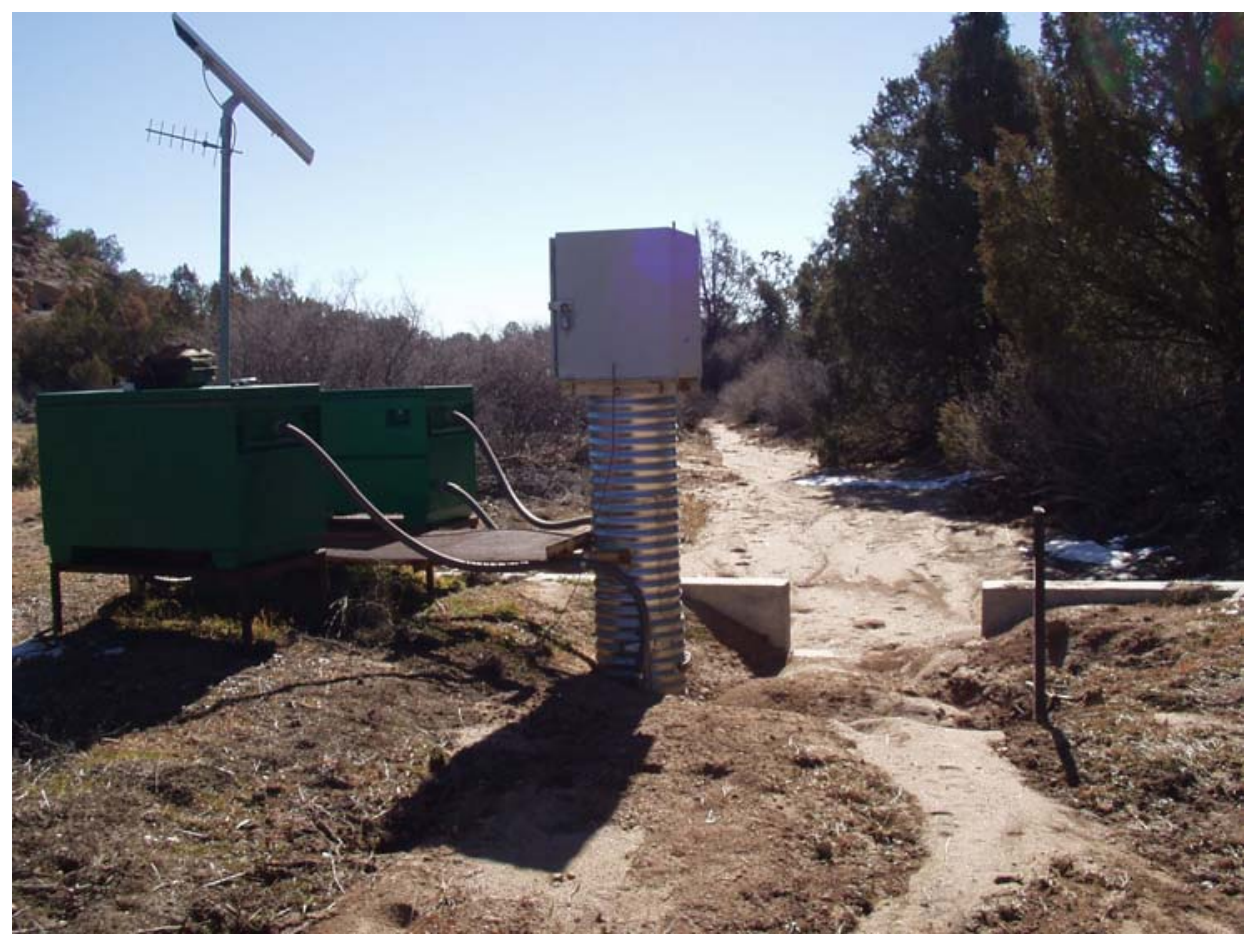




\section{E267 Potrillo Canyon above SR 4}

\section{Station Analysis}

\section{Water Year}

Equipment. Station is equipped with Sutron 8210 (5-min. interval) with shaft encoder float system (5-min. interval) and cellular phone with speech modem. The system is powered by a solar-panel battery system housed in NEMA shelter on 18" CMP well. Station is equipped with an ISCO pump sampler for water-quality sample collection. ISCO is housed in a separate shelter, a $3^{\prime} \times 4^{\prime}$ metal box. Sampler is triggered by stage through the data logger. An outside staff is available for reference. No provision for direct discharge measurements above wading stages.

Field Work. The station was visited 19 times to conduct discharge measurements and service the instrumentation. Field inspections for the gage are listed under site history files on the Hydstra database. Discharge measurements for the gage are listed under site gauging files on the Hydstra database.

Datum Correction. None.

Gage-Height Record. The data logger referenced to the outside staff gave a complete and satisfactory record for the year.

Rating. The channel is fairly straight for $300^{\prime}$ above gage and 150 ' below. Streambed consists of mostly sand. Brush is fairly thick along stream bank. The control is a concrete broad-crested weir.

One discharge measurement (No. 4) and 19 visits of no flow were made this year.

Rating No. 1 was developed based on measurements Nos. 1-4, PZF, and theoretical computation using broad-crested weir. Rating No. 1 is considered good.

Original shape and definition of rating was by computation using weir geometry with slope area used to define peak discharge and slope of upper end. Measurements 1 and 2 were made on recession and checked with rating rather well. Measurements did shift some to the right, attributed to changing stage and sand movement from high flow. Shifts were applied by stage using discharge measurements from previous years.

Discharge. Discharge was computed by applying gage height to Rating No. 1 through shift adjustment based on one shift diagram.

Remarks. Records are good. 


\section{E267 Potrillo Canyon above SR 4}

Daily Mean Discharge in Cubic Feet per Second

Water Year October 2007 to September 2008

\begin{tabular}{|c|c|c|c|c|c|c|c|c|c|c|c|c|}
\hline DAY & OCT & NOV & DEC & JAN & FEB & MAR & APR & MAY & JUN & JUL & AUG & SEP \\
\hline 1 & 0 & 0 & 0 & 0 & 0 & 0 & 0 & 0 & 0 & 0 & 0 & 0 \\
\hline 2 & 0 & 0 & 0 & 0 & 0 & 0 & 0 & 0 & 0 & 0 & 0 & 0 \\
\hline 3 & 0 & 0 & 0 & 0 & 0 & 0 & 0 & 0 & 0 & 0 & 0 & 0 \\
\hline 4 & 0 & 0 & 0 & 0 & 0 & 0 & 0 & 0 & 0 & 0 & 0 & 0 \\
\hline 5 & 0 & 0 & 0 & 0 & 0 & 0 & 0 & 0 & 0 & 0 & 0 & 0 \\
\hline 6 & 0 & 0 & 0 & 0 & 0 & 0 & 0 & 0 & 0 & 0 & 0 & 0 \\
\hline 7 & 0 & 0 & 0 & 0 & 0 & 0 & 0 & 0 & 0 & 0 & 0 & 0 \\
\hline 8 & 0 & 0 & 0 & 0 & 0 & 0 & 0 & 0 & 0 & 0 & 0 & 0 \\
\hline 9 & 0 & 0 & 0 & 0 & 0 & 0 & 0 & 0 & 0 & 0 & 0 & 0 \\
\hline 10 & 0 & 0 & 0 & 0 & 0 & 0 & 0 & 0 & 0 & 0 & 0 & 0 \\
\hline 11 & 0 & 0 & 0 & 0 & 0 & 0 & 0 & 0 & 0 & 0 & 0 & 0 \\
\hline 12 & 0 & 0 & 0 & 0 & 0 & 0 & 0 & 0 & 0 & 0 & 0 & 0 \\
\hline 13 & 0 & 0 & 0 & 0 & 0 & 0 & 0 & 0 & 0 & 0 & 0 & 0 \\
\hline 14 & 0 & 0 & 0 & 0 & 0 & 0 & 0 & 0 & 0 & 0 & 0 & 0 \\
\hline 15 & 0 & 0 & 0 & 0 & 0 & 0 & 0 & 0 & 0 & 0 & 0 & 0 \\
\hline 16 & 0 & 0 & 0 & 0 & 0 & 0 & 0 & 0 & 0 & 0 & 0 & 0 \\
\hline 17 & 0 & 0 & 0 & 0 & 0 & 0 & 0 & 0 & 0 & .03 & 0 & 0 \\
\hline 18 & 0 & 0 & 0 & 0 & 0 & 0 & 0 & 0 & 0 & 0 & 0 & 0 \\
\hline 19 & 0 & 0 & 0 & 0 & 0 & 0 & 0 & 0 & 0 & 0 & 0 & 0 \\
\hline 20 & 0 & 0 & 0 & 0 & 0 & 0 & 0 & 0 & 0 & 0 & 0 & 0 \\
\hline 21 & 0 & 0 & 0 & 0 & 0 & 0 & 0 & 0 & 0 & 0 & 0 & 0 \\
\hline 22 & 0 & 0 & 0 & 0 & 0 & 0 & 0 & 0 & 0 & 0 & 0 & 0 \\
\hline 23 & 0 & 0 & 0 & 0 & 0 & 0 & 0 & 0 & 0 & 0 & 0 & 0 \\
\hline 24 & 0 & 0 & 0 & 0 & 0 & 0 & 0 & 0 & 0 & 0 & 0 & 0 \\
\hline 25 & 0 & 0 & 0 & 0 & 0 & 0 & 0 & 0 & 0 & 0 & 0 & 0 \\
\hline 26 & 0 & 0 & 0 & 0 & 0 & 0 & 0 & 0 & 0 & 0 & 0 & 0 \\
\hline 27 & 0 & 0 & 0 & 0 & 0 & 0 & 0 & 0 & 0 & 0 & 0 & 0 \\
\hline 28 & 0 & 0 & 0 & .04 & 0 & 0 & 0 & 0 & 0 & 0 & 0 & 0 \\
\hline 29 & 0 & 0 & 0 & 0 & 0 & 0 & 0 & 0 & 0 & 0 & 0 & 0 \\
\hline 30 & 0 & 0 & 0 & 0 & ------ & 0 & 0 & 0 & 0 & 0 & 0 & 0 \\
\hline 31 & 0 & ----- & 0 & 0 & ------ & 0 & ----- & 0 & ----- & 0 & 0 & ----- \\
\hline Total & 0 & 0 & 0 & 0.04 & 0 & 0 & 0 & 0 & 0 & 0.03 & 0 & 0 \\
\hline Mean & 0 & 0 & 0 & .001 & 0 & 0 & 0 & 0 & 0 & .001 & 0 & 0 \\
\hline Max & 0 & 0 & 0 & .04 & 0 & 0 & 0 & 0 & 0 & .03 & 0 & 0 \\
\hline Min & 0 & 0 & 0 & 0 & 0 & 0 & 0 & 0 & 0 & 0 & 0 & 0 \\
\hline Acre-Ft & 0 & 0 & 0 & .08 & 0 & 0 & 0 & 0 & 0 & .06 & 0 & 0 \\
\hline Wtr Year & 2008 & Total & 0.07 & Mean & & 0 & hax & .04 & Min & 0 & Acre-Ft & 0.14 \\
\hline Cal Year & 2007 & Total & 0.05 & Mean & & 0 & Иax & .05 & Min & 0 & Acre-Ft & 0.10 \\
\hline
\end{tabular}


Location. Lat $35^{\circ}$ 49' 38", long $106^{\circ}$ 16' 36", Sec. 35, T. 19 N., R. 6 E., Ramon Vigil Grant, Santa Fe National Forest.

Drainage Area. $0.061 \mathrm{mi}^{2}$.

Period of Record. October 1, 2006, to September 30, 2008.

Gage. Data logger and 9" Parshall flume, as well as rain gage with cellular telemetry. Elevation of gage is $6,858 \mathrm{ft}$ above NGVD.

Remarks. Records are good. Records for this site existed before published period but are not reliable. Legal location based on projected values.

Extremes for Period of Record. Maximum discharge, $0.99 \mathrm{ft}^{3} / \mathrm{s}$, January 28, 2008, gage height $0.45 \mathrm{ft}$. No flow most of the time.

Extremes for Current Year. Maximum discharge, $0.99 \mathrm{ft}^{3} / \mathrm{s}$ at $0415 \mathrm{~h}$, January 28, gage height $0.45 \mathrm{ft}$. No peak above base of $1.0 \mathrm{ft}^{3} / \mathrm{s}$. No flow most of the time.

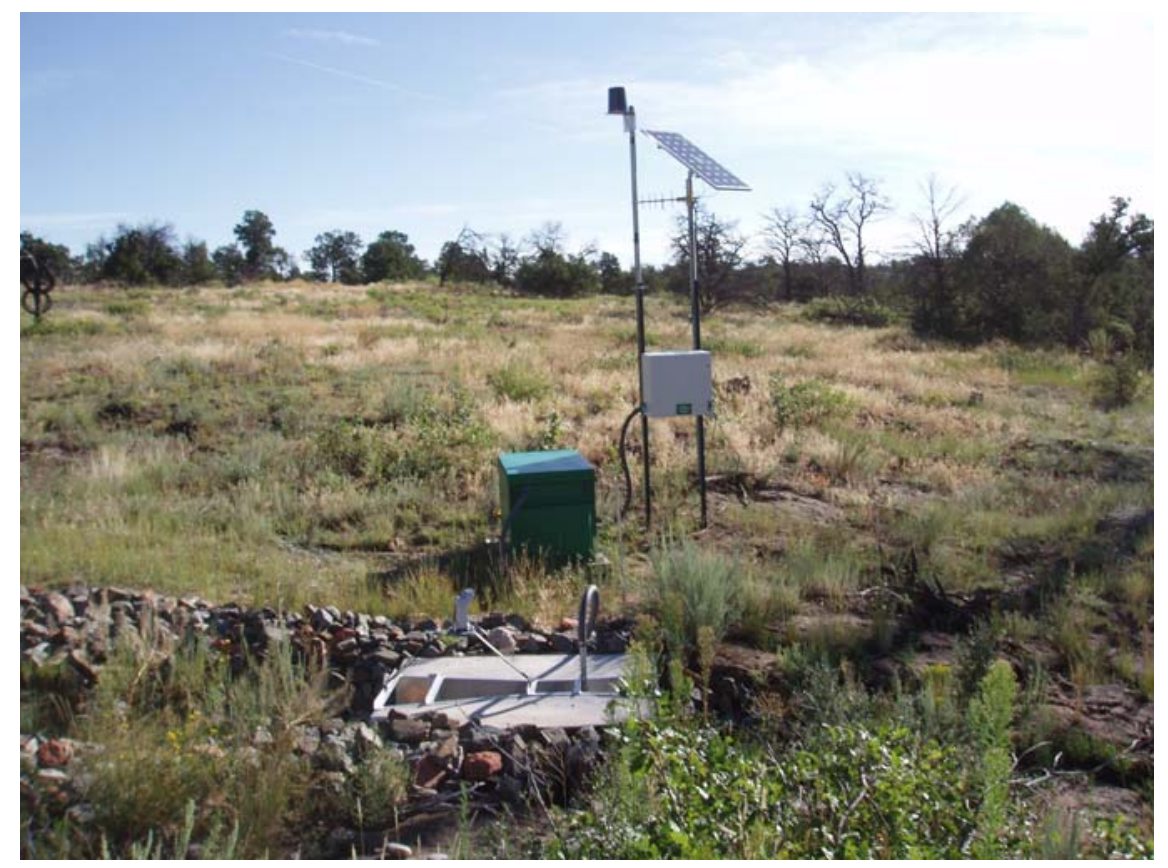




\section{E2674 TA-36 Minie Site}

\section{Station Analysis}

\section{Water Year}

Equipment. Station is equipped with Sutron 8210 data logger (5-min. interval) and milltronics sonic probe mounted on a 9" Parshall flume and cellular phone with speech modem. Phone upgraded June 16 from analog to digital service. The system is powered by a solar-panel battery system housed in a NEMA shelter. Station is equipped with an ISCO pump sampler for water-quality sample collection. ISCO is housed in a separate shelter, a $3^{\prime} \times 4^{\prime}$ metal box. Sampler is triggered by stage through the data logger. The staff in the 9" Parshall flume is the reference gage. No provision for discharge measurements above wading stage.

Station is also equipped with a tipping bucket rain gage, Rain Collection II. All equipment is powered with a solar-panel battery-charging system. Rain data are seasonal.

Field Work. This station was visited 19 times to conduct discharge measurements and service the instrumentation. Field inspections for the gage are listed under site history files on the Hydstra database. Discharge measurements for the gage are listed under site gauging files on the Hydstra database.

Datum Correction. None.

Gage-Height Record. The data logger referenced to the outside staff gave a complete and satisfactory record except for the periods of November 14 to January 22, April 18 to May 19, and August 5-15 when the data logger lost power.

Rating. The channel is straight above and below the gage for 100'. The channel near the gage is lined with angular rock. The streambed consists of mostly sand.

Nineteen inspections of no flow were made this year.

Rating No. 1 was developed based on the computation of 9" Parshall flume. Point of zero flow is 0.00 gage height.

Discharge. Discharge was computed by applying gage height to Rating No. 1 directly. Those days estimated at zero flow were based on precipitation and nearby gage stations for verification.

Remarks. Records are good. 
E2674 TA-36 Minie Site

Daily Mean Discharge in Cubic Feet per Second

Water Year October 2007 to September 2008

\begin{tabular}{|c|c|c|c|c|c|c|c|c|c|c|c|c|}
\hline DAY & OCT & NOV & DEC & JAN & FEB & MAR & APR & MAY & JUN & JUL & AUG & SEP \\
\hline 1 & 0 & 0 & $0^{*}$ & $0^{*}$ & 0 & 0 & 0 & $0^{*}$ & 0 & 0 & 0 & 0 \\
\hline 2 & 0 & 0 & $0^{*}$ & $0^{*}$ & 0 & 0 & 0 & $0^{*}$ & 0 & 0 & 0 & 0 \\
\hline 3 & 0 & 0 & $0^{*}$ & $0^{*}$ & 0 & 0 & 0 & $0^{*}$ & 0 & 0 & 0 & 0 \\
\hline 4 & 0 & 0 & $0^{*}$ & $0^{*}$ & 0 & 0 & 0 & $0^{*}$ & 0 & 0 & .01 & 0 \\
\hline 5 & 0 & 0 & $0^{*}$ & $0^{*}$ & 0 & 0 & 0 & $0^{*}$ & 0 & 0 & $0^{*}$ & 0 \\
\hline 6 & 0 & 0 & $0^{*}$ & $0^{*}$ & 0 & 0 & 0 & $0^{*}$ & 0 & 0 & $0^{*}$ & 0 \\
\hline 7 & 0 & 0 & $0^{*}$ & $0^{*}$ & 0 & 0 & 0 & $0^{*}$ & 0 & 0 & $0^{*}$ & 0 \\
\hline 8 & 0 & 0 & $0^{*}$ & $0^{*}$ & 0 & 0 & 0 & $0^{*}$ & 0 & 0 & $0^{*}$ & 0 \\
\hline 9 & 0 & 0 & $0^{*}$ & $0^{*}$ & 0 & 0 & 0 & $0^{*}$ & 0 & 0 & $0^{*}$ & 0 \\
\hline 10 & 0 & 0 & $0^{*}$ & $0^{*}$ & 0 & 0 & 0 & $0^{*}$ & 0 & 0 & $0^{*}$ & 0 \\
\hline 11 & 0 & 0 & $0^{*}$ & $0^{*}$ & 0 & 0 & 0 & $0^{*}$ & 0 & 0 & $0^{*}$ & 0 \\
\hline 12 & 0 & 0 & $0^{*}$ & $0^{*}$ & 0 & 0 & 0 & $0^{*}$ & 0 & 0 & $0^{*}$ & 0 \\
\hline 13 & 0 & 0 & $0^{*}$ & $0^{*}$ & 0 & 0 & 0 & $0^{*}$ & 0 & 0 & $0^{*}$ & 0 \\
\hline 14 & 0 & $0^{*}$ & $0^{*}$ & $0^{*}$ & 0 & 0 & 0 & $0^{*}$ & 0 & 0 & $0^{*}$ & 0 \\
\hline 15 & 0 & $0^{*}$ & $0^{*}$ & $0^{*}$ & 0 & 0 & 0 & $0^{*}$ & 0 & 0 & $0^{*}$ & 0 \\
\hline 16 & 0 & $0^{*}$ & $0^{*}$ & $0^{*}$ & 0 & 0 & 0 & $0^{*}$ & 0 & 0 & 0 & 0 \\
\hline 17 & 0 & $0^{*}$ & $0^{*}$ & $0^{*}$ & 0 & 0 & 0 & $0^{*}$ & 0 & 0 & 0 & 0 \\
\hline 18 & 0 & $0^{*}$ & $0^{*}$ & $0^{*}$ & 0 & 0 & $0^{*}$ & $0^{*}$ & 0 & 0 & 0 & 0 \\
\hline 19 & 0 & $0^{*}$ & $0^{*}$ & $0^{*}$ & 0 & 0 & $0^{*}$ & $0^{*}$ & 0 & 0 & 0 & 0 \\
\hline 20 & 0 & $0^{*}$ & $0^{*}$ & $0^{*}$ & 0 & 0 & $0^{*}$ & 0 & 0 & 0 & 0 & 0 \\
\hline 21 & 0 & $0^{*}$ & $0^{*}$ & $0^{*}$ & 0 & 0 & $0^{*}$ & 0 & 0 & 0 & 0 & 0 \\
\hline 22 & 0 & $0^{*}$ & $0^{*}$ & $0^{*}$ & 0 & 0 & $0^{*}$ & 0 & 0 & 0 & 0 & 0 \\
\hline 23 & 0 & $0^{*}$ & $0^{*}$ & 0 & 0 & 0 & $0^{*}$ & 0 & 0 & 0 & .01 & 0 \\
\hline 24 & 0 & $0^{*}$ & $0^{*}$ & 0 & 0 & 0 & $0^{*}$ & 0 & 0 & 0 & 0 & 0 \\
\hline 25 & 0 & $0^{*}$ & $0^{*}$ & 0 & 0 & 0 & $0^{*}$ & 0 & 0 & 0 & 0 & 0 \\
\hline 26 & 0 & $0^{*}$ & $0^{*}$ & 0 & 0 & 0 & $0^{*}$ & 0 & 0 & 0 & 0 & 0 \\
\hline 27 & 0 & $0^{*}$ & $0^{*}$ & 0 & 0 & 0 & $0^{*}$ & 0 & 0 & 0 & 0 & 0 \\
\hline 28 & 0 & $0^{*}$ & $0^{*}$ & .05 & 0 & 0 & $0^{*}$ & 0 & 0 & 0 & 0 & 0 \\
\hline 29 & 0 & $0^{*}$ & $0^{*}$ & 0 & 0 & 0 & $0^{*}$ & 0 & 0 & 0 & 0 & 0 \\
\hline 30 & 0 & $0^{*}$ & $0^{*}$ & 0 & ------ & 0 & $0^{*}$ & 0 & 0 & 0 & 0 & 0 \\
\hline 31 & 0 & ------ & $0^{*}$ & 0 & ------ & 0 & ------ & 0 & ----- & 0 & 0 & ------ \\
\hline Total & 0 & 0 & 0 & 0.05 & 0 & 0 & 0 & 0 & 0 & 0 & 0.02 & 0 \\
\hline Mean & 0 & 0 & 0 & .002 & 0 & 0 & 0 & 0 & 0 & 0 & .001 & 0 \\
\hline Max & 0 & 0 & 0 & .05 & 0 & 0 & 0 & 0 & 0 & 0 & .01 & 0 \\
\hline Min & 0 & 0 & 0 & 0 & 0 & 0 & 0 & 0 & 0 & 0 & 0 & 0 \\
\hline Acre-Ft & 0 & 0 & 0 & .10 & 0 & 0 & 0 & 0 & 0 & 0 & .04 & 0 \\
\hline Wtr Year & 2008 & Total & 0.07 & Mean & & 0 & $\operatorname{lax}$ & .05 & Min & 0 & Acre-Ft & 0.14 \\
\hline Cal Year & 2007 & Total & 0.05 & Mean & & 0 & $\operatorname{lax}$ & .02 & Min & 0 & Acre-Ft & 0.10 \\
\hline
\end{tabular}

*Estimate 
E2674 TA-36 Minie Site

Daily Total Rainfall in Inches

Water Year October 2007 to September 2008

\begin{tabular}{|c|c|c|c|c|c|c|c|c|c|c|c|c|}
\hline DAY & ОСТ & NOV & DEC & JAN & FEB & MAR & APR & MAY & JUN & JUL & AUG & SEP \\
\hline 1 & & & & & & & & $0^{*}$ & 0 & .25 & 0 & .05 \\
\hline 2 & & & & & & & & $0^{*}$ & 0 & 0 & 0 & 0 \\
\hline 3 & & & & & & & & $0^{*}$ & 0 & 0 & 0 & .01 \\
\hline 4 & & & & & & & & $0^{*}$ & 0 & .03 & $1.59^{\star}$ & .01 \\
\hline 5 & & & & & & & & $0^{*}$ & 0 & .10 & $0^{*}$ & 0 \\
\hline 6 & & & & & & & & $0^{*}$ & 0 & 0 & $.08^{\star}$ & 0 \\
\hline 7 & & & & & & & & $0^{*}$ & 0 & .14 & $.10^{\star}$ & 0 \\
\hline 8 & & & & & & & & $0^{*}$ & 0 & .05 & $.15^{\star}$ & 0 \\
\hline 9 & & & & & & & & $0^{*}$ & 0 & 0 & $.01^{*}$ & .01 \\
\hline 10 & & & & & & & & $0^{*}$ & 0 & 0 & $.49^{\star}$ & .01 \\
\hline 11 & & & & & & & & $0^{*}$ & 0 & .01 & $0^{*}$ & 0 \\
\hline 12 & & & & & & & & $0^{*}$ & 0 & 0 & $0^{\star}$ & .27 \\
\hline 13 & & & & & & & & $0^{*}$ & 0 & 0 & $0^{*}$ & 0 \\
\hline 14 & & & & & & & & $.15^{\star}$ & 0 & 0 & $.10^{\star}$ & 0 \\
\hline 15 & & & & & & & & $.55^{\star}$ & 0 & .25 & $.04^{*}$ & 0 \\
\hline 16 & & & & & & & & $0^{*}$ & 0 & .08 & .20 & 0 \\
\hline 17 & & & & & & & & $0^{*}$ & 0 & .12 & 0 & 0 \\
\hline 18 & & & & & & & & $0^{*}$ & 0 & 0 & .01 & 0 \\
\hline 19 & & & & & & & & $0^{*}$ & 0 & 0 & 0 & 0 \\
\hline 20 & & & & & & & & 0 & 0 & 0 & 0 & .30 \\
\hline 21 & & & & & & & & .01 & 0 & .41 & .01 & 0 \\
\hline 22 & & & & & & & & .01 & 0 & 0 & 0 & .01 \\
\hline 23 & & & & & & & & .07 & 0 & .01 & .82 & .01 \\
\hline 24 & & & & & & & & 0 & 0 & 0 & .34 & 0 \\
\hline 25 & & & & & & & & 0 & 0 & 0 & .01 & 0 \\
\hline 26 & & & & & & & & 0 & 0 & .07 & .02 & .26 \\
\hline 27 & & & & & & & & 0 & 0 & .07 & .01 & 0 \\
\hline 28 & & & & & & & & .05 & 0 & .03 & 0 & 0 \\
\hline 29 & & & & & & & & 0 & 0 & 0 & 0 & 0 \\
\hline 30 & & & & & ------ & & & 0 & 0 & 0 & 0 & 0 \\
\hline 31 & & ------ & & & ------ & & ------ & 0 & ----- & 0 & .72 & ------ \\
\hline Total & & & & & & & & 0.14 & 0 & 1.62 & 3.74 & 0.94 \\
\hline $\operatorname{Max}$ & & & & & & & & .07 & 0 & .41 & 1.6 & .30 \\
\hline Wtr Year & 2008 & Total & 6.44 & Mean & & .052 & Max & 1.6 & Min & 0 & InstMax & .24 \\
\hline Cal Year & 2007 & Total & 3.77 & Mean & & .048 & Max & .68 & Min & 0 & InstMax & .40 \\
\hline
\end{tabular}

*Estimate 


\section{E274 North Fork Ancho Canyon below SR 4}

Location. Lat $35^{\circ}$ 47' 12", long $106^{\circ}$ 15' 4.7", Sec. 13, T. 18 N., R. 6 E., Ramon Vigil Grant, Los Alamos County.

Drainage Area. $1.53 \mathrm{mi}^{2}$.

Period of Record. October 1, 2007, to September 30, 2008.

Gage. Data logger. Elevation of gage is 6,242 ft above NGVD from GPS survey.

Remarks. Records are good. Records for this site existed before period of record but are not reliable.

Extremes for Period of Record. Maximum discharge, $89 \mathrm{ft}^{3} / \mathrm{s}$, gage height $2.79 \mathrm{ft}$, August 4, 2008. No flow most of the time.

Extremes for Current Year. Peak discharge above base of $5 \mathrm{ft}^{3} / \mathrm{s}$ and maximum (*):

\begin{tabular}{|c|c|c|c|}
\hline Date & Time & Discharge $\left.\mathbf{( f t}^{\mathbf{3}} \mathbf{s}\right)$ & Gage Height (ft) \\
\hline May 10 & 0335 & 7.4 & 1.38 \\
\hline August 4 & 1800 & $89^{*}$ & $2.79^{*}$ \\
\hline
\end{tabular}

No flow most of the time.

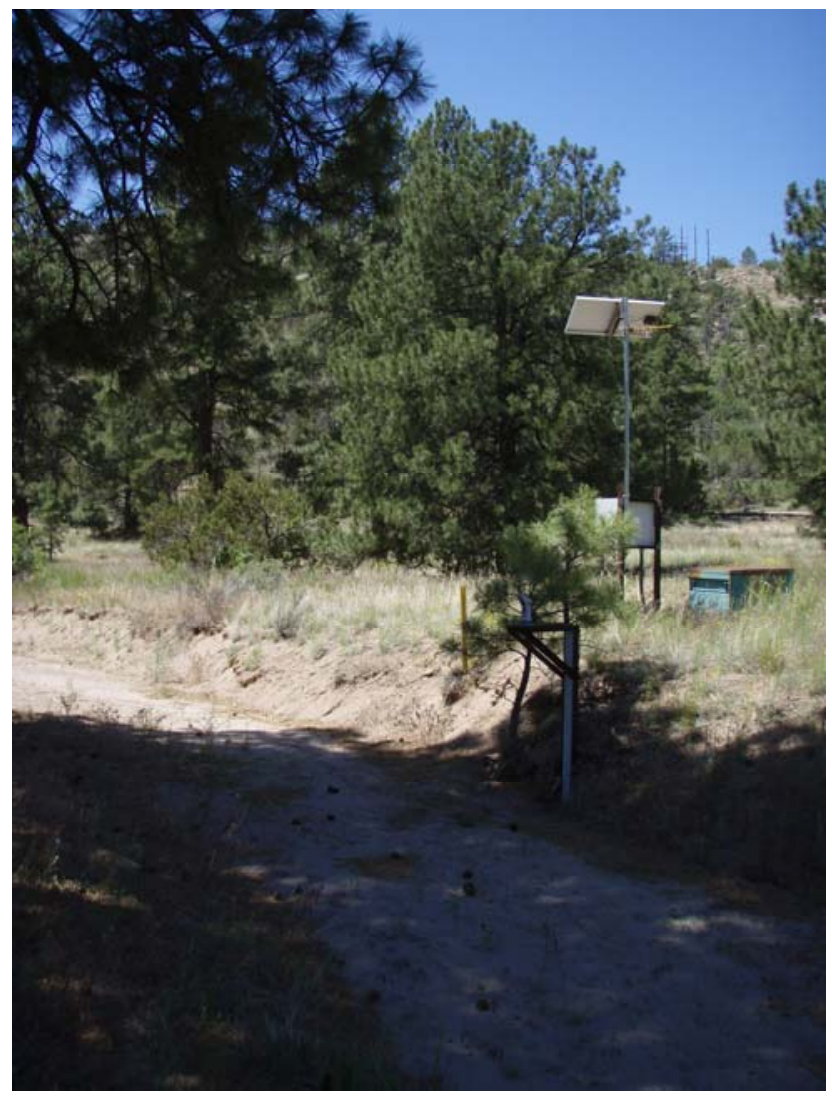




\section{E274 North Fork Ancho Canyon below SR 4}

\section{Station Analysis}

\section{Water Year}

Equipment. Station is equipped with Sutron 8210 data logger (5-min. interval) and milltronics sonic probe. The system is powered by a solar-panel battery system housed in a NEMA shelter. Station is equipped with an ISCO pump sampler for water-quality sample collection. ISCO is housed in a separate shelter, a $3^{\prime} \times 4^{\prime}$ metal box. Sampler is triggered by stage through the data logger. Outside staff is available for reference. No provision for measurements above wading stage.

Field Work. The station was visited 17 times to conduct discharge measurements and service the instrumentation. Field inspections for the gage are listed under site history files on the Hydstra database. Discharge measurements for the gage are listed under site gauging files on the Hydstra database.

Datum Corrections. None.

Gage-Height Record. The data logger referenced to the outside gage gave a complete and satisfactory record for the year.

Rating. The channel is straight above and below the gage. Streambed consists of mostly sand.

Seventeen inspections of no flow were made this year.

Discharge. Discharge was computed by applying gage height to Rating No. 1 directly.

Remarks. Records are good. 
E274 North Fork Ancho below SR 4

Daily Mean Discharge in Cubic Feet per Second

Water Year October 2007 to September 2008

\begin{tabular}{|c|c|c|c|c|c|c|c|c|c|c|c|c|}
\hline DAY & ОСт & NOV & DEC & JAN & FEB & MAR & APR & MAY & JUN & JUL & AUG & SEP \\
\hline 1 & 0 & 0 & 0 & 0 & 0 & 0 & 0 & 0 & 0 & 0 & 0 & 0 \\
\hline 2 & 0 & 0 & 0 & 0 & 0 & 0 & 0 & 0 & 0 & 0 & 0 & 0 \\
\hline 3 & 0 & 0 & 0 & 0 & 0 & 0 & 0 & 0 & 0 & 0 & 0 & 0 \\
\hline 4 & 0 & 0 & 0 & 0 & 0 & 0 & 0 & 0 & 0 & 0 & 1.3 & 0 \\
\hline 5 & 0 & 0 & 0 & 0 & 0 & 0 & 0 & 0 & 0 & 0 & 0 & 0 \\
\hline 6 & 0 & 0 & 0 & 0 & 0 & 0 & 0 & 0 & 0 & 0 & 0 & 0 \\
\hline 7 & 0 & 0 & 0 & 0 & 0 & 0 & 0 & 0 & 0 & 0 & 0 & 0 \\
\hline 8 & 0 & 0 & 0 & 0 & 0 & 0 & 0 & 0 & 0 & 0 & 0 & 0 \\
\hline 9 & 0 & 0 & 0 & 0 & 0 & 0 & 0 & 0 & 0 & 0 & 0 & 0 \\
\hline 10 & 0 & 0 & 0 & 0 & 0 & 0 & 0 & .04 & 0 & 0 & 0 & 0 \\
\hline 11 & 0 & 0 & 0 & 0 & 0 & 0 & 0 & 0 & 0 & 0 & 0 & 0 \\
\hline 12 & 0 & 0 & 0 & 0 & 0 & 0 & 0 & 0 & 0 & 0 & 0 & 0 \\
\hline 13 & 0 & 0 & 0 & 0 & 0 & 0 & 0 & 0 & 0 & 0 & 0 & 0 \\
\hline 14 & 0 & 0 & 0 & 0 & 0 & 0 & 0 & 0 & 0 & 0 & 0 & 0 \\
\hline 15 & 0 & 0 & 0 & 0 & 0 & 0 & 0 & 0 & 0 & 0 & 0 & 0 \\
\hline 16 & 0 & 0 & 0 & 0 & 0 & 0 & 0 & 0 & 0 & 0 & 0 & 0 \\
\hline 17 & 0 & 0 & 0 & 0 & 0 & 0 & 0 & 0 & 0 & 0 & 0 & 0 \\
\hline 18 & 0 & 0 & 0 & 0 & 0 & 0 & 0 & 0 & 0 & 0 & 0 & 0 \\
\hline 19 & 0 & 0 & 0 & 0 & 0 & 0 & 0 & 0 & 0 & 0 & 0 & 0 \\
\hline 20 & 0 & 0 & 0 & 0 & 0 & 0 & 0 & 0 & 0 & 0 & 0 & 0 \\
\hline 21 & 0 & 0 & 0 & 0 & 0 & 0 & 0 & 0 & 0 & 0 & 0 & 0 \\
\hline 22 & 0 & 0 & 0 & 0 & 0 & 0 & 0 & 0 & 0 & 0 & 0 & 0 \\
\hline 23 & 0 & 0 & 0 & 0 & 0 & 0 & 0 & 0 & 0 & 0 & 0 & 0 \\
\hline 24 & 0 & 0 & 0 & 0 & 0 & 0 & 0 & 0 & 0 & 0 & 0 & 0 \\
\hline 25 & 0 & 0 & 0 & 0 & 0 & 0 & 0 & 0 & 0 & 0 & 0 & 0 \\
\hline 26 & 0 & 0 & 0 & 0 & 0 & 0 & 0 & 0 & 0 & 0 & 0 & 0 \\
\hline 27 & 0 & 0 & 0 & 0 & 0 & 0 & 0 & 0 & 0 & 0 & 0 & 0 \\
\hline 28 & 0 & 0 & 0 & 0 & 0 & 0 & 0 & 0 & 0 & 0 & 0 & 0 \\
\hline 29 & 0 & 0 & 0 & 0 & 0 & 0 & 0 & 0 & 0 & 0 & 0 & 0 \\
\hline 30 & 0 & 0 & 0 & 0 & ------ & 0 & 0 & 0 & 0 & 0 & 0 & 0 \\
\hline 31 & 0 & ------ & 0 & 0 & ------ & 0 & ------ & 0 & ------ & 0 & 0 & ----- \\
\hline Total & 0 & 0 & 0 & 0 & 0 & 0 & 0 & 0.04 & 0 & 0 & 1.3 & 0 \\
\hline Mean & 0 & 0 & 0 & 0 & 0 & 0 & 0 & .001 & 0 & 0 & .042 & 0 \\
\hline Max & 0 & 0 & 0 & 0 & 0 & 0 & 0 & .04 & 0 & 0 & 1.3 & 0 \\
\hline Min & 0 & 0 & 0 & 0 & 0 & 0 & 0 & 0 & 0 & 0 & 0 & 0 \\
\hline Acre-Ft & 0 & 0 & 0 & 0 & 0 & 0 & 0 & .08 & 0 & 0 & 2.6 & 0 \\
\hline Wtr Year & 2008 & Total & 1.34 & Mean & & 04 & hax & 1.3 & Min & 0 & Acre-Ft & 2.7 \\
\hline Cal Year & 2007 & Total & 0 & Mean & & 0 & hax & 0 & Min & 0 & Acre-Ft & 0.0 \\
\hline
\end{tabular}




\section{E275 Ancho Canyon below SR 4}

Location. Lat $35^{\circ}$ 46' 54", long $106^{\circ}$ 14' 42", Sec. 19, T. 18 N., R. 7 E., Ramon Vigil

Grant, Los Alamos County.

Drainage Area. $4.75 \mathrm{mi}^{2}$.

Period of Record. December 1993 to September 30, 2008.

Revised Record. Drainage area (2006).

Gage. Data logger with cellular telemetry and concrete stabilized natural control. Elevation of gage is 6,190 ft above NGVD from GPS survey.

Remarks. Records are good. Legal location based on projected values.

Average Discharge. $13 \mathrm{yr}, 0.014 \mathrm{ft}^{3} / \mathrm{s}, 10.14$ acre-ft/yr.

Extremes for Period of Record. Maximum discharge, $536 \mathrm{ft}^{3} / \mathrm{s}$, gage height $2.89 \mathrm{ft}$, August 4, 2008 (from flood marks). No flow most of the time.

Extremes for Current Year. Peak discharge above base of $15 \mathrm{ft}^{3} / \mathrm{s}$ and maximum (*):

\begin{tabular}{|c|c|c|c|}
\hline Date & Time & Discharge $\left.\mathbf{( f t}^{\mathbf{3}} / \mathbf{s}\right)$ & Gage Height (ft) \\
\hline August 4 & 1815 & $536^{*}$ & $2.89^{*}$ \\
\hline August 23 & 1730 & 23 & 1.85 \\
\hline
\end{tabular}

No flow most of the time.

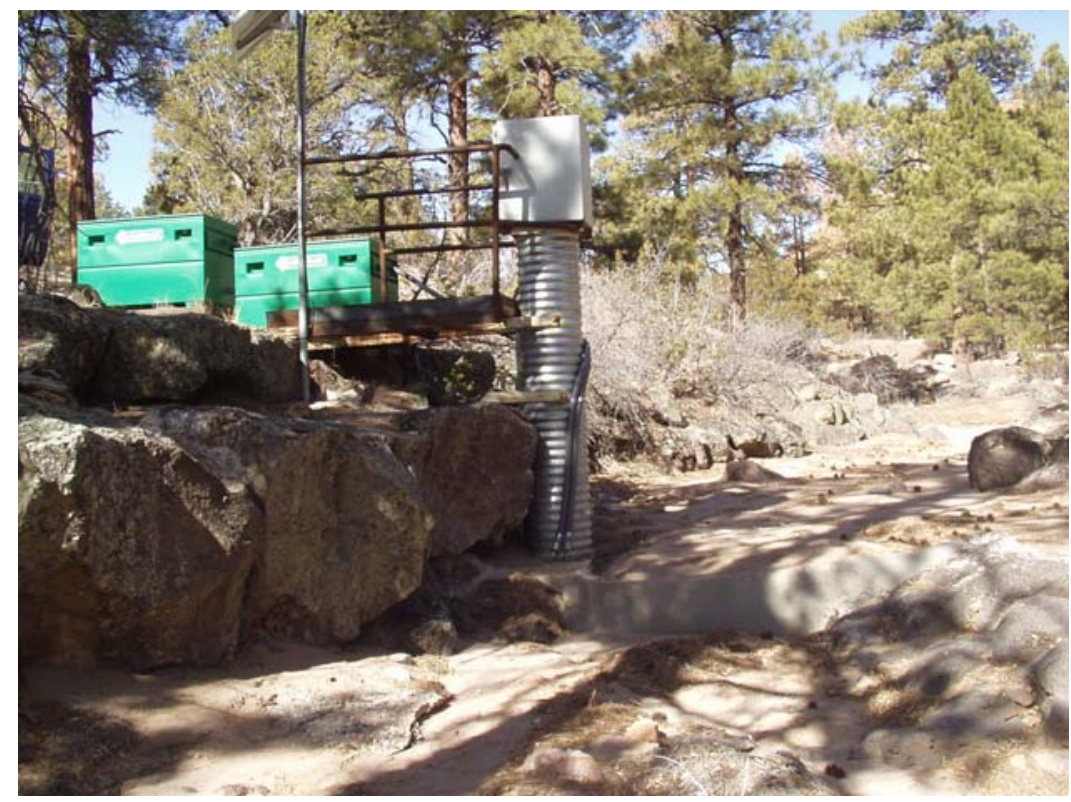




\section{E275 Ancho Canyon below SR 4}

\section{Station Analysis}

\section{Water Year}

Equipment. Station is equipped with Sutron 8210 data logger (5-min. interval) with shaft encoder float system (5-min. interval) and cellular telemetry with speech modem. Phone upgraded May 14 from analog to digital service. The system is powered by a solar-panel battery system housed in a NEMA shelter. Station is equipped with an ISCO pump sampler for water-quality sample collection. ISCO is housed in a separate shelter, a 3' X 4' metal box. Sampler is triggered by stage through the data logger. Outside staff is available for reference. No provision for measurements above wading stage.

Field Work. The station was visited 19 times to conduct discharge measurements and service the instrumentation. Field inspections for the gage are listed under site history files on the Hydstra database. Discharge measurements for the gage are listed under site gauging files on the Hydstra database.

Datum Corrections. None from levels.

Gage-Height Record. The data logger referenced to the outside gage gave a complete and satisfactory record for the year except for the period of January 18 to February 26 when ice affected gage height.

Rating. Streambed consists of a series of outcrops and sand pockets with moderate sand movement on flow events. High-water channel is straight for 200' upstream. Flow below gage goes into supercritical flow as fall increases radically below station. Onefourth mile upstream channel has very low banks and may spread out to large widths. It contracts markedly from there to the gage. The control consists of a natural rock outcrop stabilized by concrete.

One discharge measurement (No. 2) and 18 inspections of no flow were made this year.

Rating No. 1 was developed from PZF and previous measurement and slope-area measurement made on the event in 1996 Rating No. 1 was extended from $1.85 \mathrm{ft}$ to 2.75 ft from logarithmic plotting.

Discharge. Discharge computed by applying gage height to Rating No. 1 using variable diagram (-0.10 at lower end).

Remarks. Records are good. 
E275 Ancho Canyon below SR 4

Daily Mean Discharge in Cubic Feet per Second

Water Year October 2007 to September 2008

\begin{tabular}{|c|c|c|c|c|c|c|c|c|c|c|c|c|}
\hline DAY & OCT & NOV & DEC & JAN & FEB & MAR & APR & MAY & JUN & JUL & AUG & SEP \\
\hline 1 & 0 & 0 & $0^{*}$ & 0 & $0^{*}$ & 0 & 0 & 0 & 0 & 0 & 0 & 0 \\
\hline 2 & 0 & 0 & 0 & 0 & $0^{*}$ & 0 & 0 & 0 & 0 & 0 & 0 & 0 \\
\hline 3 & 0 & 0 & 0 & 0 & $0^{*}$ & 0 & 0 & 0 & 0 & 0 & 0 & 0 \\
\hline 4 & 0 & 0 & 0 & 0 & $0^{*}$ & 0 & 0 & 0 & 0 & 0 & 15 & 0 \\
\hline 5 & 0 & 0 & 0 & 0 & $0^{*}$ & 0 & 0 & 0 & 0 & 0 & 0 & 0 \\
\hline 6 & 0 & 0 & 0 & 0 & $0^{*}$ & 0 & 0 & 0 & 0 & 0 & 0 & 0 \\
\hline 7 & 0 & 0 & 0 & 0 & $0^{*}$ & 0 & 0 & 0 & 0 & 0 & 0 & 0 \\
\hline 8 & 0 & 0 & 0 & 0 & $0^{*}$ & 0 & 0 & 0 & 0 & 0 & 0 & 0 \\
\hline 9 & 0 & 0 & 0 & 0 & $0^{*}$ & 0 & 0 & 0 & 0 & 0 & 0 & 0 \\
\hline 10 & 0 & 0 & 0 & 0 & $0^{*}$ & 0 & 0 & 0 & 0 & 0 & 0 & 0 \\
\hline 11 & 0 & 0 & 0 & 0 & $0^{*}$ & 0 & 0 & 0 & 0 & 0 & 0 & 0 \\
\hline 12 & 0 & 0 & 0 & 0 & $0^{*}$ & 0 & 0 & 0 & 0 & 0 & 0 & 0 \\
\hline 13 & 0 & 0 & 0 & 0 & $0^{*}$ & 0 & 0 & 0 & 0 & 0 & 0 & 0 \\
\hline 14 & 0 & 0 & 0 & 0 & $0^{*}$ & 0 & 0 & 0 & 0 & 0 & 0 & 0 \\
\hline 15 & 0 & 0 & 0 & 0 & $0^{*}$ & 0 & 0 & 0 & 0 & 0 & 0 & 0 \\
\hline 16 & 0 & 0 & 0 & 0 & $0^{*}$ & 0 & 0 & 0 & 0 & 0 & 0 & 0 \\
\hline 17 & 0 & 0 & 0 & 0 & $0^{*}$ & 0 & 0 & 0 & 0 & 0 & 0 & 0 \\
\hline 18 & 0 & 0 & 0 & $0^{*}$ & $0^{*}$ & 0 & 0 & 0 & 0 & 0 & 0 & 0 \\
\hline 19 & 0 & 0 & 0 & $0^{*}$ & $0^{*}$ & 0 & 0 & 0 & 0 & 0 & 0 & 0 \\
\hline 20 & 0 & 0 & 0 & $0^{*}$ & $0^{*}$ & 0 & 0 & 0 & 0 & 0 & 0 & 0 \\
\hline 21 & 0 & 0 & 0 & $0^{*}$ & $0^{*}$ & 0 & 0 & 0 & 0 & 0 & 0 & 0 \\
\hline 22 & 0 & 0 & 0 & $0^{*}$ & $0^{*}$ & 0 & 0 & 0 & 0 & 0 & 0 & 0 \\
\hline 23 & 0 & 0 & 0 & $0^{*}$ & $0^{*}$ & 0 & 0 & 0 & 0 & 0 & .85 & 0 \\
\hline 24 & 0 & 0 & 0 & $0^{*}$ & $0^{*}$ & 0 & 0 & 0 & 0 & 0 & .19 & 0 \\
\hline 25 & 0 & 0 & 0 & $0^{*}$ & $0^{*}$ & 0 & 0 & 0 & 0 & 0 & .38 & 0 \\
\hline 26 & 0 & 0 & 0 & $0^{*}$ & $0^{*}$ & 0 & 0 & 0 & 0 & 0 & 0 & 0 \\
\hline 27 & 0 & 0 & 0 & $0^{*}$ & 0 & 0 & 0 & 0 & 0 & 0 & 0 & 0 \\
\hline 28 & 0 & 0 & 0 & $.10^{*}$ & 0 & 0 & 0 & 0 & 0 & 0 & 0 & 0 \\
\hline 29 & 0 & 0 & 0 & $0^{*}$ & 0 & 0 & 0 & 0 & 0 & 0 & .11 & 0 \\
\hline 30 & 0 & 0 & 0 & $0^{*}$ & ------ & 0 & 0 & 0 & 0 & 0 & 0 & 0 \\
\hline 31 & 0 & ----- & 0 & $0^{*}$ & ------ & 0 & ------ & 0 & ----- & 0 & 0 & ------ \\
\hline Total & 0 & 0 & 0 & 0.10 & 0 & 0 & 0 & 0 & 0 & 0 & 16.53 & 0 \\
\hline Mean & 0 & 0 & 0 & .003 & 0 & 0 & 0 & 0 & 0 & 0 & .53 & 0 \\
\hline Max & 0 & 0 & 0 & .10 & 0 & 0 & 0 & 0 & 0 & 0 & 15 & 0 \\
\hline Min & 0 & 0 & 0 & 0 & 0 & 0 & 0 & 0 & 0 & 0 & 0 & 0 \\
\hline Acre-Ft & 0 & 0 & 0 & .20 & 0 & 0 & 0 & 0 & 0 & 0 & 33 & 0 \\
\hline Wtr Year & 2008 & Total & 16.63 & Mean & & & hax & 15 & Min & 0 & Acre-Ft & 33 \\
\hline Cal Year & 2007 & Total & 0.37 & Mean & & 1 & hax & .32 & Min & 0 & Acre-Ft & 0.73 \\
\hline
\end{tabular}

*Estimate 


\section{E350 Rito de los Frijoles at Bandelier}

Location. Lat $35^{\circ}$ 46' 37", long $106^{\circ}$ 16' 09", Sec. 23, T. 18 N., R. 6 E., Ramon Vigil Grant, Sandoval County, in Bandelier National Monument.

Drainage Area. $18.35 \mathrm{mi}^{2}$.

Period of Record. July 1963 to September 1969; July 1977 to September 1982; May 1993 to September 1996; and October 1998 to September 30, 2008.

Revised Record. Drainage area (2006).

Gage. Data logger and concrete control. Elevation of gage is 6,046 ft above NGVD from GPS survey.

Remarks. Records are good except for winter period, which is fair. Small diversion from left bank approximately 1.0 mi upstream for irrigation of small orchard. The La Mesa fire, which occurred during mid-June 1977, burned approximately $40 \%$ of the forest covering this watershed. Legal location based on projected values.

Average Discharge. $10 \mathrm{yr}$ (1999-2008), $1.30 \mathrm{ft}^{3} / \mathrm{s}, 935$ acre-ft/yr.

Extremes for Period of Record. Maximum discharge, 3,030 $\mathrm{ft}^{3} / \mathrm{s}$, July 21, 1978, gage height $6.34 \mathrm{ft}$ site and datum then in use. Minimum daily discharge, $0 \mathrm{ft}^{3} / \mathrm{s}$, July $16-19$ and 26, 2003.

Extremes for Current Year. Peak discharge above base of $20 \mathrm{ft}^{3} / \mathrm{s}$ and maximum (*):

\begin{tabular}{|c|c|c|c|}
\hline Date & Time & Discharge $\left(\mathbf{f t}^{\mathbf{3}} / \mathbf{s}\right)$ & Gage Height (ft) \\
\hline December 1 & 0730 & 34 & 2.67 \\
\hline January 28 & 0530 & 352 & 4.04 \\
\hline August 4 & 1930 & $513^{*}$ & $4.34^{*}$ \\
\hline August 23 & 1700 & 134 & 3.35 \\
\hline August 24 & 2000 & 62 & 2.95 \\
\hline
\end{tabular}

Minimum daily discharge, $0.38 \mathrm{ft}^{3} / \mathrm{s}$, June 28 .

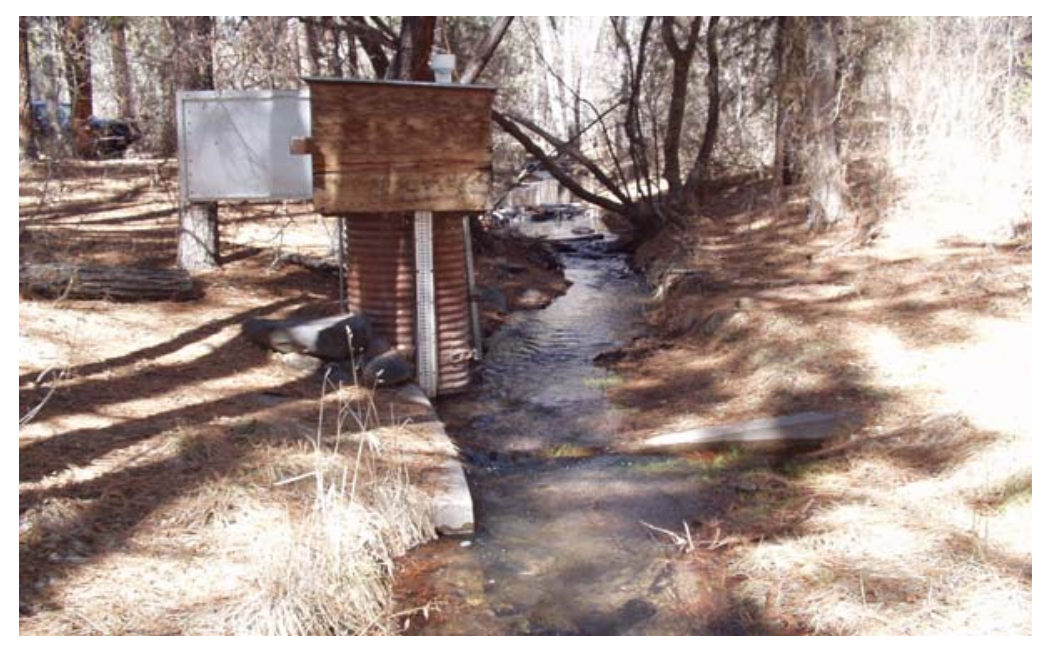




\section{E350 Rito de los Frijoles at Bandelier}

\section{Station Analysis}

\section{Water Year}

Equipment. Station is equipped with Sutron 8210 data logger (5-min. interval) with shaft encoder float system (5-min. interval) housed in a $4^{\prime} \times 4^{\prime}$ metal shelter over a $24^{\prime \prime}$ CMP stilling well on right bank. An outside staff is available for reference. Wading measurement are made $30^{\prime}-40^{\prime}$ upstream from gage. High-flow measurement can be made up from bridge upstream from gage 200' above gage.

Field Work. The station was visited 20 times to conduct discharge measurements and service the instrumentation. Field inspections for the gage are listed under site history files on the Hydstra database. Discharge measurements for the gage are listed under site gauging files on the Hydstra database.

Datum Correction. None from levels.

Gage-Height Record. The data logger referenced to the outside staff gave a complete and satisfactory record for the year except for the period of December 2-19 when ice affected gage height.

Rating. The channel is approximately $10^{\prime}$ wide and straight for about 150' upstream and downstream of gage. Low-water control consists of a concrete tapered notch with low point on right bank. The channel bed through this reach consists of gravel and cobbles and should be stable. Vegetation is grasses and fairly sparse.

Twelve discharge measurements (Nos. 187-198), with measurement No. 198 being a slope-area measurement of flood of August 4.

Rating No. 5 was developed based on the measurements and the slope-conveyance measurement made from peak of year. The shifts were distributed based on time. Rating No. 5 is considered good.

Discharge. Discharge was computed by applying gage height to Rating No. 5 through a shift based on time. Records estimated because gage heights were based on field notes and weather record.

Remarks. Records are good except for estimated daily discharges, which are fair. 
E350 Rito de los Frijoles at Bandalier

Daily Mean Discharge in Cubic Feet per Second

Water Year October 2007 to September 2008

\begin{tabular}{|c|c|c|c|c|c|c|c|c|c|c|c|c|}
\hline DAY & OCT & NOV & DEC & JAN & FEB & MAR & APR & MAY & JUN & JUL & AUG & SEP \\
\hline 1 & 1.1 & .84 & 14 & 1.0 & 5.1 & 5.4 & 12 & 3.4 & 1.5 & .45 & .40 & 2.7 \\
\hline 2 & 1.1 & .85 & $4.7^{*}$ & 1.1 & 4.9 & 5.7 & 12 & 3.4 & 1.5 & .52 & .37 & 1.9 \\
\hline 3 & .99 & .86 & $2.1^{*}$ & 1.1 & 4.5 & 5.7 & 11 & 3.4 & 1.5 & .46 & .83 & 1.9 \\
\hline 4 & .96 & .86 & $1.3^{*}$ & 1.2 & 4.4 & 5.5 & 11 & 3.3 & 1.4 & .45 & 30 & 1.8 \\
\hline 5 & .96 & .85 & $1.2^{\star}$ & 1.2 & 4.1 & 5.5 & 11 & 3.2 & 1.4 & .50 & 1.7 & 1.4 \\
\hline 6 & .91 & .86 & $1.0^{*}$ & 1.2 & 4.1 & 5.6 & 10 & 3.2 & 1.0 & .51 & 1.0 & 1.4 \\
\hline 7 & .89 & .84 & $.96^{\star}$ & 1.3 & 4.1 & 5.5 & 9.5 & 3.1 & .82 & .71 & .88 & 1.3 \\
\hline 8 & .92 & .80 & $.91^{*}$ & 1.3 & 4.3 & 5.3 & 9.5 & 3.1 & .83 & .72 & 1.3 & 1.2 \\
\hline 9 & .92 & .80 & $.95^{*}$ & 1.4 & 4.0 & 5.2 & 9.7 & 3.0 & .86 & .80 & 1.7 & 1.2 \\
\hline 10 & .90 & .82 & $.91^{*}$ & 1.4 & 3.8 & 5.3 & 9.3 & 2.9 & .88 & .58 & 1.5 & 1.1 \\
\hline 11 & .89 & .82 & $.93^{\star}$ & 1.4 & 3.8 & 5.3 & 8.4 & 2.9 & .89 & .53 & .72 & 1.1 \\
\hline 12 & .84 & .82 & $.94^{*}$ & 1.5 & 4.0 & 5.5 & 7.9 & 2.8 & .91 & .64 & .72 & 1.1 \\
\hline 13 & .82 & .83 & $.96^{*}$ & 1.5 & 4.1 & 5.7 & 7.4 & 2.8 & .93 & .53 & .72 & 1.0 \\
\hline 14 & .85 & .83 & $.97^{*}$ & 1.6 & 4.8 & 6.0 & 6.5 & 3.2 & .95 & .52 & .72 & .90 \\
\hline 15 & .85 & .85 & $.94^{*}$ & 1.5 & 5.4 & 6.0 & 5.6 & 3.0 & .96 & .56 & .79 & .85 \\
\hline 16 & .86 & .86 & $.93^{*}$ & 1.4 & 5.2 & 6.0 & 5.3 & 2.1 & 1.0 & .51 & .84 & .81 \\
\hline 17 & .86 & .86 & $.97^{\star}$ & 1.4 & 4.9 & 6.0 & 5.3 & 1.9 & 1.0 & .83 & .82 & .78 \\
\hline 18 & .85 & .86 & $.96^{\star}$ & 1.3 & 4.6 & 6.0 & 5.1 & 1.9 & 1.1 & .67 & .68 & .76 \\
\hline 19 & .87 & .85 & $.90^{\star}$ & 1.7 & 4.5 & 6.1 & 4.9 & 1.8 & 1.0 & .62 & .62 & .78 \\
\hline 20 & .86 & .84 & .84 & 2.3 & 4.4 & 6.1 & 4.7 & 1.7 & 1.1 & .53 & .61 & .76 \\
\hline 21 & .78 & .83 & .97 & 2.1 & 4.3 & 6.4 & 4.5 & 1.7 & 1.2 & .86 & .56 & .76 \\
\hline 22 & .85 & .85 & 1.1 & 1.6 & 4.3 & 6.8 & 4.4 & 1.8 & 1.2 & .78 & .48 & .76 \\
\hline 23 & .88 & .96 & 1.0 & 1.4 & 4.6 & 7.4 & 4.3 & 2.0 & .75 & .68 & 7.9 & .76 \\
\hline 24 & .86 & .93 & 1.1 & 1.4 & 4.6 & 7.9 & 4.1 & 1.9 & .44 & .53 & 5.7 & .76 \\
\hline 25 & .86 & .73 & 1.1 & 1.4 & 5.5 & 8.5 & 4.0 & 1.8 & .42 & .48 & 2.4 & .76 \\
\hline 26 & .85 & .94 & 1.1 & 1.3 & 5.1 & 9.3 & 3.9 & 1.7 & .39 & .67 & 1.6 & .76 \\
\hline 27 & .84 & .94 & 1.0 & 1.3 & 5.0 & 10 & 3.8 & 1.6 & .39 & .94 & 1.6 & .76 \\
\hline 28 & .84 & .97 & 1.0 & 57 & 5.0 & 11 & 3.8 & 1.7 & .38 & .71 & 1.7 & .76 \\
\hline 29 & .83 & .90 & .99 & 7.0 & 5.1 & 11 & 3.7 & 1.6 & .41 & .56 & 1.6 & .76 \\
\hline 30 & .82 & 1.3 & .94 & 5.9 & ------ & 12 & 3.5 & 1.5 & .43 & .49 & 1.5 & .76 \\
\hline 31 & .82 & ------ & .98 & 5.3 & ------ & 12 & ------ & 1.5 & ------ & .44 & 2.5 & ------ \\
\hline Total & 27.43 & 26.15 & 48.65 & 113.5 & 132.5 & 215.7 & 206.1 & 74.9 & 27.54 & 18.78 & 74.46 & 32.34 \\
\hline Mean & .88 & .87 & 1.57 & 3.66 & 4.57 & 6.96 & 6.87 & 2.42 & .92 & .61 & 2.40 & 1.08 \\
\hline Max & 1.1 & 1.3 & 14 & 57 & 5.5 & 12 & 12 & 3.4 & 1.5 & .94 & 30 & 2.7 \\
\hline Min & .78 & .73 & .84 & 1.0 & 3.8 & 5.2 & 3.5 & 1.5 & .38 & .44 & .37 & .76 \\
\hline Acre-Ft & 54 & 52 & 96 & 225 & 263 & 428 & 409 & 149 & 55 & 37 & 148 & 64 \\
\hline Wtr Year & 2008 & Total & 998.05 & & & 2.73 & Max & 57 & Min & .37 & Acre-Ft & 1980 \\
\hline Cal Year & 2007 & Total & 435.31 & & & 1.19 & Max & 17 & Min & .11 & Acre-Ft & 863 \\
\hline
\end{tabular}

*Estimate 
Location. Lat $35^{\circ} 51^{\prime} 1 "$ ", long $106^{\circ} 20^{\prime} 23^{\prime \prime}, 30 \mathrm{ft}$ upstream from the SWSC line crossing of Cañon de Valle in LANL TA-16.

Gage. Data logger with $90^{\circ}$ weir. Elevation of gage is $7,437 \mathrm{ft}$ above NGVD from survey.

Period of Record. October 1, 1996, to September 30, 2008.

Remarks. Water discharge records are good. This spring is in the Cañon de Valle drainage.

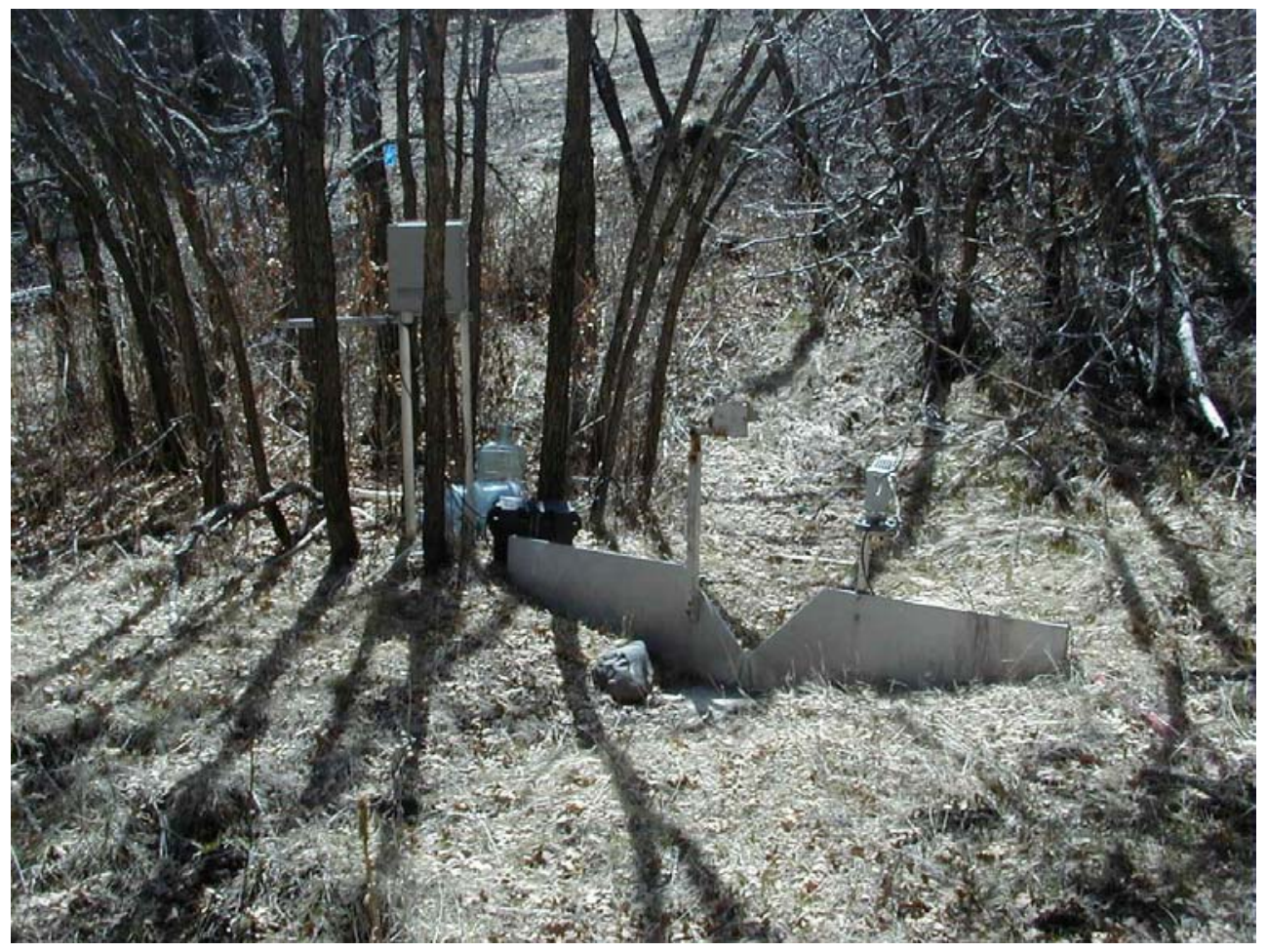


S001 SWSC Line Spring at TA-16

Daily Mean Discharge in Cubic Feet per Second

Water Year October 2007 to September 2008

\begin{tabular}{|c|c|c|c|c|c|c|c|c|c|c|c|c|}
\hline DAY & OCT & NOV & DEC & JAN & FEB & MAR & APR & MAY & JUN & JUL & AUG & SEP \\
\hline 1 & 0 & 0 & 0 & 0 & 0 & 0 & 0 & 0 & 0 & 0 & 0 & 0 \\
\hline 2 & 0 & 0 & 0 & 0 & 0 & 0 & 0 & 0 & 0 & .25 & 0 & 0 \\
\hline 3 & 0 & 0 & 0 & 0 & 0 & 0 & 0 & 0 & 0 & .28 & 0 & 0 \\
\hline 4 & 0 & 0 & 0 & 0 & 0 & 0 & 0 & 0 & 0 & 0 & 0 & 0 \\
\hline 5 & 0 & 0 & 0 & 0 & 0 & 0 & 0 & 0 & 0 & 0 & 0 & 0 \\
\hline 6 & 0 & 0 & 0 & 0 & 0 & 0 & 0 & 0 & 0 & 0 & 0 & 0 \\
\hline 7 & 0 & 0 & 0 & 0 & 0 & 0 & 0 & 0 & 0 & 0 & 0 & 0 \\
\hline 8 & 0 & 0 & 0 & 0 & 0 & 0 & 0 & 0 & 0 & 0 & 0 & 0 \\
\hline 9 & 0 & 0 & 0 & 0 & 0 & 0 & 0 & 0 & 0 & 0 & 0 & 0 \\
\hline 10 & 0 & 0 & 0 & 0 & 0 & 0 & 0 & 0 & .01 & 0 & 0 & .11 \\
\hline 11 & 0 & 0 & 0 & 0 & 0 & 0 & 0 & 0 & .02 & 0 & 0 & 0 \\
\hline 12 & 0 & 0 & 0 & $0^{*}$ & 0 & 0 & 0 & 0 & .03 & 0 & 0 & 0 \\
\hline 13 & 0 & 0 & 0 & $0^{*}$ & 0 & 0 & 0 & 0 & .04 & 0 & 0 & 0 \\
\hline 14 & 0 & 0 & 0 & $0^{*}$ & 0 & 0 & 0 & 0 & 0 & 0 & 0 & 0 \\
\hline 15 & 0 & 0 & 0 & $0^{*}$ & 0 & 0 & .01 & 0 & 0 & 0 & 0 & 0 \\
\hline 16 & 0 & 0 & 0 & $0^{*}$ & .01 & .01 & 0 & 0 & 0 & 0 & 0 & 0 \\
\hline 17 & 0 & 0 & 0 & $0^{*}$ & 0 & .01 & .01 & 0 & 0 & 0 & 0 & 0 \\
\hline 18 & 0 & 0 & 0 & 0 & 0 & .01 & 0 & 0 & 0 & 0 & 0 & 0 \\
\hline 19 & 0 & 0 & 0 & 0 & 0 & .01 & 0 & 0 & 0 & 0 & 0 & 0 \\
\hline 20 & 0 & 0 & 0 & 0 & 0 & 0 & 0 & 0 & 0 & 0 & 0 & 0 \\
\hline 21 & 0 & 0 & 0 & 0 & 0 & 0 & 0 & 0 & 0 & 0 & 0 & 0 \\
\hline 22 & 0 & 0 & 0 & 0 & 0 & 0 & 0 & 0 & 0 & 0 & 0 & 0 \\
\hline 23 & 0 & 0 & 0 & 0 & 0 & 0 & 0 & 0 & 0 & 0 & 0 & 0 \\
\hline 24 & 0 & 0 & 0 & 0 & 0 & 0 & 0 & 0 & 0 & 0 & 0 & 0 \\
\hline 25 & 0 & 0 & 0 & 0 & 0 & 0 & 0 & 0 & 0 & 0 & 0 & 0 \\
\hline 26 & 0 & 0 & 0 & 0 & 0 & 0 & 0 & 0 & 0 & 0 & 0 & 0 \\
\hline 27 & 0 & 0 & 0 & 0 & 0 & 0 & 0 & 0 & 0 & 0 & 0 & 0 \\
\hline 28 & 0 & 0 & 0 & 0 & 0 & 0 & 0 & 0 & 0 & 0 & 0 & 0 \\
\hline 29 & 0 & 0 & 0 & 0 & 0 & 0 & 0 & 0 & .12 & 0 & 0 & 0 \\
\hline 30 & 0 & 0 & 0 & 0 & ------ & 0 & 0 & 0 & 0 & 0 & 0 & 0 \\
\hline 31 & 0 & ----- & 0 & 0 & ------ & 0 & ----- & 0 & ----- & 0 & 0 & ------ \\
\hline Total & 0 & 0 & 0 & 0 & 0.01 & 0.04 & 0.02 & 0 & 0.22 & 0.53 & 0 & 0.11 \\
\hline Mean & 0 & 0 & 0 & 0 & 0 & .001 & .001 & 0 & .007 & .017 & 0 & .004 \\
\hline Max & 0 & 0 & 0 & 0 & .01 & .01 & .01 & 0 & .12 & .28 & 0 & .11 \\
\hline Min & 0 & 0 & 0 & 0 & 0 & 0 & 0 & 0 & 0 & 0 & 0 & 0 \\
\hline Acre-Ft & 0 & 0 & 0 & 0 & .02 & .08 & .04 & 0 & .44 & 1.1 & 0 & .22 \\
\hline Wtr Year & 2008 & Total & 0.93 & Mean & & 03 & Max & .28 & Min & 0 & Acre-Ft & 1.8 \\
\hline Cal Year & 2007 & Total & 37.29 & Mean & & 10 & Max & 1.5 & Min & 0 & Acre-Ft & 74 \\
\hline
\end{tabular}

*Estimate 


\section{S002 Burn Ground Spring at TA-16}

Location. Lat $35^{\circ} 50^{\prime} 58^{\prime \prime}$, long $106^{\circ} 20^{\prime} 17^{\prime \prime}, 450 \mathrm{ft}$ downstream from the SWSC line crossing of Cañon de Valle in LANL TA-16.

Gage. Data logger with $90^{\circ}$ weir. Elevation of gage is $7,420 \mathrm{ft}$ above NGVD from survey.

Period of Record. October 1, 1996, to September 30, 2008.

Remarks. Water discharge records are fair. This spring is in the Cañon de Valle drainage.

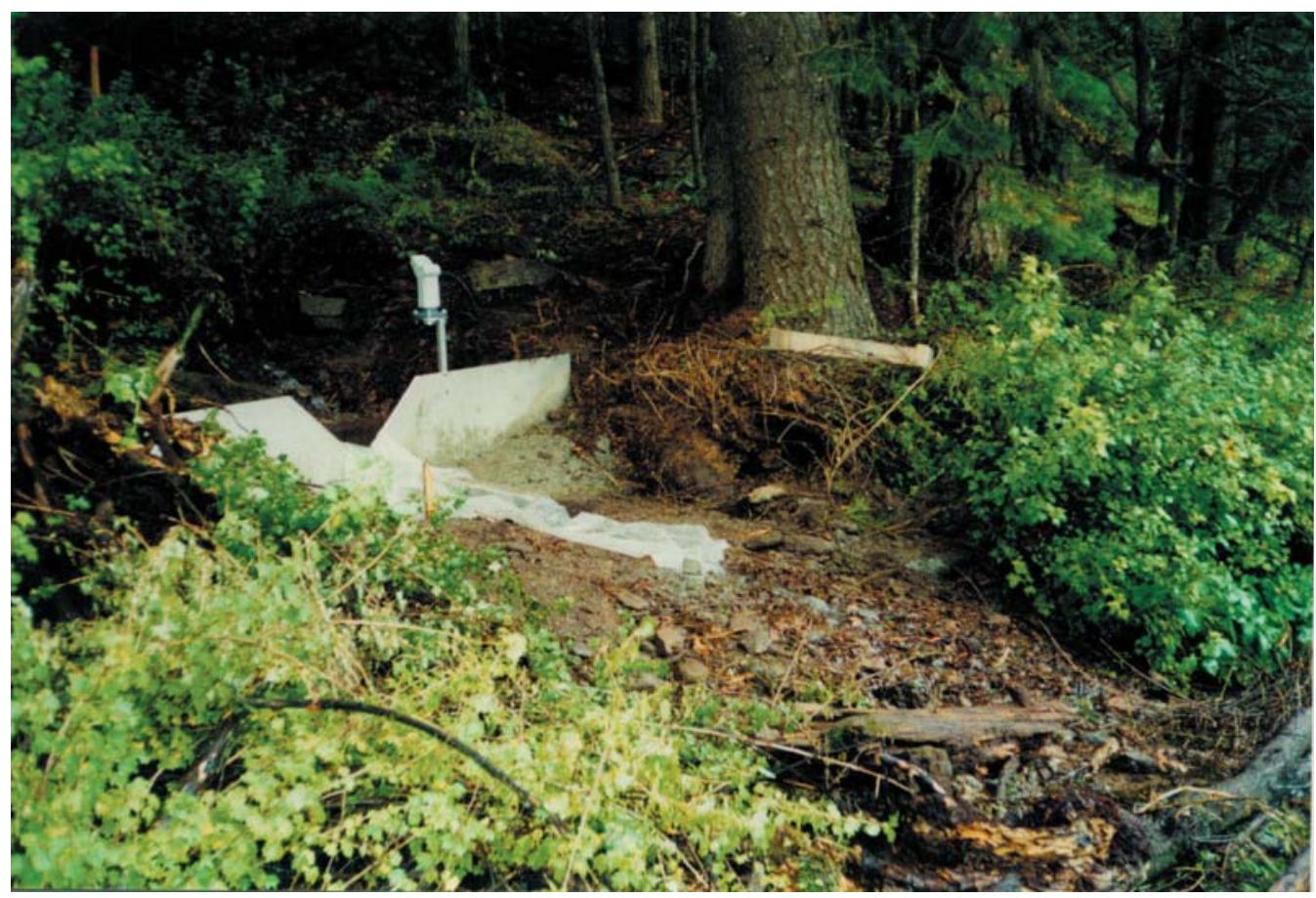


S002 Burn Ground Spring at TA-16

Daily Mean Discharge in Cubic Feet per Second

Water Year October 2007 to September 2008

\begin{tabular}{|c|c|c|c|c|c|c|c|c|c|c|c|c|}
\hline DAY & OCT & NOV & DEC & JAN & FEB & MAR & APR & MAY & JUN & JUL & AUG & SEP \\
\hline 1 & $.01^{*}$ & .01 & .01 & .01 & .01 & .02 & .02 & $.01^{*}$ & .01 & .01 & 0 & .01 \\
\hline 2 & $.01^{*}$ & .01 & .01 & .01 & .01 & .02 & .02 & $.01^{*}$ & .01 & .01 & .01 & .01 \\
\hline 3 & $.01^{*}$ & .01 & .01 & .01 & .01 & .02 & .02 & $.01^{*}$ & .01 & .01 & 0 & .01 \\
\hline 4 & $.01^{*}$ & .01 & .01 & .01 & .02 & .02 & .02 & $.01^{*}$ & .01 & .01 & .01 & .01 \\
\hline 5 & .01 & .01 & .01 & .01 & .02 & .02 & .02 & $.01^{*}$ & .01 & .01 & .01 & .01 \\
\hline 6 & .01 & .01 & .01 & .01 & .01 & .02 & .02 & $.01^{*}$ & .01 & .01 & .01 & .01 \\
\hline 7 & .01 & .01 & .01 & .01 & .01 & .02 & .02 & $.01^{*}$ & .02 & .01 & .01 & .01 \\
\hline 8 & .01 & .01 & .01 & .01 & .01 & .02 & .02 & $.01^{*}$ & .01 & .01 & .01 & .01 \\
\hline 9 & .01 & .01 & .02 & .01 & .01 & .02 & .02 & $.01^{*}$ & .01 & .01 & .01 & .01 \\
\hline 10 & .01 & .01 & .02 & .01 & .01 & .02 & .02 & $.01^{*}$ & .01 & .01 & .01 & .01 \\
\hline 11 & .01 & .01 & .02 & .01 & .01 & .02 & .02 & $.01^{*}$ & .01 & .01 & .01 & .01 \\
\hline 12 & .01 & .01 & .02 & .01 & .01 & .02 & .02 & $.01^{*}$ & .01 & .01 & .01 & .01 \\
\hline 13 & .01 & .01 & .02 & .01 & .01 & .03 & .02 & $.01^{\star}$ & .01 & .01 & .01 & .01 \\
\hline 14 & .01 & .01 & .02 & .01 & .01 & .02 & .02 & $.01^{*}$ & .01 & .01 & .01 & .01 \\
\hline 15 & .01 & .01 & .01 & .01 & .01 & .03 & .02 & $.01^{*}$ & .01 & .01 & .01 & .01 \\
\hline 16 & .01 & .01 & .01 & .01 & .01 & .02 & .02 & $.01^{*}$ & .01 & .01 & .01 & .01 \\
\hline 17 & .01 & .01 & .01 & .01 & .01 & .02 & .02 & $.01^{\star}$ & .01 & .01 & .01 & .01 \\
\hline 18 & .01 & .01 & .01 & .01 & .01 & .02 & $.01^{*}$ & $.01^{*}$ & .01 & .01 & .01 & .01 \\
\hline 19 & .01 & .01 & .01 & .01 & .01 & .02 & $.01^{*}$ & $.01^{*}$ & .01 & .01 & .01 & .01 \\
\hline 20 & .01 & .01 & .01 & .01 & .01 & .02 & $.01^{*}$ & $.01^{*}$ & .01 & .01 & .01 & .01 \\
\hline 21 & .01 & .01 & .01 & .01 & .01 & .02 & $.01^{*}$ & $.01^{*}$ & .01 & .01 & .01 & .01 \\
\hline 22 & .01 & .01 & .01 & .01 & .01 & .02 & $.01^{*}$ & $.01^{*}$ & .01 & .01 & .01 & .01 \\
\hline 23 & .01 & 0 & .01 & .01 & .02 & .02 & $.01^{*}$ & $.01^{*}$ & .01 & .01 & .01 & .01 \\
\hline 24 & .01 & 0 & .01 & .01 & .02 & .02 & $.01^{*}$ & $.01^{*}$ & .01 & .01 & .01 & .01 \\
\hline 25 & .01 & .01 & .01 & .01 & .02 & .02 & $.01^{*}$ & $.01^{*}$ & .01 & .01 & .01 & .01 \\
\hline 26 & .01 & 0 & .01 & .01 & .02 & .02 & $.01^{*}$ & $.01^{*}$ & .01 & .01 & .01 & .01 \\
\hline 27 & .01 & .01 & .01 & .01 & .02 & .02 & $.01^{*}$ & $.01^{*}$ & .01 & .01 & .01 & .01 \\
\hline 28 & .01 & .01 & .01 & .01 & .02 & .02 & $.01^{*}$ & .01 & .01 & .01 & .01 & .01 \\
\hline 29 & .01 & 0 & .01 & .01 & .02 & .02 & $.01^{*}$ & .01 & .01 & .01 & .01 & .01 \\
\hline 30 & .01 & .01 & .01 & .01 & ----- & .02 & $.01^{*}$ & .01 & .01 & .01 & .01 & 0 \\
\hline 31 & .01 & ------ & .01 & .01 & ----- & .02 & ----- & .01 & ----- & .01 & .01 & ----- \\
\hline Total & 0.31 & 0.26 & 0.37 & 0.31 & 0.38 & 0.64 & 0.47 & 0.31 & 0.31 & 0.31 & 0.29 & 0.29 \\
\hline Mean & .010 & .009 & .012 & .010 & .013 & .021 & .016 & .010 & .010 & .010 & .009 & .010 \\
\hline Max & .01 & .01 & .02 & .01 & .02 & .03 & .02 & .01 & .02 & .01 & .01 & .01 \\
\hline Min & .01 & 0 & .01 & .01 & .01 & .02 & .01 & .01 & .01 & .01 & 0 & 0 \\
\hline Acre-Ft & .61 & .52 & .73 & .61 & .75 & 1.3 & .93 & .61 & .61 & .61 & .58 & .58 \\
\hline Wtr Year & 2008 & Total & 4.25 & Mean & & 12 & Max & .03 & Min & 0 & Acre-Ft & 8.4 \\
\hline Cal Year & 2007 & Total & 2.33 & Mean & & 06 & Max & .03 & Min & 0 & Acre-Ft & 4.6 \\
\hline
\end{tabular}

*Estimate 


\section{S003 Martin Spring at TA-16}

Location. Lat $35^{\circ} 50^{\prime} 32^{\prime \prime}$, long $106^{\circ} 20^{\prime} 11^{\prime \prime}, 0.25$ mi south of Building 344 at LANL TA-16.

Gage. Data logger with $90^{\circ}$ weir. Elevation of gage is 7,429 ft above NGVD from survey.

Period of Record. October 1, 1996, to September 30, 2008.

Remarks. Water discharge records are good. This spring is in the Water Canyon drainage.

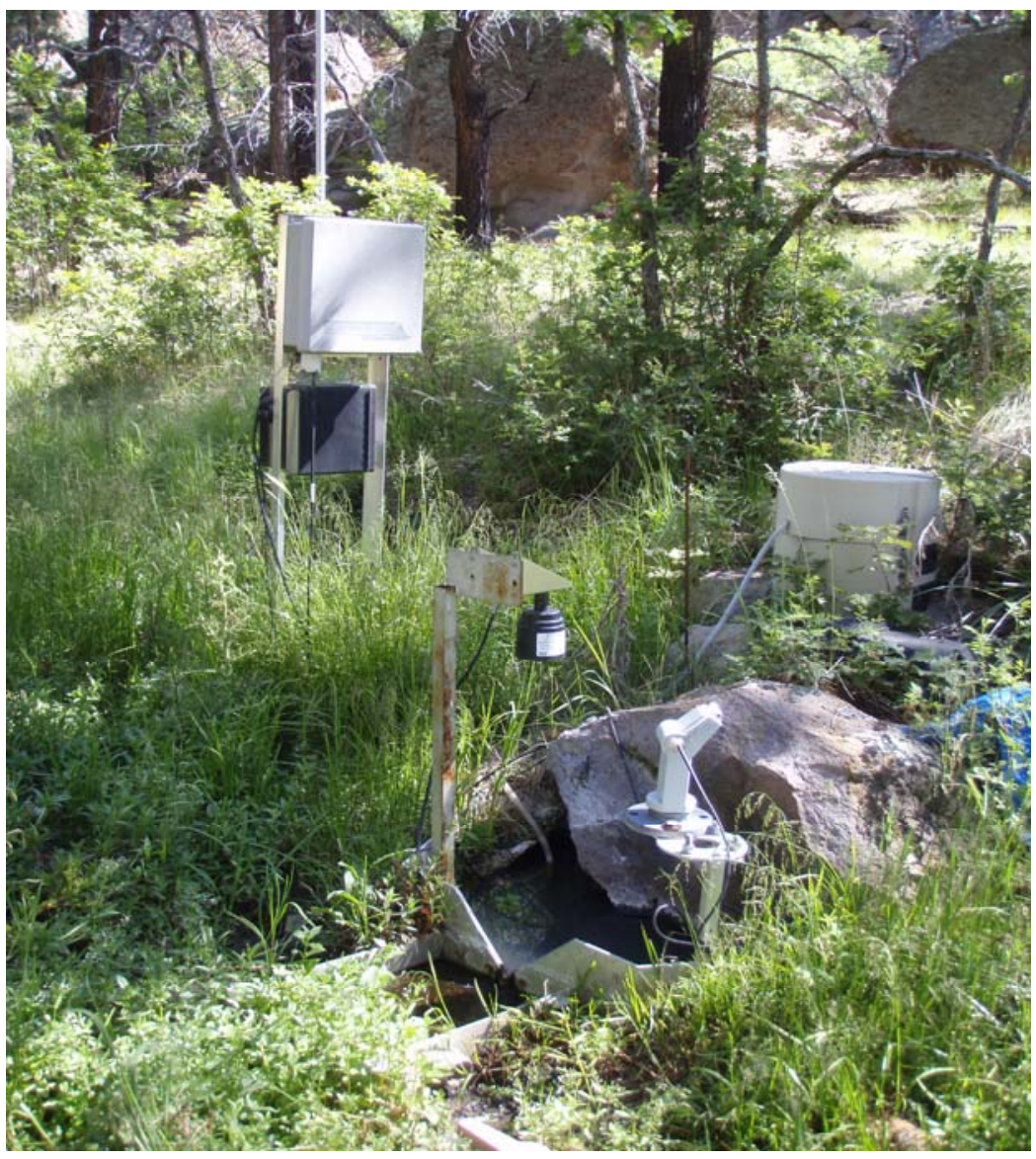


S003 Martin Spring at TA-16

Daily Mean Discharge in Cubic Feet per Second

Water Year October 2007 to September 2008

\begin{tabular}{|c|c|c|c|c|c|c|c|c|c|c|c|c|}
\hline DAY & OCT & NOV & DEC & JAN & FEB & MAR & APR & MAY & JUN & JUL & AUG & SEP \\
\hline 1 & 0 & 0 & 0 & 0 & 0 & 0 & 0 & 0 & 0 & 0 & 0 & 0 \\
\hline 2 & 0 & 0 & 0 & 0 & 0 & 0 & 0 & 0 & 0 & 0 & 0 & 0 \\
\hline 3 & 0 & 0 & 0 & 0 & 0 & 0 & 0 & 0 & 0 & 0 & 0 & 0 \\
\hline 4 & 0 & 0 & 0 & 0 & 0 & 0 & 0 & 0 & 0 & 0 & 0 & 0 \\
\hline 5 & 0 & 0 & 0 & 0 & 0 & 0 & 0 & 0 & 0 & 0 & 0 & 0 \\
\hline 6 & 0 & 0 & 0 & 0 & 0 & 0 & 0 & 0 & 0 & 0 & 0 & .01 \\
\hline 7 & 0 & 0 & 0 & 0 & 0 & 0 & 0 & 0 & 0 & 0 & 0 & .01 \\
\hline 8 & 0 & 0 & 0 & 0 & 0 & 0 & 0 & 0 & 0 & 0 & .01 & .01 \\
\hline 9 & 0 & 0 & 0 & 0 & 0 & 0 & 0 & 0 & 0 & $0^{*}$ & .01 & .01 \\
\hline 10 & 0 & 0 & 0 & 0 & 0 & 0 & 0 & 0 & 0 & $0^{*}$ & .01 & .01 \\
\hline 11 & 0 & 0 & 0 & 0 & 0 & 0 & 0 & 0 & 0 & $0^{*}$ & .01 & .01 \\
\hline 12 & 0 & 0 & 0 & 0 & 0 & 0 & 0 & 0 & 0 & 0 & .01 & .01 \\
\hline 13 & 0 & 0 & 0 & 0 & 0 & 0 & 0 & 0 & 0 & 0 & .01 & 0 \\
\hline 14 & 0 & 0 & 0 & 0 & 0 & 0 & 0 & 0 & 0 & 0 & .01 & 0 \\
\hline 15 & 0 & 0 & 0 & 0 & 0 & 0 & 0 & 0 & 0 & 0 & .01 & 0 \\
\hline 16 & 0 & 0 & 0 & 0 & 0 & 0 & 0 & 0 & 0 & 0 & .01 & 0 \\
\hline 17 & 0 & 0 & 0 & 0 & 0 & 0 & 0 & 0 & 0 & 0 & .01 & 0 \\
\hline 18 & 0 & 0 & 0 & 0 & 0 & 0 & 0 & 0 & 0 & 0 & .01 & .01 \\
\hline 19 & 0 & 0 & 0 & 0 & 0 & 0 & 0 & 0 & 0 & 0 & 0 & 0 \\
\hline 20 & 0 & 0 & 0 & 0 & 0 & 0 & 0 & 0 & 0 & 0 & 0 & 0 \\
\hline 21 & 0 & 0 & 0 & 0 & 0 & 0 & 0 & 0 & 0 & 0 & 0 & .01 \\
\hline 22 & 0 & 0 & 0 & 0 & 0 & 0 & 0 & 0 & 0 & 0 & 0 & 0 \\
\hline 23 & 0 & 0 & 0 & 0 & 0 & 0 & 0 & 0 & 0 & 0 & 0 & 0 \\
\hline 24 & 0 & 0 & 0 & 0 & 0 & 0 & 0 & 0 & 0 & 0 & .01 & .01 \\
\hline 25 & 0 & 0 & 0 & 0 & 0 & 0 & 0 & 0 & 0 & 0 & 0 & .01 \\
\hline 26 & 0 & 0 & 0 & 0 & 0 & 0 & 0 & 0 & 0 & 0 & 0 & 0 \\
\hline 27 & 0 & 0 & 0 & 0 & 0 & 0 & 0 & 0 & 0 & 0 & 0 & .01 \\
\hline 28 & 0 & 0 & 0 & 0 & 0 & 0 & 0 & 0 & 0 & 0 & 0 & .01 \\
\hline 29 & 0 & 0 & 0 & 0 & 0 & 0 & 0 & 0 & 0 & 0 & 0 & .01 \\
\hline 30 & 0 & 0 & 0 & 0 & ------ & 0 & 0 & 0 & 0 & 0 & 0 & .01 \\
\hline 31 & 0 & ----- & 0 & 0 & ------ & 0 & ------ & 0 & ----- & 0 & 0 & ------ \\
\hline Total & 0 & 0 & 0 & 0 & 0 & 0 & 0 & 0 & 0 & 0 & 0.12 & 0.15 \\
\hline Mean & 0 & 0 & 0 & 0 & 0 & 0 & 0 & 0 & 0 & 0 & .004 & .005 \\
\hline Max & 0 & 0 & 0 & 0 & 0 & 0 & 0 & 0 & 0 & 0 & .01 & .01 \\
\hline Min & 0 & 0 & 0 & 0 & 0 & 0 & 0 & 0 & 0 & 0 & 0 & 0 \\
\hline Acre-Ft & 0 & 0 & 0 & 0 & 0 & 0 & 0 & 0 & 0 & 0 & .24 & .30 \\
\hline Wtr Year & 2008 & Total & 0.27 & Mean & & b1 & hax & .01 & Min & 0 & Acre-Ft & 0.54 \\
\hline Cal Year & 2007 & Total & 0.12 & Mean & & 0 & hax & .02 & Min & 0 & Acre-Ft & 0.24 \\
\hline
\end{tabular}




\section{Gage Stations Omitted from this Publication}

For existing stations that were omitted from this publication, information was extracted from existing raw or partially reduced data files using the following procedure.

All field-collected electronic data are transferred into a commercial program referred to as "HYDSTRA". These data were accessed via the "Hydstra Data Manager's Workbench." "Raw Level" and, where available, discharge files were evaluated for relative change to the recorded stage. If visually obvious, then it was considered to be a day where some stream flow was recorded. The highest relative change was assumed to be the peak for the water year. Documented missing data or gaps in station's electronic record were tallied to estimate the days without available data. A number of these stations have been recently upgraded with equipment that should reduce the gaps in station data and provide a better record for future publication.

\begin{tabular}{|c|c|c|c|c|}
\hline Station & $\begin{array}{l}\text { Estimated } \\
\text { days with } \\
\text { flow }\end{array}$ & $\begin{array}{l}\text { Estimated } \\
\text { date of } \\
\text { peak flow }\end{array}$ & $\begin{array}{l}\text { Gap in } \\
\text { record } \\
\text { (days) }\end{array}$ & Comments \\
\hline E049 (LA Weir) & $\begin{array}{l}\text { (level } \\
\left.>2.5^{\prime}\right) 29\end{array}$ & $9 / 06$ & 0 & $\begin{array}{l}\text { Raw Stage (i.e., water level } \\
\text { behind low head weir) }\end{array}$ \\
\hline $\begin{array}{l}\text { E070 (Bayo Canyon at } \\
\text { Pueblo Canyon) }\end{array}$ & & & & Rating curve in development \\
\hline $\begin{array}{l}\text { E089 (Guaje Canyon } \\
\text { above Mouth of } \\
\text { Rendija Canyon) }\end{array}$ & 2 & $9 / 16$ & 0 & $\begin{array}{l}\text { Will publish in WY } 200 \text { ? To } \\
\text { include previous water years }\end{array}$ \\
\hline $\begin{array}{l}\text { E099 (Guaje Canyon } \\
\text { below SR 502) }\end{array}$ & 5 & $8 / 17$ & 0 & Rating curve in development \\
\hline $\begin{array}{l}\text { E124 (Sandia Canyon } \\
\text { Truck Route) }\end{array}$ & 40 & $11 / 25$ & 0 & $\begin{array}{l}\text { Record flow above } 0.62 \text { gage } \\
\text { height. No rating curve }\end{array}$ \\
\hline $\begin{array}{l}\text { E2455 Pajarito above } \\
\text { Three Mile) }\end{array}$ & 27 & $8 / 10$ & 0 & Rating curve in development \\
\hline $\begin{array}{l}\text { E2525 (Water above S- } \\
\text { Site Canyon) }\end{array}$ & 20 & $8 / 29$ & 0 & Rating curve in development \\
\hline E2624 (Phermex) & & & & New station, partial year. \\
\hline $\begin{array}{l}\text { E266 (Potrillo Canyon } \\
\text { above Discharge Sink) }\end{array}$ & 1 & $01 / 28$ & 53 & Rating curve in development \\
\hline E2733(TA-39-57) & 1 & $8 / 4$ & 0 & Rating curve in development \\
\hline E2737 (TA-39-6) & 3 & $8 / 4$ & 12 & Rating curve in development \\
\hline $\begin{array}{l}\text { E338 (Chaquehui } \\
\text { Canyon South Site) }\end{array}$ & 1 & $1 / 28$ & 16 & Rating curve in development \\
\hline $\begin{array}{l}\text { E340 (Chaquehui } \\
\text { Canyon Main Site) }\end{array}$ & 2 & $8 / 4$ & 8 & Rating curve in development \\
\hline
\end{tabular}




\section{Monthly Precipitation (inches) \\ Los Alamos National Laboratory Meteorological Stations \\ Water Year 2007 (October 2007-September 2008)}

Data Source: LANL Weather Machine; for further documentation and information, http://weather.lanl.gov

$\begin{array}{lrrrrrr}\text { Month } & \text { TA-6 } & \text { TA-49 } & \text { TA-53 } & \text { TA-54 } & \text { NCOM }^{\mathbf{1}} & \text { PJMT }^{\mathbf{2}} \\ \text { October } & 0.32 & 0.28 & 0.23 & 0.22 & 0.37 & 0.16 \\ \text { November } & 1.61 & 2.2 & 1.29 & 1.42 & 1.62 & 0.12 \\ \text { December } & 2.74 & 2.68 & 2.29 & 2.3 & 3.12 & 1.38 \\ \text { January } & 1.39 & 1.43 & 1.19 & 1.02 & 1.75 & 0.68 \\ \text { February } & 1.11 & 0.9 & 0.98 & 0.91 & 1.3 & 0.48 \\ \text { March } & 0.46 & 0.71 & 0.53 & 0.88 & 0.31 & 0.08 \\ \text { April } & 0.13 & 0.14 & 0.13 & 0.16 & 0.24 & 0.06 \\ \text { May } & 1.66 & 1.03 & 0.86 & 0.84 & 1.73 & 0.71 \\ \text { June } & 0.04 & 0.03 & 0.13 & 0.04 & 0.31 & 0.1 \\ \text { July } & 2.59 & 2.63 & 1.22 & 1.84 & 2.07 & 3.18 \\ \text { August } & 6.01 & 7.76 & 3.96 & 2.01 & 5.33 & 3.25 \\ \text { September } & 0.33 & 0.36 & 0.26 & 0.54 & 0.34 & 0.32 \\ \text { Total } & \mathbf{1 8 . 3 9} & \mathbf{2 0 . 1 5} & \mathbf{1 3 . 0 7} & \mathbf{1 2 . 1 8} & \mathbf{1 8 . 4 9} & \mathbf{1 0 . 5 2}\end{array}$

${ }^{\mathbf{1}}$ North Community

${ }^{2}$ Pajarito Mountain 
This page left blank intentionally. 
This page left blank intentionally. 
This page left blank intentionally. 
This report has been reproduced directly from the best available copy. It is available electronically on the Web (http://www.doe.gov/bridge).

Copies are available for sale to U.S. Department of Energy employees and contractors from:

Office of Scientific and Technical Information

P.O. Box 62

Oak Ridge, TN 37831

(865) 576-8401

Copies are available for sale to the public from: National Technical Information Service

U.S. Department of Commerce

5285 Port Royal Road

Springfield, VA 22161

(800) 553-6847 
- Los Alamos NATIONAL LABORATORY

EST.1943 